Prepared for the U.S. Department of Energy

under Contract DE-AC05-76RL01830

\title{
Large-Scale Spray Releases: Initial Aerosol Test Results
}

PP Schonewill

PA Gauglitz

JR Bontha

RC Daniel

DE Kurath

HE Adkins, Jr.

JM Billing

CA Burns
JM Davis

CW Enderlin

CM Fischer

JJ Jenks

CD Lukins

PJ MacFarlan

JI Shutthanandam

DM Smith

December 2012

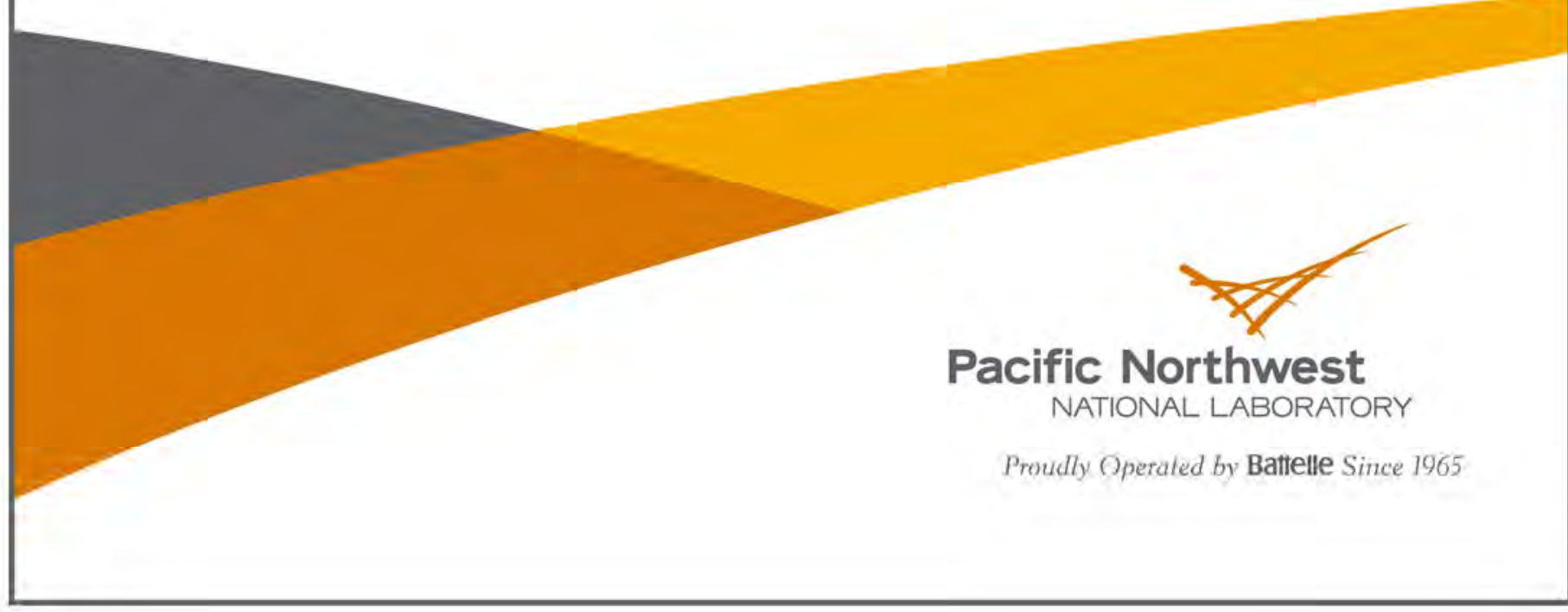




\title{
DISCLAIMER
}

This report was prepared as an account of work sponsored by an agency of the United States Government. Neither the United States Government nor any agency thereof, nor Battelle Memorial Institute, nor any of their employees, makes any warranty, express or implied, or assumes any legal liability or responsibility for the accuracy, completeness, or usefulness of any information, apparatus, product, or process disclosed, or represents that its use would not infringe privately owned rights. Reference herein to any specific commercial product, process, or service by trade name, trademark, manufacturer, or otherwise does not necessarily constitute or imply its endorsement, recommendation, or favoring by the United States Government or any agency thercof, or Battelle Memorial Institute. The views and opinions of authors expressed herein do not necessarily state or reflect those of the United States Government or any agency thereof.

\author{
PACIFIC NORTHWEST NATIONAI, I.ABORATORY \\ operated by \\ BAI'I'ELLE \\ for the \\ UNITED STATES DFPARTMENT OF FNFRGY \\ under Contract $D E-A C 05-76 R L 01830$ \\ Printed in the United States of America \\ Available to DOE and DOE contractors from the \\ Office of Scientific and Technical Information, \\ P.O. Box 62, Oak Ridge, TN 37831-0062; \\ ph: (865) 576-8401 \\ fax: (865) 576-5728 \\ email: reports@adonis.osti.gov
}

\begin{abstract}
Available to the public from the National Technical Information Service, U.S. Department of Commerce, 5285 Port Roy al Rd., Springfield, VA 22161 ph: $(800) 553-6847$ fax: $(703) 605-6900$ email: orders(a) ntis.fedworld.gov online ordering: http://www.ntis.gov/ordering.htm
\end{abstract}

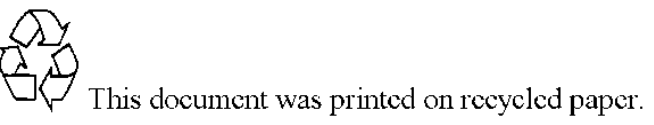

(9/2003) 


\title{
Large-Scale Spray Releases: Initial Aerosol Test Results
}

\author{
PP Schonewill \\ JM Davis \\ PA Gauglitz \\ JR Bontha \\ RC Daniel \\ CW Enderlin \\ CM Fischer \\ DE Kurath \\ JJ Jenks \\ HE Adkins, Jr. \\ CD Lukins \\ JM Billing \\ PJ MacFarlan \\ CA Burns \\ JI Shutthanandam \\ DM Smith
}

December 2012

Test Specification: None

Work Authorization: WA42AM01

Test Plan: TP-WTPSP-031, Rev. 0.2

Test Exceptions: None

Prepared for

the U.S. Department of Energy

under Contract DE-AC05-76RL01830

Pacific Northwest National Laboratory

Richland, Washington 99352 

WTP-RPT-217 Rev. 0

\section{Completeness of Testing}

This report describes the results of work and testing specified by Test Plan TP-WTPSP-031, Rev. 0.2. The work followed the quality assurance requirements outlined in the Test Plan. The descriptions provided in this report are an accurate account of both the conduct of the work and the data collected. Test plan results are reported. Also reported are any unusual or anomalous occurrences that are different from expected results. The test results and this report have been reviewed and verified.

\section{Approved:}

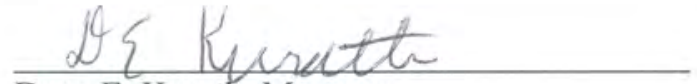

Dean E. Kurath, Manager

WTP R\&T Support Project

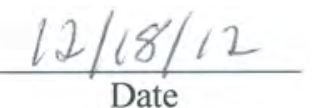





\section{Testing Summary}

One of the events postulated in the hazard analysis at the Waste Treatment and Immobilization Plant (WTP) and other U.S. Department of Energy (DOE) nuclear facilities is a breach in process piping that produces aerosols with droplet sizes in the respirable range. The current approach for predicting the size and concentration of aerosols produced in a spray leak involves extrapolating from correlations reported in the literature. These correlations are based on results obtained from small engineered spray nozzles using pure liquids with Newtonian fluid behavior. The narrow ranges of physical properties on which the correlations are based do not cover the wide range of slurries and viscous materials that will be processed in the WTP and across processing facilities in the DOE complex.

Two key technical areas were identified where testing results were needed to improve the technical basis by reducing the uncertainty due to extrapolating existing literature results. The first technical need was to quantify the role of slurry particles in small breaches where the slurry particles may plug and result in substantially reduced, or even negligible, respirable fraction formed by high-pressure sprays. The second technical need was to determine the aerosol droplet size distribution and volume from prototypic breaches and fluids, specifically including sprays from larger breaches with slurries where data from the literature are scarce.

To address these technical areas, small- and large-scale test stands were constructed and operated with simulants to determine aerosol release fractions and generation rates from a range of breach sizes and geometries. The properties of the simulants represented the range of properties expected in the WTP process streams and included water, sodium salt solutions, slurries containing boehmite or gibbsite, and a hazardous chemical simulant. The effect of antifoam agents was assessed with most of the simulants. Orifices included round holes and rectangular slots. The round holes ranged in size from 0.2 to $4.46 \mathrm{~mm}$. The slots ranged from (width $\times$ length) $0.3 \times 5$ to $2.74 \times 76.2 \mathrm{~mm}$. Most slots were oriented longitudinally along the pipe, but some were oriented circumferentially. In addition, a limited number of multi-hole test pieces were tested in an attempt to assess the impact of a more complex breach. Much of the testing was conducted at pressures of 200 and 380 psi, but some tests were conducted at 100 psi. Testing the largest postulated breaches was deemed impractical because of the much larger flow rates and equipment that would be required.

The purpose of this report is to present the experimental results and analyses for the aerosol measurements obtained in the large-scale test stand. The report includes a description of the simulants used and their properties, equipment and operations, data analysis methodology, and test results. The results of tests investigating the role of slurry particles in plugging of small breaches are reported in Mahoney et al. (2012a). The results of the aerosol measurements in the small-scale test stand are reported in Mahoney et al. (2012b).

\section{S.1 Objective}

Table S.1 provides a summary of each large-scale aerosol test objective, whether the objective was met, and a discussion of the test results. Other objectives identified in Test Plan TP-WTPSP-031, Rev. 0.2 apply to orifice plugging and small-scale aerosol testing. These objectives and the results of studies to address them are discussed by Mahoney et al. (2012a, 2012b). 
Table S.1. Summary of Test Objectives and Results

\begin{tabular}{|c|c|c|}
\hline Test Objective & $\begin{array}{c}\text { Objectiv } \\
\text { Met? }\end{array}$ & Discussion \\
\hline $\begin{array}{l}\text { Determine the size distribution of } \\
\text { aerosol droplets and the total droplet } \\
\text { volume concentration as a fraction of } \\
\text { the total spray volume for a range of } \\
\text { circular and rectangular breach } \\
\text { sizes (up to the largest postulated } \\
\text { breach in a 3-in. pipe) with water. }\end{array}$ & Yes & $\begin{array}{l}\text { Malvern Insitec-S instruments were used to measure the size } \\
\text { distribution and volume concentration of the aerosols for a } \\
\text { range of circular and rectangular orifices using water. The } \\
\text { circular orifices had target diameters that ranged from } 1 \text { to } \\
4.46 \mathrm{~mm} \text {. The rectangular slots had target dimensions that } \\
\text { ranged from } 0.5 \times 5 \text { to } 2.74 \times 76 \mathrm{~mm} \text { (width } \times \text { length). Most } \\
\text { slots were oriented longitudinally along the pipe but some were } \\
\text { oriented circumferentially. A limited number of multi-hole tests } \\
\text { were completed to assess the impact of a more complex breach. } \\
\text { In addition, the total spray volume was calculated using mass } \\
\text { measurements and time data recorded by a data acquisition } \\
\text { system. The results of the aerosol measurements are reported in } \\
\text { Chapter } 8 \text {. }\end{array}$ \\
\hline $\begin{array}{l}\text { Determine the size distribution of } \\
\text { aerosol droplets and the total droplet } \\
\text { volume concentration as a fraction of } \\
\text { the total spray volume for a range of } \\
\text { circular and rectangular breach } \\
\text { sizes (up to the largest postulated } \\
\text { breach in a 3-in. pipe) with } \\
\text { non-hazardous slurries(such as } \\
\text { gibbsite) and liquids (such as sodium } \\
\text { nitrate solutions). }\end{array}$ & Yes & $\begin{array}{l}\text { Malvern Insitec-S instruments were used to measure the size } \\
\text { distribution and volume concentration of the aerosol using a } \\
\text { boehmite in water slurry simulant (nominally } 8 \text { and } 20 \mathrm{wt} \% \text { ) } \\
\text { and sodium thiosulfate solutions. The total spray volume was } \\
\text { calculated using differential mass and flow measurements } \\
\text { recorded by a data acquisition system. The results of the } \\
\text { aerosol measurements are reported in Chapter } 8 .\end{array}$ \\
\hline
\end{tabular}

\section{S.2 Results and Performance Against Success Criteria}

The success criteria for achieving the large-scale aerosol test objectives are discussed in Table S.2.

Table S.2. Success Criteria for Large-Scale Plugging Tests

\begin{tabular}{|c|c|}
\hline Success Criteria & How Testing Did or Did Not Meet Success Criteria \\
\hline \multicolumn{2}{|l|}{ Objectives 2,3 } \\
\hline $\begin{array}{l}\text { Measure the droplet size distribution, total volume } \\
\text { concentration of droplets, and total volume sprayed } \\
\text { for each of the breaches and simulants tested. }\end{array}$ & $\begin{array}{l}\text { Met: Malvern Insitec-S instruments were used to measure } \\
\text { the size distribution and volume concentration of the aerosol. } \\
\text { The total spray volume was calculated using differential } \\
\text { mass and flow measurements. }\end{array}$ \\
\hline Measure the pressure and flow in the piping. & $\begin{array}{l}\text { Met: The pressure and flow in the piping were measured and } \\
\text { recorded with a data acquisition system. }\end{array}$ \\
\hline $\begin{array}{l}\text { Characterize the viscosity or rheology, particle size } \\
\text { distribution, bulk density, and surface tension of } \\
\text { each simulant tested. }\end{array}$ & $\begin{array}{l}\text { Met: The simulants tested were characterized prior to testing } \\
\text { and in many cases after testing (Chapter 4). }\end{array}$ \\
\hline $\begin{array}{l}\text { Calculate the test chamber volume from internal } \\
\text { dimensions. }\end{array}$ & $\begin{array}{l}\text { Met: The volume of the test chamber was calculated using } \\
\text { the AutoCAD software and as-built measurements. }\end{array}$ \\
\hline
\end{tabular}




\section{S.3 Quality Requirements}

The Pacific Northwest National Laboratory (PNNL) Quality Assurance (QA) Program is based on the requirements defined in the DOE Order 414.1D, Quality Assurance, and 10 CFR 830, Energy/Nuclear Safety Management, and Subpart A-Quality Assurance Requirements (a.k.a., the Quality Rule). PNNL has chosen to implement the following consensus standards in a graded approach:

- ASME NQA-1-2000, Quality Assurance Requirements for Nuclear Facility Applications, Part 1, Requirements for Quality Assurance Programs for Nuclear Facilities

- ASME NQA-1-2000, Part II, Subpart 2.7, Quality Assurance Requirements for Computer Software for Nuclear Facility Applications

- ASME NQA-1-2000, Part IV, Subpart 4.2, Graded Approach Application of Quality Assurance Requirements for Research and Development.

The procedures necessary to implement the requirements are documented through PNNL's "How Do I...?” (HDI) ${ }^{1}$ system.

The Waste Treatment Plant Support Project (WTPSP) implements an NQA-1-2000 Quality Assurance Program, graded on the approach presented in NQA-1-2000, Part IV, Subpart 4.2. The WTPSP Quality Assurance Manual (QA-WTPSP-0002) describes the technology life cycle stages under the WTPSP Quality Assurance Plan (QA-WTPSP-0001). The technology life cycle includes the progression of technology development, commercialization, and retirement in process phases of basic and applied research and development $(\mathrm{R} \& \mathrm{D})$, engineering and production and operation until process completion. The life cycle is characterized by flexible and informal QA activities in basic research, which becomes more structured and formalized through the applied R\&D stages.

The work described in this report has been completed under the QA technology level of Developmental Work. WTPSP addresses internal verification and validation activities by conducting an Independent Technical Review of the final data report in accordance with WTPSP's procedure QA-WTPSP-601, Document Preparation and Change. This review verifies that the reported results are traceable, that inferences and conclusions are soundly based, and that the reported work satisfies the test plan objectives.

\section{S.4 Simulant Use}

A broad range of simulants were developed and tested in the small-scale aerosol test stand (Mahoney et al. 2012b). However, because of the hazards and costs associated with some of the simulants developed, only a small subset of simulants was used in the large-scale test stand. Table S.3 presents a list of the simulants tested in the large-scale test stand, their physical and rheological property ranges, and their relevance to the WTP operations. The properties important to aerosol generation include particle size distribution (PSD), viscosity, Bingham parameters, bulk density, weight percent undissolved solids (UDS), and surface tension. The actual simulant properties are reported in Chapter 4.

\footnotetext{
${ }^{1} \mathrm{HDI}$ is a web-based system for managing the delivery of PNNL policies, requirements, and procedures.
} 
Table S.3. Target Simulants Tested in the Large-Scale Test Stand and the WTP Process Stream Categories

\begin{tabular}{|c|c|c|c|}
\hline Material/Description & Alias & Target Property Range & $\begin{array}{l}\text { WTP Process } \\
\text { Stream Categories }\end{array}$ \\
\hline Water & Water & $\begin{array}{l}\text { Viscosity } 1 \mathrm{mPa} \cdot \mathrm{s}(1 \mathrm{cP}) \\
\text { Density } 1000 \mathrm{~kg} / \mathrm{m}^{3} \\
\text { Surface tension } 73 \mathrm{mN} / \mathrm{m}\end{array}$ & - Ultrafilter permeate/treated \\
\hline $\begin{array}{l}\text { Solutions of water and } \\
\text { sodium thiosulfate }\end{array}$ & $\mathrm{Na}_{2} \mathrm{~S}_{2} \mathrm{O}_{3}$ & Viscosity $2.5 \mathrm{cP}$ & $\begin{array}{l}\text { - Cs ion exchange eluate } \\
\text { - Recycle streams }\end{array}$ \\
\hline $\begin{array}{l}\text { Small treated Hanford } \\
\text { waste PSD; boehmite } \\
\text { particulates in water }\end{array}$ & STR & $\begin{array}{l}\text { The PSDs of slurries were selected } \\
\text { to match small treated Hanford } \\
\text { slurries with } 8 \text { and } 20 \text { wt } \% \text { solids }\end{array}$ & Newtonian slurries \\
\hline
\end{tabular}

\section{S.5 Summary of Results}

The large-scale spray release test system included a large, reconfigurable enclosure (10- to 20-ft long) for investigating aerosol formation from breach sizes up to the largest postulated breach in a 3-in. pipe. This system included a large-scale recirculating flow loop for slurries and was capable of testing a range of non-hazardous slurries and liquid spray leaks up to $100 \mathrm{gpm}$. Higher spray flows up to 160 gpm were achieved, but needed to be conducted in a dead-end flow configuration to maintain adequate pressure. A wide variety of orifice sizes and geometries could be inserted into the test section of the flow loop using 3-in. pipe spools. The distance of the orifice from the splash wall was varied from 43 to $227 \mathrm{in}$. by inserting pipe extensions. Two large impeller-agitated vessels were used for simulant receipt, storage, and feed delivery with an operating capacity of approximately 600 gal. A third vessel was available for waste receipt. Aerosol measurements were obtained in real time, primarily using three Malvern Insitec-S instruments. A second aerosol instrument also was used, but the results from this instrument were used primarily to check the Insitec-S results. The aerosol instruments could be placed at most locations in the aerosol enclosure but were most commonly placed in the upper third of the chamber with one near each end of the enclosure and one near the center. Mixing fans placed near the bottom center of the chamber minimized inhomogeneities in the aerosol concentrations. Additional instruments provided real time measurements of flow rate, simulant density, flow loop pressure, and temperature that were recorded with a data acquisition system. Approximately 245 separate spray release tests were conducted including replicates.

The tests were conducted by first achieving the target flow rate and pressure in the flow loop, and then opening a spray release lever. The aerosol data were generally collected for $2 \mathrm{~min}$. Still images and video recordings of the sprays were collected separately from the aerosol tests. The feed vessel temperatures were maintained at $75 \pm 10^{\circ} \mathrm{F}$ to minimize any effects that may be caused by condensation or evaporation. Samples were collected from the feed vessel before and after testing each day when testing with water and after each test when testing with the boehmite slurry or sodium thiosulfate simulants. Selected samples were analyzed to determine the PSD, rheological parameters, bulk density, weight percent of UDS, and surface tension.

The experimental method consisted of measuring the rate of increase in the aerosol concentration in the closed chamber of known volume. Using a simple material balance, the rate of concentration increase gives the aerosol generation rate from a spray. Because the chamber is a closed system with no purge 
flow, the aerosol concentration is initially zero and builds up to a steady-state concentration at which point the generation of aerosol is equal to the aerosol losses.

A key component of the approach of using the increase in concentration is to have a concentration measurement for the chamber that is representative of the entire chamber. An advantage of using a chamber is that it allows for isolation of the spray, providing a safe testing platform for spraying simulants with chemical hazards. This methodology also allows testing to be performed in different sized chambers where a larger chamber could accommodate larger sprays but overall experimental and data analysis approach would be the same. Creating sprays inside a chamber also allows the spray to impact the walls of the chamber and generate aerosol droplets by splatter. This additional mechanism of droplet formation is typical of an actual spray, and adds to the total aerosol formation within the chamber. The approach used to measure the concentration increase in a closed chamber also allows the role of changing the orifice-to-wall distance to be determined. Because of the size and configuration of WTP piping, sprays could impact system components (e.g., walls, pipes, valves, etc.) at distances ranging from inches to hundreds of feet. In addition, because the same experimental method was used in both the small- and large-scale tests, results can be compared to one another and extrapolated to longer distances. For the reasons discussed above and because of the overall method flexibility, the primary method for determining aerosol generation rate was to measure the aerosol generation rate based on the initial rate of concentration increase in a closed chamber.

A two-part approach was used to analyze data collected during large-scale spray release testing. The first part used data from the process instruments to determine the average conditions during each test and calculate the spray leak flow rate. The flow rate was estimated either by a differential mass method or a differential flow rate method. In most cases, there is little difference in the results obtained by the two approaches; however, the differential mass method is more accurate for cases in which flow rate is small (e.g., small orifice area and/or low pressure). Therefore, the differential mass method was selected for use in calculating release fraction.

The second part used data from the aerosol instruments, namely the three Malvern Insitec-S instruments. The aerosol size distribution data were converted to a volume basis (ppmv) and corrected for laser transmission drift, and the three measured concentrations then were averaged. Both differential and cumulative concentration data were analyzed. The averaged data were aligned in time and then fit with an exponential model to determine the aerosol generation rate. The generation rate was divided by the spray leak flow rate to obtain estimates of the release fraction for the experiments.

A series of tests were conducted to identify the best equipment configuration and operating test conditions. These tests accomplished the following:

- Verified that target pressures were achieved and the spray leak flow rate estimates were consistent with expectations. In general the actual test pressures were very close to the target pressures and were off by no more than 11 psig.

- Demonstrated that the chamber mixing fans employed during the testing promoted mixing and did not lead to additional inhomogeneities in the chamber aerosol concentrations.

- Demonstrated that the Malvern Insitec-S aerosol instrument locations used in testing gave accurate representations of the chamber concentration by comparing to data obtained at other locations. 
- Multiple tests confirmed the Malvern aerosol results with a secondary aerosol instrument, the Process Particle Counter (PPC), with tests indicating agreement between the instruments to within a factor of 2. In many cases, the instruments appear to agree within the range of reproducibility for the Malvern Insitec-S ( 30 percent) as determined by replicate testing. Optimum aerosol instrument configurations also were determined and included: the distance between the laser and the detector in the Insitec-S, air purge flow rates (Insitec-S) and draw tube flow rates (PPC), and the data sampling and recording rate.

- Assessed the reproducibility of the tests and estimated an approximate test-to-test uncertainty. As a first approximation, the release fraction for any given test should be expected to have a minimum 95-percent confidence level of \pm 30 percent of the stated value at any particular aerosol droplet diameter. This uncertainty is primarily based on results using water, and may be greater with other fluids.

Once the equipment configuration and operating test conditions were established, comparisons between large-scale test data and WTP model predictions were made for several different parameters. This effort yielded the following conclusions:

- Orifice coefficients $\left(C_{D}\right)$ were determined and found to be consistent with the value of 0.62 used in the WTP model. The overall average orifice coefficient for all tests was determined to be 0.66 and 0.62 based on differential mass and flow rate measurements, respectively. Disregarding several outliers $\left(\mathrm{C}_{\mathrm{D}}<0.4\right.$ and $\mathrm{C}_{\mathrm{D}}>0.9$, which occurred in 11 of the 246 tests based on the mass measurements), the overall average orifice coefficients were 0.65 and 0.63 for the two methods.

- As pressure increases, the cumulative release fraction increases. This increase with pressure was shown to be greater than the rate of increase in the WTP model, especially at smaller droplet sizes.

- As orifice area increases, the cumulative release fraction decreases while the cumulative generation rate increases because of the increase in total spray flow with increasing orifice area. The rate of decrease in the release fraction test data, with increasing orifice area, is smaller than the decrease projected by the WTP model. The dependence on area was found to hold regardless of orifice shape (circular hole or slot or multiple adjacent holes) and slot orientation did not have an appreciable effect on the release fraction. Considering the uncertainty in the results, release fractions and generation rates from the test data approached the WTP model at an orifice area in the range of 30 to $100 \mathrm{~mm}^{2}$.

- As viscosity and density increased for a salt solution, the cumulative release fraction and generation rate varied by a slight amount that depended on droplet size. Considering the uncertainty in the test data, there was essentially no difference in the test results for water and the salt solution. The WTP model including both the density and viscosity changes predicted only slight differences between water and the salt solutions, and the data are consistent with this prediction. Not enough data was collected to determine the separate roles of viscosity or density.

- As the weight fraction of solids increases, the cumulative release fraction is unaffected for droplets $>10 \mu \mathrm{m}$. This is consistent with the WTP model, because it accounts for the presence of solids only by changes in the physical properties of the fluid. There was some deviation from water at droplet sizes $<10 \mu \mathrm{m}$, but differences below this droplet size are of minor concern for spray release accident analyses.

- As the distance from the splash wall increases, the cumulative release fraction increases. For the five different orifices and two spray pressures tested, the largest release fraction always occurred when the 
spray traveled the full length of the chamber. The WTP model does not consider the effect of obstructions such as walls which impacts the distance the spray travels.

- In-spray measurements are difficult to interpret as they are strong functions of position, pressure, orifice size, and Malvern analysis settings. They do, however, represent an upper bound on the release fraction for a particular spray in the absence of spatter on a nearby object and can be used as an estimate of the largest release fractions.

Overall, the results indicate a straightforward functionality of the cumulative release fraction on pressure and orifice area. Viscosity and the weight fraction of solids had a negligible effect. The configuration and geometry of the spray also is important, as evidenced by the splash wall distance and in-spray aerosol tests. In general, the WTP model has the correct qualitative behavior, but either does not have a quantitatively correct dependence on a quantity (pressure, orifice area) or ignores a quantity altogether (distance from a splash wall). The data indicate that the model may not be conservative for all sprays.

The comparison of release fraction results for the small and large test chambers, when the sprays traveled the full length of the chambers, showed the difference in release fraction varying from none to as much as fivefold for droplets between 10 and $100 \mu \mathrm{m}$ (large-scale tests gave lower release fractions for droplets below about $30 \mu \mathrm{m}$ and higher release fractions above this size). With only two chamber sizes, these results are insufficient for estimating the uncertainty in the extrapolations to larger chambers and spray distances. This is a significant finding of the testing, and demonstrates that the technical approach of using the transient droplet concentrations in closed chambers gives results that characterize the aerosol generation rate of the spray and that can be used to estimate the release fractions in larger chambers or facilities. The generally similar release fractions in the two different size chambers, when a spray traveled the entire length of the chamber, suggests a spray that spans the full length of an even larger chamber will be similar to results obtained in this study. Although uncertain, the testing results suggest that estimates for larger chambers and longer sprays may increase for droplets above about $30 \mu \mathrm{m}$ and decrease for droplets below $30 \mu \mathrm{m}$.

The large-scale test results show clear trends with increasing orifice area for both the release fraction and generation rate. The small-scale results do not show much of a trend with increasing orifice area, but they still generally agree with the large-scale results within the range of area that was tested. Overall, this comparison of small- and large-scale test results confirms the overall trend of increasing generation rate with increasing orifice area for both circular holes and slots. The results also show that the large-scale test results, which span a wider range of orifice areas than the small-scale tests, provide the best data for extrapolating to larger slots.

Assuming the WTP model uses the fluid properties of water for all the sprays, the combined extrapolations indicate that the cumulative release fractions from long-distance sprays from the largest postulated orifice of a Bingham plastic fluid at the upper rheological boundary are estimated to exceed the WTP model by factor of 2 to 4 for droplets that are $<10 \mu \mathrm{m}$. For larger droplets, the release fraction is expected to exceed the WTP model, but the magnitude is more uncertain. The most significant uncertainty in this estimated release fraction is due to the role of chamber size and an assumed $100-\mathrm{ft}$ spray distance. An upper bound release fraction from an in-spray PSD is slightly higher than the extrapolated large-scale chamber result for droplets $<10 \mu \mathrm{m}$, but the difference becomes progressively larger with increasing droplet size and the upper bound is as much as a factor of 50 above the WTP model 
for droplets $<100 \mu \mathrm{m}$. Additional testing will be needed to reduce the uncertainty in these estimates. Additional testing of non-Newtonian slurries sprayed through large orifices in the large-scale chamber also is suggested as a means of reducing the uncertainty.

A number of factors affect the overall uncertainty in the measured aerosol generation rates and release fractions. While a systematic quantitative evaluation of all the combined factors has not been performed, a qualitative overall uncertainty can be estimated, and the overall uncertainty in the reported release fractions and generation rates for the large-scale test chamber is probably about a factor of 2 to 3 (the reported results have not been adjusted to account for this uncertainty). The comparison of release fraction results for the small and large test chambers showed the difference in release fraction varying from none to as much as fivefold, which is important for understanding the uncertainty in extrapolations to larger chambers and spray distances. With test results for only two chamber sizes, not enough data are available to estimate the uncertainty in extrapolations to larger chambers.

\section{S.6 Discrepancies and Follow-on Tests}

The suggested follow-on tests are discussed below. No discrepancies associated with the large-scale testing were identified.

Initial testing to obtain aerosol release fraction and generation rates has recently been completed in small- and large-scale test stands and are discussed in this report and in the small-scale aerosol report (Mahoney et al. 2012b). These tests were conducted with simulants representing the expected WTP process stream properties over a range of orifice sizes and geometries and line pressures. The initial effort was directed at developing data to provide a technical basis for the WTP model predictions with the assumption that the model would be shown to be conservative. Because of the large size of the actual WTP equipment, it has been necessary to extrapolate the results from the smaller test stands to full scale. Since there is considerable uncertainty in the extrapolations, a number of follow-on tests and related investigations are proposed below.

Testing with the spray at different distances from the splash wall demonstrated that longer distance sprays yield a higher release fraction (unless the spray is very close to the splash wall, i.e., 1 in.). In-spray data has provided an upper bound for selected breaches, and although there is uncertainty in the in-spray data, some of the in-spray data exceeds the WTP model. Conducting spray release tests in a larger chamber would help reduce the uncertainty in the upper-bound spray releases. Data obtained with longer sprays also would reduce the uncertainty associated with extrapolation to longer sprays, which is the largest source of uncertainty associated with the extrapolations. Additional emphasis on in-jet measurements is warranted to provide a more direct measurement of the release fractions.

Testing with the chemical simulant in the small-scale chamber showed that these thicker slurries gave release fractions that are most likely to exceed values obtained from the WTP model, and are higher than expected. While the undissolved solids concentration exceeded the WTP concentration limit the rheological properties did not attain the upper end of the WTP rheological boundary because of simulant fabrication and testing difficulties. Additional testing with a chemical simulant that meets or slightly exceeds the rheological boundaries should be considered. 
While testing with the chemical simulant in the small-scale chamber showed that thicker slurries gave higher release fractions, large-scale testing demonstrated that the aerosol generation from the largest slots are the most likely to exceed results from the WTP model. Accordingly, there is a need to test slurries exhibiting a non-Newtonian rheology in large-slot sprays to compare this worst-case spray with the WTP model. In the large-scale system, clay slurries with rheological parameters spanning the WTP waste rheological boundaries should be considered. Testing of the clay slurries in the small-scale system will be needed to confirm similarity between the chemical simulant and the clay slurry. Testing with the chemical simulant in the large-scale test stand is precluded by the cost and hazards associated with the simulant. The large-scale system does not have the permits needed to handle hazardous materials.

The WTP is considering increasing the UDS limit from 20 to $27 \mathrm{wt} \%$. While the solids concentration during testing with the chemical simulant exceeded $27 \mathrm{wt} \%$ UDS, testing with gibbsite and boehmite slurries in water was conducted at an upper limit of $20 \mathrm{wt} \%$ UDS. Accordingly, some testing with a solids loading of $27 \mathrm{wt} \%$ for these slurries would provide a more complete set of data that would span the full range of expected waste properties.

The current testing used particles that have particle densities ranging from about 2.4 to $3.6 \mathrm{~g} / \mathrm{mL}$. Actual Hanford waste has particles that exceed this range (e.g., $\mathrm{PuO}_{2}$ ). Aerosol testing with a slurry simulant that contains a fraction of higher density particles is needed to provide test results for simulants that span the full range of expected waste properties.

While conducting additional tests, alternative lenses for the Malvern aerosol instrument capable of measuring a wider range of PSDs should be considered. The lenses used for the current work have an effective PSD range of 0.5 to $200 \mu \mathrm{m}$. This range was selected because it was focused around the PSD range of greatest interest to WTP (10 to $80 \mu \mathrm{m})$. This limited range does not capture the largest droplets and leads to an overestimate of the release fraction for in-spray measurements. With an increased interest in obtaining in-spray measurements, it is desirable to obtain different lenses with a PSD range of 2.5 to $2500 \mu \mathrm{m}$. This will allow the PSD measurements to more directly and accurately represent the release fractions.

While several efforts were made to validate and check the aerosol measurements obtained from the Malvern Insitec-S, there is still some residual uncertainty, especially in the concentration values. The Insitec-S typically is used primarily for particle sizing in process applications. For spray release testing, it was used to determine the aerosol concentration in addition to the PSD. Because use of the Insitec-S for aerosol concentration measurement is not the typical application, some caution with respect to the accuracy of the concentration result is advisable. Another uncertainty was introduced by the apparent presence of a bi-modal distribution with one of the peaks occurring below a particle size of $10 \mu \mathrm{m}$. The peak at the smaller particle sizes is not consistent with expected spray release aerosol generation, and appears to be dependent on the instrument settings and experimental conditions. Efforts undertaken to check the Insitec-S included weekly performance checks of the instruments with a reticle (i.e., a physical standard), comparison of the Insitec-S and the PPC measurements, and an evaluation of the solid-in-liquid dispersions on a similar Malvern instrument. While these efforts indicate that the Insitec-S results are reasonable, additional activities should be considered to increase confidence in the aerosol measurements.

Method validation tests in which a well characterized spray is introduced to the chamber should also be considered. These tests would apply the same measurement and analysis methods to sprays with 
known aerosol generation rate and size distribution to determine how well the estimates of aerosol generation rate match the expected values.

Considering the primary sources of uncertainty, the overall estimated uncertainty in the release fractions and generation rates for the large-scale test stand is on the order of a factor of 2 to 3 (the reported results have not been adjusted to account for this uncertainty). The primary sources of uncertainty are test-to-test variation, non-uniform concentrations in the chamber, and the aerosol measurements. Additional testing in an expanded large-scale chamber is suggested in order to better define these uncertainties and provide better extrapolations to full-scale conditions. These results should allow a better assessment of the available margin in the WTP Documented Safety Analysis. 


\section{Acknowledgments}

The authors would like to thank all the Pacific Northwest National Laboratory staff who assisted with this test program. The technical discussions and support from Lenna Mahoney and Jeremy Blanchard were invaluable. The careful and thorough technical review of this report by John Geeting was instrumental in shaping the document. We also thank Kirsten Meier for her QA support. The technical editing and formatting of this report by Cary Counts, Mike Parker, and Kathy Neiderhiser was greatly appreciated. Additionally, we owe many thanks for the technical review efforts of Rebecca Erikson, Jeromy Jenks, Justin Billing, Naveen Karri, Janani Shutthanandan, Susan Sande, Amanda Casella, Lanée Snow, and Gourihar Kulkarni.

We also sincerely appreciate the support, advice, and guidance from Mike Epstein (Fauske and Associates), Ameer Hassan (WTP Technical Lead), Susan Omberg-Carro (WTP), Andy Larson (WTP), Chris Harrington (DOE-WTP), Joel Fox (DOE-WTP), Ralph Crowe (Sludge Treatment Project K-Basin) and Bruce Zimmerman (Hanford Tank Farms).

Funding for this effort was provided by the U.S. Department of Energy's Hanford Waste Treatment and Immobilization Plant Project. 



\section{Acronyms and Abbreviations}

AFA

APEL

ASME

CFD

CSTR

CWS

DAS

DOE

DSA

FER

HDI

LAR

LAW

LRB

ORP

PEP

PLC

PNNL

PPC

PRV

PSD

QA

R\&D

RI

RPP-WTP

SAR

STP

STR

TAR

TDP

TS

UDS

VFD

WTP

WTPSP antifoam agent

Applied Process and Engineering Laboratory

American Society of Mechanical Engineers

computational fluid dynamics

continuously stirred tank reactor

Coal-Water Slurry

data acquisition system

U.S. Department of Energy

Documented Safety Analysis

Fe-rich (iron-rich)

"How Do I," the standards-based management system for PNNL

large as-received

high-volume low-activity waste

Laboratory Record Book

DOE Office of River Protection

Pretreatment Engineering Platform

programmable logic controller

Pacific Northwest National Laboratory

process particle counter

pressure relief valve

particle size distribution

Quality Assurance

research and development

refractive index

River Protection Project - Waste Treatment Plant

small as-received

Sludge Treatment Project

small treated

typical as-received

test data package

total solids

undissolved solid

variable frequency drive

Hanford Tank Waste Treatment and Immobilization Plant

Waste Treatment Plant Support Project 



\section{Contents}

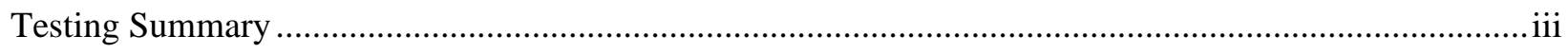

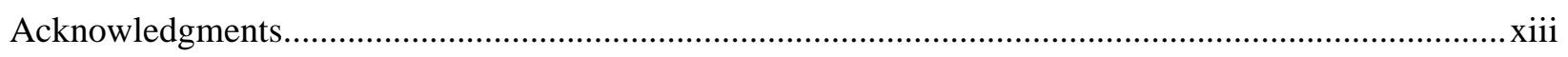

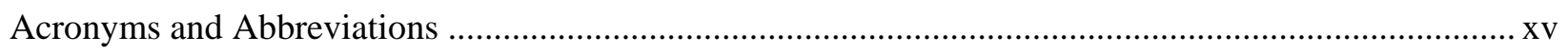

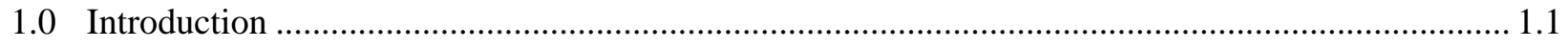

1.1 WTP Model for Estimating Aerosol Release Fraction and Generation ................................... 1.2

1.2 Technical Approach for Calculating Aerosol Release Fraction ............................................. 1.4

1.3 Literature on Aerosol Formation Related to the WTP Model .................................................. 1.6

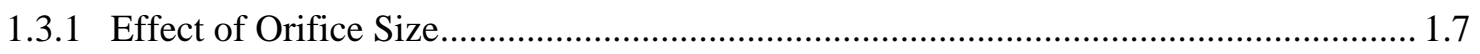

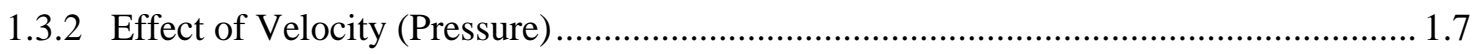

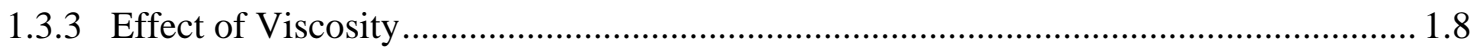

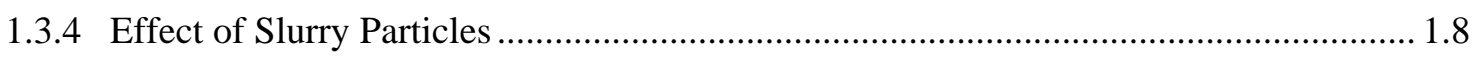

1.3.5 Effect of Surface Tension and Antifoam Agents ..................................................... 1.8

1.3.6 Effect of Non-Newtonian Rheology ..................................................................... 1.10

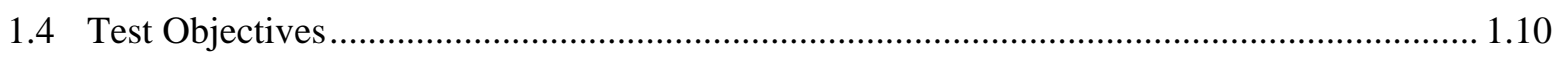

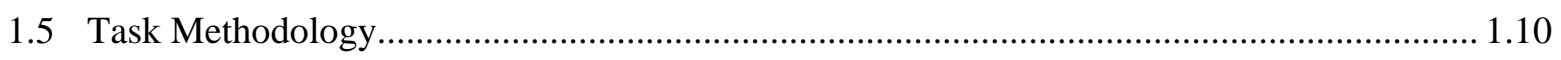

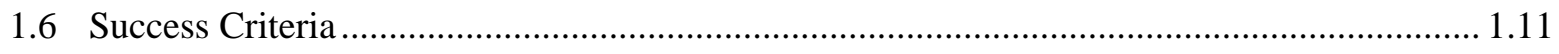

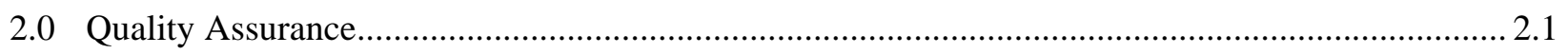

3.0 Technical Basis for Estimating Aerosol Release Fraction........................................................... 3.1

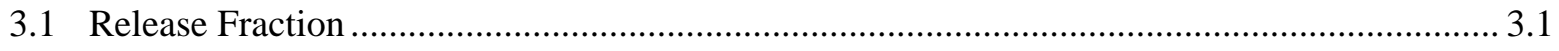

3.2 Determination of Fraction of Spray Aerosolized .............................................................. 3.1

3.3 Conceptual Model for Evaluating Generation with Loss ..................................................... 3.3

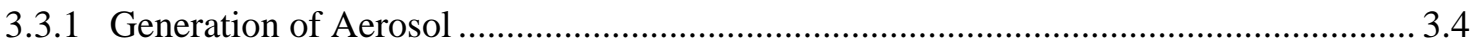

3.3.2 Convective Transport, Deposition, and Settling........................................................ 3.5

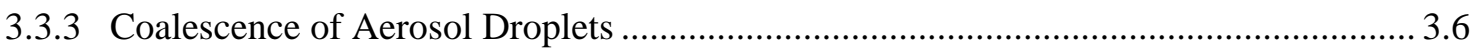

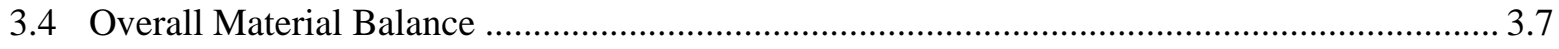

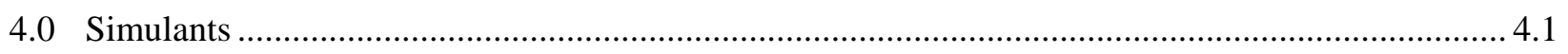

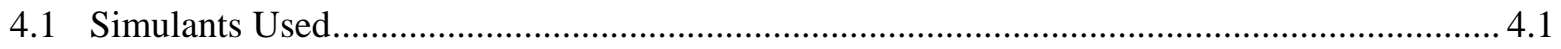

4.2 Simulant Properties ...................................................................................................... 4.4

4.2.1 Analytical Techniques....................................................................................... 4.4

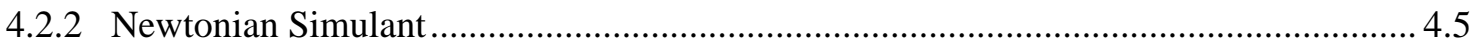

4.2.3 Small Treated (STR) Hanford Waste Simulant.............................................................. 4.5

5.0 Test Equipment and Instrumentation Description .................................................................... 5.1

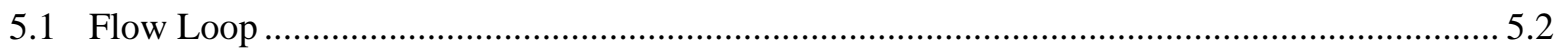

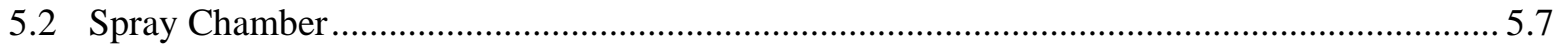




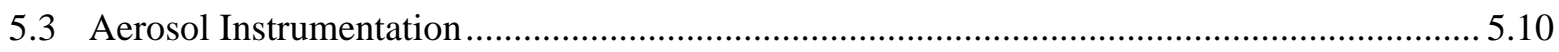

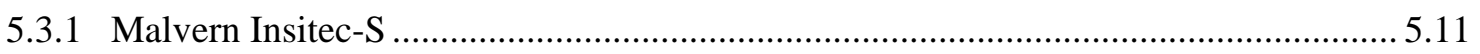

5.3.2 Aerosol Concentration by Ensemble Laser Diffraction ................................................. 5.15

5.3.3 Process Particle Counter........................................................................................ 5.20

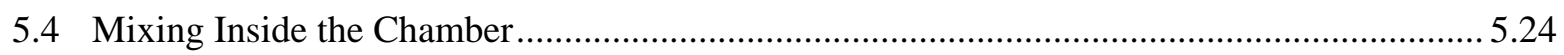

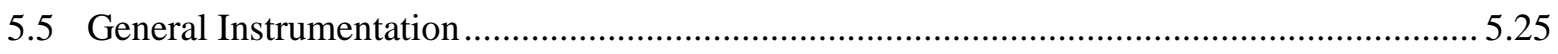

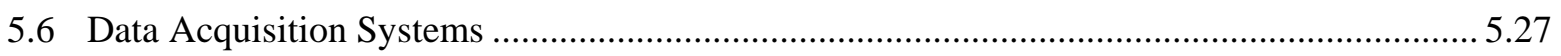

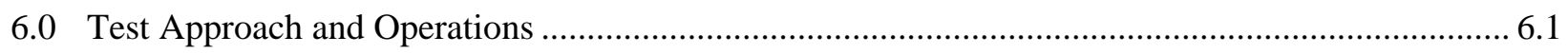

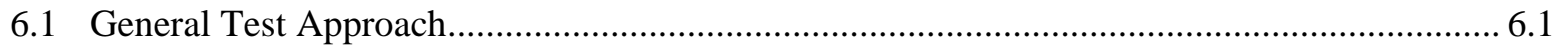

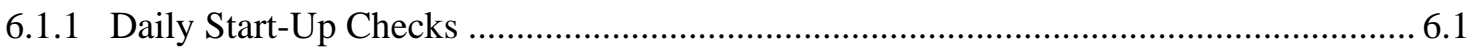

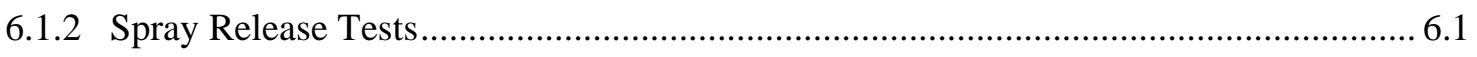

6.1.3 Daily Close-Out Checks.......................................................................................... 6.3

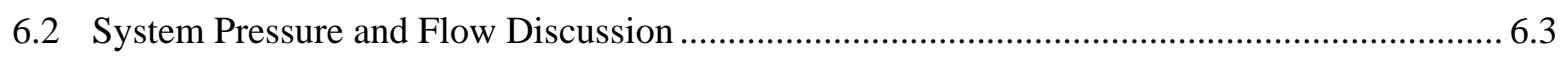

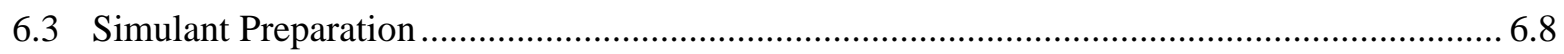

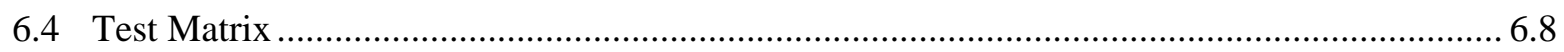

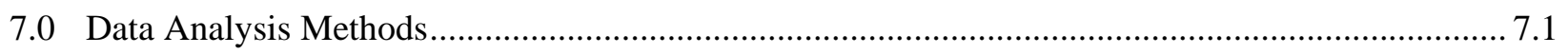

7.1 Analysis of Process Instrument Data................................................................................ 7.1

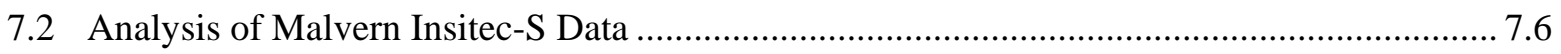

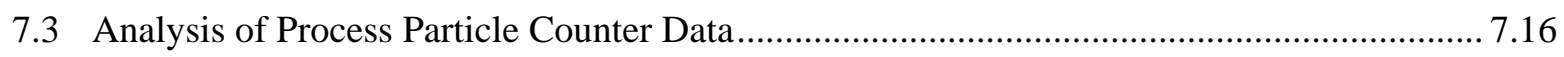

7.4 Analysis Challenges .................................................................................................. 7.18

7.4.1 Synchronization of Events Recorded by DAS-1 and DAS-2 …................................... 7.18

7.4.2 Laser Power Drift in Insitec-S Aerosol Measurements................................................... 7.19

7.4.3 Rapid Concentration Rise........................................................................................ 7.21

7.4.4 Selection of Refractive Index for Laser Diffraction Analysis...................................... 7.21

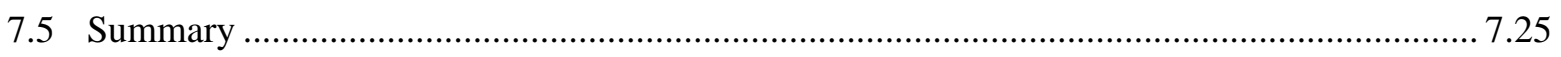

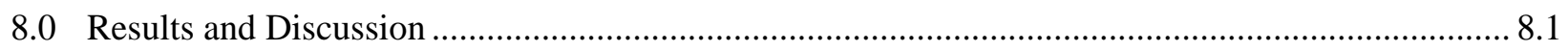

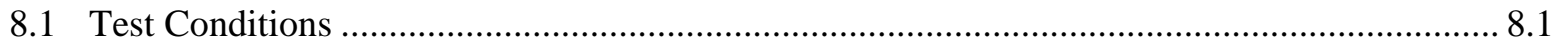

8.1.1 Process Conditions ............................................................................................ 8.2

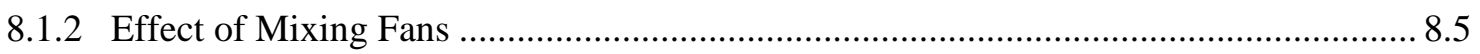

8.1.3 Aerosol Instrument Location in Chamber ................................................................... 8.6

8.1.4 Optimization of PPC Vacuum Flow Rate ………………......................................... 8.9

8.1.5 Comparison of Insitec-S and PPC Measurement Data................................................... 8.10

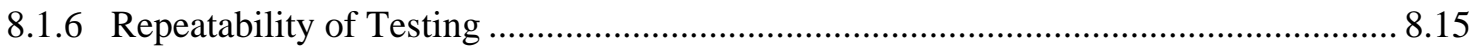




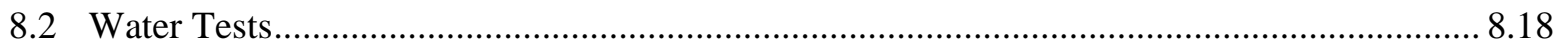

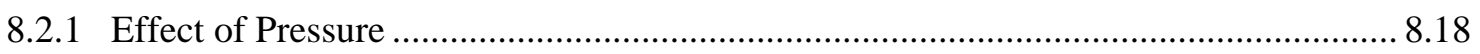

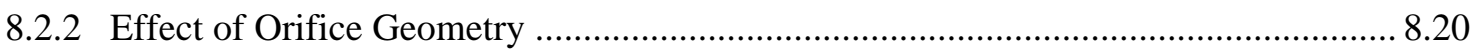

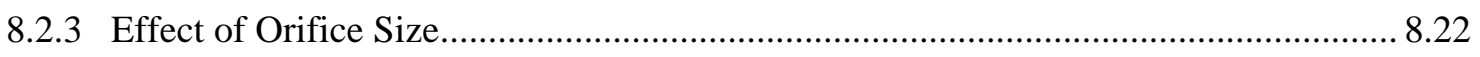

8.2.4 Effect of Aspect Ratio ............................................................................................ 8.24

8.2.5 Overall Effect of Orifice Area on Generation Rate and Release Fraction ................... 8.26

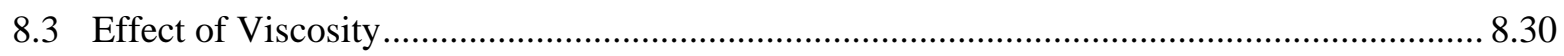

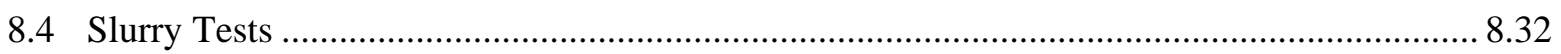

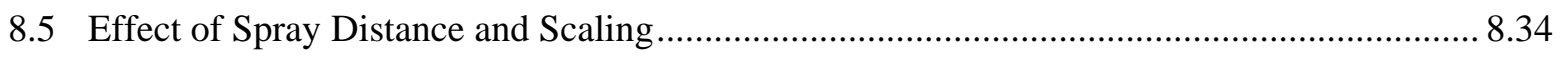

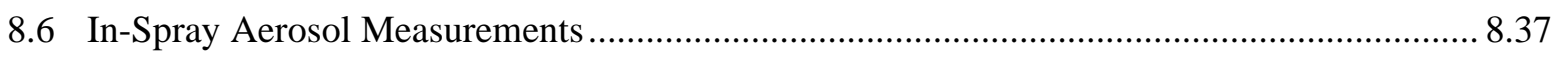

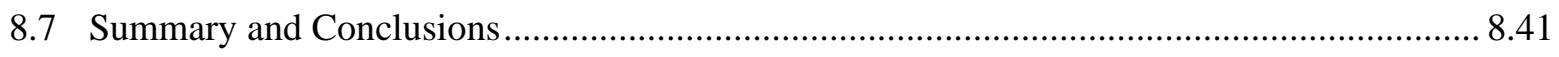

9.0 Comparison of Small- and Large-Scale Test Results and Extrapolation of Results to Larger

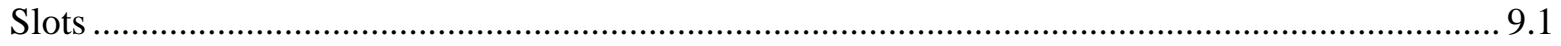

9.1 Comparison of Concentrations and Release Fractions and the Role of Chamber Size............. 9.1

9.2 Comparison of the Effect of Circular Orifice and Slot Size................................................... 9.8

9.3 Comparison of the Effect of Slurry Particles .......................................................................... 9.13

9.4 Comparison of the Effect of Increased Viscosity and Density ............................................. 9.15

9.5 Comparison of the Effect of Spray Length ....................................................................... 9.17

9.6 Extrapolation to Largest Postulated Slot and Long-Distance Spray (Worst-Case Single-

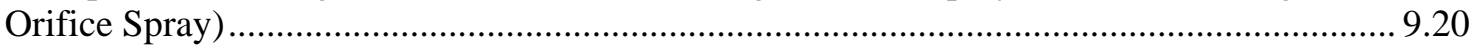

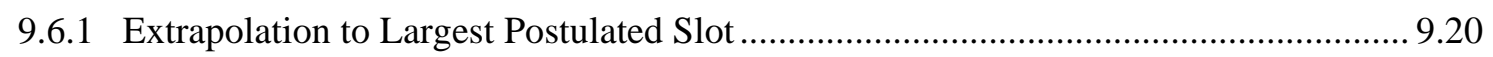

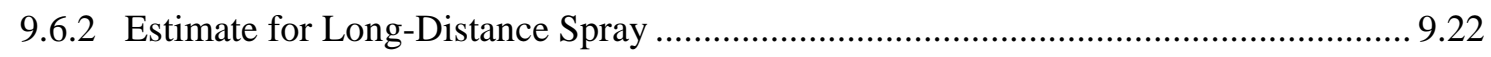

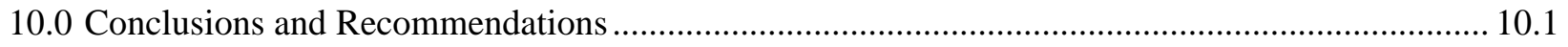

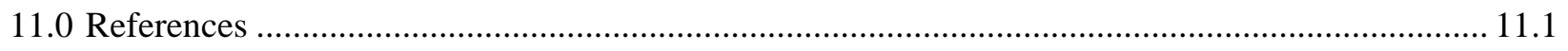

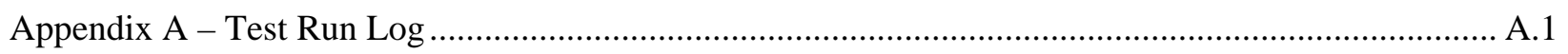

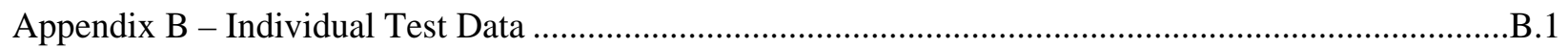

Appendix C - Basis for Concentration Averaging …..........................................................................1 


\section{Figures}

1.1. Schematic of Aerosol Concentration Increasing with Time, Where the Aerosol Generation

Rate is Calculated from the Initial Slope .....

3.1. Schematic Representation of Spray Generation and Transport and Instrument Control Volumes for Simplified Rate of Generation and Loss Material Balances ...................................... 3.3

4.1. Flowing Sonicated PSDs for Post-Caustic Leached and Washed Waste

4.2. Average Cumulative Percent Undersize Measured at 1 Min Recirculation for STR 8 and STR 20 Simulants, Unsonicated, with a Comparison to the STR Simulant Target

5.1. Schematic of the Large-Scale Test System ............................................................................. 5.1

5.2. Schematic of the Large-Scale Spray Release Test System ........................................................ 5.3

5.3. Schematic of the Flow Loop with Centrifugal Pumps on the Pump Skid...................................... 5.5

5.4. Schematic of the Feed and Storage Tanks .................................................................................... 5.6

5.5. Schematic of the Capture/Waste Tank ......................................................................................... 5.7

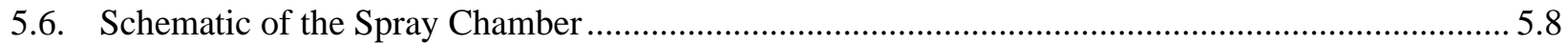

5.7. Schematic of Spool 1, with Orifices A - D From Left to Right in the Schematic ........................ 5.10

5.8. Model of the Release Assembly Used for Initiating the Spray .................................................. 5.10

5.9. Schematic of the Basic Insitec-S Aerosol Analyzer Setup and Operating Principle ..................... 5.11

5.10. Two Schematic Views Showing Key Dimensions Used for Measuring the Locations of the Three Malvern Insitec-S Analyzers Installed in the Large-Scale Test Chamber ........................... 5.13

5.11. Concentration Curve Comparison for a Glass Bead Dispersion in Water as Measured by the

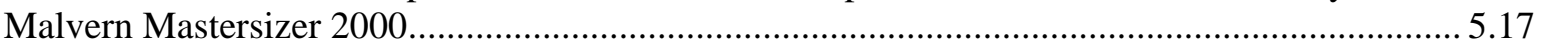

5.12. Analysis of Insitec-S PSD Measurement Reproducibility Using a Physical Standard ................. 5.19

5.13. Schematic Showing the Basic Components and Principle of Operation for the PPC................... 5.21

5.14. Top View of Malvern Instruments and Fan Arrangement ........................................................ 5.25

6.1. Pump Performance Curve for Pumps 1, 2, and 3 ........................................................................ 6.4

6.2. Slot with $0.5 \times 5 \mathrm{~mm}$ Dimensions (100 psi water test) ............................................................. 6.5

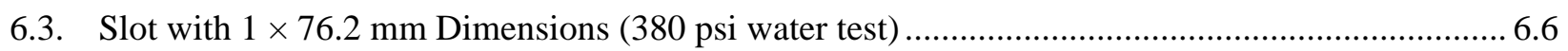

6.4. Slot with $2.74 \times 76.2 \mathrm{~mm}$ Dimensions (380 psi water test [dead head]) …................................... 6.7

7.1. Approximate Locations of the Six Primary Instruments Described in Table 7.1 ........................... 7.2

7.2. Flow Rates Measured During Test W56 (water, 100 psi, $3 \times 10$-mm orifice) from FE1 and

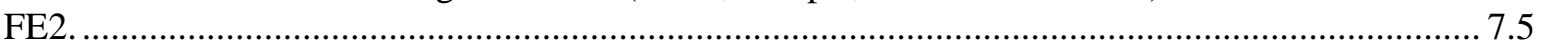

7.3. Comparison of the Laser Power to the Background Laser Power for Test W81, Malvern

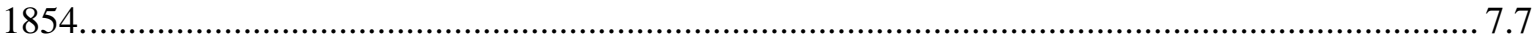

7.4. Comparison of the Laser Power to the Background Laser Power for Test W81, Malvern

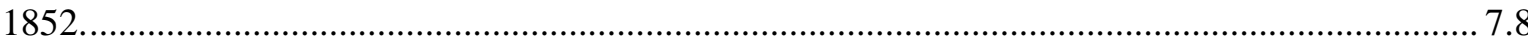

7.5. Concentration of Droplets between 27.37 and $32.28 \mu \mathrm{m}$ in Diameter Measured During Test W81 (2-mm hole, 380 psi, water) as a Function of Elapsed Time by Each Malvern Insitec-S Instrument Compared to the Average

7.6. Selected Cumulative and Differential Concentration Data from Test W81 (2-mm hole, 380 psi, water) as a Function of Elapsed Time. 
7.7. Model Fit to the Cumulative Concentration Data for Test W81 (2-mm hole, 380 psi, water). ..... 7.14

7.8. Release Fractions Calculated from the Cumulative and Differential Concentration Data Compared to the WTP Model Prediction for Test W81 (2-mm hole, 380 psi, water) ................... 7.16

7.9. Four Possible Laser Drift Scenarios During Malvern Insitec-S Measurements............................ 7.20

8.1. Pressure and Temperature Data from Test W81 (2-mm orifice, 380 psi, water) ........................... 8.2

8.2. Orifice Coefficients Determined from Analysis of Tank Masses and Coriolis Flow Rate for All 245 Experiments That Had Useable Data. ........................................................................ 8.3

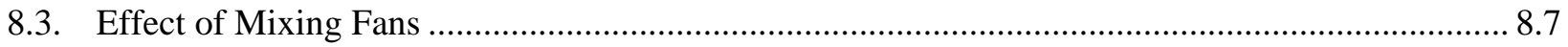

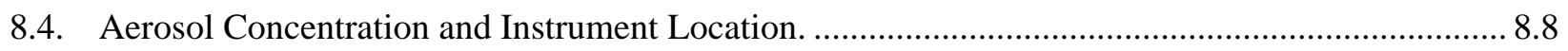

8.5. Average Release Fraction and Aerosol Instrument Location...................................................... 8.9

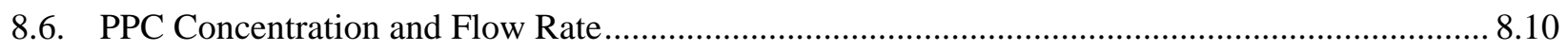

8.7. Comparison of Insitec-S and Normalized PPC Cumulative Volume Fraction Undersize Distributions for Five Large-Scale Spray Tests ............................................................................ 8.14

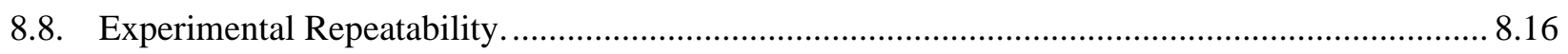

8.9. Uncertainty in the Cumulative Release Fraction..................................................................... 8.17

8.10. Effect of Pressure on Cumulative Release Fractions .................................................................. 8.19

8.11. Effect of Pressure on Cumulative Release Fractions ............................................................... 8.20

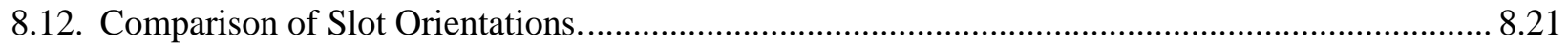

8.13. Comparison of Release Fractions for Single and Multi-Hole Orifices ...................................... 8.22

8.14. Effect of Orifice Area on Generation Rate for Round Orifices. .................................................. 8.23

8.15. Effect of Orifice Area on Generation Rate for Rectangular Orifices.......................................... 8.24

8.16. Effect of Slot Length on Release Fraction. ............................................................................ 8.25

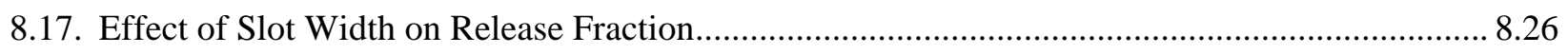

8.18. Effect of Cross-Sectional Area on Release Fraction ................................................................ 8.28

8.19. Effect of Cross-Sectional Area on Generation Rate................................................................. 8.29

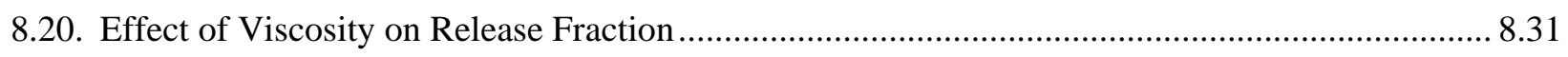

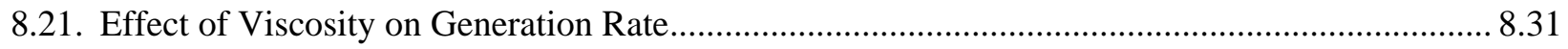

8.22. Effect of the Selection of Refractive Index on Cumulative Release Fraction.............................. 8.33

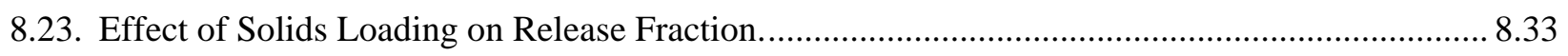

8.24. Effect of Spray Distance from the Splash Wall. ........................................................................ 8.34

8.25. Effect of Spray Distance from the Splash Wall. ........................................................................... 8.35

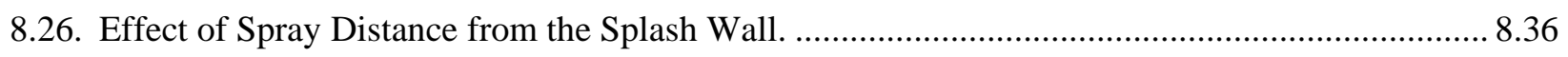

8.27. Effect of Spray Distance from the Splash Wall. ....................................................................... 8.36

8.28. Cumulative Generation Rate as a Function of Distance from the Splash Wall............................ 8.37

8.29. Normalized Droplet Size Distribution as a Function of Elapsed Time....................................... 8.38

8.30. Comparison of In-Spray Cumulative PSDs from Duplicate Tests .............................................. 8.39

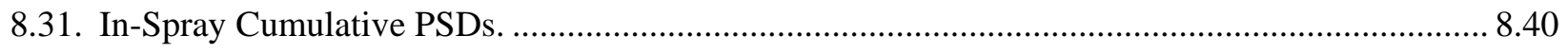

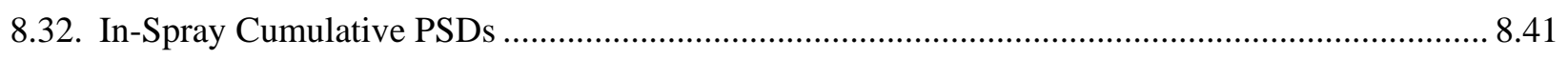

9.1. Comparison of the Transient Cumulative Droplet Concentrations in the Small and Large Chambers for 380 psi Water Sprays from 1-mm Circular Orifices 
9.2. Comparison of Release Fractions in the Small and Large Chambers for 380 psi Water Sprays from a 1-mm Circular Orifice Where the Sprays Traveled Essentially the Full Length of Each Chamber

9.3. Comparison of Release Fractions in the Small and Large Chambers for 380 psi Water Sprays form a 2-mm Circular Orifice Where the Sprays Traveled Essentially the Full Length of Each Chamber

9.4. Comparison of Release Fractions in the Small and Large Chambers for 380 psi Water Sprays from a $0.5 \times 5-\mathrm{mm}$ Slot Where the Sprays Traveled Essentially the Full Length of Each Chamber ...

9.5. Comparison of Release Fractions in the Small and Large Chambers for $8 \mathrm{wt} \%$ STR Slurry Sprays at 380 psi from a $0.5 \times 5$-mm Slot Where the Sprays Traveled Essentially the Full Length of Each Chamber

9.6. Comparison of Release Fractions in the Small and Large Chambers for $20 \mathrm{wt} \%$ STR Slurry Spray at 380 psi from a $0.5 \times 5-\mathrm{mm}$ Slot Where the Sprays Traveled Essentially the Full Length of Each Chamber

9.7. Comparison of Release Fractions in the Small and Large Chambers for 380 psi Water Sprays from a 1-mm Circular Orifice Where the Spray Length is Approximately 42 in. in Each Chamber.

9.8. Comparison of Release Fractions in the Small and Large Chambers for 380 psi Water Sprays from a 2-mm Circular Orifice Where the Spray Length is approximately 42 in. in Each Chamber ....

9.9. Comparison of the Effect of Orifice Area on Release Fractions in the Small- and Large-

Scale Chambers for 380 psig Water Sprays from Circular Orifices

9.10. Comparison of the Effect of Orifice Area on Cumulative Generation Rate in the Small- and Large-Scale Chambers for 380 psig Water Sprays from Circular Orifices

9.11. Comparison of the Effect of Orifice Area on Release Fractions in the Small- and Large-Scale Chambers for 380 psig Water Sprays from Slots

9.12. Comparison of the Effect of Orifice Area on Cumulative Generation Rate in the Small- and Large-Scale Chambers for 380 psig Water Sprays from Slots

9.13. Comparison of Slurry Sprays for the STR Simulant at 8 and 20 wt\% with Water Sprays in the Small-Scale Chamber.

9.14. Comparison of Slurry Sprays for the STR Simulant at 8 and $20 \mathrm{wt} \%$ with Water Sprays in the Large-Scale Chamber.

9.15. Effect of Increased Viscosity (and Density) on the Cumulative Aerosol Generation Rate for Water $(\sim 1 \mathrm{mPa} \bullet \mathrm{s})$, Sodium Nitrate $(\sim 1.5 \mathrm{mPa} \bullet)$, and Sodium Thiosulfate $(\sim 2.5 \mathrm{mPa} \bullet \mathrm{s})$ in the Small-Scale Chamber

9.16. Effect of Increased Viscosity (and Density) on the Cumulative Aerosol Generation Rate for Water $(\sim 1 \mathrm{mPa} \bullet \mathrm{s})$ and Sodium Thiosulfate $(\sim 2.6 \mathrm{mPa} \bullet \mathrm{s})$ in the Large-Scale Chamber

9.17. Effect of Distance from Spray to Splash Wall on the Cumulative Release Fraction for a 1-mm Orifice in Both the Small and Large Chambers.

9.18. Effect of Spray Length on Release Fraction for Droplets $<10 \mu \mathrm{m},<32 \mu \mathrm{m}$, and $<102 \mu \mathrm{m}$ for Various Circular Orifices and Slots and at 200 and 380 psi

9.19. Effect of Orifice Area on Cumulative Release Fraction for Slots Tested in the Large-Scale Chamber and the Extrapolation of Data to Larger Slots 
9.20. Cumulative Release Fraction for Progressively Larger Slots and the Estimated Release Fraction for a $4.64 \times 127-\mathrm{mm}\left(589-\mathrm{mm}^{2}\right)$ Slot with a Spray that Travels the Full Length of a 20 -ft Chamber.

9.21. Comparison of In-Spray PSDs for Circular Orifices with a Range of Sizes

9.22. Comparison of Release Fractions for a Spray Traveling the Full Length of the 20-ft Chamber (227 in.) and In-Spray PSDs for a 380 psig Water Spray from a 2-mm Circular Orifice

9.23. Comparison of Extrapolated Release Fractions for a Spray Traveling the Full Length of the 20 -ft Chamber (227 in.) and In-Spray PSDs for a 380 psig Water Spray from a 1-mm Circular Orifice

9.24. Extrapolated Release Fractions for the Largest Postulated Slot for Water in a 20-ft Chamber, an Increased Release Fraction for the Highest Postulated Bingham Yield Stress, and Comparison to a Representative In-Spray PSD and the WTP Model Assuming Fluid Properties for Water. 


\section{Tables}

S.1 Summary of Test Objectives and Results ................................................................................. iv

S.2 Success Criteria for Large-Scale Plugging Tests ........................................................................... iv

S.3 Target Simulants Tested in the Large-Scale Test Stand and the WTP Process Stream

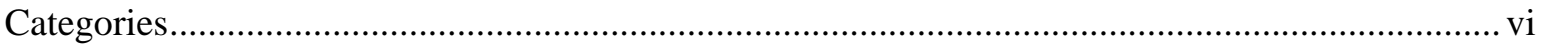

4.1 WTP Process Stream Categories and Representative Fluid Properties.......................................... 4.1

4.2 Target Simulants and the WTP Process Stream Categories........................................................... 4.2

4.3 Newtonian Simulant Properties for Large-Scale Aerosol Tests...................................................... 4.5

4.4 Small Treated Simulant Properties for Aerosol Tests .................................................................. 4.6

4.5 Summary of Selected Percentile Values for Measurements Taken at 1 Min Recirculation Time, Unsonicated, STR 8 and STR 20 Simulants with a Comparison to the Target PSD ............. 4.7

4.6 Cumulative Percent Undersize Measured at 1 Min Recirculation Time, Unsonicated, STR 8

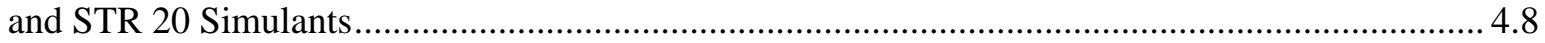

5.1 Breaches and Geometries Used for Large-Scale Testing ........................................................... 5.9

5.2 Values for Key Dimensions Listed in Figure 5.10 …............................................................ 5.13

5.3 Insitec-S Instrument Configuration and Software Parameters Used in Typical Measurements

5.4 Results of Insitec-S Performance Reproducibility Analysis Using a Physical Standard for Select Measurement Parameters................................................................................................ 5.18

5.5 Instrumentation used for Process Parameter Measurements ........................................................ 5.26

6.1 Planned and Actual Large-Scale Aerosol Testing Done with Water ..............................................10

6.2 Large-Scale Aerosol Testing of Sodium Thiosulfate and Simulants of Small Treated

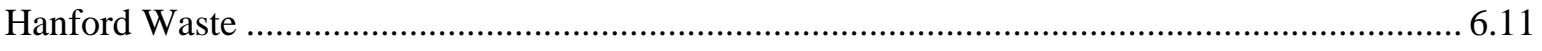

7.1 Six Primary Instruments From DAS-1 Used in Large-Scale Data Analysis.................................. 7.2

7.2 Refractive Indices for Test Systems Used in Both Small- and Large-Scale Spray Leak

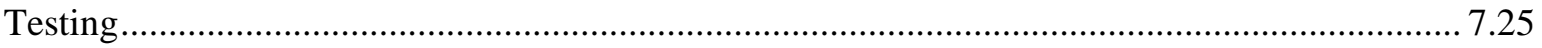

8.1 Summary of the Pressure and Temperature Data Measured during Aerosol Tests......................... 8.3

8.2 Comparison of Total Aerosol Concentration Measurements by the Malvern Insitec-S and

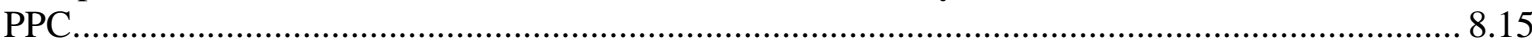

8.3 Average Total Volume Concentration for In-Spray Measurements for the 2-mm Orifice ............ 8.39 


\subsection{Introduction}

One of the events postulated in the hazard analysis for the Waste Treatment and Immobilization Plant (WTP) is a breach in process piping that produces a spray of aerosols with droplet sizes in the respirable range. The piping breaches are postulated to result from a number of causes, including jumper connection misalignment, pipe erosion/corrosion, mechanical impact, seal/gasket failures, and the like. The breaches are expected to be rough in texture and irregular in shape.

At Hanford, the generation rate and size distribution of aerosol droplets produced in a spray leak have generally been predicted by using correlations reported in the literature (Larson and Allen 2010; McAllister 2010). These correlations are based on results obtained from small engineered spray nozzles using liquids that do not contain solids. However, fluids processed at WTP include slurries and high-viscosity liquids with properties very different than the properties of liquids used to develop the correlations currently employed to evaluate spray leaks. The range of geometries postulated for random breaches differs from the geometries of the engineered spray nozzles used to develop the correlation in terms of both aspect ratio and area. Therefore, the correlations used to model spray leaks from process piping may not accurately represent spray leak conditions at WTP (or elsewhere on the Hanford site).

The amount of aerosol produced is a function of the dimensions of the opening, which affect both the total amount of flow and the fraction that becomes respirable aerosol. In some predictive correlations for aerosol generation, the respirable fraction is insensitive to the dimensions of the opening (Epstein and Plys 2006). In other correlations, the respirable fraction increases significantly as the dimensions of the opening decrease (Hey and Leach 1994). The maximum opening size postulated for WTP spray modeling depends on the pipe size, and for a pipe diameter up to 3 in. the maximum opening has a length equal to the pipe diameter and width equal to one-half of the pipe wall thickness (Larson and Allen 2010). Most models in use on the Hanford site set a minimum breach dimension based either on the gas Weber number $\left(\mathrm{We}_{\mathrm{g}}\right)^{2}$ or on plugging considerations. Arguments have been made, for example, that openings with $\mathrm{We}_{\mathrm{g}}<60$ do not support significant jet breakup and, therefore, do not result in significant aerosol production (Zimmerman 2003), or that openings with minimum dimension $<0.7 \mathrm{~mm}$ would be plugged by slurries that contain relatively large particles, such as K-Basin slurries (HNF-SD-WM-SAR-062). In practice, a plugging assumption that may determine a minimum breach size is only useful if the correlation used to model aerosol predicts greatly increased respirable droplet production as the breach size decreases. If the respirable fraction does not increase strongly enough with decreasing orifice size, the corresponding total aerosol generation and consequence in the hazards analysis also decreases.

These considerations indicated two key technical areas in which testing results are needed to improve the WTP methodology (Larson and Allen 2010) by reducing the uncertainty that results from extrapolating results from the literature. The first technical need is to quantify the role of slurry particles in small breaches where the slurry particles may plug the hole and prevent high pressure sprays. The second technical need is to determine the aerosol droplet size distribution and total droplet volume from prototypic breaches and fluids, including sprays from larger breaches and sprays of slurries where data from the literature are scarce. These needs are addressed by small- and large-scale testing using simulants that mimic the relevant physical properties projected for actual WTP process streams.

\footnotetext{
${ }^{2}$ The gas Weber number is $\rho_{g} u_{0}^{2} d_{0} / \sigma$ where $\rho_{g}$ is the gas density, $u_{0}$ the liquid velocity at the orifice, $d_{0}$ the diameter of the orifice, and $\sigma$ the surface tension.
} 
The purpose of the study described in this report is to provide experimental data and analyses for the second key technical area, determining the aerosol droplet size distribution and total droplet volume from prototypic breaches and fluids (ultimately resulting in the determination of generation rate and release fraction), by performing large-scale tests using a range of orifice sizes and orientations representative of the WTP conditions. The other technical area, breach plugging, is addressed in Mahoney (2012a).

A broad range of simulants were developed and tested in the small-scale aerosol test stand (Mahoney et al. 2012b). However, because of the hazards and costs associated with some of the simulants developed, only a small subset of the simulants was used in the large-scale test stand. The simulants used on the large-scale test stand consisted of 1) water and sodium thiosulfate in water solutions to mimic the ultrafilter permeate, Cs ion exchange eluate, and dilute recycle streams, and 2) boehmite slurries in water with solids loadings of 8 and $20 \mathrm{wt} \%$ to mimic slurries processed at the WTP that are essentially Newtonian fluids.

This chapter provides an introduction and a discussion of related tests found in the literature, and also gives details on the WTP model for estimating the aerosol release fraction and generation that will be used to compare with the experimental results. Chapter 2 details the basis of the Pacific Northwest National Laboratory (PNNL) Quality Assurance (QA) Program as applied to the RPP-WTP quality requirements. Chapter 3 describes the technical basis for the aerosol measurements. Chapter 4 describes the liquid and slurry simulants used in testing, and the basis for their choice. Chapter 5 provides a description of the equipment and instruments, and Chapter 6 summarizes the test approach and operations. Chapter 7 provides the data analysis methodology, and Chapter 8 discusses the results. Chapter 9 contains a discussion of the combined small- and large-scale test results and the extrapolation of the current results to a worst-case spray scenario. Chapter 10 describes the conclusions and recommendations of the study, and the references are listed in Chapter 11. The appendices provide a run log and process condition data (Appendix A), individual test data, namely release fractions (Appendix B), and a basis for using average aerosol concentrations (Appendix C).

\subsection{WTP Model for Estimating Aerosol Release Fraction and Generation}

Larson and Allen (2010) summarize the methodology used by the WTP for estimating the aerosol release fraction and generation rate of spray releases, and McAllister (2010) provides additional details on the equations and method. The method uses the theoretically-based correlation by Dombrowski and Johns (1963) for estimating the Sauter mean diameter (SMD) and then estimates the aerosol droplet size distributions using the Rosin and Rammler (1933) distribution. An overall objective of the current study is to collect data to determine the range of applicability of the method. The Dombrowski and Johns (1963) equations used in the WTP methodology for estimating the SMD are

$$
\begin{gathered}
d_{l}=0.9614\left(\frac{\mathrm{K}^{2} \sigma^{2}}{\rho_{l} \rho_{a} U^{4}}\right)^{1 / 6}\left[1+2.6 \mu\left(\frac{\mathrm{K} \rho_{a}^{4} U^{7}}{72 \rho_{l}^{2} \sigma^{5}}\right)^{1 / 3}\right]^{1 / 5} \\
d_{d}=1.882 d_{l}\left[1+\frac{3 \mu}{\left(\rho_{l} d_{l} \sigma\right)^{1 / 2}}\right]^{1 / 6} \\
S M D=0.63 d_{d}
\end{gathered}
$$


where

$$
\begin{aligned}
d_{l} & =\text { the theoretical ligament diameter }(\mathrm{m}) \\
d_{d} & =\text { the theoretical droplet diameter }(\mathrm{m}) \\
\mu & =\text { the liquid viscosity }\left(\mathrm{Pa}^{*} \mathrm{~s}\right)^{3} \\
\rho_{l} & =\text { the liquid density }\left(\mathrm{kg} / \mathrm{m}^{3}\right) \\
\rho_{a} & =\text { the air density }\left(\mathrm{kg} / \mathrm{m}^{3}\right) \\
\sigma & =\text { the surface tension of the liquid }(\mathrm{N} / \mathrm{m}) \\
\mathrm{U} & =\text { the fluid velocity at the breach }(\mathrm{m} / \mathrm{s}) \\
\mathrm{K} & =\text { the spray nozzle parameter }\left(\mathrm{m}^{2}\right) .
\end{aligned}
$$

The K parameter is determined with the (McAllister 2010) relationship

$$
\mathrm{K}=\frac{0.5 \mathrm{~A}}{\sin (\theta / 2)}
$$

where $A$ is the area of the breach for all shapes and $\theta$ is the full spray angle and assumed to be the maximum value of $150^{\circ}$ for a fan spray. Using the assumed maximum value of the spray angle, $\sin (\theta / 2)$ is practically unity (0.97) and $K$ is approximately $A / 2$.

Other applications of the Dombrowski and Johns (1963) model for spray release evaluations have used models for the $\mathrm{K}$ parameter that distinguish between breaches with different shapes, rather than just using the area for all breaches (Crowe 2010; Williams 2000).

The SMD for a particular spray can be determined using Equations (1.1) to (1.4). To determine the fraction of a spray contained in droplets below any particular size for a spray release accident analysis, a relationship is needed for the droplet size distribution. For the WTP methodology, Larson and Allen (2010) use the Rosin and Rammler (1933) distribution and further assume that the release fraction of a spray (RF) is equal to the droplet size distribution. The following equations give the RF for sprays used in the WTP methodology:

$$
\begin{gathered}
R F=1-\exp \left[-\left(\frac{S M D}{X} \frac{D}{S M D}\right)^{q}\right] \\
\frac{S M D}{X}=[\Gamma(1-1 / q)]^{-1}
\end{gathered}
$$

where $\quad D=$ the droplet size

$q=$ a fitting constant that provides a measure of the spread in the droplet size distribution

$R F=$ the fraction of the total spray volume contained in drops of diameter less than $\mathrm{D}$

$S M D=$ the Sauter mean diameter

$X=$ a characteristic diameter

$\Gamma=$ the gamma function.

Larson and Allen (2010) evaluated the value of $q$ and chose $q=2.4$, and also noted that this gives $\mathrm{SMD} / \mathrm{X}=0.65415$.

\footnotetext{
${ }^{3}$ McAllister (2010) has a typographical error and shows incorrect units for viscosity (uses kinematic viscosity units), but correctly uses the correct viscosity and units in the example calculation.
} 
To determine the aerosol generation rate from a spray, the flow rate of the spray is needed in addition to the release fraction given by Equation (1.5). The generation rate $\mathrm{G}$ is given by

$$
\mathrm{G}=\mathrm{Q}_{\text {spray }} * \mathrm{RF}
$$

where $Q_{\text {spray }}$ is the volumetric flow of the spray $\left(\mathrm{m}^{3} / \mathrm{s}\right)$ and $\mathrm{G}$ is the generation rate $\left(\mathrm{m}^{3} / \mathrm{s}\right)$ of droplets less than diameter D. It is important to note that for the WTP model, the entire volumetric flow of the spray is assumed to aerosolize (break up into droplets), and thus, at a certain droplet size, the release fraction will become equal to unity. Mathematically, the model predicts that at a particular droplet size (which will depend on the physical parameters of the system), $G=Q_{\text {spray. }}$.

For the experiments presented in this report, both a net $\mathrm{G}$ and $\mathrm{Q}_{\text {spray }}$ were measured for individual sprays and the results will be presented either as the generation rate or as the release fraction. Note that the net generation rate is the total spray generation rate minus losses caused by the impact at the splash wall and other effects of constrained geometry. For the remainder of this report, experimentally determined generation rates or release fractions should be understood to be concerned with the net generation rate.

For use in Equation (1.1), the velocity of the liquid leaving the orifice can be determined from the pressure difference $(\Delta P)$ with an orifice flow equation (for example, see Denn 1980), and McAllister (2010) uses the following orifice flow equation with a typical value of 0.62 for the orifice coefficient:

$$
\mathrm{U}=0.62\left(\frac{2 \Delta P}{\rho_{l}}\right)^{1 / 2}
$$

In the WTP model, the volumetric flow for calculating the total release with Equation (1.7) is simply the spray velocity times the area of the orifice.

$$
\mathrm{Q}_{\text {spray }}=\mathrm{U} * \mathrm{~A}
$$

The equations presented above represent the WTP model. Predictions from this model will be compared with the experimental results in Chapters 8 and 9. The model predictions presented in Chapter 8 will show the quantitative dependence on the various parameters in the Dombrowski and Johns (1963) correlation.

\subsection{Technical Approach for Calculating Aerosol Release Fraction}

Three experimental methods were considered to measure the aerosol generation rate and release fraction: 1) direct in-spray measurements, 2) steady-state aerosol concentration measurements in a chamber with different volumetric purge rates, and 3) transient aerosol concentration measurements in a chamber with no purge flow. The benefits and drawbacks of each method will be discussed and the selected method will be described in this section. Further details regarding the aerosol measurement methodology can be found in Chapters 3 and 7.

The first experimental method measures the aerosol directly in the spray, providing an explicit measurement of the aerosol droplet size distribution at a specific position. If the in-spray droplet size

distribution is assumed to represent total spray, the droplet size distribution is then equal to the release 
fraction for the spray. However, Epstein and Plys (2006) showed that in-spray measurements at a reasonable distance ( 0.5 to $1 \mathrm{~m}$ ) become difficult for larger orifices due to the core of the jet remaining intact. In particular, they had to measure the droplet size distribution a short distance from the center of the spray for the largest slot they tested $(1.2 \times 10 \mathrm{~mm})$ to avoid the liquid core. In addition, the measured in-spray droplet size distribution does not account for the spray moving at different velocities and having a spatial variation of droplet concentrations within the spray and hence within the region where the aerosol instrument measures the droplet size distribution. This effect gives uncertainty in equating the spray release fraction with the measured droplet size distribution. The literature gives methods for determining the spatial variation in droplet concentration (Boyaval and Dumouchel 2001) and for determining the velocity distribution across a spray (see for example Levy et al. 1997), but these studies also indicate that determining spatial variation in concentration and velocity is quite challenging. Finally, in-spray measurements do not account for droplet formation by splatter should a high-pressure spray impact on surfaces. Due primarily to the expected difficulty in obtaining in-spray measurement for larger orifices and to not including the effect of splatter, the in-spray method was not chosen as the primary measurement method.

The second experimental method considered for determining the aerosol generation rate was to generate a steady spray and measure the steady-state concentration within a chamber with varying flow rates of clean air introduced into the chamber to dilute the aerosol. The generation rate could then be calculated from the measured aerosol concentration with different purge rates. However, during shakedown testing it was determined that the steady-state aerosol concentrations were only slightly above the minimum detection limit of the aerosol instrument and that dilution of the aerosol by this method would likely give concentrations that could not be measured. In addition, for some of the larger sprays the volume of liquid sprayed into the chamber could become quite large and the accumulation of liquid in the chamber would become an experimental challenge for longer-duration steady-state tests. This experimental approach is still reasonable, but for these reasons it was not selected as the primary measurement method.

The third, and selected, experimental method consists of measuring rate of increase in aerosol concentration in a closed chamber of known volume. Using a simple material balance, the rate of concentration increase gives the aerosol generation rate from a spray. Figure 1.1 shows a conceptual example of concentration increase with time, for different cumulative droplet volumes, where the initial rate of increase can be estimated from the initial rise in concentration. Eventually, the concentrations no longer increase linearly with time and approach steady-state values. This behavior is due to aerosol losses occurring in the chamber, and an analysis including this behavior and the method used to determine the initial slope are discussed in Chapters 3 and 7. 


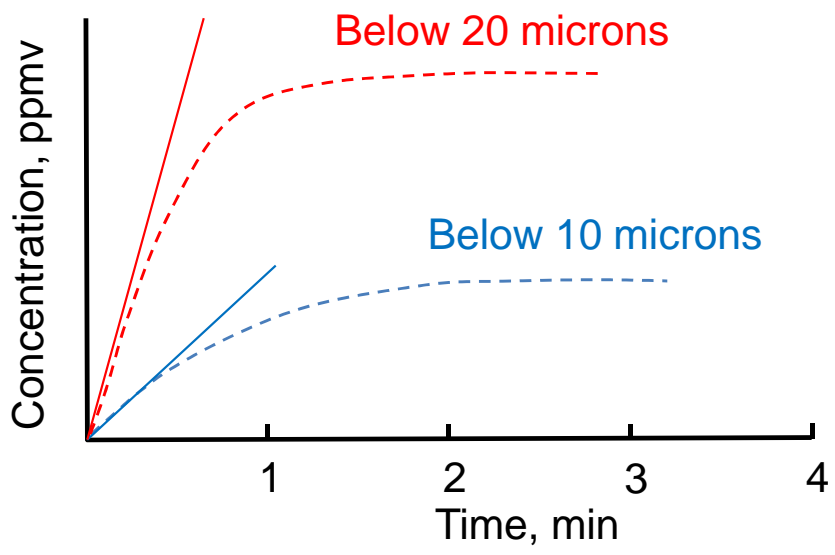

Figure 1.1. Schematic of Aerosol Concentration Increasing with Time, Where the Aerosol Generation Rate is Calculated from the Initial Slope (solid lines)

A key component of the approach of using the increase in concentration is to have a concentration measurement in the chamber that is representative of the entire chamber. An advantage of using a chamber is that it allows for isolation of the spray, providing a safe testing platform for spraying simulants with chemical hazards. This methodology also allows for testing to be performed in chambers with different sizes where larger chambers could accommodate larger sprays but overall experimental and data analysis approach would be the same. Creating sprays inside a chamber also allows the spray to impact on the walls of the chamber and generate aerosol droplets by splatter. This additional mechanism of droplet formation is prototypic of an actual spray, and adds to the total aerosol formation within the chamber. The approach of measuring the concentration increase in a closed chamber also allows for determining the role of changing the orifice-to-wall distance. Due to the size and configuration of WTP piping, sprays could impact systems (e.g., walls, pipes, valves) at distances ranging from inches to hundreds of feet. In addition, because the same experimental method will be used in both the small- and large-scale tests, results can be compared to one another and extrapolated to longer distances with more confidence than previously available. For the reasons discussed above and because of the overall method flexibility, the primary method for determining aerosol generation rate is to measure the aerosol generation rate based on the initial rate of concentration increase in a closed chamber.

\subsection{Literature on Aerosol Formation Related to the WTP Model}

The formation of aerosols for sprays from a range of nozzles and fluids has been widely studied and good summaries are available in textbooks (Lefebvre 1989; Nasr et al. 2002; Ashgriz 2011) and review articles (Eggers and Villermaux 2008). For spray release estimates, the U.S. Department of Energy (DOE) Handbook (DOE 1994) provides a compilation of information and guidance for release fractions, but these estimates were based on a limited amount of experimental data. There are a number of correlations in the literature; however, these correlations sometimes give quite different results for the effect of breach size and shape, fluid properties, and spray velocity.

A number of recent studies have evaluated the existing correlations of aerosol formation from high-pressure sprays and also have developed improved release fraction estimates. As discussed previously in Section 1.1, Larson and Allen (2010) discussed differences in the available correlations and 
specifically compared the selected approach, based on the correlation in Dombrowski and Johns (1963), with the predicted droplet size from the modified Merrington and Richardson correlation given by Lefebvre (1989). Crowe (2010) and Williams (2000) both compared the Dombrowski and Johns (1963) correlation to other correlations, and Lefebvre (1989) provided a useful summary of a number of additional correlations for plane orifice atomizers.

Epstein and Plys (2006) gave a broad summary of available correlations and, in particular, discussed the different predictions for the effect of orifice size. Epstein and Plys (2006) also reported new experimental results for high-pressure water sprays. This work is essentially the only study that provides drop size distributions, generation rates, and release fractions for orifices and pressures that specifically address the data needs for evaluating sprays that result from accidental breaches. The specific findings from Epstein and Plys (2006) will be discussed below in the subsections on the effect of orifice size and pressure. Additional subsections give brief summaries of the literature on the additional parameters evaluated in this report.

\subsubsection{Effect of Orifice Size}

The most notable difference in the existing correlations is for the effect of orifice size. Epstein and Plys (2006) measured droplet size distributions from circular holes with diameters ranging from 0.3 to $2.38 \mathrm{~mm}$ and from two rectangular slots with dimensions of $0.3 \times 10 \mathrm{~mm}$ and $1.2 \times 10 \mathrm{~mm}$. For all but the largest slot, the measured droplet size distribution did not depend on the size or shape of the orifice. Measurements from the largest slot were different, but this slot generated a long liquid core that interfered with size distribution measurement. This finding agreed with the original correlation from Merrington and Richardson (1947) that also showed no effect of orifice size on the SMD and, hence, no effect on the release fraction (see Equation (1.5)), for the spray. If the release fraction is a constant with increasing orifice diameter or area, Equations (1.7) and (1.9) show that the total aerosol generated increases with orifice size simply because of the increase in flow rate through the orifice.

As will be shown with the experimental results in Chapter 7, the WTP method predicts a decrease in the RF with increasing orifice area, but the total aerosol generated (see Equation (1.7)) will still increase with increasing orifice area because of the increase in flow rate. In contrast, the modified Merrington and Richardson correlation given by Lefebvre (1989) yields an SMD that increases and, hence, a release fraction through Equation (1.5) that decreases more with increasing orifice diameter. For the modified Merrington and Richardson correlation, the decrease in release fraction is sufficiently large so the total generation is predicted to decrease with increasing orifice diameter and flow.

The differences in the existing correlations are sufficient to make it uncertain whether larger or smaller orifices will have the largest total aerosol generation rate. Accordingly, one of the primary objectives of the current experimental measurements is to obtain data for a sufficient range of orifice sizes to reduce the uncertainty on the effect of orifice size.

\subsubsection{Effect of Velocity (Pressure)}

Increasing the spray pressure increases the liquid velocity exiting the orifice (see Equation (1.8)), and this affects aerosol formation two ways. First, the increased jet velocity reduces the droplet size and, hence, increases the release fraction. The recent work of Epstein and Plys (2006) showed the SMD to be 
proportional to $\mathrm{U}^{-0.558}$, which is similar to the Dombrowski and Johns result given by Equation (1.1), assuming the last group of terms in Equation (1.1) is small compared to one, in which case the SMD is proportional to $\mathrm{U}^{-2 / 3}$. The second effect of spray pressure is to increase the total flow of the spray and, hence, the total aerosol generation rate as given by Equation (1.7).

\subsubsection{Effect of Viscosity}

There a number of studies, including the original data and correlation of Merrington and Richardson (1947) and the Dombrowski and Johns (1963) correlation, that evaluated the effect of viscosity on droplet formation. These correlations, and others in the literature (see summaries by Lefebvre [1989] and Epstein and Plys [2006]), generally showed that increasing the viscosity increases the droplet size and, hence, decreases the release fraction; however, the dependence is generally small.

\subsubsection{Effect of Slurry Particles}

There have been a few previous studies that have evaluated the role of slurry particles on aerosol droplet formation. While there are many specific observations, there are some general overall findings. One group of studies evaluated the role of slurry particle size and generally determined that, if the slurry particles are smaller than the droplets, the slurry particles do not tend to affect the droplet distribution (Mulhem et al. 2006, 2003, 2001; Fritsching et al. 2009). When the slurry particles become progressively larger and specifically larger than droplets that would be generated in the absence of the slurry particles, droplet formation is naturally influenced. Droplets smaller than the slurry particles can still be formed, but these droplets will not contain slurry particles, and the larger droplets will contain the slurry particles. Breitling et al. (2001) presented both computational fluid dynamics (CFD) and experimental results for shear-thinning lime slurries sprayed from hollow-cone pressure swirl nozzles. The results showed little difference between the slurry and water, but there was no information on the size of the lime particles. Hecht et al. (2007) found that increasing solids loading resulted in a small decrease in the droplet size. Son and Kihm (1998) studied the effect of coal slurry particles on spray formation. The coal slurries were non-Newtonian and had progressively higher apparent viscosities for progressively smaller coal particles. The aerosol results showed that larger aerosol droplets were generated as the coal particle size became smaller, and Son and Kihm (1998) suggested that the primary reason was the increase in apparent viscosity for the smaller particle slurries. Dombrowski and Munday (1968) found that a small volume fraction of wetting particles did not change the breakup of a fan jet but that high particle concentration in the slurry changed the behavior markedly and resulted in larger drops. Finally, Hecht and Bayly (2009) discussed how aerosol formation from concentrated non-Newtonian slurries is affected by a range of phenomena associated with the particles, their interaction, and the complicated rheology.

Overall, the literature on aerosol formation with slurries suggests that the slurry particles can affect droplet formation in ways that can both increase and decrease the size of the droplets.

\subsubsection{Effect of Surface Tension and Antifoam Agents}

The effect of reduced surface tension resulting from the use of antifoam agents (AFA) (i.e., blends of surface-active agents) was addressed by testing in the small-scale test system (Mahoney et al. 2012b), which is the companion to this study. The breakup of fast moving droplets into smaller droplets depends on the magnitude of the aerodynamic forces acting on the droplet. A number of previous studies 
(e.g., Hinze 1955, Pilch and Endman 1987, Eggers and Villermaux 2008) showed that the droplet breakup mechanism depends on the gas Weber number, $\mathrm{We}_{\mathrm{g}}$, which is the ratio of the disruptive aerodynamic force to the stabilizing surface tension force

$$
W e_{g}=\frac{\rho_{g} V^{2} D}{\sigma}
$$

where

$$
\begin{aligned}
\rho_{g} & =\text { the gas density } \\
V & =\text { the droplet velocity } \\
D & =\text { the droplet diameter } \\
\sigma & =\text { the surface tension of the fluid. }
\end{aligned}
$$

In general, increasing the Weber number causes breakup into smaller droplets (Pilch and Endman 1987).

For pure fluids, reducing the surface tension increases the Weber number, and this is expected to decrease the size of droplets formed by breakup. When surface-active agents such as AFAs are present and cause a reduction in the surface tension, it is important to consider the time scale for the interface formation. When the formation of an interface is rapid compared to the time it takes surface-active species to diffuse to and adsorb at the interface, the surface tension is different than the equilibrium value, and this time dependent surface tension often is called the dynamic surface tension (Berg 2010). The dynamic surface tension approaches that of the pure fluid as the time scale for interface formation becomes progressively shorter. Recently, Gauglitz et al. (2011) reviewed the literature on droplet breakup and estimated that the time scale for droplet breakup would be about $0.001 \mathrm{~s}$ and that the dynamic surface tension at this time scale would be expected to be essentially equal to that of water for aqueous systems. Accordingly, the conclusion of this review was that small quantities of surface-active species would not affect aerosol formation.

Dombrowski and Munday (1968) discussed the role of surfactants on droplet formation from fan sprays, and they similarly commented that the timescale for the breakup of the liquid sheet was less than $0.001 \mathrm{~s}$ and that the majority of the fan had a surface tension equal to that of water. The surfactant solutions studies by Dombrowski and Munday (1968) used hard water that generated a fine precipitate, so the tests showing the effect of adding surfactant actually demonstrated the combined effect of potential surface tension reduction and the presence of fine particulates. For the two surfactant concentrations reported, the lowest concentration ( 0.05 percent) gave larger droplet sizes when compared to water, and the higher concentration ( 0.25 percent) did not appear to change the droplet size. Bühler et al. (2001) studied the effect of two surfactants on droplet formation from hollow-cone and laval nozzles. These tests used different surfactant concentrations and generated sprays at pressures varying from $2 \times 10^{5}$ to $4 \times 10^{6} \mathrm{~Pa}$ (30 to $600 \mathrm{psig}$ ). They used a bubble tensiometer to determine the dynamic surface tension of the solutions from time scales below 0.01 to $0.4 \mathrm{~s}$. They commented that the droplet formation in this study was on the order of $0.01 \mathrm{~s}$, and at this time scale, all the solutions had a surface tension very near that of pure water. The aerosol size distributions were determined with a Malvern light-scattering instrument, and the results showed that adding surfactants caused a small or negligible increase of the droplet mean diameter. The conclusion from Bühler et al.'s (2001) work was that the addition of surfactants caused little change in droplet size distribution, compared to that for water, because the surface tension was equal to that of water at the time scale for droplet formation. 
Based on these results, it is expected that the addition of an AFA, which will reduce equilibrium surface tension, will have a negligible effect on droplet formation. Because the small-scale testing showed essentially no effect of AFA on aerosol formation (Mahoney et al. 2012b), the testing summarized in this report did not investigate the effect of AFA.

\subsubsection{Effect of Non-Newtonian Rheology}

As noted by Nasr et al. (2002), there are very little systematic data showing how non-Newtonian fluid properties affect aerosol formation in comparison to Newtonian fluids. Concentrated slurries will typically show non-Newtonian fluid properties, and can often be described as Bingham plastics (if they have a yield stress) or shear-thinning fluids (Poloski et al. 2004). Breitling et al. (2001) presented both CFD and experimental results for shear-thinning lime slurries sprayed from hollow-cone pressure swirl nozzles. The results showed little difference between the slurry and water. Mansour and Chigier (1995) evaluated air-blast atomization of shear-thinning visco-elastic and visco-inelastic polymer solutions. The shear-thinning visco-inelastic solutions, which can be considered similar to non-Newtonian slurries, generally showed behavior similar to Newtonian fluids with comparable viscosity. For these fluids, the SMD could be correlated with the high shear-rate limit of the viscosity and the SMD increased with increasing viscosity. Overall, these results suggest that high-pressure sprays of non-Newtonian slurries, with generally small particles, should behave similarly to Newtonian fluids with viscosities that match the high shear-rate viscosities of the slurries.

\subsection{Test Objectives}

Of the five objectives identified in the Spray Release Methodology Project Test Plan TP-WTPSP-031, Rev. 0.2, Objectives 4 and 5 specifically pertain to large-scale testing and are discussed below.

Objective 4: Determine the size distribution of aerosol droplets and the total droplet volume concentration as a fraction of the total spray volume for a range of circular and rectangular breach sizes (up to the largest postulated breach in a 3-in. pipe) with water.

Objective 5: Determine the size distribution of aerosol droplets and the total droplet volume concentrations as a fraction of the total spray volume for a range of circular and rectangular breach sizes (up to the largest postulated breach in a 3-in. pipe) with non-hazardous slurries (such as gibbsite/boehmite slurries in water) and liquids (such as sodium nitrate solutions).

\subsection{Task Methodology}

The task methodology designed to accomplish the objectives presented above includes a large-scale test system that was designed and built to investigate breach sizes up to the largest WTP postulated breach in a 3-in pipe. The system uses 3-in pipe because this is the most common pipe size in the WTP, and it is suitable for achieving the desired flow rates and associated pressures for studying large spray leaks. The system approach is described below:

- Injecting spray from the breach into an enclosed area (referred to as the chamber or enclosure) for a prescribed duration (assuming shorter durations for conditions generating larger breach flow rates) 
- Measuring the aerosol concentration and size distribution in real time via instrumentation within the enclosure

- Terminating flow from the breach

- Performing a mass balance using load cells to determine the flow rate through the breach

- Determining the release fraction using the chamber volume, the measured aerosol volume concentration, and material density to calculate the mass of suspended aerosols.

To evaluate the impact of associated parameters, the test setup was designed to accommodate the following:

- Flow rates of $>140$ gpm (i.e., a line velocity of $>6.5 \mathrm{ft} / \mathrm{sec}$ in a 3-in., schedule 40 pipe) upstream of the breach section for pipeline pressures ranging from 100 to 380 psig

- Variation in the distance between the breach and the end wall of the enclosure to allow an assessment of spray impingement on the aerosol results

- Variation in the total volume of the chamber to provide variation in the chamber concentration for a given breach flow condition and duration

- Supplemental mixing of the chamber air space to create a uniform aerosol concentration within the enclosure

- Documentation of the visual behavior of the sprays via video recordings and/or still pictures of the discharge from the breach.

\subsection{Success Criteria}

The testing will be deemed successful if the following criteria are met:

- The droplet size distribution, total volume sprayed, and release fraction are determined for each combination of breach size, flow condition, and simulant tested.

- The corresponding pressure and flow in the piping for each condition tested is measured and recorded.

- Each simulant tested is characterized for rheology, particle size distribution (PSD), bulk density, and surface tension. 



\subsection{Quality Assurance}

The PNNL QA program is based upon the requirements defined in the DOE 414.1D, Quality Assurance, and 10 CFR 830, Energy/Nuclear Safety Management, and Subpart A-Quality Assurance Requirements (a.k.a., the Quality Rule). PNNL has chosen to implement the following consensus standards in a graded approach:

- ASME NQA-1-2000, Quality Assurance Requirements for Nuclear Facility Applications, Part 1, Requirements for Quality Assurance Programs for Nuclear Facilities

- ASME NQA-1-2000, Part II, Subpart 2.7, Quality Assurance Requirements for Computer Software for Nuclear Facility Applications

- ASME NQA-1-2000, Part IV, Subpart 4.2, Graded Approach Application of Quality Assurance Requirements for Research and Development.

The procedures necessary to implement the requirements are documented through PNNL's "How Do I...?” $\left(\mathrm{HDI}^{4}\right)$ system.

The Waste Treatment Plant Support Project (WTPSP) implements an NQA-1-2000 Quality Assurance Program, graded on the approach presented in NQA-1-2000, Part IV, Subpart 4.2. The WTPSP Quality Assurance Manual (QA-WTPSP-0002) describes the technology life cycle stages under the WTPSP Quality Assurance Plan (QA-WTPSP-0001). The technology life cycle includes the progression of technology development, commercialization, and retirement in process phases of basic and applied research and development $(\mathrm{R} \& \mathrm{D})$, engineering and production, and operation until process completion. The life cycle is characterized by flexible and informal QA activities in basic research, which becomes more structured and formalized through the applied R\&D stages.

The work described in this report has been completed under the QA technology level of Developmental Work. WTPSP addresses internal verification and validation activities by conducting an Independent Technical Review of the final data report in accordance with WTPSP procedure QA-WTPSP-601, Document Preparation and Change. This review verifies that the reported results are traceable, that inferences and conclusions are soundly based, and that the reported work satisfies the test plan objectives.

\footnotetext{
${ }^{4} \mathrm{HDI}$ is a web-based system for managing the delivery of PNNL policies, requirements, and procedures.
} 



\subsection{Technical Basis for Estimating Aerosol Release Fraction}

\subsection{Release Fraction}

The primary goal of aerosol tests in both small- and large-scale test chambers is to determine the fraction of spray aerosolized as a function of orifice size and geometry, spray pressure, and fluid properties. The overall release fraction, $R F$, is defined as the ratio of volumetric rate of spray aerosolized to the total volumetric rate that spray is released through the engineered orifice. The mathematical expression (a rearrangement of Equation (1.7)) follows

$$
R F=\frac{\mathrm{G}}{Q_{\text {spray }}}
$$

where $G$ and $Q_{\text {spray }}$ are the aerosolized and total spray leak volumetric rates, respectively. Spray will generate a broad distribution of aerosol droplet size. To capture this size distribution, release fraction may be expressed in terms of a cumulative volume fraction undersize that has been aerosolized by spray as expressed below:

$$
R F_{k}=\frac{G_{k}}{Q_{\text {spray }}}
$$

where $R F_{k}$ is the cumulative release fraction under size classification $k$ and $G_{k}$ is the total volumetric rate of spray aerosolized that generate particles at or below size classification $k$. For a system with $\mathrm{N}$ total size classifications

$$
R F_{N}=R F
$$

Measurement of release fraction then requires that the overall rate of spray, $Q_{\text {spray }}$, from the engineered orifice be determined, which can be accomplished using differential mass or flow measurements. Both measurement approaches are used in large-scale testing. Determination of aerosolized volume, $G$ and $G_{k}$, relies on the available aerosol instrumentation. In the large-scale test setup, four aerosol analyzers (three Malvern Insitec-S instruments and one Process Particle Counter [PPC]) are used to measure local aerosol concentration and size distribution.

\subsection{Determination of Fraction of Spray Aerosolized}

The large-scale test chamber is an enclosed space into which a high-velocity jet is sprayed. Under the right conditions, the spray will aerosolize and circulate throughout the chamber. Continued spray yields continued aerosol production, thus facilitating a buildup of aerosol particles in the chamber volume over time. The rate of aerosol-particle generation from the spray represents the term $G$ that is sought by release fraction analysis. Aerosol particles generated by a spray remain in the system until they leave the test volume through convection, deposit onto the system boundaries (such as the test chamber wall) or internal surfaces (such as aerosol measuring equipment), or coalesce with other droplets. Overall, the rate of generation and loss yield a transient increase in the aerosol concentration in the chamber that eventually reaches an equilibrium value. 
The mechanism outlined above can be written as an aerosol balance equation for particles of a given size. The balance is best written for a single size classification of particles because size is a key parameter that determines how the particles are lost from the system. Let the size distribution of the system be classified into $\mathrm{N}$ differential size bins, $i$. Then the rate of change in the volume of spray aerosolized takes the following mathematical form:

$$
\frac{d v_{a, i}}{d t}=g_{i}-q_{r, i}
$$

where $v_{a, i}$ is the volume of spray aerosolized and $g_{i}$ and $q_{r, i}$ are the rate $\left(\mathrm{m}^{3} \mathrm{~s}^{-1}\right)$ at which spray are generated (aerosolized) and removed from the test chamber, respectively. All quantities are for a differential size bin, $i$. The aerosol analytical instruments measure local concentration of aerosol in terms of volume fraction dispersed phase. Thus,

$$
V \frac{d\left\langle x_{i}\right\rangle}{d t}=g_{i}-q_{r, i}
$$

where $V$ is the chamber volume $\left(\mathrm{m}^{3}\right)$ and $\left\langle x_{i}\right\rangle$ is the average concentration of aerosol in size bin $i$ in the chamber ( $\mathrm{m}^{3}$ of aerosol $/ \mathrm{m}^{3}$ of space).

Without adding a conceptual model of the behavior of aerosol in the test chamber, there is little additional specificity that can be added to Equation (3.5). However, it can be recognized that the processes yielding loss of aerosol generally depend on aerosol concentration. At the beginning of spraying, the processes that yield aerosol loss may be considered small relative to generation. This yields

$$
g_{i}=V \lim _{t \rightarrow 0} \frac{d\left\langle x_{i}\right\rangle}{d t}
$$

Equation (3.6) indicates the general methodology for evaluating the rate of aerosol generation in the large-scale test chamber. Having expressed all quantities using cumulative volume undersize, the following similar expression can be written:

$$
G_{k}=V \lim _{t \rightarrow 0} \frac{d\left\langle X_{k}\right\rangle}{d t}
$$

where $\left\langle X_{k}\right\rangle$ is the cumulative volume concentration of aerosol at and below size $k$. Equations (3.6) and (3.7) may be applied to data by assuming a constant rate of generation. Under these circumstances, the rate of generation is the slope of the linear rise in aerosol concentration at low durations. Although the approach is simple and straightforward, practical limitations prevent its application under all circumstances. Its key limitations are described below:

- For large sprays, the linear portion of the transient concentration may occur at durations that cannot be resolved by the aerosol instrumentation.

- The data may contain noise that prevents assessment of linearity and linear slope.

- For systems with high-loss potential, loss of aerosol may occur before sufficient data are available to evaluate the linear slope. 
For these reasons, it may be necessary to analyze aerosol concentration data in regions where loss of aerosol occurs. To facilitate this analysis, a simple conceptual model has been developed to account for system losses when determining generation rate.

\subsection{Conceptual Model for Evaluating Generation with Loss}

To develop a conceptual model for analyzing spray release data, the large-scale test chamber is considered a fully enclosed chamber into which the jet is sprayed. The conceptual model schematic is shown in Figure 3.1. Here, spray of a jet into the enclosed chamber creates an inhomogeneous liquid concentration profile, with the concentration of liquid being high in the vicinity of the liquid jet core and other fast moving droplets near the core and falling off with radial distance from the jet. A secondary source of spray occurs as the unbroken portion of the liquid core impacts the wall adjacent to spray generation.

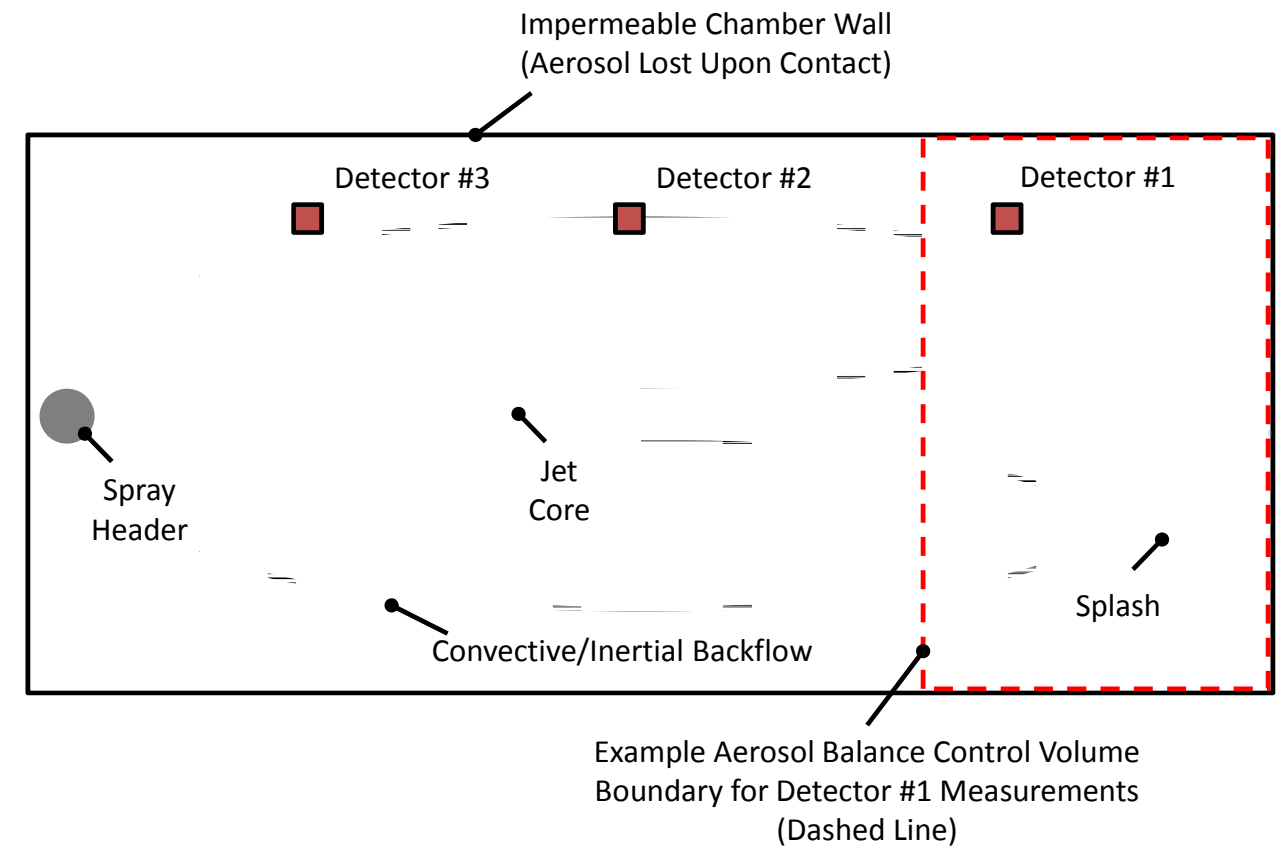

Figure 3.1. Schematic Representation of Spray Generation and Transport and Instrument Control Volumes for Simplified Rate of Generation and Loss Material Balances. Three detectors for measuring aerosol concentration and size distribution are shown. High-pressure spray generates aerosol both in-flight and after impact on the splash wall. The spray velocity sets up a convective back transport of aerosol through the test chamber. In addition, turbulence yields localized mixing. Impact of aerosol on test chamber walls and on the detector surfaces will lead to loss of droplets from the aerosolize volume.

To avoid the complexity of jet breakup mechanics, the model only considers the far-field concentration of aerosol; that is, the concentration of aerosol far from the region where inertial breakup (or other jet instability) or splatter generates spray droplets. When considered far from the spray, aerosol generation may be treated as a constant influx of spray droplets of given size to the control volume; the sizes of these droplets do not further decrease through additional breakup processes. 
Breakup of the jet both in-flight and upon impact with the splash wall yields a distribution of aerosol droplets of different size. This size distribution can change with time as aerosols are preferentially retained or removed from the system. If no mechanism for droplet loss exists, the aerosol concentration will increase linearly with time. For bounded systems, the aerosol concentration will increase until some equilibrium aerosol concentration is reached.

Aerosol loss derives from convection of aerosol out of the control volume considered and contact of aerosol with surfaces inside the control volume. Convection is driven by the spray; specifically, the jet induces air movement inside the chamber that can yield a convective back-transport and recirculation of the aerosolized fraction that can also disperse/mix the aerosol generated (through both turbulence and back-transport) throughout the chamber.

Changes to the far-field concentration occur through continued generation of aerosol or through loss of aerosol from the control volume. Loss is assumed to occur through several means:

- Convective transport of aerosol across the control volume boundary

- Deposition of aerosol on the surfaces of the test chamber, where contact is caused by convective transport of aerosol

- Settling of aerosol out of the control volume

- Coalescence or aggregation of aerosols into larger aerosol structures.

To determine rate equation forms for these losses, the far-field test chamber is treated as a continuously stirred tank reactor (CSTR). In the CSTR approximation, the control volume in which aerosol measurement is made is assumed to be homogeneous. Homogeneity results from instantaneous and complete mixing of the contents of the control volume. For the large-scale test chamber, control volume homogeneity is an assumption that was expected to be reasonable given the turbulent mixing provided by the high-velocity jet.

In addition, the form and functionality of loss equations (and even the loss mechanisms considered) will depend on the control volume assumed. For example, a control volume that considers the partial volume outlined in Figure 3.1 will need to consider convective transport of aerosol out of the control volume and loss of aerosol to the walls. If the entire box volume is considered, then only loss to the walls must be considered. For the latter case, difficulties arise as the assumption of CSTR suggests that the concentration at all three detectors should be the same, which is not observed in actual measurements.

To avoid the difficulties in setting an appropriate control volume for each detector, a general equation is derived in the sections that follow that will allow aerosol concentration and size data to be fit and analyzed. This necessitates consideration of all loss mechanisms derived above. It also requires assigning a representative (i.e., homogeneous as described above) concentration to the chamber, which was accomplished by averaging the detector concentrations together. The basis for the concentration averaging is provided in Appendix C.

\subsubsection{Generation of Aerosol}

Aerosol generation derives from the breakup mechanics of a spray through an engineered orifice. The rate of aerosol generation at any given instance may not exceed the volumetric flow rate of the leak, 
$Q_{\text {spray. }}$ In addition, aerosol generation may occur by one of two mechanisms: 1) breakup of the spray in-flight, and 2) breakup upon impact at the far end of the chamber (termed the splash wall) if the jet has sufficient velocity to reach the splash wall or break up upon impact with some other termination point (such as an obstruction in the path of the jet or the floor of the chamber). Depending on how the control volume is selected, both mechanisms might contribute to the rate of spray generation so that the generation rate, $g_{i}$, for particles of size classification $i$, is expressed as:

$$
g_{i}=\left(b_{f} f_{f, i}+b_{m} f_{m, i}\right) Q_{\text {spray }}
$$

Here, $b_{f}$ and $b_{m}$ represent the fraction of the total spray volumetric rate aerosolized in-flight and by impact, respectively. In-flight and impact breakup events generate an aerosol with volumetric size distributions represented by $f_{f, i}$ and $f_{m, i}$, respectively ( $\mathrm{m}^{3}$ of aerosol with size $i / \mathrm{m}^{3}$ of total aerosol). In general, in-flight and impact breakup events may not be distinguishable, and as such, the rate of generation in the far field is simplified as follows:

$$
g_{i}=b f_{i} Q_{\text {spray }}
$$

While this notation is not necessary for determining generation rates from modeling, it does have implications for the interpretation of release fraction. The cumulative generation of droplets is

$$
G_{k}=b F_{k} Q_{\text {spray }}
$$

where $F_{k}$ is the cumulative volume fraction undersize $k\left(\mathrm{~m}^{3}\right.$ of aerosol with size less than $k / \mathrm{m}^{3}$ of total aerosol). When $F_{k}=1, G_{k}=G$ because all the droplets sizes are cumulated in the generation rate. Thus, substitution of Equation (3.10) into Equations (3.1) and (3.2), respectively, yields

$$
\begin{gathered}
R F=b \\
R F_{k}=b F_{k}
\end{gathered}
$$

These relationships demonstrate that the overall release fraction represents, as expected, the fraction of spray aerosolized, and the cumulative undersize release fraction is the product of the PSD generated by the spray and the fraction of spray aerosolized. Equations (3.11) and (3.12) will form the basis for simplified methods for estimating release fractions for sprays where the time to reach equilibrium occurs more quickly than aerosol measurements can be made.

\subsubsection{Convective Transport, Deposition, and Settling}

Convective transport across control volume boundaries results from directional convection such as that from displacement of chamber air space by the volume of slurry sprayed and by flow fields (such as rotational flow set up by the jet). For convective deposition, aerosol is assumed to be lost from the dispersed phase when it comes into contact with a wall. There are two basic mechanisms for contact: 1) convective transport of aerosol into a wall, and 2) settling of aerosol particles under the influence of gravity. Under the CSTR assumption, the loss rates from convective transport, deposition, and settling all take similar form

$$
q_{r, i}(\text { transport })=U_{c} A_{x} x_{i}
$$




$$
\begin{gathered}
q_{r, i}(\text { deposition })=\phi_{d} U_{c} A_{s} x_{i} \\
q_{r, i}(\text { settling })=U_{s, i} A_{f} x_{i}
\end{gathered}
$$

For the transport equation, $U_{c}$ is the convective velocity and $\underline{A}_{\underline{X}}$ is the cross-section area for convective flow. For deposition, $\phi_{\mathrm{d}}$ is a coefficient that captures the fraction of aerosol impinged on the total surface area of the control volume and $A_{s}$ is the effective deposition area. It should be noted that the effective deposition is not limited to hard fixed surfaces, but may also include droplet interactions with the unbroken core of the jet. Finally, for settling $U_{s, i}$, is the settling velocity of a particle in size bin $i$, and $A_{f}$ is the effective cross-section area onto which the aerosol droplets settle. An important common feature of these convective losses is that they are all first order with respect to aerosol concentration $x_{i}$.

\subsubsection{Coalescence of Aerosol Droplets}

Apparent aerosol droplet loss can result from coalescence of aerosol drops. Coalescence/aggregation requires that two or more aerosol particles collide and possess energetically favorable kinetics for merging into a single droplet or aggregate particle. Particle collisions can occur both as a result of convective flow of the aerosol carrier gas (typically as a result of flow turbulence) or as a result of settling, whereby fast settling large particles sweep up and capture smaller droplets in their settling path. Coalescence is generally a two-particle interaction, and for a single coalescence event involving two particles in size classes $i$ and $j$, it nominally exhibits second-order kinetics, such that

$$
q_{r, i}(\text { coalescence })=k_{c, i j} x_{i} x_{j}
$$

Here $q_{r, i}$ represents the loss particles in size bin $i$. Loss of particles in size classification $i$ is also accompanied by a similar loss of particles in size class $j$,

$$
q_{r, \mathrm{j}}(\text { coalescence })=k_{c, i j} x_{i} x_{j}
$$

Coalescence of two particles generates a single larger particle in size class $k$. In these two equations, the constant $\mathrm{k}_{\mathrm{c}, \mathrm{ij}}$ is related to the frequency by which two-particle interactions yield coalescence for size classes $i$ and $j$, and $x_{i}$ and $x_{j}$ are the volumetric concentration of particles in these two size classes. In the absence of other loss mechanisms, coalescence yields a net decrease in the number of total aerosol particles but does not yield an overall decrease in the total volume of aerosol droplets. More specifically, coalescence represents a transfer of aerosol volume to larger size classifications rather than a true source of aerosol generation or loss from the control volume. This means that the cumulative loss/generation of aerosol volume resulting from coalescence is always zero.

Coalescence is nominally second-order, as expressed in Equations (3.16) and (3.17), such that the rate of coalescence depends on the concentration of both size class droplets. However, when expressed in terms of drop volume concentration in a binned size system, these second-order equations may not always be achieved, especially when a significant size difference exists in the droplets that collide. Consider a large droplet that travels through the chamber under the influence of settling. As it falls, it sweeps up and coalesces with several smaller droplets in its settling path. If the large droplet is $100 \mu \mathrm{m}$ and absorbs ten $10 \mu \mathrm{m}$ droplets, the size of the large droplet only increases to $100.3 \mu \mathrm{m}$. For typical size bins used in aerosol analysis, both 100 and $100.3 \mu \mathrm{m}$ will fall into the same size classification. As a result, only one 
size classification (i.e., that which contains the $10 \mu \mathrm{m}$ droplets) will show a loss of droplet volume rather than the two size classifications shown in Equations (3.16) and (3.17). In addition, if the volume concentration of either species is large relative to the other, then coalescence will not impact the concentration of the major species, yielding apparent first-order kinetics. Finally, the coalescence process does not continue indefinitely in either large- or small-scale test enclosures. If an aerosol droplet formed by many subsequent coalescence events is not removed from the system by deposition or convective transport, then coalescence will eventually yield a droplet size that is subject to removal by inertial or settling. As such, the extent to which coalescence can transfer aerosol volume to progressively larger size classifications will be arrested by processes that remove those large droplets. Overall, the exact rate equation required for transfer and loss that results from coalescence is not well defined for systems with binned size classes. It may appear as second-order with loss from two size classes, second-order with loss from one size classification, or as apparent first-order if one size classification dominates.

Accounting for coalescence loss and generation for individual size classifications greatly increases the complexity of the material-balance equations. The need to include coalescence terms in the model depends on the overall droplet concentrations being examined. Typical large-scale tests evidence far-field total aerosol concentrations on the order of $10^{-6}$ (volume aerosol droplets per total chamber volume). Aerosol concentrations for specific size classifications are typically at least an order-of-magnitude lower (if not more). At these concentrations, the frequency of second-order actions will likely be low relative to first-order reactions. This, combined with the fact that the overall impact of coalescence on the cumulative volume concentration is zero, means that particle loss to aerosol generation can likely be neglected in far-field considerations of aerosol dynamics.

\subsection{Overall Material Balance}

The overall aerosol balance is derived by considering the sum of generation and loss terms. Based on the arguments outlined above, the overall aerosol material balance is

$$
V_{m} \frac{d x_{i, m}}{d t}=g_{i}-\left[U_{c} A_{x} x_{i, m}+\phi_{d} U_{c} A_{s} x_{i, m}+U_{s, i} A_{f} x_{i, m}\right]
$$

Here, the subscript $m$ refers to a local aerosol concentration measurement, where the value of $m(1-4)$ refers to the particular Insitec-S or PPC aerosol analyzer that made the measurement. The volume, $V_{m}$, represents the control volume associated with aerosol instrument $m$. Because quantifying the amount lost via each mechanism is not necessary for the data analysis, the loss terms may be lumped into a single term for simplicity without losing information relevant to determining the aerosol generation rate. As such,

$$
V_{m} \frac{d x_{i, m}}{d t}=g_{i}-\kappa_{i} x_{i, m}
$$

where $\kappa_{i}$ is the lumped loss coefficient. The subscript $i$ is included to indicate that the loss coefficient depends on particle size. The solution to this differential equation is

$$
x_{i, m}=\frac{h_{i}}{\lambda_{i}}\left(1-e^{-\lambda_{i} t}\right)
$$

where $h_{i}=g_{i} / V_{m}$ and $\lambda_{i}=\kappa_{i} / V_{m}$. An initial aerosol concentration of $x_{i, m}(0)=0$ has been assumed. From Equation (3.20), it can be seen that the equilibrium concentration is $x_{i, m}^{e q}=h_{i} / \lambda_{i}$. 
Equation (3.20) can be used to analyze the dynamics of aerosol concentration at all times, including the initial period when the concentration is increasing and during the approach to equilibrium. It is useful for sprays in which the aerosol concentration increases more rapidly than the aerosol instrumentation can measure so the initial slope cannot be accurately assessed from data.

Equation (3.20) expresses the aerosol material balance in differential form. The cumulative aerosol concentration is given by

$$
X_{k, m}=\sum_{i=1}^{k} x_{i, m}
$$

Substitution of Equation (3.20) yields

$$
X_{k, m}=X_{k, m}^{e q}\left(1-\frac{1}{X_{k, m}^{e q}} \sum_{i=1}^{k} x_{i, m}^{e q} e^{-\lambda_{i} t}\right)
$$

The range of exponentials in the sum typically can be approximated well by a single rate constant. If a single rate constant is assigned, Equation (3.22) is reduced to

$$
X_{k, m}=\frac{H_{k}}{\Lambda_{k}}\left(1-e^{-\Lambda_{k} t}\right)
$$

where Equation (3.23) has adopted the notation of Equation (3.20). Here, $H_{k}=G_{k} / V_{m}$ and $\Lambda_{k}=\mathrm{K}_{k} / V_{m}$. 


\subsection{Simulants}

This chapter lists the waste simulants used in the large-scale aerosol tests, states the basis for their selection, describes the physical property measurement methods, and reports the measured physical properties of the simulants. The simulants and the basis for selection are discussed in Section 4.1. The physical property measurement methods and the resulting measurements are reported in Section 4.2.

\subsection{Simulants Used}

Table 4.1 summarizes the WTP process streams and typical ranges for important fluid properties. ${ }^{5}$ The ranges of properties and descriptions are only representative, and specific waste examples may vary from these generalizations. These are the process streams that were represented by simulants in the spray release testing. The non-Newtonian simulants represent slurries that are expected to be in the vessels commonly referred to as the non-Newtonian vessels. These include the ultrafiltration feed vessels (UFP-VSL-00002 A/B) and the high-level waste lag storage and blend vessels (HLP-VSL-0027 A/B and -0028). During some of the process steps the slurries in the ultrafiltration feed vessel are expected to exhibit a Newtonian rheology. Some of the other vessels are expected to contain Newtonian slurries and include (but are not limited to), the high-level waste receipt vessel (HLP-VSL-00022) and the ultrafiltration feed preparation vessels (UFP-VSL-0001 A/B).

Table 4.1. WTP Process Stream Categories and Representative Fluid Properties

\begin{tabular}{|c|c|c|c|}
\hline WTP Process Stream Categories & Particles & Composition & Viscosity Rheology \\
\hline $\begin{array}{l}\text { Ultrafilter permeate treated low activity } \\
\text { waste (LAW) }\end{array}$ & Negligible & $\begin{array}{l}\text { Caustic solution } \\
\text { 5-10 M Na }\end{array}$ & $\begin{array}{l}\text { Newtonian } \\
2-3 \mathrm{cP}\end{array}$ \\
\hline Cs ion exchange eluate & Negligible & $\begin{array}{c}\mathrm{Na}, \mathrm{K}, \mathrm{Cs} \text { ions with } \\
0.5 \mathrm{M} \mathrm{HNO}_{3}\end{array}$ & $\begin{array}{l}\text { Newtonian } \\
0.5 \mathrm{cP} \text { and above }\end{array}$ \\
\hline Recycle streams & $<2 \mathrm{wt} \%$ & $0.2-2 \mathrm{M} \mathrm{Na}$ & $\begin{array}{l}\text { Newtonian } \\
0.5 \mathrm{cP} \text { and above }\end{array}$ \\
\hline Newtonian slurries & $\begin{array}{l}\text { Approximately } \\
\text { 2-16 wt\% }{ }^{(\mathrm{a})}\end{array}$ & Up to $8 \mathrm{M} \mathrm{Na}$ & $\begin{array}{l}\text { Newtonian }^{(\mathrm{b})} \\
\text { about } 1-3 \mathrm{cP}\end{array}$ \\
\hline Non-Newtonian slurries & $\begin{array}{l}\text { Up to } \\
\sim 20 \mathrm{wt} \%\end{array}$ & $0.2-2 \mathrm{M} \mathrm{Na}$ & $\begin{array}{c}\text { Non-Newtonian } \\
6 \mathrm{cP} / 6 \mathrm{~Pa} \text { to } \\
30 \mathrm{cP} / 30 \mathrm{~Pa}\end{array}$ \\
\hline
\end{tabular}

(a) The upper limit of $16 \mathrm{wt} \%$ corresponds to a limit of $200 \mathrm{~g} / \mathrm{L}$ in the waste acceptance criteria (ICD-19 2011). A new upper limit of $144 \mathrm{~g} / \mathrm{L}$ in a $7 \mathrm{M}$ Na feed has been recommended and this corresponds to about $10 \mathrm{wt} \%$ solids (Campbell et al. 2010).

(b) This category also could be a weak non-Newtonian fluid based on the feed acceptance criterion that allows up to $1 \mathrm{~Pa}$ Bingham yield stress slurries to be delivered to the WTP (ICD-19 2011).

WTP = Hanford Waste Treatment and Immobilization Plant.

\footnotetext{
${ }^{5}$ These categories and ranges of process parameters were provided by the WTP client as guidance for proposal preparation.
} 
Table 4.2 lists the target simulants presented in the test plan (TP-WTPSP-031) for the spray release testing effort. ${ }^{6}$ The four simulant classes and materials were chosen to represent the range of wastes shown in Table 4.1. The last column in Table 4.2 shows how each simulant represents one or more of the WTP process stream categories. Of the four classes of wastes listed, three were tested in the large-scale tests. Water was used for shakedown and baseline testing. One aqueous sodium thiosulfate solution was chosen to represent process streams in the WTP that are Newtonian fluids but with a higher viscosity. These include the ultrafilter permeate/treated LAW, Cs ion exchange eluate, and recycle streams. The non-hazardous slurry that was tested was 8 and $20 \mathrm{wt} \%$ boehmite in water. No chemical slurry simulant was tested in the large-scale system. The types of simulants tested were selected carefully to provide test data for each simulant class within the schedule, budget, and safety considerations associated with 500 gal batches of simulant. These constraints included chemical cost, disposal cost, time for preparation, time for cleaning, and chemical hazards. A greater number of simulants were tested in the small-scale spray leak tests, including the chemical slurry simulant (Mahoney et al. 2012a, 2012b).

Table 4.2. Target Simulants and the WTP Process Stream Categories

\begin{tabular}{|c|c|c|c|}
\hline Simulant Class & Material & Target Property Range & $\begin{array}{c}\text { WTP Process } \\
\text { Stream Categories }\end{array}$ \\
\hline Baseline & Water & $\begin{array}{l}\text { Viscosity } 1 \mathrm{mPa} \cdot \mathrm{s}(1 \mathrm{cP}) \\
\text { Density } 1000 \mathrm{~kg} / \mathrm{m}^{3} \\
\text { Surface tension } 73 \mathrm{mN} / \mathrm{m}\end{array}$ & Rinse waters \\
\hline $\begin{array}{l}\text { Range of } \\
\text { Newtonian } \\
\text { viscosity }\end{array}$ & $\begin{array}{l}\text { Solutions of water and } \\
\text { non-hazardous salts (sodium } \\
\text { nitrate and sodium thiosulfate) }\end{array}$ & $\begin{array}{l}\text { Viscosities of } ~ 1.5, \\
\sim 2.5 \mathrm{mPa} \cdot \mathrm{s}(1.5,2.5 \mathrm{cP})\end{array}$ & $\begin{array}{l}\text { Ultrafilter permeate/ } \\
\text { treated LAW } \\
\text { Cs ion exchange eluate } \\
\text { recycle streams }\end{array}$ \\
\hline $\begin{array}{l}\text { Range of } \\
\text { slurries (non- } \\
\text { hazardous) }\end{array}$ & $\begin{array}{l}\text { Gibbsite, boehmite, or other } \\
\text { non-hazardous particulates in } \\
\text { water or dilute salt solutions }\end{array}$ & $\begin{array}{l}\text { PSDs of slurries were selected } \\
\text { to match Hanford waste PSDs } \\
8 \text { and } 20 \mathrm{wt} \% \text { solids }\end{array}$ & $\begin{array}{l}\text { Newtonian and } \\
\text { non-Newtonian slurries }\end{array}$ \\
\hline $\begin{array}{l}\text { Washed and } \\
\text { leached } \\
\text { chemical slurry } \\
\text { simulant }\end{array}$ & $\begin{array}{l}\text { A washed and leached version of } \\
\text { the simulant used in } \\
\text { Pretreatment Engineering } \\
\text { Platform (PEP) testing (Kurath } \\
\text { et al. 2009) }\end{array}$ & $\begin{array}{l}\text { Solids loading were adjusted } \\
\text { in an attempt to meet target } \\
\text { Bingham yield stresses of } 6 \\
\text { and } 30 \mathrm{~Pa}\end{array}$ & Non-Newtonian slurries \\
\hline
\end{tabular}

The non-hazardous slurry simulant chosen for large-scale testing, small treated Hanford waste (STR), was based on the PSD of the post-caustic leached and washed M12 samples from Wells et al. (2011). Figure 4.1 shows the sonicated PSDs for this material by waste group or mixtures of waste groups. Because treatment of the samples removed solids that were susceptible to leaching and washing, all samples for which there were data, including the saltcake groups were considered. The individual PSDs for the waste groups differ from each other to a significant extent as shown in Figure 4.1. The PSD of the Group 1/2 Mixture is noticeably the smallest of all of the PSDs. Because the slurry with the smallest PSD is least likely to plug a breach, the Group 1/2 Mixture was considered to be a conservative and appropriate target PSD to consider for spray release behavior from treated waste.

\footnotetext{
${ }^{6}$ Gauglitz PA. 2011. Test Plan for Spray Leak Quantification to Support WTP Spray Release Methodology, TP-WTPSP-031, R0.2, Pacific Northwest National Laboratory, Richland, Washington.
} 


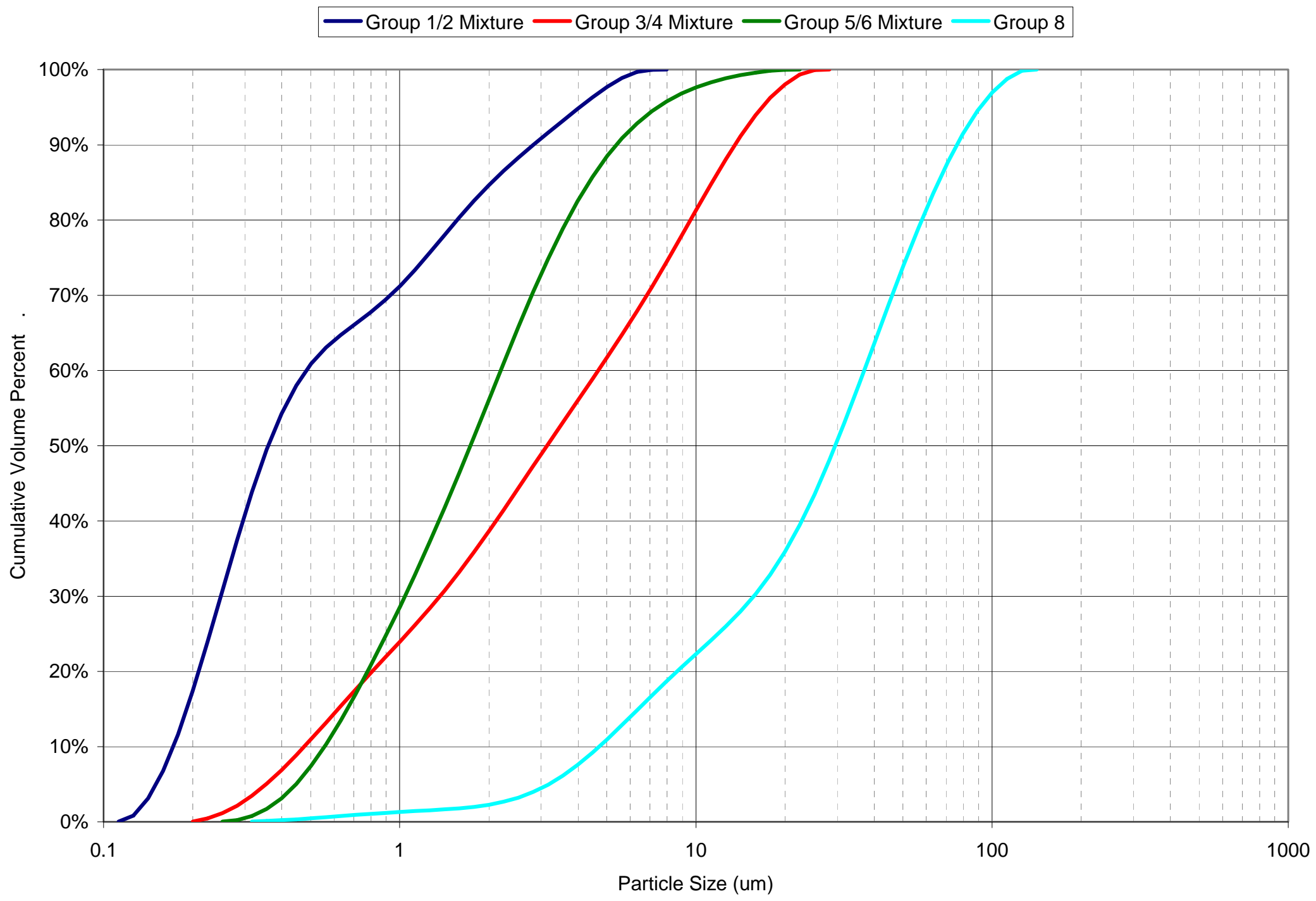

Figure 4.1. Flowing Sonicated PSDs for Post-Caustic Leached and Washed Waste (Exception: Group 8 was measured using flowing unsonicated instrument settings) 


\subsection{Simulant Properties}

The physical properties were measured from samples collected before, during, and after testing for the various simulants. The measurement techniques are described in Section 4.2.1, and the results are presented for the Newtonian simulants and the small treated simulant in Sections 4.2.2 and 4.2.3, respectively.

\subsubsection{Analytical Techniques}

Samples were characterized for physical and rheological properties including PSD, surface tension, bulk density, yield stress, and consistency. Sample analyses, except surface tension measurements, were performed according to the test procedure RPL-COLLOID-02, Rev. 2, Measurement of Physical and Rheological Properties of Solutions, Slurries and Sludges. Surface tension measurements were conducted according to operating procedure OP-WTPSP-035, Rev. 0, Measurement of Static Surface Tension of Liquids, Dispersions, and Slurries.

The PSD measurements were made using a Mastersizer 2000 Particle Size Analyzer (Malvern Instruments, Inc.) with a Hydro S wet-dispersion accessory. The particle size measurement range was nominally 0.02 to $2000 \mu \mathrm{m}$. The Mastersizer 2000 Particle Size Analyzer uses laser diffraction technology. The Hydro S wet-dispersion accessory consisted of a 150-mL sonic dispersion unit coupled with a sample flow cell, allowing the flow, stirring rate, and sonication to be controlled and altered during measurement. The PSD measurements were performed on slurry samples containing solids dispersed in a liquid, and were made with and without sonication.

Rheological characterizations were performed using a Haake RS600 rheometer operated with RheoWin Software (Thermo Electron Corporation). The RS600 rheometer is equipped with a low-inertia torque motor and coaxial cylinder measurement geometry. The drive shaft of the motor is centered by an air bearing that ensures an almost frictionless transmission of the applied torque to the sample. Unless specified otherwise, all rheological analyses were conducted at $25^{\circ} \mathrm{C}$. Each rheogram (i.e., flow curve) was obtained by shearing the sample at a controlled rate from zero to $1000 \mathrm{~s}^{-1}$ for $5 \mathrm{~min}$, holding constant at $1000 \mathrm{~s}^{-1}$ for $1 \mathrm{~min}$, and then shearing at a controlled rate from $1000 \mathrm{~s}^{-1}$ to zero for $5 \mathrm{~min}$. Prior to measuring a flow curve, the sample was gently shaken by hand and sheared at a constant $250 \mathrm{~s}^{-1}$ rate for $3 \mathrm{~min}$. The purpose of pre-measurement mixing was to homogenize the material being analyzed; thereby obtaining a representative sample.

More detail about flow curve measurement and PSD methods can be found in Appendix E, Sections E.1.2.3 and E.1.2.4 of Kurath et al. (2009).

Surface tension measurements were performed using a commercial force-balance K-12 MK6 Tensiometer (Kruss USA). The tensiometer consisted of a K-12 MK6 tensiometer processor unit, a force measuring unit (the balance), a Wilhelmy platinum plate, and a quartz sample vessel. Static surface tension of a sample was measured using the plate method (also known as the Wilhelmy method), which is based on a force measurement. A platinum plate with a well-known geometry was vertically suspended above the sample liquid. The lower edge of the plate then was brought into contact with the sample liquid surface. The sample liquid wets the plate and pulls it slightly into the liquid by the Wilhelmy force, which results from the wetting. The Wilhelmy force is measured by moving the plate up to the level of 
the sample liquid surface. The resulting force was determined from the weight measured by the balance. Surface tension measurements for all samples were carried out at room temperature.

\subsubsection{Newtonian Simulant}

Table 4.3 documents the composition and properties of the two Newtonian simulants used during aerosol testing. The simulants were a solution of tap water from the Richland, Washington, municipal water system and $\mathrm{Na}_{2} \mathrm{~S}_{2} \mathrm{O}_{3}$ salt as described in the table. As expected, the solids in the salt simulant were observed to be fully dissolved and neither of the Newtonian simulants was analyzed for PSD or total solids (TS). The surface tension of the tap water was measured as $72.25 \pm 0.07 \mathrm{mN} \cdot \mathrm{m}^{-1}$, which agrees with values documented in the literature. For the sodium thiosulfate $\left(\mathrm{Na}_{2} \mathrm{~S}_{2} \mathrm{O}_{3}\right)$ simulant, the measured viscosity was $2.60 \pm 0.01 \mathrm{mPa} \cdot \mathrm{s}$ and the measured surface tension was $78.30 \pm 0.26 \mathrm{mN} \cdot \mathrm{m}^{-1}$.

Table 4.3. Newtonian Simulant Properties for Large-Scale Aerosol Tests

\begin{tabular}{lcc}
\hline \multicolumn{1}{c}{ Properties/Simulant ID } & Water & $\mathrm{Na}_{2} \mathrm{~S}_{2} \mathrm{O}_{3}$ \\
\hline $\mathrm{Na}_{2} \mathrm{~S}_{2} \mathrm{O}_{3} \cdot 5 \mathrm{H}_{2} \mathrm{O}(\mathrm{kg})$ & --- & 1013 \\
Richland city water $(\mathrm{kg})$ & $1800-2250$ & 1343 \\
Target solution density $(\mathrm{kg} / \mathrm{L})$ & $0.998^{(\text {a) }}$ & 1.245 \\
Measured solution density $(\mathrm{kg} / \mathrm{L})$ & Not measured & $1.244 \pm 0.002$ \\
Reference surface tension $(\mathrm{mN} / \mathrm{m})$ & 72.14 & $77.32(\mathrm{est})$ \\
Measured surface tension $(\mathrm{mN} / \mathrm{m})$ & $72.25 \pm 0.07$ & $78.30 \pm 0.26^{(\text {b) }}$ \\
Target viscosity (mPa·s) & $1.002^{(\text {a) }}$ & 2.50 \\
Viscosity (Newtonian fluid) $(\mathrm{mPa} \cdot \mathrm{s})$ & Not measured & $2.60 \pm 0.01$ \\
\hline (a) Water bulk density and viscosity at $20^{\circ} \mathrm{C}$ from the CRC Handbook, $56^{\text {th }}$ Edition, \\
pp. F-11 and F- 47. & & \\
(b) Surface tension for $\mathrm{Na}_{2} \mathrm{~S}_{2} \mathrm{O}_{3}$ is the surface tension of the filtered simulant.
\end{tabular}

\subsubsection{Small Treated (STR) Hanford Waste Simulant}

Table 4.4 displays the composition and properties of the STR simulants used during large-scale aerosol testing. First, the STR waste simulant was blended from Richland city water and boehmite particles (80 wt\% Nabaltec Actilox B60 ${ }^{7}$ and $20 \mathrm{wt} \%$ NOAH Technologies R6000) at a concentration of $20 \mathrm{wt} \%$ solids (STR 20). The simulant was then diluted to achieve $8 \mathrm{wt} \%$ solids (STR 8) according to Test Instruction TI-WTPSP-063.

The STR 8 simulant exhibited a Bingham yield stress of $1.27 \pm 0.13 \mathrm{~Pa}$ and a Bingham consistency of $2.81 \pm 0.10 \mathrm{mPa} \cdot \mathrm{s}$. At the higher concentration, the STR 20 simulant appeared to be a Newtonian fluid with a Bingham yield stress of $0.05 \pm 0.01 \mathrm{~Pa}$ and a Bingham viscosity of $1.59 \pm 0.02 \mathrm{mPa} \cdot \mathrm{s}$. This is contrary to the expected behavior in which an increasing solids concentration leads to a greater yield stress and a higher consistency. These results were confirmed by analyzing replicate aliquots of several samples of the simulant materials. Similar results also were noted for an early batch of the STR simulant used in small-scale testing (Mahoney et al. 2011). There is no obvious explanation for these results, but

\footnotetext{
${ }^{7}$ Nabaltec Actilox B60 is equivalent to Apyral AOH60. They are different trade names of the same product.
} 
the difference may be related to the shearing of the simulants within the large-scale spray release system. The wt\% TS was close to the targeted values for both concentrations of the STR simulant with values of $8.16 \pm 0.03$ and $19.68 \pm 0.06 \mathrm{wt} \%$ TS for STR8 and STR 20, respectively. The bulk densities were in agreement with the calculated theoretical densities. The surface tension of the simulant supernates was measured after centrifuging and filtering aliquots of the supernate through a $0.45 \mu \mathrm{m}$ syringe filter. The surface tension was found to be slightly less than that of water.

Table 4.4. Small Treated (STR) Simulant Properties for Aerosol Tests

\begin{tabular}{lcc}
\hline \multicolumn{1}{c}{ Component/Property } & STR 8 & STR 20 \\
\hline Nabaltec Actilox B60 boehmite (kg) & 127 & 349 \\
NOAH Technologies R6000 boehmite (kg) & 31.8 & 87.4 \\
Richland city water (kg) & 1840 & 1750 \\
Targeted wt \% TS (\%) & 8.00 & 20.0 \\
Measured wt \% TS (\%) & $8.16 \pm 0.03$ & $19.68 \pm 0.06$ \\
Calculated slurry density (kg/L) & 1.056 & 1.154 \\
Measured slurry density (kg/L) & $1.055 \pm 0.002$ & $1.149 \pm 0.002$ \\
Surface tension (mN/m) & $71.05 \pm 0.23^{(\mathrm{a})}$ & $69.68 \pm 0.18^{(\mathrm{a})}$ \\
Bingham yield stress (Pa) & $1.27 \pm 0.13$ & $0.05 \pm 0.01$ \\
Bingham consistency (mPa·s) & $2.81 \pm 0.10$ & $1.59 \pm 0.02$ \\
\hline
\end{tabular}

(a) Surface tension for STR is the surface tension of the filtered supernate.

The PSDs of the STR 8 and STR 20 simulants are presented according to selected percentiles in Table 4.5 along with the target PSD of the Group 1/2 Mixture. The STR 8 and STR 20 results are based on the average of nine and twelve PSD measurements, respectively, generated from two aliquots each of two different samples of each simulant. The simulant particle sizes were measured under conditions of flow and sonication, as well as unsonicated and post-sonicated. The unsonicated data was selected for comparison and reporting because the unsonicated measurements were judged to be the most consistent across all samples. The sonicated and post-sonication results had varying populations of unexpected large particles (>100 $\mu$ m diameter) that tended to skew the cumulative results. Such populations can result from bubble and agglomerate formation during sonication.

The measured PSDs are in good agreement between the two simulant concentrations and are consistently higher than the target PSD over the range of percentiles. Figure 4.2 shows the measured cumulative volume percent undersize of the 8 and $20 \mathrm{wt} \%$ STR simulants (unsonicated) together with the target PSD for the STR simulant (the Group 1/2 PSD shown in Figure 4.1). The cumulative volume percent undersize data also are presented in Table 4.6. All representations of the results and comparisons to the target suggest that the measured PSD of the STR simulants was representative of the target PSD of the Group 1/2 Mixture. 
Table 4.5. Summary of Selected Percentile Values for Measurements Taken at 1 Min Recirculation Time, Unsonicated, STR 8 and STR 20 Simulants with a Comparison to the Target PSD

\begin{tabular}{cccc}
\hline & Target & STR 8 & \multicolumn{1}{c}{ STR 20 } \\
\cline { 2 - 4 } & PSD & 8 wt\% & 20 wt\% \\
\cline { 2 - 4 } Percentiles & Sonicated $^{(\mathrm{a})}$ & Unsonicated $^{(\mathrm{a})}$ & Unsonicated $^{(\mathrm{a})}$ \\
\cline { 2 - 4 } $\mathrm{d}(0.01)$ & 0.127 & Percentile Size, $\mu \mathrm{m}$ \\
$\mathrm{d}(0.05)$ & 0.150 & 0.389 & 0.337 \\
$\mathrm{~d}(0.10)$ & 0.172 & 0.498 & 0.422 \\
$\mathrm{~d}(0.25)$ & 0.229 & 0.595 & 0.498 \\
$\mathrm{~d}(0.50)$ & 0.360 & 1.33 & 0.697 \\
$\mathrm{~d}(0.75)$ & 1.22 & 2.30 & 1.08 \\
$\mathrm{~d}(0.90)$ & 2.83 & 5.19 & 1.86 \\
$\mathrm{~d}(0.95)$ & 4.06 & 13.1 & 4.10 \\
$\mathrm{~d}(0.99)$ & 5.73 & 56.9 & 7.17 \\
$\mathrm{~d}(1.00)$ & 7.96 & 267 & 36.4 \\
\hline
\end{tabular}

(a) Targeted PSD is based on a flowing sonicated PSD of the Group 1/2 Mixture actual waste because this approach gives the most representative and conservative PSD. However, when approaching the simulant samples the unsonicated data give the most consistent and accurate results.

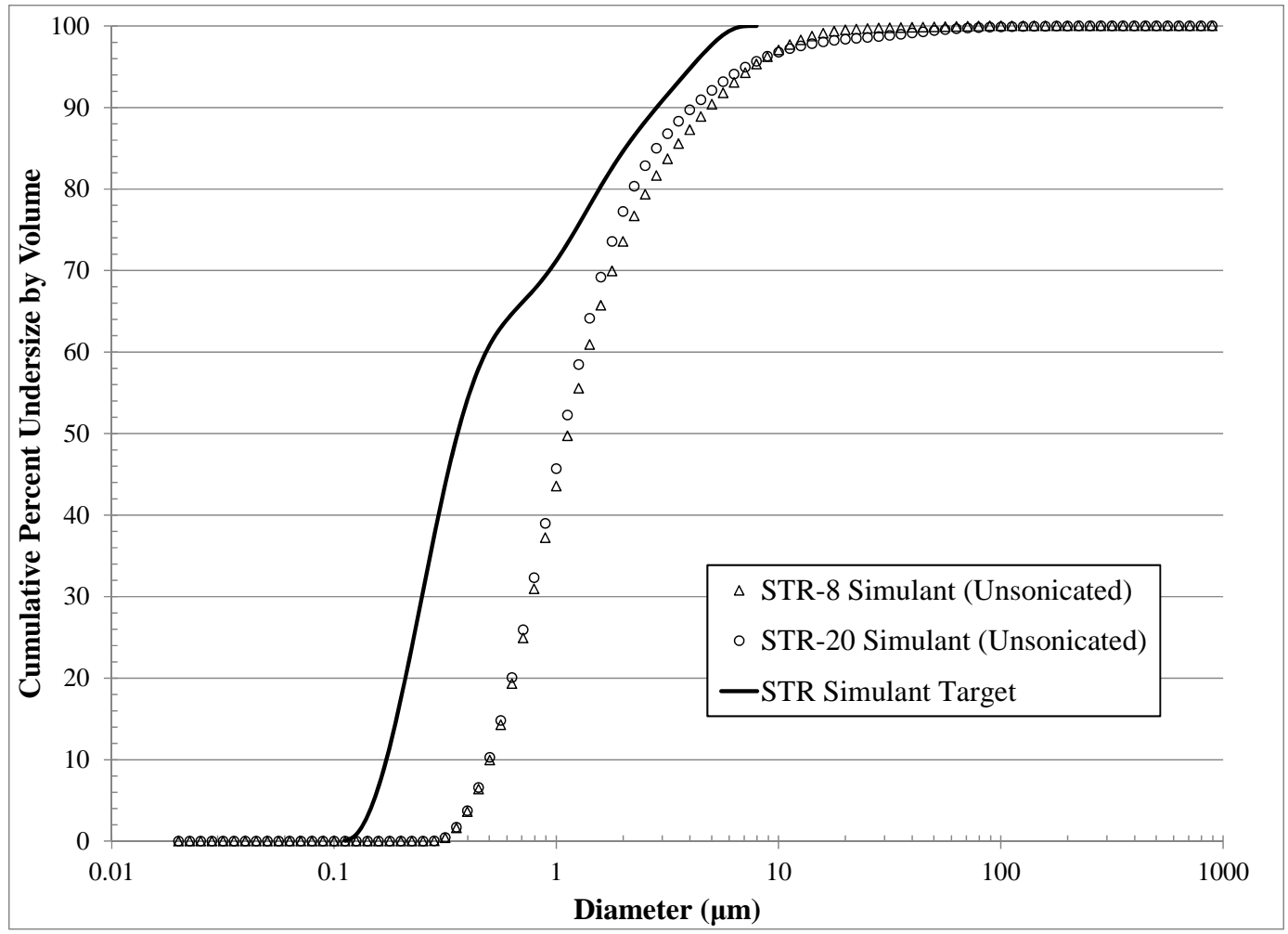

Figure 4.2. Average Cumulative Percent Undersize Measured at 1 Min Recirculation for STR 8 and STR 20 Simulants, Unsonicated, with a Comparison to the STR Simulant Target (Group 1/2 Mixture) 
Table 4.6. Cumulative Percent Undersize Measured at 1 Min Recirculation Time, Unsonicated, STR 8 and STR 20 Simulants

\begin{tabular}{ccc}
\hline & STR 8 & STR 20 \\
\cline { 2 - 3 } Size, $\mu \mathrm{m}$ & 8 wt\% & $20 \mathrm{wt} \%$ \\
\cline { 2 - 3 } 0.05 & Unsonicated $^{(\mathrm{a})}$ & Unsonicated $^{(\mathrm{a})}$ \\
0.5 & 0.00 & 0.00 \\
0.8 & 5.20 & 10.28 \\
1 & 22.08 & 32.31 \\
2 & 34.18 & 45.69 \\
5 & 69.90 & 77.24 \\
10 & 89.73 & 92.10 \\
20 & 93.82 & 96.79 \\
50 & 96.40 & 98.36 \\
100 & 98.72 & 99.42 \\
200 & 99.62 & 99.86 \\
400 & 99.91 & 99.99 \\
\hline
\end{tabular}

(a) Targeted PSD is based on a flowing sonicated PSD of the Group 1/2 Mixture actual waste because this approach gives the most representative and conservative PSD. However, when approaching the simulant samples the unsonicated data give the most consistent and accurate results. 


\subsection{Test Equipment and Instrumentation Description}

The large-scale test system was designed and built to accomplish the test objectives discussed in Chapter 1. During the design of the system, considerable flexibility was incorporated to address experimental uncertainties. One of the main uncertainties was associated with the aerosol concentrations that would be present in the test chamber over the broad range of orifices that were to be evaluated. This issue was addressed by constructing an aerosol chamber that could be reconfigured to different sizes. The smaller sizes increase the aerosol concentration while the larger sizes accommodate larger sprays. Another way this uncertainty issue was addressed was by procuring two types of aerosol instruments, one of which has a much lower detection limit. The second instrument also provides some measure of validation of the aerosol measurements. A second uncertainty was the ability to accurately measure spray leak rates over a wide range of orifice sizes. This issue was addressed by installing load cells on the vessels and Coriolis flow meters upstream and downstream of the orifice. This configuration provides the capability to determine the spray leak flow rate by using the difference in mass and flow rates during testing.

A schematic of the large-scale test system designed and built in Laboratory 184 of PNNL's Applied Process and Engineering Laboratory (APEL) is shown in Figure 5.1. The system consists of the following major components: 1) flow loop, 2) test chamber, 3) aerosol instrumentation, 4) general instrumentation, and 5) data acquisition systems (DAS). This section presents a detailed description of the major components of the large-scale test system.

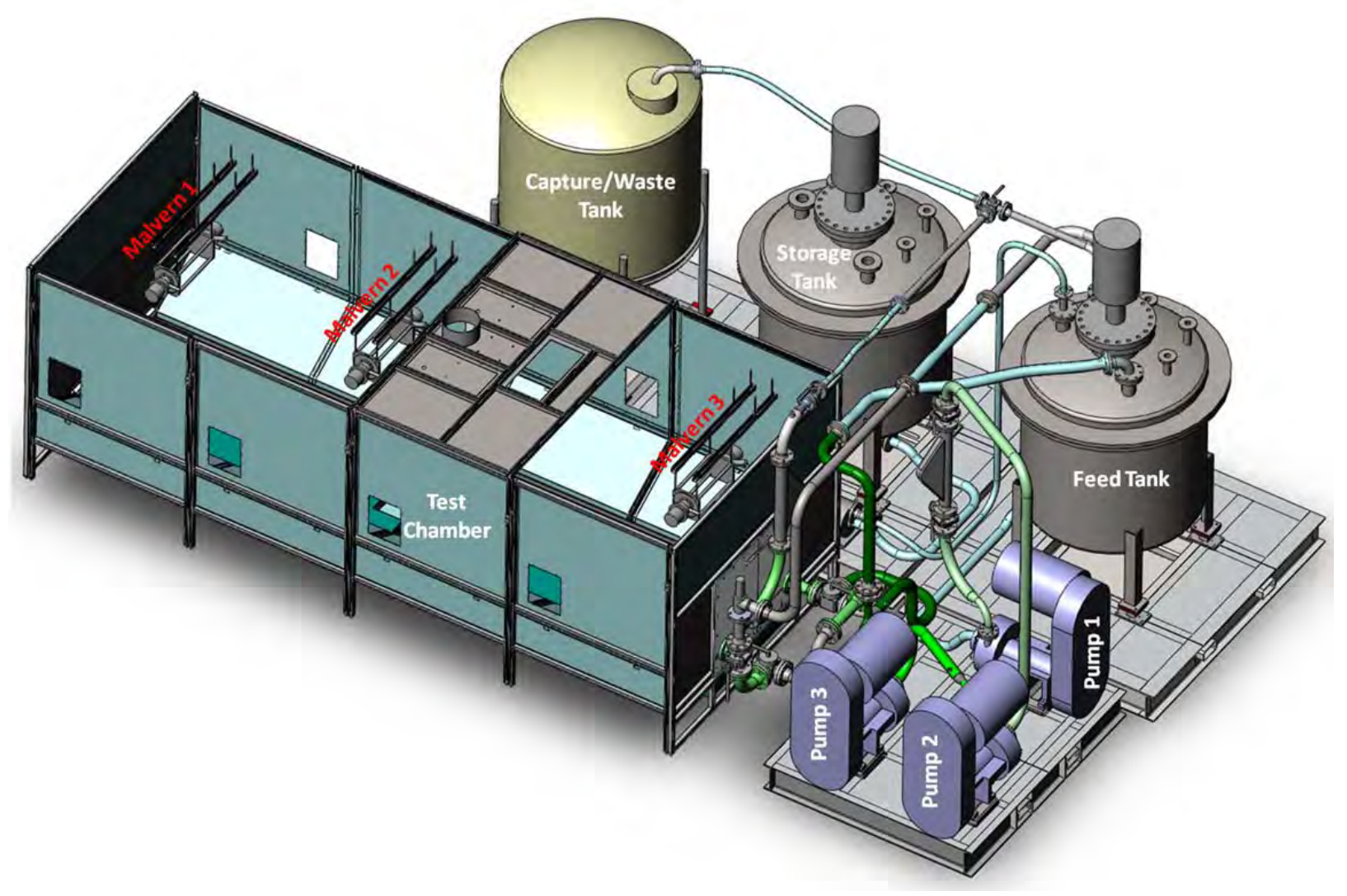

Figure 5.1. Schematic of the Large-Scale Test System 


\subsection{Flow Loop}

The flow loop is composed of the primary loop section, which includes the breached pipe, pumps, and ancillary equipment such as simulant feed/storage tanks, transfer pumps, and agitators. The flow loop was designed and built on the four skid-mounted units identified below:

1. Pump skid assembly

2. Feed tank skid assembly

3. Storage skid assembly

4. Capture/waste tank skid assembly.

The loop was designed to produce sprays from prefabricated spool pieces, called "test sections," with prototypic breach sizes varying from the smallest to the largest postulated breach in a 3-in., schedule-40 pipe. Except for the largest orifice, the loop can maintain fluid velocities at or above $6.5 \mathrm{ft} / \mathrm{s}$ with pressures from 100 to 380 psig during sprays.

All skids are plumbed together as shown in Figure 5.2, with the test section protruding from the pump skid into the test chamber. All sections of the loop, except for the return line, vessel-to-vessel lines, and feed vessel-to-pump transfer lines, were constructed primarily of 3-in., schedule-40, stainless steel pipe; this is the same piping used throughout the majority of WTP. The feed tank (TK-2) is plumbed directly to the loop, and a second tank (TK-1), labeled "Storage Tank" (see Figure 5.1, and Figure 5.2 for vessel labeling), connected to the feed tank through a transfer pump, provides extra capacity for handling large sprays ( 160 gpm loss through the breached section) for up to 5 or 6 min. The transfer pump, which is a Carver centrifugal pump controlled by a Honeywell variable frequency drive (VFD) and an ultrasonic level sensor located in the feed vessel enabled the test operators to maintain the fluid level in the feed tank if desired. Fluid exits the bottom of the feed tank via a 3-in. flexible hose connected to the inlet of the upstream pump on the pump skid.

The flow path continues through the first pump (P-1), the upstream Coriolis flow meter (FE-1), the next two pumps in series (P-2 and P-3), and into either the test section or the bypass leg as controlled by manual 3-way T-port valves (3V-1, 3V-2). The bypass leg was originally included in the loop to facilitate setup of the conditions for a test before the flow was directed through the breached section and aerosol measurements made. The bypass leg was not used during testing because the target flow conditions (fluid flow rate and pressure) could be set when flow was occurring through the test section prior to spray initiation. Therefore, throughout all testing, the 3-way (T-port) valves were set with the flow going only through the test section. The flow exits the test section and enters the downstream Coriolis flow meter (FE-2). There are two pressure relief devices included in the loop-the 450-psi rupture disk (RD-1) at the exit of P-3 and the 275-psi pressure relief valve (PRV-1) upstream of FE-2. The rupture disk was installed primarily to prevent over-pressurization of the loop, whereas the PRV was installed to prevent over-pressurization of the downstream Coriolis meter.

After exiting FE-2, the fluid can be diverted to either a waste/capture tank (TK-3) or back to the feed tank (as is the case during testing). This type of flow-loop configuration allows the system to be an open loop to the capture tank or a closed loop flowing back to the feed tank. 


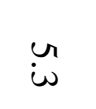

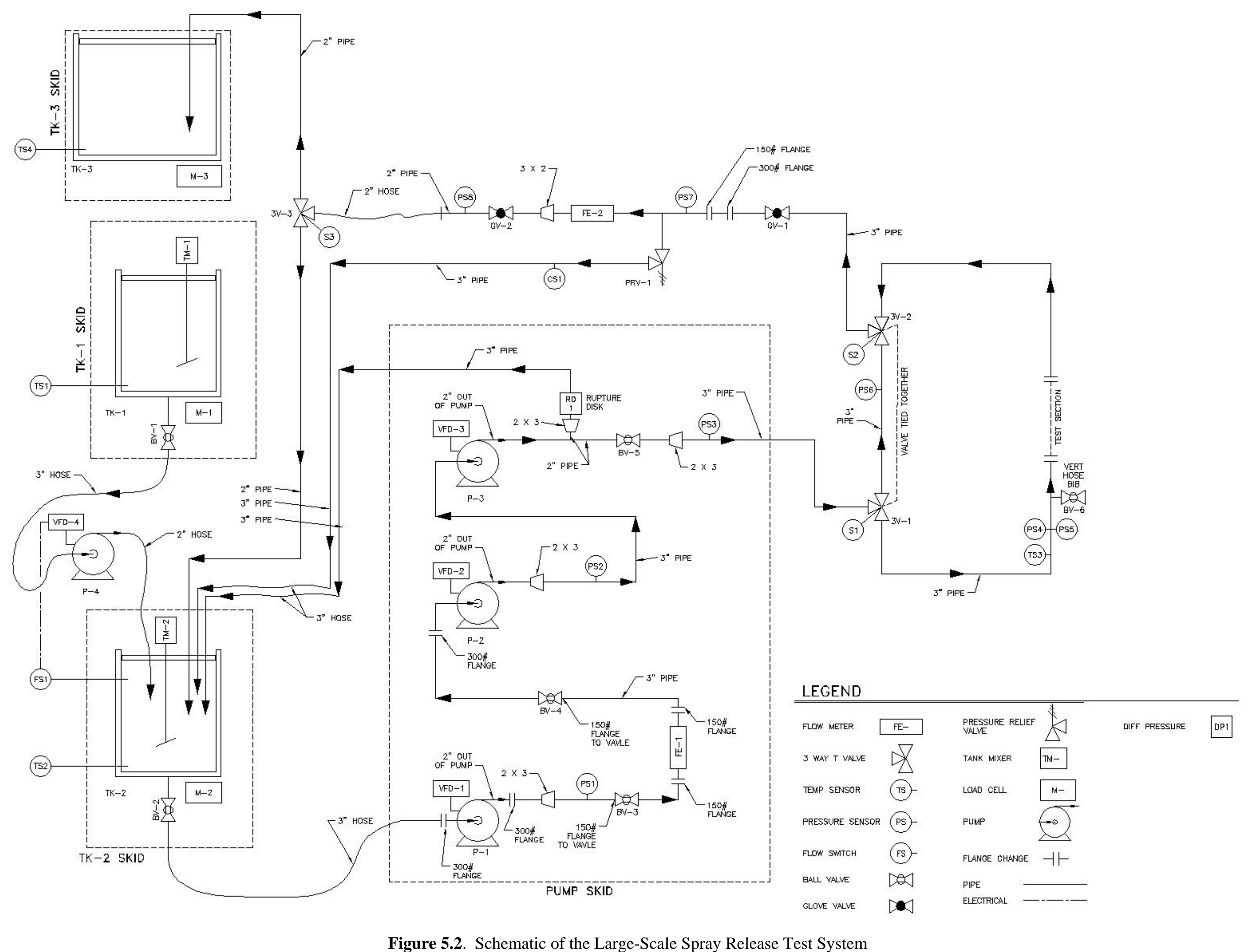



In addition to the primary flow system, extension piping was added to the loop for selected test cases to move the test section from one end of the test chamber to either the center or to $\sim 43 \mathrm{in}$. from the back wall (or splash wall) of the spray chamber. These extension pieces allowed the test section to protrude $77 \mathrm{in}$. and $184 \mathrm{in}$. further into the test chamber. This flexibility was used to evaluate the effect of spray distance and distance from the splash wall on the aerosol concentration and PSD.

The desired flow rates in the loop of $>6.5 \mathrm{ft} / \mathrm{sec}$ and pressures of up to $380 \mathrm{psi}$ at the test section were achieved using three Krebs millMAX centrifugal pumps connected in series as illustrated in Figure 5.3. Each pump consisted of a 50-hp motor, 200-gpm slurry pumps capable of producing 133 psig (with water) and handling non-Newtonian fluids with a Bingham rheology (consistency: 6-cP/yield stress:6-Pa) with $50 \mu \mathrm{m}, 2.5$ specific gravity particles at a solids loading of $20 \mathrm{wt} \%$. The flow rate through the pumps is controlled using Honeywell VFDs. The VFDs were connected in a master/slave configuration with the downstream pumps frequencies slaved to match the frequency of the upstream or master pump. Pressure in the loop is regulated using two globe valves that are located downstream of the test/bypass sections.

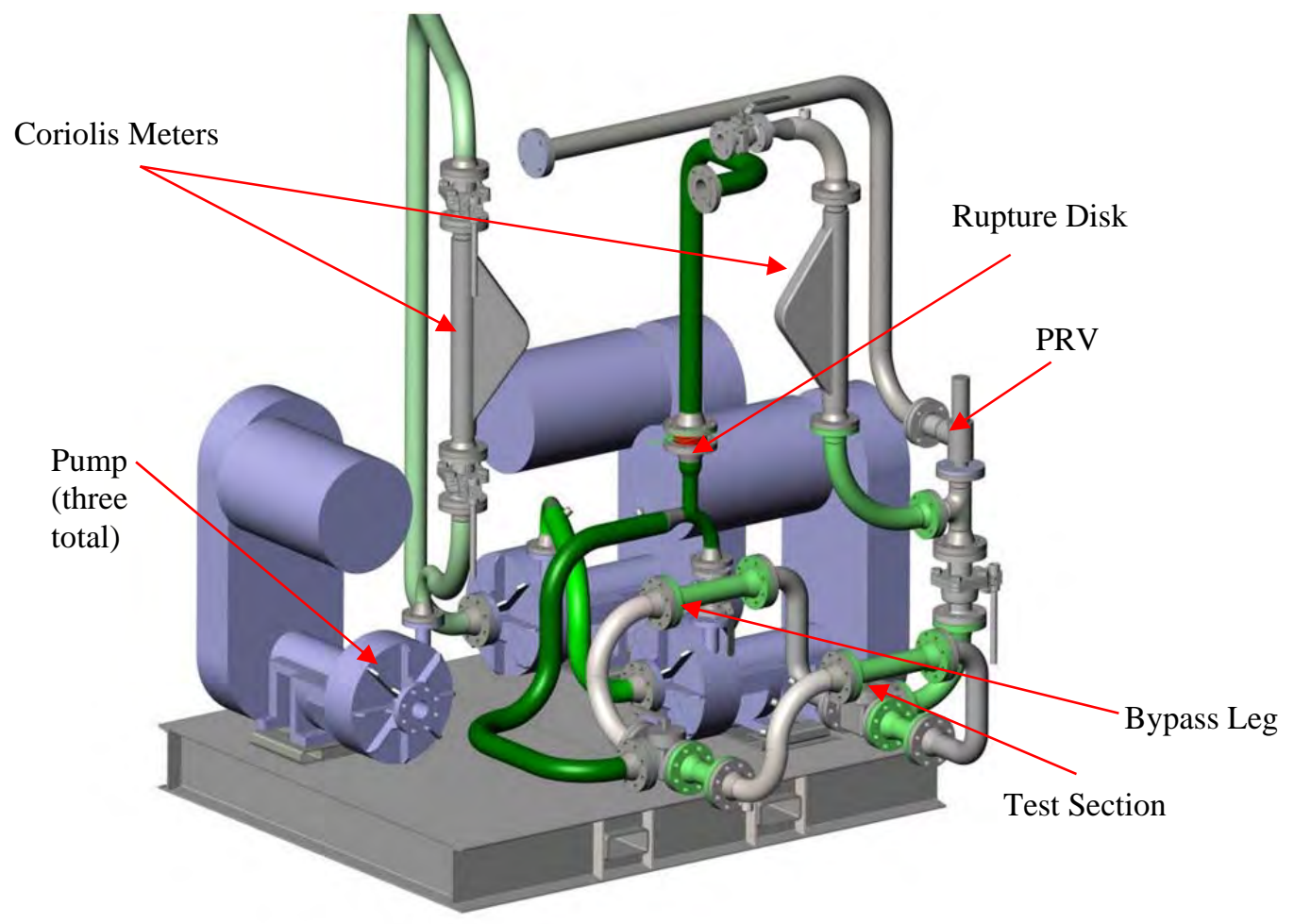

Figure 5.3. Schematic of the Flow Loop with Centrifugal Pumps on the Pump Skid

The flow rate through the loop is measured both upstream and downstream of the breach using two Coriolis mass flow meters. The locations where the Coriolis meters were installed provides for sufficient pressure to minimize interference with the meter readings from entrained air/gas. The downstream Coriolis meter is located between two pressure regulating globe valves (GV-1 and GV-2) and downstream of a PRV used to protect the meter from pressures $>270$ psig. The location of the flow meters allowed the spray leak flow rate to be determined from the differences in the flow rates during the tests. In addition to the mass flow rates, the loop also has instrumentation at several locations to measure temperature (via remote temperature detectors) and pressure (via absolute pressure transducers). 
The pump skid is plumbed to three supporting tanks, all of which are mounted on load cells, and each tank is contained on a separate skid. The load cells were provided to determine the mass of simulant sprayed during each test and, thereby, determine the spray leak flow rate.

The feed tank shown in Figure 5.4 is plumbed directly upstream of the pumps and receives the discharge from the recirculation flow from the test loop. As previously described, the feed tank contains a level switch system used to control any makeup flow from the storage tank. The working capacity of the tank is approximately 600 gal and a 2-hp mixer connected to a VFD provides agitation to the tank.

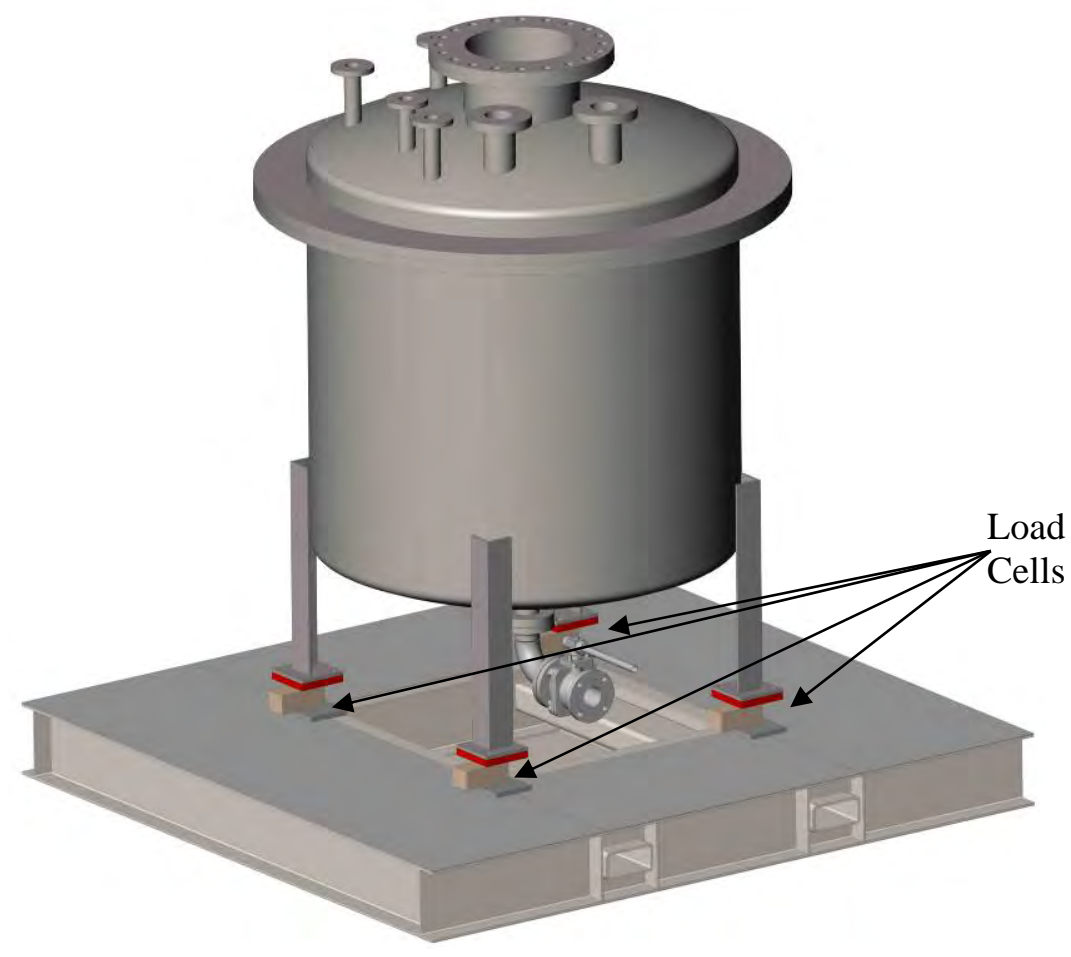

Figure 5.4. Schematic of the Feed and Storage Tanks

The storage tank is plumbed upstream of the feed vessel and supplies makeup fluid to the feed vessel via the transfer pump. The primary purpose of the storage vessel is to keep the net positive suction head of the pumps constant by keeping the hydrostatic head in the feed vessel constant within a few inches. The working capacity of the vessel is approximately 600 gal, and a 2-hp mixer connected to a VFD provides agitation to the tank.

The main function of the capture/waste vessel (see Figure 5.5) is to support slurry capture and loop flush operations. The working capacity of the vessel is approximately 1000 gal. Also, this vessel can receive the loop discharge when testing with an open flow loop configuration (if required). Although the open loop configuration was never used during actual test runs, it was always used during flush operations when cleaning the loop. When the loop is in the recirculation configuration, non-discharged fluid is recycled back to the feed tank. The initial and final load cell readings from the storage and feed tank are used to calculate the total flow discharged during each test. 


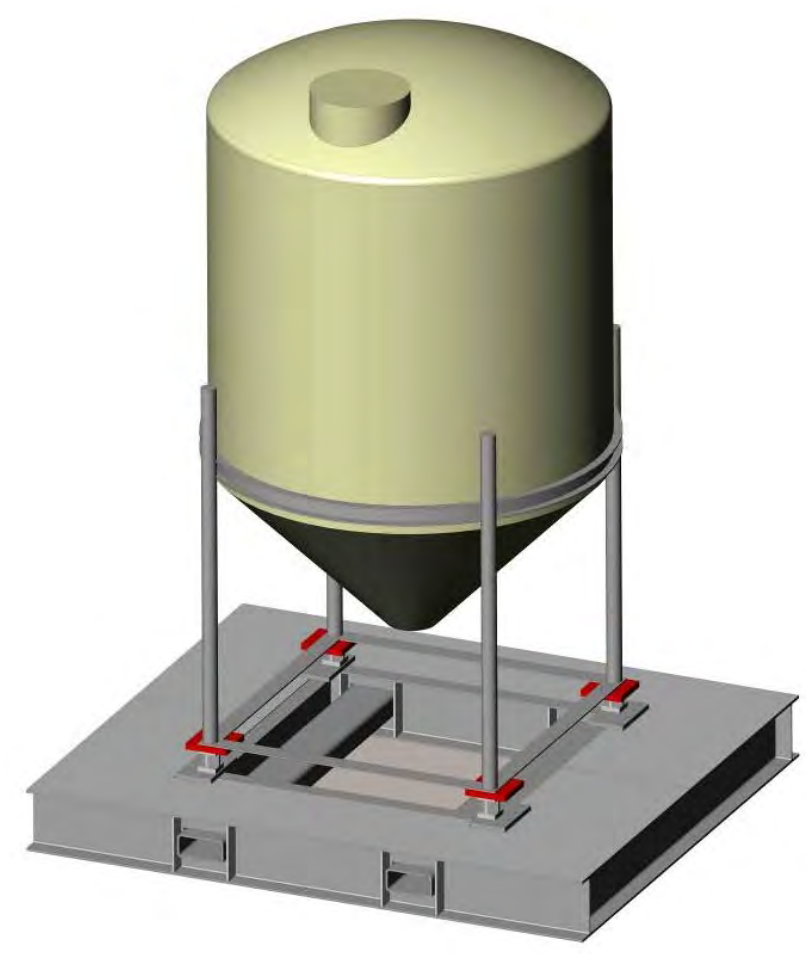

Figure 5.5. Schematic of the Capture/Waste Tank

The load cells on which the feed, storage, and capture/waste tanks are mounted enable proper accounting of the mass of fluid lost through the orifice during each test. Mechanical agitators and baffles in the feed and storage tanks provide uniform solids concentration in the tanks before and during each test. Also, both the feed and storage tanks are jacketed and connected to a chiller to remove mechanical heat gain during loop operation and maintain a constant temperature during the test. Finally, diffusers are used to decrease the flow velocity of the fluid entering the feed tank. The diffuser consists of flow expansion sections from the top tank inlet to the bottom of the tank. These expansion sections increase the pipe diameter from 2 to 6 in. A flat plate is mounted at the end of the diffuser to spread the fluid horizontally and minimize vortexing caused by the returning fluid. The diffusers are well submerged in the tank to minimize splashing and air entrainment.

\subsection{Spray Chamber}

The test/spray chamber, shown schematically in Figure 5.6, is the chamber in which aerosols from the test section are contained and characterized to determine the size distribution of aerosol droplets and the total droplet volume concentration as a fraction of the total spray volume. 


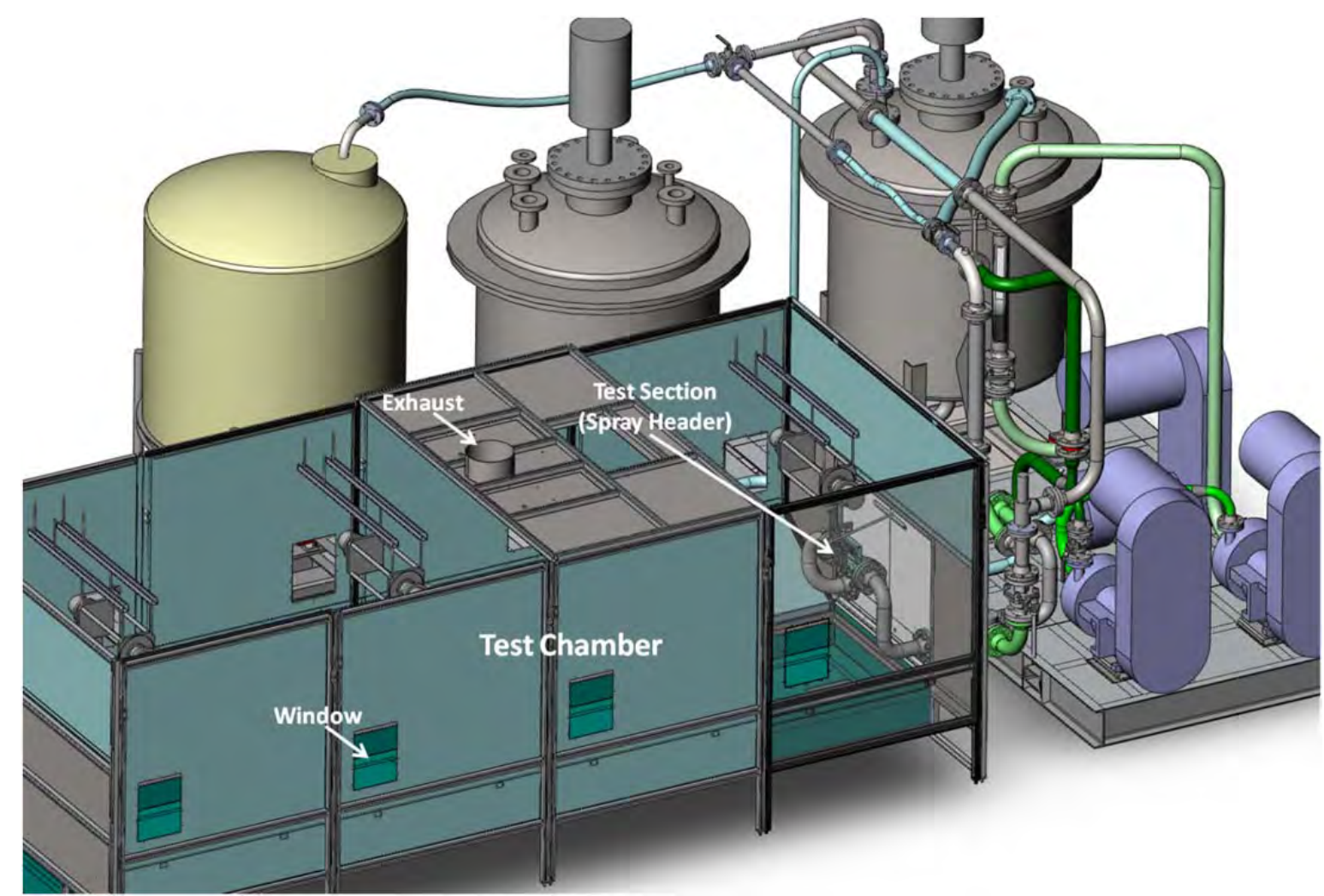

Figure 5.6. Schematic of the Spray Chamber

The spray chamber is designed to meet the following requirements:

- It is adjustable from a minimum size of $\sim 4 \mathrm{ft}$ wide $\times 8 \mathrm{ft}$ high $\times 5 \mathrm{ft}$ long ( $\sim 160 \mathrm{ft}^{3}$ volume) to a maximum size of approximately $8 \mathrm{ft}$ wide $\times 8 \mathrm{ft}$ high $\times 20 \mathrm{ft}$ long. The test chamber volume is incrementally adjustable in increments of $5 \mathrm{ft}$ in length and $2 \mathrm{ft}$ in width. The dimensions presented above are approximate, as the chamber is not a rectangle. The bottom third of the chamber walls, for instance, are sloped inward towards a collection pan that is $4 \mathrm{ft}$ wide. A vast majority of tests were performed in the $\sim 8 \mathrm{ft}$ wide $\times 8 \mathrm{ft}$ high $\times 20 \mathrm{ft}$ long chamber, the volume of which was $970.3 \mathrm{ft}^{3}$ $\left(27.48 \mathrm{~m}^{3}\right)$ after accounting for the slope in chamber walls, smaller collection pan, and the internals. A schematic of this largest chamber used is shown in Figure 5.6. Although the tests were generally conducted with the largest available chamber size, the ability to reconfigure the size was provided to accommodate low aerosol concentrations.

- It is made of materials (primarily stainless steel sheets) that have minimal affinity for attracting or interacting with aerosols (e.g., developing high static charges) generated during the testing.

- Non-aerosolized liquid is directed to a collection vessel.

- It is accessible for mounting aerosol characterization instruments.

- It has viewing ports for visually observing and video/still camera recording of the discharging spray.

- The enclosed volume is easily calculated.

- It is easily cleaned of simulant materials when not in use. 
- It has an exhaust system for clearing/evacuating aerosols between tests.

Throughout testing, the fluid volume that collects at the bottom of the chamber was minimized via sump pump transfer from the chamber to a tank vessel or drain. The windows were covered during testing to preclude any potential light interference with the aerosol measuring instruments (discussed in Section 5.3). In the absence of sprays, the back panel (wall) could be removed easily to allow operators to enter the chamber if needed.

The test section is a spool piece that has breaches (i.e., circular holes and slots) through which the fluid is discharged to create the spray leak for characterization (i.e., release fraction and PSD). For the testing discussed in this section, a broad range of circular holes (ranging from 1 to $\sim 4.5 \mathrm{~mm}$ ) and slots (ranging from $0.5 \mathrm{~mm} \times 5 \mathrm{~mm}$ to $2.74 \mathrm{~mm} \times 76.2 \mathrm{~mm}$ ) were tested to establish the release fraction and PSD for the largest anticipated breach in a 3-in. pipe at the WTP. The various breaches used during testing are detailed in Table 5.1. A total of seven spools were fabricated.

Table 5.1. Breaches and Geometries Used for Large-Scale Testing

\begin{tabular}{cllcc}
\hline $\begin{array}{c}\text { Spool } \\
\text { Number/ID }\end{array}$ & Orifice Shape & $\begin{array}{c}\text { Target Size }- \text { Diameter or Length } \\
\times \text { Width }(\mathrm{mm})\end{array}$ & Measured Size $(\mathrm{mm})$ & Area $\left(\mathrm{mm}^{2}\right)$ \\
\hline S1A & Circular & 1 & 1.00 & 0.78 \\
S1B & Circular & 4.46 & 4.67 & 17.13 \\
S1C & Circular & 2.74 & 2.74 & 5.89 \\
S1D & Circular & 2 & 2.11 & 3.50 \\
S2A & Slot & $0.5 \times 5$ horizontal (axial) & $0.43 \times 4.93$ & 2.14 \\
S2B & Slot & $1.5 \times 7$ horizontal (axial) & $1.41 \times 6.63$ & $\mathrm{n} / \mathrm{a}^{\mathrm{a}}$ \\
S2C & Slot & $1 \times 8$ horizontal (axial) & $0.99 \times 7.93$ & $\mathrm{n} / \mathrm{a}^{\mathrm{a}}$ \\
S2D & Slot & $0.5 \times 9$ horizontal (axial) & $0.44 \times 9.07$ & $\mathrm{n} / \mathrm{a}^{\mathrm{a}}$ \\
S3A & Slot & $2.74 \times 76.2$ horizontal (axial) & $2.78 \times 76.16$ & 211.82 \\
S4A & Slot & $1.0 \times 76.2$ horizontal (axial) & $0.96 \times 76.11$ & 73.14 \\
S5A & Slot & $1.0 \times 10$ vertical (circumferential) & $10.03 \times 0.97$ & 9.77 \\
S5B & Multiple & Five 1-mm holes with 1-mm gaps & $0.94,0.96,0.97,0.99$, and & $3.69^{\mathrm{b}}$ \\
& Circular & spaced horizontally & 0.99 & 19.35 \\
S5C & Slot & $1.0 \times 20$ horizontal (axial) & $0.97 \times 20.02$ & 49.02 \\
S6A & Slot & $1.0 \times 50$ horizontal (axial) & $0.98 \times 50.06$ & 8.72 \\
S7A & Slot & $1.0 \times 10$ horizontal (axial) & $0.87 \times 10.00$ & 19.00 \\
S7B & Slot & $2.0 \times 10$ horizontal (axial) & $1.91 \times 9.97$ & 30.83 \\
S7C & Slot & $3.0 \times 10$ horizontal (axial) & $3.08 \times 10.01$ & \\
\hline a) The area of this orifice was not calculated because it was not used in the testing described in this report. \\
b) The area of the multiple circular orifice (S5B) is the total area of the five 1-mm holes. & \\
\hline
\end{tabular}

A typical test section is shown in Figure 5.7. This section consists of an 18.24-in.-long, 3-in., schedule-40 pipe spool. As shown in the figure, smaller breaches allowed multiple orifices to be machined on one spool piece. 

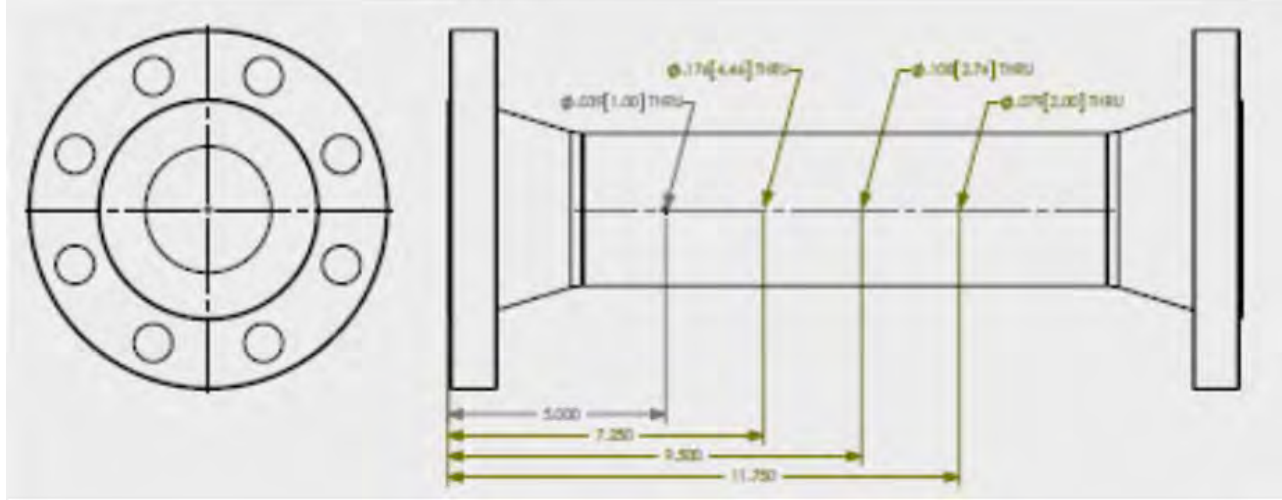

Figure 5.7. Schematic of Spool 1, with Orifices A - D From Left to Right in the Schematic

To facilitate spray initiation and cessation for each test, individual orifices were sealed with a specially designed release assembly that allowed the orifice to be opened when the desired conditions in the loop were met. The release assembly consisted of an over-center lever positioned at each orifice. Each lever was opened using a manual pull-rod from outside the test chamber. The release assembly is shown in Figure 5.8 attached to a test spool.

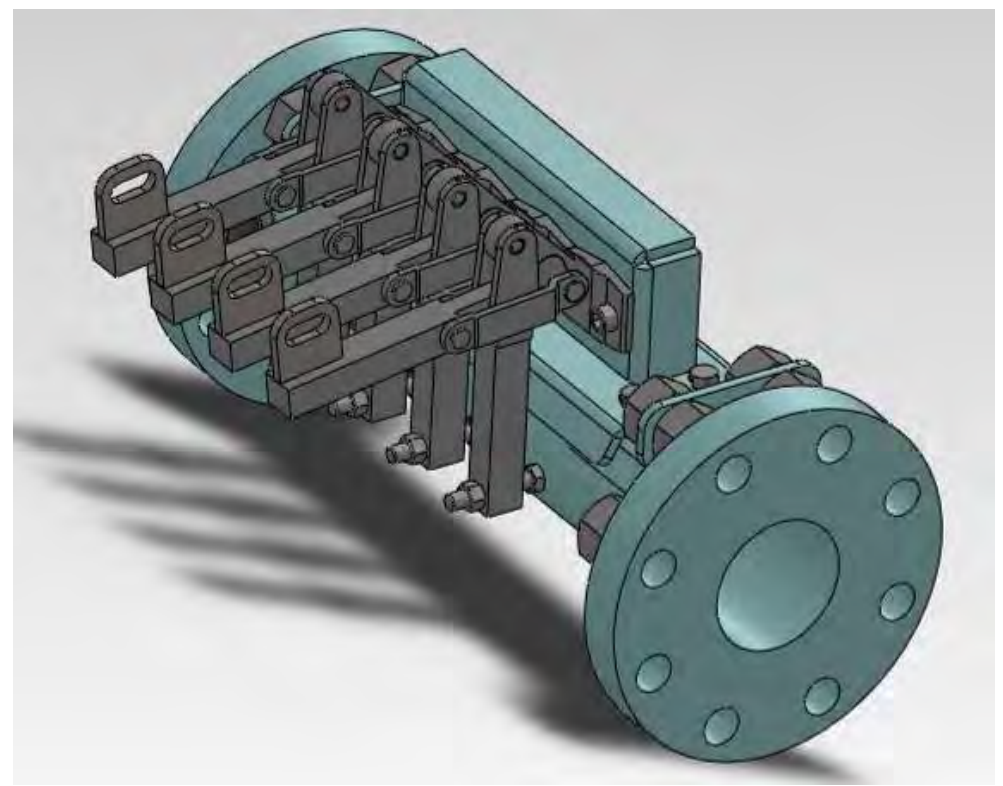

Figure 5.8. Model of the Release Assembly Used for Initiating the Spray

\subsection{Aerosol Instrumentation}

The large-scale spray leak test chamber employed two instruments for measuring aerosol concentration and size distribution: a Malvern Insitec-S open-frame process aerosol analyzer (Malvern Instruments, Ltd.) and a Process Metrix PPC (Process Metrix). Both instruments operate on the principle of laser diffraction, and both systems have proprietary DASs that convert the measured signals into aerosol concentration and size. 
The rationale for using multiple aerosol concentration and PSD measuring sensors was based on the uncertainties associated with 1) the anticipated aerosol concentrations for the range of orifices that would be used in the test chamber, and 2) use of the full size of the test chamber to assess aerosol generated through jet breakup and/or splash mechanisms. During shakedown testing, both instruments were tested using breaches of 1-mm and 2-mm holes to identify the best sensor and experimental approach (i.e., spray duration and chamber size) to achieve repeatable measurements of the aerosol concentrations. The results, which are discussed in detail in Chapter 8, indicated that the Insitec-S instrument was best suited as the primary aerosol measuring instrument. However, the PPC data was collected throughout the entire testing to provide validation of the Insitec-S data with small breaches as well as a redundant measurement technique for aerosols that were $<70 \mu \mathrm{m}$ in size.

In the sections that follow, the principle of measurement and operational specifications for the Insitec-S and PPC aerosol instruments are described in detail. These discussions include information on instrument locations and installation configurations in the large-scale test chamber, data collection/ sampling rates, and the primary measurement input parameters (e.g., refractive index).

\subsubsection{Malvern Insitec-S}

Three Malvern Insitec-S aerosol analyzers were purchased for use in large-scale spray leak testing. The Insitec-S analyzer is an open-frame aerosol size analyzer that uses laser diffraction to determine aerosol size and concentration. The basic instrument setup and operation principle are illustrated in Figure 5.9.. The Insitec-S consists of a relatively broad 10-mm-diameter laser source positioned opposite a photodetector (i.e., a photomultiplier tube) assembly. The volume swept by the laser beam as it passes from the laser source to the photodetector assembly defines the Insitec-S measuring volume. The distance between the laser source and the photodetector is set by spacer bars that typically range in length from 150 to $500 \mathrm{~mm}$. For 150 - and 500-mm spacer bar lengths, the measuring volume swept by the laser is $\sim 5.5$ and $\sim 33 \mathrm{~cm}^{3}$, respectively.

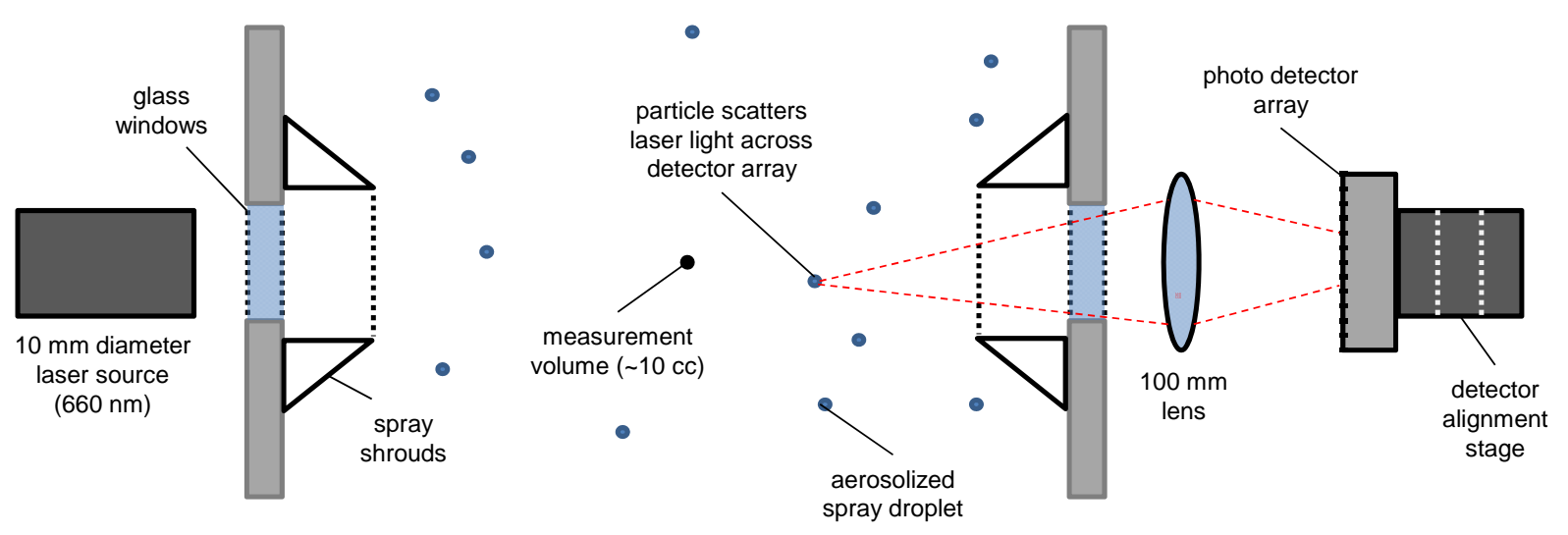

Figure 5.9. Schematic of the Basic Insitec-S Aerosol Analyzer Setup and Operating Principle

The photodetector assembly consists of a Fourier lens that focuses the laser onto a ring photodetector array. During operation, undeflected laser light is focused onto a laser power detector at the center of the ring detector. Laser light that is deflected is focused onto one of 32 photodetector rings on the surface of 
the photodetector assembly. The displacement of the deflected light from the center of the ring detector is proportional to the angle at which the laser light has been diffracted.

The Insitec-S functions by continuously monitoring and recording both the intensity of undeflected laser light striking the central detector relative to the background intensity when no aerosol is present (termed the transmission) and the diffraction pattern generated by scattering of laser light by aerosol in the measuring zone. When an aerosol particle enters the measuring volume, it scatters light in all directions. However, the intensity of scattered light varies as a function of scattering angle and generates a diffraction pattern that is unique to particles of that size and morphology. As the measuring volume of the Insitec-S is large, the instrument is capable of simultaneously measuring multiple particle scattering events in a single measurement. Using Mie scattering theory and given the nominal optical properties of the aerosol and the suspending phase, the diffraction pattern generated by the particle ensemble can be analyzed to determine the size distribution of aerosol particles. Diffraction of the laser beam yields a loss of intensity of the laser beam that strikes the central detector relative to the intensity measured when no aerosol is present (termed the background laser intensity). This decrease in intensity, along with the size distribution estimated from the diffraction pattern, can be used to determine the number of scatters in the measuring volume and hence the concentration of aerosol.

The specific size and concentration range of the Insitec-S as configured for large-scale aerosol testing are as follows:

- Size Range - The nominal size range that can be measured by the Insitec-S is defined by the focal length of the Fourier lens used to focus laser light on the photodetector. For large-scale testing, a 100 -mm lens was employed. This lens yields a nominal measuring range of 0.5 to $200 \mu \mathrm{m}$.

However, the instrument is able to interpret a maximum range of 0.1 to $450 \mu \mathrm{m}$ with this lens (with the caveat that the volume fraction of particles outside the nominal measuring range may not be accurately determined).

- Concentration - The nominal range of concentration that can be detected by the Insitec-S strongly depends on the instrument setup and spray configuration. In general, the Insitec-S can measure aerosols in the range of 0.01 to 1000 ppmv (parts per million by volume). The specific range achievable in a given experimental setup is determined primarily by the length of the spacer bars and the geometry of the spray. Longer spacer bars allow lower aerosol concentrations to be probed, but also reduce the aerosol concentration at which the detector saturates. The geometry of the spray also has a strong impact on the range of aerosols that can be detected by the instrument. For example, high aerosol concentrations can be measured if the aerosol is confined to a limited portion of the measurement volume, such as fan sprays. However, correct interpretation of aerosol concentration for such sprays requires knowledge of the spray geometry relative to the measurement volume. The Insitec-S measurement software allows input of spray geometry and spray concentration profiles to correctly determine the concentration of localized sprays.

The three Malvern Insitec-S analyzers were installed at different locations in the large-scale test chamber to allow profiling of the aerosol concentration as a function of test position. In general, an Insitec-S analyzer was placed near the spray header, at the "middle" of the test chamber, and near the splash wall. All Insitec analyzers were vertically positioned midway between the core of the spray (the orifices in the spray header were approximately 36 in. from the chamber floor) and the top of the chamber (the center of the Insitec's measurement zone was approximately 59.5 in. from the chamber floor). 
Several key dimensions and lengths were measured to precisely locate the instrument with respect to the test chamber walls. These key dimensions are illustrated in Figure 5.10, and their values are listed in Table 5.2. These locations were not universal. The specific instrument test locations were varied to determine the concentration behavior in different locations, such as in-spray measurements. A detailed list of large-scale chamber aerosol instrument configurations is provided in Appendix A of this report.
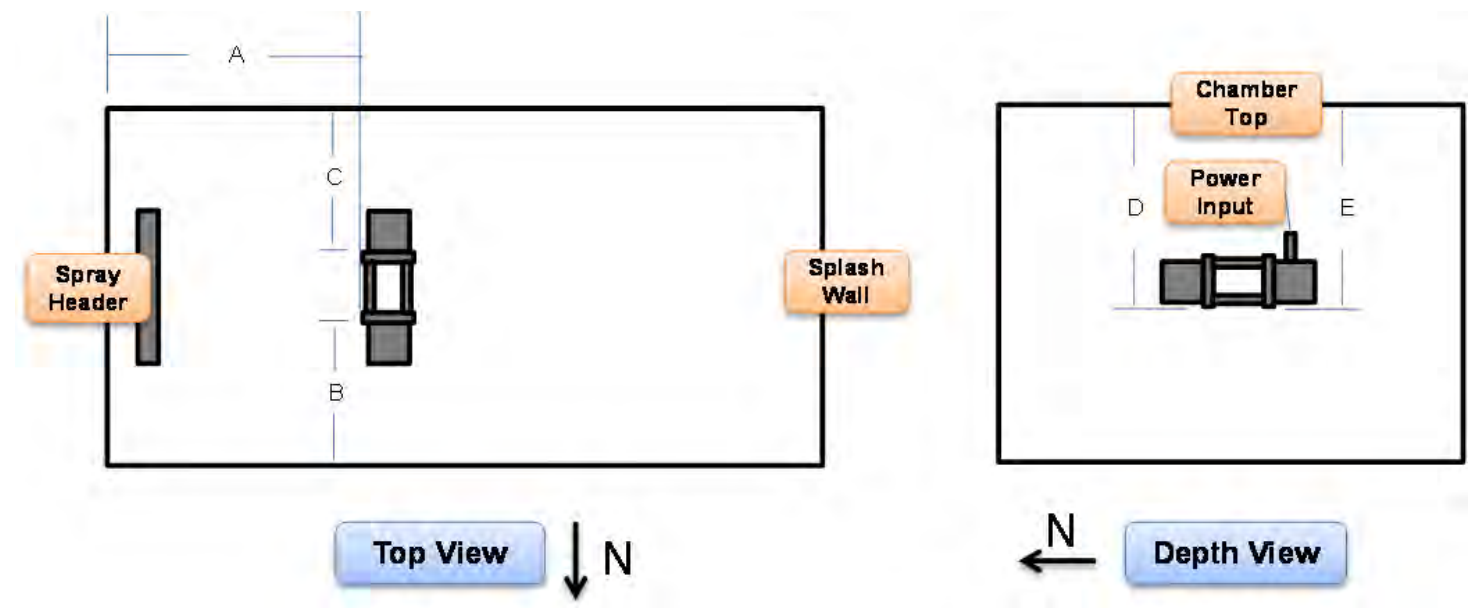

Figure 5.10. Two Schematic Views Showing Key Dimensions Used for Measuring the Locations of the Three Malvern Insitec-S Analyzers Installed in the Large-Scale Test Chamber: a top view and a depth (front to back) view. For reference, north is indicated on the figure.

Table 5.2. Values for Key Dimensions Listed in Figure 5.10

\begin{tabular}{ccccccc}
\hline Position ID & Malvern Serial Number & A (ft) & B (ft) & C (ft) & D (ft) & E (ft) \\
\hline 1 & MAL1855 & 13.3 & 3.2 & 3.2 & 2.2 & 2.2 \\
2 & MAL1852 & 6.2 & 3.2 & 3.2 & 2.2 & 2.2 \\
3 & MAL1854 & 3.3 & 3.2 & 3.2 & 2.2 & 2.2 \\
\hline
\end{tabular}

As the Insitec-S aerosol analyzers are located inside the test chamber, the instrument surfaces come into direct contact with the aerosol generated by the spray. While the instruments have been positioned above the spray, jet formation and breakup is generally a turbulent process, and there may be times where the spray may pass through the Insitec-S measuring volume or strike the analyzer itself. Although the electronic components are protected inside specialized enclosures for the laser and detector, the laser and scattered light still pass through optical windows to generate the measuring volume (see Figure 5.9). Deposition of aerosol or the presence of contaminants will impact the quality of the measurement. Any minor surface contamination or surface imperfections (such as scratches) can be accounted for by measuring the "background" scattering intensity generated by the laser prior to aerosol generation (i.e., when the measurement volume is free of aerosol). Gross contamination or damage to the windows found during these background measurements may be corrected by cleaning the windows (with a lens paper and an appropriate solvent) or by replacing the windows entirely. However, for the current set of spray testing, contamination or damage to the windows was never severe enough to require window replacement, and simple cleaning operations with water restored background measurements to appropriate levels. 
Contamination of the window during aerosol generation is of significant concern, as particles and aerosol that attach to the window will yield a static scattering signal that will remain through the measurement. To prevent contamination of the windows by direct spray impact, all Malvern instruments are equipped with a 40-mm cylindrical spray shroud. The spray shroud protects the optics from direct impact, but does not provide protection against diffusive or convective transport of aerosol particles. As such, the Insitecs are also equipped with a purge system that blows air across the windows and pushes aerosol out of the volume enclosed by the spray shrouds. In general, the purge air flow rate was set at $\sim 1 \mathrm{scfm} /$ window (a total of $\sim 2 \mathrm{scfm} /$ instrument) for all aerosol tests.

The three Insitec-S analyzers are interfaced to a single control computer through a multiport local communications interface box (part MPS2991-OP10045353). Each instrument is operated through a separate instantiation of Malvern's RTSizer software (Version 7.40). This software collects, analyzes, and reports the aerosol data collected by the instrument. The primary program outputs are aerosol size and concentration; other parameters - including raw data, such as the raw light-scattering signal, laser transmission, and background, and other calculated parameters, such as the SMD—also are measured.

Measurements made with the Insitec-S analyzer were set up and controlled through the RTSizer software. The typical instrument configuration and software settings used for analysis are listed in Table 5.3. A complete description of these parameters is given in the RTSizer user manual (Malvern Instruments Ltd. 2010). The first three parameters - the lens, gain, and update period—must be set before any data are collected because these parameters cannot be changed by post-analysis properties. The lens must be set to match the focal length of the lens installed in the instrument; for all large-scale testing, the focal length of the lens was $100 \mathrm{~mm}$. The gain is a photodiode multiplier that determines the instrument response to scattered light. The highest gain setting of $2 \times$ was employed for large-scale testing because aerosol concentrations were expected to be low (i.e., 10 ppmv or less based on initial estimates). The update period (or accumulation period) determines the time period over which results are integrated. Longer update periods tend to smooth variations in aerosol concentration with time, thus yielding smoother data, but may also time-average over periods during which aerosol concentration transience is of interest (such as the initial increase in concentration from which release fraction estimates are made). Shorter accumulation periods can be selected to capture fast transients; however, this also tends to yield an increase in noise-to-signal ratio. For large-scale testing, two update periods were employed. Initial testing used a 1-s update period, which adequately captured dynamics on sprays generated on most smallto medium-sized orifices. For larger orifices, the initial increase in concentration typically occurred on time scales of order $1 \mathrm{~s}$, and as such, a lower update period of $0.25 \mathrm{~s}$ was used in later testing. 
Table 5.3. Insitec-S Instrument Configuration and Software Parameters Used in Typical Measurements

\begin{tabular}{ll}
\hline \multicolumn{1}{c}{ Parameter } & \\
\hline Lens & $100 \mathrm{~mm}$ \\
Gain & $2 \times$ \\
Update period & Either $1 \mathrm{~s}$ or $0.25 \mathrm{~s}$, depending on time resolution needs \\
Particulate Refractive Index & Varies depending on test slurry \\
Media Refractive Index & Air: $1.00+0.00 \mathrm{i}$ \\
Particle Density & 1.00 gm/cc (typically not set to true density, as this parameter \\
& is used only in specific surface area calculations) \\
Spray Properties & Measured: \\
& $\bullet \quad$ Uniform spray concentration in measurement volume \\
& $\bullet \quad$ Path length for 500-mm spacers is $420 \mathrm{~mm}$ \\
& $\bullet \quad$ Path length for 150-mm spacers is 70 mm. \\
\hline
\end{tabular}

Other properties, such as the particulate and media refractive index (RI) and scattering threshold, may be changed by post-analysis processing to evaluate the effect of these parameters on the instrument results. From the set of parameters that can be changed post-process, two important parameter selections are refractive index and the spray properties. The refractive index is a complex number that specifies how light refracts through a material (real component) and how the material attenuates or absorbs light (imaginary component). As all aerosols are tested in air, the media refractive index is always set to that of air $(1.00+0 \mathrm{i})$. The particulate refractive index depends on the material being tested. Selection of particulate refractive index for measuring large-scale aerosols is discussed in a separate section of this report (see Chapter 7). The spray properties allow the user of the RTSizer software to define the shape, size, and concentration profile of the spray as it passes through the measurement volume. The shape and profile options are outlined in the RTSizer software manual (Malvern Instruments, Ltd. 2010).

\subsubsection{Aerosol Concentration by Ensemble Laser Diffraction}

The Malvern Insitec-S, which operates on the principle of ensemble laser diffraction, provides a rapid method for assessing aerosol PSD over several orders of magnitude in size. For typical process applications, the primary purpose of the Insitec-S is simply particle sizing. However, for spray leak testing, the Insitec-S has been used to determine both aerosol PSD and aerosol concentration. Aerosol concentration is directly calculated by the Insitec-S RTSizer software using the Lambert-Beer law. However, because measurement of aerosol concentration is not the typical use of the Insitec-S, some caution should be taken with the result provided by the instrument software. In an attempt to assess the quality of the aerosol concentration and size distribution result provided by the Insitec-S, PNNL has performed several assessments of instrument performance. These include:

- Evaluation of solid-in-liquid dispersions on similar Malvern instruments; in particular, evaluation of a dispersion concentration measurement on the Malvern Mastersizer 2000 (which employs the same principle of measurement as the Insitec-S)

- Examination of Malvern Insitec-S performance check data. 
In addition, PNNL has also assessed the performance of the Insitec-S against that of a secondary aerosol analyzer used in large-scale testing (i.e., PPC). The results of this comparison are discussed in Chapter 8 of this report.

The two checks of instrument performance described in the sections that follow (Sections 5.3.2.1 and 5.3.2.2) generally confirm the accuracy of the concentration measurement to within 40 percent. Repeat measurements of Insitec performance that employ the same physical standard indicate a baseline reproducibility of 5 percent in terms of aerosol concentration. Likewise, reproducibility of the size measurement is good to within 1 percent for the most commonly reported size percentiles, namely the $10^{\text {th }}, 50^{\text {th }}$, and $90^{\text {th }}$ percentiles. However, significant divergence in measured size appears to occur below cumulative volume undersize of 0.5 percent. The data supporting these conclusions are discussed in the following two sections. It should be noted that significant emphasis has been placed on understanding the accuracy and reproducibility of Insitec-S measurements, as this instrument was the primary means of obtaining aerosol size and concentration data. Similar treatment has not been given to the PPC.

\subsubsection{Evaluation of Laser Diffraction Performance in Solid-Liquid Dispersions}

As a basic test of the ability to determine dispersed phase concentration by laser diffraction, a concentration curve was developed for a solid dispersion of glass spheres in liquid. For this test, dispersions of 15- to 150- $\mu \mathrm{m}$ glass bead size standard provided by Malvern Instruments (as Quality Audit Standard QAS3002) were prepared in de-ionized water at several concentrations. Specifically, the standard 2.5-g vial of glass beads provided by the vendor was split into five separate 0.5 -g shots and added stepwise to a pre-measured mass of water. At each step, the dispersion size and concentration were measured on a Malvern Mastersizer 2000 (which operates using the same ensemble laser diffraction methods at the Insitec-S) equipped with Malvern Hydro G 2000 dispersion unit. Instrument control, data analysis, and data reporting were carried out using the Malvern Mastersizer 2000 software (Version 5.60). The dispersion concentration measured by the Mastersizer 2000 was then compared to the expected concentration of the dispersion determined by mass balance. The results of this comparison are shown in Figure 5.11. The results indicate excellent linearity in the concentration result; however, analysis of the linear slope of the concentration comparison curve indicates that the Mastersizer 2000 measures a dispersion concentration that is approximately 38 percent higher than that determined by mass balance. While the exact cause of the difference in concentration readings is unknown for the current test, it does provide a baseline measure of accuracy (in this case $\pm 40 \%$ ) for concentration measurements by laser diffraction. 


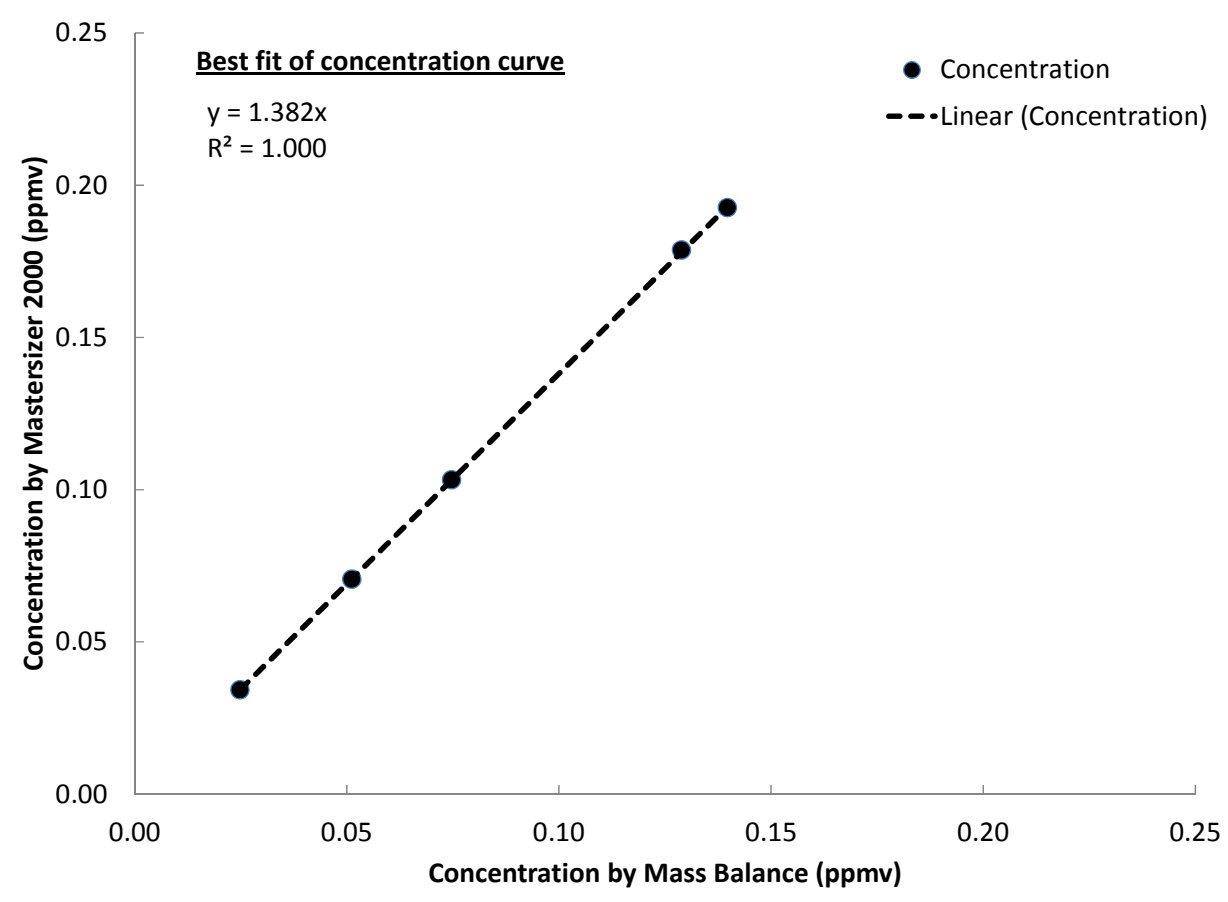

Figure 5.11. Concentration Curve Comparison for a Glass Bead Dispersion in Water as Measured by the Malvern Mastersizer 2000. Performance of the Mastersizer 2000 should be indicative of the Insitec-S aerosol analyzer performance, as both instruments employ the same ensemble laser diffraction methodology to measured dispersion concentration.

With regard to impact of this result on concentration measurements made for the purpose of estimating release fraction, the $\sim 40$ percent positive bias in the aerosol concentration measurement is not prohibitive for two reasons: 1) the error is conservative in that it will lead to an over-estimate (rather than under-estimate) of the release fraction, and 2) as will be shown in Chapter 8, the typical test-to-test variation in aerosol concentration measurements for spray leak tests is on the order 30 percent. Finally, it should be noted that the concentration curves shown in Figure 5.11 only inform on the performance of the total aerosol concentration measurement, i.e., they illustrate that the use of the Beer-Lambert Law (see Equation 7.8) is a reasonable method to approach concentration. The reproducibility of the PSD measurement is evaluated in the next section.

\subsubsection{Evaluation of Insitec-S Performance Data}

Performance of the Malvern Insitec-S was routinely checked using an RS-3 reticle, which is a physical standard purchased directly from Malvern for the purpose of validating instrument performance. The reticle consists of a fixed number of micrometer sized opaque circles that range in size from 4 to $95 \mu \mathrm{m}$ and that are deposited onto a glass plate. The reticle provides a fixed physical standard against which instrument performance can be routinely assessed. For all performance checks, each Insitec-S instrument met the accuracy requirements specified by the reticle certificate of analysis. These requirements, listed in Table 5.4, specify that the diameters corresponding to the $10^{\text {th }}, 50^{\text {th }}$, and $90^{\text {th }}$ cumulative volume percent undersize must be within $\pm 3 \%$ of $33.36 \mu \mathrm{m}, \pm 2 \%$ of $44.37 \mu \mathrm{m}$, and $\pm 10 \%$ of $60.94 \mu \mathrm{m}$, respectively. These performance checks provide a baseline measure of instrument accuracy with respect to PSD against which data may be assessed. 
Because the same physical standard is used for every performance check, the performance check data also provide a means by which the instrument reproducibility may be assessed for aerosols that possess similar size distributions as the reticle. During the course of large-scale testing, approximately nine performance checks were conducted on each of the three Insitecs. Other performance checks were run on individual instruments; however, sets of performance check data where all three instruments were tested is considered for the current reproducibility analysis. Considering complete performance sets for all three instruments, 27 measures of Insitec performance were recorded for large-scale testing. By averaging these data sets, a measure of reproducibility can be determined for measurements of total aerosol concentration and select size percentiles. The results for reproducibility analysis for these parameters are shown in Table 5.4. In addition, averaging also yields a measure of reproducibility of the reticle PSD. For this analysis, averages of the size distribution are considered on a logarithmic concentration scale to avoid over-weighting high concentration results. The results of PSD reproducibility analysis are shown in Figure 5.12.

Table 5.4. Results of Insitec-S Performance Reproducibility Analysis Using a Physical Standard for Select Measurement Parameters

\begin{tabular}{lcccc}
\hline Parameter (Unit) & Average & Standard Deviation & RPSD $^{(\mathrm{a})}$ & $\begin{array}{c}\text { Certified Value } \\
\left(\text { Range }^{(\mathrm{b})}\right)\end{array}$ \\
\hline $\mathrm{C}_{\mathrm{v}}(\mathrm{ppmv})$ & 2400 & 100 & $5 \%$ & $\mathrm{n} / \mathrm{a}$ \\
$\mathrm{d}_{\mathrm{v}}[10](\mu \mathrm{m})$ & 32.8 & 0.2 & $0.5 \%$ & $33.36( \pm 3 \%)$ \\
$\mathrm{d}_{\mathrm{v}}[50](\mu \mathrm{m})$ & 44.0 & 0.3 & $0.6 \%$ & $44.37( \pm 2 \%)$ \\
$\mathrm{d}_{\mathrm{v}}[90](\mu \mathrm{m})$ & 61.0 & 0.6 & $1 \%$ & $60.94( \pm 10 \%)$ \\
\hline
\end{tabular}

(a) Relative percent standard deviation (of all Insitec-S reticle measurements).

(b) The range is the allowed percent variation of individual measurements from the certified value.

The results in Table 5.4 demonstrate Insitec-S performance for common measurement parameters. It shows that concentration measurements can be generally reproduced to within 5 percent assuming no changes in the physical system being measured. For the most commonly used percentile values, namely the $\mathrm{d}[10], \mathrm{d}[50]$, and $\mathrm{d}[90]$, measurement reproducibility is $\sim 1$ percent or better. With respect to the measured PSD, Figure 5.12 demonstrates excellent reproducibility at cumulative volume undersize concentrations greater than $10^{-2}$. As the $10^{-2}$ cutoff corresponds to the first percentile (i.e., the d[1]), this observation is consistent with the reproducibility results for the $\mathrm{d}[10], \mathrm{d}[50]$, and $\mathrm{d}[90]$ results listed in Table 5.4. At volume fractions under $10^{-2}$, significant variation between repeat measurements is observed. This variation is associated with the measurement and is observed to occur even for repeat performance checks on the same Insitec (i.e., the variation does not simply occur between instruments). Divergence becomes significant below cumulative undersize fractions of $0.5 \times 10^{-3}$. This means that size measurements and fraction aerosol concentration measurements below $0.5 \mathrm{vol} \%$ of total aerosol concentration (which occurs at $\sim 23 \mu \mathrm{m}$ for the Insitec reticle) are not reproducible to the same extent that measurements in the typical operating range of the Insitec (generally considered between the $5^{\text {th }}$ and $95^{\text {th }}$ percentiles) can be reproduced.

The result shown in Figure 5.12 must be approached with caution, as the presentation of results suggest greater sensitivity to low volume fractions of small particles than the Insitec-S can actually achieve. In particular, the reticle PSD presented shows cumulative volume fraction undersize that spans 
10 orders of magnitude (i.e., $10^{-10}$ to 1 ). In contrast, certified reticle diameter results are only provided from $10^{-1}$ to 0.90 . Because certified diameters are provided for a limited portion of the reticle curve, accuracy of individual Insitec-S can only be assessed down to the $10^{-1}$ when comparing the measured result to the reticle's certificate of analysis. Microscopic analysis of the reticle, as documented in the certification documents that accompany the reticle, suggest lower and upper dot diameters of 4 and $95 \mu \mathrm{m}$. The PSD produced by the Insitec-S falls within this range for the volume fraction undersize shown in Figure 5.12. However, the result produced by the Malvern covers the full range of aerosol diameters selected for analysis, which is 0.1 to $1500 \mu \mathrm{m}$ for the default instrument settings. The full result indicates aerosol particles in the 0.1 to $3 \mu \mathrm{m}$ size range are observed over $10^{-30}$ to $10^{-10}$ fractional undersizes. Such results are produced by inversion of the mathematical fit of the scattering pattern and do not likely correspond to actual dots of this size on the reticle. Moreover, if small particles in this size range do exist at volume fractions lower than $10^{-2}$, either as dust on the windows or background aerosol in the environment, it is unlikely that the Insitec would be able to measure background particulate either during aerosol spray or during background measurements.

The ability to report such low fractional volumes (e.g., $10^{-30}$ ) is a result of averaging a significant number of observations, which generally range from 600-2400 individual PSD observations for each Insitec-S performance check. For single un-averaged measurements, such as those employed in analysis of large-scale aerosols produced by spray, results are reported to $\sim 10^{-10}$. This is still well below the point at which measurement precision appears to diverge. As such, the limiting factor in instrument precision appears to be measurement divergence at $\sim 0.5 \times 10^{-3}$.

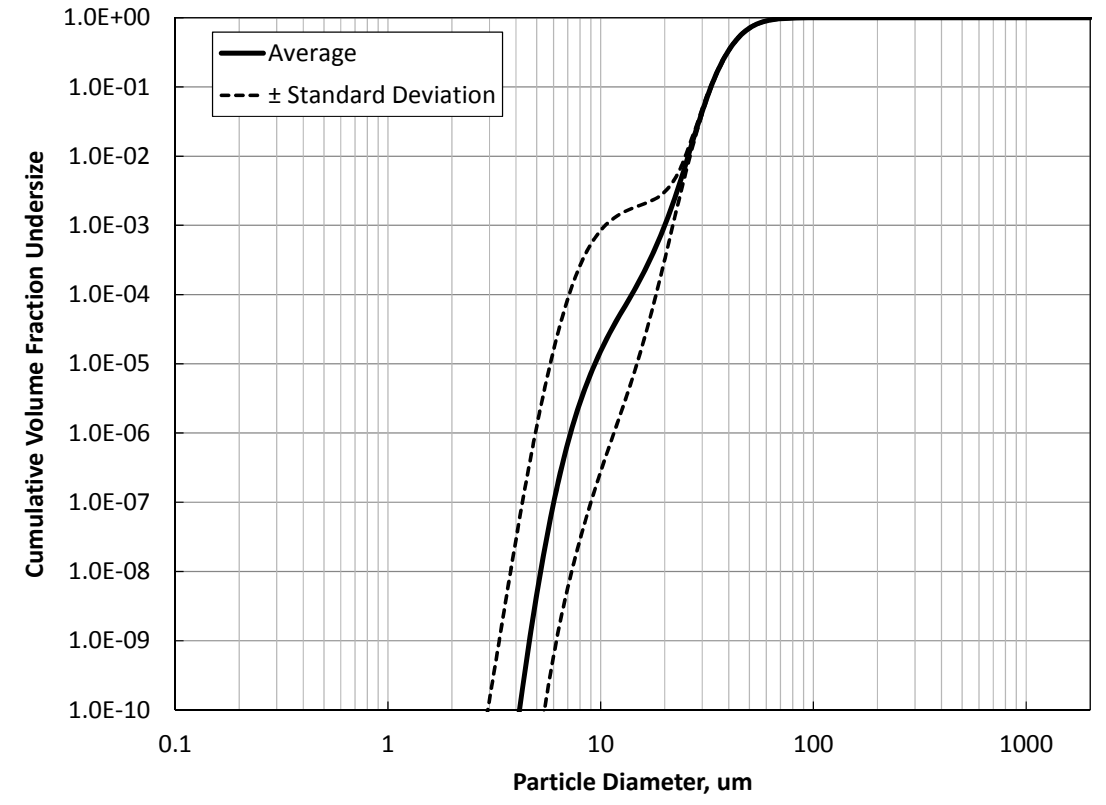

Figure 5.12. Analysis of Insitec-S PSD Measurement Reproducibility Using a Physical Standard (Note: the standard deviation curves indicate variation in the cumulative volume percent undersize at a fixed diameter) 
The arguments made in preceding paragraphs with respect to Insitec-S precision apply only to measurements of cumulative volume fraction undersize as a function of diameter (which we represent as $F_{v, k}$ for aerosol diameters at and below $d_{k}$ ). Care must be taken not to confuse volume fraction undersize (which is a measure of particle size distribution) and its associated uncertainty with measurements of aerosol release fraction. As discussed in Chapter 3, release fraction represents the product of the fraction of spray aerosolized, $b$, and the cumulative volume fraction undersize generated by the spray, $F_{k}$. Both quantities that determine release fraction (i.e., $b$ and $F_{k}$ ) are determined from the initial rise rate in concentration as a function of particle diameter. Because $F_{k}$ is a particle size distribution, it reasonable to expect that its precision as a function of particle diameter will follow the same trends as the PSD of the reticle shown in Figure 5.12. As such, loss of precision in the inferred PSD generated by spray, $F_{k}$, is expected to occur when $F_{k}<0.5 \times 10^{-3}$. It is postulated that loss of measurement precision in the PSD is also expected to yield a similar loss of precision in the release fraction. Such loss will occur when the release fraction, $\mathrm{RF}$, falls below $\sim\left(0.5 \times 10^{-3}\right) \cdot b$. Replicate RF measurements, such as those shown in Appendix B Figures B.1 and B.3, appear to support this assertion. It should also be noted that because $R F$ is the product of two numbers, uncertainty in the $R F$ result as a function of diameter will also depend on the uncertainty in $b$, which will cause variation throughout the $R F$ curve. Such variation manifests as differences in repeat measurements of the RF asymptote as large-particle diameters.

\subsubsection{Process Particle Counter}

A single PPC was purchased from Process Metrix for large-scale testing. Use of the PPC was intended to provide secondary measurement of aerosol concentration and size distribution. Initially, it also was intended to provide measurements at aerosol concentrations below the minimum detection limit for the Insitec-S analyzers; however, reconfiguration of the Insitec-S analyzers used for large-scale testing allowed measurement of aerosol concentrations for most test configurations and orifice sizes, and as such, the PPC was not needed for sprays that generated low aerosol concentrations.

The PPC is similar to the Insitec-S in that it measures aerosol size and concentration using laser diffraction. As shown in the basic PPC instrument schematic in Figure 5.13, the PPC consists of two relatively small diameter (35- and 150- $\mu \mathrm{m}$ ) lasers that pass through a measuring zone filled with aerosol. The lasers operate in sequence over a specified duty cycle (e.g., 2 s) so only one laser is active at a given period in time, and light scattering may be attributed entirely to scattering as particles enter the active beam diameter. The measuring volume defined by the collection optics, slit, and laser beams is on the order of $10^{-4} \mathrm{~cm}^{3}$ and is roughly trapezoidal in shape. Laser interactions for particles that do not pass within the measuring volume are either not collected by the optical lens or are screened by the slit positioned between the collection optics and photodiode. Scattered light is only collected and focused on the photodetector for particles that enter the measuring volume. Both small and large laser beams strike the collection optics off-center, with undeflected laser angles of 8 and 3 degrees, respectively. A photomask blocks low angle diffraction so the solid angle over which scattered light is collected is an off-center circular segment. The range of scattered angles collected for the small and large beams is 6.75 to 18.5 degrees and 1.75 to 13.5 degrees, respectively. 


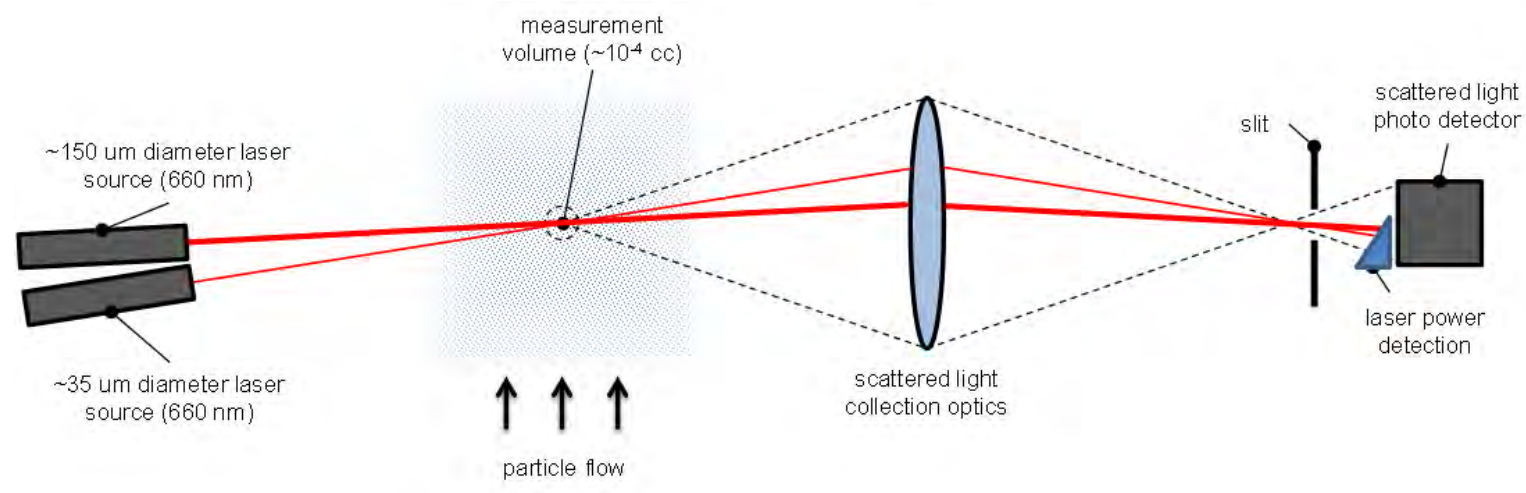

Figure 5.13. Schematic Showing the Basic Components and Principle of Operation for the PPC. The PPC is a two laser aerosol size and concentration analyzer that operates on the principle of laser diffraction. Interaction of the laser with single particles yields a diffraction pattern. The diffraction pattern is collected over a fixed range of scattering angles and focused onto a single photodetector. The power of scattered light, along with the frequency of scattering events, is accumulated over a fixed data collection period and translated into aerosol concentration and size distribution.

The collected light is focused onto a photodetector that converts laser power into an equivalent voltage signal. The measuring volume is sufficiently small that, for concentrations $<10^{4}$ particles $/ \mathrm{cm}^{3}$, it may be assumed that only one particle is in the measuring volume at a given time. Thus, each scattering event resolved by the photodetector can be attributed to a single particle passing through the measurement volume. The laser intensity is not uniform across the cross-section of the beam; rather, the beam profile is Gaussian and gradually decreases from its peak intensity at the center of the beam to zero at large radial distances from the beam center. Because of this variation in beam intensity, the size cannot directly be inferred from a single-scattering event. However, if enough scattering events are collected (typically 1000 to 10000 particle interactions), it can be assumed that all particle scattering configurations have been sampled, and the size and concentration of the accumulated scattering ensemble may be calculated. The accumulation period necessary to collect a statistically significant number of scattering events is concentration dependent, with higher particle concentrations requiring lower accumulation times relative to lower particle concentrations. As such, the accumulation period nominally should be selected to provide the required level of statistical significance. The PPC allows the user to set accumulation from as low as $5 \mathrm{~s}$ up to several hours. It should be noted that accumulation will time-average any transient conditions that occur during the accumulation period. This will smooth any variations in the data, but may also damp transients of interest, such as the initial rise in concentration from which release fraction is determined. For the purposes of large-scale testing, the minimum available accumulation period of $5 \mathrm{~s}$ was selected to minimize the time-averaging that occurs in the initial rise in concentration.

The PPC measures concentration by first counting the number of individual scattering events caused by particles passing through the measurement volume that occur within the given sampling period. This signal count measurement is supplemented by analysis of the duration of each scattering event to determine the transit time of particles through the laser beam. The transit time provides a measure of particle (or gas carrier phase) velocity which, along with the known cross-sectional area of measuring volume, can be used to calculate the volume of aerosol sampled in a given measurement period. Finally, 
particle concentration is determined by dividing the particle count by the calculated volume of aerosol sampled.

Particle count and concentration measurements are subject to inaccuracy associated with poor or unrepresentative sampling of aerosols. In addition, the number of particles will be underestimated should more than one particle frequently pass through the measuring volume at the same time. In this case, the PPC will be unable to distinguish the multiple scattering events caused by more than one particle in the measuring zone from a single scattering event. Multiple scattering events occur at high concentrations $\left(>10^{4}\right.$ particles $\left./ \mathrm{cm}^{3}\right)$ and pose an upper working concentration boundary beyond which the PPC cannot determine aerosol concentration with accuracy.

Based on the characterization methodology described in the preceding paragraph, accurate measurement of aerosol by the PPC is subject to 1) ensuring a statistical sampling of all aerosol paths through the laser beam sampling volume, and 2) associating a given detector response (or distribution of responses) to a unique particle size. The first condition may be met simply by allowing for sufficient aerosol accumulation time. The latter condition poses some difficulties, as it depends on the optical properties of the system and on the fixed optical configuration of the PPC. In general, the PPC optical configuration has been optimized to yield a response curve that typically increases for increasing aerosol diameter. That is, a large aerosol passing directly through the center of the PPC laser will generally yield a higher detection response voltage than a small particle also passing directly through the center of the laser beam. However, for particles within the nominal PPC size range (3.5 to $67 \mu \mathrm{m}$ as given in following paragraphs), the response curve can be flat for certain diameters. This means that two particles of similar (but not the same) diameter may give the same response (even when uncertainty introduced by measurement noise is excluded) and will be interpreted as two particles of the same size, biasing the concentration in the size range interpreted by the PPC signal analysis routine. It is postulated that this uncertainty is reduced somewhat by smoothing algorithms employed in inversion of the accumulated voltage response. Difficulty is also encountered when large particles pass through the laser beam. As the particle size becomes significant relative to the laser beam width (i.e., at about 50\%), the scattered laser intensity collected by the PPC optics tends to decrease. This phenomenon is termed "roll-off", and its impact to the data is that should a large particle pass through the PPC laser beam, it may be interpreted as a particle of smaller diameter. This will bias high the volume fraction of particles whose true signal corresponds to the attenuated signal of larger particles. The overall impact of "roll-off" is increased uncertainty in the PSD measured by the PPC. It should be noted that concentration measurements result from direct counting of the scattering events observed by the PPC and, unlike the Insitec-S, will not be subject to uncertainty in the measured PSD. This uncertainty does not impact the accuracy of release fraction calculations presented in this report, as the PPC was not used in release fraction calculations.

As configured for large-scale aerosol chamber measurements, the specific size and concentration range of the PPC are as follows:

- Size Range - The large (150- $\mu \mathrm{m}$ diameter) beam is used to characterize particles that range from 3.5 to $67 \mu \mathrm{m}$, while the small (35- $\mu \mathrm{m}$ diameter) beam is used to characterize particles that range from 0.5 to $2.6 \mu \mathrm{m}$ (Process Metrix 2007). This configuration yields an overall dynamic size range of 0.5 to $67 \mu \mathrm{m}$ for PPC measurements. This size range may be impacted should insufficient sample counts be reached.

- Concentration - The PPC has an upper concentration limit of $\sim 10^{4}$ particles $/ \mathrm{cm}^{3}$. At higher concentrations, the probability that more than one particle may simultaneously exist in the measuring 
volume increases, and the instrument software cannot correctly interpret simultaneous scattering of two or more particles. The exact volume concentration that this upper limit corresponds to depends strongly on the PSD. For monodisperse systems with particle diameters of 20, 50, and $100 \mu^{8}$, the concentration limits correspond to approximately 40, 700, and 5000 ppmv, respectively. Because the PPC can detect and accumulate single-scattering events, there is no theoretical limit (at least with respect to the principle of operation) on the lower concentration limit of the instrument. However, for large-scale spray testing, there are practical limits with respect to the reasonable accumulation periods. Specifically, at low concentrations, the number of laser interactions that occur during the set accumulation period may not be sufficient to yield acceptable size and count statistics. In addition, although lower concentrations may be achieved by increasing the accumulation period, the accumulation period may not be set longer than the expected rise time for concentration to reach equilibrium without losing the ability to resolve aerosol generation rates. In particle terms, this introduced a lower concentration limit of approximately 0.01 ppmv for large-scale testing. Overall, these constraints generally limited the concentration range measurable by the PPC from approximately 0.01 to $1000 \mathrm{ppmv}$.

For large-scale testing, the PPC was co-located with the Malvern instrument located in position 2 (see Table 5.2 for the dimensions of that location). The PPC was not directly installed in the chamber, but was rather set on the top-outside of the chamber. It was connected to a 0.75 -in. inner diameter conductive draw tube that was inserted $2 \mathrm{ft}$ down from the top of the chamber (placing the sample location for the PPC at approximately the same level as that for the Insitec-S instruments). Because the PPC is not installed in the chamber, use of a vacuum pump to draw aerosol from the aerosol chamber into the PPC was necessary. A PPC vacuum gas flow rate of $1.5 \mathrm{scfm}$ (standard cubic feet per minute) was used for all large-scale tests. Because the PPC draws aerosol from the chamber, it has the potential to impact in-chamber aerosol readings made in the vicinity of the draw tube inlet. Indeed, PPC vacuum draw had been observed to impact aerosol concentration measurements made by the Insitec-S system in small-scale scoping tests. As such, care was taken to offset the PPC draw tube from the Insitec-S test location to prevent influence of aerosol sampling by the PPC on the Insitec-S result. Finally, because the PPC measuring volume is located out of the large-scale test chamber, there is no concern about contamination of the optical surfaces inside the system by direct aerosol impact. However, it is possible to contaminate the PPC optics through diffusion or convection. As such, the PPC is also equipped with purge flow (with the gas flow rate set at a total of $\sim 2 \mathrm{SCFH}$ ) to prevent window contamination.

PPC control and data acquisition is controlled through computer using the Process Particle Counter Version 2.30.001 software provided by Process Metrix. The main software parameters that can be adjusted to control experiments (that cannot be changed during post-measurement analysis) are 1) the accumulation period, 2) the frequency that results are reported, and 3) the laser duty cycle. As discussed above, the accumulation period was set to $5 \mathrm{~s}$ for all large-scale measurements. This is the lowest accumulation period that can be selected and was chosen to allow resolution of the initial rise in concentration (from which generation rates must be estimated) and to minimize the impacts of time-averaging for this initial rise. A result-report interval of $2 \mathrm{~s}$ was selected for all measurements and is the highest frequency at which results may be reported by the software. The laser duty cycle selected was also $2 \mathrm{~s}$, with 80 percent (1.6 s) of the cycle employing the 150 - $\mu \mathrm{m}$ laser and 20 percent $(0.4 \mathrm{~s})$ using the 35- $\mu \mathrm{m}$ laser.

${ }^{8}$ The $100 \mu \mathrm{m}$ concentration limit is for illustrative purposes only since the PPC has an upper particle size limit of $67 \mu \mathrm{m}$. 
In practice, the reporting and accumulation settings mean results will be reported every $2 \mathrm{~s}$ and represent an accumulation of the last $5 \mathrm{~s}$ of recorded scatting events. Prior to the start of spray, the PPC measurement is activated so that the initial rise in concentration is captured. However, this means that when the spray is started, the first measurements at $\sim 2$ and $\sim 4 \mathrm{~s}$ also contain fractional accumulation periods during which no aerosol was present. As stated in the preceding paragraphs, this has the effect of smoothing (time-averaging) the initial rise in concentration. If the concentration rise time is fast relative to the 5-s accumulation period, inclusion of pre-spray measurements will "slow" the apparent rate of aerosol generation. For this reason, the PPC was not well suited for determining aerosol generation rates from concentration rise in many tests, where the time scales observed were comparable to the 5-s accumulation period employed by the PPC. Its use in large-scale aerosol testing was primarily to support size and concentration measurement made by the Malvern Insitec-S aerosol analyzers.

The Insitec-S and PPC sample different dispersion volumes for each measurement period, because of their different installation locations (in-chamber versus out-of-chamber), different measurement volumes ( $\sim 5$ to $33 \mathrm{~cm}^{3}$ versus $10^{-4} \mathrm{~cm}^{3}$ ), and different accumulation periods ( 1 or $4 \mathrm{~Hz}$ versus $0.2 \mathrm{~Hz}$ ). Rough estimates of the volume of dispersion suggest that in the $5 \mathrm{~s}$ measurement interval, the PPC generally observes about $2 \mathrm{~mL}$ of dispersion. Likewise, rough estimates indicate that the Insitec-S samples on the order of $100 \mathrm{~mL}$ and $400 \mathrm{~mL}$ of dispersion at sampling rates of 4 and $1 \mathrm{~Hz}$, respectively. These estimates suggest that individual PSD and concentration measurements produced by the Insitec-S are more statistically significant (i.e., are more representative of the actual aerosol).

Finally, it should also be noted that the PPC employed in large-scale testing was factory configured for slurry-test systems and uses a hard-coded refractive index (RI) of 1.5+0.5i for particulate systems and $1.0+0 \mathrm{i}$ for the media refractive index. In addition, the PPC software does not provide a means of easily changing the refractive index during post-measurement analysis. While the hard-coded refractive index does not pose significant difficulties when analyzing opaque systems (e.g., aerosols generated from slurry systems), it does limit comparison of PPC data collected for water sprays to accompanying measurements collected by the Insitec-S analyzers.

\subsection{Mixing Inside the Chamber}

At the start of testing, there was significant concern regarding the uniformity of the aerosol concentration throughout the chamber, particularly when the full 20-ft length of the chamber was used. To create a more homogenous aerosol concentration within the chamber, multiple fan configurations were considered. These included 1) two open fans at approximately the center of the first 10 - $\mathrm{ft}$ section and 1-ft below the spray header, and 2) four "shrouded" Detmar fans located at approximately the center of the 20 - $\mathrm{ft}$ chamber and just above the collection pan of the chamber. A series of spray release tests were conducted using a 2-mm hole to identify the fans and fan settings that gave the best mixing and repeatable measurements of the aerosol concentrations within the chamber. The results, which will be discussed in Chapter 8, indicated that only the center two shrouded Detmar fans in the "Fan Array" shown in Figure 5.14 were required to achieve adequate mixing. These 4 -in. fans were installed with flexible ducting that moved the inlet closer to the bottom of the chamber and spray header to preclude any influence on the jet. 


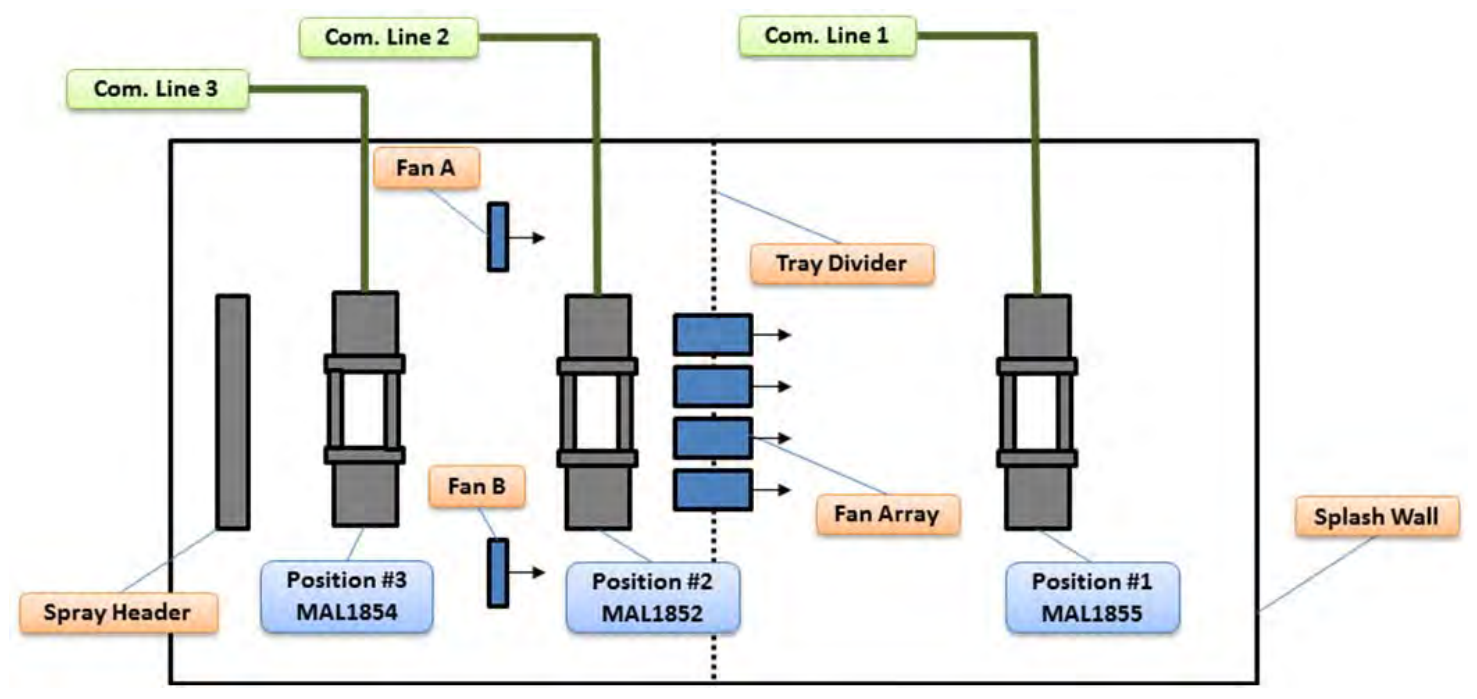

Figure 5.14. Top View of Malvern Instruments and Fan Arrangement

Figure 5.14 and Table 5.2 show the location of each Malvern instrument relative to the spray header in the standard configuration (i.e., without the extensions).

\subsection{General Instrumentation}

Several instruments were used to acquire data for the test operating conditions. Refer to Figure 5.2 for a schematic showing the various instruments used in the large-scale test configuration. These instruments are listed in Table 5.5 along with the manufacturer, model, and accuracy specifications. Also listed in the table is the data quality associated with each of these instruments. Here "NQA-1" implies that the data meets the NQA-1 requirements for the project by ensuring that the instrument has been either calibrated by an approved vendor or through approved PNNL procedures. Instruments that are listed under the category "For Information Only" are those that are used to provide information to the test operator.

It should be noted that the instruments listed in Table 5.5 and the schematic in Figure 5.2 do not show the aerosol concentration measurement devices that are included in the spray chamber. These were previously discussed. A measuring and test equipment list that includes calibration information was maintained separately for the project. This list tracked any changes to the instruments that were added or removed from the large-scale test system.

Data from the sensors listed in Table 5.5 were logged on a DAS running Allen Bradley Programmable Logic Controller (PLC) data acquisition and control software. The PLC-based DAS was chosen for this application because of its robustness and versatility; that is, it would allow expansion to an automated control system if desired. 
Table 5.5. Instrumentation Used for Process Parameter Measurements

\begin{tabular}{|c|c|c|c|c|c|}
\hline Measurement & Relative Test Location & Manufacturer & Model & Accuracy & Data Quality \\
\hline \multirow{3}{*}{ Weight } & Storage Tank (TK-1) & \multirow{3}{*}{ Hardy Instruments } & HI HLPS (18000\#) & $\pm 4.5 \mathrm{lb}$ & NQA-1 \\
\hline & Feed Tank (TK-2) & & HI HLPS (18000\#) & $\pm 4.5 \mathrm{lb}$ & NQA-1 \\
\hline & Capture/Waste Tank (TK-3) & & HI HLPS (9000\#) & $\pm 2.25 \mathrm{lb}$ & NQA-1 \\
\hline \multirow{8}{*}{ Pressure } & Bypass Leg (Absolute) (PS6) & \multirow{8}{*}{ Omega } & \multirow{8}{*}{ PX44E0-500GI } & \multirow{8}{*}{ \pm 2.5 PSI } & NQA-1 \\
\hline & Test Section (Absolute) (PS4) & & & & NQA-1 \\
\hline & Test Section (Backup) (PS5) & & & & NQA-1 \\
\hline & Feed Pump 1 (PS-1) & & & & For Information Only \\
\hline & Feed Pump 2 (PS-2) & & & & For Information Only \\
\hline & Feed Pump 3 (PS-3) & & & & For Information Only \\
\hline & Flow Meter 2 (PS-6 and PS-7) & & & & For Information Only \\
\hline & Globe Valve 2 (PS-8) & & & & For Information Only \\
\hline \multirow{3}{*}{ Humidity } & Test Chamber (RH-1) & \multirow{3}{*}{ Omega } & \multirow{3}{*}{ HX93DAC-RP1-F } & \multirow{3}{*}{ $\pm 2.5 \% \mathrm{RH}$} & For Information Only \\
\hline & Test Chamber (RH-2) & & & & For Information Only \\
\hline & Ambient (RH-3) & & & & For Information Only \\
\hline \multirow{6}{*}{ Temperature } & Test Chamber (TS-5) & \multirow{6}{*}{ Omega } & \multirow{6}{*}{ PR-13-2-100-1/4 } & \multirow{6}{*}{ $\pm 1^{\circ} \mathrm{C}$} & NQA-1 \\
\hline & Ambient (TS-6) & & & & NQA-1 \\
\hline & Storage Tank (TS-1) & & & & NQA-1 \\
\hline & Feed Tank (TS-2) & & & & NQA-1 \\
\hline & Capture/Waste Tank (TS-4) & & & & NQA-1 \\
\hline & Test Section (TS-3) & & & & NQA-1 \\
\hline \multirow[b]{2}{*}{ Flow Rate } & Downstream of Pump 1 (FE-1) & \multirow[b]{2}{*}{ MicroMotion } & \multirow[b]{2}{*}{ F300S } & \multirow[b]{2}{*}{ \pm 0.027 gal } & NQA-1 \\
\hline & $\begin{array}{l}\text { Downstream of Primary Pres. } \\
\text { Reg. Valve (FE-2) }\end{array}$ & & & & NQA-1 \\
\hline \multirow[b]{2}{*}{ Density } & Downstream of Pump 1 (FE-1) & \multirow[b]{2}{*}{ MicroMotion } & \multirow[b]{2}{*}{ F300S } & \multirow[b]{2}{*}{ $\pm 0.0001 \mathrm{~g} / \mathrm{cm}^{3}$} & NQA-1 \\
\hline & $\begin{array}{l}\text { Downstream of Primary Pres. } \\
\text { Reg. Valve (FE-2) }\end{array}$ & & & & NQA-1 \\
\hline
\end{tabular}




\subsection{Data Acquisition Systems}

Several different DAS systems were used for this testing. The first system includes the PLC DAS that collects raw data from the various devices that measure pressure, temperature, humidity, mass, and density, and logs the measurements into a file in $0.1-\mathrm{s}(10-\mathrm{Hz})$ intervals. The data were post-processed (i.e., converted to engineering units and analyzed) to determine the mass and volume of fluid lost during the spray. Logging the data at $10-\mathrm{Hz}$ required user coding and, therefore, was verified and validated in accordance with the QA-WTPSP-303, Software Control (SCP) Developmental Work, procedure before any reportable data was collected from the sensors. Calculations used to convert from analog $\mathrm{mA} /$ voltage data logged by the DAS to engineering units complied with QA-WTPSP-0304 requirements. The Malvern Insitec-S and the PPC aerosol concentration and PSD measuring devices come with their own commercial off-the-shelf software/DAS and were used as provided by the supplier without further verification and validation of the software. In addition, procedures were developed for both systems to conduct performance checks.

Commercial off-the-shelf software such as MATLAB and Microsoft Excel were used during post-test data calculations and data manipulations. All MATLAB outputs and spreadsheets generated during test data analyses were reviewed per the requirements of QA-WTPSP-0304.

Data and observations were collected in Laboratory Record Books, Test Instructions, and bench sheets. Hand-recorded data complied with QA-WTPSP-1702.

Orifice dimensions were measured by an optical microscope system that was calibrated with a NIST-traceable standard.

All electronic data were write-protected and backed up (in two storage devices) from the DAS computers at APEL Laboratory 184 on the day a test was completed. Secondary storage backups consisted of an extra internal hard drive on one of the DAS computers and a portable hard drive. An additional backup is maintained on a PNNL share drive specifically assigned to the project.

Limited spray release video recordings and/or photographs were collected for selected conditions for information only. High-quality video and still photographs were limited by the chamber size, view angle from the ports, and poor lighting. 



\subsection{Test Approach and Operations}

Chapter 5 presented a detailed description of the large-scale test configuration and equipment. This chapter presents the general methodology that was used throughout all of the testing and representative pressure and flow profiles to demonstrate system operation. The approach used to prepare the simulants used during testing also is presented. Finally, a matrix of the various tests that were performed is presented.

\subsection{General Test Approach}

Each test was conducted per an approved Test Instruction and all data and necessary information was recorded electronically, in Laboratory Record Books or in the Test Instruction itself. Operationally, each test consisted of the following checks:

- Daily start-up checks

- Spray release tests

- Daily close-out checks.

In addition, weekly performance checks were conducted for the Malvern Insitec-S and PPC aerosol measurement instruments. These checks involved removing the instruments from the chamber and using calibrated standards called reticles to determine if the measurements were within acceptable ranges.

\subsubsection{Daily Start-Up Checks}

Daily start-up checks involved turning on the instruments, configuring the loop for testing, checking the functionality of all components, and allowing the entire system to warm up. Key tasks that were performed during daily start-up checks are identified below:

- Turning on all instruments and DASs

- Synchronizing all DAS and, if needed, video/still camera clocks

- Performing a functional check of all key instruments

- Ensuring that ancillary equipment to the aerosol instruments are operating

- Mixing feed/storage tanks, as needed

- Verifying valve configurations

- Configuring chamber, internal components, and instrumentation as required and recording any changes in the Laboratory Record Book

- Pre-saturating the chamber and collecting baseline data.

\subsubsection{Spray Release Tests}

Spray release tests involved the actual test runs. To prevent ambient light from influencing the Malvern Insitec-S measurements, the chamber windows were covered in black plastic and the chamber 
lights were turned off. The two shrouded chamber fans were verified to be on and set to $12 \mathrm{~V}$; this was the voltage determined during the shakedown tests to attain good mixing within the chamber (see Chapter 8). At this point, baseline data needed for the Malvern and PPC instruments were collected, and then, data logging was initiated on the PLC and the Malvern Insitec-S /PPC DASs.

The desired test section pressure and flow were achieved by bringing the system up to operating conditions via the Pump-1 VFD and adjusting globe valves GV-1 and GV-2 (see Figure 5.32). Note: the fluid velocity was set to be $>6.5 \mathrm{ft} / \mathrm{s}(>140 \mathrm{gpm}$ ) in the Coriolis meter (FE-2) downstream of the test section pipe. In addition, the pressure downstream of the test section the Coriolis flow meter (FE-2) pressure was maintained at 50 psig to preclude formation of micro-bubbles that could lead to erroneous readings.

Upon achieving the required operating conditions, the spray release lever was opened for the orifice that was being evaluated. Simultaneously, after opening the spray release lever, the DAS operators were notified to record the time when the spray was started. Spray duration typically lasted $2 \mathrm{~min}$; although, occasionally, the duration was reduced (e.g., for sprays with leak rates >150 gpm) or increased (e.g., for time vs. concentration measurement tests or tests to precisely determine the mass of fluid lost from the breach). During the time the lever was open, the test supervisor constantly monitored the pressure at the test section to make sure the pressure did not drop by more than 10 psi because of the alternate flow path created by the breach. Because of slight pump leakage from the mechanical seal in Pumps 2 and 3 at high pressure (200 to $380 \mathrm{psig}$ ), the leaked fluid was collected and weighed for each test. ${ }^{9}$ The mass of the leaked fluid coupled with the mass change in the feed tank during a test was used to determine the amount of fluid lost through the breach. After the 2-min spray duration, the lever was closed to prevent fluid from further leaking through the test section. Simultaneously, the DAS operators were notified to record the time at which the lever was closed. After the spray was terminated, PLC and Malvern Insitec-S/PPC DAS data logging was terminated when the pressure and aerosol concentrations (as measured by the Malvern Insitec-S and PPC instruments) returned to approximately their baseline values. After this, the residual aerosol in the chamber was evacuated by forced ventilation of the chamber for at least 1 min.

Still images and video recordings of the sprays were collected separately from the spray tests to avoid impacting the Malvern Insitec-S laser light sensors. The lights in the chamber were turned on and the plastic window coverings were removed to allow sufficient light into the chamber for the image capture. Still images were captured by positioning a camera on top of a small Plexiglas (2-in. square) window located on the chamber wall right above the spray header. Video images were captured by opening a window along the side of the spray header.

Throughout each test, the feed vessel temperatures were maintained at $75 \pm 10^{\circ} \mathrm{F}$ to minimize any effects that may be caused by condensation or evaporation. During simulant testing, density was monitored at the Coriolis flow meters to make sure no significant changes occurred throughout the course of testing with a given simulant.

Samples were collected from the feed vessel before and after testing each day when testing with water and after each test when testing with the boehmite slurry or sodium thiosulfate simulants. The associated Test Instructions defined the naming conventions used for designating the grab samples collected during

\footnotetext{
${ }^{9}$ The fluid leak from the mechanical seals completely disappeared during slurry tests probably due to the small gibbsite particles lodging into the seal to prevent further leaks.
} 
testing. In general, the file naming convention allowed easy identification of the test conditions for which the samples were collected. For tests with water, the surface tension was measured for a subset of the samples collected. For the boehmite slurry simulants, a subset of the samples collected were analyzed for physical properties, such as viscosity and non-Newtonian rheology, PSD, bulk density, surface tension, and weight percent of undissolved solids (UDS). For the sodium thiosulfate simulant, only the viscosity, bulk density, surface tension, and weight percent of UDS were measured.

\subsubsection{Daily Close-Out Checks}

At the completion of testing each day, a series of daily close-out checks were performed to ensure that all the testing was completed as planned, video/still images were taken, data were backed up, data sheets were collected into test data packages (TDPs), and system valves were placed in a safe mode.

\subsection{System Pressure and Flow Discussion}

This section presents a brief discussion of the pressure/flow variations for the broad range of breaches that were evaluated during the testing.

When a spray was initiated for the largest orifices, the pressure and flow rate exiting the pumps decreased and increased, respectively. For all of the orifices tested, the pressure change was typically $<5$ psi and on the order of $\sim 10$ psi for the largest breaches. However the flow rate increased noticeably depending on the area of the breach. The reason for this is directly related to changing the system pressure (or head) curve when the spray is initiated. When the orifice is open to atmosphere, the system curve shifts to the right, and the point at which this intersects the pump curve shifts to the right. This is tantamount to a decrease in frictional loss and the pressure drop required to achieve a given flow. This intersection is the operating point. This can be illustrated by looking at the pump performance figure supplied by Krebs for the slurry pumps (see Figure 6.1). As an example, referring to the red arrow in the figure and using the best efficiency point as the system curve, shifting the curve to the right is representative of initiating the spray. Note that the head (and pressure drop) drops modestly from 160 to $150 \mathrm{ft}$, while the flow rate increases from approximately 280 to $350 \mathrm{gpm}$. The result is a relatively small pressure change, and an increase in the flow rate commensurate with the leak rate. This behavior provided stable test pressures that were very close to the target pressures.

A difference in the effect of slot size can be seen by comparing Figure 6.2 with Figure 6.3. Figure 6.2 shows the pressure and flow rate versus time for a small slot at a relatively low pressure, while Figure 6.3 shows a very large slot at a high pressure. Note that the change in pressure $(\approx 1.5 \mathrm{psi})$ and flow rate $(\approx 1 \mathrm{gpm})$ at spray initiation ( $\mathrm{t}=0 \mathrm{~s})$ is very small for the small-slot case; whereas, for the larger slot, this change is much larger ( $\approx 10 \mathrm{psi}, 55 \mathrm{gpm})$. As discussed previously, the pressure change is not as significant as the flow rate change upon spray initiation. 


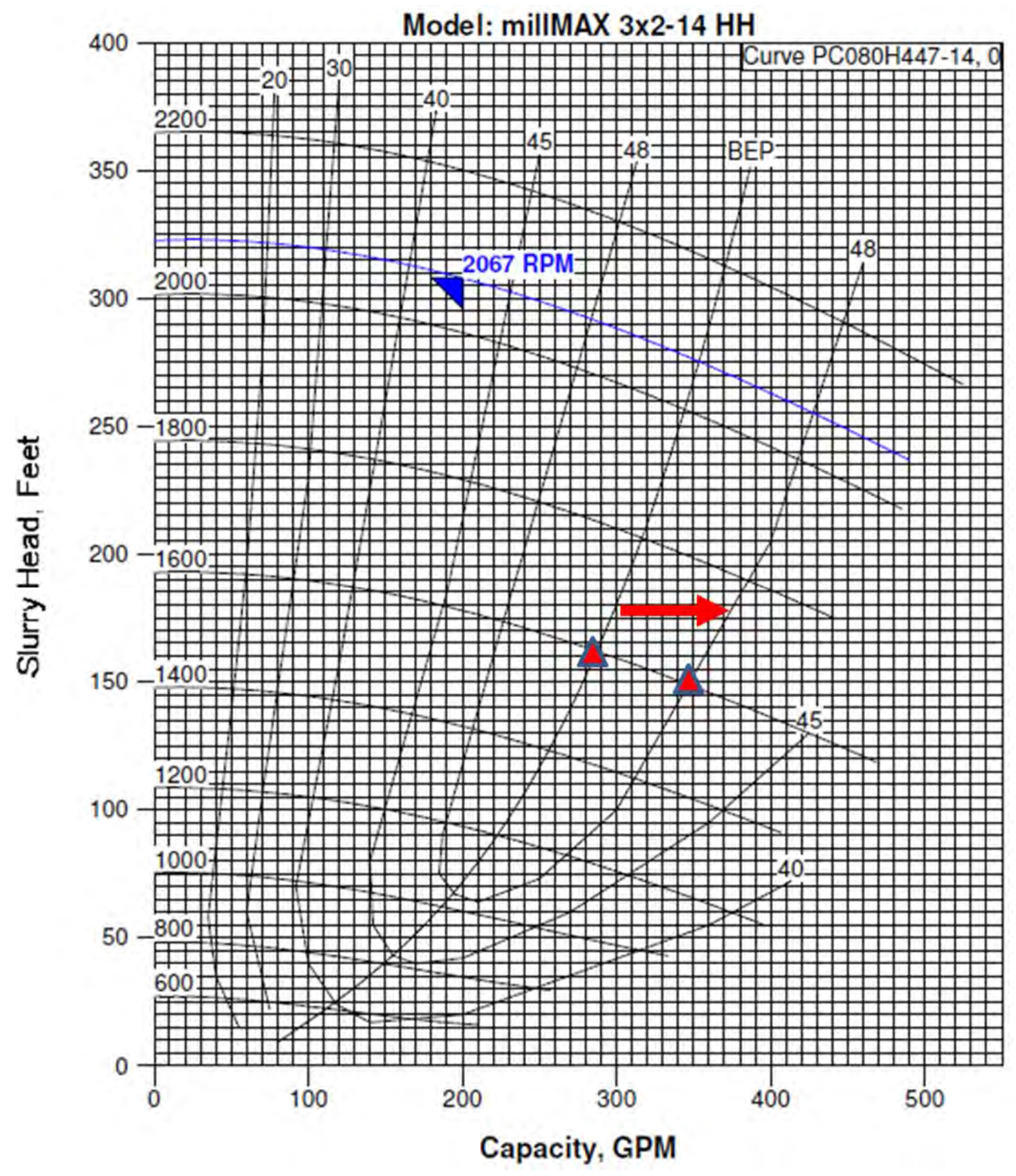

Figure 6.1. Pump Performance Curve for Pumps 1, 2, and 3 

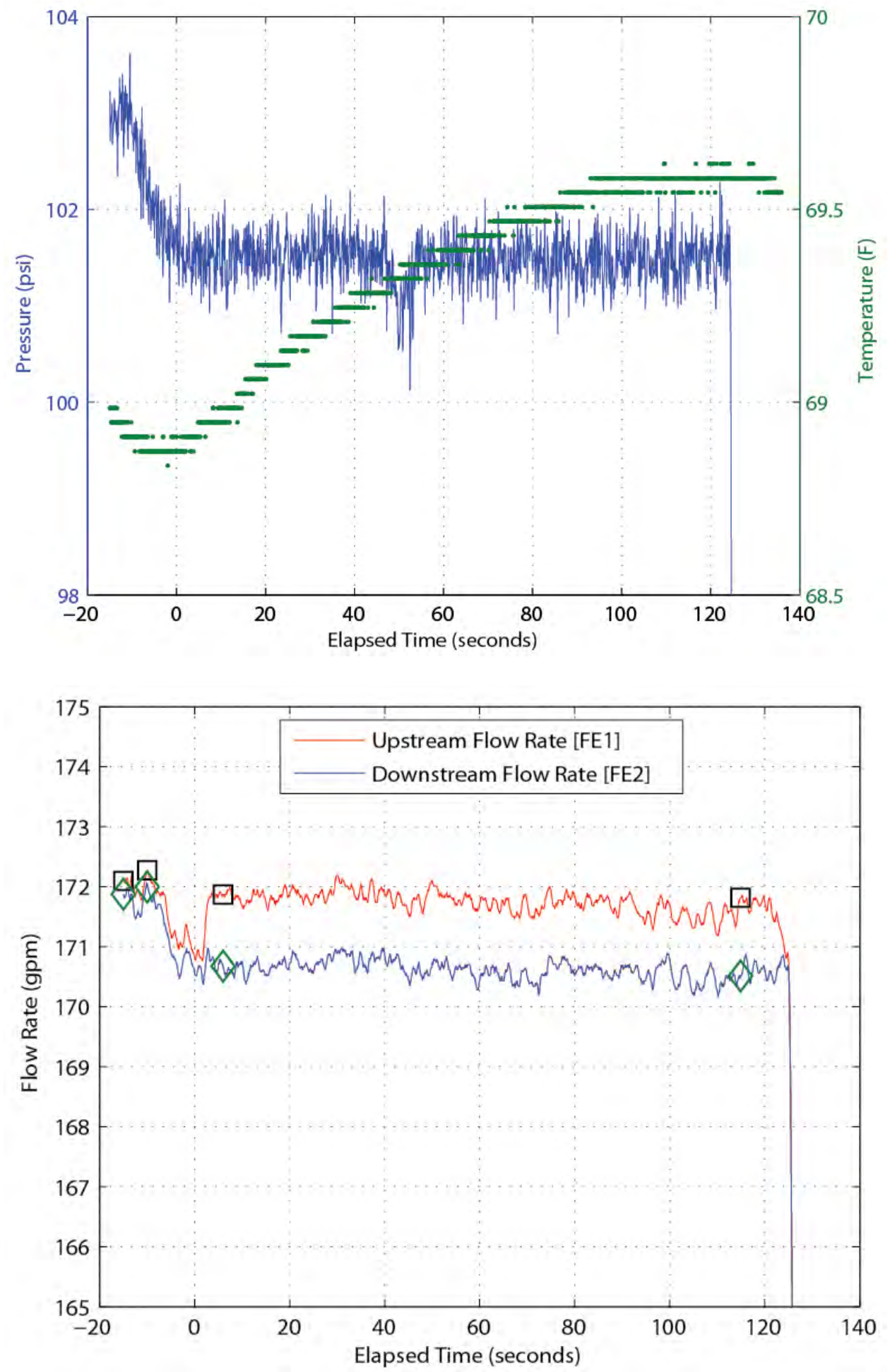

Figure 6.2. Slot with $0.5 \times 5 \mathrm{~mm}$ Dimensions (100 psi water test) 

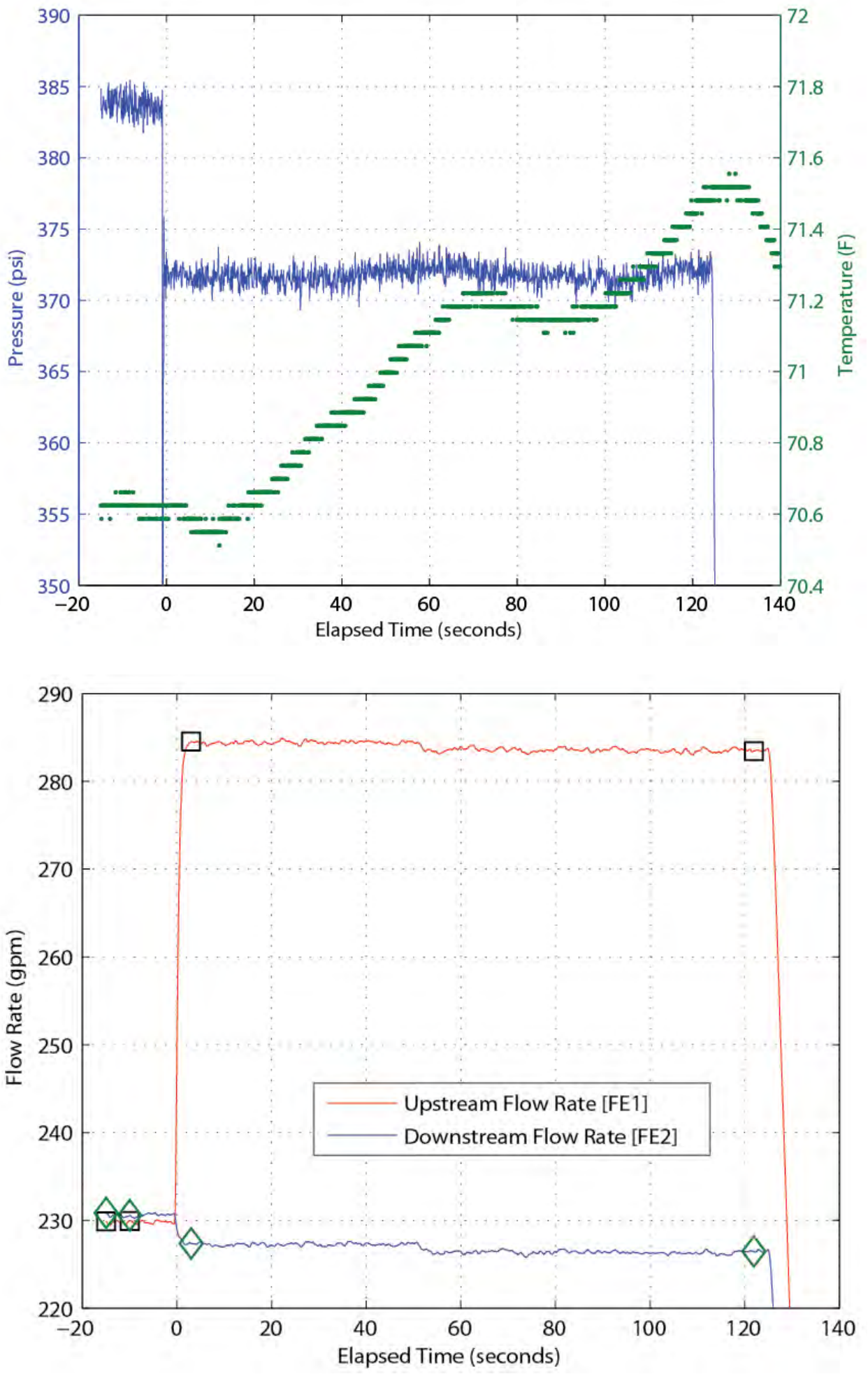

Figure 6.3. Slot with $1 \times 76.2 \mathrm{~mm}$ Dimensions (380 psi water test) 
As shown in Figure 6.4, the pressure change is negligible for this largest slot at the highest test pressure upon spray initiation. This is because at the same time the spray was initiated, the flow path was purposely restricted via a globe valve just downstream of the test section. If the flow path remained fully open, the pressure would drop well below the target pressure of 380 psig. The reason for adjusting the globe valve downstream of the test section at the exact time the spray was initiated was to prevent over-pressurization of the loop, which would cause a pressure relief flow path to open. This was a special case due to the most extreme operating conditions (i.e., the largest hole and highest operating pressure).
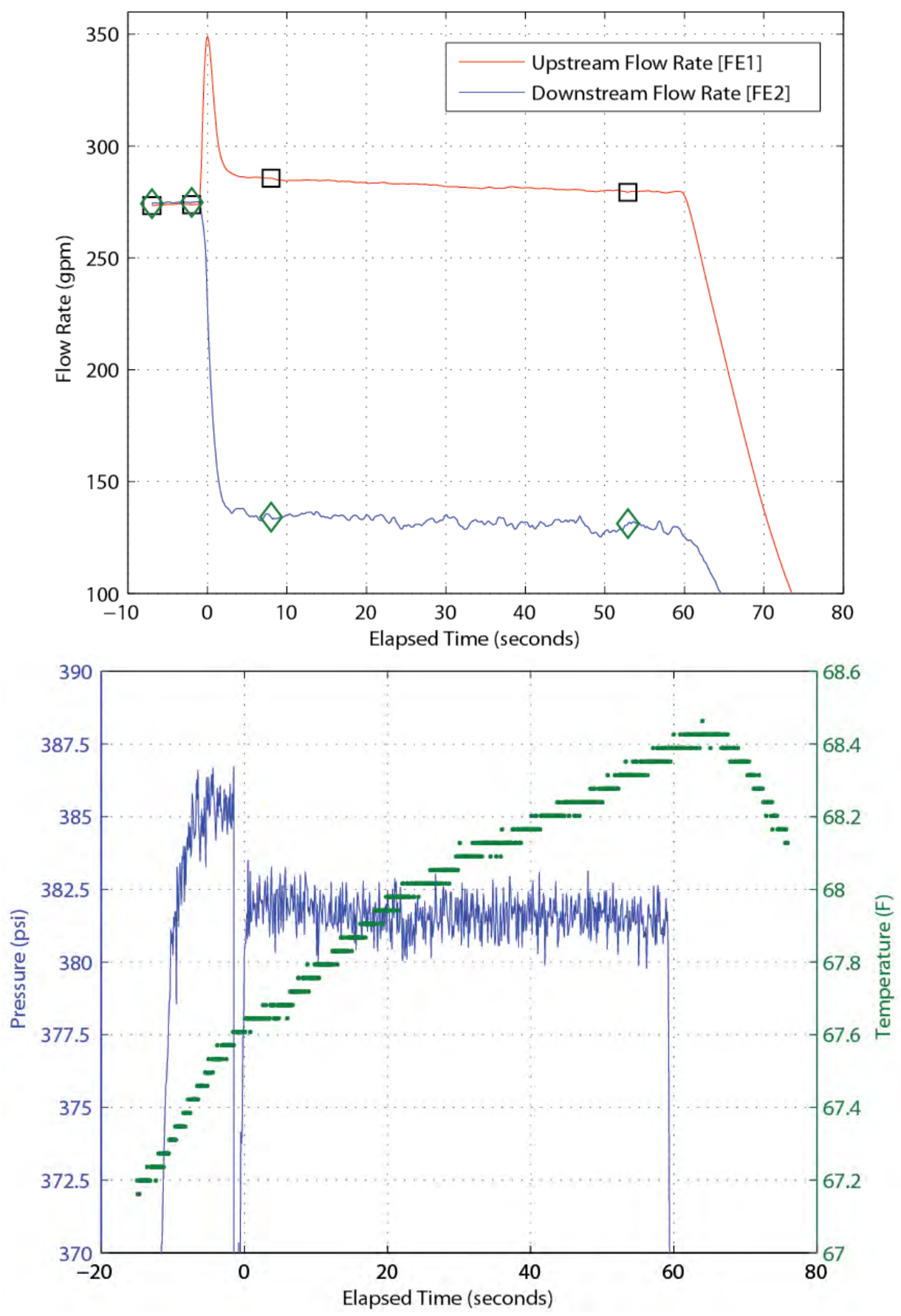

Figure 6.4. Slot with $2.74 \times 76.2 \mathrm{~mm}$ Dimensions (380 psi water test [dead head]) 


\subsection{Simulant Preparation}

As discussed in Chapter 4, two different kinds of simulants were used during large-scale testing: 1) one that primarily varied the viscosity of the fluid, and 2) another that created slightly non-Newtonian slurry. The effect of viscosity was evaluated with sodium thiosulfate and targeted to be $2.5 \mathrm{cP}$. Two varieties of boehmite were used to create the non-Newtonian simulant. The boehmite mixture was used to create 8 and 20 solid wt\% slurries. The basis for the simulant selection and the physical properties of the simulants used are presented in Chapter 4 . This section only presents the approach used to prepare the various simulants used during the large-scale testing.

Sodium thiosulfate pentahydrate was mixed with water in small $\sim 60$-gal batches using a 75 -gal mixing vessel and transferred via a pneumatic diaphragm pump to the large feed tank. Each batch was fully mixed for a minimum of 10 min before transfer to the feed tank. While in the feed tank, the large agitator was turned on and kept on overnight and until testing was complete. This continuous agitation accomplished the objective of making sure the sodium thiosulfate was fully dissolved. Samples were taken before testing to make sure the target viscosity had been reached.

The mixing process described above also was used for the $20 \mathrm{wt} \%$ boehmite slurry; however, the simulant was later diluted with water to achieve $8 \mathrm{wt} \%$ boehmite for the final round of slurry tests. To prevent the boehmite from settling and forming a hard cake, the slurry was kept in motion (i.e., the pumps and mixer ran continuously) until all slurry tests were complete. This continuous movement prevented the formation of large UDS concentration gradients within the tank or blockage in the pipes. Samples were taken before testing to make sure the target UDS concentration was achieved.

As with all testing, post-test samples were taken to make sure variations in the simulant characteristics were minimized. For example, the 8 and-20 wt $\%$ simulants remained within 0.5 percent of the initial sample taken.

\subsection{Test Matrix}

A series of 68 tests, listed in Table 6.1 and Table 6.2, were planned for determining the aerosol concentrations and release fractions at different pressures using eight orifices and three simulants (water, sodium thiosulfate, and 8 and $20 \mathrm{wt} \%$ boehmite slurries in water). These tests were planned around Groups 0 through 7 described below:

- Group 0 focused on establishing the operating conditions for spray release testing in the large-scale system.

- Group 1 focused on establishing the effect of orifice size and shape on the release fraction and size distribution.

- Group 2 focused on establishing the release fraction and size distribution for the worst-case breaches postulated in the WTP.

- Group 3 focused on establishing the impact of the slit length on release fraction and size distribution.

- Group 4 focused on establishing the impact of the slit width on release fraction and size distribution. 
- Group 5 focused on establishing the impact of obstructions in the path of the spray on release fraction and size distribution.

- Group 6 probed the impact of viscosity on release fraction and size distribution.

- Group 7 established how particulates in the slurry impact release fraction and size distribution.

As Group 0 testing began, difficulties in obtaining consistent aerosol concentration measurements were encountered because of laser drift, inadequate mixing inside the chamber, problems with establishing repeatability in measurement, etc. These problems resulted in a significant increase in the number of tests actually conducted versus those planned. Similarly, the number of tests planned for Groups 1 to 7 also significantly increased because of 1) a need for more release fraction and PSD data at $100 \mathrm{psi}, 2$ ) a need to establish variability in the test data by conducting more duplicate tests, and 3) addition of tests recommended by the peer review panel. As a result, a total of 247 tests were actually performed. The tests actually performed for each group are listed in Table 6.1 and Table 6.2. 
Table 6.1. Planned and Actual Large-Scale Aerosol Testing Done with Water

\begin{tabular}{|c|c|c|c|c|c|}
\hline \multirow{2}{*}{$\begin{array}{c}\text { Test } \\
\text { Group }\end{array}$} & \multirow{2}{*}{\multicolumn{2}{|c|}{ Orifice Shape and Target Size (mm) }} & \multirow[b]{2}{*}{ Pressure (psig) ${ }^{(a)}$} & \multicolumn{2}{|c|}{ Number of Tests } \\
\hline & & & & Planned & Actual \\
\hline 0 & Circular & ( & $100,200,380$ & 7 & 65 \\
\hline \multirow{8}{*}{1} & Circular & 1 & $100,200,380$ & 3 & 8 \\
\hline & Circular & 2 & $100,200,380$ & 3 & 6 \\
\hline & Circular & 2.74 & 200, 380 & 2 & 6 \\
\hline & Circular & 4.46 & 200, 380 & 2 & 8 \\
\hline & Slot & $0.5 \times 5$ longitudinal & $100,200,380$ & 3 & 12 \\
\hline & Slot & $1.0 \times 10$ longitudinal & $100,200,380$ & 3 & 11 \\
\hline & Circular & 1-mm holes (five) with 1-mm spacing & $100,200,380$ & 3 & 10 \\
\hline & Slot & $10 \times 1$ circumferential & $100,200,380$ & 3 & 9 \\
\hline \multirow{2}{*}{2} & Slot & $1.0 \times 76.2$ longitudinal & $100,200,380$ & 3 & 10 \\
\hline & Slot & $2.74 \times 76.2$ longitudinal & $100,200,380$ & 3 & 5 \\
\hline 3 & Slot & $1.0 \times 20$ longitudinal & $100,200,380$ & 2 & 11 \\
\hline \multirow{2}{*}{4} & \multirow{2}{*}{ Slot } & $2.0 \times 10$ longitudinal & $100,200,380$ & 3 & 5 \\
\hline & & $3.0 \times 10$ longitudinal & $100,200,380$ & 3 & 11 \\
\hline \multirow{6}{*}{5} & Hole & 1.0 backsplash distance of 117 in. & $100,200,380$ & 2 & 10 \\
\hline & Hole & 1.0 backsplash distance of 47 in. & $100,200,380$ & 2 & 10 \\
\hline & Slot & $1.0 \times 10$ longitudinal backsplash distance of $47 \mathrm{in}$. & $100,200,380$ & 0 & 5 \\
\hline & Slot & $1.0 \times 20$ longitudinal backsplash distance of 47 in. & $100,200,380$ & 0 & 4 \\
\hline & Slot & $1.0 \times 76$ longitudinal backsplash distance of 47 in. & $100,200,380$ & 0 & 4 \\
\hline & & & TOTAL & $55^{(\mathbf{b})}$ & 209 \\
\hline
\end{tabular}

(a) Pressure data highlighted in red were not included in the initial test matrix.

(b) A total of eight repeat tests were included into the total of the number of planned tests. 
Table 6.2. Large-Scale Aerosol Testing of Sodium Thiosulfate and Simulants of Small Treated Hanford Waste

\begin{tabular}{|c|c|c|c|c|c|c|}
\hline \multirow{2}{*}{$\begin{array}{c}\text { Test } \\
\text { Group }\end{array}$} & \multirow{2}{*}{\multicolumn{2}{|c|}{ Orifice Shape and Target Size (mm) }} & \multirow[b]{2}{*}{ Simulant } & \multirow[b]{2}{*}{ Pressure $^{(a)}$} & \multicolumn{2}{|c|}{ Number of Tests } \\
\hline & & & & & Planned & Actual \\
\hline \multirow{2}{*}{6} & Slot & $0.5 \times 5$ & 2.5 cP Water/Sodium-Thiosulfate & $100,200,380$ & 2 & 6 \\
\hline & Slot & $1.0 \times 10$ & 2.5 cP Water/Sodium-Thiosulfate & $100,200,380$ & 2 & 7 \\
\hline \multirow{5}{*}{7} & Slot & $0.5 \times 5$ & Small Treated, 8 wt\% & $100,200,380$ & 2 & 6 \\
\hline & Slot & $1.0 \times 10$ & Small Treated, 8 wt\% & $100,200,380$ & 2 & 6 \\
\hline & Slot & $0.5 \times 5$ & Small Treated, $20 \mathrm{wt} \%$ & $100,200,380$ & 2 & 7 \\
\hline & Slot & $1.0 \times 10$ & Small Treated, $20 \mathrm{wt} \%$ & $100,200,380$ & 2 & 6 \\
\hline & & & & TOTAL & $18^{(b)}$ & 38 \\
\hline
\end{tabular}

(a) Pressures highlighted in red were not a part of the initially planned tests.

(b) A total of three repeat tests were included into the total of the number of planned tests. 



\subsection{Data Analysis Methods}

The spray release tests were conducted to estimate the amount of aerosol generated as a function of orifice geometry, system pressure, and fluid. Ultimately, the quantity of interest is the cumulative release fraction of the aerosol, which can be compared directly to the WTP model currently in use. As described in Chapter 3, the release fraction is the volumetric generation rate of aerosol $(G)$ divided by the volumetric flow rate $\left(Q_{\text {spray }}\right)$ of the spray leak. In general, each test was conducted to measure these two quantities. The generation rate of aerosol was determined from measurement (using the Malvern Insitec-S instruments) of the volume and size distribution of the aerosol and performing a non-linear least squares fit to the data. The spray leak flow rate was calculated based on process instrument data recorded during testing. Note that any data and the subsequent determination of $G$ and $Q_{\text {spray }}$ does not include the effect of evaporation (if any) that occurs during the tests.

In the rest of this chapter, the techniques used to calculate both of these quantities, as well as other auxiliary quantities, are described. First, the analysis of process instrument data is discussed, including the approach used to estimate the spray leak flow rate. Next, the analysis of Malvern Insitec-S data is discussed, with a focus on how the generation rate was calculated for the large-scale experiments. Next, the treatment of PPC data (the secondary aerosol instrument) is briefly presented. Finally, challenges encountered during analysis to obtain meaningful results are discussed. It is important to point out that the analysis methods used here are similar (and identical in most respects) to the methods used for the small-scale aerosol data (Mahoney et al. 2012b); however, there are enough differences that the methods will be described in full.

\subsection{Analysis of Process Instrument Data}

Data from the process instruments installed on the flow loop and within the chamber (see Table 5.5) were captured by a PLC-based DAS that was designated DAS-1. Collecting process data with a DAS was not done in the small-scale tests so the analysis in this section is specifically for the large-scale data. DAS-1 recorded measurements from 33 instruments at a sampling rate of $10 \mathrm{~Hz}$ (i.e., one sample per every $0.1 \mathrm{~s}$ ). Of the 33 instruments, only six were of primary interest to accomplish the data objectives listed below:

1. Calculating an average pressure in the test section, thereby confirming that the target pressure was achieved during the test spray

2. Calculating an average temperature in the test section, thereby confirming that the temperature was kept within a tolerance of $75 \pm 10^{\circ} \mathrm{F}$

3. Calculating the mass or volume of fluid lost from the system during a spray to estimate the volumetric flow rate of the spray.

The six instruments are identified and described in Table 7.1. The approximate location of the instruments is shown schematically in Figure 7.1. Subsequently in this chapter, the instruments will usually be referred by the shorthand identifiers used in Figure 7.1 (e.g., FE1 refers to the Coriolis flow sensor upstream of the spray test section). Most of the information from the other 27 instruments was collected to troubleshoot performance problems, guide test operations, and in some cases, record 
redundant information if other analyses were required (for example, PS5 was a backup pressure transducer in the test section adjacent to PS4).

Table 7.1. Six Primary Instruments From DAS-1 Used in Large-Scale Data Analysis

\begin{tabular}{llcc}
\hline \multicolumn{1}{c}{ Instrument Type } & \multicolumn{1}{c}{ Description/Location } & Identifier & Units \\
\hline Load cell/scale & Mass of storage tank contents & M1 & $\mathrm{lb}$ \\
Load cell/scale & Mass of feed tank contents & M2 & lb \\
Coriolis flow sensor & Located upstream of test section, pre-spray flow rate & FE1 & gpm \\
Coriolis flow sensor & Located downstream of test section, post-spray flow rate & FE2 & gpm \\
Pressure transducer & Test section pressure at elbow just upstream of orifice(s) & PS4 & psig \\
Resistance temperature detector & Test section temperature, at elbow just upstream of orifice(s) & TS6 & ${ }^{\circ} \mathrm{F}$ \\
\hline
\end{tabular}

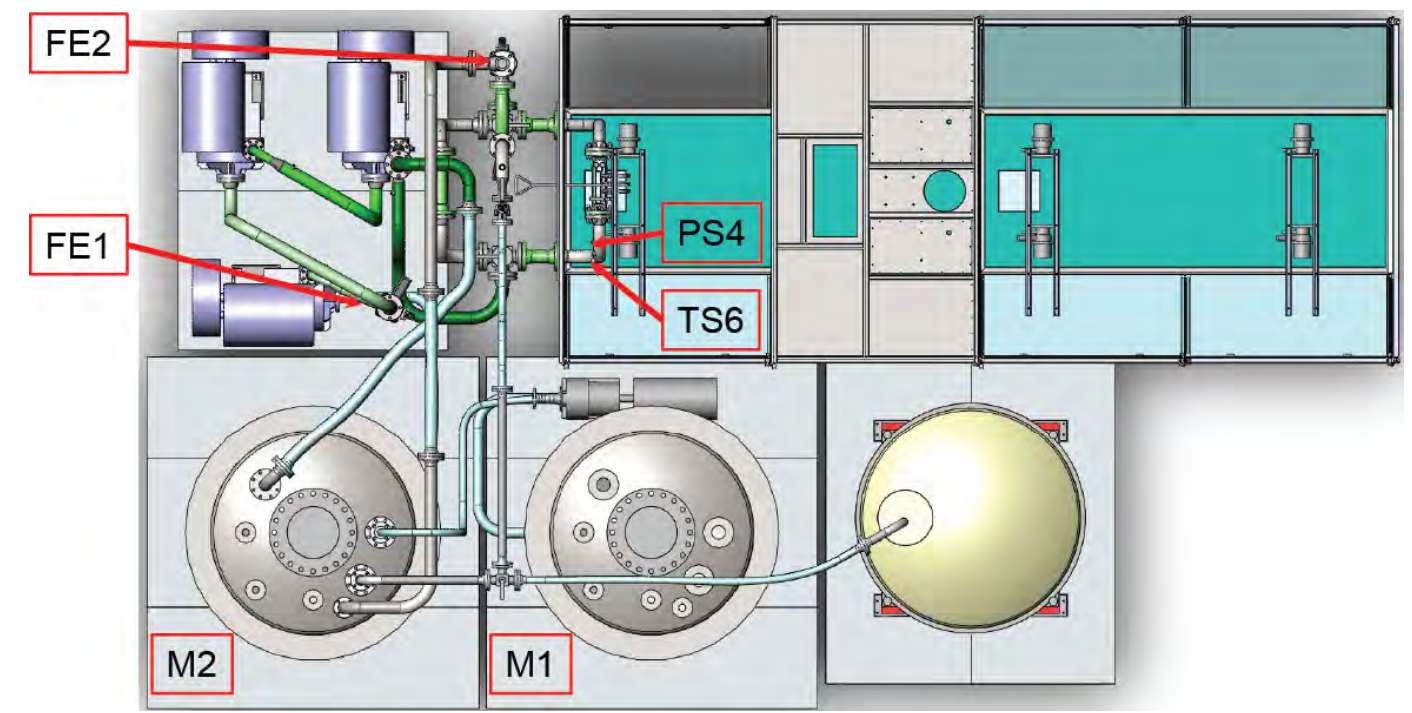

Figure 7.1. Approximate Locations of the Six Primary Instruments Described in Table 7.1

To achieve data objectives 1 and 2, the average and standard deviation of the pressure (from PS4) and temperature (from TS6) for a test were calculated by identifying the time series corresponding to a spray test and averaging the instrument data over the majority of the test. The average typically contained at least $100 \mathrm{~s}$ of data (out of a possible 120 to $125 \mathrm{~s}$ ). The average of the square root of the pressure (also from PS4) was calculated over the same time period to compare the average of the square root pressure (which determines the spray leak flow rate - see Equation (1.8)) with the square root of the average pressure.

Objective 3 was met using two different methods. The first (and primary) method was to calculate the leak flow rate using the mass of fluid present in the tanks before and after an experiment. Mathematically, this results in the simple expressions shown below

$$
M_{i}=M 1_{i}+M 2_{i},
$$




$$
\begin{gathered}
M_{f}=M 1_{f}+M 2_{f} \text {, and } \\
\Delta M=\left(M_{i}-M_{f}\right)-M_{\text {loss }},
\end{gathered}
$$

where $M 1$ and $M 2$ refer to the masses from the storage and feed tanks, respectively; the subscripts $i$ and $f$ define the initial (before the spray is initiated) and final (after the spray is stopped) values in time, and $M_{\text {loss }}$ is the mass lost via pump leakage. The fluid lost via pump leakage was collected and weighed for each experiment. It was small relative to the volume of most spray volumes, but represented an appreciable fraction of the smallest sprays (i.e., up to 5 percent of the mass). The masses from the tanks were summed to account for tests in which the tanks were in communication (e.g., higher volume sprays or situations in which one tank was used to heat/cool the contents of the other).

The initial and final masses were calculated as 5-s averages approximately 10 to $15 \mathrm{~s}$ before and after the spray start and stop times. The spray start and stop times were used to specify a spray time $\Delta t_{\text {spray }}$, and the volumetric flow rate was estimated using the following relationship

$$
Q_{\text {spray }}=\left(\frac{\Delta M}{\rho(\langle T\rangle)}\right) / \Delta t_{\text {spray }},
$$

where $\Delta M$ is given by Equation (7.3) and $\rho$ is the density of the fluid used. The average temperature from TS6 (e.g., $\langle T\rangle$ ) was used to calculate the density at the temperature of the test. All fluids tested were assumed to have the same density dependence with temperature as that of water.

The second method used the Coriolis flow sensors to calculate a net flow rate. In a typical experiment, flow was established at some pre-spray flow rate and measured by FE1 and FE2. There was a small offset (approximately $1 \mathrm{gpm}$ ) between FE1 and FE2 that had to be accounted for, especially when the flow rate was small. When the spray was initiated, FE1 quickly rose to a new, higher flow rate while FE2 remained the same. The net flow rate out of the orifice is

$$
Q_{n e t}=(\langle F E 1\rangle-\langle F E 2\rangle)+\left(F E 2_{p s}-F E 1_{p s}\right)
$$

where the first term in parentheses is the difference between the average flow rates as measured by FE1 and FE2 during the spray, and the second term is the correction for the pre-spray (thus the ps subscript) offset between the two flow sensors. The pre-spray correction was calculated using a 5-s average approximately $10 \mathrm{~s}$ before the spray was initiated, and the averages were determined in the same way as the average pressure and temperature.

As described in Chapter 6, most spray tests were conducted in the same manner, with a test duration of approximately $2 \mathrm{~min}$. Thus, the majority of the DAS- 1 data files were 4 to $6 \mathrm{~min}$ in length. The start and stop times of the spray as indicated by the DAS-1 clock were recorded during each test so the proper data could be identified. Given this information, determining the average pressure and temperature during each test should have been straightforward. Midway through the testing, we discovered that that DAS-1 clock (i.e., the time on the computer performing the data acquisition) was not the clock time recorded in the DAS-1 data files. Instead, these files were time stamped by the PLC clock. The PLC and 
DAS- 1 clocks were found differ by 1 to $10 \mathrm{~s}$, depending on the date of the test. After this problem was identified, the PLC clock was synchronized with DAS-1 clock before testing began.

Given this issue, the approach to determine the average conditions could not be based indiscriminately on the use of the recorded time stamps for the start and stop of the spray. Instead, the data needed to be assessed before performing any calculations in order to verify that the appropriate time period of the test was being used. The approach taken was to use the recorded time stamps as an initial guess and, if required, iterate to the proper averaging period. The iterative procedure for averaging data proceeded as follows:

1. Use the recorded time stamps as the initial spray start $\left(\mathrm{t}_{\mathrm{start}, 0}\right)$ and stop $\left(\mathrm{t}_{\mathrm{stop}, 0}\right)$ times

2. Plot the quantity of interest from $t_{\text {start }, 0}-15 \mathrm{~s}$ to $t_{\text {stop }, 0}+15 \mathrm{~s}$

3. Plot time markers to assess the suitability of the default time segments

a. Default for tanks mass measurements

i. Initial mass averaged between $t_{\text {start }, 0}-15 \mathrm{~s}$ and $t_{\text {start }, 0}-10 \mathrm{~s}$

ii. Final mass averaged between $t_{s t o p, 0}+10 s$ and $t_{s t o p, 0}+15 s$

b. Default for temperature and pressure

i. Average between $t_{s t a r t, 0}+2 \mathrm{~s}$ and $\mathrm{t}_{\mathrm{stop}, 0}-2 \mathrm{~s}$

c. Default for flow rate measurements

i. Pre-spray flow rates averaged between $\mathrm{t}_{\mathrm{start}, 0}-15 \mathrm{~s}$ and $\mathrm{t}_{\mathrm{start}, 0}-10 \mathrm{~s}$

ii. Average between $t_{\text {start }, 0}+2 \mathrm{~s}$ and $t_{\text {stop }, 0}-2 \mathrm{~s}$

4. Assess the data to be averaged. The start and stop of a spray can be clearly seen from the data, in particular the flow rate data, which changes immediately upon opening of the orifice.

a. If the averages look suitable, proceed with the calculations.

b. If some modifications are required, iterate to $t_{\text {start, } 1}$ and $t_{\text {stop, } 1}$ and repeat steps 2 through 4 . Sometimes additional modifications were required, such as changing the duration of the average period for a set of data.

An example of a plot used in the iteration procedure is shown in Figure 7.2. The elapsed time on the $\mathrm{x}$-axis is based on $\mathrm{t}_{\mathrm{start}, 0}$ and $\mathrm{t}_{\mathrm{stop}, 0}$, and it will align perfectly only when the recorded time and the PLC time are the same. This was not the case for this particular test because the upstream flow rate clearly increases quickly at around $t=-6$ elapsed $s$, which is when the orifice was actually opened and the spray was initiated. Figure 7.2 shows the result of the final iteration of the procedure.

Using the following expression, the spray leak flow rate also was used to estimate the orifice coefficient for each test by rearranging Equations (1.8) and (1.9) and solving for the orifice coefficient 


$$
C_{D}=\frac{\left(\frac{Q_{\text {spray }}}{A}\right)}{\left(\frac{2\langle P\rangle}{\rho(\langle T\rangle)}\right)^{\frac{1}{2}}},
$$

where $A$ is the cross-sectional area of the orifice, $\langle P\rangle$ is the average pressure during the spray, and $Q_{\text {spray }}$ is the flow rate given by either Equation (7.4) or (7.5). Equation (7.6) was the motivation for calculating the average of the square root of the pressure, in case it differed significantly from the average pressure. In the large-scale test data, the maximum difference between the square root of the average pressure and the average of the square root of the pressure was 0.016 percent. Thus, the square root of the average pressure was used in the calculations because the difference between the values was negligible.

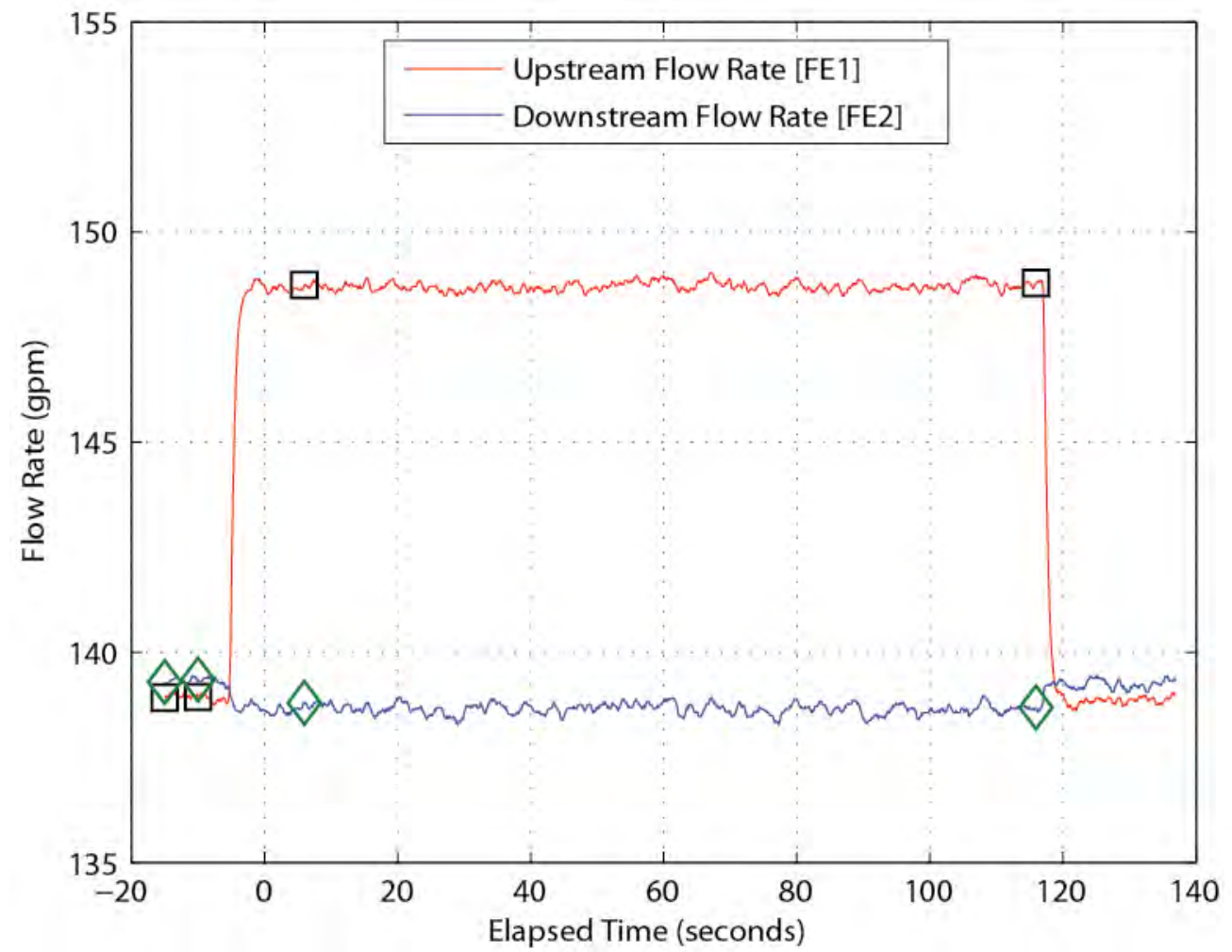

Figure 7.2. Flow Rates Measured During Test W56 (water, 100 psi, $3 \times 10$-mm orifice) from FE1 (red) and FE2 (blue). The pairs of black squares and green diamonds indicate the average periods for each set of flow rate data.

The two methods for calculating the flow rate were in agreement for most of the tests. For relatively small orifices with the smallest volumetric flows, the mass method was a superior choice because the noise in the flow meters (each one fluctuating $0.5 \mathrm{gpm}$ during the measurement period) made precise measurement of these flow rates (expected to be 1 gpm or less) challenging. For comparison, the load cells (M1 and M2) had an uncertainty of \pm 4.5 lb over the measurement range; even for the smallest flows the change in weight was 4-5 times greater than the uncertainty. 


\subsection{Analysis of Malvern Insitec-S Data}

As described in Chapter 5, the large-scale test chamber had three Malvern Insitec-S instruments installed during almost all of the spray leak tests. The data from all three instruments were collected by a single computer via commercial software that was designated DAS-2. DAS-2 recorded the measurements of the three Malvern Insitec-S instruments simultaneously every second, or in some cases, every $0.25 \mathrm{~s}$ (i.e., a 4-Hz data collection rate). The data of interest were the volume concentration of aerosol $\left(C_{v}\right)$, or the total volume of aerosol per unit spatial volume, measured in volume parts per million (ppmv), and the percent volume of aerosol as a function of predefined droplet size bins $(\phi)$, which is a differential or discrete measurement. Arrays of date/time stamps and the Malvern Insitec-S laser transmission also were required to perform the calculations.

The first step is typically a correction step in which the transmission is examined to determine if any adjustments are required to get a more accurate measurement of $C_{v}$. As described in Section 5.3, the Malvern Insitec-S measures aerosol based on scattering of laser light. The transmission is a measure of the amount the laser power, which is reduced by the presence of aerosol (or potentially other obstructions) in its path between the source and the detector. Explicitly, the transmission is written as

$$
\tau=\frac{S(t)}{S_{o}}
$$

where $S(t)$ is the undeflected laser power measured at time $t$ and $S_{o}$ is the background undeflected laser power. Ideally, the transmission should be 1 (or 100 percent if using a percentage basis), or very close to 1 , before the spray enters the chamber. To check the need for correction, the laser power is compared to the background laser power measured prior to the start of the spray. An example of a case in which a correction was not required is shown in Figure 7.3. The laser power scattering data is synonymous with the background value until the test begins, then the scattering response is observed, and the data returns to

the background value after the test. In this case the transmission is essentially equal to 1 before the test so no adjustments were made. 


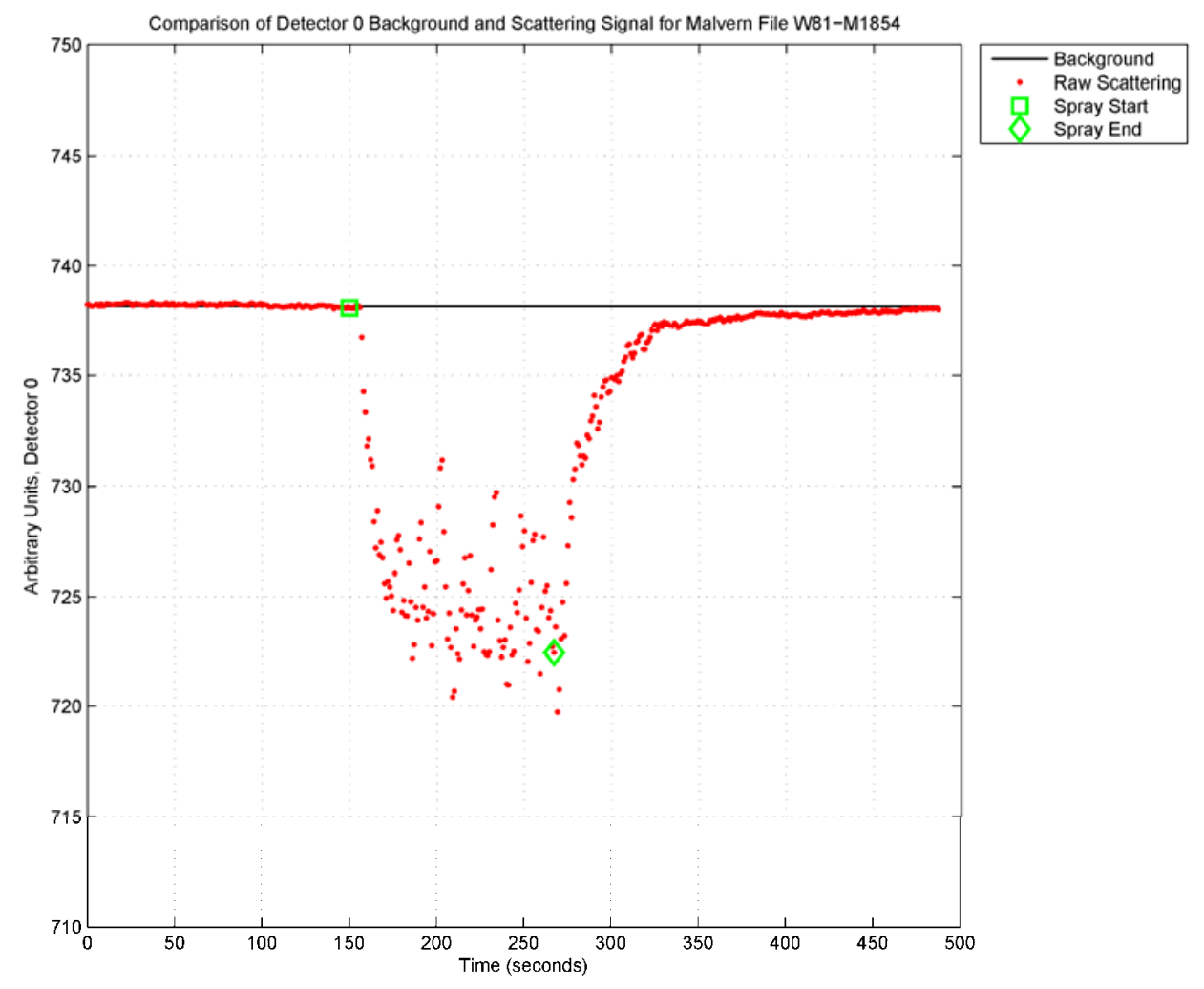

Figure 7.3. Comparison of the Laser Power (red circles) to the Background Laser Power (black line) for Test W81, Malvern 1854. The spray start time (green square) and spray end time (green diamond) are marked for convenience.

An example from the same test but a different Malvern Insitec-S instrument is shown in Figure 7.4. In this case, the laser power scattering data are less than the background values. If left uncorrected, the transmission data would yield a $C_{v}$ that was too large, provided the background is representative of the pre-test conditions. There also are cases in which the laser power data are greater than the background values. This would underestimate $C_{v}$. The adjustment that was applied to the data also is shown in the figure. The method for performing the adjustment is described in more detail below. 


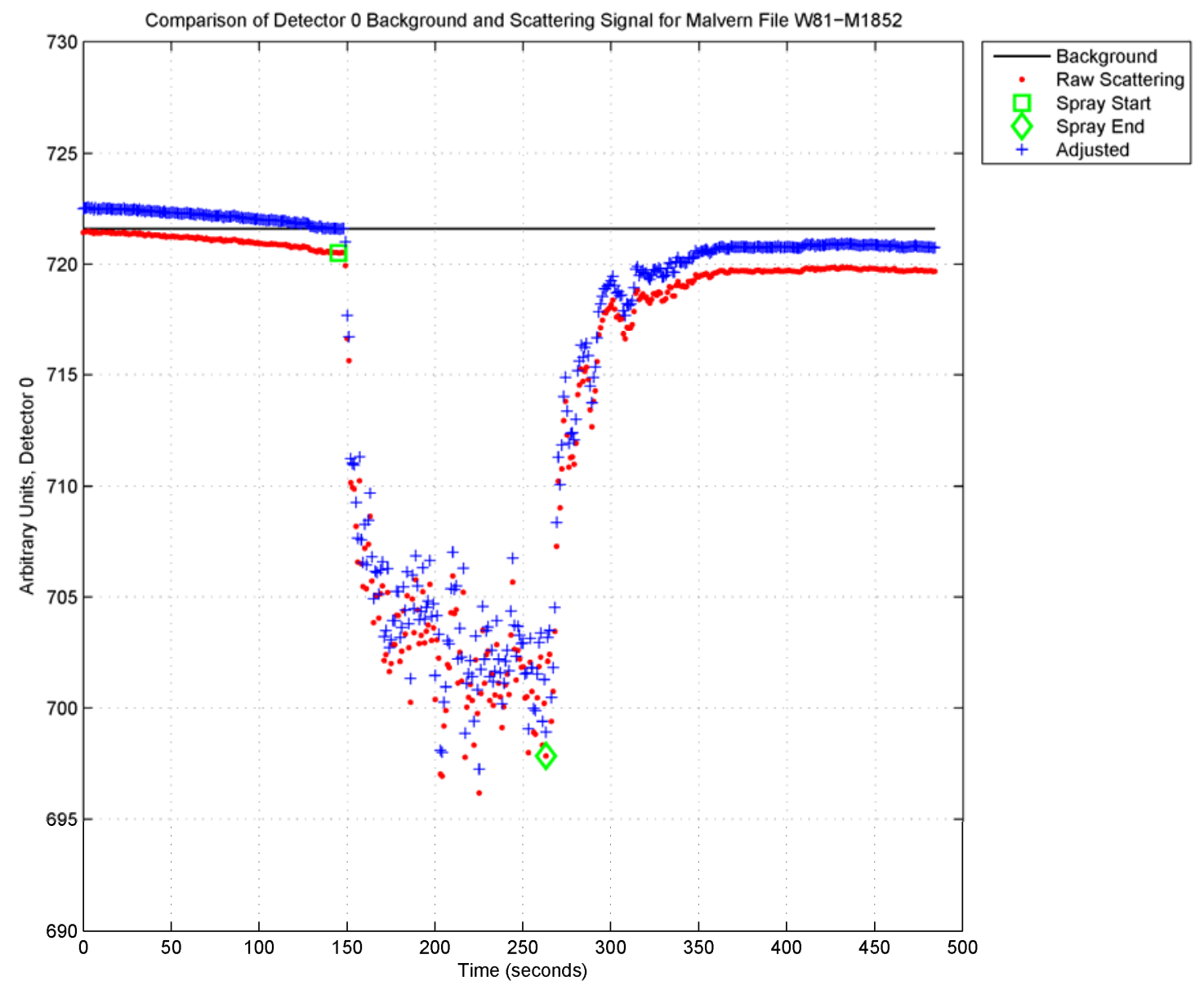

Figure 7.4. Comparison of the Laser Power (red circles) to the Background Laser Power (black line) for Test W81, Malvern 1852. The spray start time (green square) and spray end time (green diamond) are marked for convenience. The blue crosses demonstrate the data that results from performing an adjustment.

The transmission is related to $C_{v}$ via the Beer-Lambert law (Gebhart 2001), which is shown below

$$
C_{v}=-\frac{2}{3} \frac{D_{3,2}}{Q_{e} L} \ln (\tau)
$$

where $D_{3,2}$ is the SMD, $Q_{e}$ is the aerosol extinction coefficient, and $L$ is the path length of the measurement zone. In general, $Q_{e}$ is not constant (i.e., determined from Mie-Lorenz theory) so it is challenging to use Equation (7.8) outright when transmission needs to be adjusted. The Malvern Insitec-S software uses information about the scattering pattern that it measured to help determine $Q_{e}$. Equation (7.8) can be simplified by assuming that $D_{3,2}, Q_{e}$, and $L$ are constant for a given measurement and that the transmission is near unity (or 100 percent).

Consider a Taylor series expansion of $\ln (\tau)$ when $\tau$ is close to 1 , truncated to second-order accuracy:

$$
\ln (\tau)=\ln (1)+1 \cdot(\tau-1)-\frac{1}{2}(\tau-1)^{2}+\ldots
$$


The first term is zero and when $\tau \sim 1$

$$
(1-\tau)>>\frac{(1-\tau)^{2}}{2 !}
$$

therefore, when the transmission does not deviate far from 1 ( \pm 10 percent), it can be approximated as $\ln (\tau) \sim(1-\tau)$. To correct $C_{v}$, the ratio of the original measured concentration is adjusted by the apparent measured concentration, which can be written as

$$
\frac{C_{v, a d j}}{C_{v, o}}=\frac{\ln (\tau(t))}{\ln \left(\tau_{o}\right)} \approx \frac{(1-\tau(t))}{\left(1-\tau_{o}\right)}=\frac{\left(1-\frac{S(t)}{S(0)}\right)}{\left(1-\frac{S(t)}{S_{o}}\right)}
$$

where $C_{v, \text { adj }}$ is the adjusted $C_{v}$, $C_{v, o}$ is the original measured $C_{v}$, and $S(0)$ is the initial laser power at the start of aerosol generation $(t=0)$. Recall that $S_{0}$ is the laser power obtained during the background measurement (see Equation 7.7). The choice of $S(0)$ will specify the adjustment that is made. Typically, the value of the laser power just before the spray starts (e.g., $2 \mathrm{~s}$ ) was chosen to perform the adjustment. This is shown by the blue crosses in Figure 7.4. In the rest of this section, the variable $C_{v}$ will be used in a general sense to describe the volume concentration for all the tests, whether the adjustment given by Equation (7.11) was necessary or not.

The second step in the analysis of Malvern Insitec-S data is to transform the raw data into the data that can be fit to obtain the volumetric generation rate. The aerosol data needs to be on a volume basis, which can be calculated using the following equation

$$
\Phi_{k}=C_{v}\left(\frac{\phi_{k}}{100}\right)
$$

where $\Phi_{k}$ is the differential ppmv between size bin $k$ and $k-1$, and $k$ is a discrete size bin ranging from 1 to $N$. The Malvern Insitec-S instrument has 60 size bins scaled logarithmically from 0.1 to $2000 \mu \mathrm{m}$. Equation (7.12) can be cumulated to give the cumulative ppmv below a certain droplet size

$$
\Phi_{c, k}=\sum_{j=1}^{k} \Phi_{j}
$$

where the subscript $c, k$ indicates the cumulative ppmv below the droplet diameter associated with size bin $k$. The cumulative ppmv also can be normalized to calculate a PSD (volume fraction) of the aerosol. Mathematically this is expressed as

$$
\bar{\Phi}_{k}=\frac{\Phi_{c, k}}{\Phi_{c, N}} .
$$


Both the differential (Equation (7.12)) and cumulative concentrations (Equation (7.13)) were used in the analysis described in this chapter. In the data collected during the tests covered in this report, the volume contribution of the smaller droplets was very small. This resulted in very sparse and noisy data at small droplet sizes. To combat this, the differential concentrations were accumulated up to $5.25 \mu \mathrm{m}$. At this droplet size, the differential and cumulative concentrations were identical. The normalized PSD (Equation (7.14)) was occasionally of interest, but it did not support the primary objective of calculating the generation rate of aerosol.

Up to this point in the analysis, each of the Malvern Insitec-S instruments in the large-scale chamber has been treated separately. The third step in the analysis was to take either the differential or cumulative concentrations from each of the Malvern Insitec-S instruments and average them. The average of the concentrations from the three instruments is taken to be the average concentration in the chamber. Rather than attempt to specify mixing "zones” in which each Malvern Insitec-S was taking a representative sample, the average was not weighted. For a given particle size bin $k$ and time, the resulting expression is

$$
\left\langle\Phi_{c, k}\right\rangle=\frac{1}{3}\left(\Phi_{c, k}^{1}+\Phi_{c, k}^{2}+\Phi_{c, k}^{3}\right)
$$

where the superscripts indicate the Malvern Insitec-S position as described by Figure 5.10 and Table 5.2. The expression is written in terms of the cumulative concentration but would be identical for the average differential concentration. In general, the concentrations measured by the Malvern Insitec-S instruments were a function of position, such that $\Phi_{\mathrm{c}, \mathrm{k}}^{1}>\Phi_{\mathrm{c}, \mathrm{k}}^{2}>\Phi_{\mathrm{c}, \mathrm{k}}^{3}$, and $\left\langle\Phi_{\mathrm{c}, \mathrm{k}}\right\rangle \approx \Phi_{\mathrm{c}, \mathrm{k}}^{2}$. There were some experiments where it was not appropriate to average the concentrations (to isolate other variables, such as repeatability tests) or particular instances in each time series where Equation (7.15) was not used as shown. These are described in more detail in the discussion on analysis challenges later in this section. Averaging the concentration data is the most significant difference between the analysis methods used for the small-scale data and the large-scale data.

One example of the concentrations from Malvern Insitec-S instruments in a specific size bin (i.e., $27.37<\mathrm{d}_{\mathrm{p}}<32.28 \mu \mathrm{m}$ ) for the first $60 \mathrm{~s}$ of a water test is given in Figure 7.5. Although these data represent only a single size bin for a single test, the qualitative information is typical of most size bins observed over the entire suite of tests described in this report. As mentioned above, the average concentration is essentially equivalent to the concentration measured by the Malvern 1852 in position 2 . Note also how the transience in the concentration depends on position for this test: Malvern 1855, in position 1, detects aerosol of this size after $1 \mathrm{~s}$, whereas Malvern 1854, in position 3, does not detect any until $7 \mathrm{~s}$ of elapsed time. The cumulative concentration data for this test, and the majority of other tests, have similar features. Despite the similarity of the transient concentration data and relatively fast approaches to steady-state concentrations, the observed behavior indicates that the chamber was not perfectly/instantaneously mixed. Recall from Section 3.3 that perfect mixing was assumed in the development of the generation rate model; incorporation of more complex mixing phenomenon into the model was not attempted but was expected to be a good approximation. For a more detailed discussion of the basis for concentration averaging, see Appendix C. 


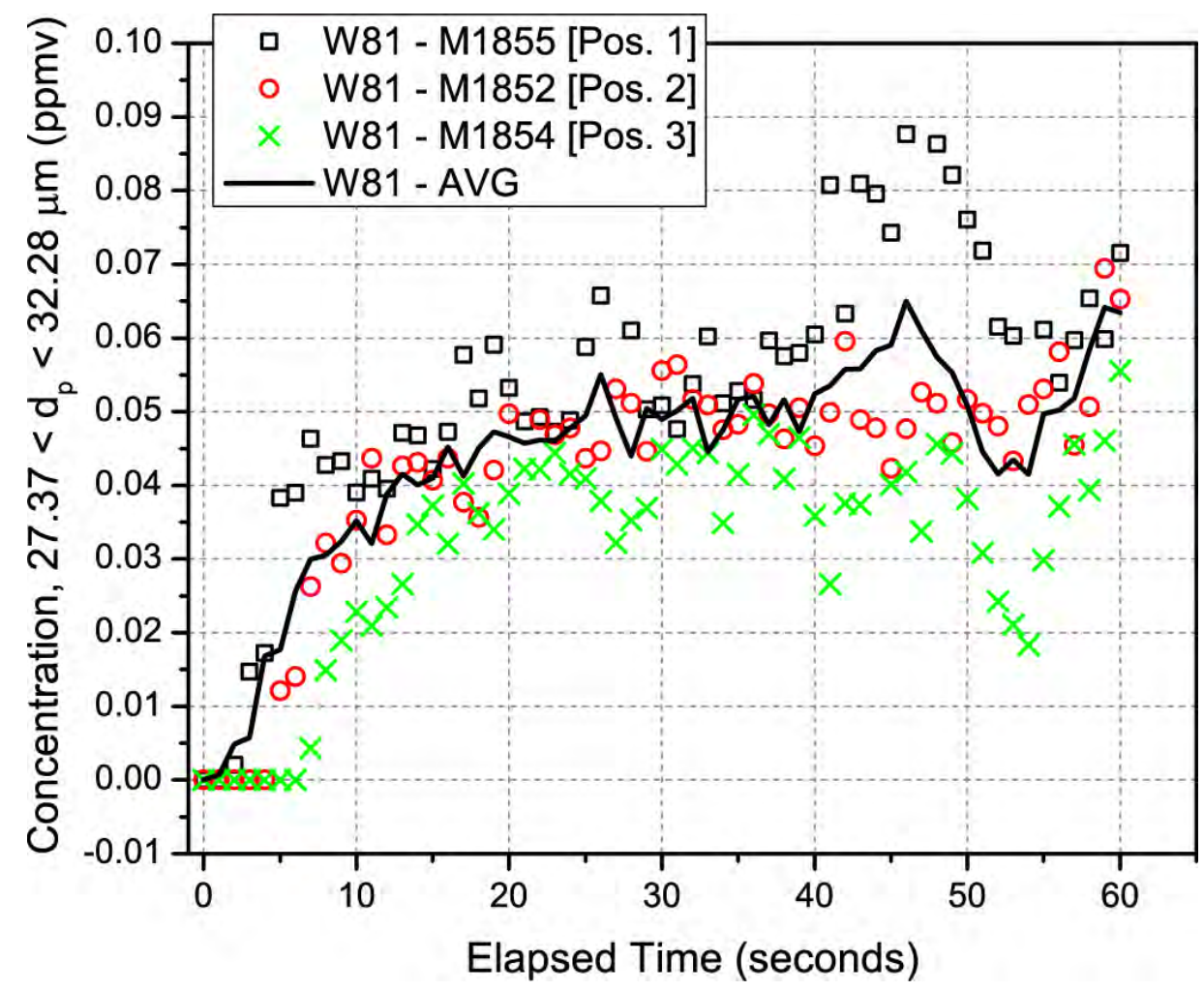

Figure 7.5. Concentration of Droplets between 27.37 and $32.28 \mu \mathrm{m}$ in Diameter Measured During Test W81 (2-mm hole, 380 psi, water) as a Function of Elapsed Time by Each Malvern Insitec-S Instrument (symbols) Compared to the Average (black line)

The average cumulative or differential concentrations were obtained as functions of time using Equation (7.15). During the time the spray was active, data were observed to increase rapidly to a steady-state concentration. The term "steady-state" should not be interpreted to mean the concentration was consistently at a precise single value, but rather that the data fluctuated around some mean concentration. In some tests, the fluctuations were sizable and in others they were not. The fluctuations usually became more significant as the orifice size increased (and consequently, the volumetric flow of the spray). Given the chaotic nature of turbulent jet flow and the data acquisition rate of the Malvern Insitec-S instruments, noise in the data was expected. A typical set of differential and cumulative concentration curves are shown in Figure 7.6 for selected droplet sizes.

The similarities between the differential and cumulative concentrations are apparent from the data shown in Figure 7.6. The plots in Figure 7.6 share very similar shapes and differ primarily in magnitude. The salient features in the data that are common to all data sets are an increase in concentration (in this case, occurring over approximately 35 to $40 \mathrm{~s}$ ), an equilibrium concentration achieved for each curve for the duration of the spray, and a sharp decay from that concentration when the spray is stopped. 

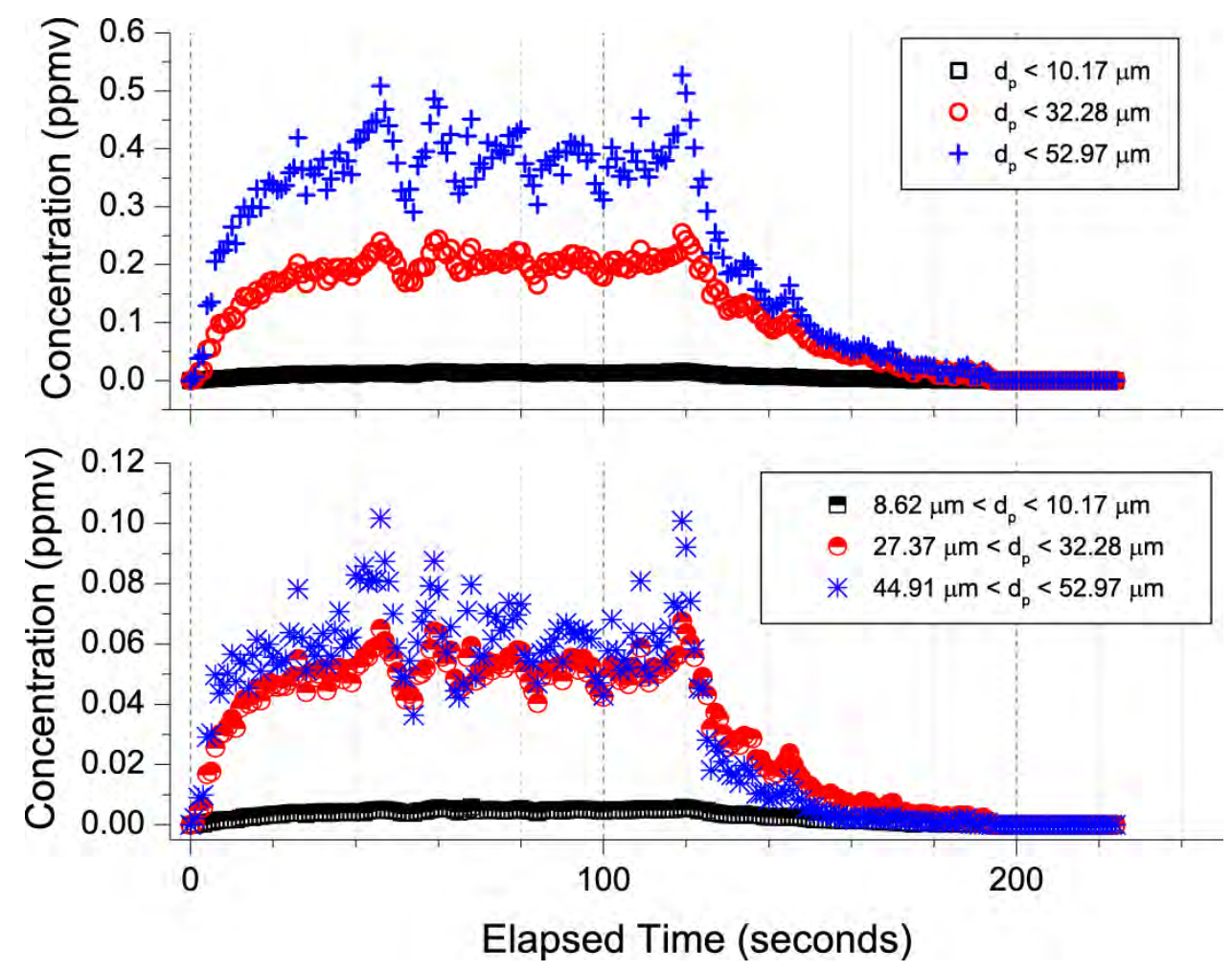

Figure 7.6. Selected Cumulative (top) and Differential (bottom) Concentration Data from Test W81 (2-mm hole, $380 \mathrm{psi}$, water) as a Function of Elapsed Time. The spray began at $t=0$ and ended at $t=119 \mathrm{~s}$.

The sheer number of particle diameter bins (recall $N=60$ ) did not permit scrutiny of each concentration curve with time when performing the analysis. A set of diameters were chosen to be representative of the entire data set, namely 5.25, 10.17, 19.67, 32.28, 52.97, and $102.5 \mu \mathrm{m}$. For the cumulative concentrations, these representative diameters describe the cumulative concentrations up to droplets of that size. For the differential concentrations, these representative diameters are the upper bound of the diameter bin, e.g., for $10.17 \mu \mathrm{m}$ the bin is $8.62-10.17 \mu \mathrm{m}$. These diameters were chosen because they covered the region of the most interest, that being droplets between 10 and $100 \mu \mathrm{m}$. Calculations, however, were performed on the entire range of valid data. As discussed earlier, only a tiny fraction of small drops (on a volume basis) were measured. Thus, analysis of cumulated concentrations less than $1.01 \mu \mathrm{m}$ was not attempted. Likewise, the Malvern Insitec-S instruments were not configured to measure droplets larger than approximately $450 \mu \mathrm{m}$, and this represented the upper bound of the analysis.

As described in the technical basis (see Section 3.4) for these tests, the quantity of interest was the volumetric generation rate of aerosol. A two-parameter exponential model based on first-order kinetics was fit to both the differential and cumulative concentration data. The model is of the form

$$
\Phi_{k}^{E}=\frac{G}{\lambda V}(1-\exp (-\lambda t))
$$

where $\quad \Phi_{k}^{E}=$ expected value of the concentration (either differential or cumulative) $G=$ generation rate $\left(\mathrm{m}^{3} \mathrm{~s}^{-1}\right)$ 


$$
\begin{aligned}
& \lambda=\text { loss coefficient }\left(\mathrm{s}^{-1}\right) \\
& V=\text { volume of the chamber }\left(\mathrm{m}^{3}\right)
\end{aligned}
$$

This was described conceptually in Chapter 3, specifically Equations (3.20) or (3.23). The model also defines the equilibrium concentration $\Phi_{k}^{e q}=G / \lambda V$. The volume of the chamber was not changed during testing and is a constant. The expected values were fit to the data using a non-linear least squares algorithm, typically for the first $60 \mathrm{~s}$ of the spray $^{10}$ (note that this is a longer time period than the typical period used in the small-scale, which was $20 \mathrm{~s}$ ). The choice was made to constrain the values of the adjustable parameters $G$ and $\lambda$, the ramification being that the algorithm used a trust-region method to solve for the best fit. In addition, the bi-square weight method was used to make the fit more robust to outliers and/or spurious noise. In the bi-square weight method, the weight given each data point in the algorithm varies depending on proximity to the current best-fit curve. Data that is outside of what would be expected based on random variation in the data is given a weight of zero.

The generation rate calculated by the algorithm also was assigned 95-percent confidence intervals, which included the uncertainty of predicting the curve based on the data and the random variation expected in a new observation. Goodness-of-fit was assessed in three ways: 1) visual observation, 2) a convergence criterion, and 3) the adjusted coefficient of determination $\left(\bar{R}^{2}\right)$. Data for which the fit did not converge were rejected, and fits that did not appear to describe the data well were flagged. Because not all the fits were examined visually, the adjusted coefficient of determination was used to screen the results, with fits of $\overline{\mathrm{R}}^{2}<0.5$ rejected. Fits that were flagged when the results were visually examined usually failed to meet the $\overline{\mathrm{R}}^{2}$ criterion. The choice of 0.5 is arbitrary and does not exclude fits with $\overline{\mathrm{R}}^{2}<0.5$ as being significantly poorer compared to those greater than 0.5 . However, it does indicate that less than 50 percent of the variability in the data is described by the model fit and it was rejected on that basis.

Despite the similarity of the differential and cumulative data, both data sets were used to obtain an estimate of generation rate. Based on the physical arguments made in Chapter 3 , the generation and loss terms in the model of Equation (7.16) should, in general, be a function of the droplet size. When the differential data is fit, the concentration is defined for very narrow droplet size ranges, and variations in kinetics with size are isolated by treating the data in each size separately. However, the differential data has some statistical drawbacks: the data are smaller in absolute magnitude than the cumulative concentration data and much noisier, particularly for the smallest droplet sizes. The noisier data are more difficult to fit, and the results have a greater uncertainty. The cumulative data are more attractive statistically but treat the droplets in aggregate. The larger the droplet size bin, the wider the range of sizes that are described by a single loss coefficient and generation rate. Rather than trade one benefit at the expense of another, both of the data sets were fit to give greater confidence in the result. An example of the fit to the cumulative concentration data is shown in Figure 7.7 for reference.

Once an estimate of the generation rate was obtained using the model fit to the data, the release fraction could be calculated using Equation (3.1). The differential concentration data, when analyzed, produce discrete release fractions that are cumulated for comparison to release fractions determined by analysis of the cumulative concentration data. The generation rate is simply the flow rate of aerosol in the

\footnotetext{
${ }^{10}$ There were occasions when this was adjusted forward or backwards depending on the nature of the data. Usually, it was shifted forward (to $\sim 30 \mathrm{~s}$ ) for very fast increases (typically larger orifices) and shifted backwards (up to $120 \mathrm{~s}$ ) when the data was sparse (typically smaller orifices).
} 
system, and it is divided by the spray leak flow rate as calculated in Section 7.1. An estimate of the uncertainty in the release fraction can be determined using the confidence intervals from the model fit to the data where $G^{+}$and $G^{-}$are the upper and lower 95-percent confidence intervals on the generation rate, respectively. When shown, the uncertainty is usually displayed as error bars in plots of release fraction
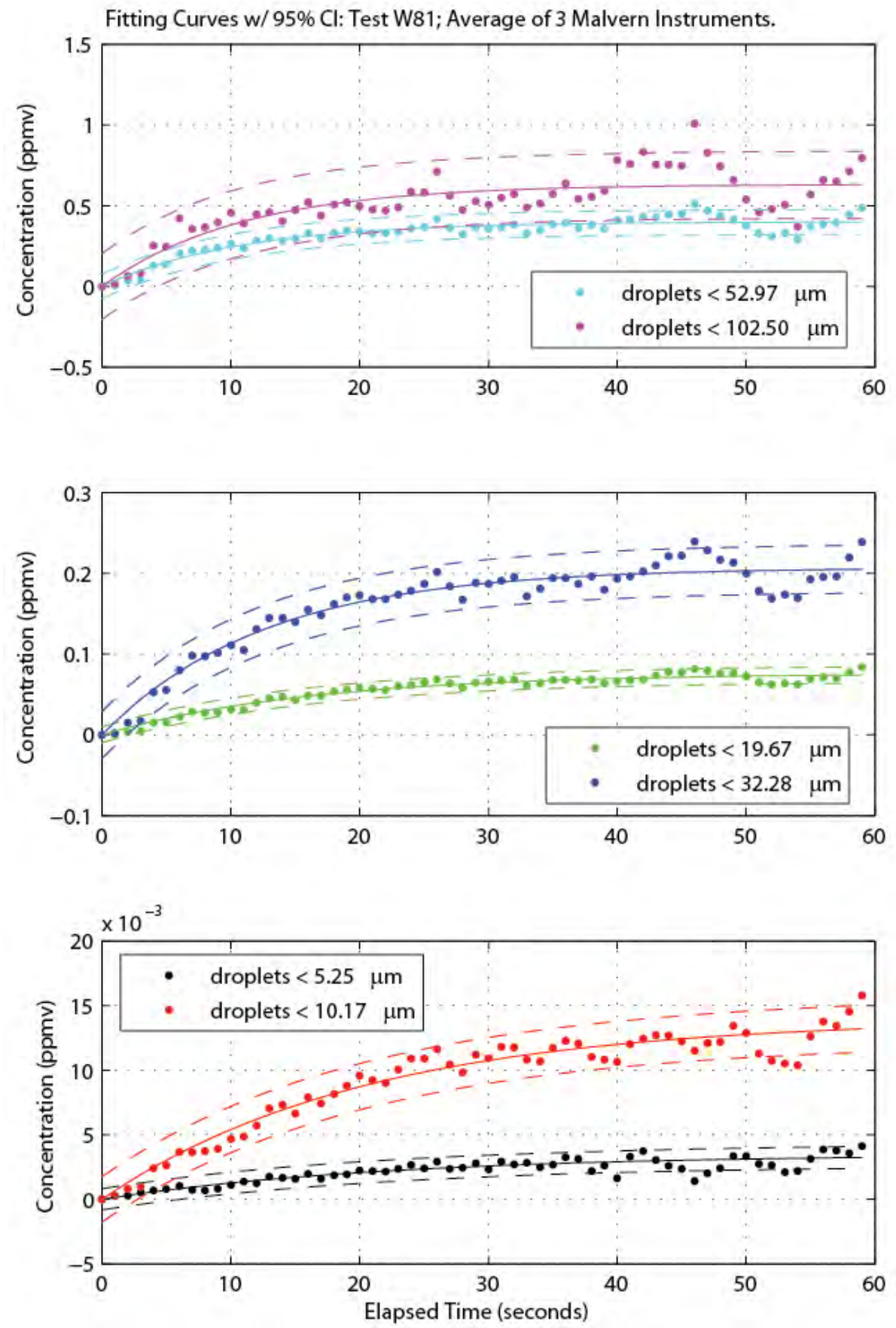

Figure 7.7. Model Fit to the Cumulative Concentration Data for Test W81 (2-mm hole, 380 psi, water). Results shown for the canonical size bins with the 95-percent confidence intervals displayed as dotted lines. 


$$
\begin{aligned}
& R F(+)=\frac{G^{+}}{Q_{\text {spray }}}-R F \\
& R F(-)=R F-\frac{G^{-}}{Q_{\text {spray }}}
\end{aligned}
$$

(as calculated by Equation (7.17)) or generation rate (using $G^{+}$and $G^{-}$directly). Other potential sources of uncertainty are not included. In Chapter 8, the uncertainties of the tests are discussed in more detail.

The end result of the analysis of the data obtained from the Malvern instrument is presented in Figure 7.8 for test W81. The figure shows the release fractions with uncertainty for both the differential and cumulative concentration data. Over the size range the Malvern instrument is configured to measure accurately (0.5 to $200 \mu \mathrm{m}$ ), the release fraction is compared to the WTP model for the same experimental conditions. The procedure used to calculate the WTP model prediction is described in detail in Section 1.1 in Equations (1.1) through (1.9). Note the excellent agreement between the release fractions derived from the differential and cumulative methods; this is typical of data from the majority of the tests. 


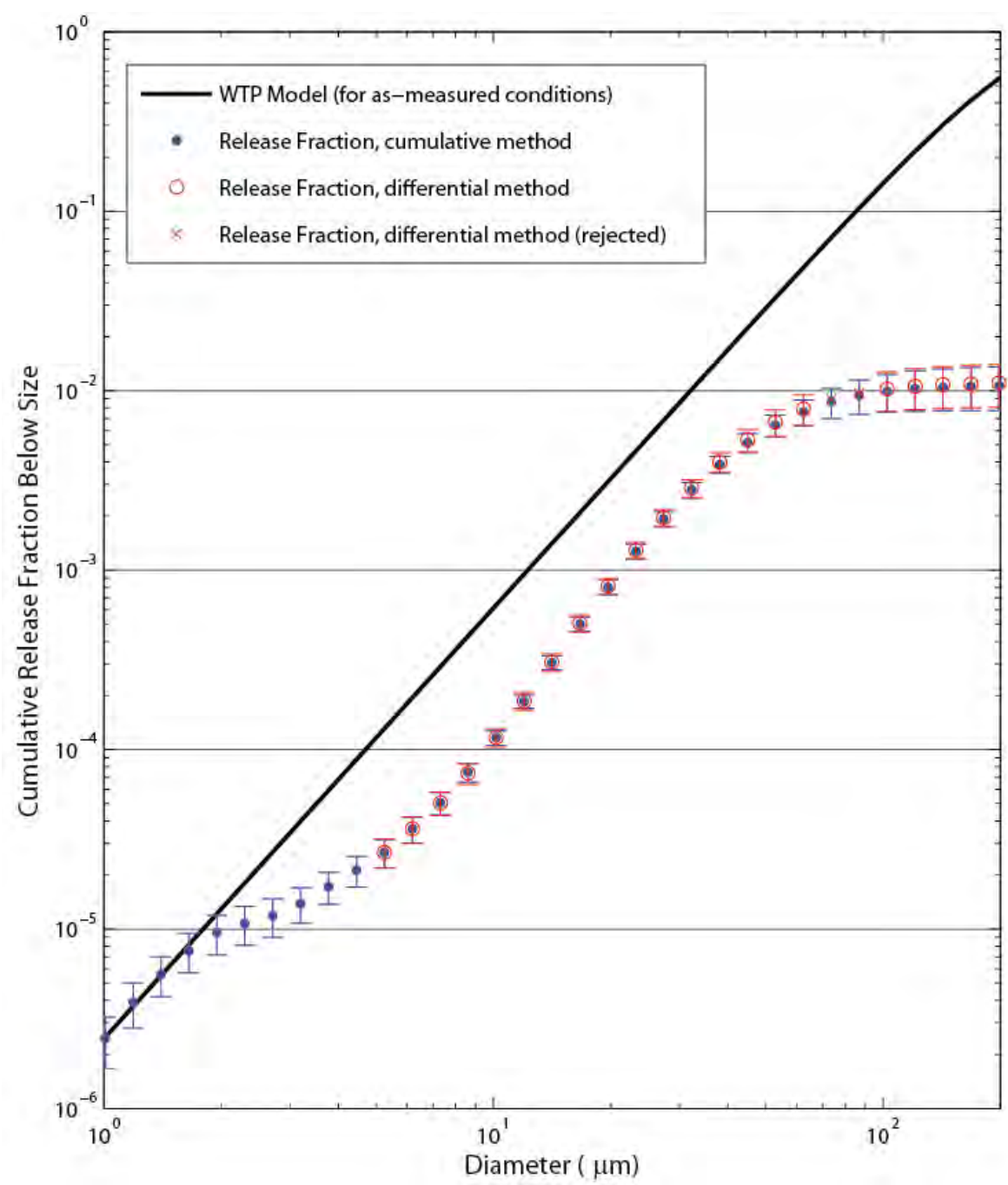

Figure 7.8. Release Fractions Calculated from the Cumulative (blue circles) and Differential (red open circles) Concentration Data Compared to the WTP Model Prediction (black line) for Test W81 (2-mm hole, 380 psi, water)

\subsection{Analysis of Process Particle Counter Data}

The PPC was co-located with the Malvern Insitec-S instrument in position 2. PPC data were collected using commercial software and recorded by DAS-2. The PPC was installed to collect backup measurements in case the aerosol concentrations were too low for the Malvern instrument to detect. Concentrations that challenged the Malvern instruments were not observed during testing, rendering the PPC data redundant to the Malvern data collected from position 2. Some analysis was still performed using the PPC data, primarily to confirm that data from the Malvern instrument in position 2 and the PPC were in agreement, giving added confidence in the accuracy of the measurements.

The method used to analyze for release fraction using PPC data would be identical to that described in Section 7.2. The only exception is that the PPC data needed to be transformed to a concentration distribution that was on the same basis as the Malvern instruments. The standard PSD output is expressed in terms of $d N_{i} / d\left(\ln d_{p, i}\right)$, which gives the differential number concentration of particles $N$ in size bin $i$ 
(e.g., number of particles per cubic centimeters) normalized to the differential change in the log of the particle diameter over bin $i$. For simplicity, we let

$$
\alpha_{i}=\frac{d N_{i}}{d \ln d_{p, i}}
$$

thus, $\alpha_{i}$ is data obtained by the PPC instrument. Particle bin counts and sizes are finite, so rearranging Equation (7.18) in a discrete form gives the following result

$$
\Delta N_{i}=\alpha_{i} \ln \left(\frac{d_{u, i}}{d_{l, i}}\right),
$$

where $\Delta N_{i}$ is the differential particle concentration in bin $i$, and $d_{u, i}$ and $d_{l, i}$ are the upper and lower particle diameters for bin $i$. The volume of a single particle in bin $i, V_{p, i}{ }^{o}$, is

$$
V_{p, i}^{o}=\frac{\pi}{6}\left\langle d_{i}\right\rangle^{3}
$$

where $\left\langle d_{i}\right\rangle$ is the average diameter of size bin $i$. The PPC size bins are scaled logarithmically so the most appropriate average of particle size is geometric. For bin $i$, the average particle diameter is

$$
\left\langle d_{i}\right\rangle=\sqrt{d_{u, i} d_{l, i}}
$$

The fractional volume concentration of aerosol in bin $i, x_{i}$, is given by Equation (7.22)

$$
x_{i}=V_{p, i}^{o} \Delta N_{i},
$$

so substituting Equations (7.19), (7.20), and (7.21) into Equation (7.22) gives the final expression for converting the PPC PSD output into volume fraction equations

$$
x_{i}=\frac{\pi}{6} \alpha_{i}\left(d_{u, i} d_{l, i}\right)^{3 / 2} \ln \left(\frac{d_{u, i}}{d_{l, i}}\right)
$$

The volume fraction given by Equation (7.23) is divided by the total at $i=N$ to give the differential volume fraction. Multiplying that result by $C_{v}$ will give the differential volume concentration. Likewise, summing the differential volume fraction to a size bin $k$ will yield the cumulative volume concentration.

Even after converting the PPC data to the same basis as the Malvern instrument data, a direct comparison is not straightforward. Two issues prevent this. First, there is a difference in the measurable particle range between the two instruments. The PPC instrument is configured to measure only between 0.5 and $67 \mu \mathrm{m}$, requiring that, to perform a comparison, data from the Malvern instrument must be adjusted for particle size bins outside of this range. Second, the default refractive index (RI) used by the PPC to analyze the scattering data is fixed to that of an opaque particle $(\mathrm{RI}=1.5+0.5 \mathrm{i})$. The refractive 
index will not represent the material used for a large portion of the testing, with the majority of the tests conducted using water. Because this refractive index is a fixed parameter in the PPC instrument (i.e., it cannot be changed), the easiest way to generate a comparison is to post-analyze Malvern data with the same RI. This condition was not representative of the test so this approach was used only for a limited set of tests undertaken to confirm the two aerosol instruments provided data that were in agreement for measurements taken at the same location.

\subsection{Analysis Challenges}

There were a handful of specific circumstances that challenged the analysis methods described in Sections 7.1 and 7.2. These challenges included:

- Time synchronization of events (such as the start of spray) recorded on DAS-1 and DAS-2

- Drift of the Insitec-S aerosol analyzer laser power from laser power reference measurements made before each test

- A rapid rise to equilibrium concentration, typically observed for large sprays, which hindered analysis of initial aerosol concentration curve slopes (and thus aerosol generation rates)

- Selection of the appropriate refractive index for multicomponent aerosols generated from spray of slurries.

The disposition of these challenges is discussed in the sections that follow.

\subsubsection{Synchronization of Events Recorded by DAS-1 and DAS-2}

Only one challenge encountered during analysis of spray data was germane to both the DAS- 1 and DAS-2 data: the relation of events in time and/or time synchronization. For the PLC DAS-1 data, this affected the calculation of average pressure, temperature, and flow rates during the test and the specification of stable tank weights before and after a test. As was described, this was mitigated by allowing for some iteration in the calculation procedure for these quantities and confirming that the points in time being used were associated with the correct data. This approach was effective because the start and stop times had a clear signature in the mass and flow rate data.

The signature was not as clear for the Malvern data because there was no signal from the instruments before the spray was initiated. For the Malvern data sets, the recorded time was used to specify $t=0$. The DAS-1 and DAS-2 clocks were synchronized, and agreement between the recorded start and stop times from each source was very good ( $\pm 1 \mathrm{~s})$. Thus, the recorded start and stop times for the Malvern instruments were assumed to be accurate and were not iterated as was done for the DAS- 1 data. ${ }^{11}$ The complication for the DAS-2 Malvern data arose from the tendency of the Malvern software to occasionally omit data points, thus leaving a $1 \mathrm{~s}$ gap in data on a single instrument. This generally occurred two to three times over the test duration per instrument. When performing the analysis, the times of each Malvern instrument could not be assumed to "align" with the others because of these gaps. When a gap was encountered, the average concentrations were calculated using only the available data.

\footnotetext{
${ }^{11}$ Recall that iteration for the DAS-1 data was necessary because the PLC clock was not always synchronized with the DAS-1 computer clock. Because the data files recorded the PLC clock time, the DAS-1 times recorded by the operator are still consistent with the recorded DAS-2 times.
} 
Therefore, approximately 5 to 7 percent of the data points during a spray contain an average of two Malvern measurements instead of three. If a Malvern instrument omitted the $t=0$ data point, all three Malvern data sets were shifted forward in time by a second (to remain conservative), and this was defined as the new $t=0$ point. This problem was specific to that data collection rate, so it did not exist when the Malvern instruments collected data at greater than $1-\mathrm{Hz}$ resolution.

\subsubsection{Laser Power Drift in Insitec-S Aerosol Measurements}

Another significant analysis challenge was related specifically to the treatment of Malvern data. The challenge of transmission drift was discussed previously, and an approach to correct data that had drifted from the background was implemented before a test began (Equation (7.11). However, that approach is limited to only certain laser power drift scenarios. As illustrated in Figure 7.9, the following four drift scenarios are possible:

1. (A) No drift - In an ideal measurement, there is no drift of the transmitted laser power from the background value so the transmitted laser power is equal to the background power before aerosol is generated and after aerosol dissipates. In this scenario, there is no systematic error in aerosol concentration determination (also see Figure 7.3).

2. (B) Negative offset - Negative offset occurs when drift yields a decrease in the laser power relative to the background power. Interpretation of the transmission data yields an aerosol concentration that is artificially high relative to the actual concentration. However, because the measured power is low relative to the background, the Malvern Insitec-S software will report concentration values for the entire period of aerosol generation (also see Figure 7.4). This is not the case when there is positive offset (i.e., for scenarios (C) and (D)).

3. (C) Slight positive offset - Positive offsets occur when laser drift yields an increase in the laser relative to the baseline value. If this increase is slight, the measured laser power will fall above the background value before aerosol generation and after aerosol has dissipated, but not during the entire period of aerosol generation and measurement. When the laser power falls above baseline, Equation (7.8) does not produce a real result, and the Malvern Insitec-S software will not report an aerosol concentration. For periods when the aerosol concentration is high enough to reduce transmitted laser power below the baseline, the Insitec-S software will report an aerosol concentration that is low relative to the actual aerosol concentration. 


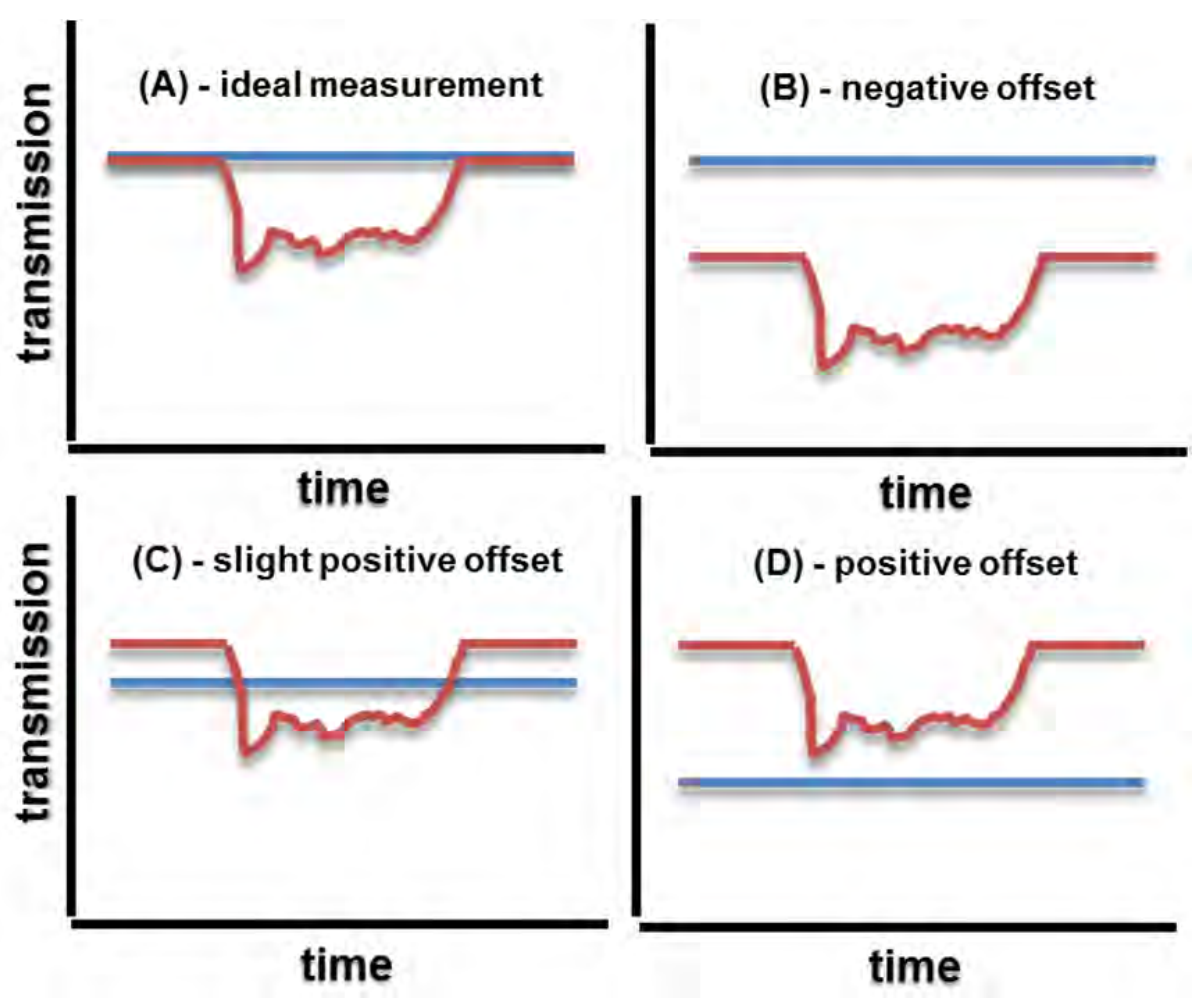

Figure 7.9. Four Possible Laser Drift Scenarios During Malvern Insitec-S Measurements. The four graphs show the potential evolution of the transmitted laser power (red curve) before, during, and after spray measurements relative to the background laser power (blue curve). A decrease in the transmitted (red) power curve occurs as a result of spray. Scenario (A) shows an ideal measurement, where the transmitted power is equal to the background before and after spray. Scenario (B) shows negative offset, where the laser power has drifted low relative to the baseline measurement. Scenarios (C) and (D) show positive offset, where the laser power has drifted high relative to the background measurement.

4. (D) Positive offset - As in case (C), positive offset occurs when the laser drift yields an increase in the laser power relative to the baseline value. In cases where positive offset is significant, the transmitted laser power always falls above the baseline power, even during periods of aerosol generation. In this case, the Insitec-S will not report aerosol concentration at all (as all concentration results derived from Equation (7.8) will be negative and have no physical meaning).

Scenarios A and B are straightforward to handle. Scenario C will have larger data gaps than actually existed, especially in the region of interest (near $t=0$ ), possibly resulting in underestimates of the generation rate. Scenario D, if present in any one Malvern instrument, will bias the average concentration data lower than the actual concentration because one instrument will be treated as measuring zero concentration for the entire experiment. Scenarios C and D, though rare, were encountered in some data sets. The accuracy of these measurements cannot be improved without a more complex expression for the extinction coefficient in Equation (7.8), from which the volume concentration could be calculated directly. Also of concern are the cases where the drift was a function of time. At the present, $C_{v}$ data that are adjusted based on the laser power are shifted a fixed amount and do not include the effect of a gradient in the drift. In large part, these concerns were allayed by the use of the Malvern instruments with longer path lengths and the replacement of two lasers, after which all tests with problematic transmission drift were repeated. 


\subsubsection{Rapid Concentration Rise}

Another analysis challenge in the Malvern data was data with a rapid initial increase. In general, the increase became more severe as the orifice area and/or pressure increased. A very rapid initial increase was difficult to fit with an exponential model, as it might have only a small number of data points indicating the rise to the equilibrium concentration. This was particularly difficult when the data were collected at $1-\mathrm{Hz}$ resolution. Several alternative approaches to estimate the generation rate were considered in light of this difficulty:

- Fitting the $C_{v}$ curve directly with an exponential model with the same form as Equation (7.16) and applying the resultant generation rate to all particle size bins unilaterally

- Approximating the initial slope of either the concentration data or the $C_{v}$ data with a linear fit in lieu of fitting an exponential model

- Integrating the $C_{v}$ data as an approximation of the total aerosol volume generated during a test.

The alternative approaches were found to provide reasonable estimates of generation rates; however, they all represent oversimplifications of the physics governing the formation of aerosol in the large-scale test system. Therefore, the approach described in Section 7.2 was used for all the large-scale test data. A large part of the difficulty associated with fitting rapid increases was mitigated when some of the problematic tests were repeated with a $4-\mathrm{Hz}$, data collection rate. Data collected at a $4-\mathrm{Hz}$ rate provided more data points in the initial time period near $t=0$, so the fit using Equation (7.16) could be used as intended. The largest orifices and pressures still challenge the technique, even with $4-\mathrm{Hz}$ collection rate, and this is reflected in the higher uncertainties for those estimates of generation rate and release fraction.

A similar problem was encountered for the smallest orifices and pressures. In these cases, data tended to be sparse, hovering just above the detection limit of the instruments. Concentration data in these tests were challenging to fit because of a lack of data specifying the slope and/or the equilibrium concentration. Without greater sensitivity in the Malvern instruments, this problem could not be easily solved. The model was usually fit for longer periods of time to give the algorithm more data points, which would improve its performance. The release fractions in the sparser data sets correspondingly have much larger uncertainties, and do not span as wide a droplet size range as in other measurements.

\subsubsection{Selection of Refractive Index for Laser Diffraction Analysis}

The aerosol systems tested in the current application consist of both pure liquids or solutions and suspensions of solid particles in pure liquids or solutions. For the majority of aerosols generated from pure liquids or suspension, determination of aerosol refractive index (RI) is straightforward, as RIs for the test solutions are available in the literature. In particular, the RI of water and solutions of sodium thiosulfate and sodium nitrate solutions can be easily obtained from general chemistry references. For the complex iron-rich simulant supernate, determination of RI is complicated by an unanticipated difference in the elemental analysis provided by the chemical manufacturer of the simulant, which indicates $\sim 10 \mathrm{wt} \%$ dissolved sodium nitrate in the as-prepared supernate, and the dissolved solids content analysis conducted by PNNL, which indicates $~ 15 \mathrm{wt} \%$ dissolved content in the as-prepared supernate. This difference suggests dissolved species other than sodium nitrate are present. However, the exact chemical makeup of the supernate has not been determined by PNNL. Because direct determination of RI of the iron-rich supernate would be prohibitive (in terms of time and cost required to set up an NQA-1 
compatible method and procedure for RI determination) and because the exact chemical makeup of the supernate is unknown, estimation of iron-rich supernate RIs have been facilitated by assuming the entire dissolved content is sodium nitrate.

While RIs are generally available for the liquids and supernates tested, assignment of appropriate values for RI for aerosols generated during spray of slurries is not straightforward. Difficulties in assigning a RI for slurry systems result from the fact that 1 ) aerosols generated by slurry sprays are heterogeneous composite particles of solids either fully or partially encapsulated in water, and 2) the composition of the composite particles is not necessarily equal to that of the test slurry as confinement and jamming of particles at the point of release may alter the composition and in-flight drag or evaporation may strip the aerosol of water content or particles. For the purpose of estimating release fractions, a single RI may be assigned to the system with the understanding that the concentration and size distribution derived from the laser diffraction aerosol instrumentation used in the current testing may not be accurate. One of three basic RIs can be assigned: 1) that of the solid component, 2) that of the liquid component, or 3) an effective RI for a particular concentration of solid in liquid. For simple simulants, solid and liquid RIs are generally available in the literature. These values can be used to bound RI of aerosol composites and are necessary inputs for estimating the effective RI of the composite liquid-solid aerosol.

Estimates of RI for composite solids can be made by using one of several mixing rules available in the literature. These rules are derived for binary mixtures where optical properties of the pure components are known. These rules are:

- Bruggeman Rule (Bruggeman, 1935)

$$
f \frac{\epsilon_{1}-\epsilon_{e f f}}{\epsilon_{1}+2 \epsilon_{e f f}}+(1-f) \frac{\epsilon_{2}-\epsilon_{e f f}}{\epsilon_{2}+2 \epsilon_{e f f}}=0
$$

- Maxwell-Garnett Rule (Garnett 1904)

$$
\frac{\epsilon_{e f f}-\epsilon_{2}}{\epsilon_{e f f}+2 \epsilon_{2}}=f \frac{\epsilon_{1}-\epsilon_{2}}{\epsilon_{1}+2 \epsilon_{2}}
$$

- Looyenga Rule (Looyenga 1965)

$$
\epsilon_{e f f}^{1 / 3}=f \epsilon_{1}^{1 / 3}+(1-f) \epsilon_{2}^{1 / 3}
$$

- Monecke Rule (Monecke 1994)

$$
\epsilon_{e f f}=\frac{2\left\{f \epsilon_{1}+(1-f) \epsilon_{2}\right\}^{2}+\epsilon_{1} \epsilon_{2}}{(1+f) \epsilon_{1}+(2-f) \epsilon_{2}}
$$

- Hollow sphere equivalent (Bohren and Huffman 1983)

$$
\epsilon_{e f f}=\epsilon_{1} \frac{(3-2 f) \epsilon_{2}+2 f \epsilon_{1}}{f \epsilon_{2}+(3-f) \epsilon_{1}}
$$


Here, $f$ is the volume fraction of component $1, \varepsilon_{\text {eff }}$ is the effective dielectric constant on the medium, and $\varepsilon_{1}$ and $\varepsilon_{2}$ are the dielectric constants of component 1 and 2 respectively. The dielectric constant is related to RI by:

$$
\epsilon=n^{2}
$$

Of these five mixing rules, two appear amenable to extension beyond binary systems. These are the Bruggeman and Looyenga mixing rules. From these rules, the following extension to multicomponent systems is proposed:

- Extended Bruggeman Rule

$$
\sum_{i} f_{i} \frac{\epsilon_{i}-\epsilon_{e f f}}{\epsilon_{i}+2 \epsilon_{e f f}}=0
$$

- Extended Looyenga Rule

$$
\epsilon_{\text {eff }}^{1 / 3}=\sum_{i} f_{i} \epsilon_{i}^{1 / 3}
$$

Here, $f_{i}$ and $\varepsilon_{i}$ are the volume fraction and dielectric constant of component $i$, respectively. These multicomponent rules produce complex RIs that are in reasonable agreement with results produced by nesting the binary rules to produce RI estimates for complex mixtures of particles.

The test matrix for both small- and large-scale efforts includes water, several salt solutions, single component solid slurries, and multicomponent slurries with both insoluble and soluble solids. The components employed in spray leak testing are described in detail in Chapter 4 of this report. To determine RIs for this diverse array of test mixtures, the approach used was to:

1. Determine, using standard chemical references, the RI for all solid components, pure liquids, and salt solutions. For the latter, the dissolved component concentration was measured directly or determined by mass balance.

2. Determine the composition of all test mixtures in terms of volume fraction by mass balance or available documentation of mixture chemical makeup.

3. Based on the composition determined in step 2 and the pure component and suspending phase RIs assigned in step 1, calculate mixture RI using the appropriate set of mixing rules (i.e., Equations (7.24) to (7.31)). The general approach used was to apply all of these mixing rules (5 for binary systems and 2 for multicomponent mixtures) to a given system. It should be noted that each mixing rule produces a slightly different RI result; however, differences in the RI produced by application of different mixing rules to the same chemical mixture typically occur in the third decimal place, and as such, analysis of Insitec-S data using the these different RI will not produce a noticeable difference in final aerosol concentration or PSD results. To produce a single RI result for analysis, the final composite RI for the mixture was calculated as the arithmetic average of the RI results produced by the individual mixing rules. 
RIs for all pure liquid and solid components and test slurries are presented in Table 7.2. In general, the absorption index (i.e., the imaginary component) of pure solids is not available in the literature. However, unless the solid particles are spherical, the imaginary component may be generally taken to be non-zero. Likewise, even spherical aerosol droplets of supernate that contain non-spherical insoluble particles will also have a non-zero absorption index. Because the exact value of solid particle absorption index is not known, it has been set to 1.000 for all solid particles, such that they are represented (in terms of Mie theory) as being completely opaque. Proper assignment of solid particle absorption index is not expected to strongly impact the size distribution result, as the diffraction result above $1 \mu \mathrm{m}$ is expected to be robust with respect to order-of-magnitude changes in absorption index, so long as the RI is reasonable for non-spherical particles (i.e., non-zero and on the order of 0.01 to 1 ). 
Table 7.2. Refractive Indices for Test Systems Used in Both Small- and Large-Scale Spray Leak Testing. The RI of pure components has been taken from reference sources available in the literature. Refractive indices for mixtures have been calculated using both binary and multicomponent mixing rules. Both the real (refractive index) and the imaginary (i.e., the absorption index) components of the complex RI are given. All RIs are listed to three decimal places to demonstrate the value of RIs provided to the Malvern software. Real RI values are typically significant to two figures, whereas imaginary RI values are significant to one figure.

\begin{tabular}{|c|c|c|c|}
\hline Component & Method $^{(a)}$ & $\begin{array}{l}\text { Refractive } \\
\text { Index (Real) }\end{array}$ & $\begin{array}{l}\text { Absorption Index } \\
\text { (Imaginary Refractive } \\
\text { Index Component) }\end{array}$ \\
\hline \multicolumn{4}{|c|}{ Pure Liquids and Solutions } \\
\hline Water (with and without AFA) & Reference & 1.330 & 0.000 \\
\hline Sodium Thiosulfate Solution (27.2 wt\%) & Reference & 1.392 & 0.000 \\
\hline Sodium Nitrate Solution (32.3 wt\%) & Reference & 1.371 & 0.000 \\
\hline \multicolumn{4}{|c|}{ Pure and Mixed Solids } \\
\hline Gibbsite & Reference & 1.580 & 1.000 \\
\hline Boehmite & Reference & 1.655 & 1.000 \\
\hline Iron-Rich/gibbsite & Calculated & 1.728 & 1.000 \\
\hline Iron-Rich/boehmite & Calculated & 1.823 & 1.000 \\
\hline \multicolumn{4}{|c|}{ Slurry Systems } \\
\hline STR 8 wt\% (with and without AFA) & Calculated & 1.342 & 0.025 \\
\hline STR 20 wt\% (with and without AFA) & Calculated & 1.363 & 0.070 \\
\hline SAR $8 \mathrm{wt} \%$ & Calculated & 1.343 & 0.033 \\
\hline SAR $20 \mathrm{wt} \%$ & Calculated & 1.364 & 0.090 \\
\hline TAR 8 wt $\%$ & Calculated & 1.343 & 0.033 \\
\hline Iron-Rich/boehmite Slurry - $6 \mathrm{~Pa}$ & Calculated & 1.371 & 0.047 \\
\hline Iron-Rich/gibbsite Slurry - $30 \mathrm{~Pa}$ & Calculated & 1.433 & 0.169 \\
\hline Iron-Rich/gibbsite Slurry - $30 \mathrm{~Pa}+400$ ppm AFA & Calculated & 1.433 & 0.170 \\
\hline Iron-Rich/gibbsite Slurry - $6 \mathrm{~Pa}+400$ ppm AFA & Calculated & 1.415 & 0.135 \\
\hline \multicolumn{4}{|c|}{ Slurry System Suspending Phase } \\
\hline STR, SAR, TAR (with and without AFA) & Reference & 1.330 & 0.000 \\
\hline Iron-Rich/boehmite Slurry - $6 \mathrm{~Pa}$ & Calculated & 1.339 & 0.000 \\
\hline Iron-Rich/gibbsite Slurry - $30 \mathrm{~Pa}$ & Calculated & 1.348 & 0.000 \\
\hline Iron-Rich/gibbsite Slurry - $30 \mathrm{~Pa}+400$ ppm AFA & Calculated & 1.348 & 0.000 \\
\hline Iron-Rich/gibbsite Slurry - $6 \mathrm{~Pa}+400$ ppm AFA & Calculated & 1.345 & 0.000 \\
\hline
\end{tabular}

(a) Method: 1) Reference - taken from a reference chemical handbook or literature source, and 2) Calculated - determined by known component indices and averaging of all mixing rules (2 for binary systems and 5 for multicomponent systems).

\subsection{Summary}

A two-step approach was used to analyze data collected during large-scale spray release testing. The first part used data from the process instruments, recorded by a PLC to DAS-1, to determine the average conditions during each test and calculate the spray leak flow rate. The flow rate was estimated either by 
a mass method or a flow rate method. In most cases, there is little difference in the results obtained by the two approaches; however, the mass method is more accurate for cases in which flow rate is small (e.g., small orifice area and/or low pressure). Therefore, the mass method was selected for use in calculating the release fraction.

The second part used data from the aerosol instruments, namely the three Malvern Insitec-S instruments. The aerosol size distribution data were converted to a volume basis (ppmv), corrected for transmission drift, and the three measured concentrations were averaged. Both differential and cumulative concentration data were analyzed. The averaged data were aligned in time and then fit with an exponential model to determine the aerosol generation rate. The generation rate was divided by the spray leak flow rate to obtain estimates of the release fraction for the experiments. Upon performing this analysis for a variety of conditions, comparisons were made to observe the effect of parameter changes on the release fraction (or generation rate). Parametric studies of this type are the subject of the Chapter 8. 


\subsection{Results and Discussion}

In this chapter, we discuss the results obtained from the analyses described in Chapter 7. The focus will be on examining the effects of various parameters on the release fraction and/or generation rate. Of particular interest are comparisons of the WTP model predictions with the large-scale test results. Although both differential and cumulative aerosol concentration data were analyzed, typically only results from the cumulative data are shown. Because the release fractions estimated from each data set are very similar (e.g., as shown in Figure 7.8), the cumulative method was chosen because it had a wider range of droplet size bins that resulted in a release fraction satisfying the $\overline{\mathrm{R}}^{2}$ criterion. As noted previously, a complete list of the specific conditions of all tests conducted is described in Appendix A. For the complete set of release fractions, calculated by both the differential and cumulative methods for each test, refer to Appendix B. Some of the figures contain composite data from multiple tests or the test number is not identified. Tables identifying individual test data contained in figures of this type are available in Appendix B.

The results are broken into six sections that are organized around a single concept or variable that was studied. In Section 8.1, basic parameters of the test system are discussed, including process conditions during the test, parameters affecting mixing of aerosol in the chamber, use of the PPC to confirm the Malvern instrument data, and an assessment of the experimental repeatability. In Section 8.2, parametric studies of pressure, orifice size, and orifice orientation are presented. These tests were all conducted with water as the working fluid. In Section 8.3, the effect of viscosity is presented by comparing water and sodium thiosulfate test results that were acquired using common orifice sizes. In Section 8.4, the effects of solids loading (e.g., 8 and $20 \mathrm{wt} \%$ ) are investigated, again using tests with common orifices sizes to compare water and slurry data. In Section 8.5, the effect of proximity to the splash wall (e.g., distance between spray jet and the splash wall) is presented using data collected when orifices were moved to different distances relative to the back wall of the aerosol chamber. In Section 8.6, in-spray aerosol measurements are discussed, and finally, a summary of this work and the conclusions are presented in Section 8.7.

\subsection{Test Conditions}

The generation of aerosol in a fixed volume chamber is governed by complex physical phenomena occurring at the free jet surface/air interface in a turbulent flow regime. To establish that the aerosol test data were collected at conditions permitting meaningful analysis of the aerosol behavior in spite of the complexity, preliminary testing was conducted to assess the quality and reproducibility of the aerosol data as a function of chamber/test configuration. Results from these tests will be discussed in this section, as well as the justification for the standard configuration that was used in subsequent testing. Some of the tests discussed in this section were conducted chronologically after the aerosol tests to confirm that similar results were achieved with the Malvern instruments in their final configuration (a consequence of switching to the 50-cm spacer bars and replacing the lasers on Malvern instruments 1854 and 1855; see Appendix A, Table A.1 for the complete test chronology). 


\subsubsection{Process Conditions}

The process conditions were assessed as described in Section 7.1. Primarily, this assessment was performed to estimate the spray leak flow rate. However, the results can also be used to confirm that the control of the pressure was performed to specification and that the test conditions during a spray were steady. An example of the pressure and temperature data from a test is shown in Figure 8.1 (note: this was the same test that was used in many of the analysis examples in Chapter 7). The noise in the pressure data is typical; some tests had decreases in pressure that were slightly larger than in the case that is shown. The variation in the temperature data also is typical, but the value of the temperature during a test was dependent on the time and sequencing of a test. Process conditions for every test are tabulated in Appendix A, Table A.4.

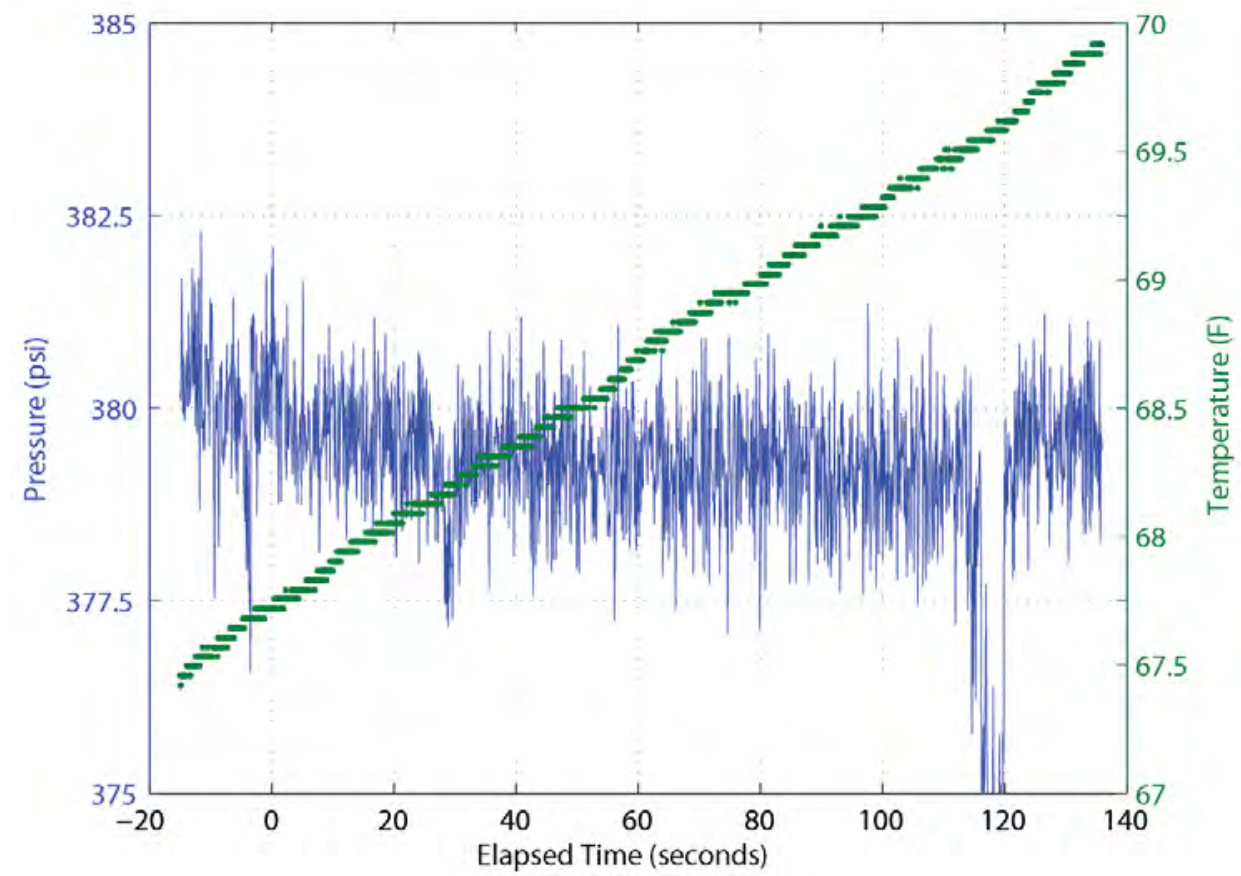

Figure 8.1. Pressure and Temperature Data from Test W81 (2-mm orifice, 380 psi, water). An elapsed time of zero is approximately when the spray was initiated.

The pressure and temperature data are summarized in the aggregate in Table 8.1. The table has the average, standard deviation of the average, minimum and maximum values measured across all the tests with a mutual target pressure. On average, the target pressure was achieved with high precision. A few of the tests with a target pressure of 380 psi and an orifice with a large cross-sectional area had pressure drops on the order of 10 to 15 psig, leading to a maximum divergence from target of 11.3 psig. The temperature was intended to be within $75 \pm 10^{\circ} \mathrm{F}$. This condition was eclipsed only three times; generally, the average temperature was between 67 and $77^{\circ} \mathrm{F}$ during a test. Average data in Table 8.1 and the corresponding individual test data in Table A.4 confirm that the pressure and temperature during a test were at or near target conditions for all tests. Since the pressure response was stable and consistent during testing, the average pressure over the spray duration was used in all calculations, i.e., both in determining orifice coefficient and WTP model predictions. This is in contrast to the approach required in the small-scale calculations, which used more than one pressure as described in Mahoney et al. 2012b. 
The other major calculation from the process instrument data is the flow rate of the spray, $Q_{\text {spray }}$. The flow rate, which was estimated by two methods, can be used to estimate the orifice coefficient using Equation (7.6). These estimates are provided for each test in Table A.4 of Appendix A. As indicated by Equation (1.8), the WTP model expects an orifice coefficient of 0.62 . The histogram of the orifice coefficients estimated from experimental data using the mass method (top) and flow rate method (bottom) is shown in Figure 8.2. All 245 tests that were conducted are shown even though the first 60 tests were later repeated and the aerosol data from them is not reported.

Table 8.1. Summary of the Pressure and Temperature Data Measured During Aerosol Tests

\begin{tabular}{|c|c|c|c|c|c|c|c|c|c|}
\hline $\begin{array}{c}\text { Test Target } \\
\text { Pressure }\end{array}$ & $\begin{array}{l}\text { No. of } \\
\text { Tests }\end{array}$ & $\begin{array}{c}\langle\mathrm{P}\rangle \\
\text { (psig) }\end{array}$ & $\begin{array}{l}\text { Std Dev. } \\
\langle\mathrm{P}\rangle \text { (psig) }\end{array}$ & $\begin{array}{l}\text { Min. P } \\
\text { (psig) }\end{array}$ & $\begin{array}{l}\text { Max. P } \\
\text { (psig) }\end{array}$ & $\langle\mathrm{T}\rangle\left({ }^{\circ} \mathrm{F}\right)$ & $\begin{array}{l}\text { Std Dev. } \\
\langle\mathrm{T}\rangle\left({ }^{\circ} \mathrm{F}\right) \\
\end{array}$ & $\begin{array}{l}\text { Min. T } \\
\left({ }^{\circ} \mathrm{F}\right)\end{array}$ & $\begin{array}{c}\text { Max. T } \\
\left({ }^{\circ} \mathrm{F}\right) \\
\end{array}$ \\
\hline 100 psig & 47 & 100.8 & 1.9 & 97.4 & 105.7 & 72.5 & 5.0 & 65.3 & 82.7 \\
\hline 200 psig & 70 & 199.6 & 1.7 & 194.7 & 204.2 & 71.7 & 3.8 & 63.3 & 82.7 \\
\hline 380 psig & 127 & 377.9 & 2.2 & 368.7 & 382.7 & 74.5 & 4.5 & 64.4 & 86.8 \\
\hline
\end{tabular}

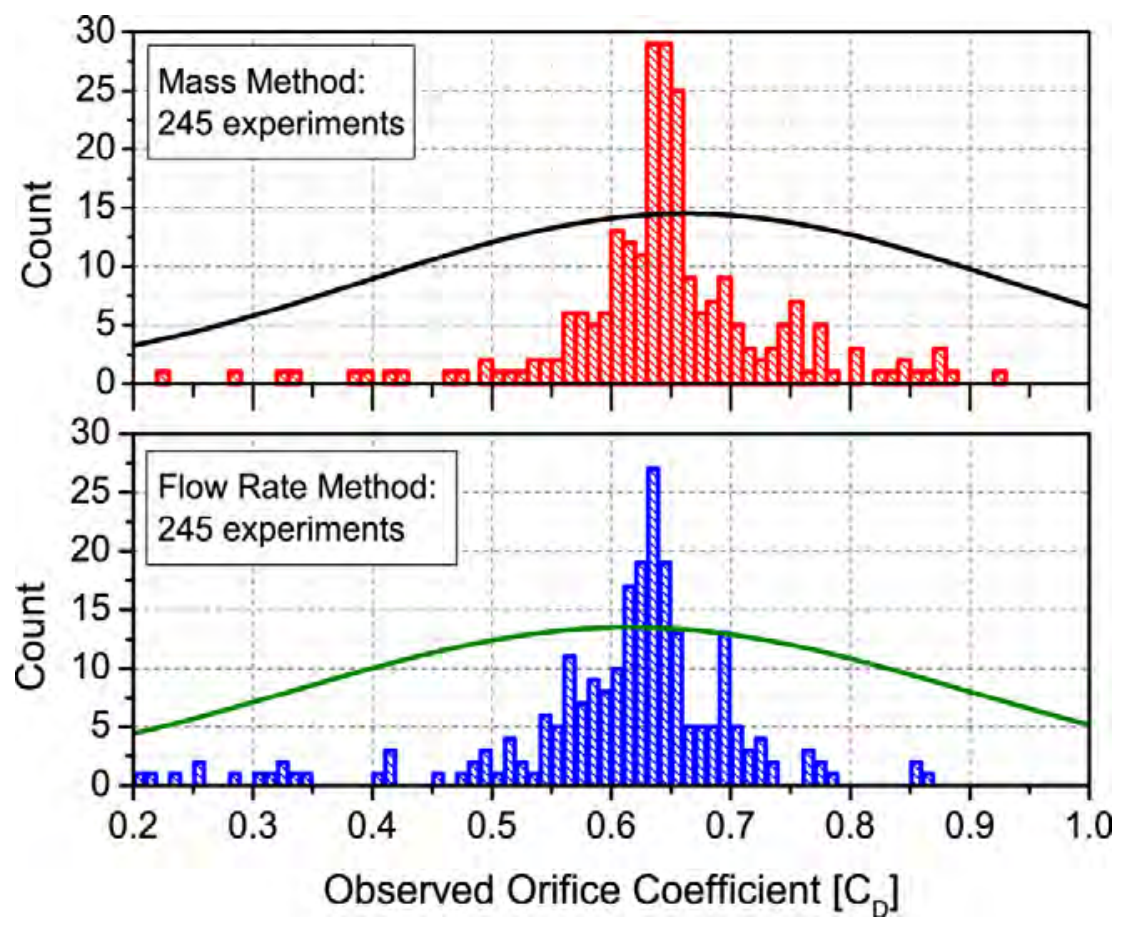

Figure 8.2. Orifice Coefficients Determined from Analysis of Tank Masses (top) and Coriolis Flow Rate (bottom) for All 245 Experiments That Had Useable Data. The black and green solid lines are normal distributions of the orifice coefficients shown at 50 percent of scale.

The orifice coefficients shown in Figure 8.2 are presented in bins of size equal to 0.01 . Note that there are several ${ }^{12}$ orifice coefficients for each method that are not shown in the figure because they are

\footnotetext{
${ }^{12}$ Specifically, for the mass method, there were four orifice coefficients that were outside of this range; for the flow meter method, there were 11 .
} 
outside of the range 0.2 to 1.0; these were considered outliers. Figure 8.2 illustrates that the $C_{D}$ values calculated by the mass method have a tighter distribution about an average value than those calculated by the flow rate method (indicated both by the spread in the data and the normal distribution curves provided on the histograms for reference). The overall average orifice coefficient for all tests was calculated to be 0.66 and 0.62 for the mass and flow rate methods, respectively. If the orifice coefficients are restricted further based on values that are within the range expected by empirical studies (i.e., $0.4 \leq C_{D} \leq 0.9$ ) ${ }^{13}$, the overall average becomes $0.65 \pm 0.08$ and $0.63 \pm 0.07$, respectively, where the uncertainty given is one standard deviation from the mean value. The general trend is that the orifice coefficient decreases slightly with increasing orifice area. Both average orifice coefficients are consistent with the value of 0.62 used in the WTP model. However, given the more direct and precise nature of the mass method, there is more confidence in the overall $\mathrm{C}_{\mathrm{D}}$ obtained using that method.

The orifice coefficients as calculated by the mass method that were higher or lower than expected are presented in Table 8.2. The aerosol data, namely RF values, obtained from the tests with $C_{D}$ values $>0.9$ and $<0.5$ are presented in this report in Appendix B but are not used in any figures or composite data sets discussed in this report. Note that the outlier $C_{D}$ values are from the smallest orifices (predominantly S1A [1 mm] and S2A [0.5 $\times 5 \mathrm{~mm}]$ ) where the measurement methods have the highest uncertainty. It is important to mention that at the conclusion of the testing, the 1-mm hole was sprayed for $10 \mathrm{~min}$ (Test W246) instead of the customary two min to get a better estimate of $Q_{\text {spray }}$, with a resulting $C_{D}=0.75$. The W246 $C_{D}$ measurement suggests that the 1-mm hole was producing flow rates consistent with expectations but the mass method was not measuring it accurately at smaller spray times. Table 8.2 also includes tests where $0.4<\mathrm{C}_{\mathrm{D}} \leq 0.5$ and $0.8 \leq \mathrm{C}_{\mathrm{D}}<0.9$ to illustrate that in the majority of tests ( $\left.>87 \%\right)$ the orifice coefficient was bounded in an expected range of $0.5<\mathrm{C}_{\mathrm{D}}<0.8$.

\footnotetext{
${ }^{13}$ See, for example, Perry's Engineering Handbook, $7^{\text {th }}$ edition, pp. 10-15 and 10-16. For the mass method, there were 11 orifice coefficients that were outside of this range; for the flow meter method, there were 23.
} 
Table 8.2. List of Outlier Orifice Discharge Coefficients

\begin{tabular}{|c|c|c|c|c|c|c|}
\hline Test & Fluid & $\begin{array}{l}\text { Pressure } \\
\text { (psig) }\end{array}$ & $\begin{array}{l}\text { Orifice (Target } \\
\text { Dimension(s)) }\end{array}$ & $\begin{array}{l}C_{D} \text { (mass } \\
\text { method) }\end{array}$ & $\begin{array}{l}C_{D} \text { (flow } \\
\text { method) }\end{array}$ & Comments \\
\hline \multicolumn{7}{|c|}{ Tests where $C_{D}$ was high relative to the global average (>0.8) } \\
\hline W04 & water & 380 & S1D (2-mm hole) & 1.859 & 1.748 & $\begin{array}{l}\text { Test later repeated, RF not } \\
\text { reported }\end{array}$ \\
\hline W47 & water & 380 & $\begin{array}{l}\text { S5B (array of } 5 \\
\text { (1) mm holes) }\end{array}$ & 4.292 & 4.008 & $\begin{array}{l}\text { Test later repeated, RF not } \\
\text { reported }\end{array}$ \\
\hline W212 & water & 200 & S1A (1-mm hole) & 0.930 & 0.726 & $\begin{array}{c}\text { In-spray measurement - W214 (a } \\
\text { duplicate test) was used in } \\
\text { figures instead }\end{array}$ \\
\hline \multicolumn{7}{|c|}{$\begin{array}{l}\text { NOTE: Tests W61 - W69, W74, W128, W133, and W138 [all water, 1-mm hole] had } 0.8 \leq C_{D} \leq 0.9 \text {. Some of } \\
\text { these tests are reported in composite plots, in particular W61 - W69 and W74. }\end{array}$} \\
\hline \multicolumn{7}{|c|}{ Tests where $C_{D}$ was low relative to the global average $(<0.5)$} \\
\hline W111 & water & 100 & $\begin{array}{c}\text { S5B (array of } 5 \\
(1) \text { mm holes) }\end{array}$ & 0.385 & 0.195 & $\begin{array}{l}\text { RF reported in Appendix B but } \\
\text { data not used in any figures }\end{array}$ \\
\hline W132 & water & 100 & S1A (1-mm hole) & 0.399 & 0.734 & $\begin{array}{l}\text { RF reported in Appendix B but } \\
\text { data not used in any figures }\end{array}$ \\
\hline W136 & water & 100 & S1A (1-mm hole) & 0.281 & 0.769 & $\begin{array}{l}\text { RF reported in Appendix B but } \\
\text { data not used in any figures }\end{array}$ \\
\hline S154 & STS & 200 & $\begin{array}{l}\text { S2A }(0.5 \times 5-\mathrm{mm} \\
\text { slot })\end{array}$ & 0.322 & 0.251 & $\begin{array}{l}\text { RF reported in Appendix B but } \\
\text { data not used in any figures }\end{array}$ \\
\hline S155 & STS & 380 & $\begin{array}{l}\text { S2A }(0.5 \times 5-m m \\
\text { slot })\end{array}$ & 0.339 & 0.348 & $\begin{array}{l}\text { RF reported in Appendix B but } \\
\text { data not used in any figures }\end{array}$ \\
\hline S156 & STS & 100 & $\begin{array}{l}\text { S2A }(0.5 \times 5-\mathrm{mm} \\
\text { slot })\end{array}$ & 0.124 & -0.002 & $\begin{array}{l}\text { RF reported in Appendix B but } \\
\text { data not used in any figures }\end{array}$ \\
\hline S157 & STS & 200 & $\begin{array}{l}\text { S2A }(0.5 \times 5-\mathrm{mm} \\
\text { slot })\end{array}$ & 0.068 & -0.057 & $\begin{array}{l}\text { RF reported in Appendix B but } \\
\text { data not used in any figures }\end{array}$ \\
\hline S158 & STS & 380 & $\begin{array}{l}\text { S2A }(0.5 \times 5-\mathrm{mm} \\
\text { slot })\end{array}$ & 0.229 & -0.132 & $\begin{array}{l}\text { RF reported in Appendix B but } \\
\text { data not used in any figures }\end{array}$ \\
\hline W126 & water & 100 & S1A (1-mm hole) & 0.426 & 0.510 & $\begin{array}{l}\text { RF reported in Appendix B but } \\
\text { data not used in any figures }\end{array}$ \\
\hline S153 & STS & 100 & $\begin{array}{l}\text { S2A }(0.5 \times 5-\mathrm{mm} \\
\text { slot })\end{array}$ & 0.467 & 0.549 & $\begin{array}{l}\text { RF reported in Appendix B but } \\
\text { data not used in any figures }\end{array}$ \\
\hline S183 & $\begin{array}{c}20 \mathrm{wt} \% \\
\text { STR }\end{array}$ & 100 & $\begin{array}{l}\text { S2A }(0.5 \times 5-\mathrm{mm} \\
\text { slot })\end{array}$ & 0.499 & 0.517 & $\begin{array}{l}\text { RF reported in Appendix B but } \\
\text { data not used in any figures }\end{array}$ \\
\hline S201 & $\begin{array}{l}8 \mathrm{wt} \% \\
\text { STR }\end{array}$ & 100 & $\begin{array}{l}\text { S2A }(0.5 \times 5-\mathrm{mm} \\
\text { slot })\end{array}$ & 0.419 & 0.405 & $\begin{array}{l}\text { RF reported in Appendix B but } \\
\text { data not used in any figures }\end{array}$ \\
\hline S202 & $\begin{array}{l}8 \mathrm{wt} \% \\
\text { STR }\end{array}$ & 200 & $\begin{array}{l}\text { S2A }(0.5 \times 5-\mathrm{mm} \\
\text { slot })\end{array}$ & 0.479 & 0.411 & $\begin{array}{l}\text { RF reported in Appendix B but } \\
\text { data not used in any figures }\end{array}$ \\
\hline S204 & $\begin{array}{l}8 \mathrm{wt} \% \\
\text { STR }\end{array}$ & 100 & $\begin{array}{l}\text { S2A }(0.5 \times 5-\mathrm{mm} \\
\text { slot })\end{array}$ & 0.493 & 0.457 & $\begin{array}{l}\text { RF reported in Appendix B but } \\
\text { data not used in any figures }\end{array}$ \\
\hline
\end{tabular}

\subsubsection{Effect of Mixing Fans}

At the outset of testing, the first 25 tests (i.e., W01 to W25) were performed to assess how different fan configurations and speeds affected the aerosol concentration and, ultimately, the release fraction calculated from the concentration data. Owing to the use of Malvern instruments with smaller spacing $(15 \mathrm{~cm})$ and lasers that drifted significantly (and were replaced prior to start of Group 1 testing), the data collected during these 25 tests were subject to significant variability. Despite this, the data did show that 
the use of fans in the chamber resulted in a release fraction that was higher than when fans were not used. This was expected, as it was assumed the fans added convective motion that helped suspend aerosol that would have otherwise fallen to the chamber floor, thereby improving the spatial distribution of aerosol in the chamber. At the time, based on the data from tests W01 to W25, the choice was made to use two bilge fans (the inner two from an array of four total) operating at $12 \mathrm{~V}$ in the rest of the large-scale tests. The data obtained at $12 \mathrm{~V}$ gave the most consistent results out of the fan speeds tested (i.e., $0,6,9,12$, and $15 \mathrm{~V})$.

Later, after a standard Malvern instrument configuration had been determined through iteration during testing, the tests were repeated to confirm the choice of the two bilge fans operating at $12 \mathrm{~V}$. These tests (W235 to W242) were conducted using the same conditions (2-mm orifice, $380 \mathrm{psi}$ ) as the earlier fan tests. The release fractions estimated from the cumulative concentration data of those tests are presented in Figure 8.3. The release fractions are all very similar, and considering the variability observed in repeated tests (see Section 8.1.6), are statistically indistinguishable from one another. The tests in which the fans were in operation at any speed do indicate a small increase in the release fraction over the test in which the fans were not used, particularly in the region of interest (10 to $100 \mu \mathrm{m})$. This finding, combined with evidence from the earlier fan tests that $12 \mathrm{~V}$ was a consistent performer, confirmed the selection of the fan configuration used in the large-scale testing.

\subsubsection{Aerosol Instrument Location in Chamber}

The effect of the mixing fans described in the previous section was established with fixed Malvern instrument locations. The intent of the fans was to enhance the convective mixing provided by the turbulent jet and promote homogeneity in the aerosol chamber. A homogeneous chamber has the following two connotations: 1) the total volume concentration is the same everywhere in the chamber, and 2) the size distribution of aerosol is the same everywhere in the chamber. Therefore, in a well-mixed chamber, the measurement of aerosol concentration will not depend on location. Even if the mixing in the chamber was such that the total volume concentration was approximately homogeneous, loss mechanisms (settling of droplets, in particular) will make obtaining the same size distribution challenging. In addition, data from the three Malvern instruments had already indicated that there was a concentration gradient within the chamber from the splash wall, where the concentration is highest, back towards the spool piece, where the concentration is lowest. The technical approach adopted to estimate the generation rate despite the presence of the observed gradient along the "spray axis" (i.e., in the direction the jet is spraying) was to average data obtained from the Malvern instruments (this is discussed in Section 7.2 and Appendix C). 


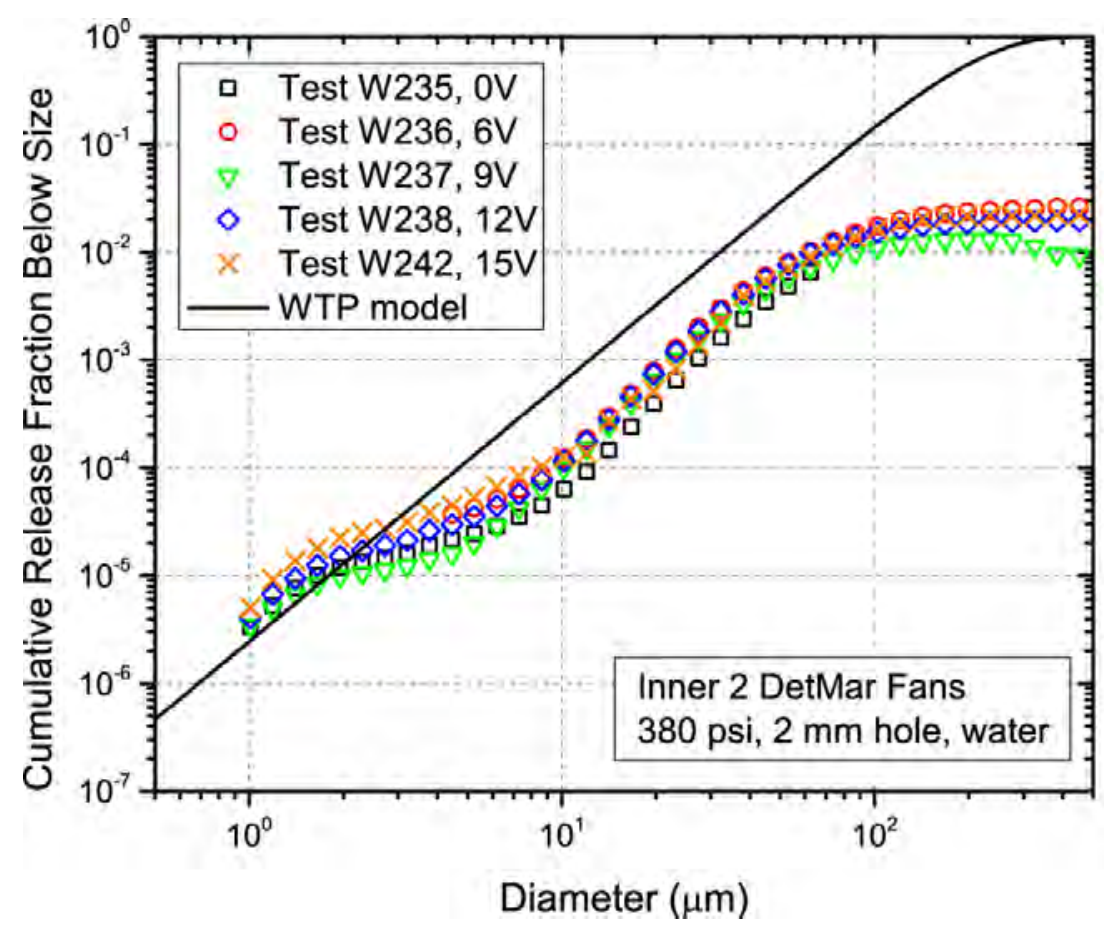

Figure 8.3. Effect of Mixing Fans. Cumulative release fraction calculated using cumulative concentration data from tests conducted to confirm selection of the inner two 4-in., bilge fans (DetMar Corporation, Detroit, Michigan) using a 2-mm orifice at 380 psi. The uncertainty bars have been removed from the data to improve clarity. Refer to Appendix B for the release fractions with uncertainty bars and the results of the differential concentration calculation.

A set of tests were conducted to assess whether the vertical position of the Malvern instruments had a significant effect on the aerosol concentration. Of particular concern was whether the vertical locations of the instruments (which are above the spray during the testing described in this report) resulted in an underestimate of the aerosol concentration, by virtue of not measuring the larger droplets that fell to the floor of the chamber. Three different vertical locations were compared, where the Malvern instruments remained in approximately the same lateral position (refer to Table 5.2, dimensions A, B, and C) and were moved collectively to a specified height. These heights, as measured from the top of the aerosol chamber to the measurement zone of the Malvern instruments, are 15.75, 23, and 62 in. ${ }^{14}$ These locations are referred to as high $[\mathrm{H}]$, medium [M], and low [L], respectively, in Figure 8.4. The medium location was in the same vertical position as the standard Malvern configuration used in the majority of testing and specified in Table 5.2. The high location was above the standard configuration, whereas the low location was far below the standard configuration and, in fact, was beneath the spray jet itself. A fourth vertical location also was studied, but the measurements were performed in the spray (between the medium and low locations), and the data are not directly comparable. The in-spray data are discussed in Section 8.6.

\footnotetext{
${ }^{14}$ In the case of the 62 in. (i.e., low) vertical location, Malvern 1855 in position 1 had to be rotated 90 degrees to accommodate its vertical location (as it would otherwise have been directly in the path of the spray). Note also that these distances are approximate and varied $\pm 0.5 \mathrm{in}$. between the various Malvern instruments.
} 
Examples of measured concentrations in droplet size bins of interest for the various vertical locations are shown in Figure 8.4. The concentration data for the 8.62- to 10.17- $\mu \mathrm{m}$ and 16.68- to 19.67- $\mu \mathrm{m}$ bins are very similar. Data from the 27.37 - to $32.28-\mu \mathrm{m}$ bin illustrate how the data sets are different as the droplet size continues to increase. On average, a small gradient in the vertical direction is evident when the concentration has reached an equilibrium value, where $\Phi_{32.28}(\mathrm{~L})>\Phi_{32.28}(\mathrm{M})>\Phi_{32.28}(\mathrm{H})$. This gradient becomes more pronounced at larger particle sizes, and because the total volume concentration is dominated by larger droplets, $\Phi_{c, \mathrm{~N}}(\mathrm{~L})>\Phi_{\mathrm{c}, \mathrm{N}}(\mathrm{M})>\Phi_{\mathrm{c}, \mathrm{N}}(\mathrm{H})$. For example, in the tests shown in Figure 8.4, the cumulative concentrations for droplets that are $<102.5 \mu \mathrm{m}$ are approximately 1.5 (low location), 0.6 (medium location), and 0.4 (high location) ppmv, respectively.

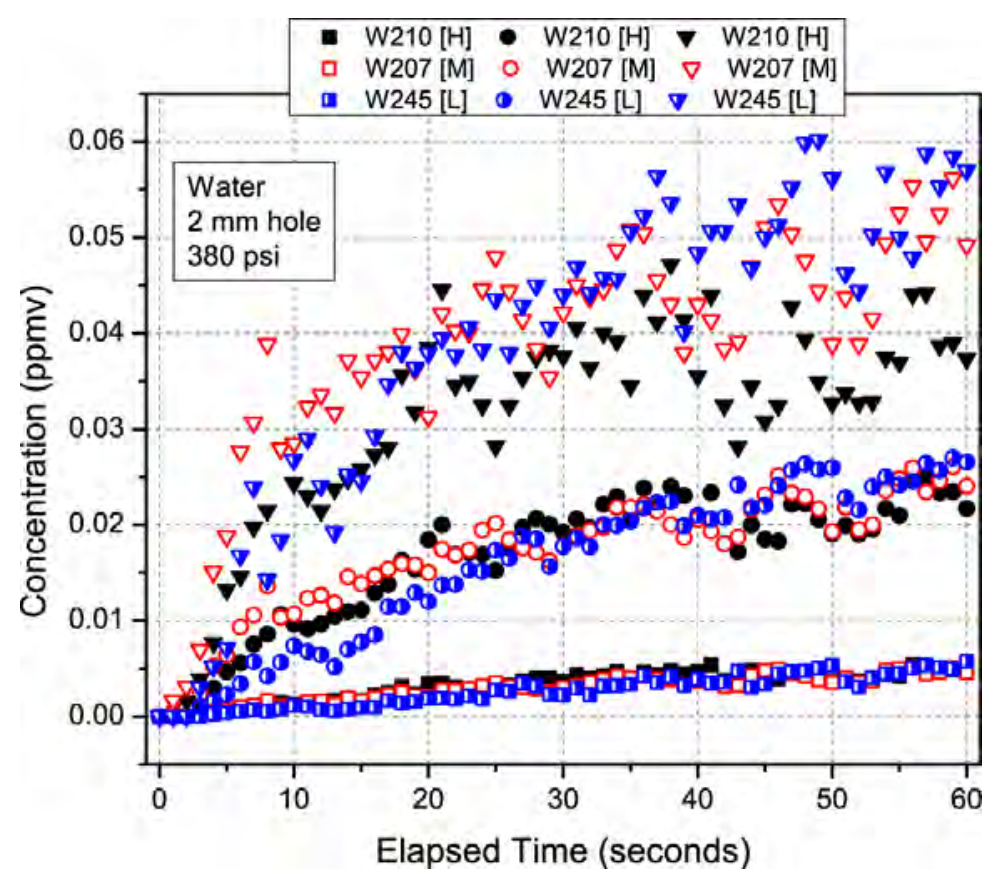

Figure 8.4. Aerosol Concentration and Instrument Location. Aerosol concentration as a function of vertical location for three discrete size bins: 8.62 to $10.17 \mu \mathrm{m}$ (squares), 16.68 to $19.67 \mu \mathrm{m}$ (circles), and 27.37 to $32.28 \mu \mathrm{m}$ (triangles). Only a single test at each location (high [H], medium [M], and low [L]) is shown for the first $60 \mathrm{~s}$ of the spray.

Data from the vertical location tests were analyzed using the methods described in Section 7.2.

Because multiple tests were performed at each vertical location, the release fractions were averaged. The average release fractions are presented in Figure 8.5. Despite the differences in concentrations of larger droplets, in the region of interest between 10 and $100 \mu \mathrm{m}$, the impact on the release fraction is small. The variation between the locations is within the variability observed in repeat experiments (see

Section 8.1.6). Significant divergence between the locations does not occur until roughly $80 \mu \mathrm{m}$. This can be explained upon re-examination of Figure 8.4. Even though data from the low location reaches a slightly larger equilibrium concentration, the data from the medium location increases faster, leading to a slightly greater release fraction. It can be surmised from these tests that the aerosol concentration at the low location is not appreciably amplified by settling until the droplets are $80 \mu \mathrm{m}$ or larger. The results from these tests provide confidence that measurements taken at the medium location are representative of 
the chamber aerosol concentration, especially for droplets $<80 \mu \mathrm{m}$ in diameter, and will not underestimate the release fraction relative to the other measurement locations in the vertical plane.

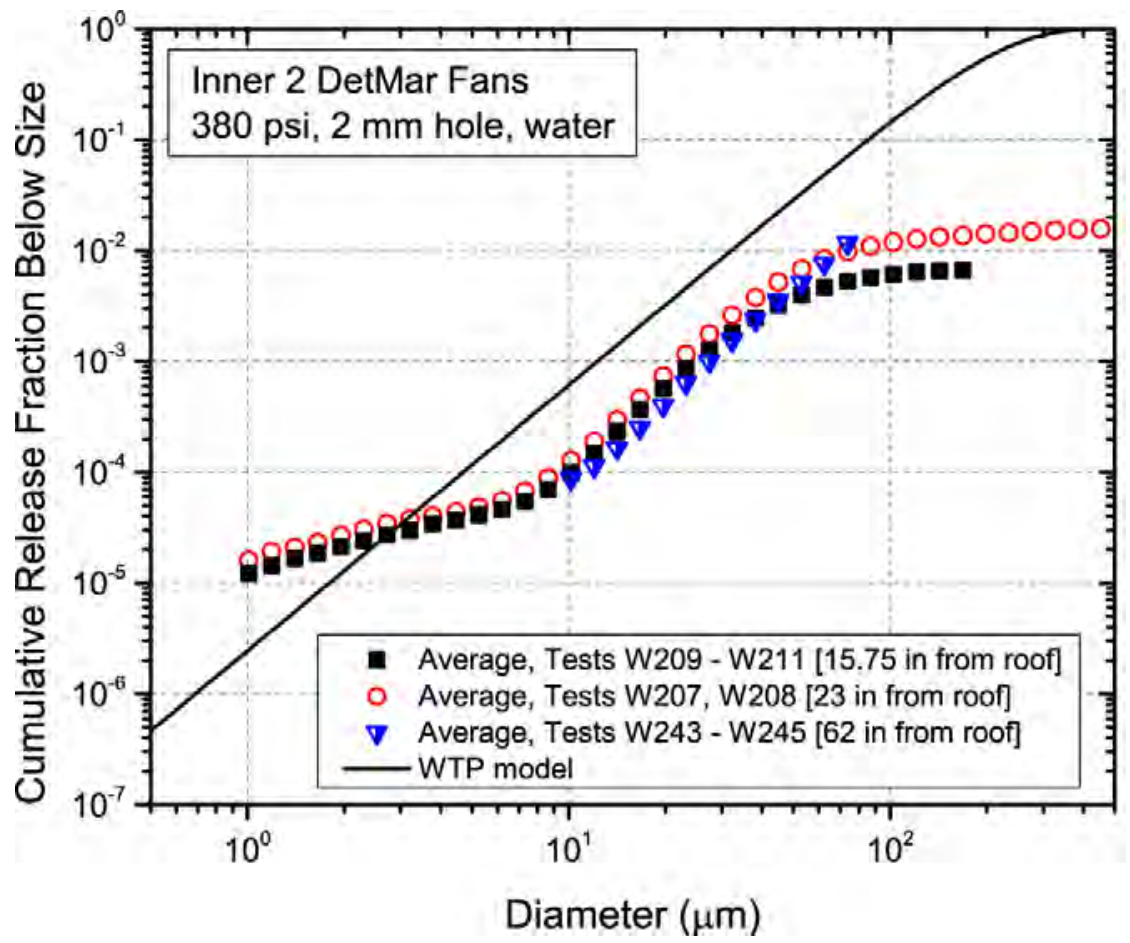

Figure 8.5. Average Release Fraction and Aerosol Instrument Location. Release fraction is estimated from the cumulative concentrations of tests conducted at three vertical locations in the chamber: high (black squares), medium (red circles), low (blue triangles). The WTP model prediction is provided for reference. The uncertainty bars have been removed from the data to improve clarity. Refer to Appendix B for the release fractions with uncertainty bars and the results of the differential concentration calculation.

\subsubsection{Optimization of PPC Vacuum Flow Rate}

The concentration ranges observed in the large-scale tests, especially for tests with smaller orifices (e.g., S1A, S2A) and/or smaller pressures (primarily 100 psig), were at or near the sensitivity limit of the Malvern instruments. This was identified as a potential limitation of the experimental system before testing began. This was the motivation for acquiring and installing a secondary (backup) aerosol instrument, the PPC, which has a narrower particle size measurement range and operates on a slightly different principle than the Malvern instrument. For a measurement to be made, a vacuum pump pulls chamber contents through the PPC. It was expected that the choice of vacuum pump flow rate would affect the results. Two tests (W70 and W72) were conducted to check this assertion.

During these tests, which were performed using 2-mm (W70) and 4.46-mm (W72) orifices, water was sprayed for $8 \mathrm{~min}$ at $380 \mathrm{psi}$. During each 2-min increment, the vacuum pump flow rate was increased, using $0.5,1.0,1.5$ and $1.8 \mathrm{scfm}$ (the maximum flow rate possible). The cumulative concentrations measured during test W70 are shown in Figure 8.6. Data from test W72 were very similar and are not 
shown. The vacuum pump rate has a clear effect. A flow rate of $0.5 \mathrm{scfm}$ is not strong enough to obtain an equilibrium concentration across the droplet sizes, even after $120 \mathrm{~s}$. Typically, concentrations were observed to achieve an equilibrium value within 30 to 60 s, depending on the orifice size (e.g., see Figure 7.5 or Figure 7.6). The concentrations were not steady until a flow rate of $1.5 \mathrm{scfm}$ was used. There was also concern that higher vacuum pump flow rates biased the measurement for the smaller particles, as evidenced by the increase in the cumulative concentration of small droplets relative to the larger ones in Figure 8.6. Based on these considerations, $1.5 \mathrm{scfm}$ was selected as the working vacuum pump flow rate for all aerosol testing. It is important to note that this was only "optimized" for these two tests and identical behavior may not be achieved for other orifices (in particular, orifices with significantly larger area).

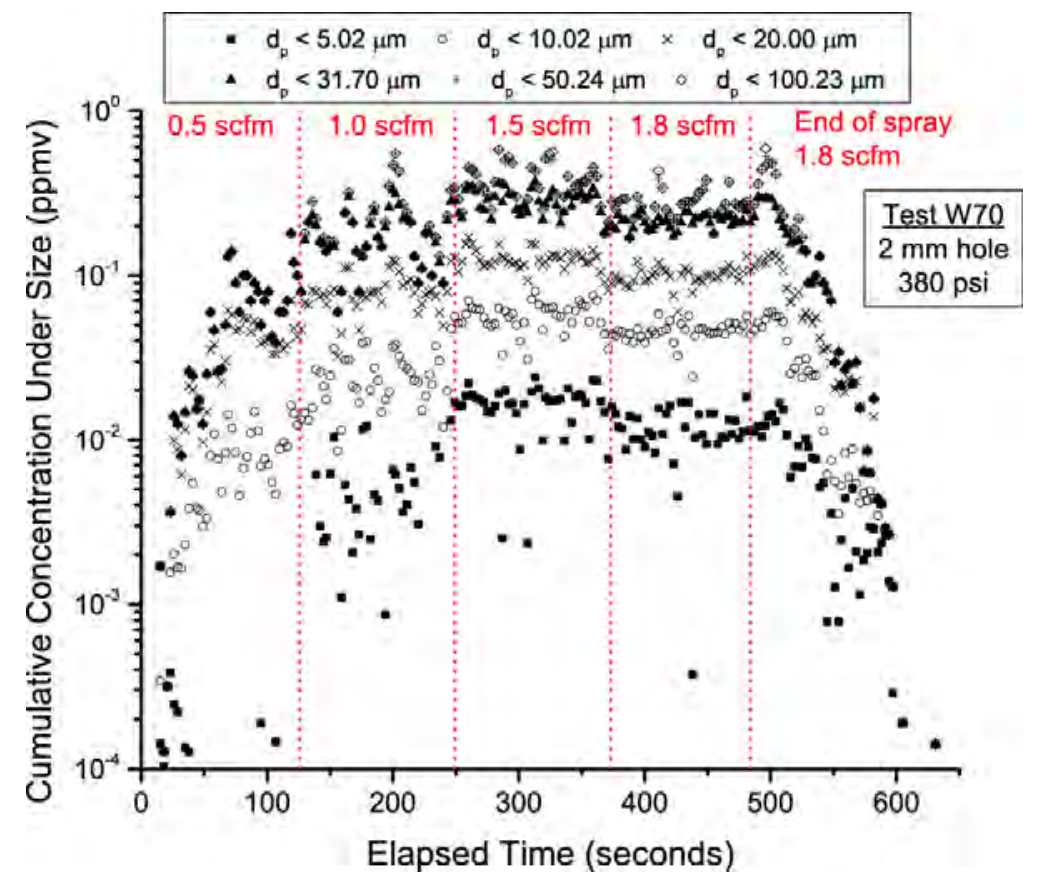

Figure 8.6. PPC Concentration and Flow Rate. Cumulative concentrations measured by the PPC during test W70 (2-mm orifice, water, 380 psi) as a function of vacuum pump flow rate (shown in red). Each period is delineated by the red dotted lines.

\subsubsection{Comparison of Insitec-S and PPC Measurement Data}

For the majority of large-scale aerosol tests, aerosol size and concentration measurements were taken using both Malvern Insitec-S and PPC analyzers. The Insitec-S was quickly identified as the primary source of aerosol measurement data for several reasons, including but not limited to:

- The Insitec-S instruments could be configured to provide measurement of aerosol concentration for the vast majority of orifice and pressure conditions through the use of $500-\mathrm{mm}$ spacer bars and appropriate selection instrument software detection thresholds.

- Installation of three Insitec-S analyzers provided large-scale chamber aerosol concentration profiling and increased measurement confidence by having three independent measurements of chamber concentration that could be averaged. 
- Direct installation of the Insitec-S analyzers in the large-scale test chamber eliminated the need to optimize sampling subsystems, whereas the PPC analyzer installation required a vacuum pump with a rate of suction that would have to be optimized for specific test conditions to ensure representative sampling of the aerosol. Such optimization would have been time consuming, because a single optimized vacuum flow rate may not have held for all tests.

- The measurable size range of droplets provided by the Insitec-S analyzer was larger than that provided by the PPC.

- The rate of aerosol data acquisition provided by the Insitec-S was faster than that provided by the PPC.

Despite the advantages of the Insitec-S relative to the PPC, the PPC was still employed to collect secondary aerosol data to provide increased confidence in the Insitec-S results, especially given the concerns that measurement of aerosol concentration is not a typical use of the Insitec-S analyzer. As such, the PPC data collected for select tests have been analyzed and compared to the corresponding results collected by the Insitec-S analyzer to assess Insitec-S performance with respect to actual spray concentration and size measurements.

While aerosol data collected by the PPC provides a relatively direct way to assess performance of the Insitec-S analyzer, it must be noted that several factors prevent direct side-to-side comparison. These include 1) different nominal size ranges for the Insitec-S $(0.5$ to $200 \mu \mathrm{m})$ and the PPC (0.5 to $67 \mu \mathrm{m})$, 2) different sampling methods (open frame versus draw tube), 3) different baseline values for refractive index, and 4) different sampling volumes for the Insitec-S $\left(\sim 10 \mathrm{~cm}^{3}\right)$ and the PPC $\left(10^{-4} \mathrm{~cm}^{3}\right)$.

The difference in Insitec-S and PPC sampling volumes will lead to different count statistics for both instruments. In particular, the PPC may struggle to achieve sufficient counts for a statistically significant result if results are considered on a point-by-point basis. To avoid this (and to avoid errors associated with comparing measurement over different measurement averaging periods), Malvern Insitec-S and PPC comparisons are made by averaging data for both instruments over the same 1-min period of aerosol generation. In addition, to avoid incorporation of transient aerosol concentrations that may bias the concentration results, only the last minute of the total two-min period of spray generation will be incorporated into the one-min average.

Differences in the nominal size range detected by the instruments will yield differences in the cumulative size distribution and the total aerosol concentration reported by the instruments. For example, the Insitec-S will (and does) observe particle sizes above the upper limit detected by the PPC (67 $\mu \mathrm{m}$ ). Since the volume contribution of these particles is included in the total aerosol concentration, the aerosol concentration reported by the Insitec-S must be larger than that reported by the PPC. In addition, the presence of larger particles in the Insitec-S size distributions also prevents direct comparisons of the normalized (fractional) PSDs reported by both instruments. For these reasons, total aerosol concentrations and PSDs reported by the instruments may not be directly compared. Instead, the concentration of particles at specific sizes that fall within the measurable size ranges of both instruments must form the basis of Insitec-S and PPC comparisons. For the current analysis, the cumulative volume concentration undersize as a function of droplet diameter are compared for both instruments, with comparisons being limited to a size range of $\sim 1$ to $70 \mu \mathrm{m}$.

The PPC uses a "hard-wired" refractive index of $1.5+0.5 \mathrm{i}$ (for a standard opaque particle) for all measurements. This value cannot be easily changed in the software that controls PPC operations and data 
analysis. To overcome this difficulty, all Insitec-S data were reanalyzed and averaged using a refractive index of $1.5+0.5 \mathrm{i}$, eliminating any differences that could result from the assumed particle refractive index.

The final source of error when comparing Insitec-S and PPC results introduced in the preceding paragraphs is the difference in instrument sampling methods. Of the two instruments, the Insitec-S open frame provides the most direct (and unobtrusive) method for sampling the aerosol in the large- and small-scale test chambers. The PPC, which sits outside the test chamber, requires use of a draw tube and vacuum to continuously pass aerosol through the PPC measuring volume. Use of a draw tube introduces the possibility of unrepresentative (non-isokinetic) sampling and aerosol deposition on the inner diameter of the draw tube before it reaches the measuring volume. It is expected that use of the draw tube further reduces the total aerosol concentration measured by the PPC and may strip large particles (through inertial deposition) from the aerosol stream prior to reaching the PPC measuring volume, which may reduce or eliminate the volume contribution of large particles in the PPC size result. No attempt has been made to correct for differences in Insitec-S and PPC size and concentration results introduced by sampling. With this in mind, comparison of the Insitec-S and PPC results should be approached with caution.

For comparison of the two instruments, only a subset of tests was examined. In total, only five large-scale chamber aerosol tests were evaluated using the 1-min averaging approach outlined in the preceding paragraphs. These five tests were selected because they produced what were considered reliable results on the Insitec-S measuring system (i.e., reasonable transmission loss relative to background and no saturation of the detector). These tests were W75, W76, W80, W84, and W91. It is important to note that all five reference tests were conducted with water. These tests were selected, as the PPC vacuum flow rate had been optimized shortly before conducting these tests (see Section 8.1.4). For all of these tests, the PPC was located near the Insitec-S located in position 2. The presence of a vacuum flow did not appear to influence the aerosol measured by the Insitec-S; this is different than what was observed in the small-scale system (Mahoney et al. (2012b).

Figure 8.7 shows a comparison of cumulative concentration measurements for tests W75, W79, W80, W84, and W91. As expected, all tests except W80 indicate divergence in measured volume concentration at droplet diameters that approach the maximum size range of the PPC. This divergence may be related to loss or deposition of large particles in the PPC draw tube and/or from poor sampling statistics for large particles. Agreement between Insitec-S and PPC concentration measurements improve at smaller droplet diameters. In particular, the agreement between aerosol instruments is excellent (i.e., the PPC and Insitec-S readings agree to within a factor of 0.5 to 2 times of each other) for droplet diameters between 10 and $30 \mu \mathrm{m}$. For droplet diameters below $10 \mu \mathrm{m}$, there is increased divergence between aerosol concentration readings for the Insitec-S and PPC, with the Insitec-S reading being high relative to that of the PPC.

For the majority of results presented in Figure 8.7, the Insitec-S concentration measurements are equal to or higher than those obtained using the PPC. Exception to this observation typically occurs for droplet diameters from 10 to $30 \mu \mathrm{m}$, where the PPC concentration is slightly higher than that produced by the Insitec-S. It should be noted that this trend is opposite of that found in small-scale comparisons of Insitec-S and PPC performance, where the PPC was found to typically produce much higher aerosol concentrations than those determined on the Insitec-S. Differences in large- and small-scale comparison results are expected, as both setups used different lengths of draw tubing for sampling, employed different 
PPC vacuum rates (1 scfm for small scale; 1.5 scfm for large scale), and had drastically different chamber volumes from which aerosol was sampled. In addition, small-scale PPC measurements were conducted without optimizing the vacuum flow rate to the PPC. As such, it is difficult to directly compare side-by-side PPC and Insitec-S concentration measurement results for the two test scales. 


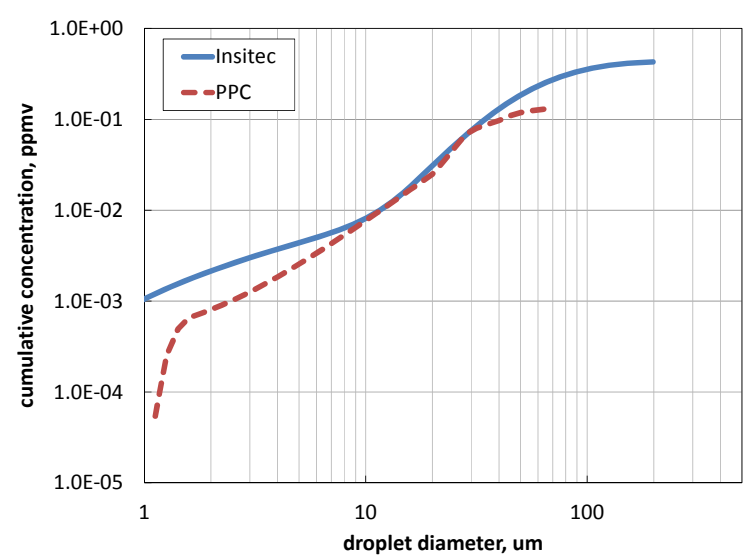

A - Test W75 (2-mm round, 200 psig)

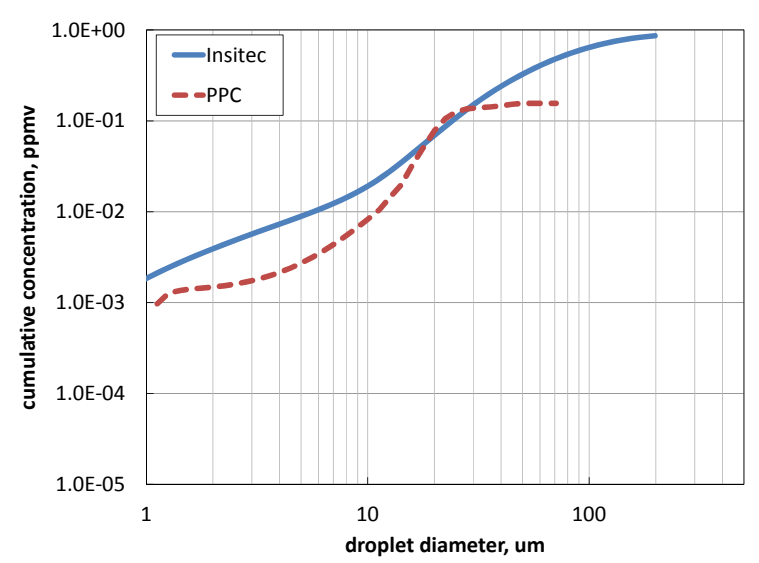

C - Test W76 (4.5-mm round, 380 psig)

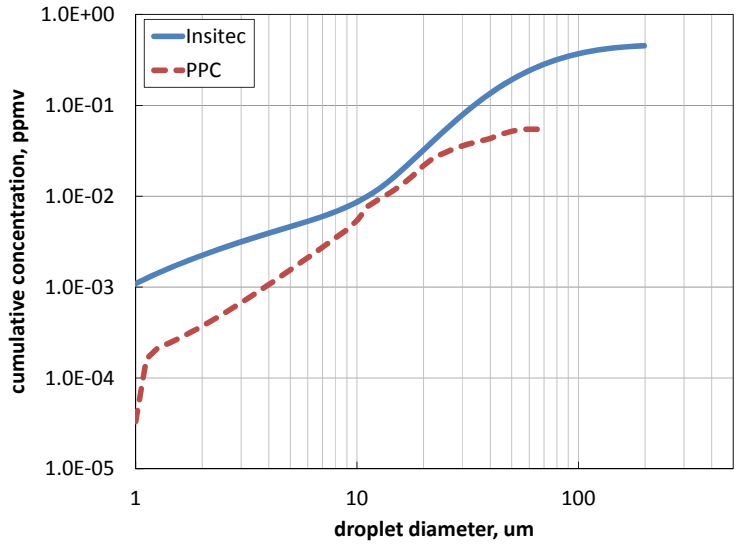

B - Test W84 (2-mm round, 200 psig)

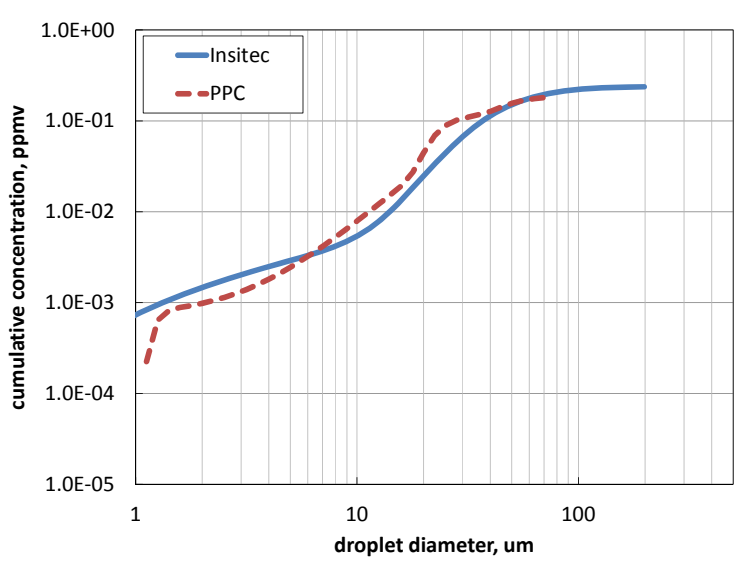

D - Test W80 (2-mm round, 200 psig)

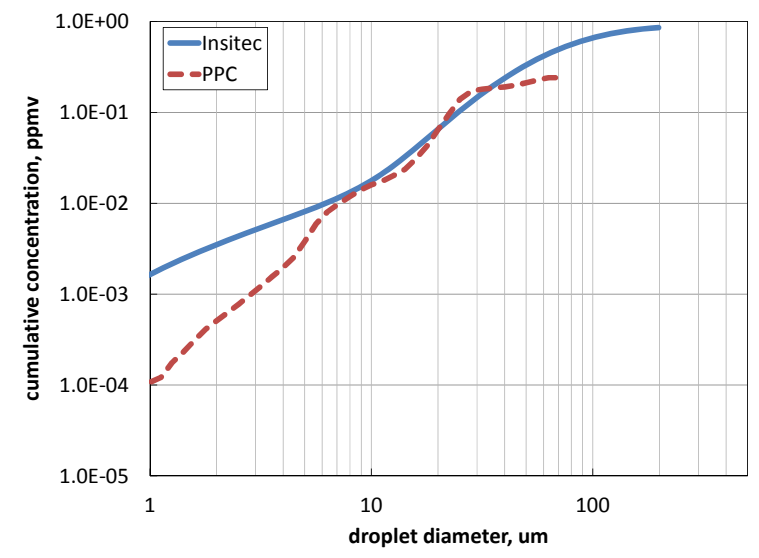

E - Test W91 (1×10-mm slot, 380)

Figure 8.7. Comparison of Insitec-S (solid line) and Normalized PPC (dashed line) Cumulative Volume Fraction Undersize Distributions for Five Large-Scale Spray Tests. The test number, orifice size and morphology, and test pressure associated with each result is listed below its corresponding figure. 
Based on Figure 8.7, the large-scale results appear to indicate reasonable agreement between Insitec-S and PPC aerosol concentration measurements. To further demonstrate this agreement, the cumulative undersize aerosol concentrations measured by Insitec-S and PPC were compared at a single diameter. For a given test, a single diameter was selected for comparison. The diameter selected was the d[50] droplet diameter measured by the PPC. This diameter was selected because it represents an approximate midpoint measurement of size for both systems, and as such, falls within the nominal operating region of the $5^{\text {th }}$ to $95^{\text {th }}$ cumulative volume percentiles of both instruments (i.e., where both instruments are expected to be reasonably accurate). Table 8.3 shows the results of this comparison for all five test systems considered. For three of the five tests, the d[50] shows excellent agreement between PPC (relative to the expected $\sim 30 \%$ difference expected for repeat Insitec-S measurements). For two tests, W80 and W84, the Insitec-S and PPC do not compare well. For test W84, the Insitec-S reads too high for the PPC, whereas for test W80, the Insitec-S reads too low for the PPC. However, for all cases examined, the Insitec-S and PPC readings agree to within a factor of two.

Table 8.3. Comparison of Total Aerosol Concentration Measurements by the Malvern Insitec-S and PPC

\begin{tabular}{|c|c|c|c|c|c|c|c|}
\hline $\begin{array}{c}\text { Test } \\
\text { ID } \\
\end{array}$ & Orifice & $\begin{array}{c}\text { Pressure } \\
\text { [psig] }\end{array}$ & Fluid & $\begin{array}{c}\mathrm{PPC} \mathrm{d}_{50} \\
{[\mu \mathrm{m}]}\end{array}$ & $\begin{array}{c}\mathrm{PPC} c_{v} \text { at } \\
\mathrm{PPC} \mathrm{d} \mathrm{d}_{50} \\
{[\mathrm{ppmv}]}\end{array}$ & $\begin{array}{c}\text { Insitec-S } c_{v} \text { at } \\
\text { PPC } d_{50} \\
{[p p m v]}\end{array}$ & Ratio $^{(a)}$ \\
\hline $\bar{W} 80$ & 2-mm round & 200 & Water & 25.6 & 0.091 & 0.047 & 1.92 \\
\hline W75 & 4.5-mm round & 200 & Water & 27.9 & 0.066 & 0.064 & 1.02 \\
\hline W84 & 4.5-mm round & 200 & Water & 22.8 & 0.027 & 0.044 & 0.63 \\
\hline W76 & 4.5-mm round & 380 & Water & 20.0 & 0.078 & 0.070 & 1.12 \\
\hline W91 & $1 \times 10-\mathrm{mm}$ slot & 380 & Water & 24.0 & 0.121 & 0.096 & 1.26 \\
\hline
\end{tabular}

Overall, comparison of Insitec-S and PPC performance in large-scale testing appears to indicate agreement between the instruments to within a factor of two. In many cases, the instruments appear to agree within the range of reproducibility for the Insitec-S ( 30\%) as determined by replicate testing. This result should be approached with caution, as there are limits to the instrument comparisons made in this section. First, comparison only applies to the common size measuring ranges for the Insitec-S and PPC ( 0.5 to $67 \mu \mathrm{m}$ ), and even within this range, the concentration results appear to diverge for certain cases at diameters $<10 \mu \mathrm{m}$ and $>30 \mu \mathrm{m}$. In addition, the comparisons only examine a small subset of all tests. Comparison of PPC and Insitec-S performance for test cases that challenge either instrument (such as sprays generated through small orifices and at low pressure) may result in much poorer agreement.

\subsubsection{Repeatability of Testing}

To assess the test-to-test variation in the large-scale spray release system, a series of repeated tests (W61 to W69) were conducted with water at 380 psig using a 1-mm orifice. In the series, triplicate experiments were performed with a single Malvern instrument (M1852) in each of the three standard positions indicated in Table 5.2. These tests were analyzed, and the resulting release fractions were compared; an example is shown in Figure 8.8 for position 2. The release fractions obtained from the three tests are nearly indistinguishable from one another, thus giving confidence that tests yield reproducible results. Agreement in results also is excellent between the cumulative method and differential method 
when the droplet size is less than about $80 \mu \mathrm{m}$; above this droplet size, the release fractions were rejected because they failed to meet the $\overline{\mathrm{R}}^{2}$ criterion. The results from the other two positions can be found in Appendix B, Figure B.1 and Figure B.2, and the agreement for those triplicate experiments is comparable to the example in Figure 8.8. The small differences in observed release fraction with position as measured by Malvern instrument M1852 for this suite of tests also confirms the previously mentioned concentration gradient behavior among Malvern instruments in locations 1, 2, and 3.
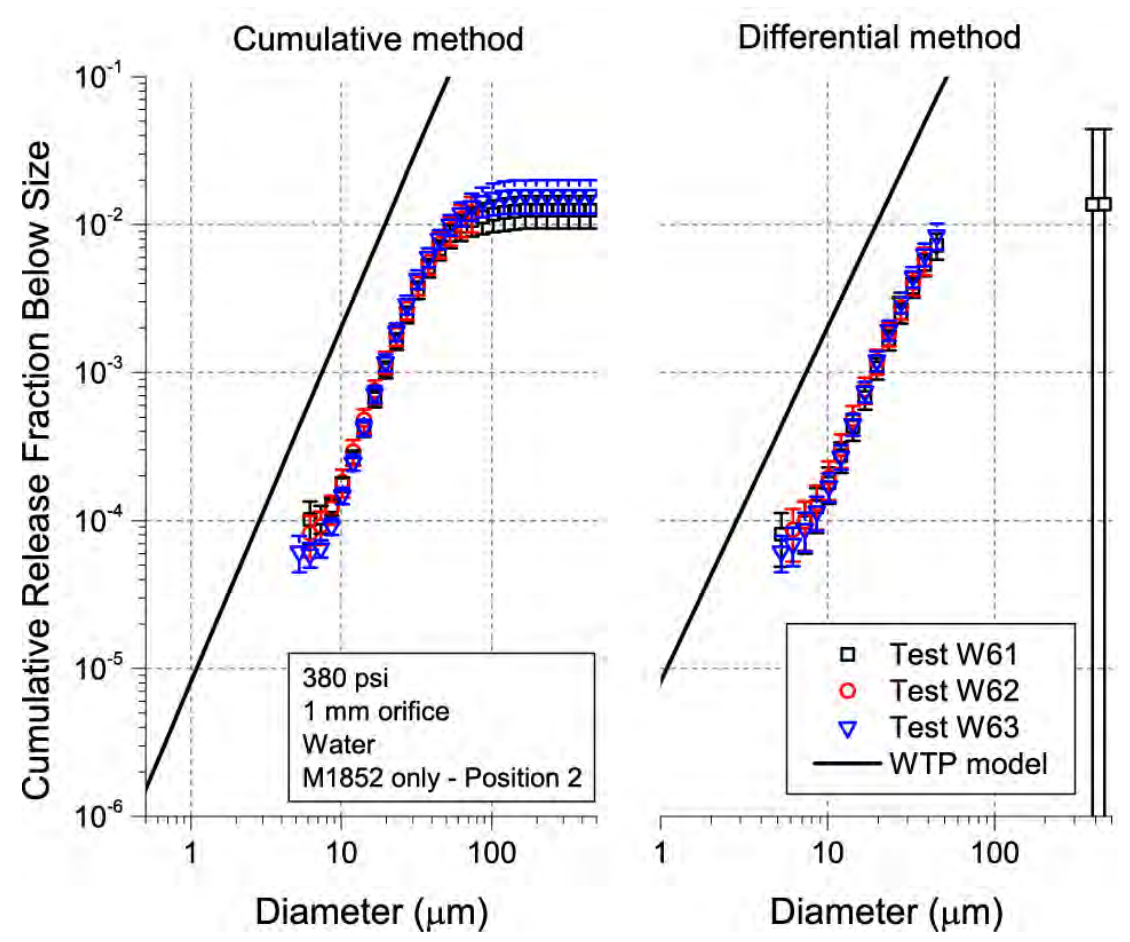

Figure 8.8. Experimental Repeatability. Release fractions determined from triplicate experiments using a single Malvern instrument (M1852) in position 2. The tests were conducted consecutively with water at 380 psig using a 1-mm orifice.

The Malvern instrument (M1852) used to assess repeatability in tests W61 to W69 was equipped with the 15-cm spacer bars. To that point, M1852 had been the most consistent performer of the three Malvern instruments, but 15-cm spacer bars were not used in the aerosol tests reported in subsequent sections. Malvern instrument 1855, which already had 50-cm spacer bars installed, also was present for six of the tests in position 1 (W61 to W66). These six tests, when used to estimate release fraction, exhibited a tight distribution of release fractions (see Appendix B, Figure B.3). Because the configuration of M1855 was more representative of the final configuration of the instruments, and there are six tests instead of three, it is the best data set to use to estimate the uncertainty due to random variability between tests.

An estimate of the 95 percent confidence level based on data from tests W61 to W66 is presented in Figure 8.9. The release fraction distribution in the figure is the average of the six tests. The standard deviation of the average was used in conjunction with the appropriate student's t value to estimate the 95-percent confidence level (shaded area), the boundaries of which are indicated by the dotted line. The 
figure inset shows the same information scaled linearly in the region of greatest interest (10 to $100 \mu \mathrm{m}$ ) so a better estimate of the uncertainty can be observed. A reasonable estimate of the 95-percent confidence level is on the order of \pm 15 percent of the mean value at any particular diameter based on this data set.

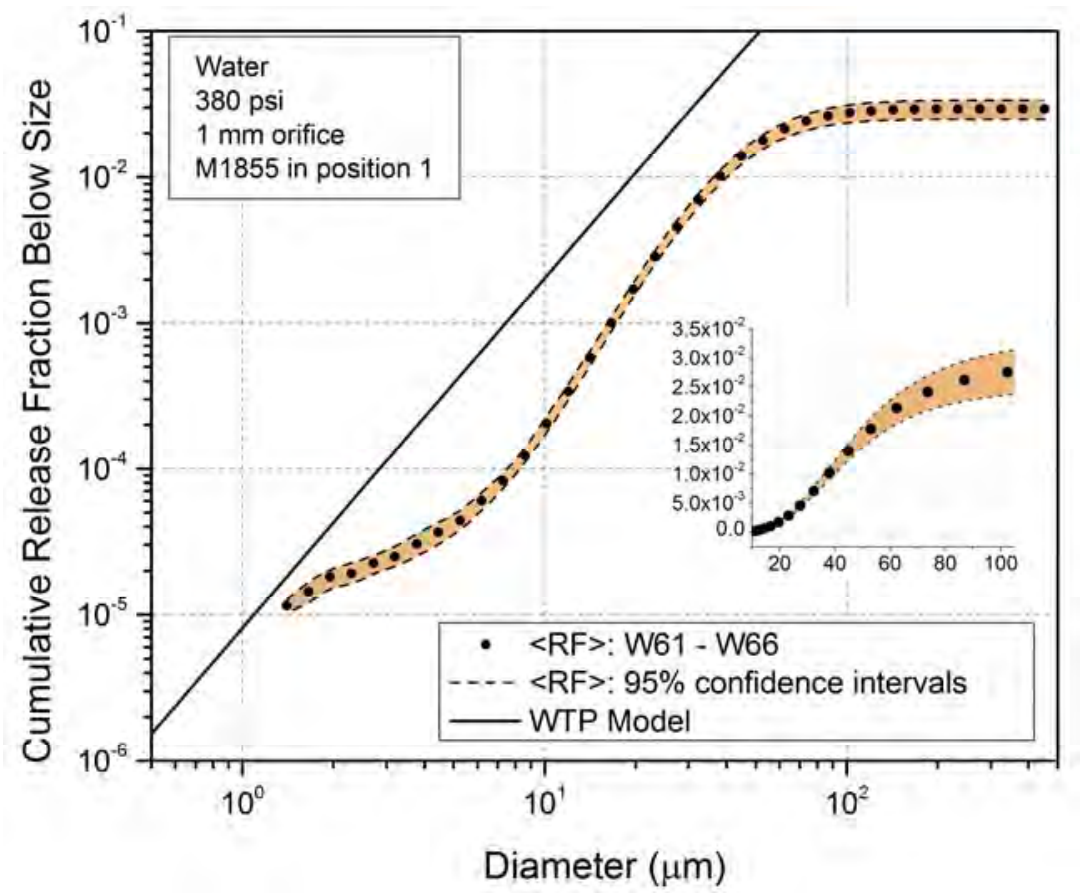

Figure 8.9. Uncertainty in the Cumulative Release Fraction. Estimate of 95-percent confidence levels for large-scale spray release testing was based on six discrete experiments (W61 to W66) conducted using water at $380 \mathrm{psig}$ and a 1-mm orifice. The average release fraction is from Malvern instrument M1855 located in position 1. The region of uncertainty is shaded for visualization purposes. The inset figure is scaled linearly in the region of interest (10 to $100 \mu \mathrm{m}$ ) to provide a clearer order-of-magnitude estimate of the width of the confidence region.

However, even though the repeatability tests provide an estimate of the expected test-to-test variation that is judged to be sound, the estimate is subject to some limitations. The tests were conducted with one of the smallest orifices ( $1 \mathrm{~mm}$ ) and the fluctuation (or noise) in the data generally increases as the orifice size increases. Another stipulation is that there is no error assumed in the calculation of the spray leak flow rate. However, as Figure 8.2 demonstrated (in a global sense), there was some deviation from the expected mean value $\left(C_{D}=0.62\right)$ in this calculation as well. The flow rate calculation was subject to greater uncertainty when the orifice was small or the fluid was not water. In light of these limitations, the majority of the tests were performed in duplicate. Other triplicate tests were found to have 95-percent confidence levels on the order of 25 to 30 percent using the standard deviation of the average release fraction. As a first approximation, the release fraction for any given test should be expected to have a minimum 95-percent confidence level of \pm 30 percent of the stated value. In general, the estimate derived from the repeatability tests (i.e., \pm 30 percent) is slightly larger than the uncertainty estimated from the 
fitting algorithm during analysis (for the tests represented by the average release fraction in Figure 8.9, the 95 -percent confidence level averages to about \pm 18 percent of the stated value). ${ }^{15}$

The uncertainty from test-to-test is primarily a measure of the precision in the experiments. There are other sources of uncertainty more difficult to assess and a rigorous estimation of the overall uncertainty in a calculated release fraction was not performed. Based on the results of the comparison between the Malvern Insitec-S and the PPC presented in Section 8.1.5, the largest difference between the d[50] concentrations was determined to be a factor of two. Thus, the overall uncertainty is estimated to be approximately a factor of two to three in the release fractions discussed in the remaining sections of this report. Note that this overall uncertainty is not applied to any of the data shown in figures; rather, only quantitatively computed uncertainties are depicted. Consequently, the overall uncertainty is likely greater that what is shown by the error bars. Other sources of uncertainty are the accuracy of the Malvern Insitec-S and the measurement of an appropriate average concentration in the aerosol chamber (i.e., obtaining a sample that is representative). A more quantitative study of these sources would likely reduce the overall uncertainty.

\subsection{Water Tests}

A significant portion of the aerosol testing in the large-scale spray release system was conducted using process water. In this section, the effect of various parameters on release fractions and/or generation rates are shown and discussed.

\subsubsection{Effect of Pressure}

As discussed in Section 1.3.2, the SMD is proportional to $U^{-2 / 3}$ when the leading term in Equation (1.1) is much greater than the other terms. The dependence of the SMD on pressure is thus $\mathrm{SMD} \sim \Delta \mathrm{P}^{-1 / 3}$, and based on Equation (1.5), this results in larger release fractions as pressure increases. In the large-scale tests, three target pressures were employed: 100, 200, and 380 psi. The effect of pressure can be observed by comparing the release fractions measured at these three pressures for a constant orifice size. Two examples are shown in Figure 8.10 (a round orifice) and Figure 8.11 (a rectangular orifice). Note that these two figures use target pressures and not actual pressures (though the excellent agreement between the two was already discussed in Section 8.1.1).

Both Figure 8.10 and Figure 8.11 present the cumulative release fractions (determined using the cumulative method) and calculated from water tests at three undersize bins: 1) 10.17, 2) 32.28, and 3) $102.50 \mu \mathrm{m}$. Henceforth, these will be referred to as $<10,<32$, and $<102 \mu \mathrm{m}$ for simplicity. The release fractions, when more than one test was performed, are averages. The error bars on the plots are assigned at the 95-percent confidence level. The estimate of the 95-percent confidence level combines the estimate of experimental variability as described in Section 8.1.6 (i.e., \pm 30 percent of the value) and the 95-percent confidence intervals from the data analysis method used to calculate the generation rate (see Section 7.2), resulting in greater uncertainties for experimental data sets that were noisier or had data closer to the

\footnotetext{
${ }^{15}$ For both estimates of uncertainty, the 95-percent confidence levels are appreciably smaller for droplet sizes between $\sim 10$ and $\sim 50 \mu \mathrm{m}$. The release fractions for tests W61 to W66 have 95-percent confidence levels of approximately \pm 11 percent (based on the standard deviation of the repeatability tests) and \pm 8 percent (based on the uncertainty estimates from the fitting algorithm) in this range. Narrower confidence intervals in this droplet size range were commonly observed for almost all tests.
} 
instrument detection limits. For other parametric plots in this and later sections, the same approach is used to present the results.

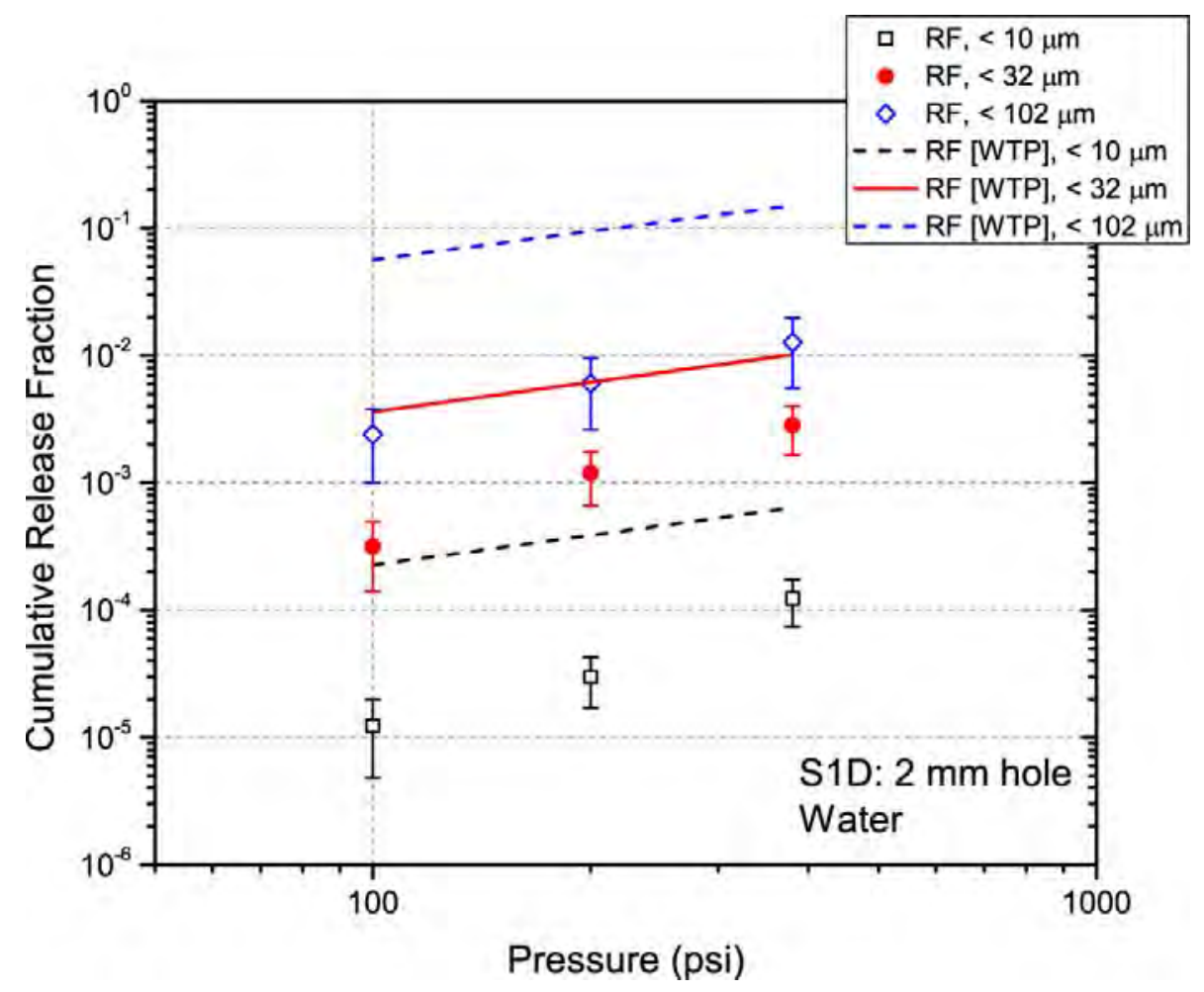

Figure 8.10. Effect of Pressure on Cumulative Release Fractions. Cumulative release fractions vs. target pressure at three droplet sizes $(<10,32$, and $102 \mu \mathrm{m})$ for tests conducted with water using a 2-mm orifice. The error bars show an estimate of the 95-percent confidence level based on the fitting confidence interval and the uncertainty due to test-to-test repeatability. The WTP model prediction is shown by the dotted lines.

Figure 8.10 and Figure 8.11 demonstrate that the release fraction measured in the large-scale system increases with increasing pressure. The release fractions obtained from round (2-mm orifice) and rectangular $(1 \times 10$-mm orifice) geometries are qualitatively similar, both in magnitude and in trend. The WTP model prediction for the three droplet bins also is shown for reference on the figures, and its results agree with the trend in the data sets. However, the experimental data increases faster with pressure than the WTP model, particularly in the smallest droplet sizes $(<10 \mu \mathrm{m})$. This behavior with pressure is representative of what was observed for all the orifices that were tested at multiple pressures. 


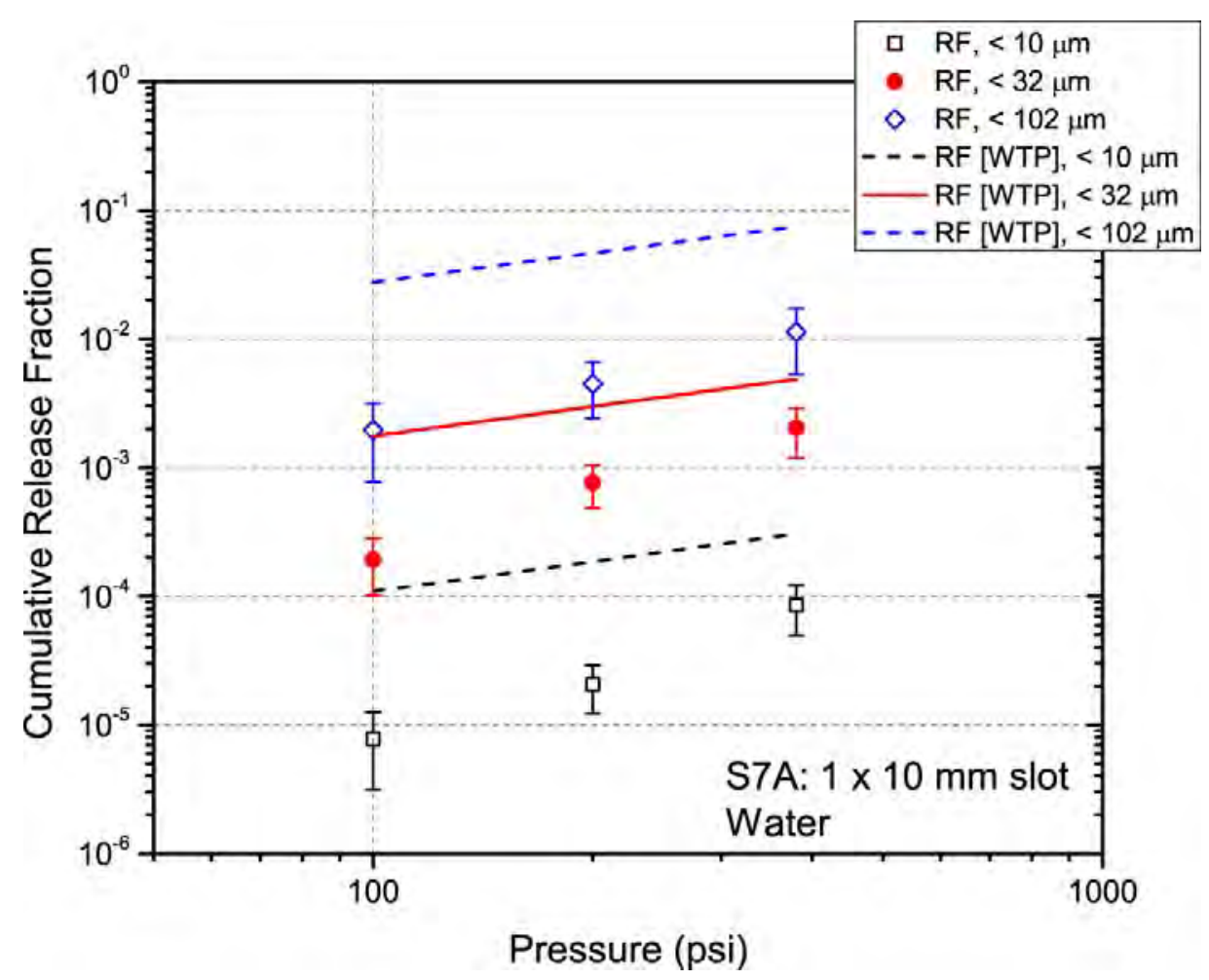

Figure 8.11. Effect of Pressure on Cumulative Release Fractions. Cumulative release fractions vs. target pressure at three droplet sizes $(<10,32$, and $102 \mu \mathrm{m})$ for tests conducted with water using a $1 \times 10$-mm orifice. The error bars show an estimate of the 95-percent confidence level based on the fitting confidence interval and the uncertainty due to test-to-test repeatability. The WTP model prediction is shown by the dotted lines.

\subsubsection{Effect of Orifice Geometry}

The geometry of a leak is not explicitly accounted for in the WTP model because the cross-sectional area of the orifice (via the $K$ parameter in Equation (1.4)) specifies the release fraction. To test this assumption, orifices of similar cross-sectional area but different orientations in the spool piece were tested at the same conditions. The orifices were approximately $10 \mathrm{~mm}^{2}$, with one orientated axially (as the orifices shown in Figure 5.7) and the other rotated 90 degrees and orientated circumferentially. If an observer was looking at the pipe spool piece from the side, the axially orientated orifice would have target dimensions of $1 \times 10 \mathrm{~mm}$, and the circumferential orientated orifice $10 \times 1 \mathrm{~mm}$.

The results from tests using water at 380 psi for these orifices of different orientation are shown in Figure 8.12. For the majority of the range of droplet sizes measurable by the Malvern instruments, the cumulative release fractions are indistinguishable from each other. The agreement is particularly good in the size region of interest (10 to $100 \mu \mathrm{m}$ ). Based on this comparison and other data obtained at lower pressure, it can be concluded that the orientation of the orifice does not have an appreciable effect on the release fraction. The similarity in the cumulative release fractions implies similarity in cumulative generation rates between the orientations because the discharge coefficients were indistinguishable (both were determined to be 0.64) for the tests shown in Figure 8.12. 


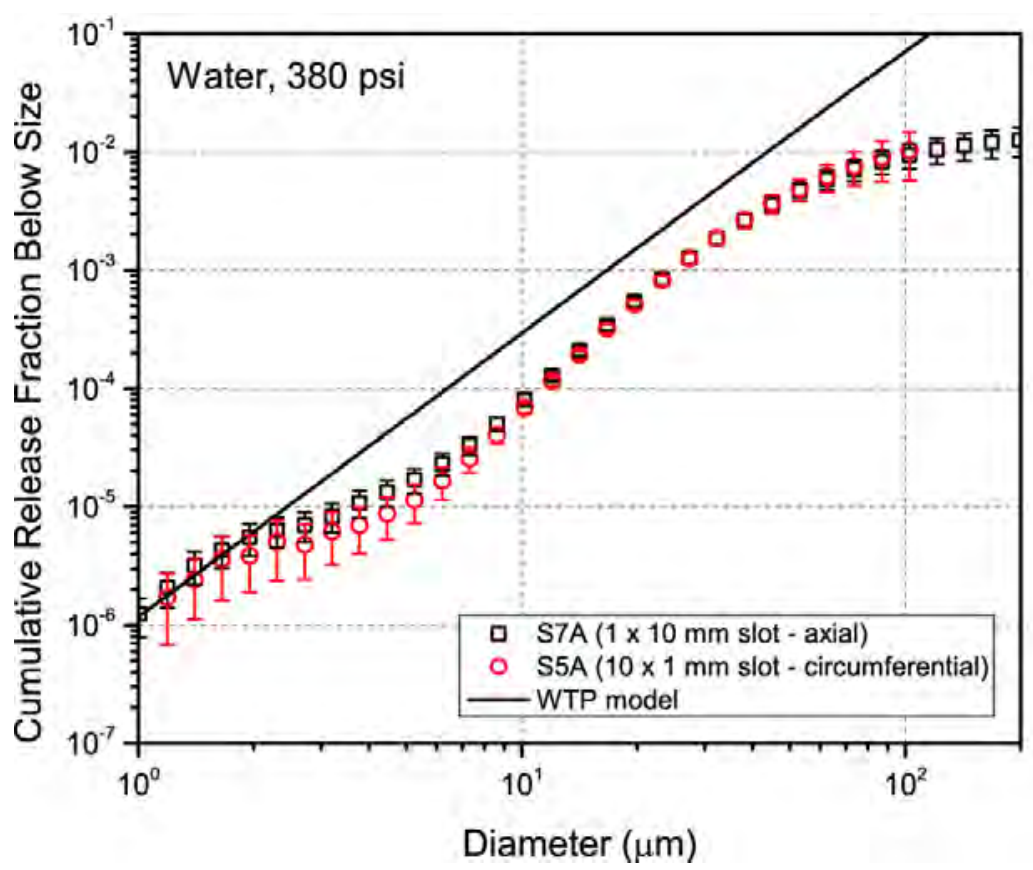

Figure 8.12. Comparison of Slot Orientations. Comparison of release fractions determined for rectangular orifices with similar cross-sectional area and different orientations from a test performed using water at 380 psi. The WTP model prediction is shown by the black line.

A second exploration of the effect of orientation was performed by testing an array of five round orifices $1 \mathrm{~mm}$ in diameter that were axially aligned, with each orifice separated by $1 \mathrm{~mm}$. These tests were conducted in an attempt to determine the impact of a breach with an irregular shape on the release fractions. Actual breaches caused by erosion, corrosion, or equipment failure are not expected to have an engineered shape such as the orifices used in the tests. Rather, they are expected to have irregular edges and varying widths. Testing with more realistic breaches was not conducted because of the challenges associated with fabricating such breaches and then characterizing the dimensions. The use of a test piece with an array of closely spaced orifices is an attempt to mimic an actual breach. The array of 1-mm orifices had a cross-sectional area that was very similar to the $2-\mathrm{mm}$ orifice ( $3.69 \mathrm{vs} .3 .50 \mathrm{~mm}^{2}$ ). The cumulative release fractions measured at $200 \mathrm{psi}$ for the 1-mm and 2-mm orifices and an array of 1-mm orifices are shown in Figure 8.13. As expected, the release fractions of the 2-mm orifice and the array of 1-mm orifices are very similar over the entire droplet size range and are higher than the release fractions measured for the 1-mm orifice. That they are in good agreement suggests that, as the WTP model predicts, only cross-sectional area is important for predicting the release fraction of aerosol. 


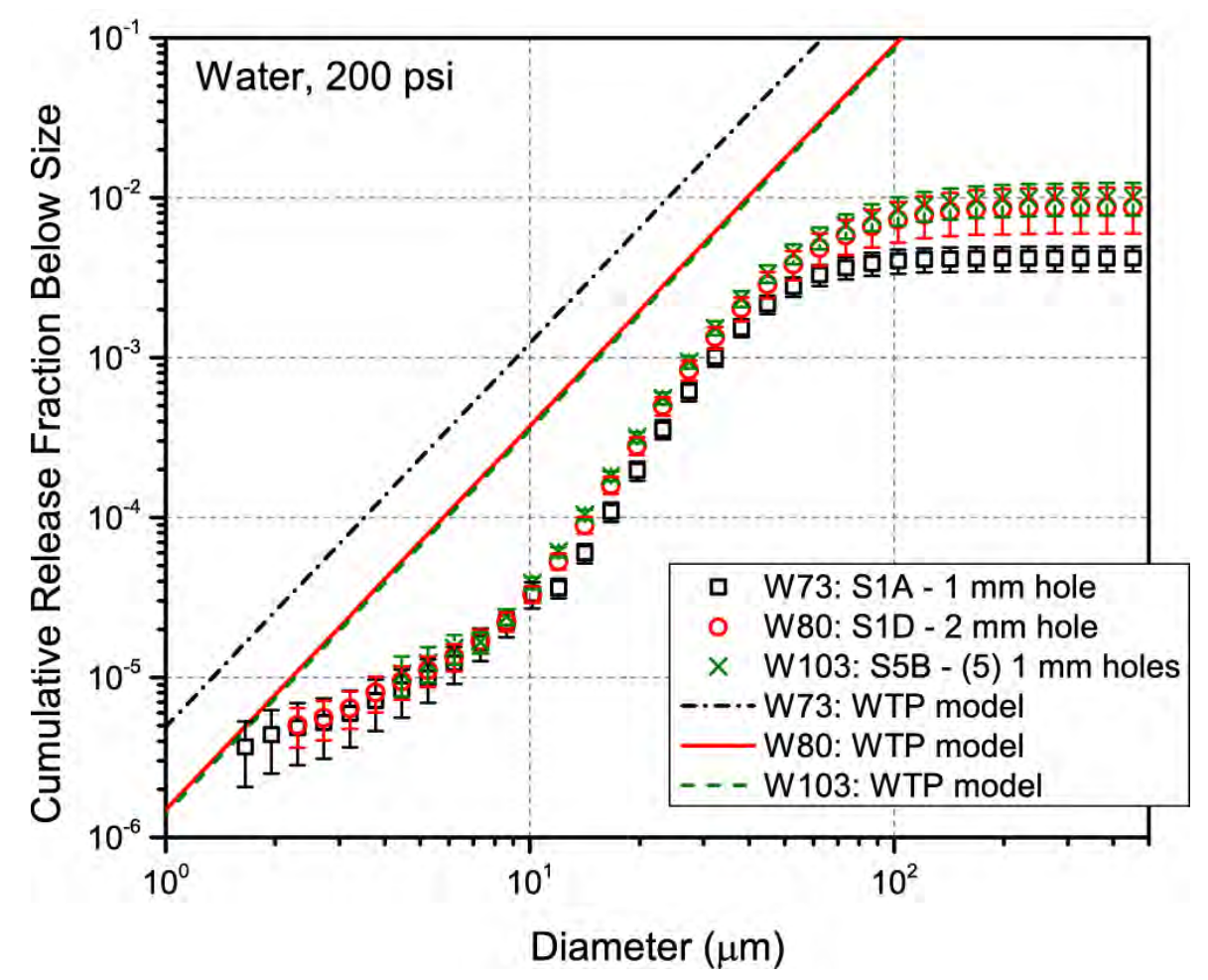

Figure 8.13. Comparison of Release Fractions for Single and Multi-Hole Orifices. Comparison of the release fractions determined for selected round orifices tested using water at 200 psi. The area of orifice S5B (an array of five 1-mm orifices) is approximately equal to the area of orifice S1D (2-mm orifice).

Further parametric studies on the effect of orientation and/or spacing between orifices were not performed, so caution should be exercised when extrapolating this observation to more disparate orifice types. For example, an array of 1-mm orifices separated by much larger distances may have a different behavior. Specifically, the jets emanating from the orifices will have more time to spread out and aerosolize before interacting with each other. At some point, orifices separated by large enough distances would have to be treated as entirely separate spray leak events. The transition between these two extremes, where geometry and orientation of the orifices influence the release fraction of aerosol, was not explored.

\subsubsection{Effect of Orifice Size}

As mentioned in the previous section, the WTP model predicts that the only geometric quantity that affects release fraction is cross-sectional area. To leading order, according to the expressions in Equations (1.1) through (1.3), the SMD $\sim \operatorname{area}^{1 / 3}$ (recall that $\mathrm{SMD} / X$ is a constant); thus, the model states that release fraction decreases with increasing cross-sectional area. The results presented in Section 8.2.2, based on a handful of tests, indicated that the release fraction is related to orifice size strictly through the cross-sectional area. Another way to think about the relationship is to use the generation rate. The dependence of generation rate on area is via the release fraction, which was just discussed, and the flow rate of the spray, which is directly proportional to cross-sectional area (see Equation (1.9)). The 
proportional relationship with flow rate is stronger than the weak functionality of the release fraction on area; therefore, the generation rate should increase with increasing area.

As shown in Figure 8.14, this was observed for all the round orifices tested in the large-scale system. The orifices represented in the figure have target dimensions of $1 \mathrm{~mm}, 2 \mathrm{~mm}, 1 \mathrm{~mm}$ in an array of five, $2.74 \mathrm{~mm}$, and $4.46 \mathrm{~mm}$, respectively, from smallest to largest. The cumulative generation rate is shown for the same undersize bins as before, with average values representing repeated tests, for tests performed at 380 psi. The generation rate predicted by the WTP model is shown for comparison. The experimental generation rates have an appreciably larger slope with area for all three droplet size bins.

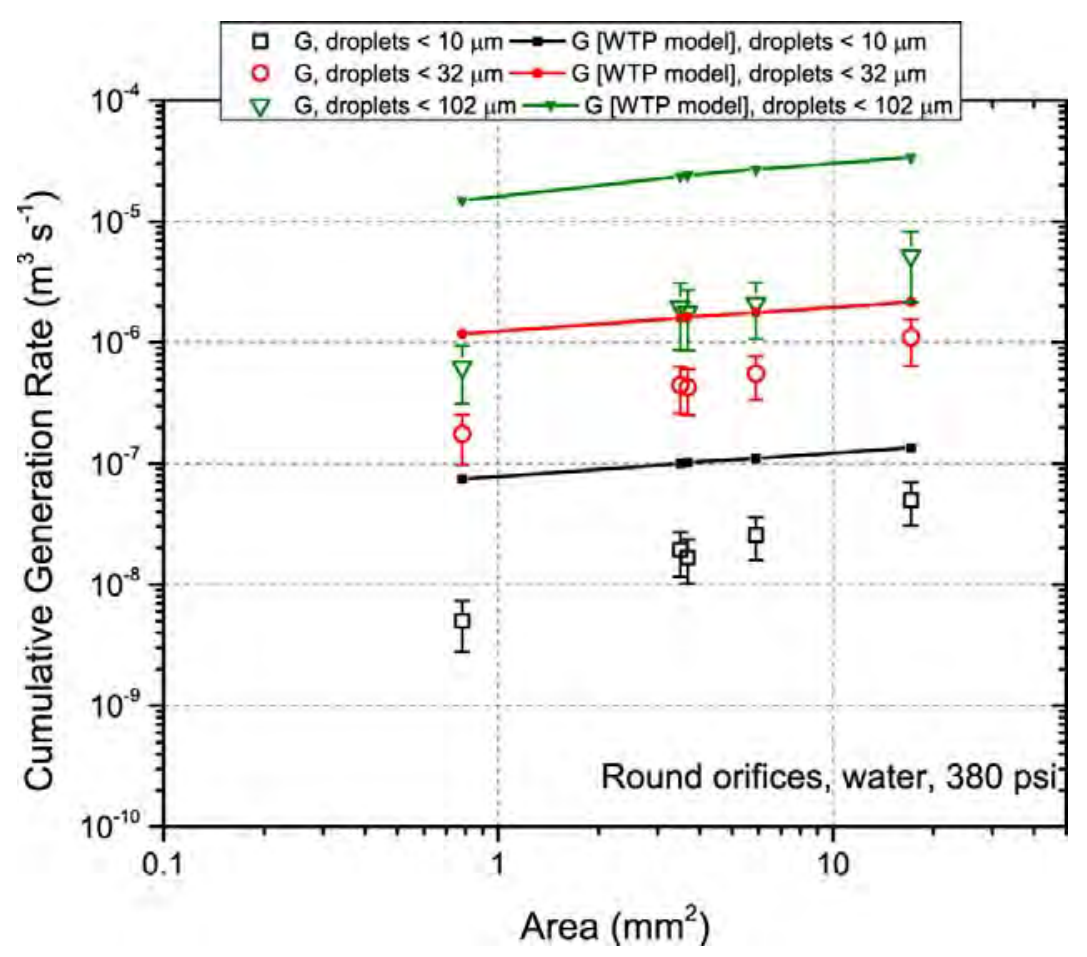

Figure 8.14. Effect of Orifice Area on Generation Rate for Round Orifices. Effect of cross-sectional area of round orifices on the observed cumulative generation rate at three droplet sizes $(<10,32$, and $102 \mu \mathrm{m})$ for tests conducted using water at 380 psi. The error bars show an estimate of the 95-percent confidence level based on the fitting confidence interval and the uncertainty due to test-to-test repeatability. The WTP model prediction is shown by the solid colored lines.

A similar relationship is observed for the rectangular orifices (see Figure 8.15) when cumulative generation rate is plotted as a function of cross-sectional area. The average data are from tests conducted at 380 psi using orifices of the following target dimensions: $0.5 \times 5 \mathrm{~mm}, 1 \times 10 \mathrm{~mm}, 10 \times 1 \mathrm{~mm}$, $2 \times 10 \mathrm{~mm}, 1 \times 20 \mathrm{~mm}, 3 \times 10 \mathrm{~mm}, 1 \times 76.2 \mathrm{~mm}$, and $2.74 \times 76.2 \mathrm{~mm}$. Again, the experimentally observed generation rates increase faster with area than the WTP model. In the case of the rectangular orifices, which explore larger cross-sectional areas than the round orifices, the WTP model is approached at approximately $30 \mathrm{~mm}^{2}$ and exceeded at larger areas. Note that the cumulative generation rate for 
droplets $<102 \mu \mathrm{m}$ could not be estimated for two orifices $(2 \times 10 \mathrm{~mm}, 1 \times 76.2 \mathrm{~mm})$ because of rejected fits $\left(\overline{\mathrm{R}}^{2}<0.5\right)$ for all tests at the stated conditions.

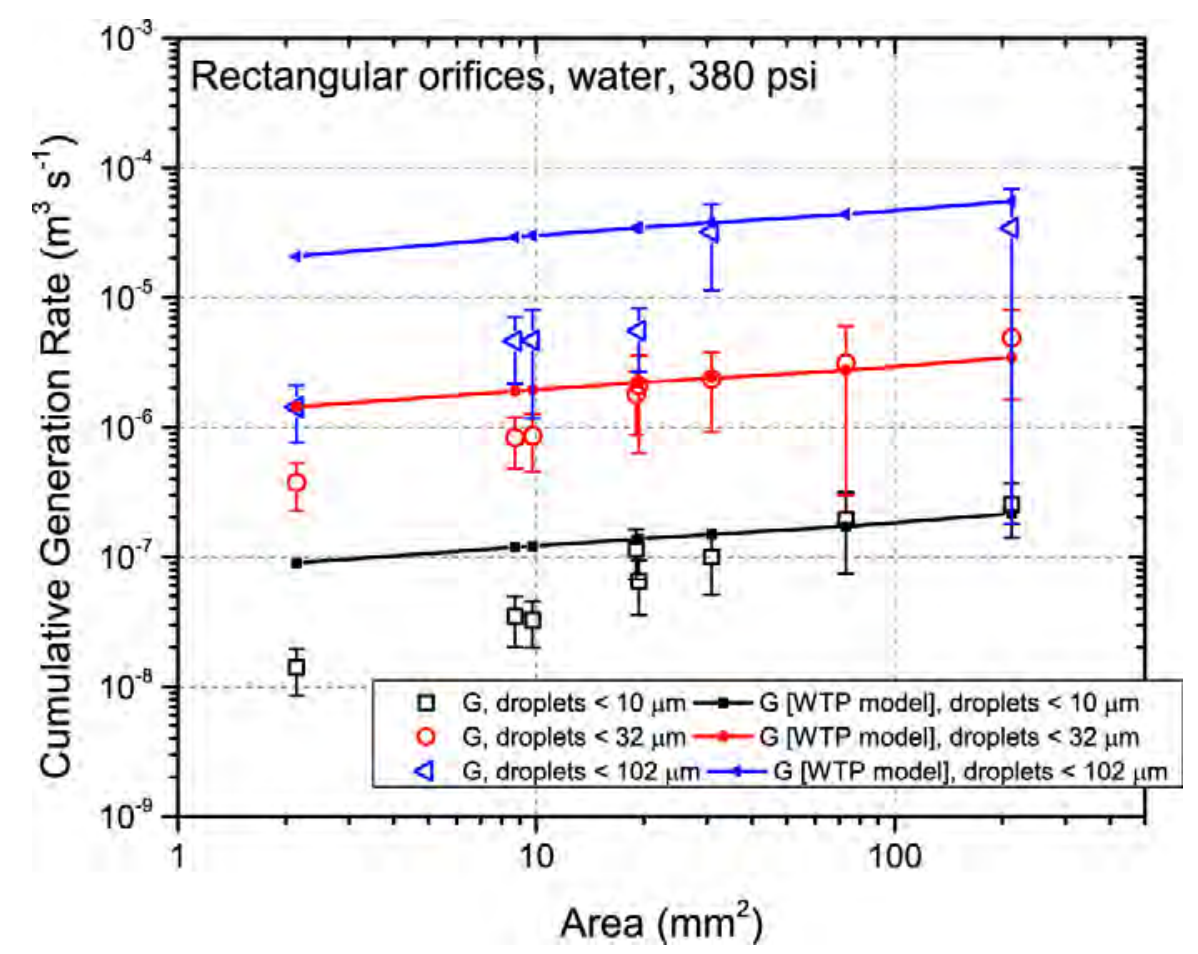

Figure 8.15. Effect of Orifice Area on Generation Rate for Rectangular Orifices. Effect of cross-sectional area of rectangular orifices on the observed cumulative generation rate at three droplet sizes $(<10,32$, and $102 \mu \mathrm{m})$ for tests conducted using water at 380 psi. The error bars show an estimate of the 95-percent confidence level based on the fitting confidence interval and the uncertainty due to test-to-test repeatability. The WTP model prediction is shown by the solid colored lines.

\subsubsection{Effect of Aspect Ratio}

The set of rectangular orifices studied in this report were selected to provide a subset that had orifices of the same width but increasing length $(1 \times 10 \mathrm{~mm}, 1 \times 20 \mathrm{~mm}, 1 \times 76.2 \mathrm{~mm})$. The data from this subset can be examined to observe the effect of changing length on the cumulative release fraction. In Figure 8.16 the variation of release fraction at two target pressures (200 and 380 psi) is shown as a function of length (plotted as area). Given what was observed in Figure 8.15, the data has trends that are expected. Figure 8.16 has plotted the release fraction instead of the generation rate against the area, so the WTP model predicts a decrease with increasing length. The experimental data, however, show a decrease that is less severe (example: the release fraction [RF] at $380 \mathrm{psi},<32 \mu \mathrm{m}$ ) or even the absence of a statistically significant decrease (example: the RF at $200 \mathrm{psi},<10 \mu \mathrm{m}$ ). 


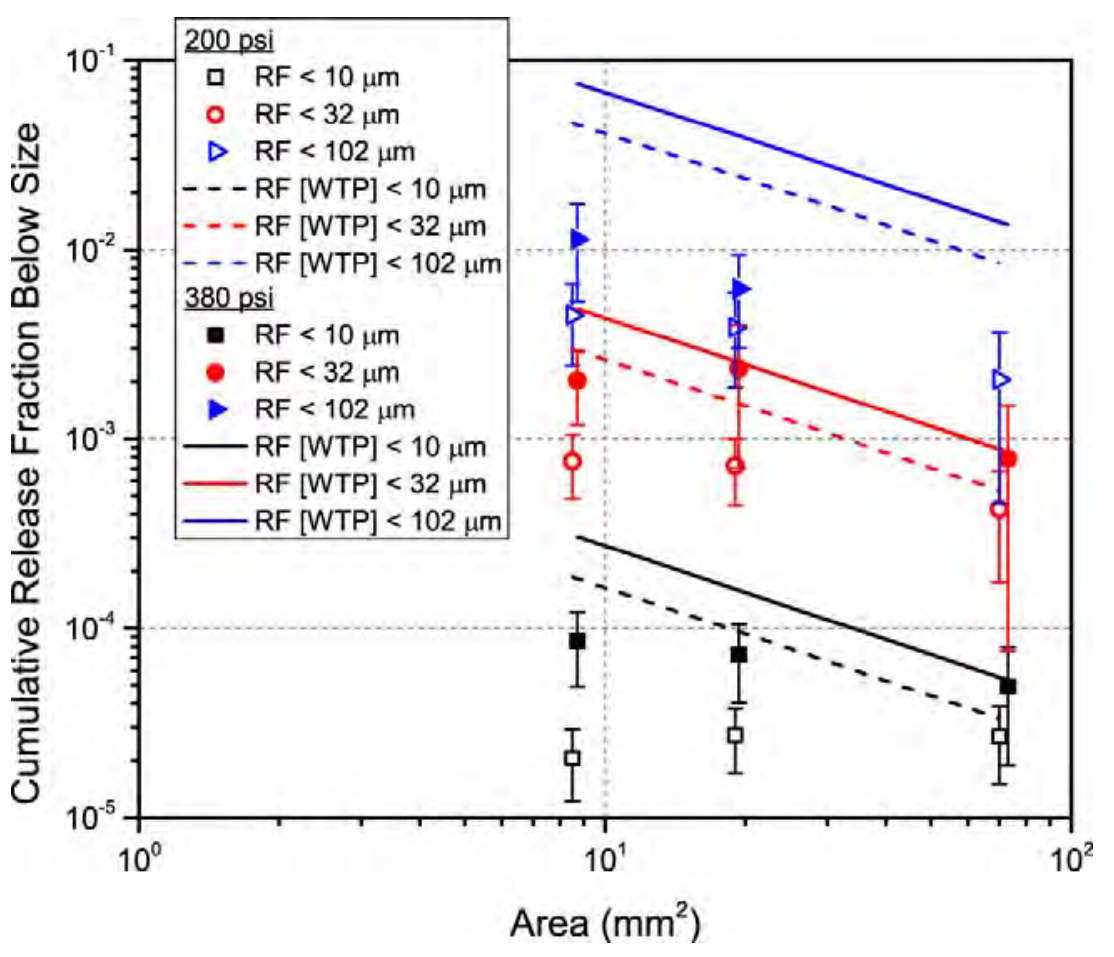

Figure 8.16. Effect of Slot Length on Release Fraction. Effect of the length of a rectangular orifice on the cumulative release fraction at 200 psi (open symbols) and 380 psi (closed symbols) for tests conducted using water at three droplet sizes $(<10,32$, and $102 \mu \mathrm{m})$. The areas correspond to orifices of target dimensions of $1 \times 10,1 \times 20$, and $1 \times 76.2 \mathrm{~mm}$, respectively (the 200 and 380 psi data have been offset slightly for better visibility of the data, with the 380 psi data located at the actual areas and the 200 psi data shifted left of the actual values to arbitrary areas which are approximately 5\% less). The error bars show an estimate of the 95-percent confidence level based on the fitting confidence interval and the uncertainty due to test-to-test repeatability. The WTP model prediction is shown by the dashed (200 psi) and solid (380 psi) lines.

Similarly, the set of rectangular orifices studied in this report also contained a subset that had orifices of the same length but increasing width $(1 \times 10 \mathrm{~mm}, 2 \times 10 \mathrm{~mm}, 3 \times 10 \mathrm{~mm})$. Figure 8.17 presents the data from that subset at 200 and 380 psi. The results imply that changes in the width of an orifice lead to deviations from the model predictions that are larger than if the length is changed. Statistically, the release fraction is unchanged as the width increases, and it could be argued that some of the RF data (e.g., $<102 \mu \mathrm{m}$ data in particular) have a small positive slope. This is directly contrasted with the negative slope of the WTP model predictions shown in the figure. The data from the tests in which width was varied also include some of the noisiest data sets, making the interpretation more challenging. However, these data suggest that the functionality of release fraction with area may change as the aspect ratio approaches unity. 


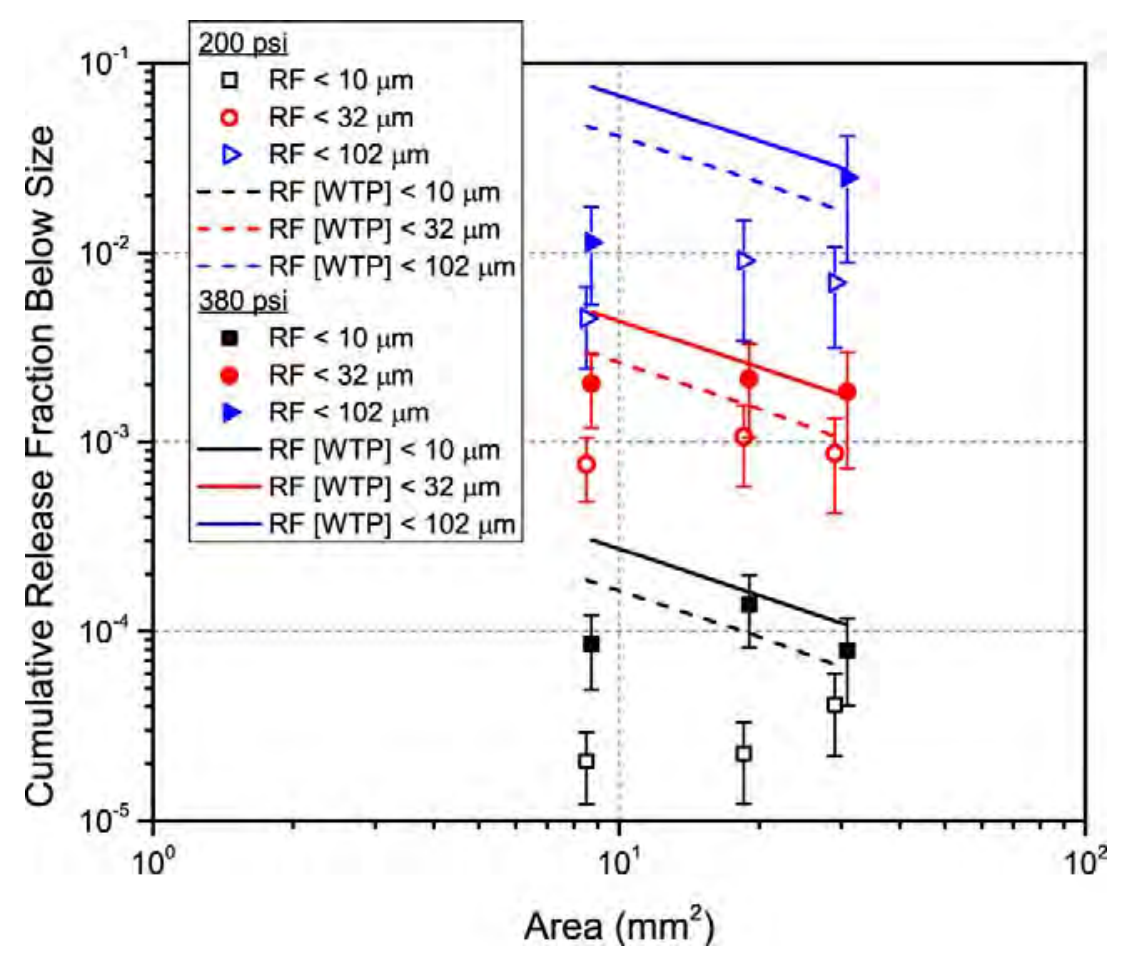

Figure 8.17. Effect of Slot Width on Release Fraction. Effect of the width of a rectangular orifice on the cumulative release fraction at 200 psi (open symbols) and 380 psi (closed symbols) for tests conducted using water at three droplet sizes $(<10,32$, and $102 \mu \mathrm{m})$. The areas correspond to orifices of target dimensions of $1 \times 10,2 \times 10$, and $3 \times 10 \mathrm{~mm}$, respectively (the 200 and 380 psi data have been offset slightly for better visibility of the data, with the 380 psi data located at the actual areas and the 200 psi data shifted left of the actual values to arbitrary areas which are approximately $5 \%$ less). The error bars show an estimate of the 95-percent confidence level based on the fitting confidence interval and the uncertainty due to test-to-test repeatability. The WTP model prediction is shown by the dashed (200 psi) and solid (380 psi) lines.

\subsubsection{Overall Effect of Orifice Area on Generation Rate and Release Fraction}

In Section 8.2.4, some weak dependence of release fraction/generation rate on aspect ratio was considered based on comparison of data sets that varied the length and width of orifices at the same conditions. The amount of data collected was not sufficient to propose any formal mathematical relationship. In part, this was not examined further because the orifice cross-sectional area is an adequate parameter to capture the effect of orifice geometry. See, for example, the effect of cross-sectional area for all orifices tested presented in Figure 8.18 and Figure 8.19. The cumulative release fraction is shown for round (solid symbols) and rectangular orifices (open symbols) at three different pressures (sub-figures a, b, and c). Differentiating between orifice types does not appear to affect the relationship with area. The experimental data consistently decrease at a lower rate than the WTP model prediction over the entire range of areas tested. This decrease is similar at each of the three pressures tested; however, lower pressure data has a smaller slope than higher pressure data, to the extent that the release fraction is nearly independent of orifice area at 100 psi. Note also that the WTP model becomes progressively more conservative as the orifice area decreases. 
An analogous conclusion was drawn from examination of the cumulative generation rate vs. the area (Figure 8.19); the data consistently increases at a greater rate than the WTP model over the entire range of areas. Moreover, the data challenges the WTP model prediction at or near $30 \mathrm{~mm}^{2}$ at all three droplet sizes at each of the three pressures tested. Note that the range of orifices sizes tested did not include the maximum breach size under consideration in the WTP safety analysis. The implications of the data for even larger orifices sizes are discussed in more detail in Chapter 9.

In general, the water tests served to confirm that the WTP model contains the qualitatively appropriate dependence of release fraction on pressure, orifice orientation, and size/area (correct trends and sign), but the actual data have some quantitative differences. The effect of pressure (increasing pressure increases RF) is greater in magnitude and the effect of area (increasing area decreases RF) is less in magnitude in the actual data. The larger orifices tested in this report approach the WTP model, indicating it may not be a conservative estimator of release fraction for those orifices. 


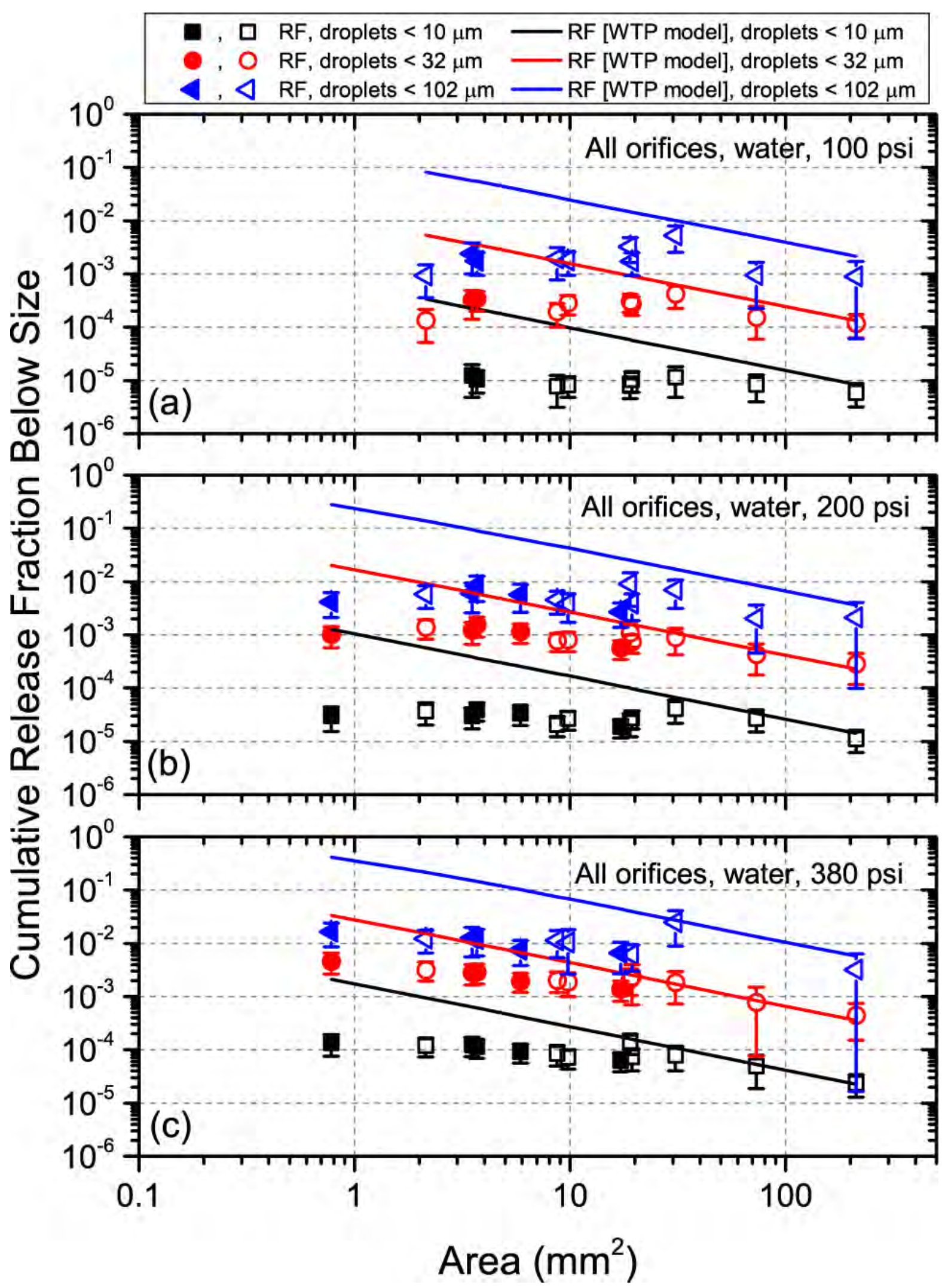

Figure 8.18. Effect of Cross-Sectional Area on Release Fraction. Observed cumulative release fraction for three droplet sizes (<10, 32, and $102 \mu \mathrm{m}$ ) at (a) 100 psi, (b) 200 psi, and (c) 380 psi. 


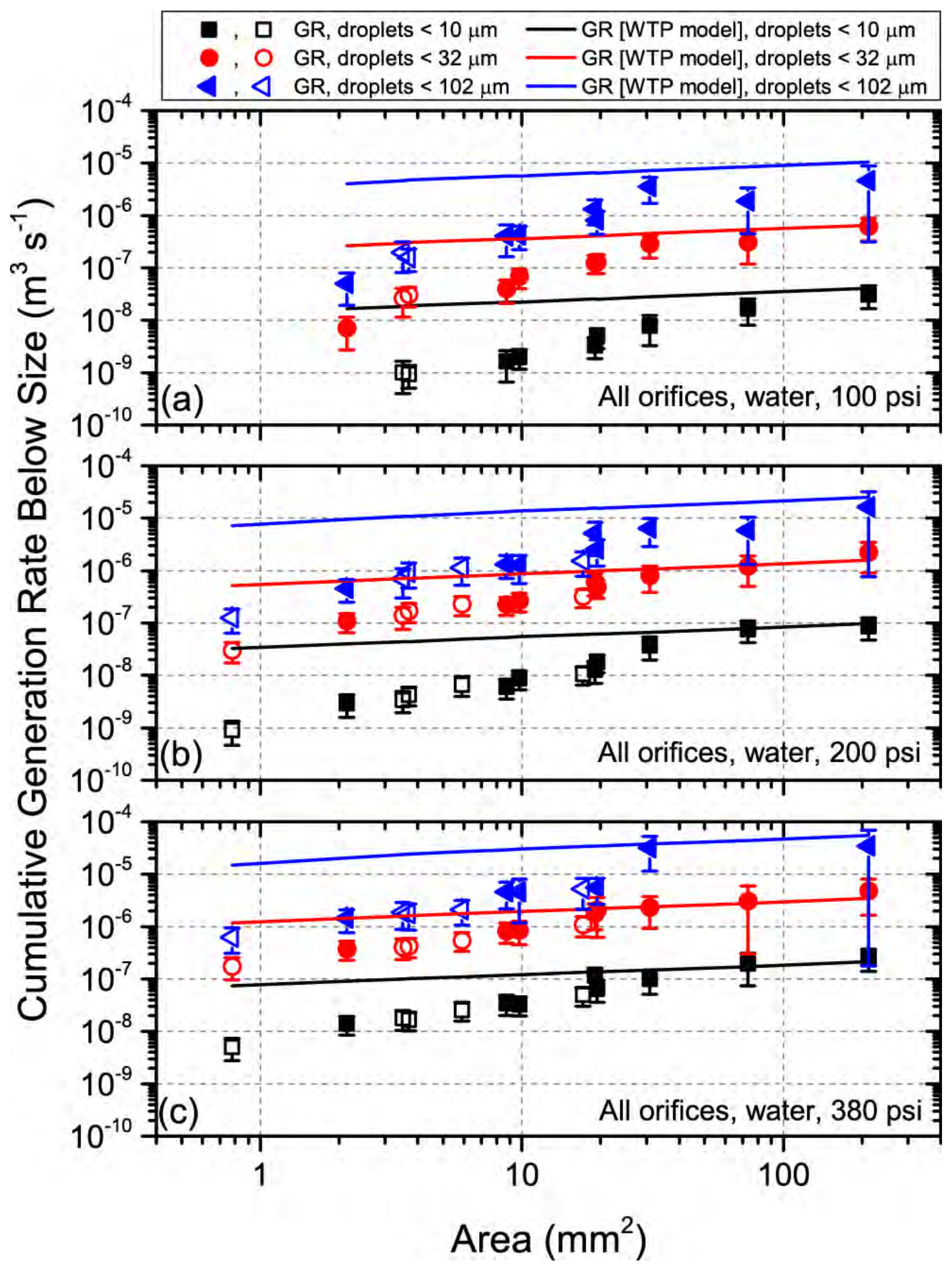

Figure 8.19. Effect of Cross-Sectional Area on Generation Rate. Observed cumulative generation rate for three droplet sizes (<10, 32, and $102 \mu \mathrm{m}$ ) at (a) 100 psi, (b) 200 psi, and (c) 380 psi. 


\subsection{Effect of Viscosity}

The WTP model includes a small effect of fluid viscosity on the release fraction. Based on Equations (1.1) and (1.2), the SMD has a weak positive dependence on viscosity (since SMD/ $X$ is a constant in the WTP model). This will result in a small decrease in release fraction with increasing viscosity if all other parameters remain the same. Increasing the viscosity without changing other physical parameters of the fluid (i.e., density, surface tension, presence of solid particles) is challenging. The sodium thiosulfate simulant used to test a fluid with a higher viscosity ( $2.6 \mathrm{mPa} \cdot \mathrm{s})$ had a density that was greater than that of water by approximately 25 percent (see Chapter 4). This reduces the certainty of any conclusions that may be drawn from the sodium thiosulfate results, because the density also affects the release fraction. The density affects both the WTP prediction (see Equations (1.1), (1.2), and (1.8)) and the experimental data (needed to calculate the spray leak volumetric flow rate as discussed in Section 7.1). Note that the small-scale tests included a pair of density-matched simulants with different viscosities that better isolate the effect of viscosity (Mahoney et al. 2012b).

The sodium thiosulfate tests were conducted in two rectangular orifices, a $0.5 \times 5$-mm slot and a $1 \times 10$-mm slot. An example of a comparison between the cumulative release fraction calculated from a water test and a sodium thiosulfate test at 380 psi is shown in Figure 8.20 in which the WTP model predictions for the two fluids are shown by the solid black and dashed blue lines. The sodium thiosulfate test has a larger release fraction across all droplet sizes. This is in contrast to the WTP model expectation; however, the spray leak flow rate $\left(Q_{\text {spray }}\right)$ measured for the sodium thiosulfate was lower than the measured $Q_{\text {spray }}$ for the water test. The difference in $Q_{\text {spray }}$ accounts for most of the difference between the release fractions shown in Figure 8.20. This difference is primarily driven by the density difference between the two fluids. On the other hand, the WTP model takes the density difference into account as well, and the difference between the model lines on the figure is smaller in magnitude than the difference between the test data.

One approach to eliminating the effect of density is to compare generation rates instead of release fractions. This comparison is made in Figure 8.21 for the $1 \times 10$-mm slot at two target pressures, 200 and 380 psi. The WTP model predicts a slight decrease in cumulative generation rate between the two fluid viscosities. The experimental data, at both pressures, are statistically unchanged as viscosity is increased. As before with pressure and orifice area, the WTP model appears to capture the qualitative effect of viscosity. Two caveats are important to note: 1 ) the density still has a small effect on the generation rate itself, so the effect of viscosity is not completely decoupled from the fluid density, and 2) the sodium thiosulfate data were less consistent than some of the other data sets, suggesting that the \pm 30 percent uncertainty based on repeatability tests (conducted with water) underestimate the uncertainty when the fluid is sodium thiosulfate. Thus, this conclusion is speculative and would require more data for corroboration. 


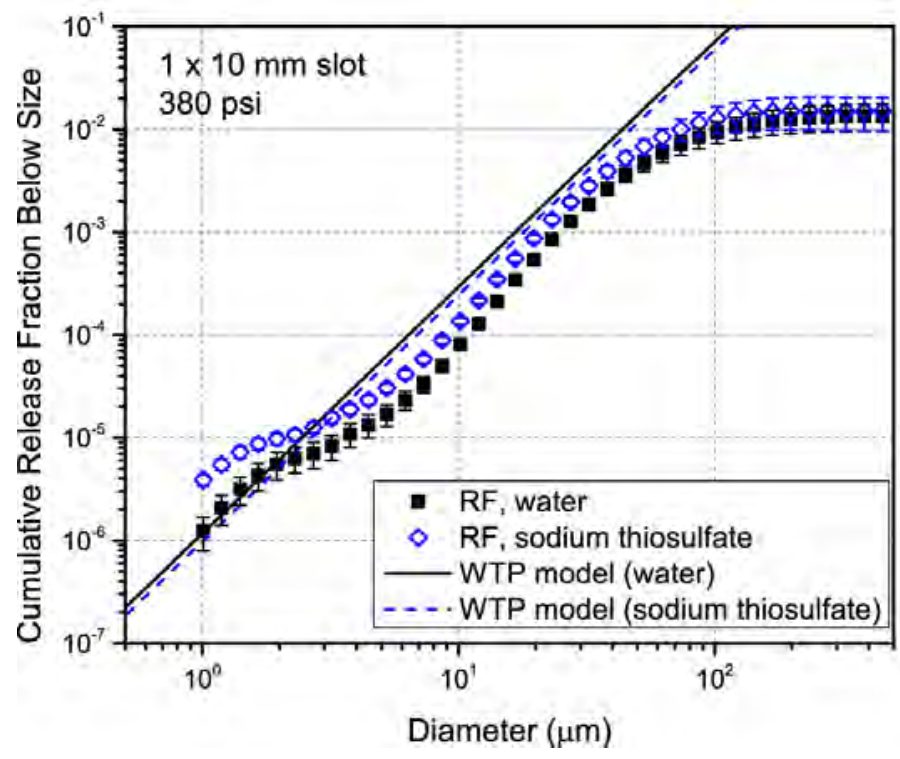

Figure 8.20. Effect of Viscosity on Release Fraction. Comparison of release fractions measured using water (black squares) and a sodium thiosulfate-water solution (blue diamonds) at $380 \mathrm{psi}$ using a $1 \times 10$-mm orifice. The WTP model predictions are shown as solid black and dashed blue lines, respectively.

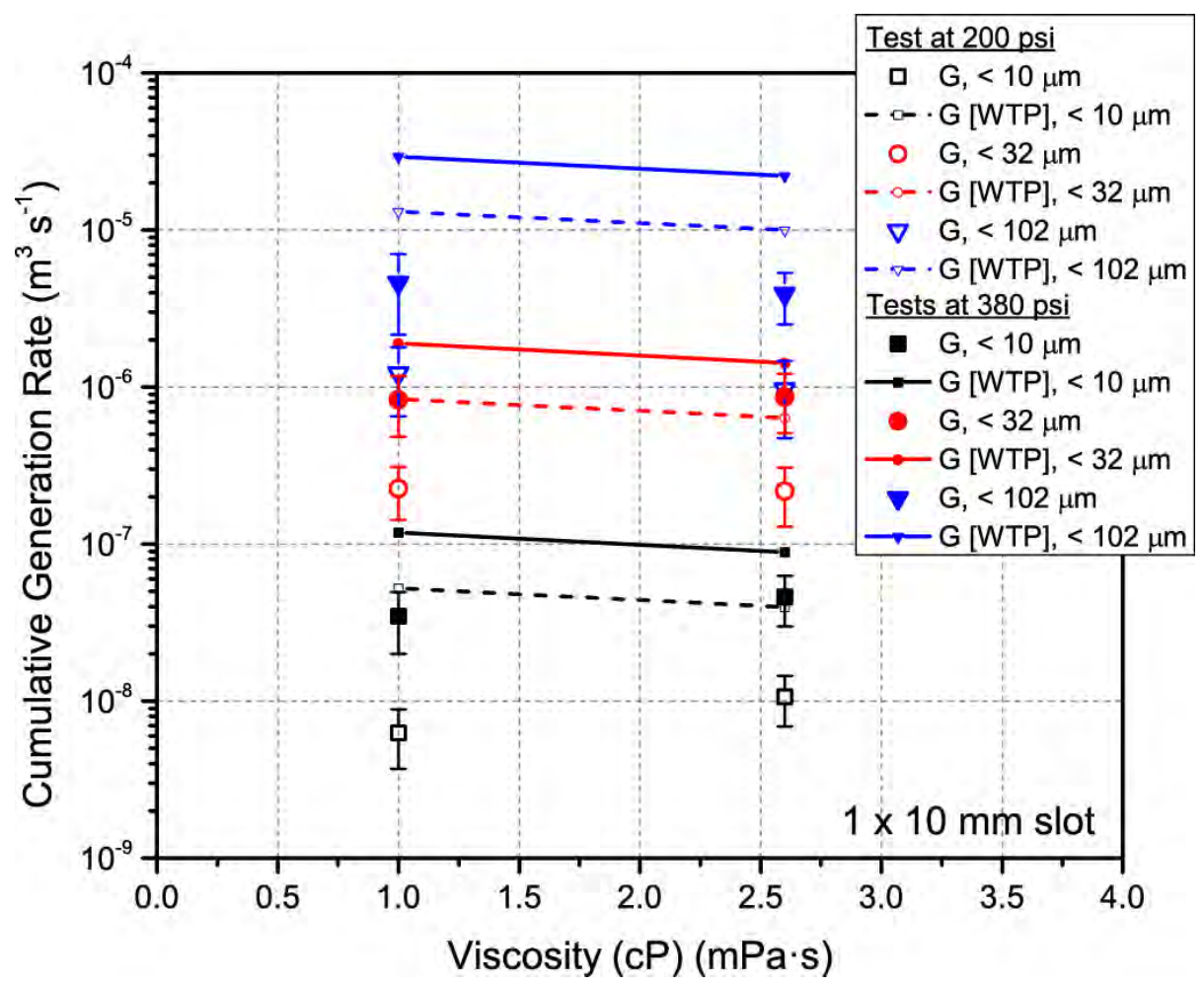

Figure 8.21. Effect of Viscosity on Generation Rate. Cumulative generation rate as a function of fluid viscosity calculated for tests conducted with a $1 \times 10$-mm orifice at $200 \mathrm{psi}$ (open symbols) and 380 psi (closed symbols) at three droplet sizes $(<10$, 32, and $102 \mu \mathrm{m})$. The error bars show an estimate of the 95-percent confidence level based on the fitting confidence interval and the uncertainty due to test-to-test repeatability. The WTP model prediction is shown by the dashed (200 psi) and solid (380 psi) lines. 


\subsection{Slurry Tests}

The effect of solids loading in the fluid was investigated in the large-scale system using the STR slurry simulant at two solids concentrations, 8 and $20 \mathrm{wt} \%$. The same two orifices $(0.5 \times 5 \mathrm{~mm}$, $1 \times 10 \mathrm{~mm}$ ) were used that were tested with sodium thiosulfate. Examination of the WTP model reveals a known and unknown impact of using STR slurries on the release fraction. The known impact is that they have different densities (though not as different as the sodium thiosulfate, see Chapter 4) and viscosities than water. The unknown impact is the presence of solid particles and their effect on breakup and atomization of the jet and the corresponding residence time as aerosol in the environment, any of which could lead to a droplet size distribution that is different than that of water. Some of these impacts are discussed in Section 1.3.4.

Because they introduce some opacity to the system, slurry particles are fundamentally different than water, which is optically transparent. Because the measuring instruments depend on the scattering of laser light, acquisition of quality data depends on proper specification of the refractive index of the material being measured. This is complicated by the possibility of aerosol generated that only contains solid, only contains liquid, or contains some mixture of both (i.e., a composite droplet). The approach used in this work was to use a composite refractive index based on mixing rules (see Section 7.4.4). However, before analysis of the slurry data was performed in full, a small number of data sets were checked for robustness to the choice of refractive index.

One such example of a robustness check is shown in Figure 8.22. The three sets of cumulative release fractions in this figure are all derived from the same test, S190, which was conducted with 20 wt\% STR simulant at 200 psi using a $1 \times 10$-mm orifice. Each set of RF data was derived from a different aerosol concentration data set that was produced using a different refractive index to process the Malvern instrument data. The three refractive indices correspond to the refractive index of the liquid component only (water), the solid component only (boehmite), and the slurry (computed using mixing rules). At droplet sizes approximately $>10 \mu \mathrm{m}$, the release fractions are nearly the same. At droplet sizes $<10 \mu \mathrm{m}$, using the refractive index of water results in the calculation of a set of release fractions that diverge rapidly from the other two sets of RF. The solid component and slurry refractive indices result in RFs that remain difficult to discriminate between. Based on the similarity of the data sets in Figure 8.22, especially at droplet sizes $>10 \mu \mathrm{m}$, the choice of refractive index is of minor concern. For the rest of the analysis, the slurry refractive index was used to analyze data because the aerosol was visually confirmed to be opaque and, thus, must have some finite absorbance of laser light (expressed as the imaginary component of the refractive index).

Cumulative release fractions for the STR simulant, at both 8 and $20 \mathrm{wt} \%$, are compared with the results from a water test in Figure 8.23. All tests were conducted at 380 psi with a $1 \times 10$-mm orifice. The WTP predictions are shown as lines on the plot. The experimental data are nearly the same until the droplet sizes fall below approximately $14 \mu \mathrm{m}$. At smaller droplet sizes, the slurry data remains indistinguishable from each other and the water data has a smaller release fraction, a gap that widens as the droplet size decreases further. Based on data shown in Figure 8.23 and from similar comparisons from other large-scale tests, it appears that the WTP model does not require further adjustment for predicting release fraction outside of what it already accounts for in the physical properties, such as density, viscosity, and surface tension. Conversely, this is a limited data set (only a single slurry was studied), and the results should be compared with the much larger data set investigated in the small-scale testing program (see Mahoney et al. 2012b). 


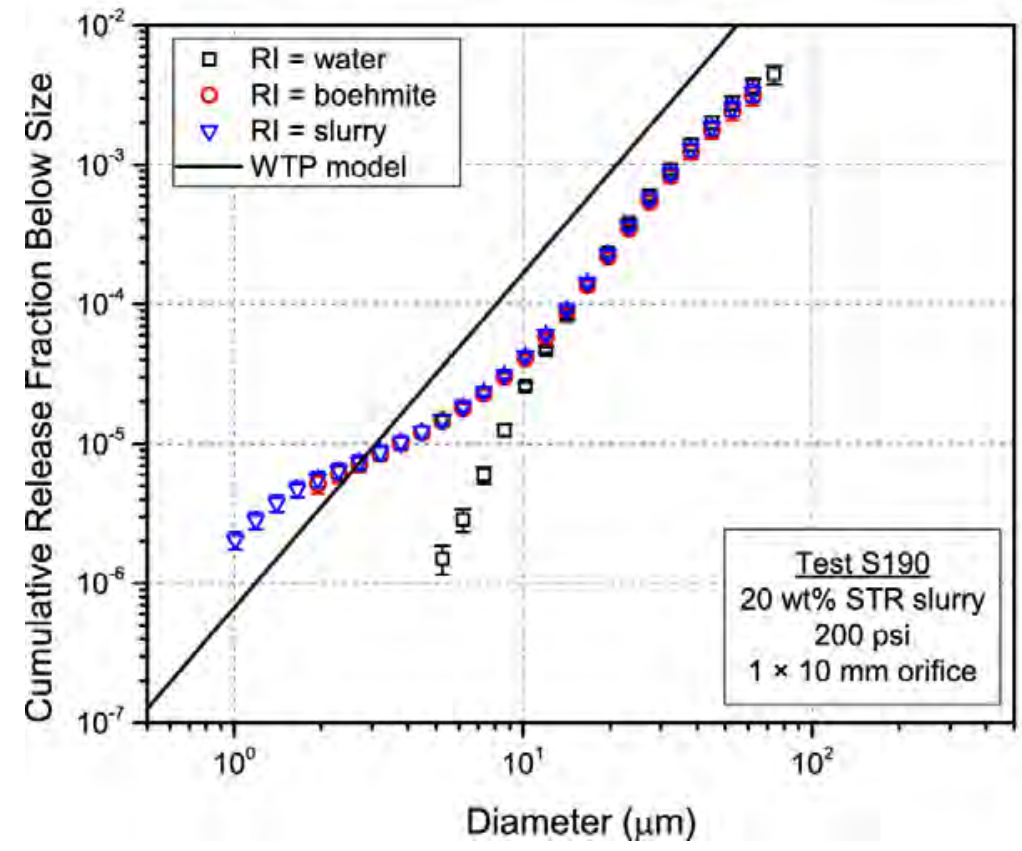

Figure 8.22. Effect of the Selection of Refractive Index on Cumulative Release Fraction. The test was conducted with $20 \mathrm{wt} \%$ STR slurry at 200 psi using a $1 \times 10$-mm orifice. The WTP model prediction is shown by the solid line.

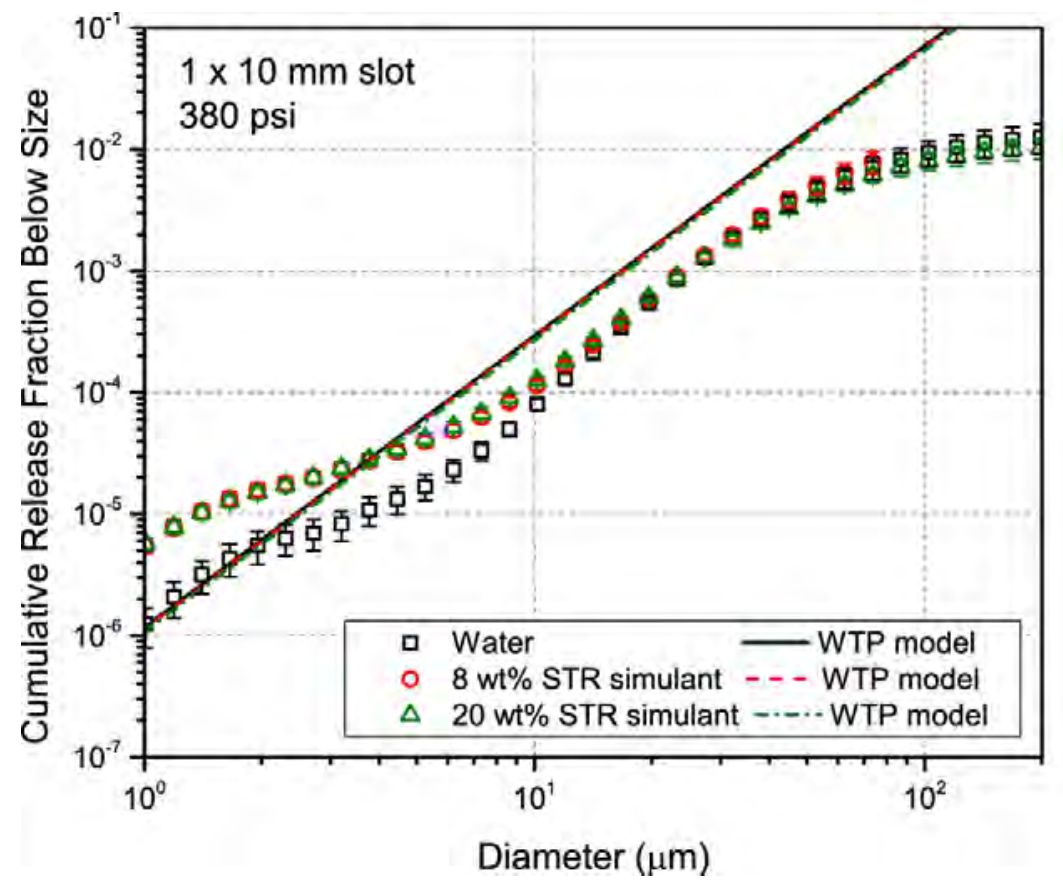

Figure 8.23. Effect of Solids Loading on Release Fraction. Comparison of cumulative release fractions estimated from tests conducted using a $1 \times 10-\mathrm{mm}$ orifice at $380 \mathrm{psi}$ with water (squares), $8 \mathrm{wt} \%$ STR slurry (circles), and $20 \mathrm{wt} \%$ STR slurry (triangles). The corresponding WTP model predictions are shown by lines: water (solid line), 8 wt\% STR (dashed line), and 20 wt\% STR (dashed-dotted line). 


\subsection{Effect of Spray Distance and Scaling}

One variable that is not accounted for in the WTP model is the fate of the spray producing the aerosol once it leaves the leak. The model assigns the entire aerosol volume created by the spray to be part of the release fraction, and thus does not adjust the release fraction for loss terms such as capture by a surface or other obstruction in the path of the jet. The dependence of the release fraction on proximity to such a surface was studied by moving the spool containing the test orifices to different distances from the rear wall (hereafter referred to as the splash wall) of the test chamber. The three distances were nominally $227.25,120.25$, and 43.25 in. The longest distance was the standard orifice location for all the other large-scale tests described in this report. The shortest distance was selected to be very similar to the standard orifice-to-splash-wall distance in the small-scale test chamber (approximately 42 in.). The location of the Malvern instruments during these tests was not changed; the position the spray originated from was the only alteration to the configuration.

The effect of the distance to the splash wall on the release fraction is shown for all three distances for a 1-mm orifice (Figure 8.24) and a 2-mm orifice (Figure 8.25) at 380 psi using water. Both of the data sets demonstrate that the release fraction increases as the distance from the wall is increased across all droplet sizes. The magnitude of the increase in release fraction as the distance from the splash wall increased from 43.25 to 227.25 in. was larger for the larger orifice, especially for the droplets that were greater than $30 \mu \mathrm{m}$. The splash wall influences aerosol generation and formation in two ways:

1. It prevents atomization of a portion of the jet by capturing the spray.

2. It promotes some aerosol generation by a portion of the jet and/or droplets breaking up at the wall and rebounding back into the measurement volume.

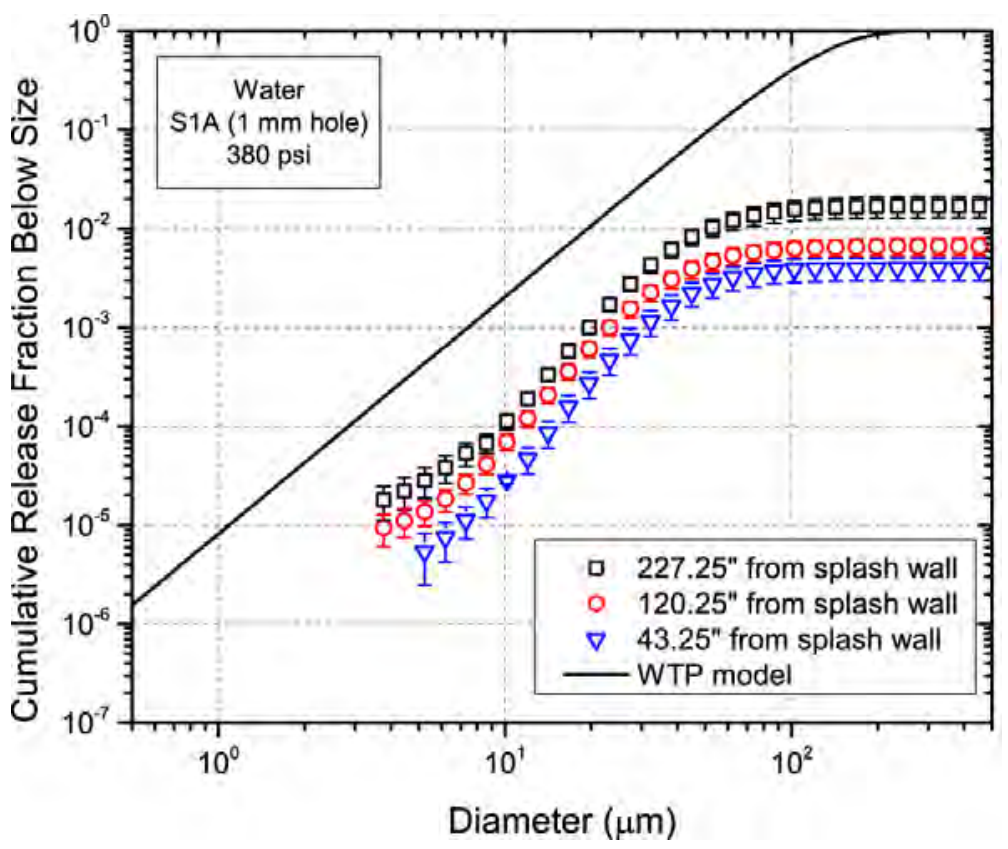

Figure 8.24. Effect of Spray Distance from the Splash Wall. Cumulative release fractions obtained from tests using water at $380 \mathrm{psi}$, with a 1-mm orifice located at different distances from the splash (rear) wall of the aerosol chamber. The WTP model prediction is shown by the solid line. 


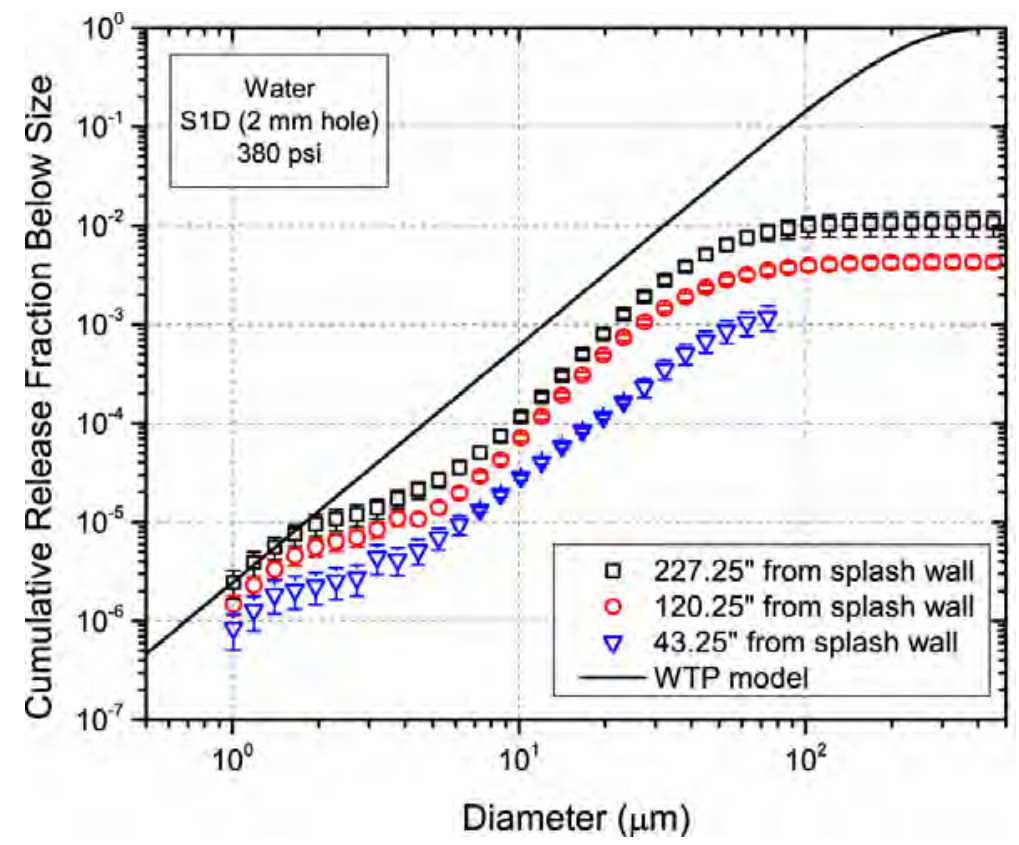

Figure 8.25. Effect of Spray Distance from the Splash Wall. Cumulative release fractions obtained from tests using water at $380 \mathrm{psi}$, with a 2-mm orifice located at different distances from the splash (rear) wall of the aerosol chamber. The WTP model prediction is shown by the solid line.

The relative magnitude of these two physical processes was unknown, but the data from Figure 8.24 and Figure 8.25 support the conclusion that the loss of aerosol by wall capture is the dominant mechanism.

The importance of this result is that the difference between the shortest distance and longest distance is a factor of approximately an order of magnitude for a large range of droplet sizes. Because the majority of the orifices tested in this report resulted in a spray jet that impinged on the rear wall of the chamber, aerosol is being lost that would otherwise be generated in the more open geometry. Thus, the increase of release fraction with distance from the splash wall suggests that the release fraction measured in the tests described in this report may underestimate the release fraction for longer sprays. The orifices shown in Figure 8.24 and Figure 8.25 are among the smallest orifices (by area). Based on the results of the splash wall tests for these geometries, a few larger orifices were tested at the shortest and longest distances from the splash wall to confirm that the same effect was observed with increasing orifice size.

Figure 8.26 presents the cumulative release fractions measured using a $1 \times 20$-mm orifice at $380 \mathrm{psi}$ using water at two distances from the splash wall, and Figure 8.27 presents the same for a $1 \times 76.2-\mathrm{mm}$ orifice. As before, the release fraction is larger when the distance from the splash wall is longer, but the magnitude of the difference for the larger slots is not as pronounced. It is worth noticing that the WTP model prediction is a single line in all of these figures. The model does not have the ability to handle geometric considerations such as distance from an impinging surface (or any other obstruction that may exist). In the case of a short distance to an obstruction, the WTP model is a larger over-prediction; however, sprays that are allowed to atomize and break up over longer distances than the large-scale chamber may result in release fractions that are even greater than those shown at $227.25 \mathrm{in}$. Sprays of that type may approach or even exceed the WTP model predictions for those conditions. 


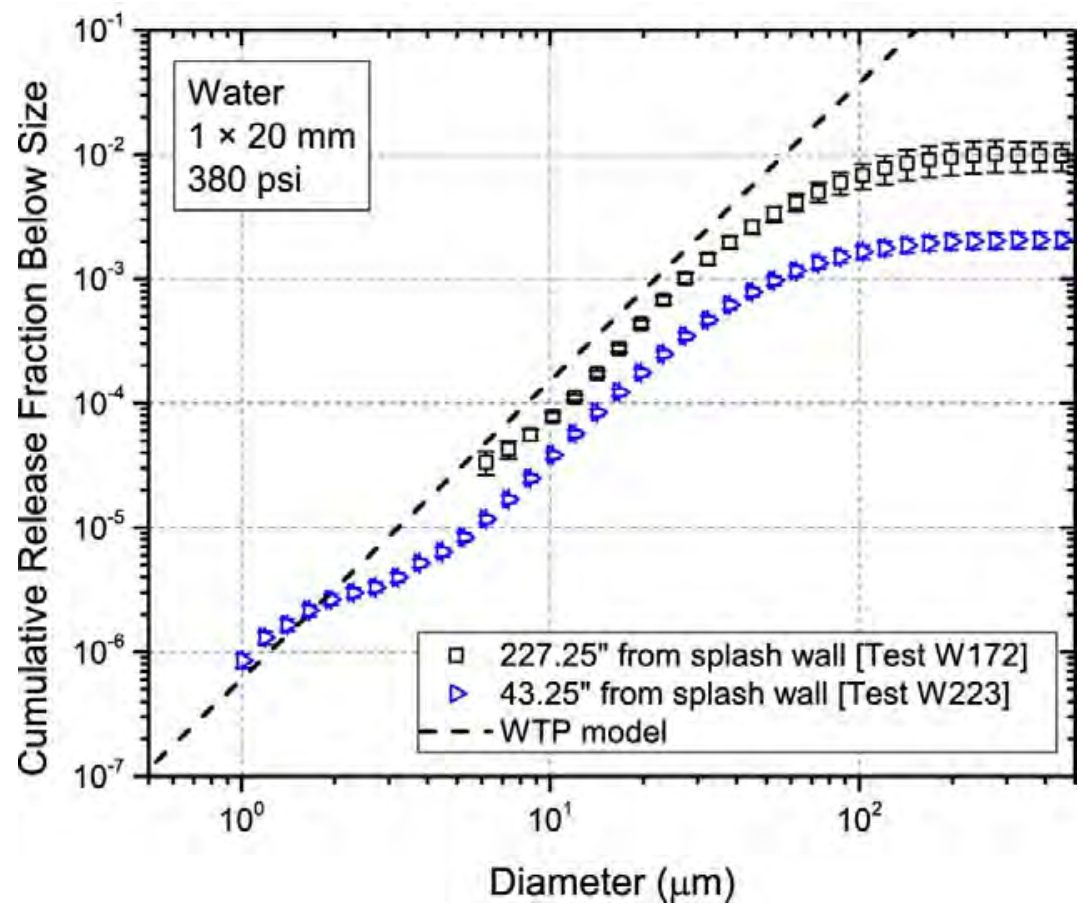

Figure 8.26. Effect of Spray Distance from the Splash Wall. Cumulative release fractions obtained from tests using water at $380 \mathrm{psi}$, with a $1 \times 20$-mm orifice located at two distances from the splash (rear) wall of the aerosol chamber. The WTP model prediction is shown by the dashed line.

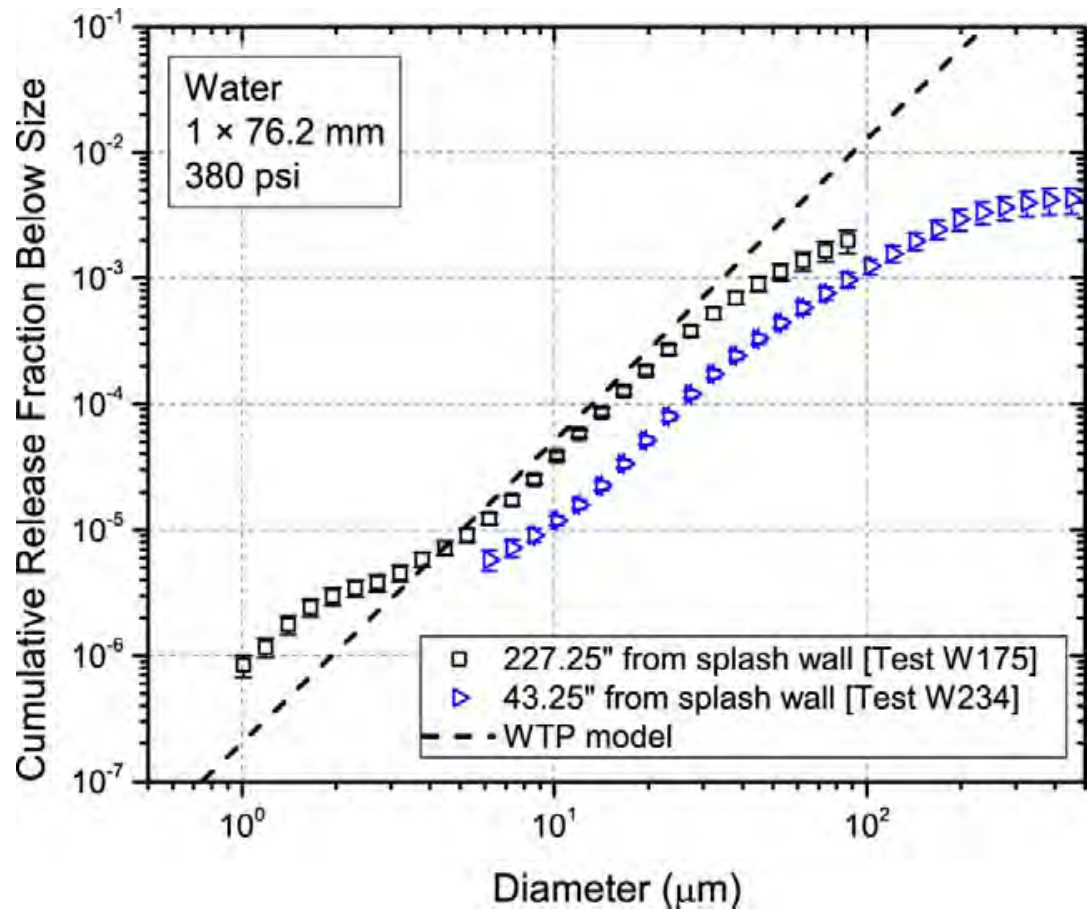

Figure 8.27. Effect of Spray Distance from the Splash Wall. Cumulative release fractions obtained from tests using water at $380 \mathrm{psi}$, with a $1 \times 76.2$-mm orifice located at two distances from the splash (rear) wall of the aerosol chamber. The WTP model prediction is shown by the dashed line. 
This is made clearer graphically in Figure 8.28. The cumulative generation rate as a function of distance to the splash wall is shown at three droplet sizes $(<10,<32$, and $<102 \mu \mathrm{m})$ for the 2 -mm round orifice at 380 psi. Again, the model does not account for the changes in configuration and is constant with distance as shown. The generation rate, meanwhile, is increasing rapidly with distance.

Extrapolating this result to longer distances is discussed in more detail in Chapter 9. Similar experiments also were performed in the small-scale system at separation distances from the splash wall down to 1 in. (Mahoney et al. 2012b). The results of those experiments thus far suggest additional testing that expands the parameter space (i.e., studying longer distances in particular than 227.25 in.) would be useful to fully assess the effect on release fractions.

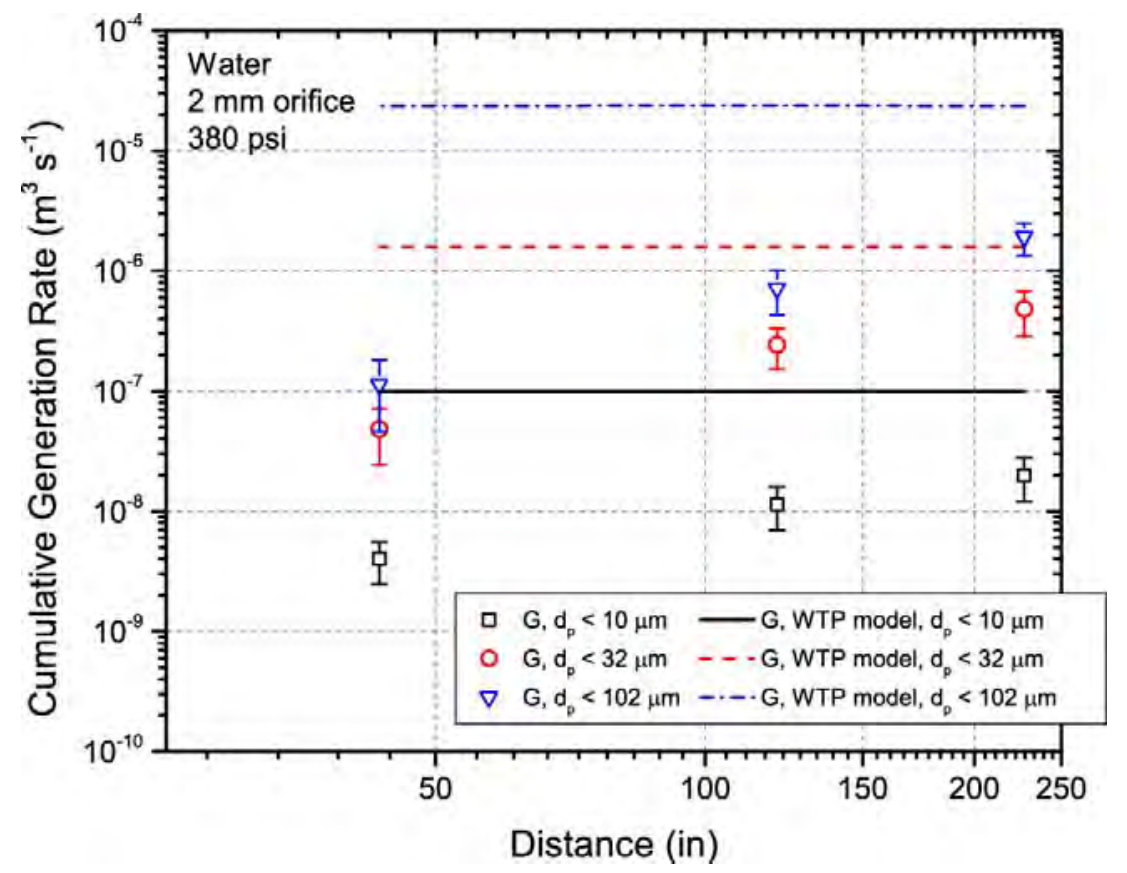

Figure 8.28. Cumulative Generation Rate as a Function of Distance from the Splash Wall. The generation rate is shown at three droplet sizes $(<10,32$, and $102 \mu \mathrm{m})$ for tests performed using water and a 2-mm orifice at 380 psi. The error bars show an estimate of the 95-percent confidence level based on the fitting confidence interval and the uncertainty due to test-to-test repeatability. The WTP model predictions are given by the various lines.

\subsection{In-Spray Aerosol Measurements}

The data from Section 8.5 indicates that performing the large-scale tests in a confined geometry results in release fractions that are underestimates relative to the concentration of aerosol that would be generated in an open system. One method to address this issue is to measure the aerosol concentration in the spray itself, assuming that the entrained aerosol contribution in the jet was small compared to the local generation. This measurement estimates the release fraction when there is no loss and no aerosol generated from splatter, and can be considered an upper bound on the RF. This type of measurement is difficult to perform in the large scale for all but the smallest orifices because of the size of the spray jet as it spreads out in the chamber. If the jet is not restricted to the instrument measurement volume it cannot be considered an upper bound. Furthermore, the concentration of aerosol is large, which complicates 
accurate measurement of the aerosol and risks flooding the system or swamping the lenses with condensate.

In-spray measurements were performed for both the 1-mm and 2-mm orifices at both 200 and 380 psi using water. Only the Malvern instruments in positions 2 and 3 could be used to obtain in-spray data. Position 1 was too far away from the jet's point of origin to permit an accurate measurement of the aerosol. Position 3 was occupied by Malvern instrument M1854, approximately 23.5 in. from the orifice, and position 2 was occupied by instrument M1852, approximately 59 in. from the orifice. The tests were conducted in the same manner as other tests. The primary difference is that all aerosols were measured directly, and so the quantity of interest is the normalized aerosol distribution (volume fraction) as a function of droplet size. The normalized distribution is the cumulative concentration of aerosol divided by the sum of the concentration across all droplet sizes. This is described by Equation (7.14).

The normalized particle distribution is shown in Figure 8.29. Data displayed in the figure is from a test that used the 2-mm orifice at $380 \mathrm{psi}$ as a function of elapsed time (where $t=0$ is when the spray starts). Each distribution shown is a 5-s average centered on the elapsed time indicated in the legend. At this in-spray measurement location (Malvern instrument M1854, 23.5 in. from the orifice) the volume fraction distribution is fully developed immediately after the spray is initiated, and there is no evidence of any significant transient behavior. Other similar data sets did not show any significant transience either, so the distribution at $10 \mathrm{~s}$ elapsed time was chosen to compare test data from different test conditions. The data of Figure 8.29 also shows that there are only a very small fraction of droplets that are less than $10 \mu \mathrm{m}$ in diameter.

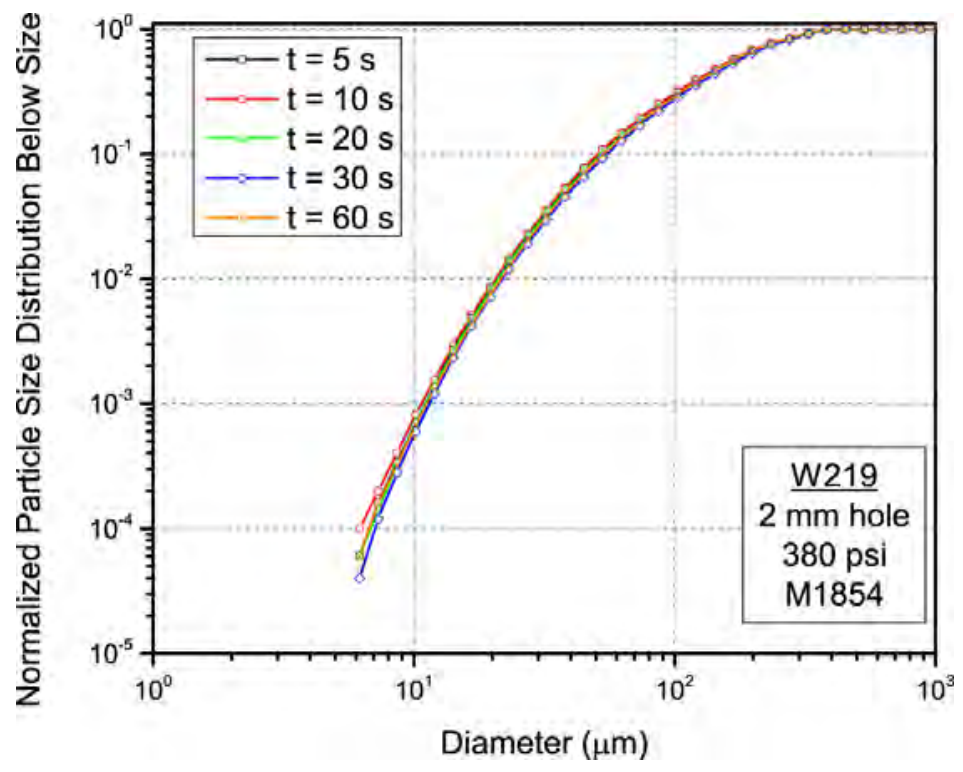

Figure 8.29. Normalized Droplet Size Distribution as a Function of Elapsed Time. The normalized size distribution is from an in-spray measurement (test W219: water, 380 psi, 2-mm orifice), and each distribution is a $5 \mathrm{~s}$ average centered on the elapsed times shown in the legend.

The repeatability of an in-spray measurement also was assessed. All in-spray tests were performed in duplicate; one such example of duplicate test data is presented in Figure 8.30. The figure shows the data 
from both Malvern instruments (M1852, M1854) located in the spray at 200 psi using water and a 2-mm orifice. The droplet size distribution is from an elapsed time at $10 \mathrm{~s}$. The agreement between the data sets is quite good. There is some divergence of the distributions measured by M1852 (59 in. from the orifice) at droplet sizes of $30 \mu \mathrm{m}$ and less. However, this measurement location is farther from the orifice and the jet has had more distance to atomize, break up, and interact with its surroundings. The turbulent nature of the jet itself introduces chaos and randomness into the spray, and it is not surprising to observe some effect of that turbulence in the in-spray data. Overall, the measurements were consistently repeatable. This can also be confirmed by looking at the average total volume concentrations over the first $60 \mathrm{~s}$ of the spray, as seen in Table 8.4. The average total volume concentration of aerosol $\left(\left\langle\mathrm{C}_{\mathrm{v}}\right\rangle\right)$ for each measurement location and target pressure are consistent for duplicate tests.

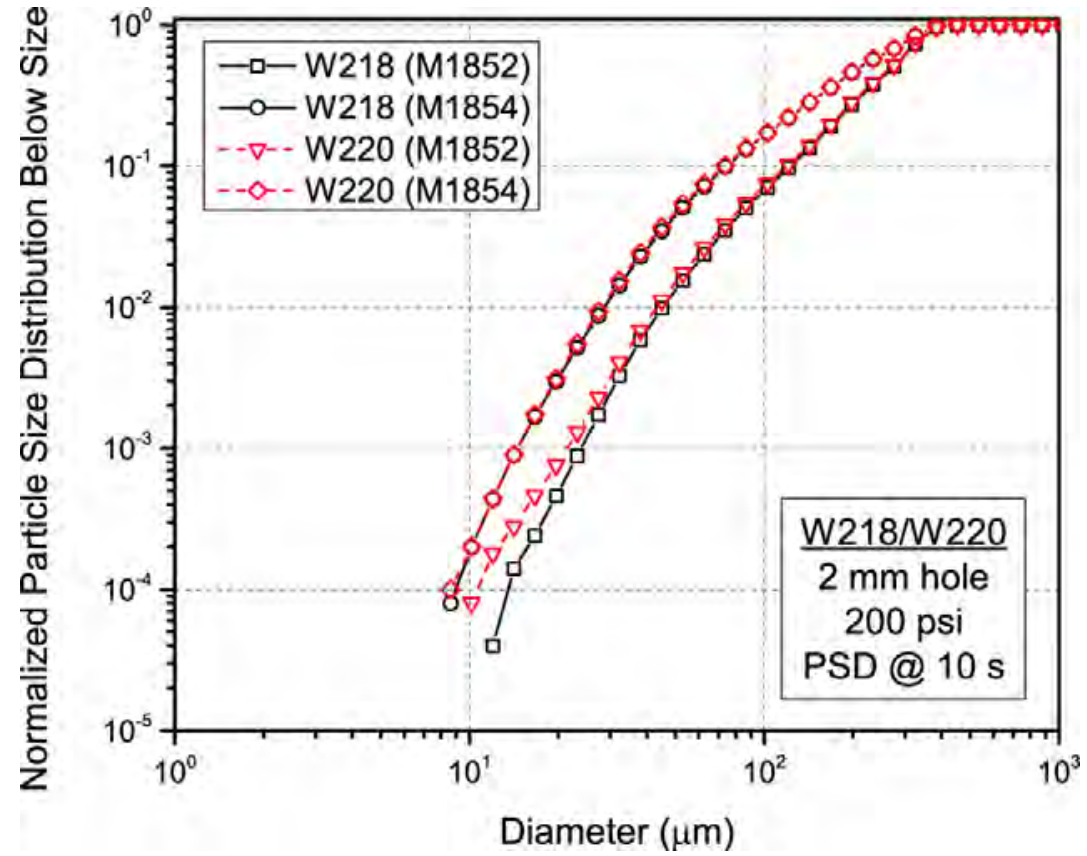

Figure 8.30. Comparison of In-Spray Cumulative PSDs from Duplicate Tests. The comparison includes two measurement locations: 1) Malvern Instrument M1854 at 23.5 in. from the spray (squares/triangles), and 2) Malvern Instrument M1852 at 59 in. from the spray (circles/diamonds). The tests were conducted at 200 psi with water using a 2-mm orifice. The PSDs are $5 \mathrm{~s}$ averages centered on an elapsed time of $10 \mathrm{~s}$ from the start of the spray test.

Table 8.4. Average Total Volume Concentration for In-Spray Measurements for the 2-mm Orifice

\begin{tabular}{cccc}
\hline Test & Conditions & $\left\langle\mathrm{C}_{\mathrm{v}}\right\rangle$, M1852 (ppmv) & $\left\langle\mathrm{C}_{\mathrm{v}}\right\rangle$, M1854 (ppmv) \\
\hline W218 & 2-mm orifice, 200 psi & 148.8 & 43.2 \\
W219 & 2-mm orifice, 380 psi & 272.9 & 90.1 \\
W220 & 2-mm orifice, 200 psi & 153.5 & 44.2 \\
W221 & 2-mm orifice, 380 psi & 278.1 & 89.7 \\
\hline
\end{tabular}


The normalized droplet size distributions for the 1-mm and 2-mm orifices at both pressures tested (200 and 380 psi) are shown in Figure 8.31 and Figure 8.32, respectively. These in-spray measurements are shown at both measurement locations using the droplet size distribution at $10 \mathrm{~s}$. The data shows, as before, that the droplet size distribution is a function of distance from the orifice. The differences in the two data sets illustrate a further impediment to evaluation of in-spray data: orifices of different size will have different functionalities. The 1-mm orifice data in Figure 8.31 indicate a small difference between the 23.5- and 59-in. distances. At both pressures, the droplet size distribution measured at $23.5 \mathrm{in}$. contains a larger fraction of small droplets below around $50 \mu \mathrm{m}$ than the droplet size distribution measured at 59 in. This difference is greatly amplified in the 2-mm orifice data shown in Figure 8.32. The droplet size distributions do not converge until the droplets are $200 \mu \mathrm{m}$ in diameter or larger. Both figures also suggest that pressure modifies the spray behavior with distance because the spread between distributions in the $380 \mathrm{psi}$ data is different in magnitude than the corresponding spread in the $200 \mathrm{psi}$ data. This illustrates the complexity of interpreting the in-spray measurements.

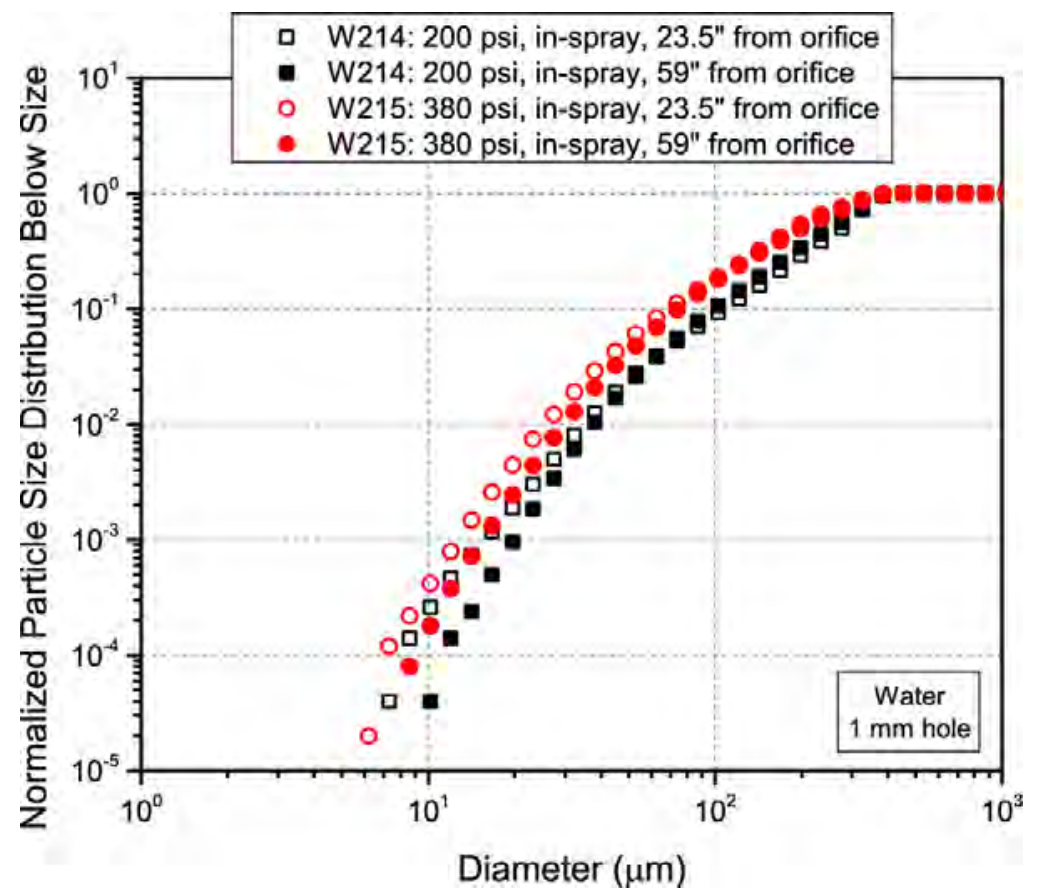

Figure 8.31. In-Spray Cumulative PSDs. Measurements are from tests using water and a 1-mm orifice at 200 psi (black squares) and 380 psi (red circles). The PSDs are $5 \mathrm{~s}$ averages centered on an elapsed time of $10 \mathrm{~s}$ from the start of the test.

The droplet size distributions obtained for 1-mm and 2-mm orifices at common measurement locations and target pressures are quite similar in shape and in magnitude. The primary difference is the effect the distance from the spray origin has on the two droplet size distributions. In general, data for the 2-mm orifices have a droplet size distribution that has a smaller fraction of droplets at any given diameter than the $1 \mathrm{~mm}$ data when the measurement is performed at $59 \mathrm{in}$. from the orifice. At $23.5 \mathrm{in}$. from the orifice, the opposite is true. The $2 \mathrm{~mm}$ data have a droplet size distribution with a larger fraction of droplets than the $1 \mathrm{~mm}$ data. Thus, the spread is wider between the two measurement locations in the $2 \mathrm{~mm}$ data because the droplet size distributions are moving in opposite directions relative to the same 
test with a 1-mm orifice. The "widening" of the data demonstrates how challenging measurements of this type would be for much larger orifices.

It is important to note that the Malvern instrument settings used to post-analyze and export the data affected the results. The effect was generally observed at volume fractions of $10^{-3}$ or less. The results presented in this section for the in-spray measurements were generated by assuming that the spray was centrally located and confined to a narrow region (i.e., a region approximately $6 \mathrm{~cm}$ in diameter). Typically, when the Malvern instruments were mounted in the chamber outside of the spray, the aerosol was assumed to be spread uniformly across the measurement zone. For the in-spray data, if a wider spray was assumed, the result was a bi-modal droplet distribution with widely separated peaks that seemed unphysical for water droplet formation. The presence of a secondary peak in the in-chamber measurements may be due to an artifact of the instrument calculation, but it is not as pronounced as it was for the in-spray data. Note also that the secondary peak usually occurs when the volume fraction or release fraction becomes small $\left(<10^{-3}\right)$, which is a region in which the Malvern Insitec-S was not as accurate and the results more variable (see Section 5.3.2.2). Understanding the effect that Insitec-S instrument configuration and properties have on the resultant data is the subject of ongoing work.

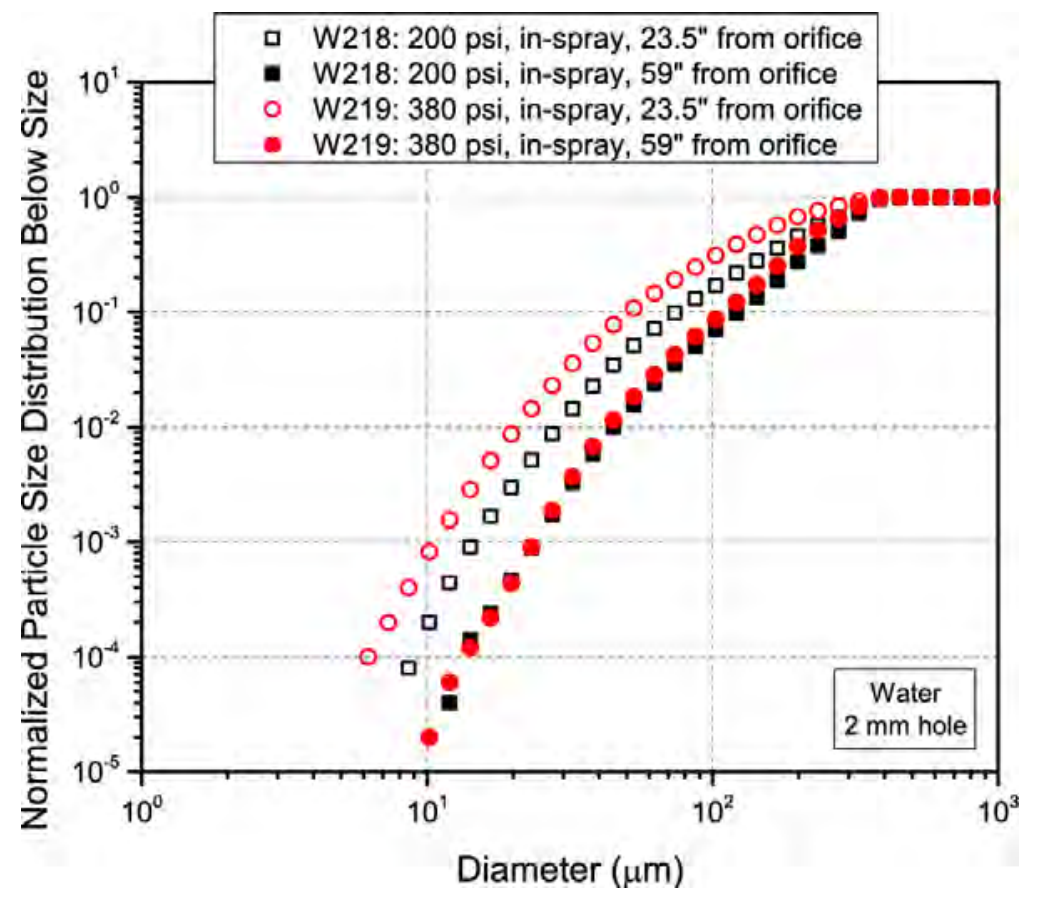

Figure 8.32. In-Spray Cumulative PSDs. Measurements are from tests using water and a 2-mm orifice at 200 psi (black squares) and 380 psi (red circles). The PSDs are 5 s averages centered on an elapsed time of $10 \mathrm{~s}$ from the start of the test.

\subsection{Summary and Conclusions}

The results presented in this section focused on the effect of parameter variations on either the cumulative release fraction or generation rate as a function of droplet diameter. Before these effects could be discussed, some results were presented supporting configuration choices that were made during the testing, namely: 
- Verifying that target pressures were achieved and the spray leak flow rate estimates were consistent with expectations

- Demonstrating that the fans employed during the testing (two 4-in. bilge fans, operated at $12 \mathrm{~V}$ ) promoted mixing and did not lead to additional chamber inhomogeneities

- Demonstrating that the Malvern instrument locations used in testing were accurate representations of the chamber concentration by comparing data obtained at other locations

- Confirming the results obtained from the Malvern instruments with a secondary aerosol instrument, the PPC

- Assessing the reproducibility of the tests and estimating an approximate test-to-test uncertainty.

Once these were established, comparisons between large-scale test data and WTP model predictions were made for several different parameters. This yielded the following conclusions:

1. As pressure increases, the cumulative release fraction increases. This increase with pressure was shown to be greater than the rate of increase in the WTP model, especially at smaller droplet sizes.

2. As orifice area increases, the cumulative release fraction decreases. The rate of decrease in the test data is smaller than the decrease projected by the WTP model. The cumulative generation rate still increases with area because the total spray flow rate is increasing. The dependence on area was found to hold regardless of orifice shape (whether the orifice was round or rectangular) and the orientation of rectangular orifices did not have an appreciable effect on the release fraction. As pressure decreases, the decrease in release fraction with increasing orifice area becomes smaller, perhaps even being independent of area. The release fractions from the test data approached the WTP model at an orifice area of approximately 30 to $100 \mathrm{~mm}^{2}$.

3. As viscosity and density of the fluid increases, the cumulative release fraction is unchanged. This is comparable to the WTP model prediction. Not enough data was collected to determine the separate roles of viscosity or density.

4. As the weight fraction of solids increases, the cumulative release fraction is unaffected for droplets $>10 \mu \mathrm{m}$. This is consistent with the WTP model, because it accounts for the presence of solids only by changes in the physical properties of the fluid. There was some deviation from water at droplet sizes $<10 \mu \mathrm{m}$; however, differences below this droplet size are of minor concern.

5. As the distance from the splash wall increases, the cumulative release fraction increases. The WTP model does not consider the effect of obstructions such as walls. Data collected to support this conclusion suggest that sprays that travel longer distances than the large-scale system permitted may approach the WTP model at even smaller orifice sizes than would be predicted by the area functionality alone.

6. In-spray measurements are difficult to interpret as they are strong functions of position, pressure, orifice size, and Malvern analysis settings. They do, however, represent an upper bound on the release fraction for a particular spray (in the absence of splatter) and can be used to estimate worst cases (see Chapter 9).

Overall, the results indicate a straightforward functionality of the cumulative release fraction on pressure and orifice area. Viscosity and the weight fraction of solids had a negligible effect. The configuration and geometry of the spray also is important, as evidenced by the splash wall distance and 
in-spray aerosol tests. In general, the WTP model has the correct qualitative behavior but either has too strong or too weak a dependence on a quantity (pressure and orifice area, respectively) or ignores a quantity altogether (distance from a splash wall). The data indicate that the model may not be an overestimate for all sprays; actual release fractions may approach the model predictions at some of the limits of the test matrix investigated in this report. 



\subsection{Comparison of Small- and Large-Scale Test Results and Extrapolation of Results to Larger Slots}

Tests were conducted in both the small- and large- scale test chambers where the tests evaluated the roles of some identical parameters, and some of the tests were conducted with identical orifices (same target dimensions), test conditions, and fluids to quantify any differences between the test results in two different size chambers. As described in Chapter 1, the overall technical approach is to measure the generation rate of aerosol droplets of different sizes for specific sprays. Ideally, this measured result will be the same in both test chambers, thereby allowing the result to be applied to any size enclosure. In Section 9.1, a comparison between the small- and large-scale test chamber results are presented for identical orifice and test conditions that show the generally similar test results for droplet generation when the sprays travel the full distance of the chamber but different release fraction results for sprays of identical length in the two different chambers. Sections 9.2 through 9.5 compare the small- and large-chamber test results for the effects of the key parameters of slot and circular orifice size, the presence of slurry particles, the increase in viscosity and density for salt solutions, and the length of the sprays within the chambers. Finally, Section 9.6 uses the current test results showing the effect of orifice size and non-Newtonian rheology to extrapolate to the largest postulated slot for a non-Newtonian slurry.

\subsection{Comparison of Concentrations and Release Fractions and the Role of Chamber Size}

For tests with identical orifices and test conditions conducted in the small and large chambers, the results should give, ideally, the same total generation rates and release fractions in both chambers. Because of the increased volume of the larger chamber, the aerosol concentrations and the rate of concentration increase will be smaller, as discussed in Chapters 1 and 7. In actual tests, the dynamics of the aerosol droplets in the different chambers will influence the test results.

Figure 9.1 shows a comparison of the transient cumulative droplet concentration in the small and large test chambers for 380 psi water sprays from 1-mm circular orifices (same target dimensions) where the sprays traveled essentially the full length of each chamber. The two results are shown with the same ranges for both the $\mathrm{x}$ - and $\mathrm{y}$-axis to allow a direct comparison. Note that, as expected, the concentrations are higher in the small-scale chamber. For the small-scale chamber, the rate of concentration increase, or the slope of the droplet concentration with time, also is much higher. For example, the concentration of droplets from small-scale testing that are $<19.7 \mu \mathrm{m}$ increased to $\sim 0.5 \mathrm{ppmv}$ in $10 \mathrm{~s}$, while the concentration from large-scale testing increases to only $0.05 \mathrm{ppmv}$ in about $30 \mathrm{~s}$. This is a thirtyfold slower rate of concentration increase in the large-scale chamber, which is essentially equal to the fortyfold difference in the large- and small-scale chamber volumes (see Chapter 5 and Mahoney et al. 2012b). 
OS7: Water, $1 \mathrm{~mm}$ axial orifice, 380 psig; Malvern in position 2

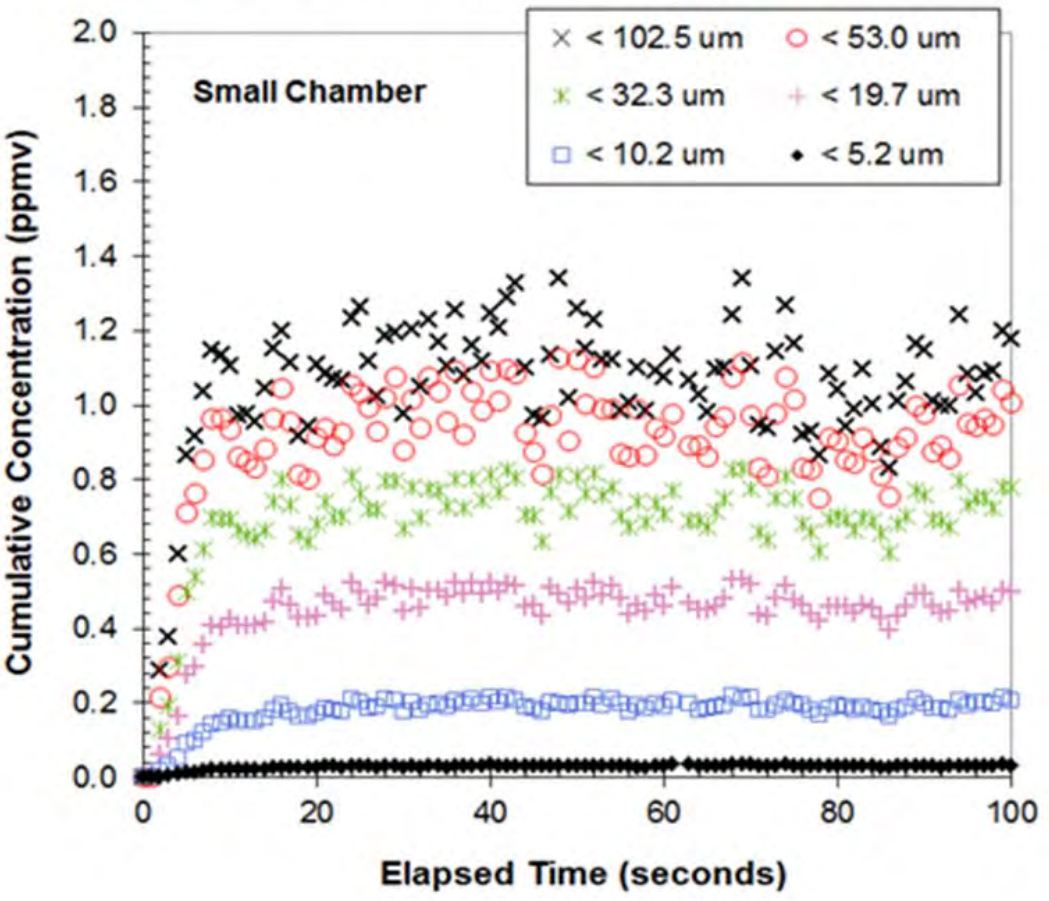

Test W74 : Average of 3 Malverns ; 380 psi ; Orifice S1A - round, 1 mm

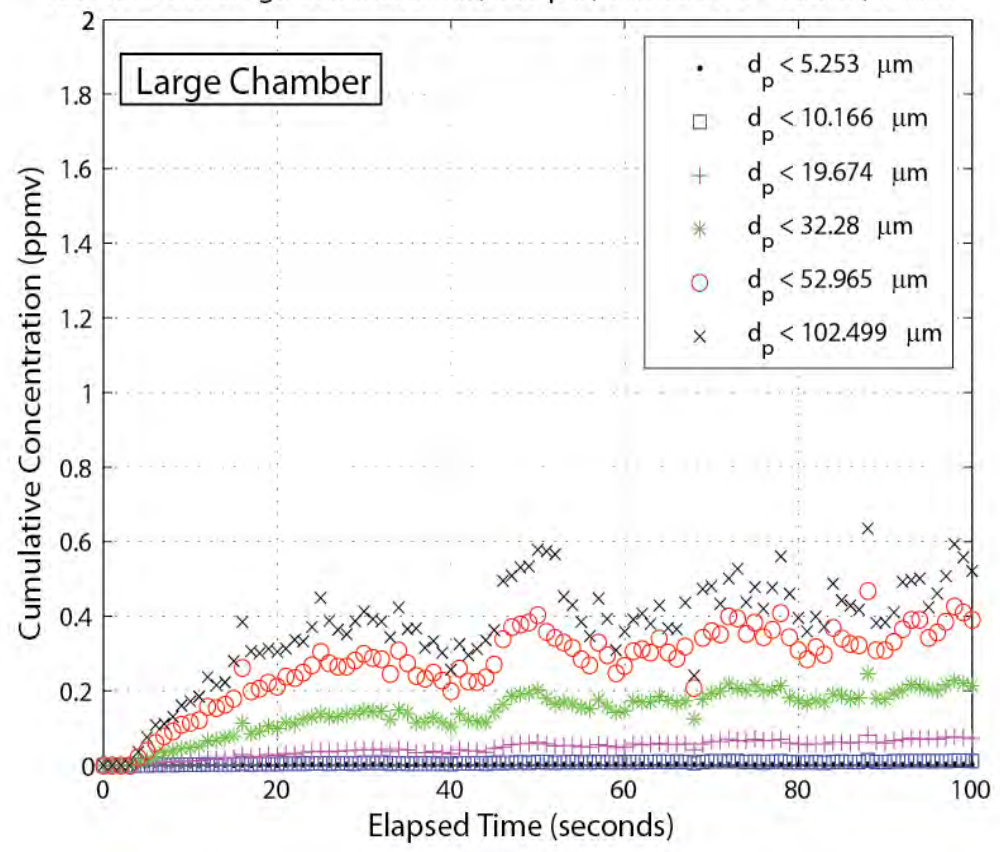

Figure 9.1. Comparison of the Transient Cumulative Droplet Concentrations in the Small and Large Chambers for 380 psi Water Sprays from 1-mm Circular Orifices 
The transient droplet concentrations can be analyzed to determine the droplet generation rate and release fractions. Comparisons between the small- and large-chamber release fractions are shown in Figure 9.2, Figure 9.3, and Figure 9.4 for 380 psi water sprays from 1-and 2-mm circular orifices and from $0.5 \times 5$-mm slots (actual dimensions for each chamber were slightly different from these target sizes). These plots also show the WTP model results - note that the model results are identical for both chamber sizes. In these comparisons, the sprays traveled essentially the full length of each chamber and so the sprays had similar lengths relative to the chamber size (additional figures below will compare sprays that had the same actual length within the small and large chambers and thus had different lengths relative to each chamber). For these water sprays, the comparison of release fraction results for the small and large test chambers shows a difference in release fraction varying from none to as much as fivefold for droplets between 10 and $100 \mu \mathrm{m}$ (large-scale tests gave lower release fractions for droplets below about $30 \mu \mathrm{m}$ and higher release fractions above this size). While differences exist between these release fraction results in the different chambers, given the fortyfold difference in chamber volume the overall agreement in the release fraction results is reasonably good.

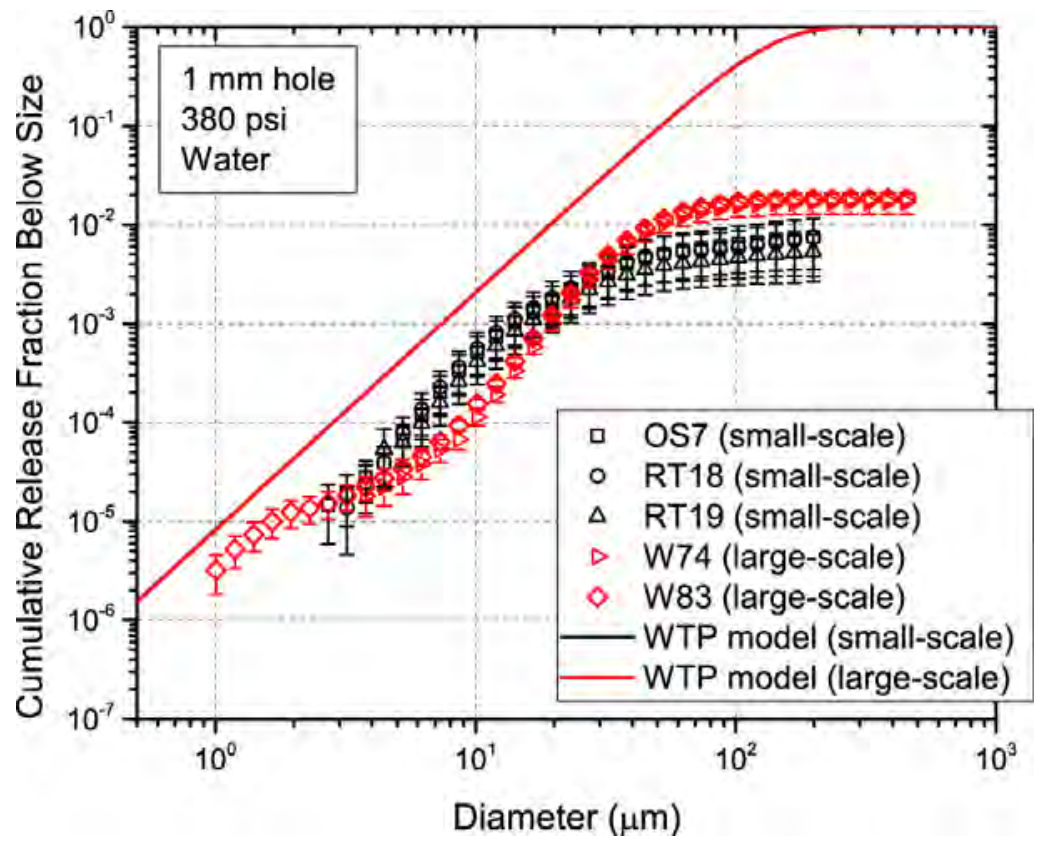

Figure 9.2. Comparison of Release Fractions in the Small and Large Chambers for 380 psi Water Sprays from a 1-mm Circular Orifice Where the Sprays Traveled Essentially the Full Length of Each Chamber

Figure 9.5 and Figure 9.6 show similar comparisons of release fractions for the small and large chambers for slurry sprays with 8 and $20 \mathrm{wt} \%$ STR simulant, respectively. For these tests, the sprays again traveled essentially the full length of each chamber. Similar to the water results discussed above, the release fractions in the small-scale chamber are higher than in the large-scale chamber for droplets below about $30 \mu \mathrm{m}$ and have lower release fractions above this droplet size. The largest difference between the small and large-scale release fractions for droplets between 10 and $100 \mu \mathrm{m}$ is again about a factor of five. 


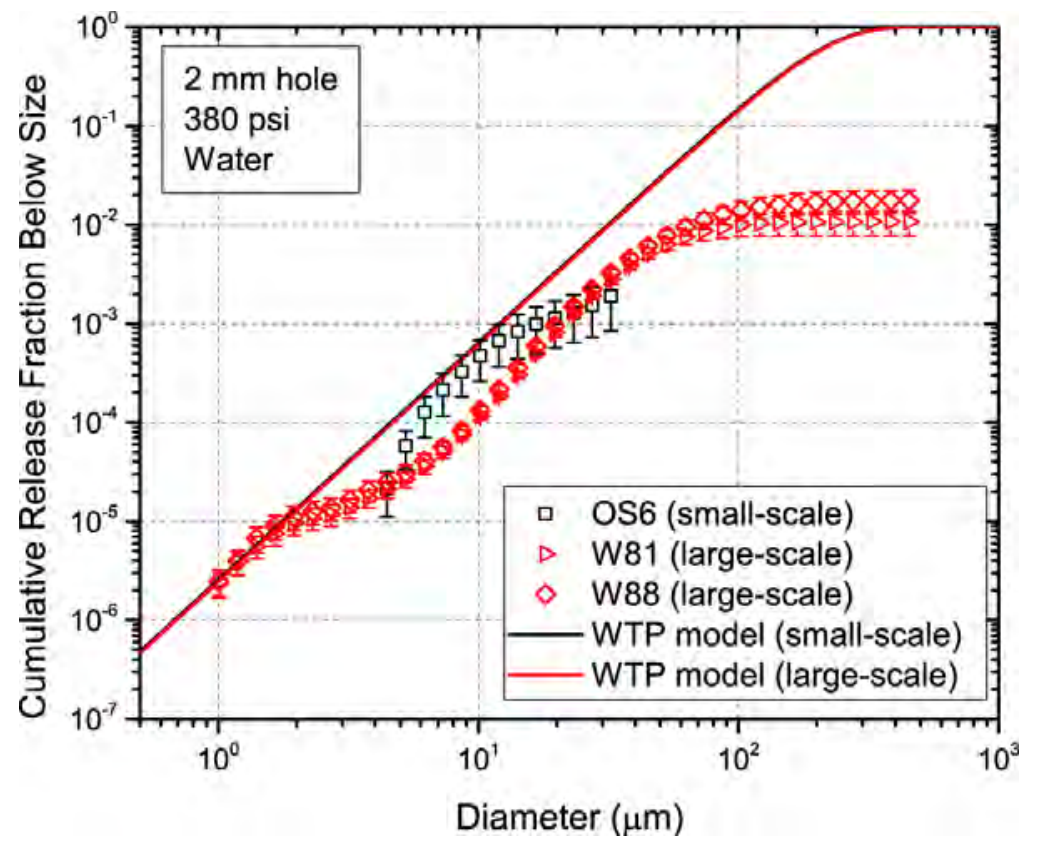

Figure 9.3. Comparison of Release Fractions in the Small and Large Chambers for 380 psi Water Sprays form a 2-mm Circular Orifice Where the Sprays Traveled Essentially the Full Length of Each Chamber

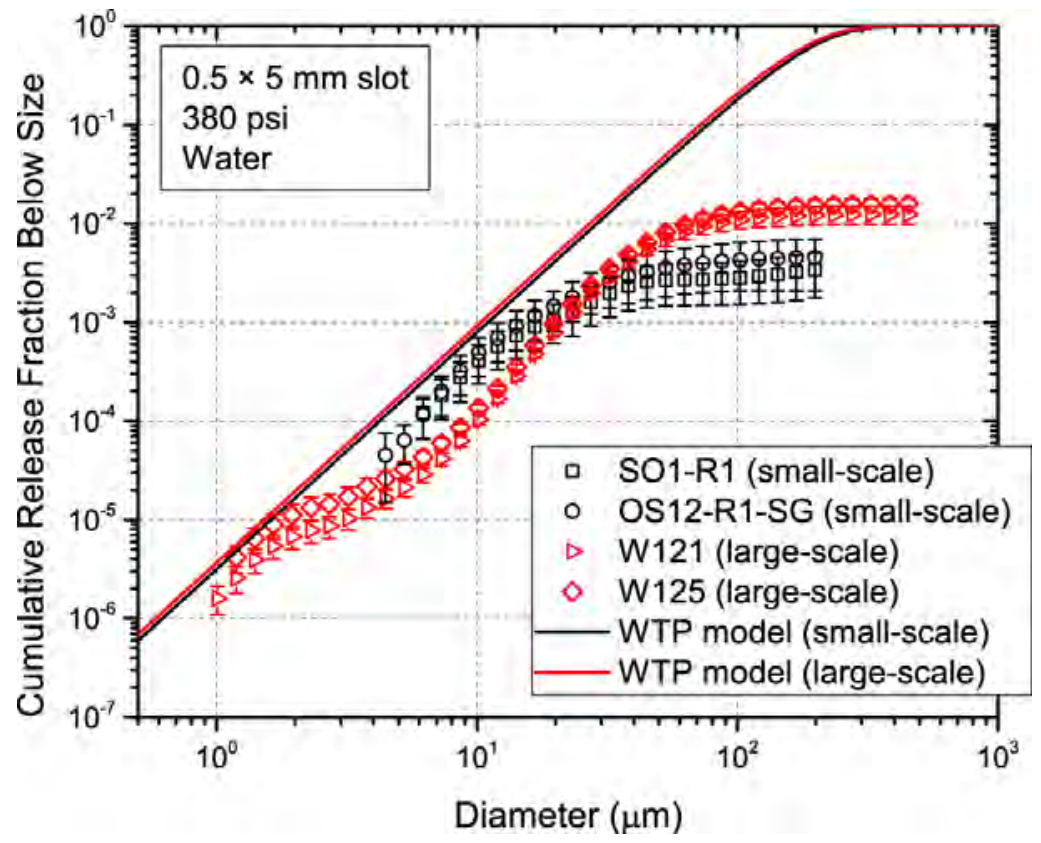

Figure 9.4. Comparison of Release Fractions in the Small and Large Chambers for 380 psi Water Sprays from a $0.5 \times 5-\mathrm{mm}$ Slot Where the Sprays Traveled Essentially the Full Length of Each Chamber 


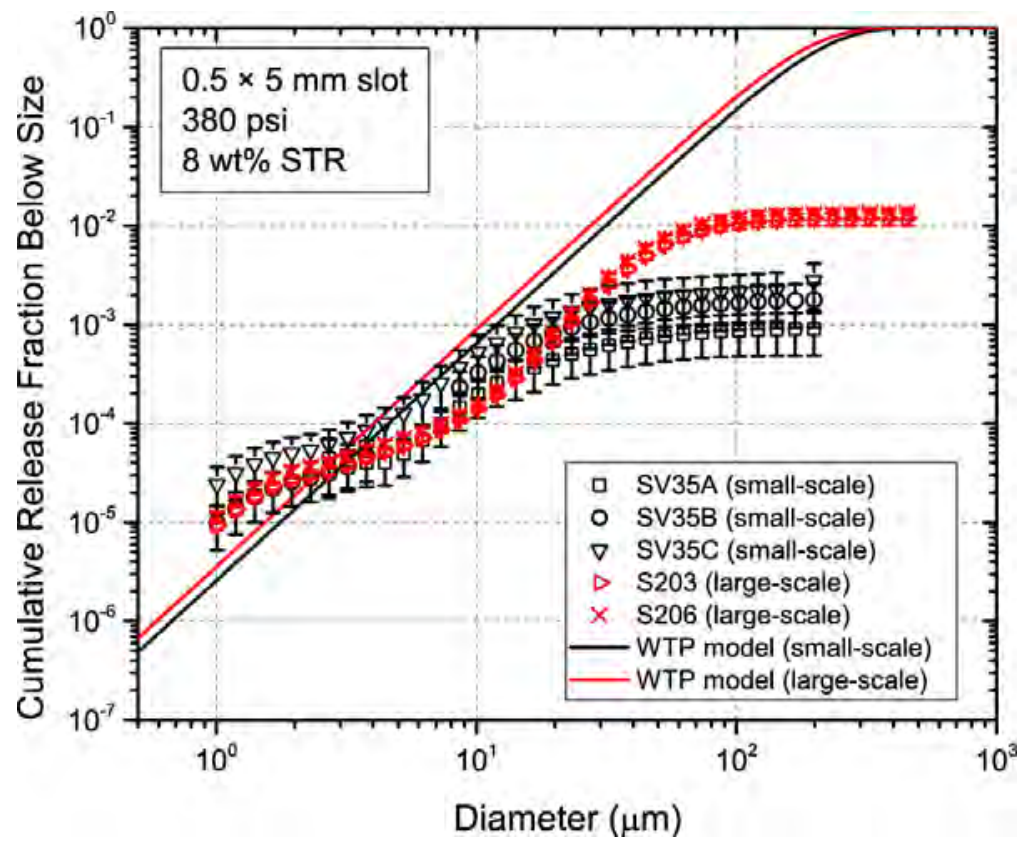

Figure 9.5. Comparison of Release Fractions in the Small and Large Chambers for $8 \mathrm{wt} \%$ STR Slurry Sprays at 380 psi from a $0.5 \times 5-\mathrm{mm}$ Slot Where the Sprays Traveled Essentially the Full Length of Each Chamber

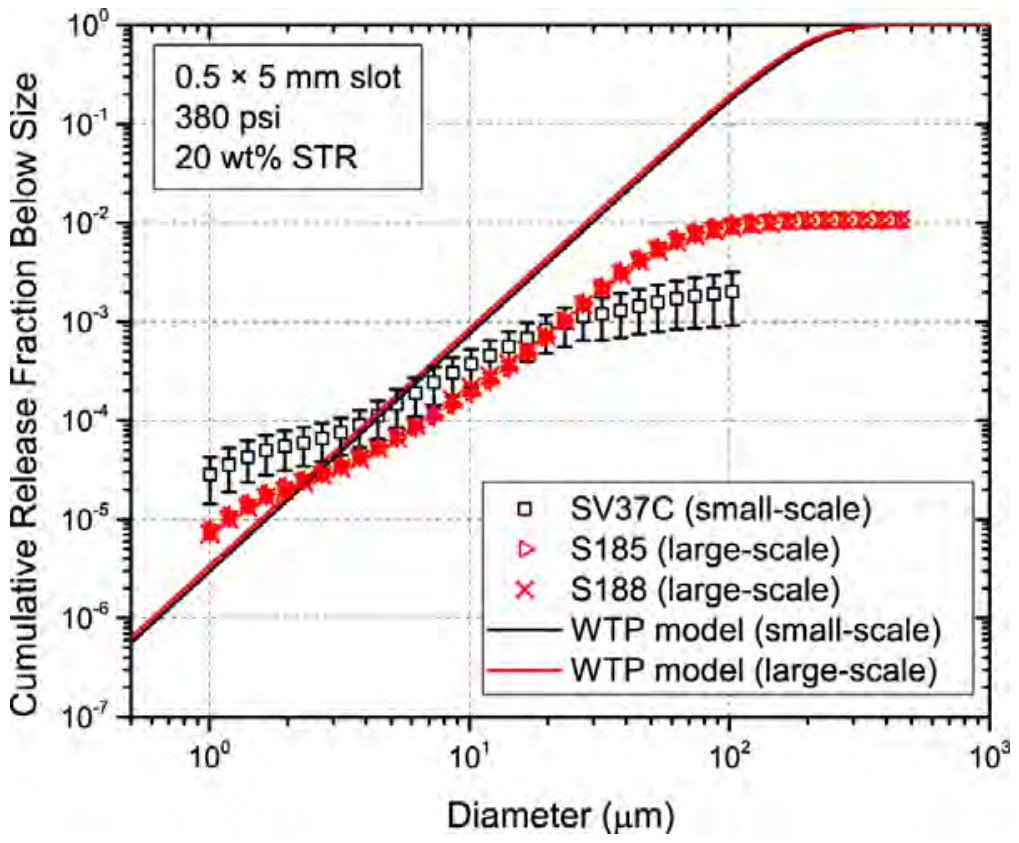

Figure 9.6. Comparison of Release Fractions in the Small and Large Chambers for $20 \mathrm{wt} \%$ STR Slurry Spray at 380 psi from a $0.5 \times 5-\mathrm{mm}$ Slot Where the Sprays Traveled Essentially the Full Length of Each Chamber 
Although testing with a systematic variation in chamber size was not conducted, it is likely that the different shapes for the release fraction curves result from the different aerosol dynamics in the different size chambers. For example, in the small chamber the larger droplets likely impact the far wall and are captured, while in the larger chamber these larger droplets have more time to slow down and fewer of these droplets may impact the wall. In the larger chamber, the aerosol instruments detect the larger droplets, and this results in a higher release fraction of the larger droplets compared to the release fraction for larger droplets in the small chamber.

For the sprays that traveled the full length of the chambers, there are differences between the release fraction results in the small- and large-scale chambers but the results also show general overall agreement for sprays with both water and STR slurries. Considering the difference in chamber size, this agreement is reasonably good and suggests that sprays in even larger chambers will have similar results. The comparison of the results also suggests that for yet larger chambers the release fractions may increase further for droplets above about $30 \mu \mathrm{m}$ and may decrease for droplets below this size. With tests results available in only two chamber sizes, estimates of release fractions in larger chambers or full-scale facilities are uncertain. Based on the reasonable comparisons in Figure 9.2 through Figure 9.6 for sprays that traveled the full length of each chamber, additional tests are being conducted for a range of chamber sizes to quantify the effect of chamber size and to reduce uncertainty in the aerosol results. One common approach in conducting scale-up experiments is to conduct tests in systems that maintain geometric similarity but are of different size (Zlokarnik 2006, Tatterson 2003). Sprays that travel the full length of the small and large chambers are geometrically similar in this sense.

As mentioned earlier in this section, tests were also conducted in the small and large chambers where sprays had the same length. The purpose in comparing identical sprays of the same length is that, ideally, the measured release fractions in different size chambers would be identical. This purpose is similar to the objective of comparing the results in different size chambers but with the sprays traveling the full length of the chamber. This alternate way of comparing release fractions in different size chambers can provide insight into the role of chamber size and how best to estimate release fractions in larger chambers. In these tests, 380 psi sprays from 1- and 2-mm holes traveled approximately 42 in. in both chambers. For the small-scale chamber, this was essentially the full length of the chamber and in the large-scale chamber the spray orifice was located 43.25 in. away from the splash wall. Figure 9.7 and Figure 9.8 show the comparison of the release fractions. For both sets of tests, the large-scale release fractions are lower for all droplet sizes.

These results show that the chamber size certainly affects the measured release fractions and the effect with sprays of the same length is larger than when the sprays traveled the full length of each chamber. Had the comparison of same-length sprays shown similar release fractions, estimates of release fractions for sprays in larger chambers or facilities may have been done by evaluating sprays of the same length. However, the comparisons of results when the sprays travel the full length of the chamber appears to provide more similar results and are preferred for estimating the release fractions in larger chambers and facilities. 


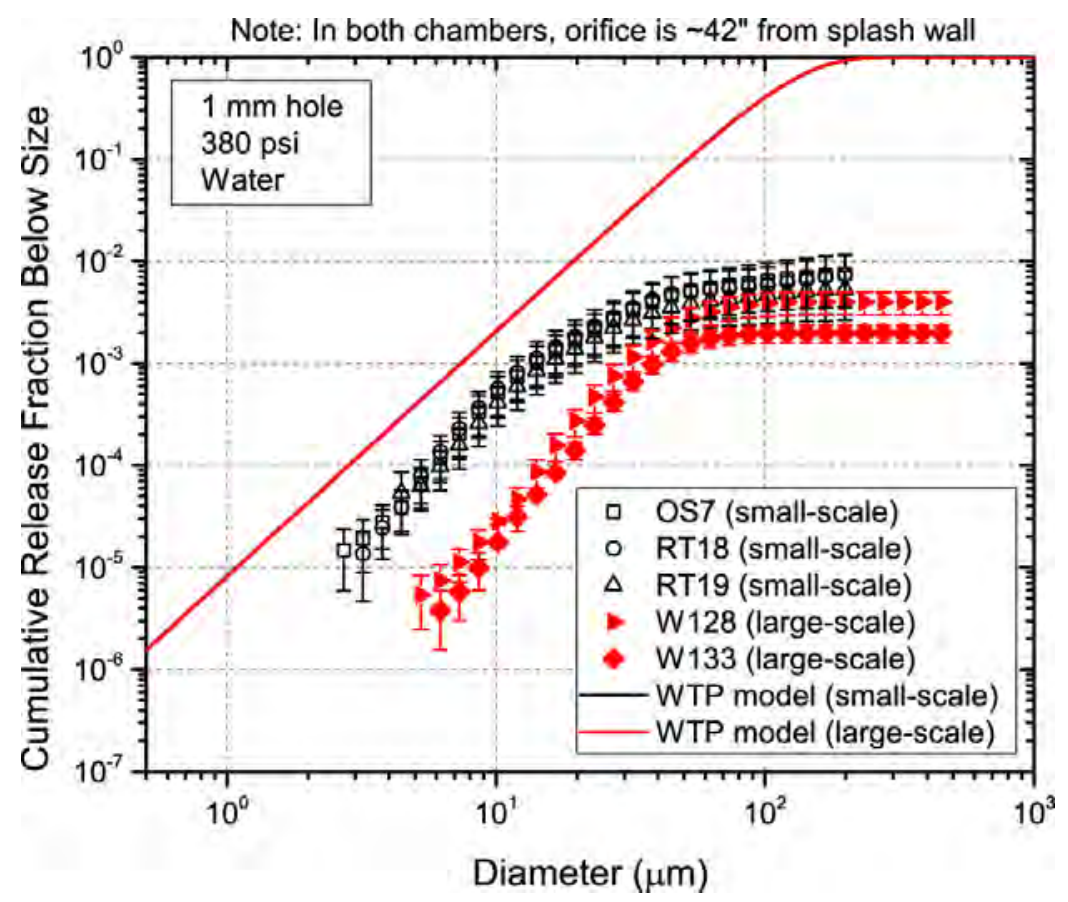

Figure 9.7. Comparison of Release Fractions in the Small and Large Chambers for 380 psi Water Sprays from a 1-mm Circular Orifice Where the Spray Length is Approximately 42 in. in Each Chamber

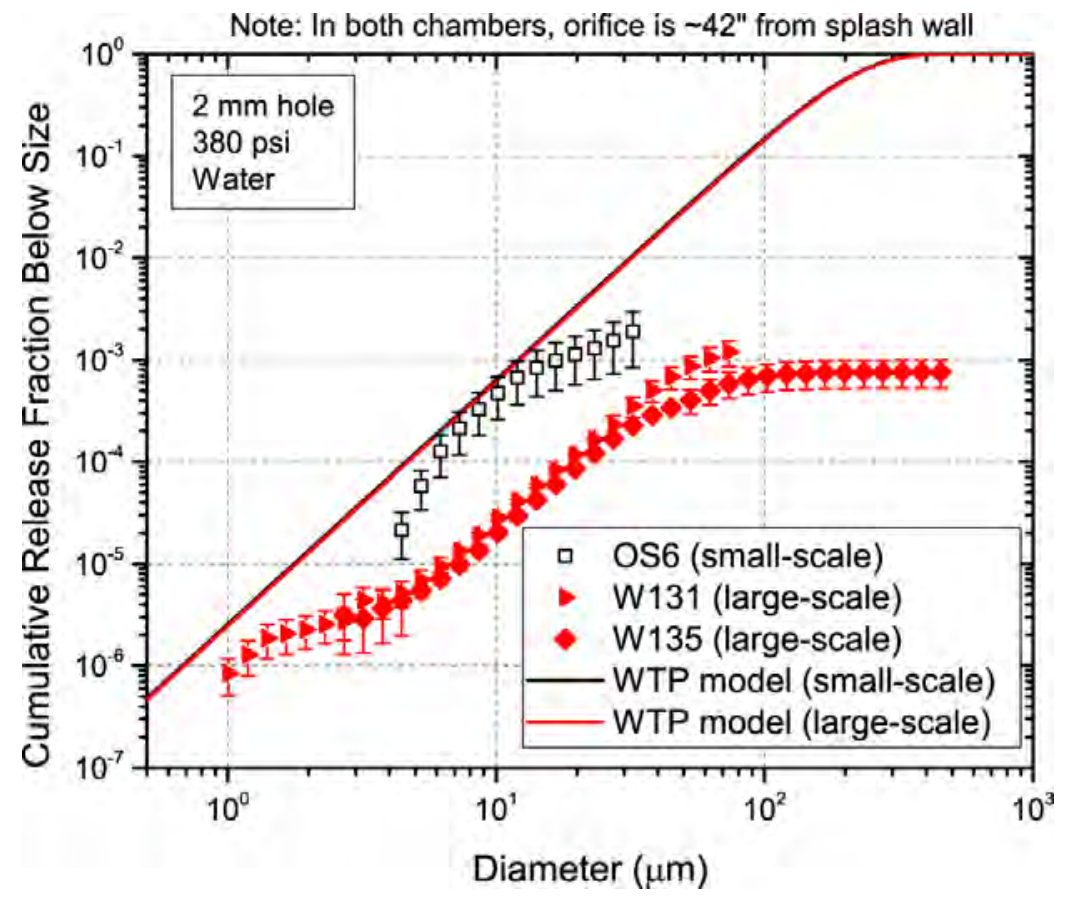

Figure 9.8. Comparison of Release Fractions in the Small and Large Chambers for 380 psi Water Sprays from a 2-mm Circular Orifice Where the Spray Length is approximately 42 in. in Each Chamber 
Overall, the comparisons of release fractions in different size chambers show generally similar release fraction results for sprays that travel the full length of the chamber. The generally similar release fractions, considering the substantially different sizes of the test chambers, is a useful and significant finding of the testing. This result is particularly important when considering how to estimate the aerosol release fraction from longer distance sprays in larger chambers or facilities. The generally similar release fractions in the two different size chambers, when the sprays traveled the entire length of the chamber, suggests that a spray that spans the full length of an even larger chamber or room will be similar to results obtained in this study. With only two chamber sizes, results are insufficient for estimating uncertainty in applying the current results to larger chambers and spray distances. The comparison of release fractions also demonstrates that the technical approach of using the transient droplet concentrations in closed chambers gives results that can be used to characterize the aerosol generation rate of sprays in other size test chambers or facilities. The topic of estimating the release fractions of sprays in larger chambers is discussed below in Section 9.6, and the different release fractions when the spray travels less than the full distance of the chamber, which was previously discussed in Section 8.5, is also discussed below.

\subsection{Comparison of the Effect of Circular Orifice and Slot Size}

The role of orifice and slot size on aerosol droplet generation rate and release fraction from sprays was discussed previously in Chapter 8 and by Mahoney et al. (2012b) for the small-scale test system. Figure 9.9 shows the comparison of the effect of orifice area on release fractions for 380 psig water sprays from circular orifices. The results are shown for three different droplet sizes selected from the release fraction results, such as those shown in Figure 9.2 and Figure 9.3. For the range of orifice sizes tested, the release fraction decreased with orifice area in a similar manner for both the small- and large-scale chambers. As noted in Section 9.1, the large-scale cumulative release fraction results tend to be less than the small-scale results for droplets that are $<10 \mu \mathrm{m}$, similar to the small-scale results for droplets $<32 \mu \mathrm{m}$, and larger than the small-scale results for droplets $<102 \mu \mathrm{m}$. These differences and the similar release fractions for droplets $<32 \mu \mathrm{m}$ are apparent in Figure 9.9. Figure 9.10 shows a similar comparison of results but in terms of the cumulative generation rate. The cumulative generation rate, as given by Equation (1.7), is the product of the flow rate from the orifice times the release fraction. As the orifice area increases, the flow from the orifice increases while the release fraction decreases. The overall combined effect is that the generation rate increases with increasing orifice area, and the small- and large-scale test results show a similar increasing trend in generation rate with increasing orifice area for the circular orifices. 


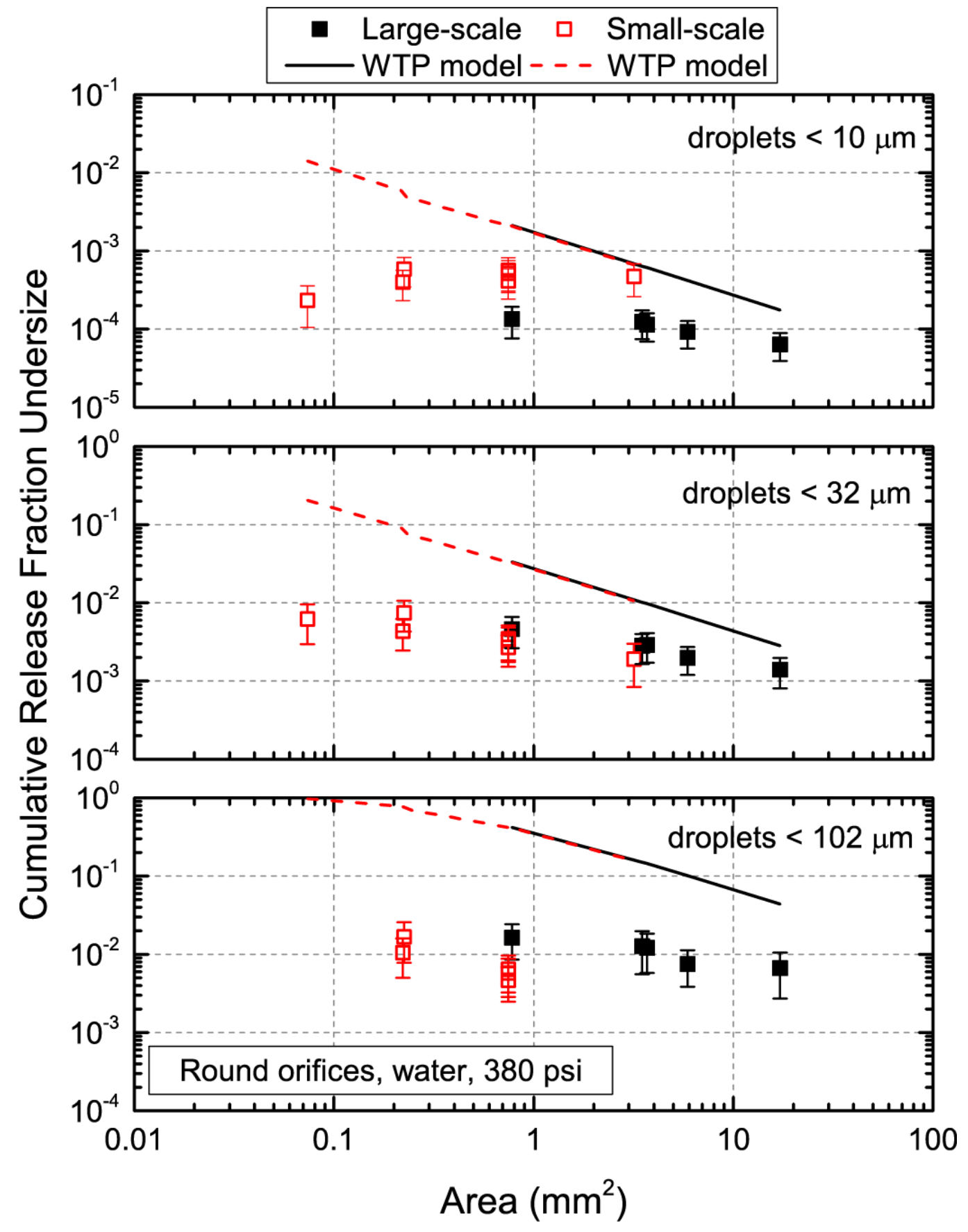

Figure 9.9. Comparison of the Effect of Orifice Area on Release Fractions in the Small- and Large-Scale Chambers for 380 psig Water Sprays from Circular Orifices (results shown separately for droplets $<10 \mu \mathrm{m}$ [top], $<32 \mu \mathrm{m}$ [middle], and $<102 \mu \mathrm{m}$ [bottom]). 


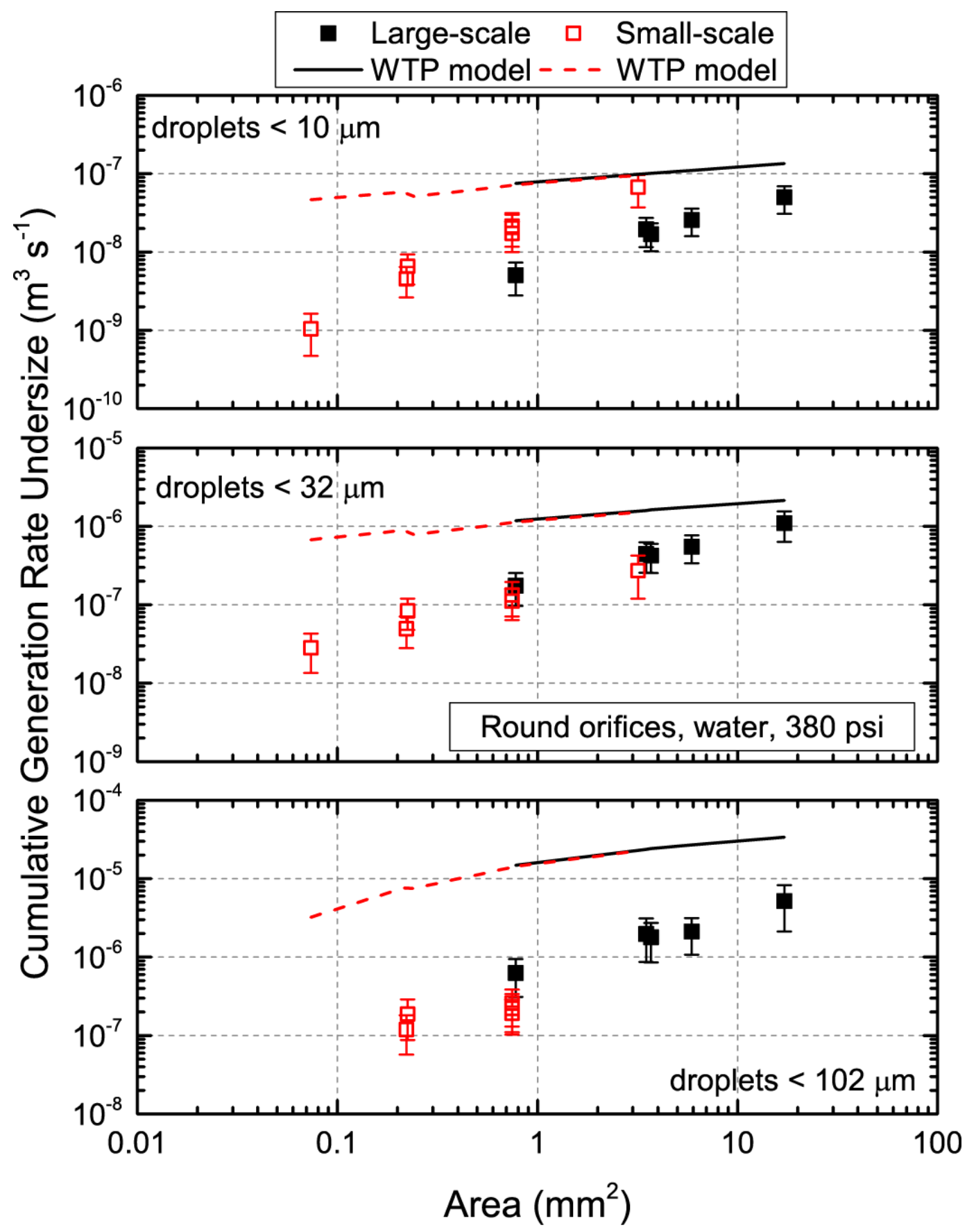

Figure 9.10. Comparison of the Effect of Orifice Area on Cumulative Generation Rate in the Small- and Large-Scale Chambers for 380 psig Water Sprays from Circular Orifices (results shown separately for droplets $<10 \mu \mathrm{m}$ [top], $<32 \mu \mathrm{m}$ [middle], and $<102 \mu \mathrm{m}$ [bottom]).

Figure 9.11 and Figure 9.12 show the comparison of small- and large-scale results for generation rate and release fraction for slots of different area. The large-scale test results show clear trends with increasing orifice area for both the release fraction and generation rate. The small-scale results do not show a trend as clearly with increasing orifice area, but they still generally agree with the large-scale results within the range of orifice area that was tested. Overall, this comparison of small- and large-scale 
test results confirms the overall trend of increasing generation rate with increasing orifice area for both circular orifices and slots. The large-scale test results are the best data for extrapolating to larger slots because the large-scale test results span a wider range of orifice areas than the small-scale tests and only need a small extrapolation to estimate the aerosol generation of the largest postulated slot. It is also apparent in Figure 9.11 and Figure 9.12 that the trends in the data, for both release fraction and generation rate, have different slopes than the WTP model. In Section 9.6 below, an extrapolation of the data to larger slots is provided and the extrapolated release fractions and generation rates exceed the WTP model. 


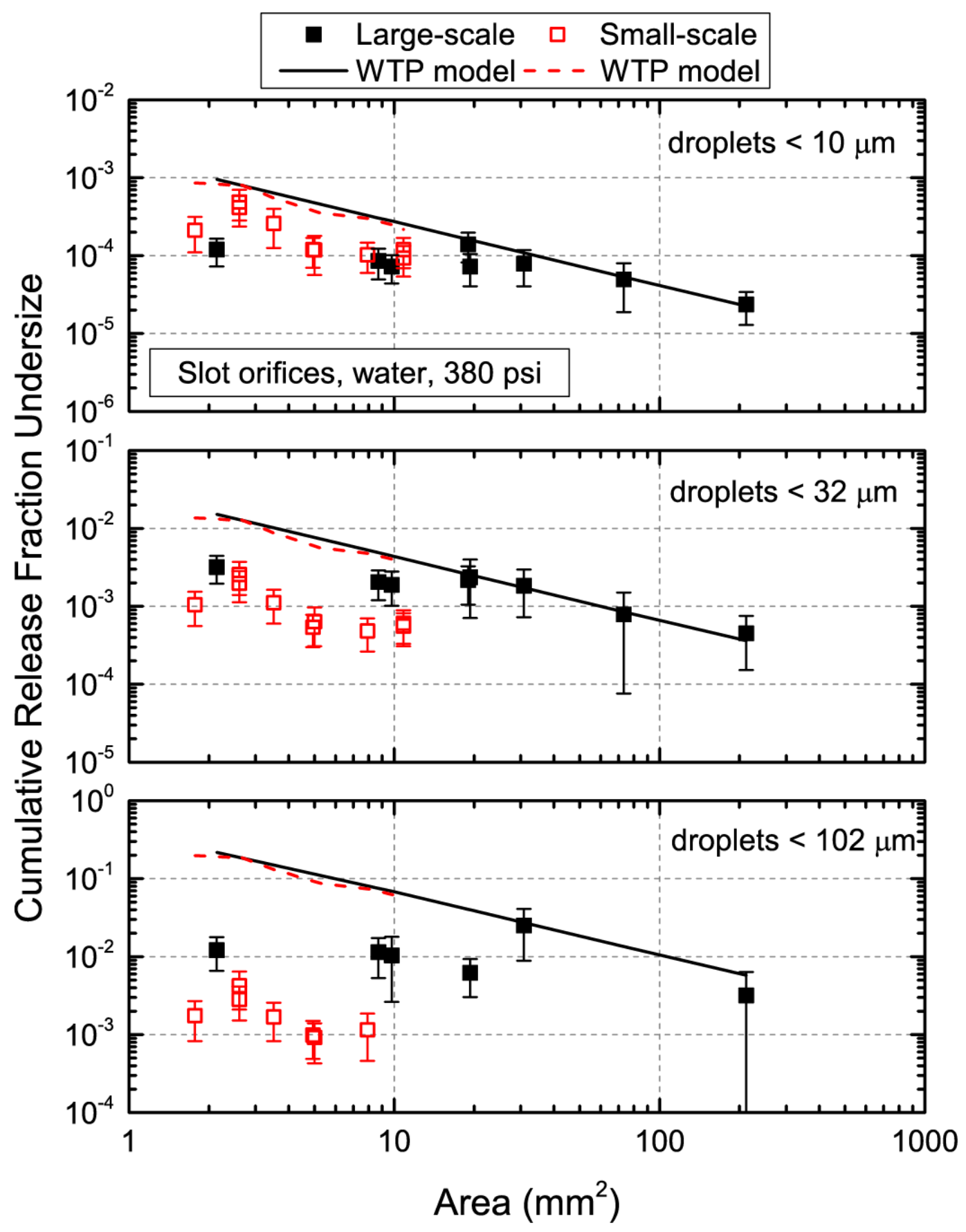

Figure 9.11. Comparison of the Effect of Orifice Area on Release Fractions in the Small- and Large-Scale Chambers for 380 psig Water Sprays from Slots (results shown separately for droplets $<10 \mu \mathrm{m}$ [(top], $<32 \mu \mathrm{m}$ [middle], and $<102 \mu \mathrm{m}$ [bottom]). 


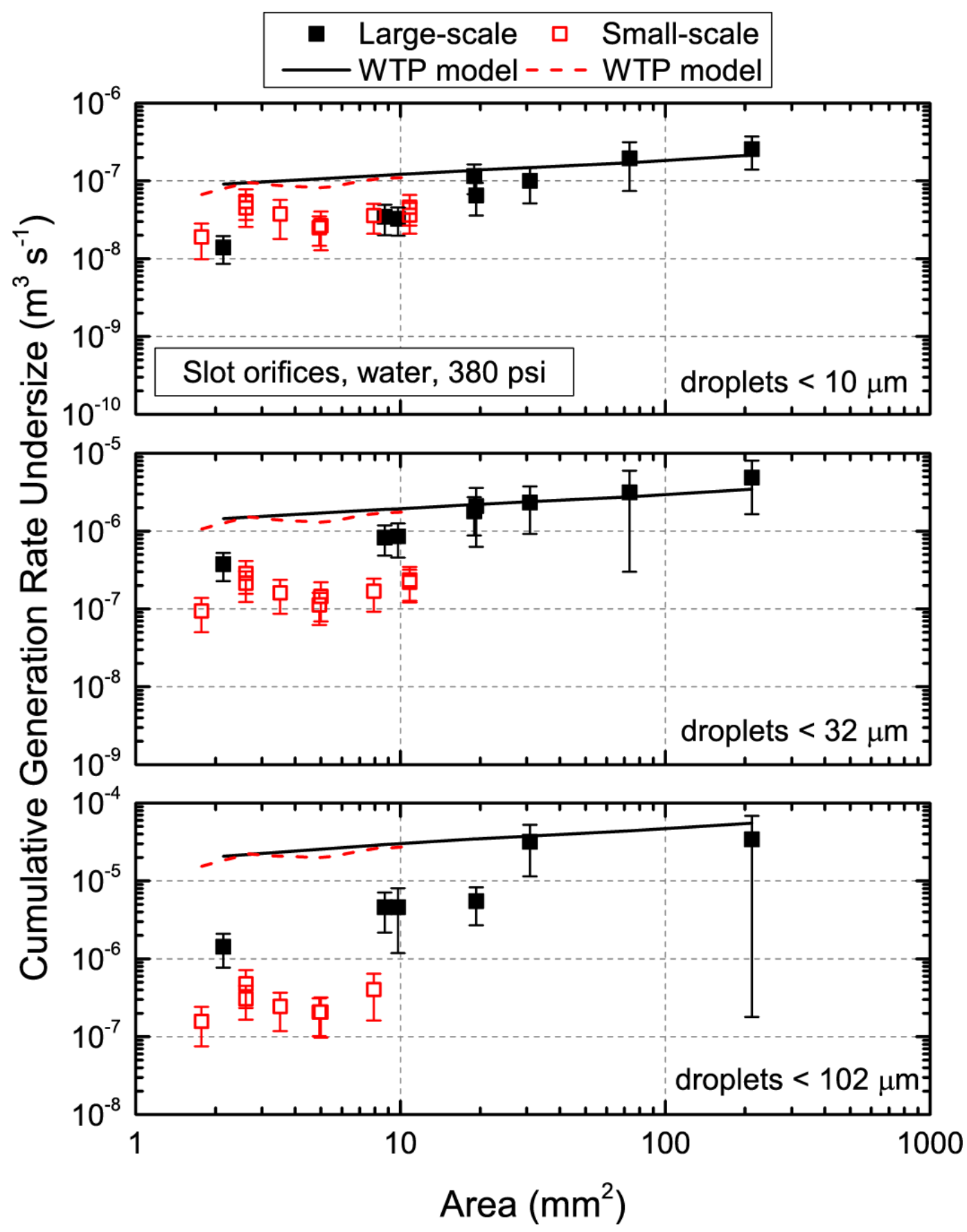

Figure 9.12. Comparison of the Effect of Orifice Area on Cumulative Generation Rate in the Small- and Large-Scale Chambers for 380 psig Water Sprays from Slots (results shown separately for droplets $<10 \mu \mathrm{m}$ [top], $<32 \mu \mathrm{m}$ [middle], and $<102 \mu \mathrm{m}$ [bottom]).

\subsection{Comparison of the Effect of Slurry Particles}

The small treated simulant (STR), which is composed of boehmite particles in water, was tested at both 8 and $20 \mathrm{wt} \%$ in both the small- and large-scale test chambers for $0.5 \times 5$-mm slots. In this section, the focus is on whether the small- and large-scale chamber results show similar comparisons between 
water and slurry sprays. Figure 9.13 shows a comparison of the release fractions between the STR simulants and water in the small-scale test chamber. Overall the release fractions are similar (other than one very low value for water at $\sim 3 \mu \mathrm{m}$ ) across the range of droplet sizes, with the STR simulants having the same or lower release fractions between 10 and $100 \mu \mathrm{m}$ droplets. Figure 9.14 shows an equivalent comparison between water and slurry sprays in the large-scale test chamber, again for the $0.5 \times 5$-mm slot. Results from the large-scale test chamber show an equivalent comparison between the water and slurry release fractions with the slurry release fractions being higher than the release fractions for water below about $20 \mu \mathrm{m}$ droplets and almost identical above $20 \mu \mathrm{m}$ droplets.

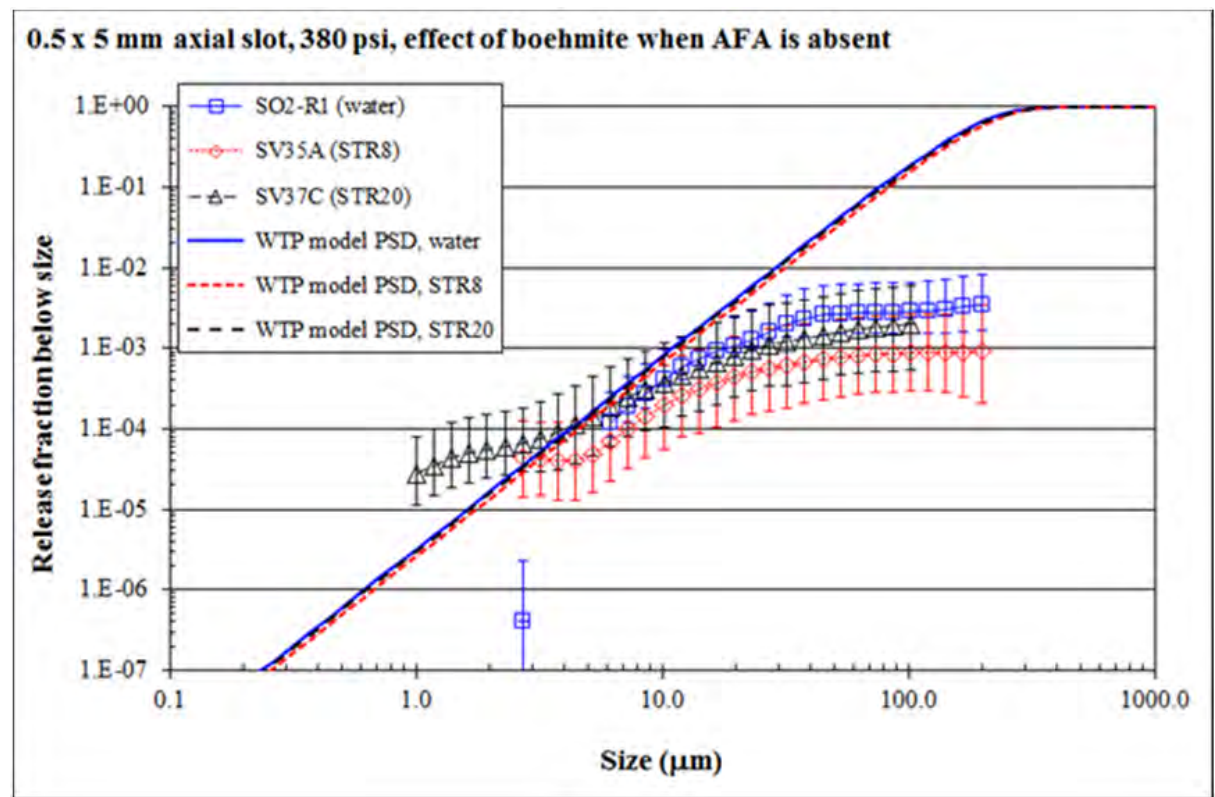

Figure 9.13. Comparison of Slurry Sprays for the STR Simulant at 8 and $20 \mathrm{wt} \%$ with Water Sprays in the Small-Scale Chamber 


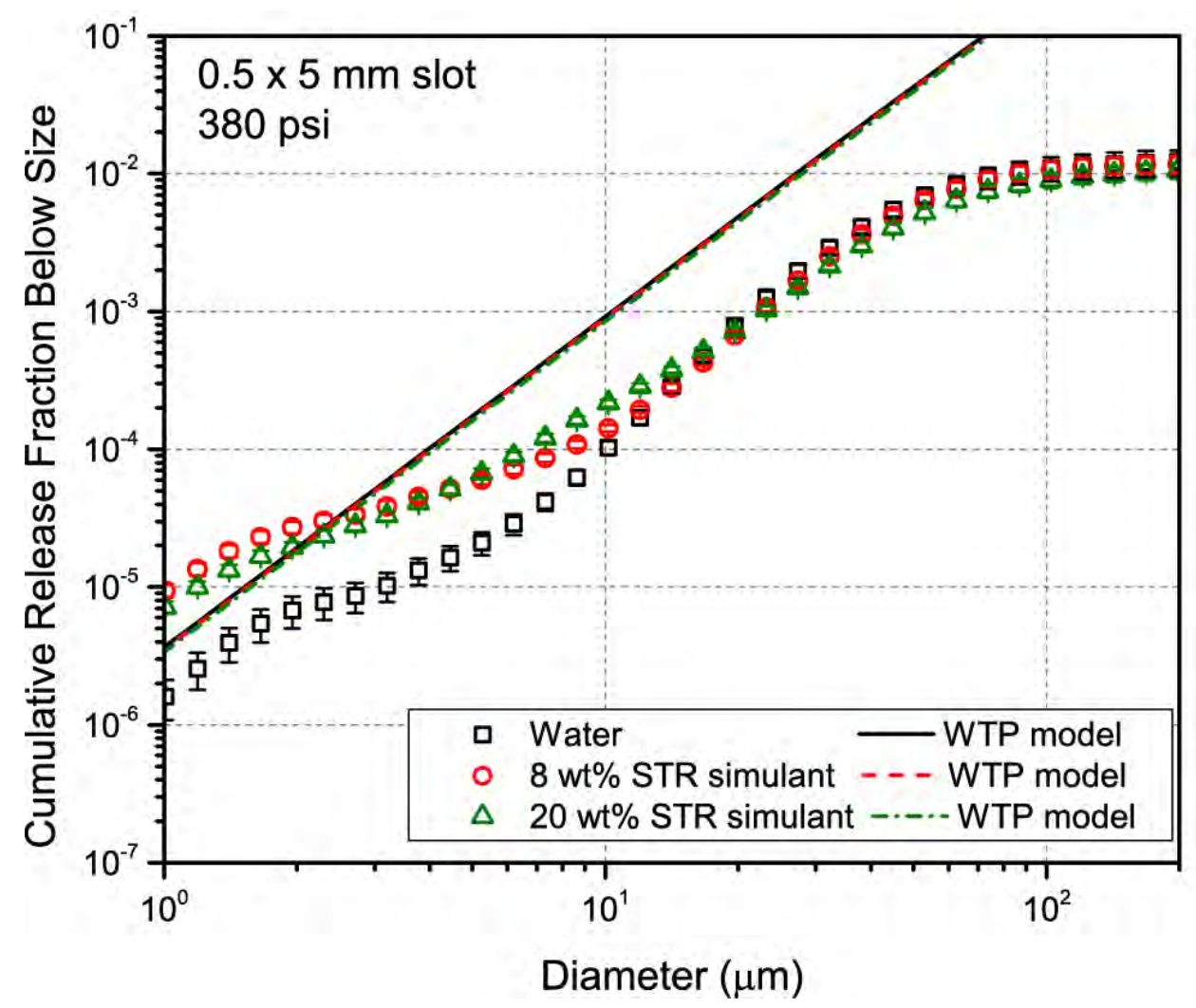

Figure 9.14. Comparison of Slurry Sprays for the STR Simulant at 8 and 20 wt $\%$ with Water Sprays in the Large-Scale Chamber

\subsection{Comparison of the Effect of Increased Viscosity and Density}

Tests were conducted in both the small- and large-scale chambers to quantify the effect of increased viscosity and density on the aerosol generation rate and release fraction, in comparison to water, for sprays of salt solutions. Figure 9.15 shows the small-scale results for the aerosol generation rate for water ( $\sim 1 \mathrm{cP}, 1 \mathrm{~g} / \mathrm{mL}$ ) and two salt solutions with nearly identical densities but different viscosities. As discussed previously in the small-scale aerosol report (Mahoney et al. 2012b), there is essentially no effect of viscosity for this small range and also essentially no difference between water and the two salt solutions. Figure 9.16 shows a similar comparison from the large-scale tests for water and sodium thiosulfate ( $2.5 \mathrm{mPa} \bullet \mathrm{s})$. Similar to the small-scale test results, there is essentially no difference in aerosol generation rates between water and the salt solution. 
Rate: 1-mm round holes, (a) 380 psig, different liquid viscosities

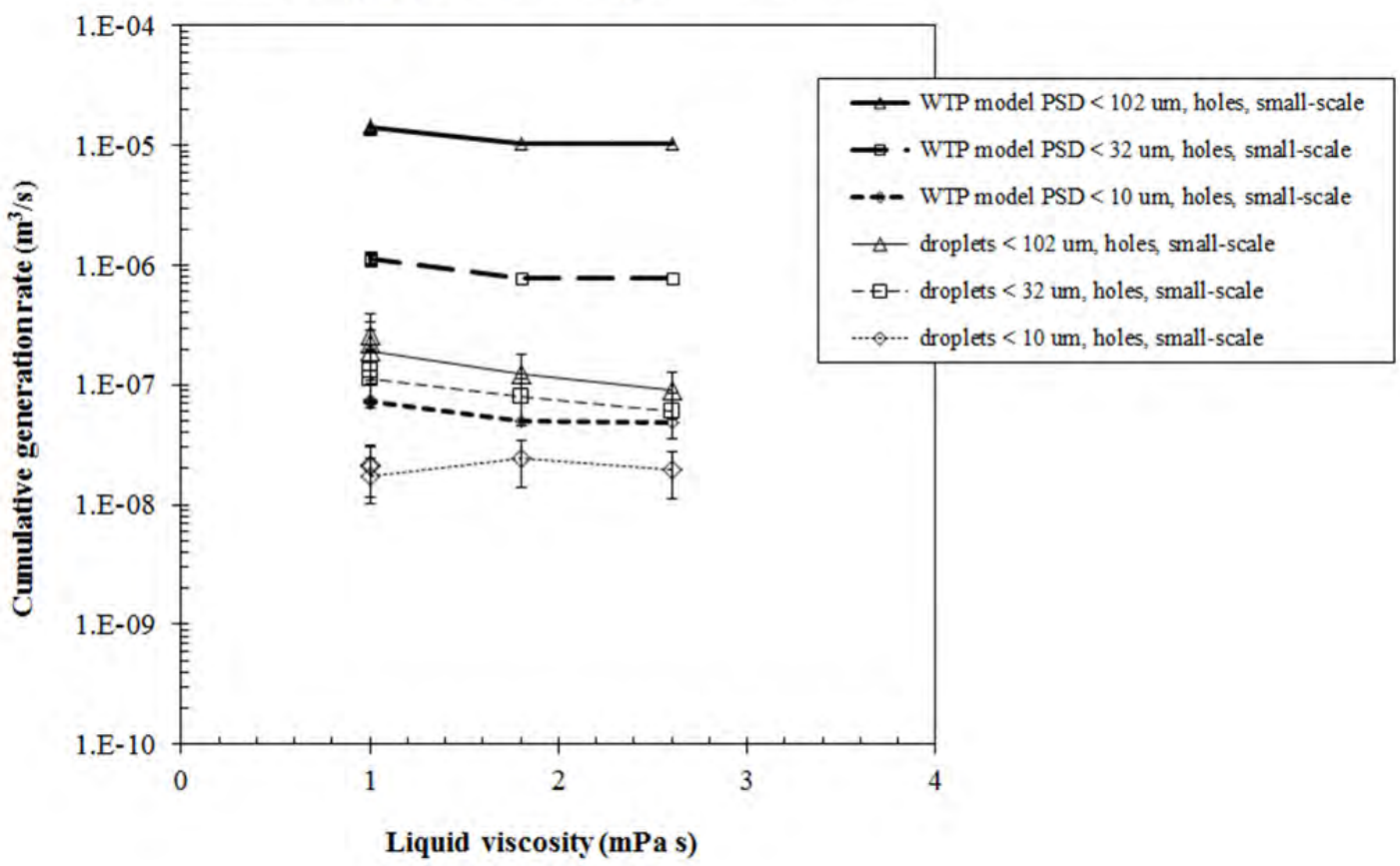

Figure 9.15. Effect of Increased Viscosity (and Density) on the Cumulative Aerosol Generation Rate for Water ( 1 mPa $\bullet$ ), Sodium Nitrate ( 1.5 mPa $\bullet$ s), and Sodium Thiosulfate $(\sim 2.5 \mathrm{mPa} \bullet \mathrm{s})$ in the Small-Scale Chamber

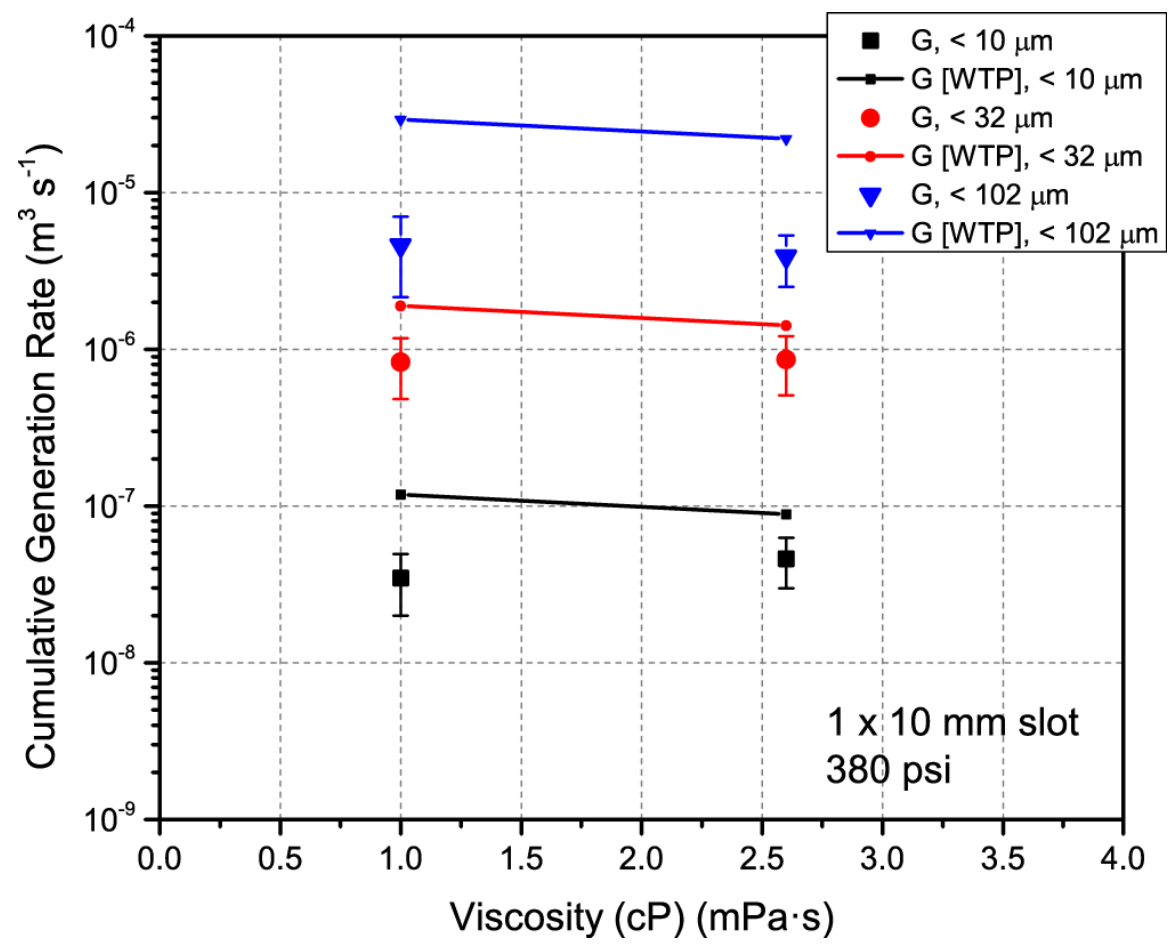

Figure 9.16. $\quad$ Effect of Increased Viscosity (and Density) on the Cumulative Aerosol Generation Rate for Water $(\sim 1 \mathrm{mPa} \cdot \mathrm{s})$ and Sodium Thiosulfate $(\sim 2.6 \mathrm{mPa} \cdot \mathrm{s})$ in the Large-Scale Chamber 


\subsection{Comparison of the Effect of Spray Length}

Tests were conducted in both the small- and large-scale chambers to quantify how the release fraction is affected by the distance between the orifices and where the sprays hit the splash wall. In the small-scale chamber, the spray lengths ranged from 1 to 42 in. and in the large-scale chamber the distances ranged from 43 to 227 in. The tests with $~ 42$-in. spray lengths in each chamber were conducted to allow a comparison of identical length sprays in chambers of different size and these results were discussed previously in Section 9.1. For the small-scale chamber, spray-length tests were only conducted with a 1-mm hole at 380 psi. In the large-scale chamber, a range of holes and slots were tested at 380 and 200 psi spray pressures to confirm the general behavior that the release fraction for a specific orifice and spray pressure is always greatest, for the spray lengths tested, when the spray travels the full length the of chamber.

Figure 9.17 shows the how the release fractions for different size droplets varied with the distance between the spray and splash wall for 380 psig water sprays from a 1-mm circular orifice in the smalland large-scale chambers. For the small-scale results, there is very little difference in the release fraction for distances between 3 and 42 in. At a 1-in. gap between the spray and the wall, the release fraction increases noticeably. As is evident from the three replicate tests, the higher release fraction for the 1-in. spray distance was repeatable. Figure 9.17 also shows results from the large-scale chamber for the same 1-mm orifice for spray distances ranging from 43 to 227 in. For these longer distances, the results show a very clear increase in release fraction as the distance between the spray and splash wall increases. As shown in Figure 9.7, the release fraction results for the large-scale tests are less than the small-scale results when the sprays have the same length, and this difference is apparent in Figure 9.17 for all droplet sizes.

To show that the release fractions increase with spray length, additional tests were conducted in the large-scale chamber for sprays with lengths between 43 and 227 in. at pressures of 200 and 380 psi using two circular orifices and three slots. These tests included sprays from the large $1 \times 76.2-\mathrm{mm}$ slot used in the extrapolation to the largest postulated slot of 589- $\mathrm{mm}^{2}$ slot (shown later in Figure 9.20). Figure 9.18 shows the release fraction results for three droplet sizes for these ten different test conditions with the symbols identifying the sprays with different lengths. These results show the longest spray (black squares) always had the largest release fractions for all of the droplet sizes. There are a few droplet sizes and test conditions, such as the 1-mm hole 200 psi test, where release fractions from shorter length sprays are comparable with the longest sprays, but the general conclusion is that largest release fraction occurs with the longest spray. 


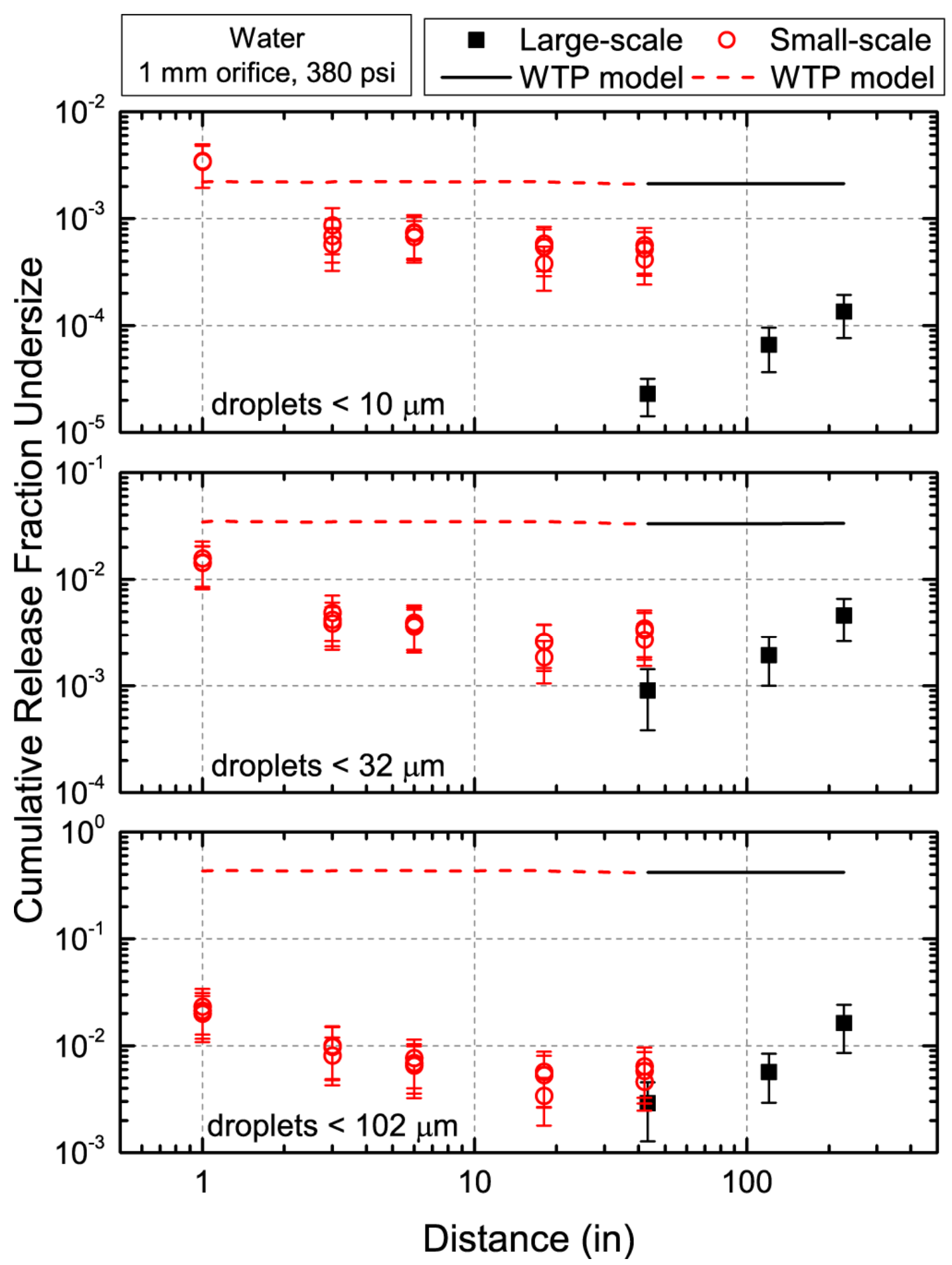

Figure 9.17. Effect of Distance from Spray to Splash Wall on the Cumulative Release Fraction for a 1-mm Orifice in Both the Small and Large Chambers (results shown separately for droplets $<10 \mu \mathrm{m}$ [top], $<32 \mu \mathrm{m}$ [middle], and $<102 \mu \mathrm{m}$ [bottom]) 


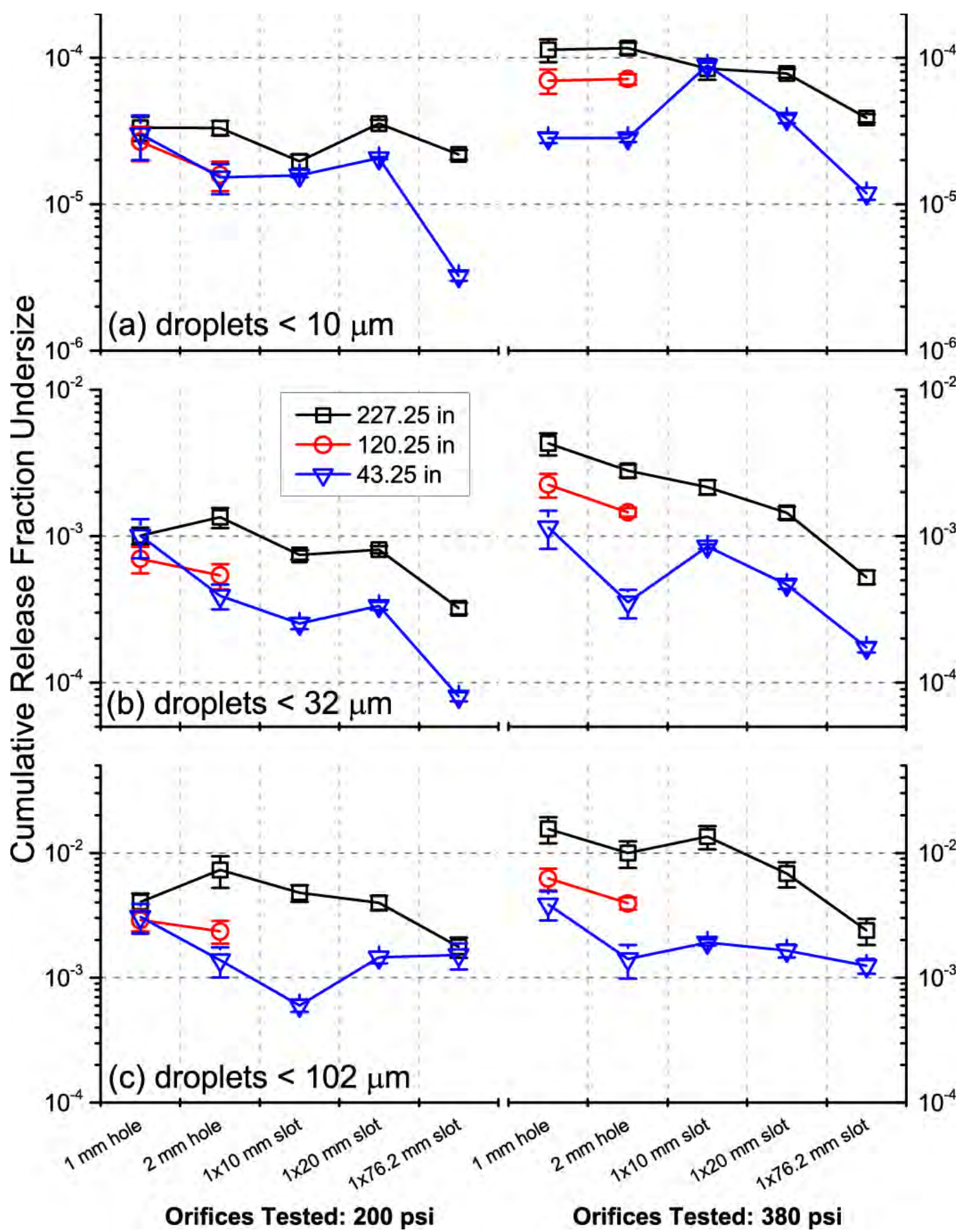

Figure 9.18. Effect of Spray Length on Release Fraction for Droplets (a) $<10 \mu \mathrm{m}$ (b) $<32 \mu \mathrm{m}$, and (c) $<102 \mu \mathrm{m}$ for Various Circular Orifices and Slots and at 200 and $380 \mathrm{psi}$ 


\subsection{Extrapolation to Largest Postulated Slot and Long-Distance Spray (Worst-Case Single-Orifice Spray)}

The largest postulated breach in a 10-in. pipe, specifically the ultrafiltration pipe loop, is currently considered the most significant spray release in terms of consequence for the WTP (Larson and Allen 2010). This breach is larger than what has been tested, and only the small-scale tests (Mahoney et al. 2012b) have evaluated slurries with Bingham parameters that approach the upper target values of $30 \mathrm{~Pa} / 30 \mathrm{cP}$ for slurries that may be in the ultrafiltration pipe loop. The spray release data that has been collected, however, allow estimates to be made for this worst potential spray. The reason for extrapolating the results is that the results in Chapter 8 and Section 9.1 show that larger breaches and possibly longer distance sprays in larger enclosures both give increased aerosol generation rates. Small-scale testing (Mahoney et al. 2012b) also has shown that the chemical slurry simulant with the highest Bingham parameters (i.e., yield stress and consistency) had a higher release fraction when compared to water. As discussed in Section 9.1, the size of the chamber also affects the release fraction and the results suggest that the release fractions may increase in larger chambers for droplets above about $30 \mu \mathrm{m}$. In combination, for sprays from a single breach the largest potential aerosol generation, and hence worst-case spray, comes from the largest postulated breach, at the highest pressure, and from the material with the highest Bingham parameters. Any extrapolation adds uncertainty beyond that in the test data used in the extrapolation, and the extrapolations presented below have an additional degree of uncertainty.

The test results collected in this study are primarily for sprays from individual orifices. Note that Section 8.2.2 discusses test results from multiple closely-spaced holes that give release fractions that are equivalent to a single hole of the same total area). Multiple individual orifices spraying simultaneously and spaced sufficiently far apart, will give a combined aerosol generation rate that is the sum of the generation rates from the individual orifices. The results presented in this report (e.g., single-orifice generation rates in Figure 8.19) can be used to determine the combined aerosol generation rate of any combination of individual orifices spraying simultaneously and can also be used to determine the number of smaller orifices that give the same total aerosol generation rate as a single larger orifice. A sufficient number of small orifices can have a generation rate equivalent to the worst-case single-orifice spray discussed in this section.

\subsubsection{Extrapolation to Largest Postulated Slot}

Figure 9.19 shows the release fraction results discussed previously in Chapter 8 for slots, and the dashed lines through the data show the extrapolation of these data to larger slots. A breach in a 10-in. pipe, specifically the ultrafiltration pipe loop, is currently considered to represent the most significant spray release for the WTP (Larson and Allen 2010). McAllister (2010) gives specific information on the dimensions for postulated breaches and pipes, and the largest breach postulated for a 10 -in. pipe is a $4.64 \times 127-\mathrm{mm}\left(589-\mathrm{mm}^{2}\right)$ slot. Figure 9.19 shows the extrapolation of the cumulative release fraction results to an orifice of this area for different droplet sizes. These data also show that the release fraction results have about the same dependence on orifice area for the three different droplet sizes shown, which spans the range of significant sizes for use in spray release estimates. The data suggest that there may be a change in the slope when the area is greater than approximately $30 \mathrm{~mm}^{2}$. Given the small number of orifices tested with areas greater than $30 \mathrm{~mm}^{2}$, it is difficult to determine if the change in slope is real. 
The extrapolation, as performed using all the data, should be considered a conservative estimate because it gives a higher release fraction than is suggested by the few largest orifices.

The cumulative release fraction for a $589-\mathrm{mm}^{2}$ orifice can be estimated by taking the release fraction for the $1 \times 76.2-\mathrm{mm}$ slot, which was the second largest slot tested, and using the fit to the data for the droplets that are $<10 \mu \mathrm{m}$ shown in Figure 9.19 to estimate how much to reduce the release fraction for the $589-\mathrm{mm}^{2}$ slot for all droplet sizes. The dotted line in Figure 9.19 for droplets that are $<10 \mu \mathrm{m}$ shows that extrapolation from a $76.2-\mathrm{mm}^{2}$ orifice $(1 \times 76.2 \mathrm{~mm})$ to a $589-\mathrm{mm}^{2}$ orifice decreases the release fraction by a factor of approximately two. Figure 9.20 shows the estimated cumulative release fraction for the $589-\mathrm{mm}^{2}$ orifice and also shows release fraction data for the $1 \times 76.2-\mathrm{mm}^{2}$ orifice and two other similar but smaller slots. The release fraction for the $589-\mathrm{mm}^{2}$ orifice represents the behavior of the largest postulated breach with an 20-ft-long spray in a 20-ft-long enclosure. This result is also shown later in Figure 9.24 below as the curve labeled as "Extrapolate using $1 \times 76.2 \mathrm{~mm}$ data."

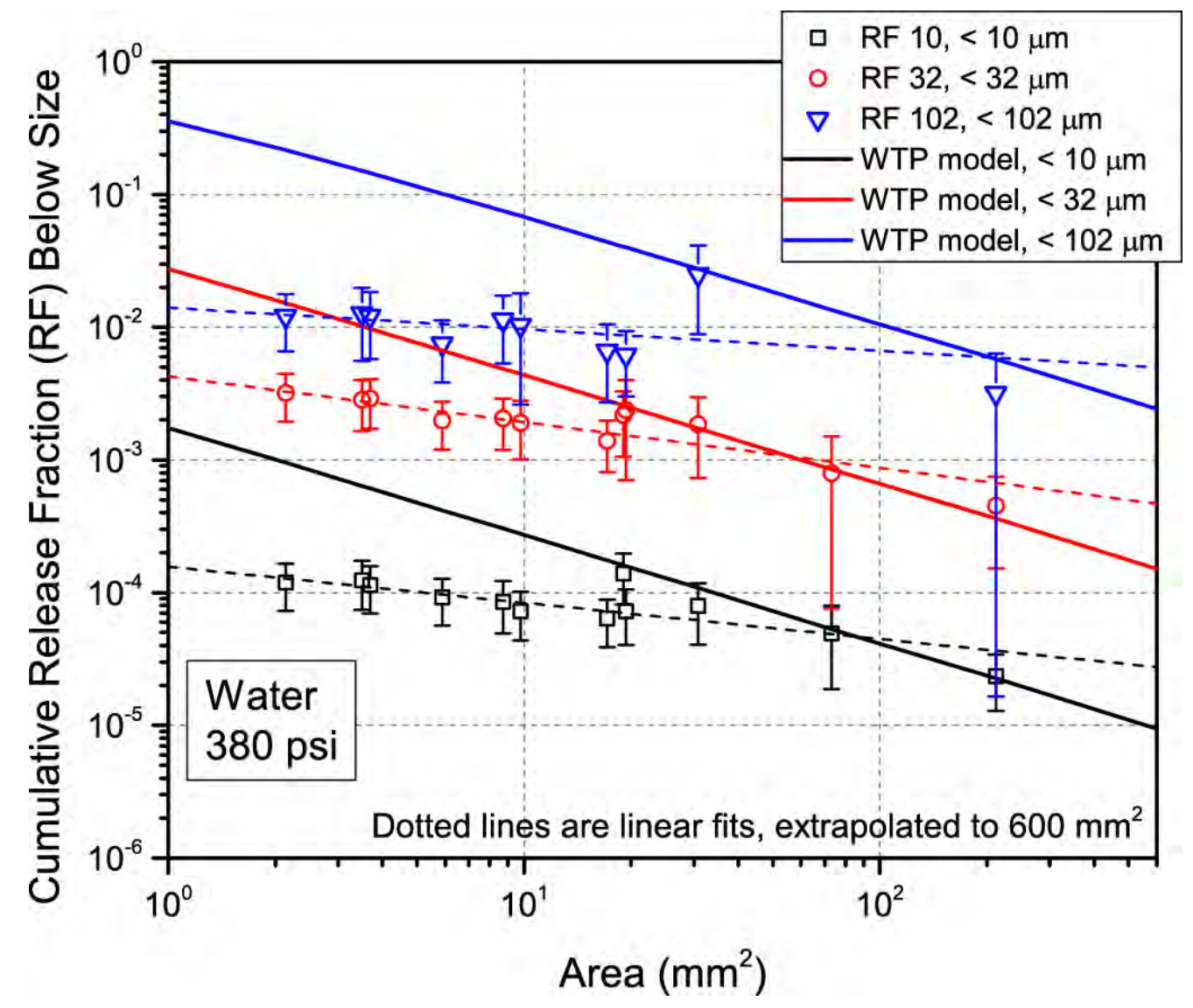

Figure 9.19. Effect of Orifice Area on Cumulative Release Fraction for Slots Tested in the Large-Scale Chamber and the Extrapolation of Data to Larger Slots (data were obtained for sprays traveling the full length of the chamber with the spray header approximately 227-in. from the splash wall) 


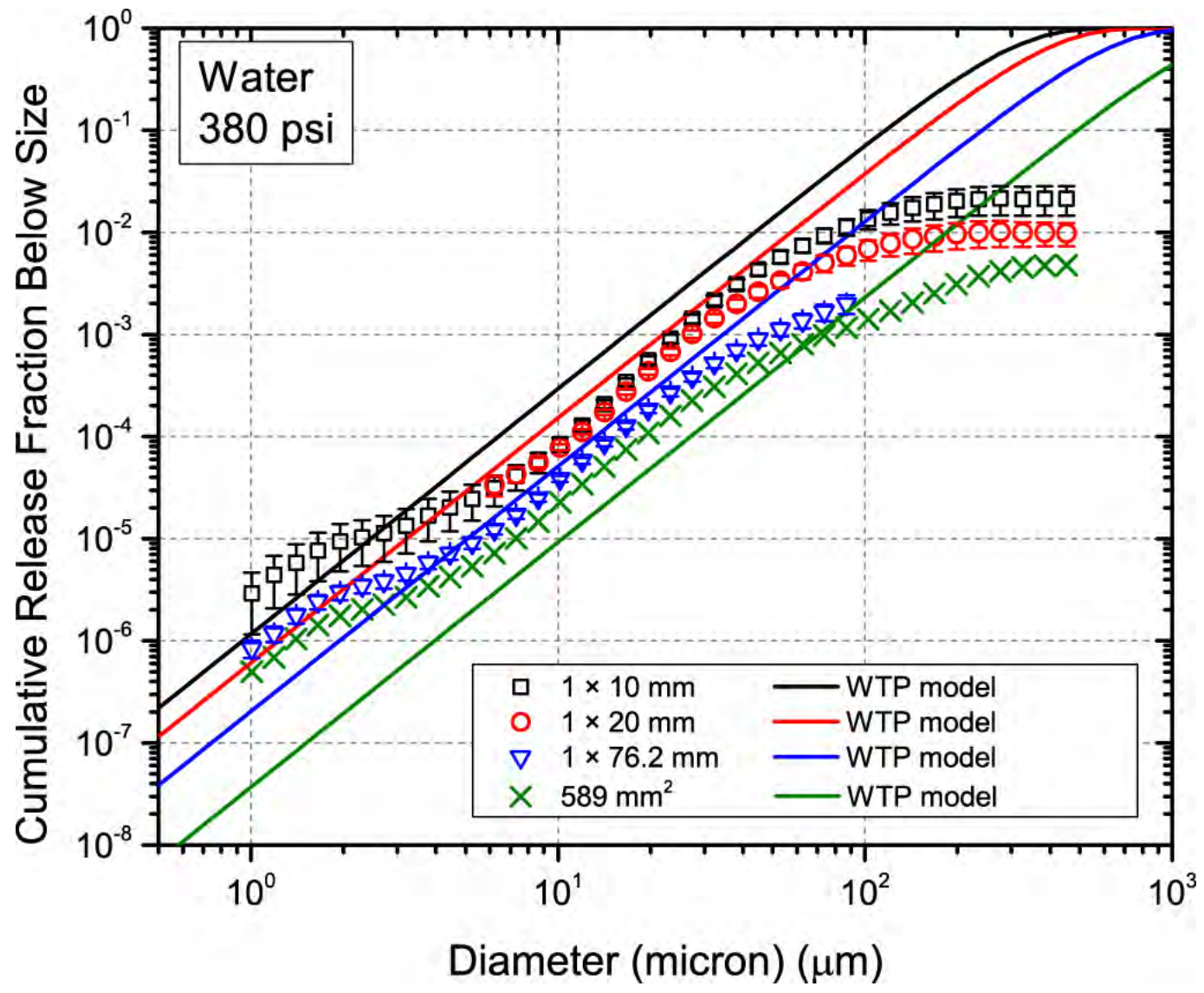

Figure 9.20. Cumulative Release Fraction for Progressively Larger Slots and the Estimated Release Fraction for a $4.64 \times 127-\mathrm{mm}\left(589-\mathrm{mm}^{2}\right)$ Slot with a Spray that Travels the Full Length of a 20 -ft Chamber

\subsubsection{Estimate for Long-Distance Spray}

The next step in estimating the release fraction for the worst-postulated spray is to estimate the release fraction for the longest likely spray in a larger chamber or facility. Given the size of hot cells and black cells containing the process piping and vessels in the WTP, it is possible for sprays to travel a long distance before impacting a wall or dropping to the floor. A quantitative analysis has not been performed, but it seems possible that a spray could travel $100 \mathrm{ft}$ in some situations, which is a longer distance than the maximum size of the 20 -ft test chamber used in the this study.

There are two potential approaches for estimating the release fraction for a long-distance spray in a larger chamber or facility based on available data, and each method has uncertainty and gives different results. The first approach is to use the comparison discussed in Section 9.1 for sprays that travel the full length of the small and large chambers to estimate the release fraction for a 100-ft chamber and spray. The second approach is to use results for the droplet PSD taken within the spray, where the in-spray PSD should represent the total droplet release fraction into a chamber that is sufficiently large such that the spray does not strike a wall and splatter. 


\subsubsection{Approach 1: Estimate Based on Chamber Size}

For the first approach, Figure 9.2 through Figure 9.6 show comparisons of the release fractions between the small and large chambers for 380 psi water sprays for two circular orifices and a slot and 380 psi slurry sprays in a slot. The generally similar release fractions, considering the substantially different sizes of the test chambers, suggest that, when the sprays travel the entire length of the chamber, the release fraction is nearly independent of chamber size. As discussed in Section 9.1, the release fraction for droplets above about $30 \mu \mathrm{m}$ appears to be increasing with increasing chamber size and spray length, but there is a limited amount of data to provide quantitative estimates of the effect of chamber size. Accordingly, the results shown in in Figure 9.20 for the estimated release fraction for a $4.64 \times 127-\mathrm{mm}\left(589-\mathrm{mm}^{2}\right)$ slot can be used as an estimate for a $100-\mathrm{ft}$ spray in a $100-\mathrm{ft}$ chamber, but it should be noted that the release fraction for droplets above about $30 \mu \mathrm{m}$ may be higher in larger chambers. Of the two approaches for estimating the release fraction for a 100-ft spray, this estimate gives the lowest result. This result is shown later in Figure 9.24 as the curve labeled "Extrapolate using $1 \times 76.2$-mm orifice data" and the arrows in this figure suggest the potential for higher release fractions for droplets above about $30 \mu \mathrm{m}$.

\subsubsection{Approach 2: Estimate Using In-Spray Measurements}

For the second approach, data were collected with the sprays going directly through the Malvern instrument to give PSDs representing the entire spray. See Section 8.6 of this report and Mahoney et al. (2012b) for a discussion of the in-spray measurements. The in-spray PSD can be assumed to represent the release fractions for sprays in the specific situation where there is no aerosol loss to the chamber walls and floor and no aerosol generation from splatter where the spray hits the far wall of the test chamber. In the absence of splatter, the in-spray PSDs represent upper bounds for the release fraction. As discussed in Section 1.2.1, Epstein and Plys (2006) showed in-spray results where there was essentially no difference in PSDs for a range of orifice sizes. They also reported that the largest slot that was tested had a lower PSD, but the spray did not fully break up and was difficult to characterize. Figure 9.21 shows the in-spray PSDs for 1-mm and 2-mm circular orifices discussed in Section 8.6, for a 0.5-mm circular orifice from the small-scale tests (Mahoney et al. 2012b), and for a 0.6-mm circular orifice from Epstein and Plys (2006). These PSD results are for distances relatively close to the orifice and selected to allow comparison of the small-scale and larger-scale results with those of Epstein and Plys. The limited results in Figure 8.32 for different distances from the orifice suggest that the in-spray PSDs may shift to larger droplets farther from the orifice. The PSDs in Figure 9.21 are generally similar, but with increasing differences for smaller droplets. For this limited amount of in-spray data, there is no apparent effect of orifice size. Assuming there is no effect of orifice size on the PSDs, these PSDs could be used to estimate the PSD of the largest postulated orifice $\left(589 \mathrm{~mm}^{2}\right)$. Alternatively, the PSD from the longer distance spray of 59 in. in Figure 8.32 for the largest orifice tested $(2 \mathrm{~mm})$ could also be used. The largest postulated orifice has an area more than 100 times larger than the 2-mm orifice, so there is uncertainty in assuming that these in-spray results will be similar to in-spray PSDs of much larger orifices. Additional in-spray data for a range of larger orifices is being collected in ongoing studies. 


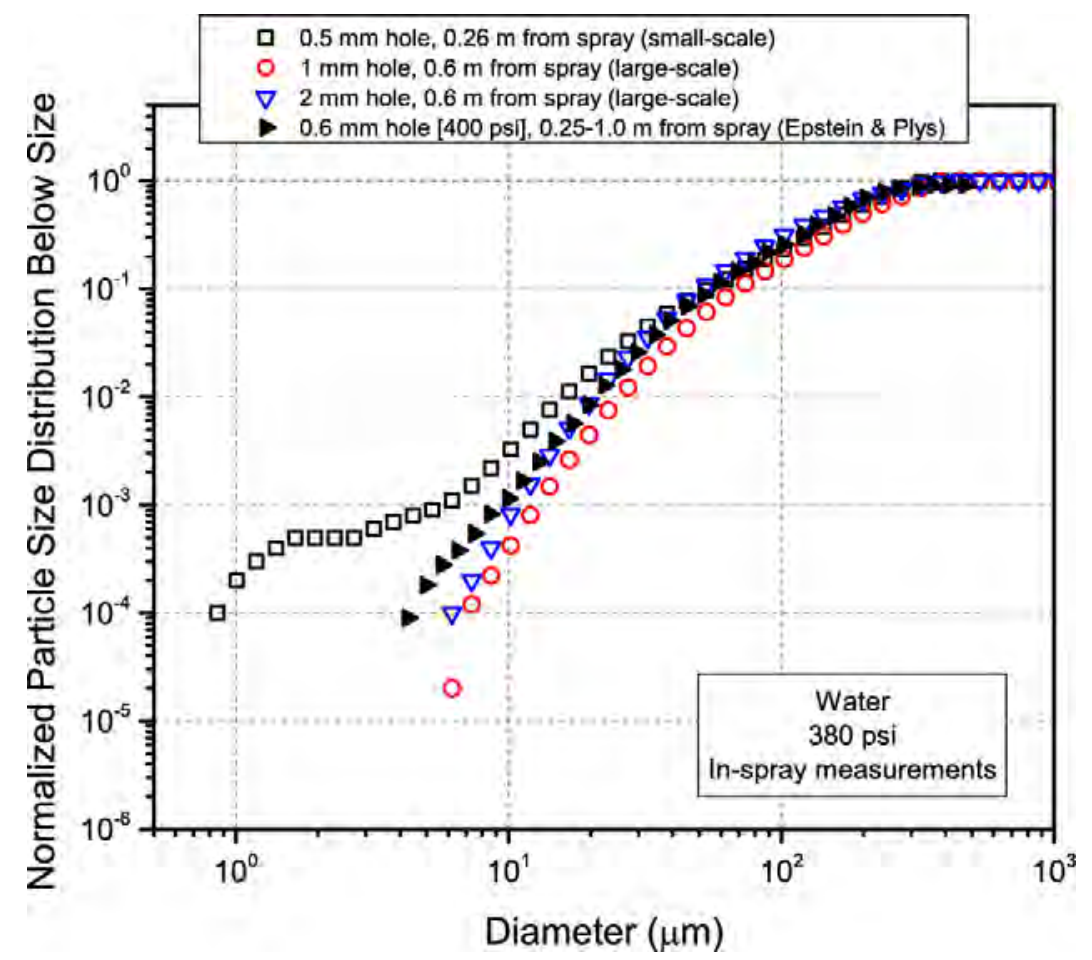

Figure 9.21. Comparison of In-Spray PSDs for Circular Orifices with a Range of Sizes

Of the two approaches for estimating the release fractions for a 100-ft spray, the in-spray PSDs give the highest estimate and thus can represent an upper bound to the release fraction, in the absence of splatter, for a particular spray. Accordingly, it is useful to compare the release fraction results from the large-scale chamber with sprays traveling the full length of the chamber with in-spray PSDs to compare these two estimates of the release fraction for long-distance sprays. Figure 9.22 shows the release fraction results for a 380 psig water spray from a 2-mm circular orifice, where the spray traveled the full length of the 20 -ft chamber, together with the in-spray PSDs at two distances from the orifice. The two in-spray PSDs are different and repeatable, as discussed in Section 8.6. The release fraction results from the large-scale chamber are similar to the in-spray PSD results at 59-in. from spray for droplets below about $30 \mu \mathrm{m}$ and the in-spray results become progressively higher for larger droplets. Figure 9.23 shows a similar comparison for a 1-mm circular orifice with a spray that traveled the full length of the 20-ft chamber and the in-spray PSDs. For this comparison, the in-spray PSDs are nearly the same as the large-scale chamber release fraction for $<10 \mu \mathrm{m}$ droplets and then progressively higher for larger droplets. 


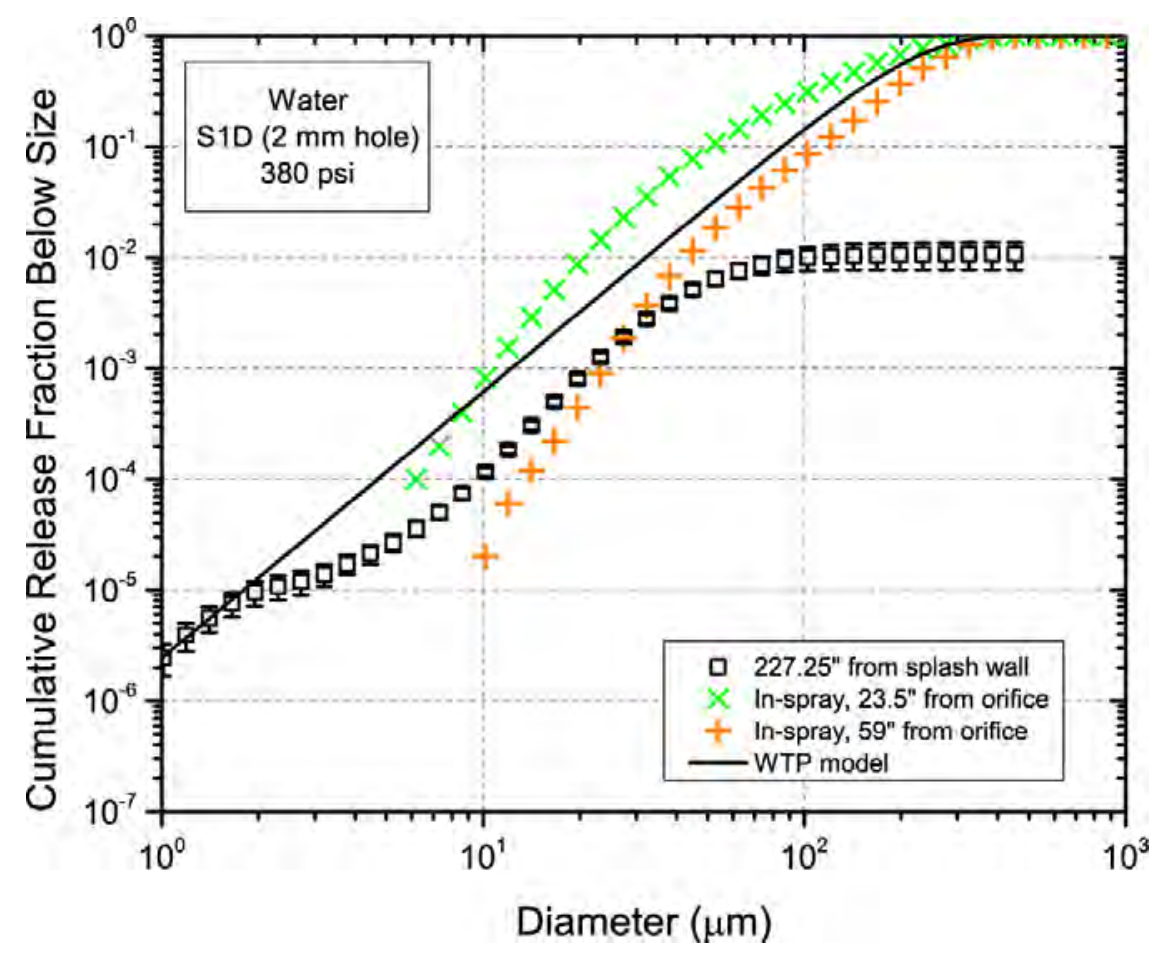

Figure 9.22. Comparison of Release Fractions for a Spray Traveling the Full Length of the $20-\mathrm{ft}$ Chamber (227 in.) and In-Spray PSDs for a 380 psig Water Spray from a 2-mm Circular Orifice

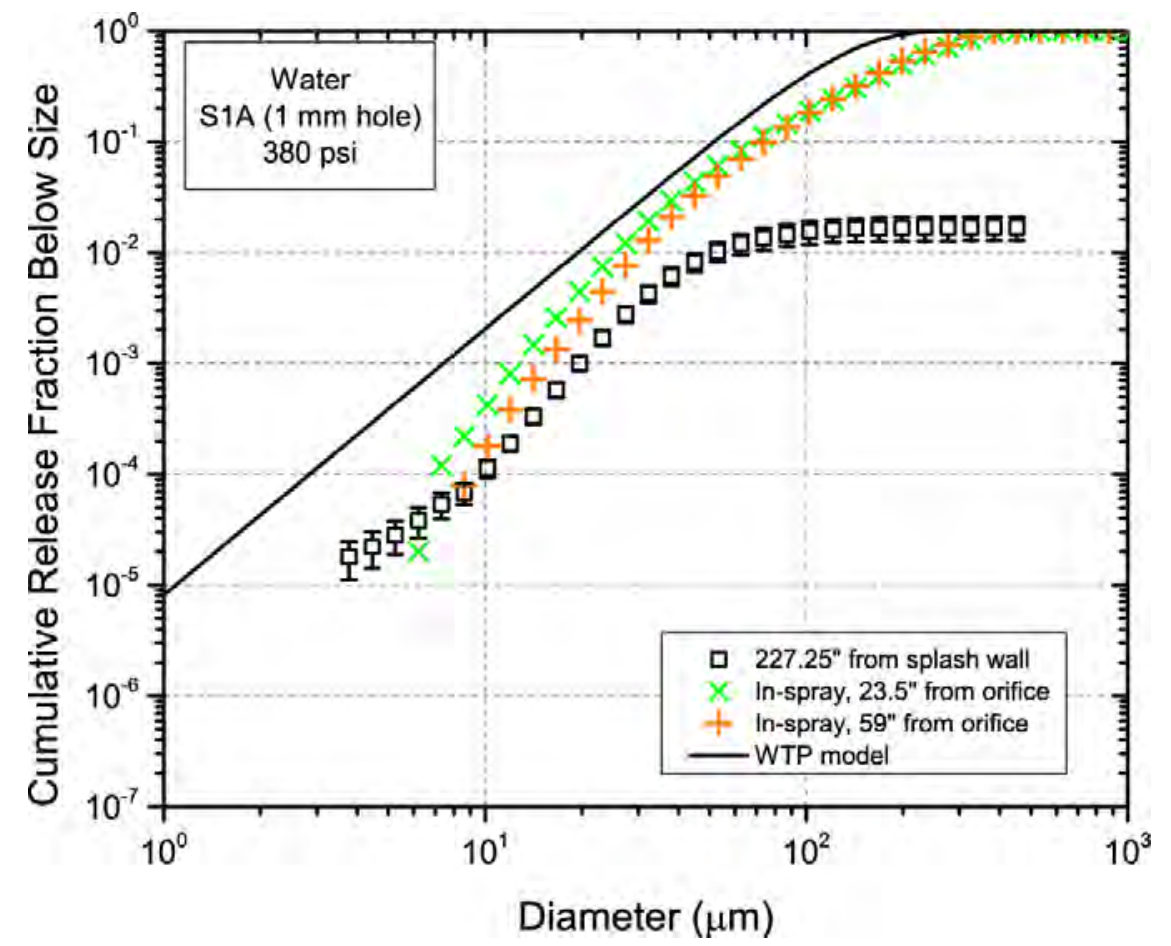

Figure 9.23. Comparison of Extrapolated Release Fractions for a Spray Traveling the Full Length of the 20-ft Chamber (227 in.) and In-Spray PSDs for a 380 psig Water Spray from a 1-mm Circular Orifice 
The comparisons in Figure 9.22 and Figure 9.23 show that the release fractions from the large-scale chamber are always less than the upper bound in-spray PSDs results. For these relatively small orifices, the in-spray and large-scale chamber results are generally similar for droplets less than about $20 \mu \mathrm{m}$. As discussed in Section 9.1, the comparison of release fraction results in the small and large chambers suggests that the release fractions in larger chambers will increase for droplets larger than about $30 \mu \mathrm{m}$. Sufficient data in a range of different size chambers is not yet available to estimate the release fractions in larger chambers, but testing is ongoing for a range of different size chambers. For sprays from orifices larger than a 2-mm circular orifice, collecting in-spray PSDs with the currently used instruments has not been attempted because the sprays maintain a liquid core for a long distance from the spray and also become too wide to fit between the laser and detector at a distance where it appears that the spray has broken up into droplets. As part of an ongoing study, instrument modifications are being made and additional in-spray data are being collected for a range of larger orifices. These results will be beneficial for providing an upper bound for the release fraction from the largest postulated slot.

\subsubsection{Comparison of Estimates of Long-Distance Sprays for Largest Postulated Slot}

In Section 9.6.1, an extrapolation was presented to estimate the release fraction for the largest postulated slot based on water sprays in the large-scale chamber. In Section 9.6.2, two different approaches were used to estimate how the release fractions may be different in larger chambers or facilities and Figure 9.24 shows the results of both the approaches. The data labeled "Extrapolate using $1 \mathrm{x} 76.2 \mathrm{~mm}$ data” gives the results for the approach of using the large-scale release fraction in the 20 - $\mathrm{ft}$ chamber together with the blue arrows noting the possible effect of larger chamber size based on the limited data for the effect of chamber size. The upper bound is provided by the in-spray PSD results for a 2-mm hole at 59 in. from the orifice. Figure 9.24 also shows the comparison to the WTP model for this water spray. Using the large-scale chamber results, the estimated release fraction exceeds the WTP model for droplet sizes below about $70 \mu \mathrm{m}$ by about a factor of two. The upper bound from the in-spray PSD is slightly higher than the large-scale chamber result for droplets $<10 \mu \mathrm{m}$, but the difference becomes progressively larger with increasing droplet size and the upper bound is as much as a factor of 50 above the WTP model for droplets $<100 \mu \mathrm{m}$. For the large-scale chamber release fraction for droplets $<100 \mu \mathrm{m}$, it is possible that the release fraction will be large in larger chambers as discussed in Section 9.1 and the release fraction may exceed the WTP model but the magnitude is uncertain. The in-spray PSD again provides an upper bound. The ongoing studies of sprays in different size chambers will help reduce the uncertainty in choosing between the large-scale (20-ft) chamber results and the in-spray upper bound. 


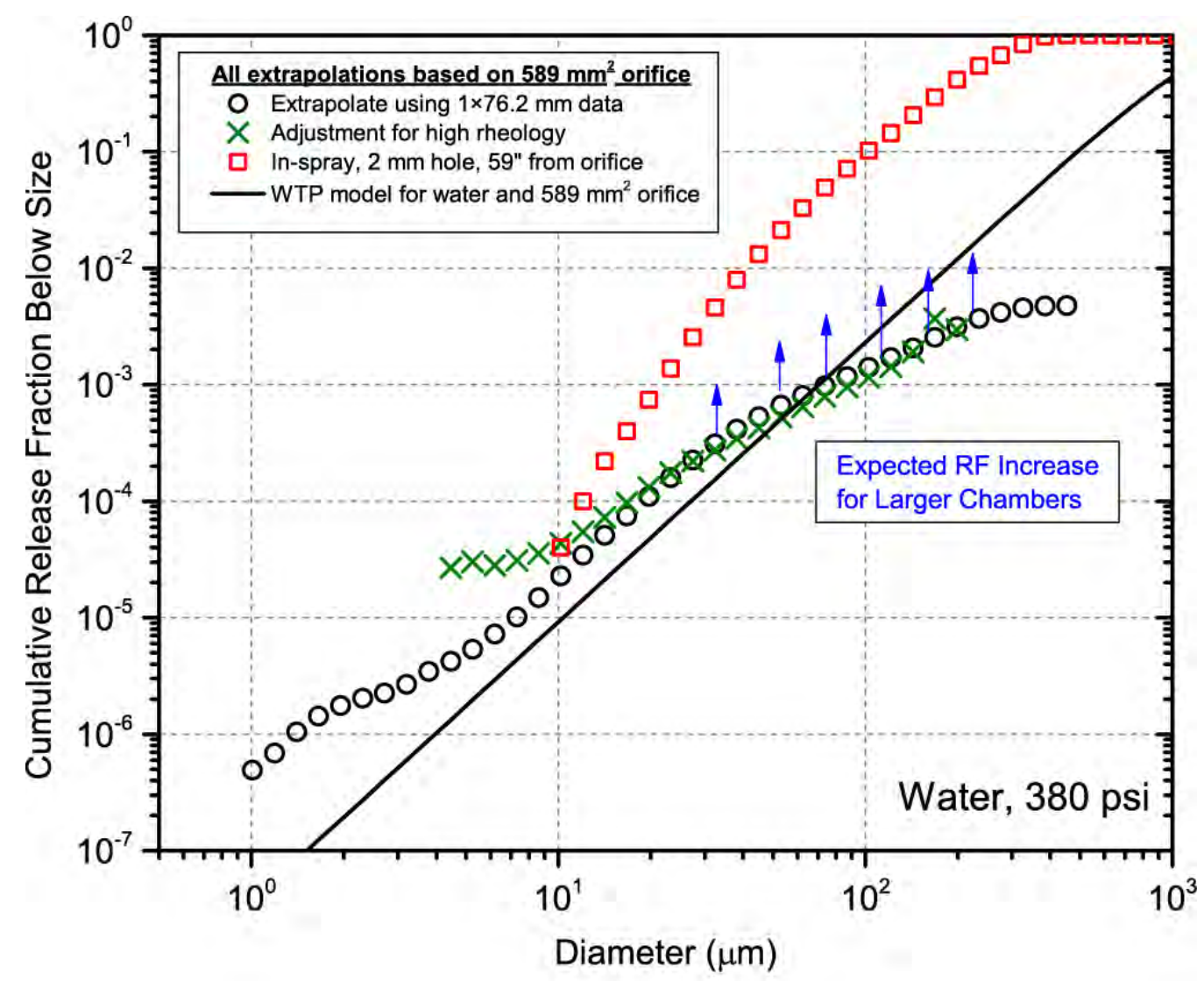

Figure 9.24. Extrapolated Release Fractions for the Largest Postulated Slot for Water in a 20-ft Chamber, an Increased Release Fraction for the Highest Postulated Bingham Yield Stress, and Comparison to a Representative In-Spray PSD and the WTP Model Assuming Fluid Properties for Water

The final consideration for this extrapolation is to select the fluid that gives the largest release fraction and, hence, represents the spray with the most significant consequence. In the small-scale tests (Mahoney et al. 2012b), release fraction results were obtained for a range of different fluids and slurries. This testing showed that the chemical slurry simulant with the highest Bingham plastic parameters (yield stress and consistency), with anti-foaming agent (AFA) present, gave the largest release fractions when compared to water (Mahoney et al. 2012b). ${ }^{16}$ For the large-scale tests, slurries with high Bingham parameters have not been tested, and for the boehmite slurries tested, the release fractions were essentially the same as water (see Figure 8.23 and Figure 9.14). Assuming that the increase in release fraction in comparison to water will be the same for both the largest postulated slot and the small-scale orifices, the release fraction results in Figure 9.24 can be increased to include the effect of spraying this worst-case fluid (note that the FER30 simulant used in calculating this increase had a UDS of about $35 \mathrm{wt} \%$, which exceeds the maximum expected range in the WTP of $20 \mathrm{wt} \%$, and had Bingham parameters of about

\footnotetext{
${ }^{16}$ The average of small-scale tests SV55A, SV55B, and SV55C was used to represent the chemical slurry/AFA simulant, and the average of tests SO2-R1 and OS12-R1-SG was used to represent water. In all these tests the target test pressure was $380 \mathrm{psi}$ and the orifice was a slot with target dimensions of 0.5 x $5 \mathrm{~mm}$. See Mahoney et al. (2012b) for more information about these tests.
} 
$12 \mathrm{~Pa} / 12$ cP, which are below the upper target of 30/30; see Mahoney et al. 2012b). This final extrapolation is labeled in Figure 9.24 as "Adjustment for high rheology," and is slightly higher that the water extrapolation. The WTP model results shown in Figure 9.24 for comparison assume the fluid properties for water. If the WTP model were to assume an increased viscosity for the highest Bingham consistency, the WTP model estimates would be below the water estimates by approximately a factor of 3.

Assuming the WTP model uses the fluid properties of water for all the sprays, the overall extrapolations shown in Figure 9.24 indicate that long-distance sprays from the largest postulated orifice are estimated to exceed the WTP model by factor of 2-4 for droplets that are $<10 \mu \mathrm{m}$. For larger droplets, the estimates become more uncertain, but the results are still expected to exceed the WTP model. The extrapolation for orifice size is based on data for a wide range of orifice sizes, and the increase in release fraction to account for the highest Bingham parameters is only about twofold, which is a relatively small adjustment. The most significant uncertainty in these estimate is from the effect of chamber size and spray length, and additional testing will be needed to reduce this uncertainty. Additional testing of non-Newtonian slurries sprayed through large orifices in the large-scale chamber also is suggested as a means of reducing the uncertainty. 


\subsection{Conclusions and Recommendations}

Aerosol generation tests were performed to quantify the release fraction and generation rate for a range of orifice sizes and shape, fluids, and spray pressures that represent expected WTP process stream properties and potential spray release scenarios. Extrapolations to postulated worst-case spray release conditions also were conducted. Both the test results and extrapolations were compared with the WTP model predictions. In addition, testing was conducted to evaluate instrument performance, variation in repeat tests, and uncertainty in aerosol measurements related to non-uniformity in the test chamber.

The tests results for determining the effect of orifice size and shape, fluid properties, spray pressures, and length of spray within the chamber can be summarized as follows:

- Orifice coefficients, $C_{D}$, were determined and found to be consistent with the value of 0.62 used in the WTP model. The overall average orifice coefficient for all tests was determined to be 0.66 and 0.62 based on differential mass and flow rate measurements respectively.

- As pressure increases, the cumulative release fraction increases. This increase with pressure was shown to be greater than the rate of increase in the WTP model, especially at smaller droplet sizes.

- As orifice area increases, the cumulative release fraction decreases while the cumulative generation rate increases because of the increase in total spray flow with increasing orifice area. The rate of decrease in the release fraction test data, with increasing orifice area, is smaller than the decrease projected by the WTP model. The dependence on area was found to hold regardless of orifice shape (circular hole or slot or multiple adjacent holes) and slot orientation did not have an appreciable effect on the release fraction. As pressure decreases, the decrease in release fraction with increasing orifice area becomes smaller, perhaps even being independent of area. Considering the uncertainty in the results, the release fractions and generation rates from the test data approached the WTP model at an orifice area in the range of 30 to $100 \mathrm{~mm}^{2}$.

- As viscosity and density increased for a salt solution, the cumulative release fraction is unchanged. This is comparable to the WTP model prediction including both the density and viscosity change. Not enough data was collected to determine the separate roles of viscosity or density.

- As the weight fraction of solids increases, the cumulative release fraction is unaffected for droplets $>10 \mu \mathrm{m}$. This is consistent with the WTP model, because it accounts for the presence of solids only by changes in the physical properties of the fluid. There was some deviation from water at droplet sizes $<10 \mu \mathrm{m}$, but differences below this droplet size are of minor concern for spray release accident analyses.

- As the distance from the splash wall increases, the cumulative release fraction increases. For the five different orifices and two spray pressures tested, the largest release fraction always occurred when the spray traveled the full length of the chamber. The WTP model does not consider the effect of obstructions such as walls which impacts the distance the spray travels.

- In-spray measurements are difficult to interpret as they are strong functions of position, pressure, orifice size, and Malvern analysis settings. They do, however, represent an upper bound, in the absence of splatter, on the release fraction for a particular spray and can be used to estimate worst cases (see Chapter 9). 
The comparison of small- and large-chamber test results can be summarized as follows:

- The comparison of release fraction results for the small and large test chambers, when the sprays traveled the full length of the chambers, showed the difference in release fraction varying from none to as much as fivefold for droplets between 10 and $100 \mu \mathrm{m}$. The large-scale tests gave lower release fractions for droplets below about $30 \mu \mathrm{m}$ and higher release fractions above this size and this suggests that for yet larger chambers the release fractions may increase further for droplets above about $30 \mu \mathrm{m}$ and may decrease for droplets below this size. With only two chamber sizes, these results are insufficient for estimating the uncertainty in the extrapolations to larger chambers and spray distances. While there are differences between the release fraction results in the different chambers, given the fortyfold difference in chamber volume the overall agreement in the release fraction results is reasonably good. This is a significant finding of the testing and demonstrates that the technical approach of using the transient droplet concentrations in closed chambers gives results that characterize the aerosol generation rate of the spray and can be used to estimate the release fraction in larger chambers and facilities.

- A comparison of release fraction results in the small and large test chambers, when the sprays had the same length in each chamber, gave noticeably different results, making this approach more uncertain for estimating the release fractions in larger chambers or facilities. Accordingly, the results for sprays that travel the full length of the chambers appear better for estimating the release fractions in larger chambers and facilities.

- Sprays of different lengths were tested in the small and large test chambers. In the small-scale chamber, the release fractions were essentially independent of the spray length except for sprays at 1-in. from the splash wall that had a larger release fraction. For the large-scale chamber, the spray distance varied from 43-in. to essentially the full length of the chamber. For five different orifices tested at pressures of 200 and 380 psi, the largest release fraction always occurred with sprays that traveled the full length of the chamber.

- The small and large test chamber results showed quite similar parametric dependence on the effect of orifice size and shape, fluid density and viscosity, and the presence of slurry particles for slurries that are essentially Newtonian fluids. For the key result of the effect of orifice size, the results also show that the large-scale test results, which span a wider range of orifice areas than the small-scale tests, show a clearer trend for orifice area and only need a small extrapolation to estimate the largest postulated slot and hence provide the best data for extrapolating to larger slots.

The extrapolations to estimate the release fraction for the postulated worst-case single-orifice spray (largest postulated orifice, largest chamber and spray distance, and highest Bingham yield stress) can be summarized as follows:

- The extrapolation of release fraction data to the largest postulated orifice predicts a small reduction in release fraction and gives an estimated cumulative release fraction that is about a factor of 2 higher than the WTP model prediction for droplets $<10 \mu \mathrm{m}$.

- Estimates of the release fractions for longer sprays in larger chambers (assumed to be $100 \mathrm{ft}$ ) are done using two different approaches. For one approach, the release fraction from the measurements of sprays that traveled the full length of the 20 -ft chamber are used, but with the recognition that the release fractions may be higher above about $30 \mu \mathrm{m}$ in larger chambers. In the second approach, an in-spray PSD is used as an upper bound for release fractions in a larger chamber and longer spray. 
- Modifying the extrapolated release fraction results for water sprays to account for spraying a fluid with the highest Bingham yield stress and consistency gives a small increase in the release fraction.

- Assuming the WTP model uses the fluid properties of water for all the sprays, the combined estimation for a long-distance spray from the largest postulated orifice with the highest Bingham yield stress and consistency gives cumulative release fraction results that are estimated to exceed the WTP model by a factor of 2 to 4 for droplets that are $<10 \mu \mathrm{m}$. The upper bound from the in-spray PSD is slightly higher than this large-scale chamber result for droplets $<10 \mu \mathrm{m}$, but the difference becomes progressively larger with increasing droplet size and the upper bound is as much as a factor of 50 above the WTP model for droplets $<100 \mu \mathrm{m}$.

A number of factors affect the overall uncertainty in the measured aerosol generation rates and release fractions. While a systematic quantitative evaluation of all the combined factors has not been performed, a qualitative overall uncertainty can be estimated and the overall uncertainty in the reported release fractions and generation rates for the large-scale test chamber is probably about a factor of 2 to 3 (the reported results have not been adjusted to account for this uncertainty). The comparison of release fraction results for the small and large test chambers showed the difference in release fraction varying from none to as much as fivefold, and this is important for understanding the uncertainty in extrapolations to larger chambers and spray distances. With test results for only two chamber sizes, there is too little data to estimate the uncertainty in extrapolations to larger chambers. This overall estimate of uncertainty is based on the following test results:

- Replicate tests for a number of test conditions were conducted to evaluate the test-to-test uncertainty. As a first approximation, the release fraction for any given test should be expected to have a minimum 95-percent confidence level of \pm 30 percent of the stated value at any particular aerosol droplet diameter.

- Multiple tests confirmed the Malvern aerosol results with a secondary aerosol instrument, the PPC, with tests indicating agreement between the instruments to within a factor of 2 . In many cases, the instruments appear to agree within the range of reproducibility for the Malvern Insitec-S ( 30\%) as determined by replicate testing.

Based on the testing results, the following recommendations address the key technical issues for which additional testing and evaluation will reduce uncertainty or provide results for important test conditions that have not been previously obtained:

- The most significant uncertainty in the extrapolation for the worst-postulated spray is for the effects of spray length and chamber size, and additional testing will be needed to reduce this uncertainty.

- Current testing did not evaluate non-Newtonian slurries sprayed through large orifices in the large-scale chamber, but this scenario most closely represents the worst-case spray. Accordingly, additional testing with non-Newtonian slurries in large orifices is needed to obtain data for this significant scenario.

- The overall uncertainty in the aerosol release fraction results depends on a number of factors, including instrument accuracy and obtaining representative aerosol samples. Experiments to evaluate the current estimates of this uncertainty will likely reduce the overall uncertainty in the spray release results. 



\subsection{References}

Ashgriz N. 2011. Handbook of Atomization and Sprays: Theory and Applications. Springer, New York, New York.

Berg JC. 2010. An Introduction to Interfaces and Colloids: The Bridge to Nanoscience. World Scientific Publishing Co., Hackensack, New Jersey.

Bohren CF and DR Huffman. 1983. Absorption and Scattering of Light by Small Particles. Wiley, New York, p. 149.

Boyaval S and C Dumouchel. 2001. "Deconvolution Technique to Determine Local Spray Drop Size Distributions - Application to High-Pressure Swirl Atomizers.” In Proceedings of the $17^{\text {th }}$ Annual Conference on Liquid Atomization and Spray Systems, September 2-6, 2001, Zurich, Switzerland.

Breitling M, S Nonnenmacher, S Schutz, and M Piesche. 2001. "Atomization of Non-Newtonian Liquids by Axial Hollow-Cone Pressure Swirl Nozzles.” In Proceedings of the $17^{\text {th }}$ Annual Conference on Liquid Atomization and Spray Systems, September 2-6, 2001, Zurich, Switzerland.

Bruggeman DAG. 1935. "Berechnung verschiedener physikalischer Konstanten von heterogenen Substanzen.” Annuals of Physics (Leipzig) 24:636-679.

Bühler F, U Gärtner, and S Clement. 2001. "Influence of the Surface Tension on the Atomization of Water.” In Proceedings of the $17^{\text {th }}$ Annual Conference on Liquid Atomization and Spray Systems, September 2-6, 2001, Zurich, Switzerland.

Campbell T, M Parker, A Moon, B Fant, K Clossey, and J Cook. 2010. EFRT Issue M3 PJM Vessel Mixing Assessment, Volume 8 - HLP-22. 24590-WTP-RPT-ENG-08-021-08, Rev. 1, River Protection Project, Waste Treatment Plant, Richland, Washington.

CRC. 1975. Handbook of Chemistry and Physics. $56^{\text {th }}$ Edition. CRC Press, Cleveland, Ohio.

Crowe RD. 2010. Sludge Treatment Project (STP) Methodology for Spray Leak Scenarios. PRC-STP-00292, Rev 0, CH2M HILL Plateau Remediation Company, Richland, Washington.

Denn MM. 1980. Process Fluid Mechanics. Prentice-Hall, Inc., Englewood Cliffs, New Jersey.

Dombrowski N and WR Johns. 1963. "The Aerodynamic Instability and Disintegration of Viscous Liquid Sheets.” Chemical Engineering Science 18:203-414.

Dombrowski N and G Munday. 1968. Biochemical and Biological Engineering Science. N Blakebrough (ed.), Chapter 16 - Spray Drying, 2:209-320. Academic Press, New York.

Eggers J and E Villermaux. 2008. "Physics of Liquid Jets.” Reports of Progress in Physics 71(3):036601. doi:10.1088/0034-4885/71/3/036601. 
Epstein M and MG Plys. 2006. Measured Drop Size Distributions with Cold Sprays Emanating from Small Leak Openings. FAI/06-55, Fauske \& Associates, LLC., Burr Ridge, Illinois.

Fritsching U, B Mulhem, O Kurt, and G Schulte. 2009. "Influence of Suspended Solid Particles on Suspension Atomization Processes.” ICLASS 2009, $11^{\text {th }}$ Triennial International Annual Conference on Liquid Atomization and Spray Systems, July 2009, Vail, Colorado.

Garnett JCM. 1904. "Colours in metal glasses and in metallic films." Philosophical Transactions of the Royal Society London 203:385-420.

Gauglitz PA, LA Mahoney, J Blanchard, and JA Bamberger. 2011. Surface Tension Estimates for Droplet Formation in Slurries with Low Concentrations of Hydrophobic Particles, Polymer Flocculants or Surface-Active Contaminants. PNNL-20466 Rev. 0, 46497-RPT15 Rev. 0, Pacific Northwest National Laboratory, Richland, Washington.

Gebhart J. 2001. “Optical Direct-Reading Techniques: Light Intensity Systems.” Aerosol Measurement: Principles, Techniques, and Applications, P Kulkarni, PA Baron, and K Willeke (eds.), Chapter 15, $2^{\text {nd }}$ Edition, Wiley, New York.

Hecht JP and AE Bayly. 2009. “Atomization for Spray Drying: Unanswered Questions and Industrial Needs.” ICLASS 2009, $11^{\text {th }}$ Triennial International Annual Conference on Liquid Atomization and Spray Systems, July 2009, Vail, Colorado.

Hecht JP, JA Stamper, and DK Giles. 2007. "Pneumatic Atomization of Laundry Detergent Slurries as Affected by Solid Particle Size and Concentration.” ILASS Americas, $20^{\text {th }}$ Annual Conference on Liquid Atomization and Spray Systems, May 2007, Chicago, Illinois.

Hey BE and DS Leach. 1994. A Model for Predicting Respirable Releases from Pressurized Leaks. WHC-SD-GN-SWD-20007, Rev. 0, Westinghouse Hanford Company, Richland, Washington.

Hinze JO. 1955. "Fundamentals of the Hydrodynamic Mechanism of Splitting in Dispersion Processes." American Institute of Chemical Engineering Journal 1(3):289-295.

ICD 19. 2011. ICD 19 - Interface Control Document for Waste Feed. 24590-WTP-ICD-MG-01-19, Rev 5, River Protection Project, Waste Treatment Plant, Richland, Washington.

Kurath DE, BD Hanson, MJ Minette, DL Baldwin, BM Rapko, LA Mahoney, PP Schonewill, RC Daniel, PW Eslinger, JL Huckaby, JM Billing, PS Sundar, GB Josephson, JJ Toth, ST Yokuda, EBK Baer, SM Barnes, EC Golovich, SD Rassat, CF Brown, JGH Geeting, GJ Sevigny, AJ Casella, JR Bontha, RL Aaberg, PM Aker, CE Guzman-Leong, ML Kimura, SK Sundaram, RP Pires, BE Wells, and OP Bredt. 2009. Pretreatment Engineering Platform Phase I Final Test Report. PNNL-18894, WTP-RPT-197, Pacific Northwest National Laboratory, Richland, Washington.

Larson AR and BT Allen. 2010. WTP Methodology for Spray Leak Scenarios.

24590-WTP-RPT-ENS-10-001, Rev. 1, River Protection Project, Waste Treatment Plant, Richland, Washington. 
Lefebvre AH. 1989. Atomization and Sprays. CRC Press, Taylor and Francis Group, Boca Raton, Florida.

Levy N, S Amara, J-C Champoussin, and N Guerrassi. 1997. "Non-Reactive Diesel Spray Computations Supported by PDA Measurements.” SAE Technical Paper 970049.

Looyenga H. 1965. “Dielectric Constants of Heterogeneous Mixtures.” Physica 31(3):401-406.

Mahoney LA, PA Gauglitz, J Blanchard, ML Kimura, DE Kurath. 2012a. Small-Scale Spray Releases:

Orifice Plugging Test Results. PNNL-21361, WTP-RPT-219 Rev. 0, Pacific Northwest National

Laboratory, Richland, Washington.

Mahoney LA, PA Gauglitz, ML Kimura, GN Brown, DE Kurath, J Blanchard, C Song, RC Daniel, BE Wells, D Tran, WC Buchmiller, CA Burns, and DM Smith. 2012b. Small-Scale Spray Releases:

Initial Aerosol Test Results. PNNL-21367, WTP-RPT-216, Rev. 0, Pacific Northwest National

Laboratory, Richland, Washington.

Malvern Instruments, Ltd. 2010. RTSizer and Insitec Analyser User Manual. MAN0467 Issue 1.0, Malvern Instruments Ltd., Malvern, Worcestershire, United Kingdom.

Mansour A and N Chigier. 1995. “Air-Blast Atomization of Non-Newtonian Liquids.” Journal of Non-Newtonian Fluid Mechanics 58:161-194.

McAllister J. 2010. Severity Level Calculations for the Pretreatment Facility Based on Updated MAR. Calculation No. 24590-PTF-Z0C-W14T-00036, Rev. B, River Protection Project, Waste Treatment Plant, Bechtel National, Inc., Richland, Washington.

Merrington AC and EG Richardson. 1947. “The Break-Up of Liquid Jets.” Proceedings of the Physical Society 59(331):1-13.

Monecke J, 1994. "Bergman Spectral Representation of a Simple Expression for the Dielectric Response of a Symmetric Two-Component Composite.” Journal of Physics: Condensed Matter 6:907-912.

Mulhem B, G Schulte, and U Fritsching. 2006. "Solid-Liquid Separation in Suspension Atomization.” Chemical Engineering Science 61:2582-2589.

Mulhem B, U Fritsching, G Schulte, and K Bauckhage. 2001. "Effect of Solid Particle Size on Suspension Atomization." In Proceedings of the $17^{\text {th }}$ Annual Conference on Liquid Atomization and Spray Systems, September 2-6, 2001, Zurich, Switzerland.

Mulhem B, U Fritsching, G Schulte, and K Bauckhage. 2003. “Effect of Solid Particle Characteristics on Suspension Atomization.” Atomization and Sprays 13:321-343.

Nasr GG, AJ Yule, and L Bendig. 2002. Industrial Sprays and Atomization: Design, Analysis and Applications. Springer, New York, New York. 
Pilch M and CA Endman. 1987. "Use of Breakup Time Data and Velocity History Data to Predict the Maximum Size of Stable Fragment for Acceleration-Induced Breakup of a Liquid Drop.” International Journal of Multiphase Flow 13(6):741-757.

Poloski AP, PA Meyer, LK Jagoda, and PR Hrma. 2004. Non-Newtonian Slurry Simulant Development and Selection for Pulse Jet Mixer Testing. PNWD-3495, WTP-RPT-111 Rev 0, Battelle--Pacific Northwest Division, Richland, Washington.

Process Metrix. 2007. Process Particle Counter: Basic Operation and Technical Specifications. Process Metrix, Pleasanton, California.

Rosin P and E Rammler. 1933. “The Laws Governing the Fineness of Powdered Coal.” Journal of the Institute of Fuel 7:29-36.

Son SY and KD Kihm. 1998. "Effect of Coal Particle Size on Coal-Water Slurry (CWS) Atomization.” Atomization and Sprays 8:503-519.

Tatterson GB. 2003. Scaleup and Design of Industrial Mixing Processes, $2^{\text {nd }}$ ed.

U.S. Department of Energy (DOE). 1994. Airborne Release Fractions/Rates and Respirable Fractions for Nonreactor Nuclear Facilities. DOE-HDBK-3010-94, U.S. Department of Energy, Washington, D.C.

Wells BE, DE Kurath, LA Mahoney, Y Onishi, JL Huckaby, SK Cooley, CA Burns, EC Buck, JM Tingey, RC Daniel, and KK Anderson. 2011. Hanford Waste Physical and Rheological Properties: Data and Gaps. PNNL-20646, EMSP-RPT-006, Pacific Northwest National Laboratory, Richland, Washington.

Williams JC. 2000. Analysis of Waste Leak and Toxic Chemical Release Accidents from Waste Feed Delivery (WFD) Dilute System. RPP-5098, Rev. 1, Fluor Hanford, Richland, Washington.

Zimmerman BD. 2003. Waste Transfer Leaks Technical Basis Document. RPP-13750, Rev. 0, CH2MHILL Hanford Group, Inc., Richland, Washington.

Zlokarnik M. 2006. Scale-up in Chemical Engineering. WILEY-VCH Verlag GmbH \& Co. KGaA, Weinheim, Germany. 
Appendix A

Test Run Log 



\section{Appendix A}

\section{Test Run Log}

The tables in this appendix present information and data from the entire testing program that was performed in the large-scale spray release system. Table A.1 presents the run log of the tests with the nominal/target conditions of each test and other notes. Refer to Table 5.1 for the actual sizes of the orifices listed in Table A.1. Tables A.2 and A.3 summarize the conventions used in Table A.1 to describe the configuration of the aerosol chamber during testing and the operating conditions of the PPC instrument, respectively. Note that the chamber configuration described as "A9" in this appendix was the most commonly used chamber configuration during testing. Configuration "A9" is the standard configuration described in Chapter 5. Finally, Table A.4 presents the results of the analysis of the process instrument data, including the actual average pressure and temperature of each test. The methods used to calculate the results in Table A.4 are described in detail in Section 7.1. Note that entries in Table A.4 are traceable to Table A.1 entries via the test number, which is a unique identifier.

Table A.1. Run Log of the Large-Scale Spray Release Tests

\begin{tabular}{|c|c|c|c|c|c|c|c|}
\hline $\begin{array}{l}\text { Test } \\
\text { No. }\end{array}$ & Date & $\begin{array}{c}\text { Pressure } \\
\text { (psig) }\end{array}$ & $\begin{array}{c}\text { Orifice } \\
\text { ID }\end{array}$ & $\begin{array}{l}\text { Orifice Type, } \\
\text { Size }\end{array}$ & Fluid & $\begin{array}{c}\text { Chamber } \\
\text { Configuration }^{(a)}\end{array}$ & Other Conditions $^{(\mathbf{b})}$ \\
\hline W01 & $11 / 23 / 2011$ & 380 & S1D & round, $2 \mathrm{~mm}$ & Water & A1 & $\begin{array}{l}\text { Motorcycle fans: } 0 \mathrm{~V} ; \\
\text { PPC cond = } 0\end{array}$ \\
\hline W02 & $11 / 23 / 2011$ & 380 & S1D & round, $2 \mathrm{~mm}$ & Water & A1 & $\begin{array}{l}\text { Motorcycle fans: } 6 \mathrm{~V} ; \\
\text { PPC cond = } 0\end{array}$ \\
\hline W03 & $11 / 23 / 2011$ & 380 & S1D & round, $2 \mathrm{~mm}$ & Water & A1 & $\begin{array}{l}\text { Motorcycle fans: } 12 \mathrm{~V} ; \\
\text { PPC cond = } 0\end{array}$ \\
\hline W04 & $11 / 29 / 2011$ & 380 & S1D & round, $2 \mathrm{~mm}$ & Water & A1 & $\begin{array}{l}\text { Motorcycle fans: } 15 \mathrm{~V} ; \\
\text { PPC cond = } 0\end{array}$ \\
\hline W05 & $12 / 1 / 2011$ & 380 & S1D & round, $2 \mathrm{~mm}$ & Water & A2 & $\begin{array}{l}\text { Motorcycle fans: } 0 \mathrm{~V} ; \\
\quad \text { PPC cond = }\end{array}$ \\
\hline W06 & $12 / 1 / 2011$ & 380 & S1D & round, $2 \mathrm{~mm}$ & Water & A2 & $\begin{array}{l}\text { Motorcycle fans: } 15 \mathrm{~V} ; \\
\text { PPC cond = } 0\end{array}$ \\
\hline W07 & $12 / 1 / 2011$ & 380 & S1D & round, $2 \mathrm{~mm}$ & Water & A1 & $\begin{array}{l}\text { Bilge fans (2 inner): 0V; } \\
\text { PPC cond = } 0\end{array}$ \\
\hline W08 & $12 / 1 / 2011$ & 380 & S1D & round, $2 \mathrm{~mm}$ & Water & A1 & $\begin{array}{l}\text { Bilge fans ( } 2 \text { inner): } 6 \mathrm{~V} ; \\
\text { PPC cond = }\end{array}$ \\
\hline W09 & $12 / 1 / 2011$ & 380 & S1D & round, $2 \mathrm{~mm}$ & Water & A1 & $\begin{array}{l}\text { Bilge fans ( } 2 \text { inner): } 12 \mathrm{~V} ; \\
\text { PPC cond = } 0\end{array}$ \\
\hline W10 & $12 / 1 / 2011$ & 380 & S1D & round, $2 \mathrm{~mm}$ & Water & A1 & $\begin{array}{l}\text { Bilge fans ( } 2 \text { inner): 15V; } \\
\text { PPC cond = } 0\end{array}$ \\
\hline W11 & $12 / 1 / 2011$ & 380 & S1D & round, $2 \mathrm{~mm}$ & Water & A1 & $\begin{array}{l}\text { Bilge fans (all 4): 6V; } \\
\text { PPC cond = } 0\end{array}$ \\
\hline W12 & $12 / 1 / 2011$ & 380 & S1D & round, $2 \mathrm{~mm}$ & Water & A1 & $\begin{array}{l}\text { Bilge fans (all 4): } 12 \mathrm{~V} \text {; } \\
\text { PPC cond = } 0\end{array}$ \\
\hline W13 & $12 / 1 / 2011$ & 380 & S1D & round, $2 \mathrm{~mm}$ & Water & A1 & $\begin{array}{l}\text { Bilge fans (all 4): } 15 \mathrm{~V} ; \\
\text { PPC cond = } 0\end{array}$ \\
\hline
\end{tabular}


Table A.1. (contd)

\begin{tabular}{|c|c|c|c|c|c|c|c|}
\hline $\begin{array}{l}\text { Test } \\
\text { No. }\end{array}$ & Date & $\begin{array}{c}\text { Pressure } \\
\text { (psig) }\end{array}$ & $\begin{array}{c}\text { Orifice } \\
\text { ID }\end{array}$ & $\begin{array}{l}\text { Orifice Type, } \\
\text { Size }\end{array}$ & Fluid & $\begin{array}{c}\text { Chamber } \\
\text { Configuration }^{(a)}\end{array}$ & Other Conditions $^{(b)}$ \\
\hline W14 & $12 / 2 / 2011$ & 380 & S1D & round, $2 \mathrm{~mm}$ & Water & A1 & $\begin{array}{l}\text { All fans (2 Motorcycle, } 4 \\
\text { Bilge): 0V; PPC cond }=0\end{array}$ \\
\hline W15 & $12 / 2 / 2011$ & 380 & S1D & round, $2 \mathrm{~mm}$ & Water & A1 & $\begin{array}{l}\text { All fans (2 Motorcycle, } 4 \\
\text { Bilge): } 6 \mathrm{~V} \text {; PPC cond }=0\end{array}$ \\
\hline W16 & $12 / 2 / 2011$ & 380 & S1D & round, $2 \mathrm{~mm}$ & Water & A1 & $\begin{array}{c}\text { All fans (2 Motorcycle, } 4 \\
\text { Bilge): } 12 \mathrm{~V} ; \text { PPC } \\
\text { cond = } 0\end{array}$ \\
\hline W17 & $12 / 2 / 2011$ & 380 & S1D & round, $2 \mathrm{~mm}$ & Water & A1 & $\begin{array}{c}\text { All fans (2 Motorcycle, } 4 \\
\text { Bilge): 15V; PPC } \\
\text { cond = } 0\end{array}$ \\
\hline W18 & $12 / 5 / 2011$ & 380 & S1D & round, $2 \mathrm{~mm}$ & Water & A1 & $\begin{array}{c}\text { Modified Bilge fans (2 } \\
\text { inner): 0V; PPC cond = } 0\end{array}$ \\
\hline W19 & $12 / 5 / 2011$ & 380 & S1D & round, $2 \mathrm{~mm}$ & Water & A1 & $\begin{array}{l}\text { Modified Bilge fans ( } 2 \\
\text { inner): } 6 \mathrm{~V} \text {; PPC cond = } 0\end{array}$ \\
\hline W20 & $12 / 5 / 2011$ & 380 & S1D & round, $2 \mathrm{~mm}$ & Water & A1 & $\begin{array}{l}\text { Modified Bilge fans (2 } \\
\text { inner): } 12 \mathrm{~V} ; \text { PPC } \\
\text { cond = } 0\end{array}$ \\
\hline W21 & $12 / 5 / 2011$ & 380 & S1D & round, $2 \mathrm{~mm}$ & Water & A1 & $\begin{array}{l}\text { Modified Bilge fans (2 } \\
\text { inner): 15V; PPC } \\
\text { cond = } 0\end{array}$ \\
\hline W22 & $12 / 5 / 2011$ & 380 & S1D & round, $2 \mathrm{~mm}$ & Water & A1 & $\begin{array}{l}\text { Modified Bilge fans (2 } \\
\text { inner): } 12 \mathrm{~V} ; \text { PPC } \\
\text { cond = } 0\end{array}$ \\
\hline W23 & $12 / 5 / 2011$ & 380 & S1D & round, $2 \mathrm{~mm}$ & Water & A1 & $\begin{array}{l}\text { Modified Bilge fans ( } 2 \\
\text { inner): 0V; PPC cond = } 0\end{array}$ \\
\hline W24 & $12 / 5 / 2011$ & 380 & S1D & round, $2 \mathrm{~mm}$ & Water & A1 & $\begin{array}{l}\text { Modified Bilge fans (2 } \\
\text { inner): 15V; PPC } \\
\text { cond = 0 }\end{array}$ \\
\hline W25 & $12 / 5 / 2011$ & 380 & S1D & round, $2 \mathrm{~mm}$ & Water & A1 & $\begin{array}{c}\text { Modified Bilge fans ( } 2 \\
\text { inner): 6V; PPC cond = } 0\end{array}$ \\
\hline W26 & $12 / 6 / 2011$ & 200 & S1A & round, $1 \mathrm{~mm}$ & Water & A1 & STD fans; PPC cond $=0$ \\
\hline W27 & $12 / 6 / 2011$ & 380 & S1A & round, $1 \mathrm{~mm}$ & Water & A1 & STD fans; PPC cond $=0$ \\
\hline W28 & $12 / 6 / 2011$ & 200 & S1B & round, $4.46 \mathrm{~mm}$ & Water & A1 & STD fans; PPC cond = 0 \\
\hline W29 & $12 / 6 / 2011$ & 380 & S1B & round, $4.46 \mathrm{~mm}$ & Water & A1 & STD fans; PPC cond = 0 \\
\hline W30 & $12 / 6 / 2011$ & 200 & S1C & round, $2.74 \mathrm{~mm}$ & Water & A1 & STD fans; PPC cond $=0$ \\
\hline W31 & $12 / 6 / 2011$ & 380 & S1C & round, $2.74 \mathrm{~mm}$ & Water & A1 & STD fans; PPC cond = 0 \\
\hline W32 & $12 / 6 / 2011$ & 200 & S1A & round, $1 \mathrm{~mm}$ & Water & A1 & STD fans; PPC cond = 0 \\
\hline W33 & $12 / 6 / 2011$ & 380 & S1A & round, $1 \mathrm{~mm}$ & Water & A1 & STD fans; PPC cond $=0$ \\
\hline W34 & $12 / 6 / 2011$ & 200 & S1B & round, $4.46 \mathrm{~mm}$ & Water & A1 & STD fans; PPC cond $=0$ \\
\hline W35 & $12 / 6 / 2011$ & 380 & S1B & round, $4.46 \mathrm{~mm}$ & Water & A1 & STD fans; PPC cond = 0 \\
\hline W36 & $12 / 6 / 2011$ & 200 & S1C & round, $2.74 \mathrm{~mm}$ & Water & A1 & STD fans; PPC cond = 0 \\
\hline W37 & $12 / 6 / 2011$ & 380 & S1C & round, $2.74 \mathrm{~mm}$ & Water & A1 & STD fans; PPC cond $=0$ \\
\hline W38 & $12 / 7 / 2011$ & 100 & S2A & slot, $0.5 \times 5 \mathrm{~mm}$ & Water & A3 & STD fans; PPC cond $=0$ \\
\hline
\end{tabular}


Table A.1. (contd)

\begin{tabular}{|c|c|c|c|c|c|c|c|}
\hline $\begin{array}{l}\text { Test } \\
\text { No. }\end{array}$ & Date & $\begin{array}{c}\text { Pressure } \\
\text { (psig) }\end{array}$ & $\begin{array}{c}\text { Orifice } \\
\text { ID }\end{array}$ & $\begin{array}{c}\text { Orifice Type, } \\
\text { Size }\end{array}$ & Fluid & $\begin{array}{c}\text { Chamber } \\
\text { Configuration }^{(a)}\end{array}$ & Other Conditions $^{(\mathbf{b})}$ \\
\hline W39 & $12 / 7 / 2011$ & 200 & S2A & slot, $0.5 \times 5 \mathrm{~mm}$ & Water & A3 & STD fans; PPC cond $=0$ \\
\hline W40 & $12 / 7 / 2011$ & 380 & S2A & slot, $0.5 \times 5 \mathrm{~mm}$ & Water & A3 & STD fans; PPC cond $=0$ \\
\hline W41 & $12 / 7 / 2011$ & 200 & S2A & slot, $0.5 \times 5 \mathrm{~mm}$ & Water & A3 & STD fans; PPC cond $=0$ \\
\hline W42 & $12 / 7 / 2011$ & 380 & S2A & slot, $0.5 \times 5 \mathrm{~mm}$ & Water & A3 & STD fans; PPC cond $=0$ \\
\hline W43 & $12 / 7 / 2011$ & 100 & S2A & slot, $0.5 \times 5 \mathrm{~mm}$ & Water & A3 & STD fans; PPC cond $=0$ \\
\hline W44 & $12 / 8 / 2011$ & 200 & S5A & slot, $10 \times 1 \mathrm{~mm}$ & Water & A3 & STD fans; PPC cond $=0$ \\
\hline W45 & $12 / 8 / 2011$ & 380 & S5A & slot, $10 \times 1 \mathrm{~mm}$ & Water & A3 & STD fans; PPC cond $=0$ \\
\hline W46 & $12 / 8 / 2011$ & 200 & S5B & round, (5x) $1 \mathrm{~mm}$ & Water & A3 & STD fans; PPC cond $=0$ \\
\hline W47 & $12 / 8 / 2011$ & 380 & S5B & round, (5x) $1 \mathrm{~mm}$ & Water & A3 & STD fans; PPC cond $=0$ \\
\hline W48 & $12 / 8 / 2011$ & 200 & S5C & slot, $1 \times 20 \mathrm{~mm}$ & Water & A3 & STD fans; PPC cond $=0$ \\
\hline W49 & $12 / 8 / 2011$ & 380 & S5C & slot, $1 \times 20 \mathrm{~mm}$ & Water & A3 & STD fans; PPC cond $=0$ \\
\hline W50 & $12 / 8 / 2011$ & 200 & S5A & slot, $10 \times 1 \mathrm{~mm}$ & Water & A3 & STD fans; PPC cond $=0$ \\
\hline W51 & $12 / 8 / 2011$ & 380 & S5A & slot, $10 \times 1 \mathrm{~mm}$ & Water & A3 & STD fans; PPC cond $=0$ \\
\hline W52 & $12 / 8 / 2011$ & 200 & S5B & round, (5x) $1 \mathrm{~mm}$ & Water & A3 & STD fans; PPC cond $=0$ \\
\hline W53 & $12 / 8 / 2011$ & 380 & S5B & round, (5x) $1 \mathrm{~mm}$ & Water & A3 & STD fans; PPC cond $=0$ \\
\hline W54 & $12 / 9 / 2011$ & 100 & S7A & slot, $1 \times 10 \mathrm{~mm}$ & Water & A3 & STD fans; PPC cond $=0$ \\
\hline W55 & $12 / 9 / 2011$ & 100 & S7B & slot, $2 \times 10 \mathrm{~mm}$ & Water & A3 & STD fans; PPC cond $=0$ \\
\hline W56 & $12 / 9 / 2011$ & 100 & S7C & slot, $3 \times 10 \mathrm{~mm}$ & Water & A3 & STD fans; PPC cond $=0$ \\
\hline W57 & $12 / 9 / 2011$ & 200 & S7A & slot, $1 \times 10 \mathrm{~mm}$ & Water & A3 & STD fans; PPC cond $=0$ \\
\hline W58 & $12 / 9 / 2011$ & 200 & S7B & slot, $2 \times 10 \mathrm{~mm}$ & Water & A3 & STD fans; PPC cond $=0$ \\
\hline W59 & $12 / 9 / 2011$ & 200 & S7C & slot, $3 \times 10 \mathrm{~mm}$ & Water & A3 & STD fans; PPC cond $=0$ \\
\hline W60 & $12 / 9 / 2011$ & 380 & S7A & slot, $1 \times 10 \mathrm{~mm}$ & Water & A3 & STD fans; PPC cond $=0$ \\
\hline W61 & $12 / 14 / 2011$ & 380 & S1A & round, $1 \mathrm{~mm}$ & Water & A4 & STD fans; PPC cond = 1 \\
\hline W62 & $12 / 14 / 2011$ & 380 & S1A & round, $1 \mathrm{~mm}$ & Water & A4 & STD fans; PPC cond = 1 \\
\hline W63 & $12 / 14 / 2011$ & 380 & S1A & round, $1 \mathrm{~mm}$ & Water & A4 & STD fans; PPC cond = 1 \\
\hline W64 & $12 / 14 / 2011$ & 380 & S1A & round, $1 \mathrm{~mm}$ & Water & A5 & STD fans; PPC cond = 1 \\
\hline W65 & $12 / 14 / 2011$ & 380 & S1A & round, $1 \mathrm{~mm}$ & Water & A5 & STD fans; PPC cond = 1 \\
\hline W66 & $12 / 14 / 2011$ & 380 & S1A & round, $1 \mathrm{~mm}$ & Water & A5 & STD fans; PPC cond = 1 \\
\hline W67 & $12 / 14 / 2011$ & 380 & S1A & round, $1 \mathrm{~mm}$ & Water & A6 & STD fans; PPC cond = 1 \\
\hline W68 & $12 / 14 / 2011$ & 380 & S1A & round, $1 \mathrm{~mm}$ & Water & A6 & STD fans; PPC cond = 1 \\
\hline W69 & $12 / 14 / 2011$ & 380 & S1A & round, $1 \mathrm{~mm}$ & Water & A6 & STD fans; PPC cond = 1 \\
\hline W70 & $12 / 15 / 2011$ & 380 & S1D & round, $2 \mathrm{~mm}$ & Water & A7 & STD fans; PPC cond $=2$ \\
\hline W71 & $12 / 15 / 2011$ & 380 & S1B & round, $4.46 \mathrm{~mm}$ & Water & A7 & $\begin{array}{l}\text { STD fans; PPC cond = 2; } \\
\text { test aborted when clamp } \\
\text { malfunctioned }\end{array}$ \\
\hline W72 & $12 / 15 / 2011$ & 380 & S1B & round, $4.46 \mathrm{~mm}$ & Water & A7 & STD fans; PPC cond = 2 \\
\hline W73 & $12 / 19 / 2011$ & 200 & S1A & round, $1 \mathrm{~mm}$ & Water & A8 & STD fans; PPC cond = 3 \\
\hline
\end{tabular}


Table A.1. (contd)

\begin{tabular}{|c|c|c|c|c|c|c|c|}
\hline $\begin{array}{l}\text { Test } \\
\text { No. }\end{array}$ & Date & $\begin{array}{c}\text { Pressure } \\
\text { (psig) }\end{array}$ & \begin{tabular}{|c|} 
Orifice \\
ID
\end{tabular} & $\begin{array}{c}\text { Orifice Type, } \\
\text { Size }\end{array}$ & Fluid & $\begin{array}{c}\text { Chamber } \\
\text { Configuration }^{(a)}\end{array}$ & Other Conditions $^{(\mathbf{b})}$ \\
\hline W74 & $12 / 19 / 2011$ & 380 & S1A & round, $1 \mathrm{~mm}$ & Water & A8 & STD fans; PPC cond = 3 \\
\hline W75 & 12/19/2011 & 200 & S1B & round, $4.46 \mathrm{~mm}$ & Water & A8 & STD fans; PPC cond $=3$ \\
\hline W76 & $12 / 19 / 2011$ & 380 & S1B & round, $4.46 \mathrm{~mm}$ & Water & A8 & STD fans; PPC cond = 3 \\
\hline W77 & $12 / 19 / 2011$ & 200 & S1C & round, $2.74 \mathrm{~mm}$ & Water & A8 & STD fans; PPC cond = 3 \\
\hline W78 & $12 / 19 / 2011$ & 380 & S1C & round, $2.74 \mathrm{~mm}$ & Water & A8 & STD fans; PPC cond = 3 \\
\hline W79 & $12 / 19 / 2011$ & 100 & S1D & round, $2 \mathrm{~mm}$ & Water & A8 & STD fans; PPC cond = 3 \\
\hline W80 & $12 / 19 / 2011$ & 200 & S1D & round, $2 \mathrm{~mm}$ & Water & A8 & STD fans; PPC cond = 3 \\
\hline W81 & $12 / 19 / 2011$ & 380 & S1D & round, $2 \mathrm{~mm}$ & Water & A8 & STD fans; PPC cond = 3 \\
\hline W82 & $12 / 19 / 2011$ & 200 & S1A & round, $1 \mathrm{~mm}$ & Water & A8 & STD fans; PPC cond = 3 \\
\hline W83 & $12 / 20 / 2011$ & 380 & S1A & round, $1 \mathrm{~mm}$ & Water & A8 & STD fans; PPC cond = 3 \\
\hline W84 & $12 / 20 / 2011$ & 200 & S1B & round, $4.46 \mathrm{~mm}$ & Water & A8 & STD fans; PPC cond = 3 \\
\hline W85 & $12 / 20 / 2011$ & 380 & S1B & round, $4.46 \mathrm{~mm}$ & Water & A8 & STD fans; PPC cond = 3 \\
\hline W86 & $12 / 20 / 2011$ & 100 & S1D & round, $2 \mathrm{~mm}$ & Water & A8 & STD fans; PPC cond = 3 \\
\hline W87 & $12 / 20 / 2011$ & 200 & S1D & round, $2 \mathrm{~mm}$ & Water & A8 & STD fans; PPC cond = 3 \\
\hline W88 & $12 / 20 / 2011$ & 380 & S1D & round, $2 \mathrm{~mm}$ & Water & A8 & STD fans; PPC cond = 3 \\
\hline W89 & $12 / 21 / 2011$ & 100 & S7A & slot, $1 \times 10 \mathrm{~mm}$ & Water & A8 & STD fans; PPC cond = 3 \\
\hline W90 & $12 / 21 / 2011$ & 200 & S7A & slot, $1 \times 10 \mathrm{~mm}$ & Water & A8 & STD fans; PPC cond = 3 \\
\hline W91 & $12 / 21 / 2011$ & 380 & S7A & slot, $1 \times 10 \mathrm{~mm}$ & Water & A8 & STD fans; PPC cond = 3 \\
\hline W92 & $12 / 21 / 2011$ & 100 & S7C & slot, $3 \times 10 \mathrm{~mm}$ & Water & A8 & STD fans; PPC cond = 3 \\
\hline W93 & $12 / 21 / 2011$ & 200 & S7C & slot, $3 \times 10 \mathrm{~mm}$ & Water & A8 & STD fans; PPC cond = 3 \\
\hline W94 & $12 / 21 / 2011$ & 380 & S7C & slot, $3 \times 10 \mathrm{~mm}$ & Water & A8 & STD fans; PPC cond = 3 \\
\hline W95 & $12 / 21 / 2011$ & 100 & S7A & slot, $1 \times 10 \mathrm{~mm}$ & Water & A8 & STD fans; PPC cond = 3 \\
\hline W96 & $12 / 21 / 2011$ & 380 & S7A & slot, $1 \times 10 \mathrm{~mm}$ & Water & A8 & STD fans; PPC cond = 3 \\
\hline W97 & $12 / 21 / 2011$ & 100 & S7C & slot, $3 \times 10 \mathrm{~mm}$ & Water & A8 & STD fans; PPC cond = 3 \\
\hline W98 & $12 / 21 / 2011$ & 380 & S7C & slot, $3 \times 10 \mathrm{~mm}$ & Water & A8 & STD fans; PPC cond = 3 \\
\hline W99 & $12 / 22 / 2011$ & 100 & S5A & slot, $10 \times 1 \mathrm{~mm}$ & Water & A9 & STD fans; PPC cond = 3 \\
\hline W100 & $12 / 22 / 2011$ & 200 & S5A & slot, $10 \times 1 \mathrm{~mm}$ & Water & A9 & STD fans; PPC cond = 3 \\
\hline W101 & $12 / 22 / 2011$ & 380 & S5A & slot, $10 \times 1 \mathrm{~mm}$ & Water & A9 & STD fans; PPC cond = 3 \\
\hline W102 & $12 / 22 / 2011$ & 100 & S5B & round, $(5 \mathrm{x}) 1 \mathrm{~mm}$ & Water & A9 & STD fans; PPC cond = 3 \\
\hline W103 & $12 / 22 / 2011$ & 200 & S5B & round, (5x) $1 \mathrm{~mm}$ & Water & A9 & STD fans; PPC cond = 3 \\
\hline W104 & $12 / 22 / 2011$ & 380 & S5B & round, (5x) $1 \mathrm{~mm}$ & Water & A9 & STD fans; PPC cond = 3 \\
\hline W105 & $12 / 22 / 2011$ & 380 & S5B & round, $(5 \mathrm{x}) 1 \mathrm{~mm}$ & Water & A9 & STD fans; PPC cond = 3 \\
\hline W106 & $12 / 22 / 2011$ & 100 & S5C & slot, $1 \times 20 \mathrm{~mm}$ & Water & A9 & STD fans; PPC cond = 3 \\
\hline W107 & $12 / 22 / 2011$ & 200 & S5C & slot, $1 \times 20 \mathrm{~mm}$ & Water & A9 & STD fans; PPC cond = 3 \\
\hline W108 & $12 / 22 / 2011$ & 380 & S5C & slot, $1 \times 20 \mathrm{~mm}$ & Water & A9 & STD fans; PPC cond = 3 \\
\hline W109 & $12 / 27 / 2011$ & 100 & S5A & slot, $10 \times 1 \mathrm{~mm}$ & Water & A9 & STD fans; PPC cond = 3 \\
\hline W110 & $12 / 27 / 2011$ & 380 & S5A & slot, $10 \times 1 \mathrm{~mm}$ & Water & A9 & STD fans; PPC cond = 3 \\
\hline
\end{tabular}


Table A.1. (contd)

\begin{tabular}{|c|c|c|c|c|c|c|c|}
\hline $\begin{array}{l}\text { Test } \\
\text { No. }\end{array}$ & Date & $\begin{array}{c}\text { Pressure } \\
\text { (psig) }\end{array}$ & $\begin{array}{c}\text { Orifice } \\
\text { ID }\end{array}$ & $\begin{array}{c}\text { Orifice Type, } \\
\text { Size }\end{array}$ & Fluid & $\begin{array}{c}\text { Chamber } \\
\text { Configuration }^{(a)}\end{array}$ & Other Conditions $^{(\mathbf{b})}$ \\
\hline W111 & $12 / 27 / 2011$ & 100 & S5B & round, (5x) $1 \mathrm{~mm}$ & Water & A9 & STD fans; PPC cond = 3 \\
\hline W112 & $12 / 27 / 2011$ & 380 & S5B & round, (5x) $1 \mathrm{~mm}$ & Water & A9 & STD fans; PPC cond = 3 \\
\hline W113 & $12 / 27 / 2011$ & 100 & S5C & slot, $1 \times 20 \mathrm{~mm}$ & Water & A9 & STD fans; PPC cond $=3$ \\
\hline W114 & $12 / 27 / 2011$ & 380 & S5C & slot, $1 \times 20 \mathrm{~mm}$ & Water & A9 & STD fans; PPC cond = 3 \\
\hline W115 & $12 / 27 / 2011$ & 100 & S4A & slot, $1 \times 76.2 \mathrm{~mm}$ & Water & A9 & STD fans; PPC cond = 3 \\
\hline W116 & $12 / 27 / 2011$ & 200 & S4A & slot, $1 \times 76.2 \mathrm{~mm}$ & Water & A9 & STD fans; PPC cond $=3$ \\
\hline W117 & $12 / 28 / 2011$ & 100 & S4A & slot, $1 \times 76.2 \mathrm{~mm}$ & Water & A9 & STD fans; PPC cond = 3 \\
\hline W118 & $12 / 28 / 2011$ & 200 & S4A & slot, $1 \times 76.2 \mathrm{~mm}$ & Water & A9 & STD fans; PPC cond = 3 \\
\hline W119 & $12 / 28 / 2011$ & 380 & S4A & slot, $1 \times 76.2 \mathrm{~mm}$ & Water & A9 & STD fans; PPC cond $=3$ \\
\hline W120 & $12 / 28 / 2011$ & 100 & S2A & slot, $0.5 \times 5 \mathrm{~mm}$ & Water & A9 & STD fans; PPC cond = 3 \\
\hline W121 & $12 / 28 / 2011$ & 380 & S2A & slot, $0.5 \times 5 \mathrm{~mm}$ & Water & A9 & STD fans; PPC cond $=3$ \\
\hline W122 & $12 / 28 / 2011$ & 200 & S2A & slot, $0.5 \times 5 \mathrm{~mm}$ & Water & A9 & STD fans; PPC cond = 3 \\
\hline W123 & $12 / 28 / 2011$ & 200 & S2A & slot, $0.5 \times 5 \mathrm{~mm}$ & Water & A9 & STD fans; PPC cond = 3 \\
\hline W124 & $12 / 28 / 2011$ & 100 & S2A & slot, $0.5 \times 5 \mathrm{~mm}$ & Water & A9 & STD fans; PPC cond = 3 \\
\hline W125 & $12 / 28 / 2011$ & 380 & S2A & slot, $0.5 \times 5 \mathrm{~mm}$ & Water & A9 & STD fans; PPC cond $=3$ \\
\hline W126 & $1 / 3 / 2012$ & 100 & S1A & round, $1 \mathrm{~mm}$ & Water & C9 & STD fans; PPC cond = 3 \\
\hline W127 & $1 / 3 / 2012$ & 200 & S1A & round, $1 \mathrm{~mm}$ & Water & C9 & STD fans; PPC cond $=3$ \\
\hline W128 & $1 / 3 / 2012$ & 380 & S1A & round, $1 \mathrm{~mm}$ & Water & C9 & STD fans; PPC cond $=3$ \\
\hline W129 & $1 / 3 / 2012$ & 100 & S1D & round, $2 \mathrm{~mm}$ & Water & C9 & STD fans; PPC cond = 3 \\
\hline W130 & $1 / 3 / 2012$ & 200 & S1D & round, $2 \mathrm{~mm}$ & Water & C9 & STD fans; PPC cond $=3$ \\
\hline W131 & $1 / 3 / 2012$ & 380 & S1D & round, $2 \mathrm{~mm}$ & Water & C9 & STD fans; PPC cond $=3$ \\
\hline W132 & $1 / 3 / 2012$ & 100 & S1A & round, $1 \mathrm{~mm}$ & Water & C9 & STD fans; PPC cond = 3 \\
\hline W133 & $1 / 3 / 2012$ & 380 & S1A & round, $1 \mathrm{~mm}$ & Water & C9 & STD fans; PPC cond = 3 \\
\hline W134 & $1 / 3 / 2012$ & 100 & S1D & round, $2 \mathrm{~mm}$ & Water & C9 & STD fans; PPC cond $=3$ \\
\hline W135 & $1 / 3 / 2012$ & 380 & S1D & round, $2 \mathrm{~mm}$ & Water & C9 & STD fans; PPC cond = 3 \\
\hline W136 & $1 / 4 / 2012$ & 100 & S1A & round, $1 \mathrm{~mm}$ & Water & B9 & STD fans; PPC cond = 3 \\
\hline W137 & $1 / 4 / 2012$ & 200 & S1A & round, $1 \mathrm{~mm}$ & Water & B9 & STD fans; PPC cond = 3 \\
\hline W138 & $1 / 4 / 2012$ & 380 & S1A & round, $1 \mathrm{~mm}$ & Water & B9 & STD fans; PPC cond = 3 \\
\hline W139 & $1 / 4 / 2012$ & 100 & S1D & round, $2 \mathrm{~mm}$ & Water & B9 & STD fans; PPC cond = 3 \\
\hline W140 & $1 / 4 / 2012$ & 200 & S1D & round, $2 \mathrm{~mm}$ & Water & B9 & STD fans; PPC cond = 3 \\
\hline W141 & $1 / 4 / 2012$ & 380 & S1D & round, $2 \mathrm{~mm}$ & Water & B9 & STD fans; PPC cond = 3 \\
\hline W142 & $1 / 4 / 2012$ & 100 & S1D & round, $2 \mathrm{~mm}$ & Water & B9 & STD fans; PPC cond = 3 \\
\hline W143 & $1 / 4 / 2012$ & 380 & S1D & round, $2 \mathrm{~mm}$ & Water & B9 & STD fans; PPC cond = 3 \\
\hline W144 & $1 / 4 / 2012$ & 100 & S1A & round, $1 \mathrm{~mm}$ & Water & B9 & STD fans; PPC cond = 3 \\
\hline W145 & $1 / 4 / 2012$ & 380 & S1A & round, $1 \mathrm{~mm}$ & Water & B9 & STD fans; PPC cond = 3 \\
\hline S146 & $1 / 5 / 2012$ & 100 & S7A & slot, $1 \times 10 \mathrm{~mm}$ & $\begin{array}{l}\text { Salt } \\
2.5\end{array}$ & A9 & STD fans; PPC cond $=3$ \\
\hline
\end{tabular}


Table A.1. (contd)

\begin{tabular}{|c|c|c|c|c|c|c|c|}
\hline $\begin{array}{l}\text { Test } \\
\text { No. }\end{array}$ & Date & $\begin{array}{c}\text { Pressure } \\
\text { (psig) }\end{array}$ & $\begin{array}{c}\text { Orifice } \\
\text { ID }\end{array}$ & $\begin{array}{c}\text { Orifice Type, } \\
\text { Size }\end{array}$ & Fluid & $\begin{array}{c}\text { Chamber } \\
\text { Configuration }^{(a)}\end{array}$ & Other Conditions $^{(\mathbf{b})}$ \\
\hline S147 & $1 / 5 / 2012$ & 200 & S7A & slot, $1 \times 10 \mathrm{~mm}$ & $\begin{array}{l}\text { Salt } \\
2.5\end{array}$ & A9 & STD fans; PPC cond = 3 \\
\hline S148 & $1 / 5 / 2012$ & 380 & S7A & slot, $1 \times 10 \mathrm{~mm}$ & $\begin{array}{l}\text { Salt } \\
2.5\end{array}$ & A9 & STD fans; PPC cond = 3 \\
\hline S149 & $1 / 5 / 2012$ & 380 & S7A & slot, $1 \times 10 \mathrm{~mm}$ & $\begin{array}{l}\text { Salt } \\
2.5\end{array}$ & A9 & STD fans; PPC cond = 3 \\
\hline S150 & $1 / 5 / 2012$ & 100 & S7A & slot, $1 \times 10 \mathrm{~mm}$ & $\begin{array}{l}\text { Salt } \\
2.5\end{array}$ & A9 & STD fans; PPC cond = 3 \\
\hline S151 & $1 / 5 / 2012$ & 200 & S7A & slot, $1 \times 10 \mathrm{~mm}$ & $\begin{array}{l}\text { Salt } \\
2.5\end{array}$ & A9 & STD fans; PPC cond = 3 \\
\hline S152 & $1 / 5 / 2012$ & 380 & S7A & slot, $1 \times 10 \mathrm{~mm}$ & $\begin{array}{l}\text { Salt } \\
2.5\end{array}$ & A9 & STD fans; PPC cond = 3 \\
\hline S153 & $1 / 6 / 2012$ & 100 & S2A & slot, $0.5 \times 5 \mathrm{~mm}$ & $\begin{array}{l}\text { Salt } \\
2.5\end{array}$ & A9 & STD fans; PPC cond = 3 \\
\hline S154 & $1 / 6 / 2012$ & 200 & S2A & slot, $0.5 \times 5 \mathrm{~mm}$ & $\begin{array}{c}\text { Salt } \\
2.5\end{array}$ & A9 & STD fans; PPC cond = 3 \\
\hline S155 & $1 / 6 / 2012$ & 380 & S2A & slot, $0.5 \times 5 \mathrm{~mm}$ & $\begin{array}{l}\text { Salt } \\
2.5\end{array}$ & A9 & STD fans; PPC cond = 3 \\
\hline S156 & $1 / 6 / 2012$ & 100 & S2A & slot, $0.5 \times 5 \mathrm{~mm}$ & $\begin{array}{l}\text { Salt } \\
2.5\end{array}$ & A9 & STD fans; PPC cond = 3 \\
\hline S157 & $1 / 6 / 2012$ & 200 & S2A & slot, $0.5 \times 5 \mathrm{~mm}$ & $\begin{array}{l}\text { Salt } \\
2.5\end{array}$ & A9 & STD fans; PPC cond = 3 \\
\hline S158 & $1 / 6 / 2012$ & 380 & S2A & slot, $0.5 \times 5 \mathrm{~mm}$ & $\begin{array}{l}\text { Salt } \\
2.5\end{array}$ & A9 & STD fans; PPC cond = 3 \\
\hline W159 & $1 / 9 / 2012$ & 100 & S7A & slot, $1 \times 10 \mathrm{~mm}$ & Water & A9 & STD fans; PPC cond = 3 \\
\hline W160 & $1 / 9 / 2012$ & 200 & S7A & slot, $1 \times 10 \mathrm{~mm}$ & Water & A9 & STD fans; PPC cond = 3 \\
\hline W161 & $1 / 9 / 2012$ & 380 & S7A & slot, $1 \times 10 \mathrm{~mm}$ & Water & A9 & STD fans; PPC cond = 3 \\
\hline W162 & $1 / 9 / 2012$ & 100 & S7B & slot, $2 \times 10 \mathrm{~mm}$ & Water & A9 & STD fans; PPC cond = 3 \\
\hline W163 & $1 / 9 / 2012$ & 200 & S7B & slot, $2 \times 10 \mathrm{~mm}$ & Water & A9 & STD fans; PPC cond = 3 \\
\hline W164 & $1 / 9 / 2012$ & 380 & S7B & slot, $2 \times 10 \mathrm{~mm}$ & Water & A9 & STD fans; PPC cond = 3 \\
\hline W165 & $1 / 9 / 2012$ & 200 & S7C & slot, $3 \times 10 \mathrm{~mm}$ & Water & A9 & STD fans; PPC cond = 3 \\
\hline W166 & $1 / 9 / 2012$ & 380 & S7C & slot, $3 \times 10 \mathrm{~mm}$ & Water & A9 & STD fans; PPC cond = 3 \\
\hline W167 & $1 / 9 / 2012$ & 200 & S7C & slot, $3 \times 10 \mathrm{~mm}$ & Water & A9 & STD fans; PPC cond = 3 \\
\hline W168 & $1 / 9 / 2012$ & 380 & S7C & slot, $3 \times 10 \mathrm{~mm}$ & Water & A9 & STD fans; PPC cond = 3 \\
\hline W169 & $1 / 10 / 2012$ & 200 & S5C & slot, $1 \times 20 \mathrm{~mm}$ & Water & A9 & STD fans; PPC cond = 3 \\
\hline W170 & $1 / 10 / 2012$ & 380 & S5C & slot, $1 \times 20 \mathrm{~mm}$ & Water & A9 & STD fans; PPC cond = 3 \\
\hline W171 & $1 / 10 / 2012$ & 200 & S5C & slot, $1 \times 20 \mathrm{~mm}$ & Water & A9 & STD fans; PPC cond = 3 \\
\hline W172 & $1 / 10 / 2012$ & 380 & S5C & slot, $1 \times 20 \mathrm{~mm}$ & Water & A9 & STD fans; PPC cond = 3 \\
\hline W173 & 1/10/2012 & 100 & S4A & slot, $1 \times 76.2 \mathrm{~mm}$ & Water & A9 & STD fans; PPC cond = 3 \\
\hline
\end{tabular}


Table A.1. (contd)

\begin{tabular}{|c|c|c|c|c|c|c|c|}
\hline $\begin{array}{l}\text { Test } \\
\text { No. }\end{array}$ & Date & $\begin{array}{c}\text { Pressure } \\
\text { (psig) }\end{array}$ & $\begin{array}{c}\text { Orifice } \\
\text { ID }\end{array}$ & $\begin{array}{c}\text { Orifice Type, } \\
\text { Size }\end{array}$ & Fluid & $\begin{array}{c}\text { Chamber } \\
\text { Configuration }^{(a)}\end{array}$ & Other Conditions $^{(\mathbf{b})}$ \\
\hline W174 & $1 / 10 / 2012$ & 200 & S4A & slot, $1 \times 76.2 \mathrm{~mm}$ & Water & A9 & STD fans; PPC cond $=3$ \\
\hline W175 & $1 / 10 / 2012$ & 380 & S4A & slot, $1 \times 76.2 \mathrm{~mm}$ & Water & A9 & STD fans; PPC cond $=3$ \\
\hline W176 & $1 / 10 / 2012$ & 200 & S4A & slot, $1 \times 76.2 \mathrm{~mm}$ & Water & A9 & STD fans; PPC cond = 3 \\
\hline W177 & $1 / 10 / 2012$ & 380 & S4A & slot, $1 \times 76.2 \mathrm{~mm}$ & Water & A9 & STD fans; PPC cond = 3 \\
\hline W178 & $1 / 11 / 2012$ & 100 & S3A & $\begin{array}{c}\text { slot, } 2.74 \times 76.2 \\
\mathrm{~mm}\end{array}$ & Water & A9 & STD fans; PPC cond = 3 \\
\hline W179 & $1 / 11 / 2012$ & 100 & S3A & $\begin{array}{c}\text { slot, } 2.74 \times 76.2 \\
\mathrm{~mm}\end{array}$ & Water & A9 & STD fans; PPC cond = 3 \\
\hline W180 & $1 / 11 / 2012$ & 200 & S3A & $\begin{array}{c}\text { slot, } 2.74 \times 76.2 \\
\mathrm{~mm}\end{array}$ & Water & A9 & STD fans; PPC cond = 3 \\
\hline W181 & $1 / 11 / 2012$ & 200 & S3A & $\begin{array}{c}\text { slot, } 2.74 \times 76.2 \\
\mathrm{~mm}\end{array}$ & Water & A9 & STD fans; PPC cond = 3 \\
\hline W182 & $1 / 11 / 2012$ & 380 & S3A & $\begin{array}{c}\text { slot, } 2.74 \times 76.2 \\
\mathrm{~mm}\end{array}$ & Water & A9 & STD fans; PPC cond = 3 \\
\hline S183 & $1 / 12 / 2012$ & 100 & S2A & slot, $0.5 \times 5 \mathrm{~mm}$ & $\begin{array}{c}\text { STR, } \\
20 \\
\text { wt } \%\end{array}$ & A9 & STD fans; PPC cond = 3 \\
\hline S184 & $1 / 12 / 2012$ & 200 & S2A & slot, $0.5 \times 5 \mathrm{~mm}$ & $\begin{array}{c}\text { STR, } \\
20 \\
\text { wt } \%\end{array}$ & A9 & STD fans; PPC cond = 3 \\
\hline S185 & $1 / 12 / 2012$ & 380 & S2A & slot, $0.5 \times 5 \mathrm{~mm}$ & $\begin{array}{c}\text { STR, } \\
20 \\
\text { wt } \%\end{array}$ & A9 & STD fans; PPC cond = 3 \\
\hline S186 & $1 / 12 / 2012$ & 100 & S2A & slot, $0.5 \times 5 \mathrm{~mm}$ & $\begin{array}{c}\text { STR, } \\
20 \\
\text { wt } \%\end{array}$ & A9 & STD fans; PPC cond = 3 \\
\hline S187 & $1 / 12 / 2012$ & 200 & S2A & slot, $0.5 \times 5 \mathrm{~mm}$ & $\begin{array}{c}\text { STR, } \\
20 \\
\text { wt } \%\end{array}$ & A9 & STD fans; PPC cond = 3 \\
\hline S188 & $1 / 12 / 2012$ & 380 & S2A & slot, $0.5 \times 5 \mathrm{~mm}$ & $\begin{array}{c}\text { STR, } \\
20 \\
\text { wt } \%\end{array}$ & A9 & STD fans; PPC cond = 3 \\
\hline S189 & $1 / 12 / 2012$ & 100 & S7A & slot, $1 \times 10 \mathrm{~mm}$ & $\begin{array}{c}\text { STR, } \\
20 \\
\text { wt } \%\end{array}$ & A9 & STD fans; PPC cond = 3 \\
\hline S190 & $1 / 12 / 2012$ & 200 & S7A & slot, $1 \times 10 \mathrm{~mm}$ & $\begin{array}{c}\text { STR, } \\
20 \\
\text { wt } \% \\
\end{array}$ & A9 & STD fans; PPC cond = 3 \\
\hline S191 & $1 / 12 / 2012$ & 380 & S7A & slot, $1 \times 10 \mathrm{~mm}$ & $\begin{array}{c}\text { STR, } \\
20 \\
\text { wt } \%\end{array}$ & A9 & STD fans; PPC cond = 3 \\
\hline
\end{tabular}


Table A.1. (contd)

\begin{tabular}{|c|c|c|c|c|c|c|c|}
\hline $\begin{array}{l}\text { Test } \\
\text { No. }\end{array}$ & Date & $\begin{array}{c}\text { Pressure } \\
\text { (psig) }\end{array}$ & $\begin{array}{c}\text { Orifice } \\
\text { ID }\end{array}$ & $\begin{array}{c}\text { Orifice Type, } \\
\text { Size }\end{array}$ & Fluid & $\begin{array}{c}\text { Chamber } \\
\text { Configuration }^{(a)}\end{array}$ & Other Conditions $^{(\mathbf{b})}$ \\
\hline S192 & $1 / 12 / 2012$ & 100 & S7A & slot, $1 \times 10 \mathrm{~mm}$ & $\begin{array}{c}\text { STR, } \\
20 \\
\text { wt } \%\end{array}$ & A9 & STD fans; PPC cond = 3 \\
\hline S193 & $1 / 12 / 2012$ & 200 & S7A & slot, $1 \times 10 \mathrm{~mm}$ & $\begin{array}{c}\text { STR, } \\
20 \\
\text { wt } \%\end{array}$ & A9 & STD fans; PPC cond = 3 \\
\hline S194 & $1 / 12 / 2012$ & 380 & S7A & slot, $1 \times 10 \mathrm{~mm}$ & $\begin{array}{c}\text { STR, } \\
20 \\
\text { wt } \%\end{array}$ & A9 & STD fans; PPC cond = 3 \\
\hline S195 & $1 / 13 / 2012$ & 100 & S7A & slot, $1 \times 10 \mathrm{~mm}$ & $\begin{array}{c}\text { STR, } 8 \\
\text { wt } \%\end{array}$ & A9 & STD fans; PPC cond = 3 \\
\hline S196 & $1 / 13 / 2012$ & 200 & S7A & slot, $1 \times 10 \mathrm{~mm}$ & $\begin{array}{c}\text { STR, } 8 \\
\text { wt } \%\end{array}$ & A9 & STD fans; PPC cond = 3 \\
\hline S197 & $1 / 13 / 2012$ & 380 & S7A & slot, $1 \times 10 \mathrm{~mm}$ & $\begin{array}{c}\text { STR, } 8 \\
\text { wt } \%\end{array}$ & A9 & STD fans; PPC cond = 3 \\
\hline S198 & $1 / 13 / 2012$ & 100 & S7A & slot, $1 \times 10 \mathrm{~mm}$ & $\begin{array}{c}\text { STR, } 8 \\
\text { wt } \%\end{array}$ & A9 & STD fans; PPC cond = 3 \\
\hline S199 & $1 / 13 / 2012$ & 200 & S7A & slot, $1 \times 10 \mathrm{~mm}$ & $\begin{array}{c}\text { STR, } 8 \\
\text { wt } \%\end{array}$ & A9 & STD fans; PPC cond = 3 \\
\hline S200 & $1 / 13 / 2012$ & 380 & S7A & slot, $1 \times 10 \mathrm{~mm}$ & $\begin{array}{c}\text { STR, } 8 \\
\text { wt } \%\end{array}$ & A9 & STD fans; PPC cond = 3 \\
\hline S201 & $1 / 13 / 2012$ & 100 & S2A & slot, $0.5 \times 5 \mathrm{~mm}$ & $\begin{array}{c}\text { STR, } 8 \\
\text { wt } \%\end{array}$ & A9 & STD fans; PPC cond = 3 \\
\hline S202 & $1 / 13 / 2012$ & 200 & S2A & slot, $0.5 \times 5 \mathrm{~mm}$ & $\begin{array}{c}\text { STR, } 8 \\
\text { wt } \%\end{array}$ & A9 & STD fans; PPC cond = 3 \\
\hline S203 & $1 / 13 / 2012$ & 380 & S2A & slot, $0.5 \times 5 \mathrm{~mm}$ & $\begin{array}{c}\text { STR, } 8 \\
\text { wt } \%\end{array}$ & A9 & STD fans; PPC cond = 3 \\
\hline S204 & $1 / 13 / 2012$ & 100 & S2A & slot, $0.5 \times 5 \mathrm{~mm}$ & $\begin{array}{c}\text { STR, } 8 \\
\text { wt } \%\end{array}$ & A9 & STD fans; PPC cond = 3 \\
\hline S205 & $1 / 13 / 2012$ & 200 & S2A & slot, $0.5 \times 5 \mathrm{~mm}$ & $\begin{array}{c}\text { STR, } 8 \\
\text { wt } \%\end{array}$ & A9 & STD fans; PPC cond = 3 \\
\hline S206 & $1 / 13 / 2012$ & 380 & S2A & slot, $0.5 \times 5 \mathrm{~mm}$ & $\begin{array}{c}\text { STR, } 8 \\
\text { wt } \%\end{array}$ & A9 & STD fans; PPC cond = 3 \\
\hline W207 & $2 / 20 / 2012$ & 380 & S1D & round, $2 \mathrm{~mm}$ & Water & A9 & STD fans; PPC cond = 3 \\
\hline W208 & $2 / 20 / 2012$ & 380 & S1D & round, $2 \mathrm{~mm}$ & Water & A9 & STD fans; PPC cond = 3 \\
\hline W209 & $2 / 20 / 2012$ & 380 & S1D & round, $2 \mathrm{~mm}$ & Water & A10 & STD fans; PPC cond = 3 \\
\hline W210 & $2 / 20 / 2012$ & 380 & S1D & round, $2 \mathrm{~mm}$ & Water & A10 & STD fans; PPC cond = 3 \\
\hline W211 & $2 / 20 / 2012$ & 380 & S1D & round, $2 \mathrm{~mm}$ & Water & A10 & STD fans; PPC cond = 3 \\
\hline W212 & $2 / 21 / 2012$ & 200 & S1A & round, $1 \mathrm{~mm}$ & Water & A11 & No fans; PPC cond = 3 \\
\hline W213 & $2 / 21 / 2012$ & 380 & S1A & round, $1 \mathrm{~mm}$ & Water & A11 & No fans; PPC cond $=3$ \\
\hline
\end{tabular}


Table A.1. (contd)

\begin{tabular}{|c|c|c|c|c|c|c|c|}
\hline $\begin{array}{l}\text { Test } \\
\text { No. }\end{array}$ & Date & $\begin{array}{c}\text { Pressure } \\
\text { (psig) }\end{array}$ & $\begin{array}{c}\text { Orifice } \\
\text { ID }\end{array}$ & $\begin{array}{l}\text { Orifice Type, } \\
\text { Size }\end{array}$ & Fluid & $\begin{array}{c}\text { Chamber } \\
\text { Configuration }^{(a)}\end{array}$ & Other Conditions $^{(\mathbf{b})}$ \\
\hline W214 & $2 / 21 / 2012$ & 200 & S1A & round, $1 \mathrm{~mm}$ & Water & A12 & No fans; PPC cond $=3$ \\
\hline W215 & 2/21/2012 & 380 & S1A & round, $1 \mathrm{~mm}$ & Water & A12 & No fans; PPC cond = 3 \\
\hline W216 & 2/21/2012 & 200 & S1A & round, $1 \mathrm{~mm}$ & Water & A12 & No fans; PPC cond $=3$ \\
\hline W217 & 2/21/2012 & 380 & S1A & round, $1 \mathrm{~mm}$ & Water & A12 & No fans; PPC cond $=3$ \\
\hline W218 & 2/21/2012 & 200 & S1D & round, $2 \mathrm{~mm}$ & Water & A13 & No fans; PPC cond $=3$ \\
\hline W219 & $2 / 21 / 2012$ & 380 & S1D & round, $2 \mathrm{~mm}$ & Water & A13 & No fans; PPC cond $=3$ \\
\hline W220 & $2 / 21 / 2012$ & 200 & S1D & round, $2 \mathrm{~mm}$ & Water & A13 & No fans; PPC cond $=3$ \\
\hline W221 & 2/21/2012 & 380 & S1D & round, $2 \mathrm{~mm}$ & Water & A13 & No fans; PPC cond $=3$ \\
\hline W222 & $2 / 23 / 2012$ & 200 & S5C & slot, $1 \times 20 \mathrm{~mm}$ & Water & C9 & STD fans; PPC cond = 3 \\
\hline W223 & $2 / 23 / 2012$ & 380 & S5C & slot, $1 \times 20 \mathrm{~mm}$ & Water & C9 & STD fans; PPC cond = 3 \\
\hline W224 & $2 / 23 / 2012$ & 200 & S5C & slot, $1 \times 20 \mathrm{~mm}$ & Water & C9 & STD fans; PPC cond = 3 \\
\hline W225 & $2 / 23 / 2012$ & 380 & S5C & slot, $1 \times 20 \mathrm{~mm}$ & Water & C9 & STD fans; PPC cond = 3 \\
\hline W226 & $2 / 23 / 2012$ & 200 & S7A & slot, $1 \times 10 \mathrm{~mm}$ & Water & C9 & STD fans; PPC cond = 3 \\
\hline W227 & $2 / 23 / 2012$ & 380 & S7A & slot, $1 \times 10 \mathrm{~mm}$ & Water & C9 & STD fans; PPC cond = 3 \\
\hline W228 & $2 / 23 / 2012$ & 200 & S7A & slot, $1 \times 10 \mathrm{~mm}$ & Water & C9 & STD fans; PPC cond = 3 \\
\hline W229 & $2 / 23 / 2012$ & 380 & S7A & slot, $1 \times 10 \mathrm{~mm}$ & Water & C9 & STD fans; PPC cond = 3 \\
\hline W230 & $2 / 23 / 2012$ & 200 & S7A & slot, $1 \times 10 \mathrm{~mm}$ & Water & C9 & STD fans; PPC cond = 3 \\
\hline W231 & $2 / 24 / 2012$ & 200 & S4A & slot, $1 \times 76.2 \mathrm{~mm}$ & Water & C9 & STD fans; PPC cond = 3 \\
\hline W232 & $2 / 24 / 2012$ & 380 & S4A & slot, $1 \times 76.2 \mathrm{~mm}$ & Water & C9 & STD fans; PPC cond = 3 \\
\hline W233 & $2 / 24 / 2012$ & 200 & S4A & slot, $1 \times 76.2 \mathrm{~mm}$ & Water & C9 & STD fans; PPC cond = 3 \\
\hline W234 & $2 / 24 / 2012$ & 380 & S4A & slot, $1 \times 76.2 \mathrm{~mm}$ & Water & C9 & STD fans; PPC cond = 3 \\
\hline W235 & $2 / 27 / 2012$ & 380 & S1D & round, $2 \mathrm{~mm}$ & Water & A9 & $\begin{array}{l}\text { Bilge fans ( } 2 \text { inner): } 0 \mathrm{~V} ; \\
\text { PPC cond = } 3\end{array}$ \\
\hline W236 & 2/27/2012 & 380 & S1D & round, $2 \mathrm{~mm}$ & Water & A9 & $\begin{array}{l}\text { Bilge fans ( } 2 \text { inner): } 6 \mathrm{~V} ; \\
\text { PPC cond = } 3\end{array}$ \\
\hline W237 & $2 / 27 / 2012$ & 380 & S1D & round, $2 \mathrm{~mm}$ & Water & A9 & $\begin{array}{l}\text { Bilge fans ( } 2 \text { inner): } 9 \mathrm{~V} ; \\
\text { PPC cond }=3\end{array}$ \\
\hline W238 & $2 / 27 / 2012$ & 380 & S1D & round, $2 \mathrm{~mm}$ & Water & A9 & $\begin{array}{l}\text { Bilge fans ( } 2 \text { inner): } 12 \mathrm{~V} ; \\
\text { PPC cond = } 3\end{array}$ \\
\hline W239 & 2/27/2012 & 380 & S1D & round, $2 \mathrm{~mm}$ & Water & A9 & $\begin{array}{l}\text { Bilge fans ( } 2 \text { inner): 15V; } \\
\text { PPC cond = } 3\end{array}$ \\
\hline W240 & $2 / 27 / 2012$ & 380 & S1D & round, $2 \mathrm{~mm}$ & Water & A9 & $\begin{array}{l}\text { Bilge fans (2 inner): } 15 \mathrm{~V} \text {; } \\
\text { PPC cond = 3, repeat }\end{array}$ \\
\hline W241 & $2 / 27 / 2012$ & 380 & S1D & round, $2 \mathrm{~mm}$ & Water & A9 & $\begin{array}{l}\text { Bilge fans ( } 2 \text { inner): } 15 \mathrm{~V} ; \\
\text { PPC cond = 3; test } \\
\text { discontinued }\end{array}$ \\
\hline W242 & $2 / 27 / 2012$ & 380 & S1D & round, $2 \mathrm{~mm}$ & Water & A9 & $\begin{array}{l}\text { Bilge fans ( } 2 \text { inner): } 15 \mathrm{~V} \text {; } \\
\text { PPC cond = 3; repeat }\end{array}$ \\
\hline W243 & $2 / 28 / 2012$ & 380 & S1D & round, $2 \mathrm{~mm}$ & Water & A14 & STD fans; PPC cond = 3 \\
\hline W244 & $2 / 28 / 2012$ & 380 & S1D & round, $2 \mathrm{~mm}$ & Water & A14 & STD fans; PPC cond = 3 \\
\hline
\end{tabular}


Table A.1. (contd)

\begin{tabular}{|c|c|c|c|c|c|c|c|}
\hline $\begin{array}{c}\text { Test } \\
\text { No. }\end{array}$ & Date & $\begin{array}{c}\text { Pressure } \\
\text { (psig) }\end{array}$ & $\begin{array}{c}\text { Orifice } \\
\text { ID }\end{array}$ & $\begin{array}{c}\text { Orifice Type, } \\
\text { Size }\end{array}$ & Fluid & $\begin{array}{c}\text { Chamber } \\
\text { Configuration }^{(\mathbf{a})}\end{array}$ & Other Conditions $^{(\mathbf{b})}$ \\
\hline W245 & $2 / 28 / 2012$ & 380 & S1D & round, $2 \mathrm{~mm}$ & Water & A14 & STD fans; PPC cond = 3 \\
\hline W246 & $2 / 28 / 2012$ & 100 & S1A & round, $1 \mathrm{~mm}$ & Water & A14 & $\begin{array}{c}\text { For determination of flow } \\
\text { rate only }\end{array}$ \\
\hline W247 & $2 / 28 / 2012$ & various & $\begin{array}{c}\text { S1A - } \\
\text { S1D }\end{array}$ & n/a & Water & A14 & $\begin{array}{c}\text { For acquiring } \\
\text { photographs of sprays }\end{array}$ \\
\hline
\end{tabular}

a) See Table A.2 for the list of configurations used in this column.

b) See Table A.3 for PPC condition definitions used in this column. The term STD fans refers to use of Modified Bilge fans, 2 inner, at $12 \mathrm{~V}$, which was the standard fan setting used in the majority of the tests.

The chamber configuration is reported as a two character code in Table A.1 and is explained below in Table A.2. The first character, a letter, indicates the location of the spool piece. The second character, a number, indicates the configuration of the Malvern instruments in the chamber. Refer to Section 5.3.1 for additional explanation of Malvern instrument locations. A general schematic of the aerosol chamber is also available in Figure 5.14. The appropriate character needs to be selected from the list below and used to interpret the configuration of each test in Table A.1.

Table A.2. Chamber Configuration States Used in Large-Scale Spray Release Testing

\begin{tabular}{|c|l|}
\hline Identifier & \multicolumn{1}{c|}{ Description } \\
\hline A & Standard spool location where the spool was approximately 226” from the splash wall \\
\hline B & $\begin{array}{l}\text { Spool location where the spool was approximately in the middle of the chamber, 119" from the } \\
\text { splash wall (using “medium” pipe extensions) }\end{array}$ \\
\hline C & $\begin{array}{l}\text { Spool location where the spool was located near the splash wall, at a distance of approximately } \\
\text { 42” (using “long” pipe extensions) }\end{array}$ \\
\hline \multirow{2}{*}{1} & $\begin{array}{l}\text { Position 1: M1854, 150 cm spacers } \\
\text { Position 2: M1852, 150 cm spacers } \\
\text { Position 3: M1855, 150 cm spacers } \\
\text { Mounted in standard (see Figure 5.10 and Table 5.2) spatial locations }\end{array}$ \\
\hline 2 & $\begin{array}{l}\text { As 1, but M1854 (Position 1) was mounted 6 in. higher (vertically) than the standard spatial } \\
\text { location. M1855 and M1852 positions were unchanged. }\end{array}$ \\
\hline 3 & $\begin{array}{l}\text { Position 1: M1855, 150 cm spacers } \\
\text { Position 2: M1852, 150 cm spacers } \\
\text { Position 3: M1854, 150 cm spacers } \\
\text { Mounted in standard (see Figure 5.10 and Table 5.2) spatial locations }\end{array}$ \\
\hline 4 & $\begin{array}{l}\text { Position 1: M1855, 500 cm spacers } \\
\text { Position 2: M1852, 150 cm spacers } \\
\text { Position 3: Unoccupied } \\
\text { Mounted in standard (see Figure 5.10 and Table 5.2) spatial locations }\end{array}$ \\
\hline 5 & $\begin{array}{l}\text { Position 1: M1855, 500 cm spacers } \\
\text { Position 2: Unoccupied } \\
\text { Position 3: M1852, 150 cm spacers } \\
\text { Mounted in standard (see Figure 5.10 and Table 5.2) spatial locations }\end{array}$ \\
\hline
\end{tabular}


Table A.2. (contd)

\begin{tabular}{|c|c|}
\hline Identifier & Description \\
\hline 6 & $\begin{array}{l}\text { Position 1: M1852, } 150 \mathrm{~cm} \text { spacers } \\
\text { Position 2: Unoccupied } \\
\text { Position 3: Unoccupied } \\
\text { Mounted in standard (see Figure } 5.10 \text { and Table 5.2) spatial locations }\end{array}$ \\
\hline 7 & $\begin{array}{l}\text { Position 1: M1855, } 500 \mathrm{~cm} \text { spacers } \\
\text { Position 2: M1852, } 150 \mathrm{~cm} \text { spacers } \\
\text { Position 3: M1854, } 500 \mathrm{~cm} \text { spacers } \\
\text { Mounted in standard (see Figure } 5.10 \text { and Table 5.2) spatial locations }\end{array}$ \\
\hline 8 & $\begin{array}{l}\text { Position 1: M1855, } 500 \mathrm{~cm} \text { spacers } \\
\text { Position 2: M1852, } 500 \mathrm{~cm} \text { spacers } \\
\text { Position 3: M1854, } 500 \mathrm{~cm} \text { spacers [replacement laser] } \\
\text { Mounted in standard (see Figure } 5.10 \text { and Table 5.2) spatial locations }\end{array}$ \\
\hline 9 & $\begin{array}{l}\text { Position 1: M1855, } 500 \mathrm{~cm} \text { spacers [replacement laser] } \\
\text { Position 2: M1852, } 500 \mathrm{~cm} \text { spacers } \\
\text { Position 3: M1854, } 500 \mathrm{~cm} \text { spacers [replacement laser] } \\
\text { Mounted in standard (see Figure } 5.10 \text { and Table 5.2) spatial locations }\end{array}$ \\
\hline 10 & $\begin{array}{l}\text { Position 1: M1855, } 500 \mathrm{~cm} \text { spacers [replacement laser] } \\
\text { Position 2: M1852, } 500 \mathrm{~cm} \text { spacers } \\
\text { Position 3: M1854, } 500 \mathrm{~cm} \text { spacers [replacement laser] } \\
\text { All three Malvern instruments were mounted approximately } 8 \text { in. higher (vertically) than the } \\
\text { standard spatial location, measured as } 107 / 8 \text { in. from the ceiling to the upper spacer rod }\end{array}$ \\
\hline 11 & $\begin{array}{l}\text { Position 1: M1855, } 500 \mathrm{~cm} \text { spacers [replacement laser] - } 38 \text { in. } \\
\text { Position 2: M1852, } 500 \mathrm{~cm} \text { spacers - } 35.5 \mathrm{in} \text {. } \\
\text { Position 3: M1854, } 500 \mathrm{~cm} \text { spacers [replacement laser] - } 36.5 \mathrm{in} \text {. } \\
\text { All three Malvern instruments were mounted to conduct an in-spray measurement for the 1-mm } \\
\text { orifice (S1A). They were centered with the orifice and mounted at vertical heights as indicated } \\
\text { above, measured from the floor of the chamber. }\end{array}$ \\
\hline 12 & $\begin{array}{l}\text { As 11, but the vertical position of M1852 (position 2) was changed to } 34.75 \text { in. from the chamber } \\
\text { floor. M1854 and M1855 positions were unchanged. }\end{array}$ \\
\hline 13 & $\begin{array}{l}\text { Position 1: M1855, } 500 \mathrm{~cm} \text { spacers [replacement laser] - } 48.25 \mathrm{in} \text {. } \\
\text { Position 2: M1852, } 500 \mathrm{~cm} \text { spacers - } 36.25 \mathrm{in} . \\
\text { Position 3: M1854, } 500 \mathrm{~cm} \text { spacers [replacement laser] - } 36.5 \mathrm{in} \text {. } \\
\text { All three Malvern instruments were mounted to conduct an in-spray measurement for the 2-mm } \\
\text { orifice (S1D). They were centered with the orifice and mounted at vertical heights as indicated } \\
\text { above, measured from the floor of the chamber. }\end{array}$ \\
\hline 14 & $\begin{array}{l}\text { Position 1: M1855, } 500 \mathrm{~cm} \text { spacers [replacement laser] - } 57.25 \text { (25) in., rotated } 90^{\circ} \\
\text { Position 2: M1852, } 500 \mathrm{~cm} \text { spacers - } 57.5(24.75) \text { in. } \\
\text { Position 3: M1854, } 500 \mathrm{~cm} \text { spacers [replacement laser] - } 58.25 \text { (24) in. } \\
\text { All three Malvern instruments were mounted to measure beneath the height of the spray. The } \\
\text { measurements listed above are the distances from the ceiling of the chamber to the top of the } \\
\text { upper spacer rod for each Malvern. The approximate distance from the floor is indicated in } \\
\text { parentheses. }\end{array}$ \\
\hline
\end{tabular}


Table A.3. PPC Conditions Used During Large-Scale Spray Release Testing

\begin{tabular}{|c|l|}
\hline PPC Identifier & \multicolumn{1}{c|}{ Operating Condition } \\
\hline 0 & PPC was not in service for the test. \\
\hline 1 & $\begin{array}{l}\text { PPC inlet was approximately } 6 \text { in. from the ceiling with a vacuum pump flow rate of } \\
1.0 \text { scfm during the test. }\end{array}$ \\
\hline 2 & $\begin{array}{l}\text { PPC inlet was 22 5/8 in. from the ceiling with a variable vacuum pump flow rate of } \\
0.5-1.8 \text { scfm that was changed throughout the test. }\end{array}$ \\
\hline 3 & $\begin{array}{l}\text { PPC inlet was 22 5/8 in. from the ceiling with a variable vacuum pump flow rate of } \\
1.5 \text { scfm during the test. }\end{array}$ \\
\hline
\end{tabular}

Table A.4, as mentioned previously, contains measured process instrument data values and calculated values derived from the data for every test performed in the large-scale test stand. The configuration of each test can be looked up in Table A.1 using the Test ID number (first column). Recall that Section 7.1 describes the calculation procedure for most of these quantities. The column headings in the table refer to the following:

- $\quad\langle\mathrm{P}\rangle$ : average pressure during the spray, measured in the spray header;

- $\quad \sigma\langle\mathrm{P}\rangle$ : standard deviation of the pressure during the spray over the average period;

- $\quad \mathrm{T}$ : average temperature of the fluid being sprayed, measured in the spray header;

- $\rho$ : average density of the fluid being sprayed over the average period, derived from the average temperature and assumed density functionality;

- $\mathrm{Q}_{\text {spray }}$ : spray leak flow rate, determined by either the mass method (Equation 7.4) or the flow rate method (Equation 7.5), presented in two different units;

- $\mathrm{C}_{\mathrm{D}}$ : orifice discharge coefficient, determined by either the mass of the flow rate method (see Equation 7.6);

- \% difference: percent that the spray leak flow rates calculated by the two methods are different from one another, calculated as $\left(\mathrm{Q}_{\text {spray }}\right.$ (mass) $-\mathrm{Q}_{\text {spray }}($ flow rate $\left.)\right) / \mathrm{Q}_{\text {spray }}$ (mass) $\times 100$.

Tests W01 through W60 are included in Table A.4 for completeness but the release fraction for those tests are not reported in Appendix B or presented in the main text. These tests were repeated later using a more appropriate Malvern configuration, including replacement of the laser diodes on two of the Malvern instruments (see Table A.2 for more details on the progression of configurations used during testing). Release fraction data for the tests in Table A.4 can be cross-referenced in Appendix B using the Test ID number given in the first column. 
Table A.4. Actual Test Conditions Obtained from Analysis of Process Instrument Data.

\begin{tabular}{|c|c|c|c|c|c|c|c|c|c|c|c|}
\hline Test ID & $\langle\mathbf{P}\rangle$ (psig) & $\begin{array}{c}\sigma \\
\langle\mathbf{P}\rangle \\
\text { (psig) }\end{array}$ & $\mathbf{T}\left({ }^{\circ} \mathbf{F}\right)$ & $\begin{array}{c}\rho \\
(g / m L)\end{array}$ & $\begin{array}{c}\mathbf{Q}_{\text {spray, by }} \\
\text { mass } \\
\text { (gpm) }\end{array}$ & $\begin{array}{c}\mathbf{Q}_{\text {spray }}, \text { by } \\
\text { mass }\left(\mathbf{m}^{3} / \mathbf{s}\right)\end{array}$ & $\begin{array}{c}C_{D} \text {, by } \\
\text { mass }\end{array}$ & $\begin{array}{c}Q_{\text {spray }}, \text { by } \\
\text { flow } \\
\text { rates } \\
\text { (gpm) }\end{array}$ & $\begin{array}{c}Q_{\text {spray }}, \text { by } \\
\text { flow rates } \\
\left(\mathrm{m}^{3} / \mathrm{s}\right)\end{array}$ & $\begin{array}{c}C_{D} \text {, by } \\
\text { flow } \\
\text { rates } \\
\end{array}$ & $\begin{array}{c}\% \\
\text { difference } \\
\text { (mass vs. } \\
\text { flow rates) }\end{array}$ \\
\hline W01 & -- & -- & -- & -- & -- & \begin{tabular}{|c|}
-- \\
\end{tabular} & -- & -- & \begin{tabular}{|c|}
-- \\
\end{tabular} & -- & -- \\
\hline W02 & 381.76 & 1.24 & 64.45 & 0.9986 & 2.64 & $1.675 \times 10^{-4}$ & 0.659 & 2.69 & $1.706 \times 10^{-4}$ & 0.671 & $-1.9 \%$ \\
\hline W03 & 382.63 & 0.80 & 65.40 & 0.9985 & 2.76 & $1.746 \times 10^{-4}$ & 0.686 & 2.90 & $1.834 \times 10^{-4}$ & 0.720 & $-5.1 \%$ \\
\hline W04 & 377.20 & 0.76 & 68.09 & 0.9982 & 7.42 & $4.701 \times 10^{-4}$ & 1.859 & 6.98 & $4.418 \times 10^{-4}$ & 1.748 & $6.0 \%$ \\
\hline W05 & 377.45 & 0.79 & 72.09 & 0.9977 & 2.54 & $1.612 \times 10^{-4}$ & 0.637 & 2.46 & $1.557 \times 10^{-4}$ & 0.616 & $3.4 \%$ \\
\hline W06 & 377.22 & 0.73 & 73.09 & 0.9976 & 2.69 & $1.703 \times 10^{-4}$ & 0.673 & 2.36 & $1.492 \times 10^{-4}$ & 0.590 & $12.4 \%$ \\
\hline W07 & 376.97 & 0.74 & 69.00 & 0.9981 & 2.47 & $1.567 \times 10^{-4}$ & 0.620 & 2.46 & $1.558 \times 10^{-4}$ & 0.616 & $0.6 \%$ \\
\hline W08 & 376.88 & 0.77 & 71.52 & 0.9978 & 2.52 & $1.598 \times 10^{-4}$ & 0.632 & 2.17 & $1.373 \times 10^{-4}$ & 0.543 & $14.1 \%$ \\
\hline W09 & 376.63 & 0.77 & 74.24 & 0.9975 & 2.57 & $1.627 \times 10^{-4}$ & 0.644 & 2.20 & $1.392 \times 10^{-4}$ & 0.551 & $14.4 \%$ \\
\hline W10 & 376.83 & 0.78 & 75.50 & 0.9973 & 2.60 & $1.644 \times 10^{-4}$ & 0.650 & 2.28 & $1.446 \times 10^{-4}$ & 0.572 & $12.0 \%$ \\
\hline W11 & 376.78 & 0.74 & 76.66 & 0.9971 & 2.58 & $1.632 \times 10^{-4}$ & 0.645 & 2.26 & $1.429 \times 10^{-4}$ & 0.565 & $12.4 \%$ \\
\hline W12 & 376.72 & 0.74 & 77.75 & 0.9970 & 2.60 & $1.648 \times 10^{-4}$ & 0.652 & 2.10 & $1.329 \times 10^{-4}$ & 0.526 & $19.4 \%$ \\
\hline W13 & 376.75 & 0.80 & 78.94 & 0.9968 & 2.55 & $1.618 \times 10^{-4}$ & 0.640 & 2.29 & $1.453 \times 10^{-4}$ & 0.575 & $10.2 \%$ \\
\hline W14 & 378.71 & 0.77 & 74.88 & 0.9974 & 2.62 & $1.659 \times 10^{-4}$ & 0.655 & 2.21 & $1.400 \times 10^{-4}$ & 0.552 & $15.6 \%$ \\
\hline W15 & 378.34 & 0.76 & 76.11 & 0.9972 & 2.63 & $1.664 \times 10^{-4}$ & 0.657 & 2.27 & $1.441 \times 10^{-4}$ & 0.569 & $13.4 \%$ \\
\hline W16 & 378.25 & 0.76 & 76.68 & 0.9971 & 2.55 & $1.613 \times 10^{-4}$ & 0.637 & 2.23 & $1.415 \times 10^{-4}$ & 0.559 & $12.2 \%$ \\
\hline W17 & 378.15 & 0.78 & 77.30 & 0.9970 & 2.65 & $1.675 \times 10^{-4}$ & 0.661 & 2.25 & $1.422 \times 10^{-4}$ & 0.562 & $15.1 \%$ \\
\hline W18 & 378.44 & 0.77 & 72.21 & 0.9977 & 2.62 & $1.660 \times 10^{-4}$ & 0.655 & 2.50 & $1.584 \times 10^{-4}$ & 0.625 & $4.6 \%$ \\
\hline W19 & 378.50 & 0.76 & 72.92 & 0.9976 & 2.57 & $1.628 \times 10^{-4}$ & 0.643 & 2.41 & $1.524 \times 10^{-4}$ & 0.602 & $6.3 \%$ \\
\hline W20 & 377.92 & 1.09 & 71.89 & 0.9978 & 2.56 & $1.624 \times 10^{-4}$ & 0.642 & 2.23 & $1.414 \times 10^{-4}$ & 0.559 & $13.0 \%$ \\
\hline W21 & 378.34 & 0.77 & 73.44 & 0.9976 & 2.61 & $1.650 \times 10^{-4}$ & 0.651 & 2.28 & $1.442 \times 10^{-4}$ & 0.569 & $12.6 \%$ \\
\hline W22 & 377.42 & 1.20 & 71.78 & 0.9978 & 2.58 & $1.631 \times 10^{-4}$ & 0.645 & 2.29 & $1.448 \times 10^{-4}$ & 0.572 & $11.2 \%$ \\
\hline W23 & 377.92 & 0.80 & 73.60 & 0.9975 & 2.64 & $1.673 \times 10^{-4}$ & 0.661 & 2.36 & $1.495 \times 10^{-4}$ & 0.591 & $10.6 \%$ \\
\hline W24 & 377.66 & 1.36 & 74.76 & 0.9974 & 2.57 & $1.630 \times 10^{-4}$ & 0.644 & 2.11 & $1.338 \times 10^{-4}$ & 0.529 & $17.9 \%$ \\
\hline W25 & 377.63 & 1.01 & 76.36 & 0.9972 & 2.52 & $1.599 \times 10^{-4}$ & 0.632 & 2.17 & $1.373 \times 10^{-4}$ & 0.543 & $14.1 \%$ \\
\hline W26 & 199.18 & 0.45 & 71.16 & 0.9978 & 0.43 & $2.751 \times 10^{-5}$ & 0.674 & 0.47 & $2.996 \times 10^{-5}$ & 0.734 & $-8.9 \%$ \\
\hline W27 & 376.91 & 0.77 & 73.12 & 0.9976 & 0.69 & $4.379 \times 10^{-5}$ & 0.780 & 0.62 & $3.937 \times 10^{-5}$ & 0.701 & $10.1 \%$ \\
\hline W28 & 198.62 & 0.42 & 72.02 & 0.9977 & 8.96 & $5.672 \times 10^{-4}$ & 0.632 & 9.01 & $5.707 \times 10^{-4}$ & 0.636 & $-0.6 \%$ \\
\hline W29 & 375.23 & 0.69 & 73.85 & 0.9975 & 12.35 & $7.820 \times 10^{-4}$ & 0.634 & 12.28 & $7.776 \times 10^{-4}$ & 0.630 & $0.6 \%$ \\
\hline W30 & 199.06 & 0.43 & 70.58 & 0.9979 & 3.20 & $2.028 \times 10^{-4}$ & 0.657 & 3.29 & $2.081 \times 10^{-4}$ & 0.674 & $-2.6 \%$ \\
\hline W31 & 375.60 & 0.75 & 72.17 & 0.9977 & 4.48 & $2.837 \times 10^{-4}$ & 0.669 & 4.38 & $2.772 \times 10^{-4}$ & 0.653 & $2.3 \%$ \\
\hline W32 & 199.44 & 0.45 & 72.52 & 0.9977 & 0.47 & $2.947 \times 10^{-5}$ & 0.722 & 0.49 & $3.126 \times 10^{-5}$ & 0.765 & $-6.1 \%$ \\
\hline W33 & 378.21 & 0.75 & 74.48 & 0.9974 & 0.69 & $4.354 \times 10^{-5}$ & 0.774 & 0.77 & $4.879 \times 10^{-5}$ & 0.867 & $-12.1 \%$ \\
\hline W34 & 198.35 & 0.46 & 74.11 & 0.9975 & 9.14 & $5.790 \times 10^{-4}$ & 0.646 & 9.04 & $5.728 \times 10^{-4}$ & 0.639 & $1.1 \%$ \\
\hline W35 & 375.69 & 0.72 & 75.37 & 0.9973 & 12.43 & $7.875 \times 10^{-4}$ & 0.638 & 12.26 & $7.762 \times 10^{-4}$ & 0.629 & $1.4 \%$ \\
\hline W36 & 198.90 & 0.51 & 75.01 & 0.9973 & 3.21 & $2.033 \times 10^{-4}$ & 0.659 & 3.19 & $2.022 \times 10^{-4}$ & 0.655 & $0.5 \%$ \\
\hline
\end{tabular}


Table A.4. (contd)

\begin{tabular}{|c|c|c|c|c|c|c|c|c|c|c|c|}
\hline Test ID & $|\mathbf{P}\rangle$ (psig) & $\begin{array}{c}\sigma \\
\langle\mathbf{P}\rangle \\
\text { (psig) }\end{array}$ & $\mathbf{T}\left({ }^{\circ} \mathbf{F}\right)$ & $\begin{array}{c}\rho \\
(\mathrm{g} / \mathrm{mL})\end{array}$ & $\begin{array}{c}\mathbf{Q}_{\text {spray, }} \text { by } \\
\text { mass } \\
\text { (gpm) }\end{array}$ & $\begin{array}{c}\mathbf{Q}_{\text {spray }}, \text { by } \\
\text { mass }_{\left(\mathbf{m}^{3} / \mathbf{s}\right)}\end{array}$ & $\begin{array}{c}C_{D} \text {, by } \\
\text { mass }\end{array}$ & $\begin{array}{c}\mathbf{Q}_{\text {spray, by }} \\
\text { flow } \\
\text { rates } \\
\text { (gpm) }\end{array}$ & $\begin{array}{c}\mathbf{Q}_{\text {spray, }} \text { by } \\
\text { flow rates } \\
\left(\mathbf{m}^{3} / \mathbf{s}\right)\end{array}$ & $\begin{array}{c}C_{D}, \text { by } \\
\text { flow } \\
\text { rates }\end{array}$ & $\begin{array}{c}\% \\
\text { difference } \\
\text { (mass vs. } \\
\text { flow rates) } \\
\end{array}$ \\
\hline W37 & \begin{tabular}{|l|}
377.23 \\
\end{tabular} & 0.82 & 76.42 & 0.9972 & 4.46 & $2.826 \times 10^{-4}$ & 0.665 & 4.16 & $2.632 \times 10^{-4}$ & 0.619 & $6.9 \%$ \\
\hline W38 & 100.20 & 0.25 & 68.79 & 0.9981 & 0.83 & $5.260 \times 10^{-5}$ & 0.661 & 0.78 & $4.931 \times 10^{-5}$ & 0.620 & $6.2 \%$ \\
\hline W39 & 198.72 & 0.46 & 69.80 & 0.9980 & 1.20 & $7.627 \times 10^{-5}$ & 0.680 & 1.21 & $7.637 \times 10^{-5}$ & 0.681 & $-0.1 \%$ \\
\hline W40 & 377.48 & 0.79 & 71.28 & 0.9978 & 1.68 & $1.065 \times 10^{-4}$ & 0.689 & 1.89 & $1.198 \times 10^{-4}$ & 0.776 & $-12.5 \%$ \\
\hline W41 & 198.64 & 0.50 & 71.33 & 0.9978 & 1.20 & $7.579 \times 10^{-5}$ & 0.676 & 1.16 & $7.352 \times 10^{-5}$ & 0.656 & $3.0 \%$ \\
\hline W42 & 377.50 & 0.78 & 72.46 & 0.9977 & 1.71 & $1.083 \times 10^{-4}$ & 0.701 & 2.09 & $1.322 \times 10^{-4}$ & 0.856 & $-22.1 \%$ \\
\hline W43 & 100.17 & 0.25 & 70.46 & 0.9979 & 0.88 & $5.590 \times 10^{-5}$ & 0.702 & 0.84 & $5.345 \times 10^{-5}$ & 0.672 & $4.4 \%$ \\
\hline W44 & 201.14 & 0.43 & 69.65 & 0.9980 & 5.24 & $3.320 \times 10^{-4}$ & 0.645 & 5.19 & $3.289 \times 10^{-4}$ & 0.639 & $0.9 \%$ \\
\hline W45 & 377.07 & 0.71 & 71.13 & 0.9979 & 7.04 & $4.460 \times 10^{-4}$ & 0.632 & 7.43 & $4.706 \times 10^{-4}$ & 0.667 & $-5.5 \%$ \\
\hline W46 & 201.32 & 0.68 & 70.90 & 0.9979 & 1.98 & $1.255 \times 10^{-4}$ & 0.644 & 2.07 & $1.309 \times 10^{-4}$ & 0.672 & $-4.2 \%$ \\
\hline W47 & 373.63 & 2.23 & 71.58 & 0.9978 & 17.99 & $1.139 \times 10^{-3}$ & 4.292 & 16.79 & $1.064 \times 10^{-3}$ & 4.008 & $6.6 \%$ \\
\hline W48 & 199.52 & 2.02 & 70.33 & 0.9980 & 10.78 & $6.825 \times 10^{-4}$ & 0.672 & 10.49 & $6.646 \times 10^{-4}$ & 0.654 & $2.6 \%$ \\
\hline W49 & 374.24 & 1.88 & 70.30 & 0.9980 & 14.26 & $9.029 \times 10^{-4}$ & 0.649 & 14.16 & $8.969 \times 10^{-4}$ & 0.645 & $0.7 \%$ \\
\hline W50 & 200.70 & 0.83 & 67.21 & 0.9983 & 5.19 & $3.285 \times 10^{-4}$ & 0.639 & 5.17 & $3.275 \times 10^{-4}$ & 0.637 & $0.3 \%$ \\
\hline W51 & 376.64 & 0.70 & 68.61 & 0.9982 & 7.11 & $4.500 \times 10^{-4}$ & 0.639 & 7.20 & $4.561 \times 10^{-4}$ & 0.647 & $-1.3 \%$ \\
\hline W52 & 201.41 & 0.45 & 67.95 & 0.9982 & 2.02 & $1.279 \times 10^{-4}$ & 0.657 & 1.89 & $1.199 \times 10^{-4}$ & 0.616 & $6.2 \%$ \\
\hline W53 & 375.18 & 4.83 & 69.17 & 0.9981 & 2.72 & $1.722 \times 10^{-4}$ & 0.648 & 2.56 & $1.620 \times 10^{-4}$ & 0.609 & $5.9 \%$ \\
\hline W54 & 98.09 & 0.26 & 82.31 & 0.9963 & 3.26 & $2.064 \times 10^{-4}$ & 0.642 & 3.30 & $2.091 \times 10^{-4}$ & 0.651 & $-1.3 \%$ \\
\hline W55 & 97.81 & 0.25 & 80.36 & 0.9966 & 6.67 & $4.222 \times 10^{-4}$ & 0.604 & 6.60 & $4.178 \times 10^{-4}$ & 0.598 & $1.0 \%$ \\
\hline W56 & 97.40 & 0.24 & 78.43 & 0.9969 & 10.45 & $6.617 \times 10^{-4}$ & 0.585 & 10.41 & $6.593 \times 10^{-4}$ & 0.583 & $0.4 \%$ \\
\hline W57 & 197.48 & 0.54 & 78.47 & 0.9969 & 4.71 & $2.981 \times 10^{-4}$ & 0.654 & 4.61 & $2.918 \times 10^{-4}$ & 0.640 & $2.1 \%$ \\
\hline W58 & 196.94 & 0.56 & 77.66 & 0.9970 & 9.20 & $5.828 \times 10^{-4}$ & 0.588 & 9.19 & $5.822 \times 10^{-4}$ & 0.587 & $0.1 \%$ \\
\hline W59 & 196.27 & 0.53 & 77.25 & 0.9970 & 14.65 & $9.278 \times 10^{-4}$ & 0.578 & 14.63 & $9.264 \times 10^{-4}$ & 0.577 & $0.2 \%$ \\
\hline W60 & 376.86 & 0.72 & 73.82 & 0.9975 & 6.29 & $3.984 \times 10^{-4}$ & 0.633 & 6.17 & $3.906 \times 10^{-4}$ & 0.621 & $2.0 \%$ \\
\hline W61 & 378.59 & 0.71 & 72.13 & 0.9977 & 0.72 & $4.544 \times 10^{-5}$ & 0.808 & 0.76 & $4.811 \times 10^{-5}$ & 0.855 & $-5.9 \%$ \\
\hline W62 & 378.17 & 0.73 & 75.20 & 0.9973 & 0.76 & $4.824 \times 10^{-5}$ & 0.858 & 0.68 & $4.297 \times 10^{-5}$ & 0.764 & $10.9 \%$ \\
\hline W63 & 378.26 & 0.72 & 75.48 & 0.9973 & 0.75 & $4.768 \times 10^{-5}$ & 0.848 & 0.44 & $2.763 \times 10^{-5}$ & 0.491 & $42.0 \%$ \\
\hline W64 & 377.93 & 0.75 & 72.39 & 0.9977 & 0.72 & $4.542 \times 10^{-5}$ & 0.808 & 0.29 & $1.853 \times 10^{-5}$ & 0.330 & $59.2 \%$ \\
\hline W65 & 378.19 & 0.73 & 73.48 & 0.9976 & 0.74 & $4.712 \times 10^{-5}$ & 0.838 & 0.63 & $4.016 \times 10^{-5}$ & 0.714 & $14.8 \%$ \\
\hline W66 & 378.10 & 0.73 & 75.15 & 0.9973 & 0.75 & $4.744 \times 10^{-5}$ & 0.843 & 0.46 & $2.921 \times 10^{-5}$ & 0.519 & $38.4 \%$ \\
\hline W67 & 378.00 & 0.75 & 75.44 & 0.9973 & 0.78 & $4.939 \times 10^{-5}$ & 0.878 & 0.57 & $3.618 \times 10^{-5}$ & 0.643 & $26.7 \%$ \\
\hline W68 & 378.04 & 0.75 & 76.95 & 0.9971 & 0.77 & $4.851 \times 10^{-5}$ & 0.862 & 0.62 & $3.946 \times 10^{-5}$ & 0.702 & $18.7 \%$ \\
\hline W69 & 378.34 & 0.74 & 78.55 & 0.9969 & 0.78 & $4.916 \times 10^{-5}$ & 0.873 & 0.53 & $3.382 \times 10^{-5}$ & 0.601 & $31.2 \%$ \\
\hline W70 & 377.82 & 0.73 & 77.05 & 0.9971 & 2.53 & $1.603 \times 10^{-4}$ & 0.633 & 2.51 & $1.590 \times 10^{-4}$ & 0.628 & $0.8 \%$ \\
\hline W71 & 375.65 & 0.67 & 78.98 & 0.9968 & 12.68 & $8.033 \times 10^{-4}$ & 0.651 & 11.90 & $7.539 \times 10^{-4}$ & 0.611 & $6.2 \%$ \\
\hline W72 & 375.61 & 0.71 & 81.30 & 0.9964 & 12.32 & $7.803 \times 10^{-4}$ & 0.632 & 12.14 & $7.687 \times 10^{-4}$ & 0.623 & $1.5 \%$ \\
\hline W73 & 199.63 & 0.44 & 63.32 & 0.9987 & 0.49 & $3.096 \times 10^{-5}$ & 0.758 & 0.50 & $3.174 \times 10^{-5}$ & 0.777 & $-2.5 \%$ \\
\hline W74 & 379.96 & 0.73 & 65.11 & 0.9986 & 0.73 & $4.646 \times 10^{-5}$ & 0.825 & 0.95 & $6.019 \times 10^{-5}$ & 1.068 & $-29.5 \%$ \\
\hline W75 & 198.82 & 0.48 & 65.65 & 0.9985 & 9.00 & $5.699 \times 10^{-4}$ & 0.635 & 9.01 & $5.706 \times 10^{-4}$ & 0.636 & $-0.1 \%$ \\
\hline W76 & 377.11 & 0.80 & 66.74 & 0.9984 & 12.45 & $7.884 \times 10^{-4}$ & 0.638 & 12.57 & $7.962 \times 10^{-4}$ & 0.644 & $-1.0 \%$ \\
\hline W77 & 199.56 & 0.52 & 67.40 & 0.9983 & 3.14 & $1.988 \times 10^{-4}$ & 0.643 & 3.10 & $1.966 \times 10^{-4}$ & 0.636 & $1.1 \%$ \\
\hline W78 & 378.91 & 0.80 & 68.47 & 0.9982 & 4.42 & $2.798 \times 10^{-4}$ & 0.657 & 4.48 & $2.840 \times 10^{-4}$ & 0.667 & $-1.5 \%$ \\
\hline W79 & 102.15 & 1.64 & 67.78 & 0.9983 & 1.29 & $8.199 \times 10^{-5}$ & 0.623 & 1.34 & $8.498 \times 10^{-5}$ & 0.646 & $-3.6 \%$ \\
\hline W80 & 199.44 & 0.51 & 67.34 & 0.9983 & 1.80 & $1.140 \times 10^{-4}$ & 0.620 & 1.71 & $1.085 \times 10^{-4}$ & 0.591 & $4.8 \%$ \\
\hline W81 & 379.28 & 0.75 & 68.69 & 0.9981 & 2.54 & $1.610 \times 10^{-4}$ & 0.635 & 2.52 & $1.597 \times 10^{-4}$ & 0.630 & $0.8 \%$ \\
\hline W82 & 199.61 & 0.45 & 68.43 & 0.9982 & 0.46 & $2.925 \times 10^{-5}$ & 0.716 & 0.45 & $2.859 \times 10^{-5}$ & 0.700 & $2.2 \%$ \\
\hline W83 & 380.00 & 2.20 & 65.06 & 0.9986 & 0.49 & $3.091 \times 10^{-5}$ & 0.549 & 0.30 & $1.887 \times 10^{-5}$ & 0.335 & $39.0 \%$ \\
\hline W84 & 199.27 & 2.07 & 65.83 & 0.9985 & 9.00 & $5.703 \times 10^{-4}$ & 0.635 & 8.99 & $5.696 \times 10^{-4}$ & 0.634 & $0.1 \%$ \\
\hline
\end{tabular}


Table A.4. (contd)

\begin{tabular}{|c|c|c|c|c|c|c|c|c|c|c|c|}
\hline Test ID & $\mid\langle\mathbf{P}\rangle$ (psig) & $\begin{array}{c}\sigma \\
\langle\mathbf{P}\rangle \\
\text { (psig) }\end{array}$ & $\mathrm{T}\left({ }^{\circ} \mathbf{F}\right)$ & $\begin{array}{c}\rho \\
(\mathrm{g} / \mathrm{mL})\end{array}$ & $\begin{array}{c}\mathbf{Q}_{\text {sprayy }} \text { by } \\
\text { mass } \\
\text { (gpm) }\end{array}$ & $\begin{array}{c}\mathbf{Q}_{\text {spray }}, \text { by } \\
\text { mass }_{\left(\mathbf{m}^{3} / \mathbf{s}\right)}\end{array}$ & $\begin{array}{c}C_{D} \text {, by } \\
\text { mass }\end{array}$ & $\begin{array}{c}\mathbf{Q}_{\text {spray, by }} \\
\text { flow } \\
\text { rates } \\
\text { (gpm) }\end{array}$ & $\begin{array}{c}\mathbf{Q}_{\text {spray }}, \text { by } \\
\text { flow rates } \\
\left(\mathbf{m}^{3} / \mathbf{s}\right)\end{array}$ & $\begin{array}{c}C_{D}, \text { by } \\
\text { flow } \\
\text { rates }\end{array}$ & $\begin{array}{c}\% \\
\text { difference } \\
\text { (mass vs. } \\
\text { flow rates) }\end{array}$ \\
\hline W85 & \begin{tabular}{|l|}
377.67 \\
\end{tabular} & 0.81 & 68.38 & 0.9982 & 12.36 & $7.829 \times 10^{-4}$ & 0.633 & 10.04 & $6.360 \times 10^{-4}$ & 0.514 & $18.8 \%$ \\
\hline W86 & 99.67 & 2.09 & 68.13 & 0.9982 & 1.33 & $8.435 \times 10^{-5}$ & 0.649 & 1.31 & $8.292 \times 10^{-5}$ & 0.638 & $1.7 \%$ \\
\hline W87 & 200.12 & 1.16 & 68.17 & 0.9982 & 1.82 & $1.151 \times 10^{-4}$ & 0.625 & 1.21 & $7.684 \times 10^{-5}$ & 0.417 & $33.3 \%$ \\
\hline W88 & 376.63 & 2.04 & 70.04 & 0.9980 & 2.50 & $1.580 \times 10^{-4}$ & 0.626 & -1.22 & $-7.726 \times 10^{-5}$ & -0.306 & $148.9 \%$ \\
\hline W89 & 100.85 & 0.26 & 67.96 & 0.9982 & 3.30 & $2.088 \times 10^{-4}$ & 0.642 & 3.31 & $2.095 \times 10^{-4}$ & 0.644 & $-0.3 \%$ \\
\hline W90 & 198.20 & 1.69 & 69.01 & 0.9981 & 4.56 & $2.890 \times 10^{-4}$ & 0.633 & 4.51 & $2.856 \times 10^{-4}$ & 0.626 & $1.2 \%$ \\
\hline W91 & 378.10 & 0.94 & 70.89 & 0.9979 & 6.38 & $4.042 \times 10^{-4}$ & 0.641 & 6.16 & $3.899 \times 10^{-4}$ & 0.619 & $3.5 \%$ \\
\hline W92 & 99.60 & 0.71 & 68.41 & 0.9982 & 10.60 & $6.712 \times 10^{-4}$ & 0.587 & 10.61 & $6.718 \times 10^{-4}$ & 0.588 & $-0.1 \%$ \\
\hline W93 & 198.64 & 0.50 & 67.70 & 0.9983 & 14.66 & $9.285 \times 10^{-4}$ & 0.575 & 14.48 & $9.171 \times 10^{-4}$ & 0.568 & $1.2 \%$ \\
\hline W94 & 375.18 & 1.19 & 68.36 & 0.9982 & 20.08 & $1.272 \times 10^{-3}$ & 0.573 & 19.85 & $1.257 \times 10^{-3}$ & 0.567 & $1.1 \%$ \\
\hline W95 & 104.68 & 1.02 & 66.00 & 0.9985 & 3.33 & $2.108 \times 10^{-4}$ & 0.636 & 3.34 & $2.114 \times 10^{-4}$ & 0.638 & $-0.3 \%$ \\
\hline W96 & 379.18 & 0.90 & 68.51 & 0.9982 & 6.38 & $4.042 \times 10-4$ & 0.641 & 6.22 & $3.938 \times 10^{-4}$ & 0.624 & $2.6 \%$ \\
\hline W97 & 101.98 & 1.80 & 67.83 & 0.9982 & 10.81 & $6.848 \times 10^{-4}$ & 0.592 & 10.61 & $6.722 \times 10^{-4}$ & 0.581 & $1.8 \%$ \\
\hline W98 & 375.52 & 0.77 & 70.20 & 0.9980 & 20.22 & $1.281 \times 10^{-3}$ & 0.577 & 19.89 & $1.260 \times 10^{-3}$ & 0.567 & $1.6 \%$ \\
\hline W99 & 99.66 & 0.68 & 68.10 & 0.9982 & 3.76 & $2.383 \times 10^{-4}$ & 0.657 & 3.96 & $2.509 \times 10^{-4}$ & 0.692 & $-5.3 \%$ \\
\hline W100 & 201.96 & 0.51 & 68.74 & 0.9981 & 5.25 & $3.324 \times 10^{-4}$ & 0.644 & 5.24 & $3.320 \times 10^{-4}$ & 0.643 & $0.1 \%$ \\
\hline W101 & 376.80 & 3.45 & 72.44 & 0.9977 & 7.09 & $4.493 \times 10^{-4}$ & 0.637 & 6.49 & $4.109 \times 10^{-4}$ & 0.583 & $8.6 \%$ \\
\hline W102 & 103.53 & 0.22 & 73.75 & 0.9975 & 1.41 & $8.915 \times 10^{-5}$ & 0.638 & 1.41 & $8.939 \times 10^{-5}$ & 0.640 & $-0.3 \%$ \\
\hline W103 & 197.86 & 0.49 & 78.85 & 0.9968 & 1.74 & $1.101 \times 10^{-4}$ & 0.570 & 1.63 & $1.031 \times 10^{-4}$ & 0.534 & $6.3 \%$ \\
\hline W104 & 381.05 & 3.45 & 81.40 & 0.9964 & 2.24 & $1.420 \times 10^{-4}$ & 0.530 & 1.36 & $8.592 \times 10^{-5}$ & 0.320 & $39.5 \%$ \\
\hline W105 & 379.46 & 2.31 & 86.80 & 0.9956 & 2.17 & $1.377 \times 10^{-4}$ & 0.514 & 2.04 & $1.289 \times 10^{-4}$ & 0.481 & $6.4 \%$ \\
\hline W106 & 99.17 & 0.27 & 82.72 & 0.9962 & 7.45 & $4.720 \times 10^{-4}$ & 0.659 & 6.72 & $4.254 \times 10^{-4}$ & 0.593 & $9.9 \%$ \\
\hline W107 & 198.76 & 1.75 & 80.92 & 0.9965 & 10.55 & $6.684 \times 10^{-4}$ & 0.659 & 10.20 & $6.459 \times 10^{-4}$ & 0.637 & $3.4 \%$ \\
\hline W108 & 378.39 & 1.46 & 80.45 & 0.9966 & 14.33 & $9.078 \times 10^{-4}$ & 0.648 & 14.36 & $9.096 \times 10^{-4}$ & 0.650 & $-0.2 \%$ \\
\hline W109 & 100.01 & 1.62 & 70.09 & 0.9980 & 3.77 & $2.390 \times 10^{-4}$ & 0.658 & 3.78 & $2.395 \times 10^{-4}$ & 0.660 & $-0.2 \%$ \\
\hline W110 & 379.40 & 0.95 & 73.39 & 0.9976 & 7.20 & $4.559 \times 10^{-4}$ & 0.644 & 7.55 & $4.782 \times 10^{-4}$ & 0.676 & $-4.9 \%$ \\
\hline W111 & 100.75 & 0.22 & 74.48 & 0.9974 & 0.84 & $5.312 \times 10^{-5}$ & 0.385 & 0.43 & $2.693 \times 10^{-5}$ & 0.195 & $49.3 \%$ \\
\hline W112 & 382.74 & 0.78 & 76.92 & 0.9971 & 2.63 & $1.668 \times 10^{-4}$ & 0.621 & 2.45 & $1.553 \times 10^{-4}$ & 0.578 & $6.9 \%$ \\
\hline W113 & 99.90 & 0.26 & 77.77 & 0.9970 & 7.53 & $4.769 \times 10^{-4}$ & 0.663 & 7.37 & $4.670 \times 10^{-4}$ & 0.649 & $2.1 \%$ \\
\hline W114 & 377.10 & 0.77 & 80.16 & 0.9966 & 13.92 & $8.814 \times 10^{-4}$ & 0.631 & 14.19 & $8.984 \times 10^{-4}$ & 0.643 & $-1.9 \%$ \\
\hline W115 & 103.10 & 1.50 & 78.49 & 0.9969 & 31.13 & $1.972 \times 10^{-3}$ & 0.714 & 30.71 & $1.945 \times 10^{-3}$ & 0.704 & $1.4 \%$ \\
\hline W116 & 194.73 & 0.34 & 70.82 & 0.9979 & 43.58 & $2.760 \times 10^{-3}$ & 0.728 & 41.84 & $2.650 \times 10^{-3}$ & 0.698 & $4.0 \%$ \\
\hline W117 & 99.77 & 0.19 & 69.98 & 0.9980 & 31.99 & $2.026 \times 10^{-3}$ & 0.746 & 29.84 & $1.890 \times 10^{-3}$ & 0.696 & $6.7 \%$ \\
\hline W118 & 199.62 & 2.02 & 68.75 & 0.9981 & 45.08 & $2.855 \times 10^{-3}$ & 0.743 & 42.12 & $2.668 \times 10^{-3}$ & 0.695 & $6.6 \%$ \\
\hline W119 & 368.73 & 0.66 & 69.71 & 0.9980 & 62.43 & $3.954 \times 10^{-3}$ & 0.757 & 57.21 & $3.623 \times 10^{-3}$ & 0.694 & $8.4 \%$ \\
\hline W120 & 99.73 & 2.42 & 65.30 & 0.9985 & 0.81 & $5.136 \times 10^{-5}$ & 0.647 & 0.88 & $5.546 \times 10^{-5}$ & 0.699 & $-8.0 \%$ \\
\hline W121 & 378.82 & 0.77 & 69.43 & 0.9981 & 1.90 & $1.203 \times 10^{-4}$ & 0.777 & 1.53 & $9.690 \times 10^{-5}$ & 0.626 & $19.4 \%$ \\
\hline W122 & 199.34 & 0.46 & 69.84 & 0.9980 & 1.27 & $8.059 \times 10^{-5}$ & 0.718 & 0.74 & $4.661 \times 10^{-5}$ & 0.415 & $42.2 \%$ \\
\hline W123 & 197.90 & 3.82 & 69.88 & 0.9980 & 1.21 & $7.688 \times 10^{-5}$ & 0.687 & 1.14 & $7.238 \times 10^{-5}$ & 0.647 & $5.9 \%$ \\
\hline W124 & 101.47 & 0.25 & 69.35 & 0.9981 & 0.85 & $5.390 \times 10^{-5}$ & 0.673 & 0.86 & $5.469 \times 10^{-5}$ & 0.683 & $-1.5 \%$ \\
\hline W125 & 378.78 & 0.71 & 71.49 & 0.9978 & 1.83 & $1.159 \times 10^{-4}$ & 0.749 & 1.77 & $1.121 \times 10^{-4}$ & 0.724 & $3.3 \%$ \\
\hline W126 & 103.47 & 0.26 & 70.22 & 0.9980 & 0.20 & $1.254 \times 10^{-5}$ & 0.426 & 0.24 & $1.500 \times 10^{-5}$ & 0.510 & $-19.6 \%$ \\
\hline W127 & 201.37 & 0.46 & 71.53 & 0.9978 & 0.39 & $2.481 \times 10^{-5}$ & 0.605 & 0.13 & $8.215 \times 10-6$ & 0.200 & $66.9 \%$ \\
\hline W128 & 379.27 & 0.73 & 74.06 & 0.9975 & 0.71 & $4.512 \times 10^{-5}$ & 0.801 & 0.49 & $3.124 \times 10^{-5}$ & 0.555 & $30.8 \%$ \\
\hline W129 & 103.36 & 0.33 & 74.58 & 0.9974 & 1.18 & $7.490 \times 10^{-5}$ & 0.566 & 1.14 & $7.198 \times 10^{-5}$ & 0.544 & $3.9 \%$ \\
\hline W130 & 200.85 & 0.45 & 75.51 & 0.9973 & 1.80 & $1.137 \times 10^{-4}$ & 0.616 & 1.42 & $8.990 \times 10^{-5}$ & 0.487 & $20.9 \%$ \\
\hline W131 & 379.41 & 0.75 & 77.62 & 0.9970 & 2.64 & $1.669 \times 10^{-4}$ & 0.658 & 2.55 & $1.616 \times 10^{-4}$ & 0.637 & $3.2 \%$ \\
\hline W132 & 102.92 & 0.25 & 78.15 & 0.9969 & 0.19 & $1.172 \times 10^{-5}$ & 0.399 & 0.34 & $2.154 \times 10^{-5}$ & 0.734 & $-83.7 \%$ \\
\hline
\end{tabular}


Table A.4. (contd)

\begin{tabular}{|c|c|c|c|c|c|c|c|c|c|c|c|}
\hline Test ID & $\mid\langle\mathbf{P}\rangle$ (psig) & $\begin{array}{c}\sigma \\
\langle\mathbf{P}\rangle \\
\text { (psig) }\end{array}$ & $\mathrm{T}\left({ }^{\circ} \mathbf{F}\right)$ & $\begin{array}{c}\rho \\
(\mathrm{g} / \mathrm{mL})\end{array}$ & $\begin{array}{c}\mathbf{Q}_{\text {spray }}, \text { by } \\
\text { mass } \\
\text { (gpm) }\end{array}$ & 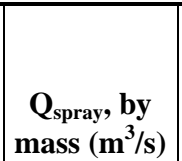 & $\begin{array}{c}C_{D} \text {, by } \\
\text { mass }\end{array}$ & $\begin{array}{c}Q_{\text {spray, by }} \\
\text { flow } \\
\text { rates } \\
\text { (gpm) }\end{array}$ & $\begin{array}{c}\mathbf{Q}_{\text {spray, }} \text { by } \\
\text { flow rates } \\
\left(\mathbf{m}^{3} / \mathbf{s}\right)\end{array}$ & $\begin{array}{c}C_{D} \text {, by } \\
\text { flow } \\
\text { rates }\end{array}$ & $\begin{array}{c}\% \\
\text { difference } \\
\text { (mass vs. } \\
\text { flow rates) }\end{array}$ \\
\hline W133 & 379.38 & 0.72 & 80.29 & 0.9966 & 0.78 & $4.933 \times 10^{-5}$ & 0.875 & 0.65 & $4.093 \times 10^{-5}$ & 0.726 & $17.0 \%$ \\
\hline W134 & 102.78 & 0.25 & 80.47 & 0.9966 & 1.28 & $8.094 \times 10^{-5}$ & 0.613 & 1.23 & $7.775 \times 10^{-5}$ & 0.589 & $3.9 \%$ \\
\hline W135 & 378.61 & 0.74 & 79.69 & 0.9967 & 2.58 & $1.636 \times 10^{-4}$ & 0.646 & 2.51 & $1.590 \times 10^{-4}$ & 0.627 & $2.8 \%$ \\
\hline W136 & 102.12 & 0.27 & 70.04 & 0.9980 & 0.13 & $8.208 \times 10-6$ & 0.281 & 0.35 & $2.248 \times 10^{-5}$ & 0.769 & $-173.8 \%$ \\
\hline W137 & 201.15 & 0.46 & 71.26 & 0.9978 & 0.38 & $2.435 \times 10^{-5}$ & 0.594 & 0.40 & $2.520 \times 10^{-5}$ & 0.614 & $-3.5 \%$ \\
\hline W138 & 379.85 & 0.74 & 74.40 & 0.9974 & 0.79 & $5.002 \times 10^{-5}$ & 0.887 & 0.57 & $3.602 \times 10^{-5}$ & 0.639 & $28.0 \%$ \\
\hline W139 & 102.13 & 0.26 & 75.08 & 0.9973 & 1.23 & $7.808 \times 10^{-5}$ & 0.593 & 1.23 & $7.760 \times 10^{-5}$ & 0.590 & $0.6 \%$ \\
\hline W140 & 200.86 & 0.45 & 76.11 & 0.9972 & 1.79 & $1.131 \times 10^{-4}$ & 0.613 & 1.65 & $1.047 \times 10^{-4}$ & 0.567 & $7.4 \%$ \\
\hline W141 & 379.55 & 0.73 & 78.68 & 0.9968 & 2.72 & $1.725 \times 10^{-4}$ & 0.680 & 2.48 & $1.568 \times 10^{-4}$ & 0.618 & $9.1 \%$ \\
\hline W142 & 101.90 & 0.26 & 79.41 & 0.9967 & 1.23 & $7.764 \times 10^{-5}$ & 0.590 & 1.33 & $8.435 \times 10^{-5}$ & 0.641 & $-8.6 \%$ \\
\hline W143 & 379.32 & 0.68 & 80.83 & 0.9965 & 2.63 & $1.667 \times 10^{-4}$ & 0.657 & 2.51 & $1.590 \times 10^{-4}$ & 0.627 & $4.6 \%$ \\
\hline W144 & 101.67 & 0.27 & 78.42 & 0.9969 & 0.25 & $1.564 \times 10^{-5}$ & 0.536 & 0.33 & $2.075 \times 10^{-5}$ & 0.711 & $-32.7 \%$ \\
\hline W145 & 379.27 & 0.73 & 78.29 & 0.9969 & 0.69 & $4.356 \times 10^{-5}$ & 0.773 & 0.90 & $5.702 \times 10^{-5}$ & 1.012 & $-30.9 \%$ \\
\hline S146 & 100.92 & 0.43 & 69.58 & 1.2443 & 2.71 & $1.715 \times 10^{-4}$ & 0.588 & 3.02 & $1.911 \times 10^{-4}$ & 0.655 & $-11.4 \%$ \\
\hline S147 & 199.89 & 0.55 & 70.86 & 1.2441 & 3.70 & $2.345 \times 10^{-4}$ & 0.571 & 3.77 & $2.387 \times 10^{-4}$ & 0.582 & $-1.8 \%$ \\
\hline S148 & 379.28 & 1.11 & 73.26 & 1.2437 & 5.64 & $3.573 \times 10^{-4}$ & 0.632 & 5.61 & $3.555 \times 10^{-4}$ & 0.629 & $0.5 \%$ \\
\hline S149 & 378.64 & 0.96 & 74.17 & 1.2436 & 5.87 & $3.718 \times 10^{-4}$ & 0.658 & 5.71 & $3.617 \times 10^{-4}$ & 0.640 & $2.7 \%$ \\
\hline S150 & 99.08 & 0.34 & 70.53 & 1.2442 & 2.72 & $1.725 \times 10^{-4}$ & 0.597 & 2.94 & $1.861 \times 10^{-4}$ & 0.644 & $-7.8 \%$ \\
\hline S151 & 199.09 & 1.06 & 70.23 & 1.2442 & 4.03 & $2.555 \times 10^{-4}$ & 0.624 & 4.10 & $2.599 \times 10^{-4}$ & 0.635 & $-1.7 \%$ \\
\hline S152 & 379.86 & 1.02 & 71.47 & 1.2440 & 5.96 & $3.776 \times 10^{-4}$ & 0.668 & 5.94 & $3.764 \times 10^{-4}$ & 0.665 & $0.3 \%$ \\
\hline S153 & 105.70 & 1.28 & 79.78 & 1.2426 & 0.54 & $3.418 \times 10^{-5}$ & 0.467 & 0.64 & $4.024 \times 10^{-5}$ & 0.549 & $-17.7 \%$ \\
\hline S154 & 202.18 & 0.92 & 81.26 & 1.2423 & 0.51 & $3.262 \times 10^{-5}$ & 0.322 & 0.40 & $2.545 \times 10^{-5}$ & 0.251 & $22.0 \%$ \\
\hline S155 & 382.19 & 3.63 & 80.57 & 1.2424 & 0.75 & $4.723 \times 10^{-5}$ & 0.339 & 0.77 & $4.846 \times 10^{-5}$ & 0.348 & $-2.6 \%$ \\
\hline S156 & 104.41 & 0.51 & 75.35 & 1.2434 & 0.14 & $9.025 \times 10^{-6}$ & 0.124 & 0.00 & $-1.722 \times 10^{-7}$ & -0.002 & $101.9 \%$ \\
\hline S157 & 204.16 & 0.55 & 75.06 & 1.2434 & 0.11 & $6.910 \times 10^{-6}$ & 0.068 & -0.09 & $-5.779 \times 10^{-6}$ & -0.057 & $183.6 \%$ \\
\hline S158 & 379.54 & 3.91 & 76.26 & 1.2432 & 0.50 & $3.172 \times 10^{-5}$ & 0.229 & $\begin{array}{l}-0.29 \\
\end{array}$ & $-1.839 \times 10^{-5}$ & -0.132 & $158.0 \%$ \\
\hline W159 & 99.60 & 0.23 & 66.74 & 0.9984 & 3.16 & $1.999 \times 10^{-4}$ & 0.618 & 3.24 & $2.054 \times 10^{-4}$ & 0.635 & $-2.7 \%$ \\
\hline W160 & 200.81 & 0.51 & 67.12 & 0.9983 & 4.69 & $2.970 \times 10^{-4}$ & 0.647 & 4.58 & $2.901 \times 10^{-4}$ & 0.632 & $2.3 \%$ \\
\hline W161 & 376.24 & 1.08 & 68.64 & 0.9982 & 6.45 & $4.088 \times 10^{-4}$ & 0.650 & 6.36 & $4.025 \times 10^{-4}$ & 0.640 & $1.5 \%$ \\
\hline W162 & 100.65 & 0.24 & 68.04 & 0.9982 & 6.43 & $4.072 \times 10^{-4}$ & 0.575 & 6.63 & $4.198 \times 10^{-4}$ & 0.593 & $-3.1 \%$ \\
\hline W163 & 201.07 & 0.53 & 68.30 & 0.9982 & 8.99 & $5.695 \times 10^{-4}$ & 0.569 & 9.08 & $5.751 \times 10^{-4}$ & 0.574 & $-1.0 \%$ \\
\hline W164 & 376.45 & 3.16 & 69.68 & 0.9980 & 13.09 & $8.292 \times 10^{-4}$ & 0.605 & 13.11 & $8.303 \times 10^{-4}$ & 0.606 & $-0.1 \%$ \\
\hline W165 & 197.57 & 0.40 & 68.05 & 0.9982 & 14.46 & $9.155 \times 10^{-4}$ & 0.568 & 14.31 & $9.065 \times 10^{-4}$ & 0.563 & $1.0 \%$ \\
\hline W166 & 374.51 & 0.66 & 73.27 & 0.9976 & 19.90 & $1.260 \times 10^{-3}$ & 0.568 & 19.26 & $1.220 \times 10^{-3}$ & 0.550 & $3.2 \%$ \\
\hline W167 & 197.17 & 0.41 & 75.28 & 0.9973 & 14.17 & $8.974 \times 10^{-4}$ & 0.558 & 14.37 & $9.099 \times 10^{-4}$ & 0.565 & $-1.4 \%$ \\
\hline W168 & 373.71 & 0.64 & 76.40 & 0.9972 & 19.86 & $1.258 \times 10^{-3}$ & 0.567 & 21.79 & $1.380 \times 10^{-3}$ & 0.623 & $-9.7 \%$ \\
\hline W169 & 198.77 & 0.42 & 70.51 & 0.9979 & 10.35 & $6.552 \times 10^{-4}$ & 0.646 & 10.18 & $6.446 \times 10^{-4}$ & 0.636 & $1.6 \%$ \\
\hline W170 & 377.42 & 0.69 & 73.31 & 0.9976 & 14.08 & $8.919 \times 10^{-4}$ & 0.638 & 14.59 & $9.238 \times 10^{-4}$ & 0.661 & $-3.6 \%$ \\
\hline W171 & 200.03 & 1.12 & 73.19 & 0.9976 & 10.34 & $6.552 \times 10^{-4}$ & 0.644 & 10.13 & $6.416 \times 10^{-4}$ & 0.631 & $2.1 \%$ \\
\hline W172 & 376.24 & 2.34 & 74.45 & 0.9974 & 14.06 & $8.908 \times 10^{-4}$ & 0.638 & 13.77 & $8.720 \times 10^{-4}$ & 0.625 & $2.1 \%$ \\
\hline W173 & 99.14 & 0.31 & 71.37 & 0.9978 & 31.48 & $1.994 \times 10^{-3}$ & 0.736 & 29.37 & $1.860 \times 10^{-3}$ & 0.687 & $6.7 \%$ \\
\hline W174 & 203.07 & 0.43 & 69.32 & 0.9981 & 45.04 & $2.853 \times 10^{-3}$ & 0.736 & 42.32 & $2.680 \times 10^{-3}$ & 0.692 & $6.0 \%$ \\
\hline W175 & 371.78 & 0.69 & 71.01 & 0.9979 & 62.56 & $3.962 \times 10^{-3}$ & 0.756 & 57.96 & $3.671 \times 10^{-3}$ & 0.700 & $7.4 \%$ \\
\hline W176 & 196.90 & 0.38 & 69.65 & 0.9980 & 45.90 & $2.907 \times 10^{-3}$ & 0.762 & 41.89 & $2.653 \times 10^{-3}$ & 0.696 & $8.7 \%$ \\
\hline W177 & 371.05 & 0.73 & 73.14 & 0.9976 & 62.45 & $3.955 \times 10^{-3}$ & 0.755 & 56.71 & $3.591 \times 10^{-3}$ & 0.686 & $9.2 \%$ \\
\hline W178 & 97.68 & 0.44 & 72.66 & 0.9977 & 81.43 & $5.157 \times 10^{-3}$ & 0.663 & 76.15 & $4.823 \times 10^{-3}$ & 0.620 & $6.5 \%$ \\
\hline W179 & 102.54 & 0.20 & 69.26 & 0.9981 & 82.22 & $5.207 \times 10^{-3}$ & 0.653 & 77.97 & $4.938 \times 10^{-3}$ & 0.619 & $5.2 \%$ \\
\hline W180 & 201.62 & 0.31 & 68.80 & 0.9981 & 122.07 & $7.731 \times 10^{-3}$ & 0.692 & 109.09 & $6.909 \times 10^{-3}$ & 0.618 & $10.6 \%$ \\
\hline
\end{tabular}


Table A.4. (contd)

\begin{tabular}{|c|c|c|c|c|c|c|c|c|c|c|c|}
\hline Test ID & $\mid\langle\mathbf{P}\rangle$ (psig) & $\begin{array}{c}\sigma \\
\langle\mathbf{P}\rangle \\
\text { (psig) }\end{array}$ & $\mathrm{T}\left({ }^{\circ} \mathbf{F}\right)$ & $\begin{array}{c}\rho \\
(\mathrm{g} / \mathrm{mL})\end{array}$ & $\begin{array}{c}\mathbf{Q}_{\text {sprayy }} \text { by } \\
\text { mass } \\
\text { (gpm) }\end{array}$ & $\begin{array}{c}\mathbf{Q}_{\text {spray }}, \text { by } \\
\text { mass }_{\left(\mathbf{m}^{3} / \mathbf{s}\right)}\end{array}$ & $\begin{array}{c}C_{D} \text {, by } \\
\text { mass }\end{array}$ & $\begin{array}{c}\mathbf{Q}_{\text {spray, by }} \\
\text { flow } \\
\text { rates } \\
\text { (gpm) }\end{array}$ & $\begin{array}{c}Q_{\text {spray, by }} \\
\text { flow rates } \\
\left(\mathbf{m}^{3} / \mathbf{s}\right)\end{array}$ & $\begin{array}{c}C_{D}, \text { by } \\
\text { flow } \\
\text { rates }\end{array}$ & $\begin{array}{c}\% \\
\text { difference } \\
\text { (mass vs. } \\
\text { flow rates) }\end{array}$ \\
\hline W181 & \begin{tabular}{|l|}
202.07 \\
\end{tabular} & 0.35 & 73.15 & 0.9976 & 123.75 & $7.837 \times 10^{-3}$ & 0.700 & 109.04 & $6.906 \times 10^{-3}$ & 0.617 & $11.9 \%$ \\
\hline W182 & 381.60 & 0.60 & 68.06 & 0.9982 & 171.22 & $1.084 \times 10-2$ & 0.705 & 151.01 & $9.564 \times 10^{-3}$ & 0.622 & $11.8 \%$ \\
\hline S183 & 99.94 & 0.29 & 67.62 & 1.1495 & 0.58 & $3.696 \times 10^{-5}$ & 0.499 & 0.60 & $3.829 \times 10^{-5}$ & 0.517 & $-3.6 \%$ \\
\hline S184 & 200.28 & 0.49 & 68.64 & 1.1494 & 1.00 & $6.328 \times 10^{-5}$ & 0.603 & 0.95 & $6.023 \times 10^{-5}$ & 0.574 & $4.8 \%$ \\
\hline S185 & 376.80 & 1.18 & 71.55 & 1.1490 & 1.37 & $8.655 \times 10^{-5}$ & 0.602 & 1.56 & $9.853 \times 10^{-5}$ & 0.685 & $-13.8 \%$ \\
\hline S186 & 99.62 & 0.26 & 70.93 & 1.1491 & 0.69 & $4.395 \times 10^{-5}$ & 0.594 & 0.75 & $4.773 \times 10^{-5}$ & 0.645 & $-8.6 \%$ \\
\hline S187 & 199.69 & 0.44 & 71.27 & 1.1490 & 0.97 & $6.141 \times 10^{-5}$ & 0.586 & 1.01 & $6.420 \times 10^{-5}$ & 0.613 & $-4.5 \%$ \\
\hline S188 & 378.14 & 0.77 & 73.34 & 1.1487 & 1.15 & $7.287 \times 10^{-5}$ & 0.506 & 1.46 & $9.236 \times 10^{-5}$ & 0.641 & $-26.7 \%$ \\
\hline S189 & 101.03 & 0.27 & 67.53 & 1.1496 & 2.90 & $1.836 \times 10^{-4}$ & 0.605 & 2.89 & $1.829 \times 10^{-4}$ & 0.603 & $0.4 \%$ \\
\hline S190 & 198.82 & 0.55 & 68.41 & 1.1494 & 4.24 & $2.685 \times 10^{-4}$ & 0.630 & 4.30 & $2.726 \times 10^{-4}$ & 0.640 & $-1.5 \%$ \\
\hline S191 & 379.21 & 0.74 & 72.29 & 1.1489 & 5.91 & $3.743 \times 10^{-4}$ & 0.636 & 5.67 & $3.589 \times 10^{-4}$ & 0.610 & $4.1 \%$ \\
\hline S192 & 99.58 & 0.28 & 67.06 & 1.1496 & 3.05 & $1.930 \times 10^{-4}$ & 0.641 & 3.02 & $1.914 \times 10^{-4}$ & 0.635 & $0.9 \%$ \\
\hline S193 & 199.06 & 0.45 & 68.05 & 1.1495 & 4.34 & $2.748 \times 10^{-4}$ & 0.645 & 4.43 & $2.803 \times 10^{-4}$ & 0.658 & $-2.0 \%$ \\
\hline S194 & 378.93 & 0.69 & 71.48 & 1.1490 & 5.85 & $3.703 \times 10^{-4}$ & 0.630 & 5.91 & $3.740 \times 10^{-4}$ & 0.636 & $-1.0 \%$ \\
\hline S195 & 98.76 & 0.24 & 68.49 & 1.0554 & 3.42 & $2.168 \times 10^{-4}$ & 0.692 & 3.22 & $2.039 \times 10^{-4}$ & 0.651 & $5.9 \%$ \\
\hline S196 & 200.14 & 0.41 & 71.62 & 1.0550 & 4.62 & $2.928 \times 10^{-4}$ & 0.657 & 4.50 & $2.849 \times 10^{-4}$ & 0.639 & $2.7 \%$ \\
\hline S197 & 378.73 & 0.70 & 77.65 & 1.0541 & 6.26 & $3.962 \times 10^{-4}$ & 0.646 & 6.36 & $4.028 \times 10^{-4}$ & 0.656 & $-1.7 \%$ \\
\hline S198 & 100.19 & 0.33 & 72.81 & 1.0548 & 3.27 & $2.074 \times 10^{-4}$ & 0.657 & 3.26 & $2.062 \times 10^{-4}$ & 0.653 & $0.6 \%$ \\
\hline S199 & 199.42 & 0.47 & 72.88 & 1.0548 & 4.63 & $2.932 \times 10^{-4}$ & 0.659 & 4.43 & $2.809 \times 10^{-4}$ & 0.631 & $4.2 \%$ \\
\hline S200 & 377.47 & 0.81 & 74.95 & 1.0545 & 6.25 & $3.960 \times 10^{-4}$ & 0.646 & 5.91 & $3.740 \times 10^{-4}$ & 0.611 & $5.6 \%$ \\
\hline S201 & 100.60 & 0.23 & 70.18 & 1.0552 & 0.51 & $3.252 \times 10^{-5}$ & 0.419 & 0.50 & $3.141 \times 10^{-5}$ & 0.405 & $3.4 \%$ \\
\hline S202 & 200.25 & 0.49 & 70.97 & 1.0551 & 0.83 & $5.243 \times 10^{-5}$ & 0.479 & 0.71 & $4.493 \times 10^{-5}$ & 0.411 & $14.3 \%$ \\
\hline S203 & 378.88 & 0.82 & 73.82 & 1.0547 & 1.33 & $8.416 \times 10^{-5}$ & 0.559 & 0.72 & $4.550 \times 10^{-5}$ & 0.302 & $45.9 \%$ \\
\hline S204 & 101.44 & 0.28 & 75.88 & 1.0544 & 0.61 & $3.842 \times 10^{-5}$ & 0.493 & 0.56 & $3.562 \times 10^{-5}$ & 0.457 & $7.3 \%$ \\
\hline S205 & 200.89 & 0.43 & 76.75 & 1.0543 & 0.95 & $6.021 \times 10^{-5}$ & 0.549 & 0.89 & $5.607 \times 10^{-5}$ & 0.511 & $6.9 \%$ \\
\hline S206 & 376.37 & 0.94 & 78.27 & 1.0540 & 1.26 & $7.956 \times 10^{-5}$ & 0.530 & 0.56 & $3.568 \times 10^{-5}$ & 0.238 & $55.2 \%$ \\
\hline W207 & 379.23 & 0.770 & 78.50 & 0.9969 & 2.42 & $1.535 \times 10^{-4}$ & 0.605 & 2.41 & $1.528 \times 10^{-4}$ & 0.603 & $0.4 \%$ \\
\hline W208 & 379.36 & 0.752 & 81.91 & 0.9963 & 2.43 & $1.541 \times 10^{-4}$ & 0.607 & 2.38 & $1.506 \times 10^{-4}$ & 0.594 & $2.3 \%$ \\
\hline W209 & 380.31 & 1.073 & 85.46 & 0.9958 & 2.82 & $1.786 \times 10^{-4}$ & 0.703 & 2.87 & $1.818 \times 10^{-4}$ & 0.715 & $-1.8 \%$ \\
\hline W210 & 380.72 & 0.720 & 81.13 & 0.9965 & $\mathrm{e}$ & $1.550 \times 10^{-4}$ & 0.610 & 1.98 & $1.255 \times 10^{-4}$ & $\mathrm{e}$ & $19.1 \%$ \\
\hline W211 & 380.61 & 0.772 & 82.27 & 0.9963 & 2.46 & $1.558 \times 10^{-4}$ & 0.613 & 2.42 & $1.531 \times 10^{-4}$ & 0.602 & $1.7 \%$ \\
\hline W212 & 197.84 & 0.430 & 78.77 & $\mathrm{e}$ & $\mathrm{e}$ & $3.785 \times 10^{-5}$ & 0.930 & 0.47 & $2.956 \times 10^{-5}$ & $\mathrm{e}$ & $21.9 \%$ \\
\hline W213 & 380.20 & 0.710 & 78.41 & 0.9969 & 0.66 & $4.195 \times 10^{-5}$ & 0.744 & 0.25 & $1.603 \times 10^{-5}$ & 0.284 & $61.8 \%$ \\
\hline W214 & 200.61 & 0.424 & 77.12 & 0.9971 & 0.43 & $2.739 \times 10^{-5}$ & 0.668 & 0.51 & $3.214 \times 10^{-5}$ & 0.784 & $-17.4 \%$ \\
\hline W215 & 380.06 & 0.714 & 78.11 & 0.9969 & 0.62 & $3.938 \times 10^{-5}$ & 0.698 & -0.10 & $-6.196 \times 10^{-6}$ & \begin{tabular}{|l|}
-0.110 \\
\end{tabular} & $115.7 \%$ \\
\hline W216 & 200.57 & 0.441 & 76.70 & 0.9971 & 0.49 & $3.075 \times 10^{-5}$ & 0.750 & 0.45 & $2.842 \times 10^{-5}$ & $\mathrm{e}$ & $7.6 \%$ \\
\hline W217 & 379.75 & 1.094 & 78.30 & 0.9969 & 0.66 & $4.168 \times 10^{-5}$ & 0.739 & 0.23 & $1.453 \times 10^{-5}$ & 0.258 & $65.1 \%$ \\
\hline W218 & 199.07 & 0.424 & 74.59 & 0.9974 & 1.79 & $1.136 \times 10^{-4}$ & 0.619 & 1.84 & $1.162 \times 10^{-4}$ & 0.633 & $-2.3 \%$ \\
\hline W219 & 379.49 & 0.839 & 76.47 & 0.9971 & 2.48 & $1.572 \times 10^{-4}$ & 0.620 & 1.89 & $1.199 \times 10^{-4}$ & 0.473 & $23.7 \%$ \\
\hline W220 & 199.19 & 0.443 & 76.54 & 0.9971 & 1.77 & $1.119 \times 10^{-4}$ & 0.609 & 1.77 & $1.120 \times 10^{-4}$ & 0.609 & $-0.1 \%$ \\
\hline W221 & 379.64 & 0.692 & 78.17 & 0.9969 & 2.46 & $1.560 \times 10^{-4}$ & 0.615 & 1.97 & $1.248 \times 10^{-4}$ & 0.492 & $20.0 \%$ \\
\hline W222 & 202.86 & 0.770 & 69.95 & 0.9980 & 11.07 & $7.010 \times 10^{-4}$ & 0.684 & 10.31 & $6.532 \times 10^{-4}$ & 0.638 & $6.8 \%$ \\
\hline W223 & 379.74 & 0.715 & 72.81 & 0.9976 & 15.11 & $9.569 \times 10^{-4}$ & 0.683 & 13.94 & $8.828 \times 10^{-4}$ & 0.630 & $7.7 \%$ \\
\hline W224 & 201.45 & 0.739 & 73.20 & 0.9976 & 11.16 & $7.067 \times 10^{-4}$ & 0.692 & 9.98 & $6.320 \times 10^{-4}$ & 0.619 & $10.6 \%$ \\
\hline W225 & 379.49 & 0.689 & 74.87 & 0.9974 & 15.48 & $9.804 \times 10^{-4}$ & 0.700 & 13.97 & $8.845 \times 10^{-4}$ & 0.631 & $9.8 \%$ \\
\hline W226 & 199.08 & 0.451 & 71.42 & 0.9978 & 4.95 & $3.134 \times 10^{-4}$ & 0.685 & 4.75 & $3.011 \times 10^{-4}$ & 0.658 & $3.9 \%$ \\
\hline W227 & 380.50 & 0.709 & 73.20 & 0.9976 & 6.89 & $4.366 \times 10^{-4}$ & 0.690 & 6.34 & $4.016 \times 10^{-4}$ & 0.635 & $8.0 \%$ \\
\hline W228 & 199.10 & 0.449 & 73.46 & 0.9976 & 4.98 & $3.157 \times 10^{-4}$ & 0.690 & 4.70 & $2.979 \times 10^{-4}$ & 0.651 & $5.6 \%$ \\
\hline
\end{tabular}


Table A.4. (contd)

\begin{tabular}{|c|c|c|c|c|c|c|c|c|c|c|c|}
\hline Test ID & $|\mathbf{P}\rangle$ (psig) & $\begin{array}{c}\sigma \\
\langle\mathbf{P}\rangle \\
\text { (psig) }\end{array}$ & $\mathrm{T}\left({ }^{\circ} \mathrm{F}\right)$ & $\begin{array}{c}\rho \\
(\mathrm{g} / \mathrm{mL})\end{array}$ & $\begin{array}{c}\mathbf{Q}_{\text {spray, }} \text { by } \\
\text { mass } \\
\text { (gpm) }\end{array}$ & $\begin{array}{c}\mathbf{Q}_{\text {spray }}, \text { by } \\
\text { mass }\left(\mathbf{m}^{3} / \mathrm{s}\right)\end{array}$ & $\begin{array}{c}C_{D} \text {, by } \\
\text { mass }\end{array}$ & $\begin{array}{c}\mathbf{Q}_{\text {spray }}, \text { by } \\
\text { flow } \\
\text { rates } \\
\text { (gpm) }\end{array}$ & $\begin{array}{c}\mathbf{Q}_{\text {sprayy }} \text { by } \\
\text { flow rates } \\
\left(\mathbf{m}^{3} / \mathbf{s}\right)\end{array}$ & $\begin{array}{l}C_{D} \text {, by } \\
\text { flow } \\
\text { rates }\end{array}$ & $\begin{array}{c}\% \\
\text { difference } \\
\text { (mass vs. } \\
\text { flow rates) }\end{array}$ \\
\hline W229 & \begin{tabular}{|l|}
380.80 \\
\end{tabular} & 0.728 & 74.97 & 0.9974 & 6.94 & $4.397 \times 10^{-4}$ & 0.695 & 6.23 & $3.944 \times 10^{-4}$ & 0.623 & $10.3 \%$ \\
\hline W230 & 197.78 & 3.165 & 75.20 & 0.9973 & 5.03 & $3.183 \times 10^{-4}$ & 0.698 & 4.48 & $2.836 \times 10^{-4}$ & 0.622 & $10.9 \%$ \\
\hline W231 & 197.19 & 0.344 & 70.82 & 0.9979 & 45.57 & $2.886 \times 10^{-3}$ & 0.756 & 41.78 & $2.646 \times 10^{-3}$ & 0.693 & $8.3 \%$ \\
\hline W232 & 370.10 & 0.889 & 74.98 & 0.9974 & 63.96 & $4.051 \times 10^{-3}$ & 0.774 & 58.35 & $3.696 \times 10^{-3}$ & 0.706 & $8.8 \%$ \\
\hline W233 & 201.26 & 0.392 & 70.43 & 0.9979 & 45.76 & $2.898 \times 10^{-3}$ & 0.751 & 42.11 & $2.667 \times 10^{-3}$ & 0.691 & $8.0 \%$ \\
\hline W234 & 376.27 & 0.673 & 71.10 & 0.9979 & 65.59 & $4.154 \times 10^{-3}$ & 0.788 & 58.24 & $3.688 \times 10^{-3}$ & 0.699 & $11.2 \%$ \\
\hline W235 & 379.02 & 0.662 & 70.38 & 0.9979 & 2.50 & $1.584 \times 10^{-4}$ & 0.625 & 2.26 & $1.429 \times 10^{-4}$ & 0.564 & $9.8 \%$ \\
\hline W236 & 378.38 & 0.708 & 73.30 & 0.9976 & 2.44 & $1.547 \times 10^{-4}$ & 0.611 & 2.19 & $1.388 \times 10^{-4}$ & 0.548 & $10.3 \%$ \\
\hline W237 & 378.53 & 0.901 & 75.36 & $\mathrm{e}$ & 2.49 & $1.579 \times 10^{-4}$ & 0.623 & 2.66 & $1.688 \times 10^{-4}$ & 0.666 & $-6.9 \%$ \\
\hline W238 & 378.41 & 0.740 & 77.32 & 0.9970 & 2.46 & $1.558 \times 10^{-4}$ & 0.615 & 1.27 & $8.021 \times 10^{-5}$ & 0.317 & $48.5 \%$ \\
\hline W239 & 378.04 & 0.873 & 78.98 & 0.9968 & 2.41 & $1.527 \times 10^{-4}$ & 0.603 & $\mathrm{e}$ & $1.474 \times 10^{-4}$ & 0.582 & $3.5 \%$ \\
\hline W240 & 377.35 & 0.742 & 81.09 & 0.9965 & 2.42 & $1.533 \times 10^{-4}$ & 0.606 & 2.39 & $1.513 \times 10^{-4}$ & 0.598 & $1.3 \%$ \\
\hline W241 & -- & & -- & -- & -- & -- & -- & -- & -- & -- & -- \\
\hline W242 & 377.37 & 0.755 & 85.67 & 0.9957 & 2.49 & $1.578 \times 10^{-4}$ & 0.623 & 0.88 & $5.561 \times 10^{-5}$ & 0.220 & $64.7 \%$ \\
\hline W243 & 377.47 & 0.673 & 80.03 & 0.9966 & 2.42 & $1.531 \times 10^{-4}$ & 0.605 & 2.43 & $1.540 \times 10^{-4}$ & 0.608 & $-0.6 \%$ \\
\hline W244 & 376.64 & 0.996 & 81.59 & 0.9964 & 2.47 & $1.562 \times 10^{-4}$ & 0.618 & 2.43 & $1.537 \times 10^{-4}$ & 0.608 & $1.6 \%$ \\
\hline W245 & 377.03 & 0.704 & 83.18 & 0.9961 & 2.51 & $1.588 \times 10^{-4}$ & 0.628 & 2.50 & $1.586 \times 10^{-4}$ & 0.627 & $0.2 \%$ \\
\hline W246 & 99.00 & 1.818 & 81.66 & 0.9964 & 0.34 & $2.158 \times 10^{-5}$ & 0.750 & 0.52 & $3.273 \times 10^{-5}$ & 1.137 & $-51.6 \%$ \\
\hline W247 & -- & -- & -- & -- & -- & -- & -- & -- & -- & -- & -- \\
\hline
\end{tabular}


Appendix B

Individual Test Data 



\section{Appendix B}

\section{Individual Test Data}

In this appendix, the release fractions estimated for every test is presented chronologically (in order of unique test identification number from smallest to largest). Each figure has the release fraction calculated by the cumulative and differential methods, the corresponding $95 \%$ confidence intervals of the fit algorithm, and the WTP model prediction for the experimental conditions of the test. The release fractions calculated by the algorithm that were rejected based on the adjusted coefficient of determination being less than 0.5 are also shown (as " $x$ ") without any confidence intervals. Confidence intervals that are not connected to the release fractions represent instances where the lower bound could not be estimated. Information about the test conditions are shown in the title of each figure. Note that the figures are not captioned and thus can only be referenced via the unique test number and other information provided in the title. Tests W01 through W60 are not included in the appendix because they were repeated in later tests using a more sensitive (and appropriate) Malvern Insitec configuration, including replacement of the laser diodes on two of the Malvern instruments (see Table A.2 for more details on the progression of configurations used during testing).

Three other figures are also provided as part of this appendix. These figures are part of the investigation into repeatability that is discussed in detail in Section 8.1.6. They are comparisons of repeated tests performed in either triplicate (Figure B.1 and Figure B.2) or six times (Figure B.3). Figure B.1 shows triplicate tests conducted with Malvern M1852 in position 1, and Figure B.2 shows triplicate tests with Malvern M1852 in position 3. Figure B.3 presents the release fraction of six repeated tests based on data from Malvern M1855 located in position 1. All of these tests were conducted using water at 380 psi using a 1-mm orifice.

Preceding these figures, a series of tables is presented. The tables refer to specific figures in the main text, in particular Chapters 8 and 9. These figures either were organized by some parameter such as a physical property or contained composite data built from averaging or combining test data. The tables are provided to assist the reader in locating individual tests that appear in figures of this type. The individual tests are referred to by their test number, e.g., W50. Using this test number, the relevant configuration and process operating data (see Appendix A) or resulting release fraction data (this appendix starting at page B.14) can be referenced for a particular test. The tables refer to the relevant figure(s) in their corresponding titles. For reference, the following figures are included in a table in this appendix:

- Figures $8.10-8.12$

- $\quad$ Figures $8.14-8.21$

- Figures $8.23-8.24$

- $\quad$ Figure 8.28

- Figures $9.9-9.12$

- $\quad$ Figure 9.14

- Figures 9.16 - 9.20

- $\quad$ Figures $9.22-9.23$ 
Figures that do not appear on this list are considered to have enough information either in the figure or the supporting text to identify the individual test data.

Table B.1. Figure 8.10 Data Sets

\begin{tabular}{|c|c|c|c|c|}
\hline $\begin{array}{l}\text { Composite } \\
\text { Data Set }\end{array}$ & $\begin{array}{l}\text { Orifice / Target } \\
\text { Dimensions }\end{array}$ & Fluid & $\begin{array}{c}\text { Tests Included in Composite } \\
\text { Data Set }\end{array}$ & Notes \\
\hline $100 \mathrm{psi}$ & \multirow{3}{*}{$\begin{array}{l}\text { S1D (2-mm } \\
\text { hole) }\end{array}$} & \multirow{3}{*}{ Water } & W79, W86 & \multirow{3}{*}{$\begin{array}{l}\text { RFs for }<10 \mu \mathrm{m},<32 \mu \mathrm{m},<102 \mu \mathrm{m} \\
\text { were averaged; error bars generated } \\
\text { according to description in } \\
\text { Section } 8.1 .6\end{array}$} \\
\hline 200 psi & & & W80, W87 & \\
\hline $380 \mathrm{psi}$ & & & $\begin{array}{l}\text { W81, W88, W207, W208, } \\
\text { W238 }\end{array}$ & \\
\hline
\end{tabular}

Table B.2. Figure 8.11 Data Sets

\begin{tabular}{|c|c|c|c|c|}
\hline $\begin{array}{l}\text { Composite } \\
\text { Data Set }\end{array}$ & $\begin{array}{l}\text { Orifice / Target } \\
\text { Dimensions }\end{array}$ & Fluid & $\begin{array}{c}\text { Tests Included in Composite } \\
\text { Data Set }\end{array}$ & Notes \\
\hline $100 \mathrm{psi}$ & \multirow{3}{*}{$\begin{array}{l}\text { S7A }(1 \times 10-\mathrm{mm} \\
\text { slot })\end{array}$} & \multirow{3}{*}{ Water } & W89, W95, W159 & \multirow{3}{*}{$\begin{array}{l}\text { RFs for }<10 \mu \mathrm{m},<32 \mu \mathrm{m},<102 \mu \mathrm{m} \\
\text { were averaged; error bars generated } \\
\text { according to description in } \\
\text { Section 8.1.6 }\end{array}$} \\
\hline 200 psi & & & W90, W160 & \\
\hline $380 \mathrm{psi}$ & & & W91, W96, W161 & \\
\hline
\end{tabular}

Table B.3. Figure 8.12 Data Sets

\begin{tabular}{|c|c|c|c|c|}
\hline Data Set & Fluid & Pressure & Test in Data Set & Notes \\
\hline S7A $(1 \times 10-\mathrm{mm}$ slot $)$ & \multirow[t]{3}{*}{ Water } & \multirow[t]{3}{*}{$380 \mathrm{psi}$} & W91 & \multirow{3}{*}{$\begin{array}{l}\text { Only one WTP model was shown; } \\
\text { due to the similarity in area the } \\
\text { curves are essentially the same }\end{array}$} \\
\hline S5A $(10 \times 1-\mathrm{mm}$ slot $)$ & & & W101 & \\
\hline WTP model & & & $\begin{array}{l}\text { Prediction based } \\
\text { on W91 }\end{array}$ & \\
\hline
\end{tabular}

Table B.4. Figure 8.14 Data Sets

\begin{tabular}{|c|c|c|c|c|}
\hline $\begin{array}{l}\text { Composite } \\
\text { Data Set }\end{array}$ & $\begin{array}{l}\text { Orifice / Target } \\
\text { Dimensions }\end{array}$ & $\begin{array}{l}\text { Fluid / } \\
\text { Pressure }\end{array}$ & $\begin{array}{c}\text { Tests Included in Composite } \\
\text { Data Set }\end{array}$ & Notes \\
\hline $0.78 \mathrm{~mm}^{2}$ & S1A (1-mm hole) & \multirow{5}{*}{$\begin{array}{l}\text { Water, } \\
380 \text { psi }\end{array}$} & W74, W83 & \multirow{5}{*}{$\begin{array}{l}\text { GRs for }<10 \mu \mathrm{m},<32 \mu \mathrm{m} \text {, } \\
<102 \mu \mathrm{m} \text { were averaged if } \\
\text { more than one test is } \\
\text { included in the data set; } \\
\text { error bars generated } \\
\text { according to description in } \\
\text { Section } 8.1 .6\end{array}$} \\
\hline $3.50 \mathrm{~mm}^{2}$ & S1D (2-mm hole) & & $\begin{array}{l}\text { W81, W88, W207, W208, } \\
\text { W238 }\end{array}$ & \\
\hline $3.69 \mathrm{~mm}^{2}$ & $\begin{array}{l}\text { S5B (array of five } \\
\text { 1-mm holes) }\end{array}$ & & W104, W105, W112 & \\
\hline $5.89 \mathrm{~mm}^{2}$ & S1C (2.74-mm hole) & & W78 & \\
\hline $17.13 \mathrm{~mm}^{2}$ & S1B (4.46-mm hole) & & W76, W85 & \\
\hline
\end{tabular}


Table B.5. Figure 8.15 Data Sets

\begin{tabular}{|c|c|c|c|c|}
\hline $\begin{array}{l}\text { Composite } \\
\text { Data Set }\end{array}$ & $\begin{array}{l}\text { Orifice / Target } \\
\text { Dimensions }\end{array}$ & $\begin{array}{c}\text { Fluid / } \\
\text { Pressure }\end{array}$ & $\begin{array}{l}\text { Tests Included in } \\
\text { Composite Data Set }\end{array}$ & Notes \\
\hline $2.14 \mathrm{~mm}^{2}$ & $\begin{array}{l}\text { S2A }(0.5 \times 5-\mathrm{mm} \\
\text { slot })\end{array}$ & \multirow{8}{*}{$\begin{array}{c}\text { Water, } 380 \\
\text { psi }\end{array}$} & W121, W125 & \multirow{8}{*}{$\begin{array}{l}\text { GRs for }<10 \mu \mathrm{m},<32 \mu \mathrm{m} \text {, } \\
<102 \mu \mathrm{m} \text { were averaged if } \\
\text { more than one test is } \\
\text { included in the data set; } \\
\text { error bars generated } \\
\text { according to description in } \\
\text { Section 8.1.6 }\end{array}$} \\
\hline $8.72 \mathrm{~mm}^{2}$ & $\begin{array}{l}\text { S7A }(1 \times 10-\mathrm{mm} \\
\text { slot })\end{array}$ & & W91, W96, W161 & \\
\hline $9.77 \mathrm{~mm}^{2}$ & $\begin{array}{l}\text { S5A }(10 \times 1-\mathrm{mm} \\
\text { slot })\end{array}$ & & W101, W110 & \\
\hline $19.00 \mathrm{~mm}^{2}$ & S7B $(2 \times 10-\mathrm{mm}$ slot $)$ & & W164 & \\
\hline $19.35 \mathrm{~mm}^{2}$ & S5C $(1 \times 20-m m$ slot $)$ & & W108, W114, W170, W172 & \\
\hline $30.83 \mathrm{~mm}^{2}$ & S7C $(3 \times 10-m m$ slot $)$ & & W94, W98, W166, W168 & \\
\hline $73.14 \mathrm{~mm}^{2}$ & $\begin{array}{c}\text { S4A }(1 \times 76.2-\mathrm{mm} \\
\text { slot })\end{array}$ & & W119, W175, W177 & \\
\hline $211.82 \mathrm{~mm}^{2}$ & $\begin{array}{c}\text { S3A }(2.74 \times 76.2-\mathrm{mm} \\
\text { slot })\end{array}$ & & W182 & \\
\hline
\end{tabular}

Table B.6. Figure 8.16 Data Sets

\begin{tabular}{|c|c|c|c|c|}
\hline $\begin{array}{l}\text { Composite } \\
\text { Data Set* }\end{array}$ & $\begin{array}{l}\text { Orifice / Target } \\
\text { Dimensions }\end{array}$ & $\begin{array}{c}\text { Fluid / } \\
\text { Pressure }\end{array}$ & $\begin{array}{c}\text { Tests Included in } \\
\text { Composite Data Set }\end{array}$ & Notes \\
\hline $8.72 \mathrm{~mm}^{2}$ & $\begin{array}{c}\text { S7A }(1 \times 10-\mathrm{mm} \\
\text { slot })\end{array}$ & \multirow{3}{*}{$\begin{array}{c}\text { Water, } 200 \\
\text { psi }\end{array}$} & W90, W160 & \multirow{6}{*}{$\begin{array}{l}\text { RFs for }<10 \mu \mathrm{m},<32 \mu \mathrm{m} \text {, } \\
<102 \mu \mathrm{m} \text { were averaged if } \\
\text { more than one test is } \\
\text { included in the data set; } \\
\text { error bars generated } \\
\text { according to description in } \\
\text { Section } 8.1 .6\end{array}$} \\
\hline $19.35 \mathrm{~mm}^{2}$ & S5C $(1 \times 20-m m$ slot $)$ & & W107, W169, W171 & \\
\hline $73.14 \mathrm{~mm}^{2}$ & $\begin{array}{c}\text { S4A }(1 \times 76.2-\mathrm{mm} \\
\text { slot })\end{array}$ & & W116, W118, W174, W176 & \\
\hline $8.72 \mathrm{~mm}^{2}$ & $\begin{array}{c}\text { S7A }(1 \times 10-\mathrm{mm} \\
\text { slot })\end{array}$ & \multirow{3}{*}{$\begin{array}{l}\text { Water, } 380 \\
\text { psi }\end{array}$} & W91, W96, W161 & \\
\hline $19.35 \mathrm{~mm}^{2}$ & S5C $(1 \times 20-\mathrm{mm}$ slot $)$ & & W108, W114, W170, W172 & \\
\hline $73.14 \mathrm{~mm}^{2}$ & $\begin{array}{c}\text { S4A }(1 \times 76.2-\mathrm{mm} \\
\text { slot })\end{array}$ & & W119, W175, W177 & \\
\hline
\end{tabular}

Table B.7. Figure 8.17 Data Sets

\begin{tabular}{|c|c|c|c|c|}
\hline $\begin{array}{l}\text { Composite } \\
\text { Data Set* }\end{array}$ & $\begin{array}{l}\text { Orifice / Target } \\
\text { Dimensions }\end{array}$ & $\begin{array}{c}\text { Fluid / } \\
\text { Pressure }\end{array}$ & $\begin{array}{c}\text { Tests Included in } \\
\text { Composite Data Set }\end{array}$ & Notes \\
\hline $8.72 \mathrm{~mm}^{2}$ & $\begin{array}{l}\text { S7A }(1 \times 10-\mathrm{mm} \\
\text { slot })\end{array}$ & \multirow{3}{*}{$\begin{array}{c}\text { Water, } 200 \\
\text { psi }\end{array}$} & W90, W160 & \multirow{6}{*}{$\begin{array}{l}\text { RFs for }<10 \mu \mathrm{m},<32 \mu \mathrm{m} \text {, } \\
<102 \mu \mathrm{m} \text { were averaged if } \\
\text { more than one test is } \\
\text { included in the data set; } \\
\text { error bars generated } \\
\text { according to description in } \\
\text { Section 8.1.6 }\end{array}$} \\
\hline $19.00 \mathrm{~mm}^{2}$ & S7B $(2 \times 10-\mathrm{mm}$ slot $)$ & & W163 & \\
\hline $30.83 \mathrm{~mm}^{2}$ & S7C $(3 \times 10-m m$ slot $)$ & & W93, W165, W167 & \\
\hline $8.72 \mathrm{~mm}^{2}$ & $\begin{array}{c}\text { S7A }(1 \times 10-\mathrm{mm} \\
\text { slot })\end{array}$ & \multirow{3}{*}{$\begin{array}{c}\text { Water, } 380 \\
\text { psi }\end{array}$} & W91, W96, W161 & \\
\hline $19.00 \mathrm{~mm}^{2}$ & S7B $(2 \times 10-\mathrm{mm}$ slot $)$ & & W164 & \\
\hline $30.83 \mathrm{~mm}^{2}$ & S7C $(3 \times 10-\mathrm{mm}$ slot $)$ & & W94, W98, W166, W168 & \\
\hline
\end{tabular}


Table B.8. Figure 8.18a / 8.19a Data Sets

\begin{tabular}{|c|c|c|c|c|}
\hline $\begin{array}{l}\text { Composite } \\
\text { Data Set }\end{array}$ & $\begin{array}{l}\text { Orifice / Target } \\
\text { Dimensions }\end{array}$ & $\begin{array}{l}\text { Fluid / } \\
\text { Pressure }\end{array}$ & $\begin{array}{l}\text { Tests Included in } \\
\text { Composite Data Set }\end{array}$ & Notes \\
\hline $2.14 \mathrm{~mm}^{2}$ & $\begin{array}{l}\text { S2A }(0.5 \times 5-\mathrm{mm} \\
\text { slot })\end{array}$ & \multirow{10}{*}{$\begin{array}{l}\text { Water, } 100 \\
\text { psi }\end{array}$} & W120, W124 & \multirow{10}{*}{$\begin{array}{l}\text { RFs (8.18a) and GRs (8.19a) } \\
\text { for }<10 \mu \mathrm{m},<32 \mu \mathrm{m}, \\
<102 \mu \mathrm{m} \text { were averaged if } \\
\text { more than one test is } \\
\text { included in the data set; } \\
\text { error bars generated } \\
\text { according to description in } \\
\text { Section 8.1.6 }\end{array}$} \\
\hline $3.50 \mathrm{~mm}^{2}$ & S1D (2-mm hole) & & W79, W86 & \\
\hline $3.69 \mathrm{~mm}^{2}$ & $\begin{array}{l}\text { S5B (array of five } \\
\text { 1-mm holes) }\end{array}$ & & W102 & \\
\hline $8.72 \mathrm{~mm}^{2}$ & $\begin{array}{l}\text { S7A }(1 \times 10-\mathrm{mm} \\
\text { slot })\end{array}$ & & W89, W95, W159 & \\
\hline $9.77 \mathrm{~mm}^{2}$ & $\begin{array}{l}\text { S5A }(10 \times 1-\mathrm{mm} \\
\text { slot })\end{array}$ & & W99, W109 & \\
\hline $19.00 \mathrm{~mm}^{2}$ & S7B $(2 \times 10-m m$ slot $)$ & & W162 & \\
\hline $19.35 \mathrm{~mm}^{2}$ & S5C $(1 \times 20-m m$ slot $)$ & & W106, W113 & \\
\hline $30.83 \mathrm{~mm}^{2}$ & S7C $(3 \times 10-\mathrm{mm}$ slot $)$ & & W92, W97 & \\
\hline $73.14 \mathrm{~mm}^{2}$ & $\begin{array}{c}\text { S4A }(1 \times 76.2-\mathrm{mm} \\
\text { slot })\end{array}$ & & W115, W117, W173 & \\
\hline $211.82 \mathrm{~mm}^{2}$ & $\begin{array}{l}\text { S3A }(2.74 \times 76.2-\mathrm{mm} \\
\text { slot })\end{array}$ & & W178, W179 & \\
\hline
\end{tabular}

Table B.9. Figure 8.18b / 8.19b Data Sets

\begin{tabular}{|c|c|c|c|c|}
\hline $\begin{array}{l}\text { Composite } \\
\text { Data Set }\end{array}$ & $\begin{array}{l}\text { Orifice / Target } \\
\text { Dimensions }\end{array}$ & $\begin{array}{c}\text { Fluid / } \\
\text { Pressure }\end{array}$ & $\begin{array}{c}\text { Tests Included in } \\
\text { Composite Data Set }\end{array}$ & Notes \\
\hline $0.78 \mathrm{~mm}^{2}$ & S1A (1-mm hole) & \multirow{13}{*}{$\begin{array}{c}\text { Water, } 200 \\
\text { psi }\end{array}$} & W73, W82 & \multirow{13}{*}{$\begin{array}{l}\text { RFs }(8.18 \mathrm{~b}) \text { and GRs } \\
(8.19 \mathrm{~b}) \text { for }<10 \mu \mathrm{m} \text {, } \\
<32 \mu \mathrm{m},<102 \mu \mathrm{m} \text { were } \\
\text { averaged if more than one } \\
\text { test is included in the data } \\
\text { set; error bars generated } \\
\text { according to description in } \\
\text { Section 8.1.6 }\end{array}$} \\
\hline $2.14 \mathrm{~mm}^{2}$ & $\begin{array}{l}\text { S2A }(0.5 \times 5-\mathrm{mm} \\
\text { slot })\end{array}$ & & W122, W123 & \\
\hline $3.50 \mathrm{~mm}^{2}$ & S1D (2-mm hole) & & W80, W87 & \\
\hline $3.69 \mathrm{~mm}^{2}$ & $\begin{array}{l}\text { S5B (array of five } \\
\text { 1-mm holes) }\end{array}$ & & W103 & \\
\hline $5.89 \mathrm{~mm}^{2}$ & S1C (2.74-mm hole) & & W77 & \\
\hline $8.72 \mathrm{~mm}^{2}$ & $\begin{array}{c}\text { S7A }(1 \times 10-\mathrm{mm} \\
\text { slot })\end{array}$ & & W90, W160 & \\
\hline $9.77 \mathrm{~mm}^{2}$ & $\begin{array}{c}\text { S5A }(10 \times 1-\mathrm{mm} \\
\text { slot })\end{array}$ & & W100 & \\
\hline $17.13 \mathrm{~mm}^{2}$ & S1B (4.46-mm hole) & & W75, W84 & \\
\hline $19.00 \mathrm{~mm}^{2}$ & S7B $(2 \times 10-m m$ slot $)$ & & W163 & \\
\hline $19.35 \mathrm{~mm}^{2}$ & S5C $(1 \times 20$-mm slot $)$ & & W107, W169, W171 & \\
\hline $30.83 \mathrm{~mm}^{2}$ & S7C $(3 \times 10$-mm slot $)$ & & W93, W165, W167 & \\
\hline $73.14 \mathrm{~mm}^{2}$ & $\begin{array}{c}\text { S4A }(1 \times 76.2-\mathrm{mm} \\
\text { slot })\end{array}$ & & W116, W118, W174, W176 & \\
\hline $211.82 \mathrm{~mm}^{2}$ & $\begin{array}{l}\text { S3A (2.74 } \times 76.2-\mathrm{mm} \\
\text { slot) }\end{array}$ & & W180, W181 & \\
\hline
\end{tabular}


Table B.10. Figure 8.18c / 8.19c Data Sets

\begin{tabular}{|c|c|c|c|c|}
\hline $\begin{array}{c}\text { Composite } \\
\text { Data Set }\end{array}$ & $\begin{array}{c}\text { Orifice / Target } \\
\text { Dimensions }\end{array}$ & $\begin{array}{c}\text { Fluid / } \\
\text { Pressure }\end{array}$ & $\begin{array}{c}\text { Tests Included in } \\
\text { Composite Data Set }\end{array}$ & Notes \\
\hline $0.78 \mathrm{~mm}^{2}$ & S1A (1-mm hole) & \multirow{13}{*}{$\begin{array}{c}\text { Water, } 380 \\
\text { psi }\end{array}$} & W74, W83 & \multirow{13}{*}{$\begin{array}{l}\text { RFs }(8.18 \mathrm{c}) \text { and GRs }(8.19 \mathrm{c}) \\
\text { for }<10 \mu \mathrm{m},<32 \mu \mathrm{m} \text {, } \\
<102 \mu \mathrm{m} \text { were averaged if } \\
\text { more than one test is } \\
\text { included in the data set; } \\
\text { error bars generated } \\
\text { according to description in } \\
\text { Section } 8.1 .6\end{array}$} \\
\hline $2.14 \mathrm{~mm}^{2}$ & $\begin{array}{c}\text { S2A }(0.5 \times 5-\mathrm{mm} \\
\text { slot })\end{array}$ & & W121, W125 & \\
\hline $3.50 \mathrm{~mm}^{2}$ & S1D (2-mm hole) & & $\begin{array}{l}\text { W81, W88, W207, W208, } \\
\text { W238 }\end{array}$ & \\
\hline $3.69 \mathrm{~mm}^{2}$ & $\begin{array}{c}\text { S5B (array of five } \\
\text { 1-mm holes) }\end{array}$ & & W104, W105, W112 & \\
\hline $5.89 \mathrm{~mm}^{2}$ & S1C (2.74-mm hole) & & W78 & \\
\hline $8.72 \mathrm{~mm}^{2}$ & $\begin{array}{c}\text { S7A }(1 \times 10-\mathrm{mm} \\
\text { slot })\end{array}$ & & W91, W96, W161 & \\
\hline $9.77 \mathrm{~mm}^{2}$ & $\begin{array}{c}\text { S5A }(10 \times 1-\mathrm{mm} \\
\text { slot })\end{array}$ & & W101, W110 & \\
\hline $17.13 \mathrm{~mm}^{2}$ & S1B (4.46-mm hole) & & W76, W85 & \\
\hline $19.00 \mathrm{~mm}^{2}$ & S7B $(2 \times 10-\mathrm{mm}$ slot $)$ & & W164 & \\
\hline $19.35 \mathrm{~mm}^{2}$ & S5C $(1 \times 20-m m$ slot $)$ & & W108, W114, W170, W172 & \\
\hline $30.83 \mathrm{~mm}^{2}$ & S7C $(3 \times 10-m m$ slot $)$ & & W94, W98, W166, W168 & \\
\hline $73.14 \mathrm{~mm}^{2}$ & $\begin{array}{c}\text { S4A }(1 \times 76.2-\mathrm{mm} \\
\text { slot })\end{array}$ & & W119, W175, W177 & \\
\hline $211.82 \mathrm{~mm}^{2}$ & $\begin{array}{c}\text { S3A }(2.74 \times 76.2-\mathrm{mm} \\
\text { slot })\end{array}$ & & W182 & \\
\hline
\end{tabular}

Table B.11. Figure 8.20 Data Sets

\begin{tabular}{|c|c|c|c|c|}
\hline Data Set & Fluid & $\begin{array}{c}\text { Pressure / } \\
\text { Orifice }\end{array}$ & Test in Data Set & Notes \\
\hline RF, water & Water & \multirow{4}{*}{$\begin{array}{c}380 \text { psi, } \\
\text { S7A (1× } \\
10-m m \text { slot) }\end{array}$} & W91 & \multirow{4}{*}{$\begin{array}{l}\text { "STS" is an abbreviation } \\
\text { for the sodium thiosulfate } \\
\text { simulant, described in } \\
\text { Chapter } 4 \text { (see Table 4.3) }\end{array}$} \\
\hline RF, sodium thiosulfate & STS & & S148 & \\
\hline WTP model (water) & Water & & Prediction based on W91 & \\
\hline WTP model (sodium thiosulfate) & STS & & Prediction based on S148 & \\
\hline
\end{tabular}

Table B.12. Figure 8.21 Data Sets

\begin{tabular}{|c|c|c|c|c|}
\hline Data Set & Fluid & Viscosity & Tests in Data Set & Notes \\
\hline \multirow{2}{*}{$\mathrm{G},<10 \mu \mathrm{m}, 200 \mathrm{psi}, 1 \times 10-\mathrm{mm}$ slot } & Water & $1.0 \mathrm{cP}$ & W90 & \multirow{12}{*}{$\begin{array}{l}\text { "STS" is an abbreviation } \\
\text { for the sodium thiosulfate } \\
\text { simulant, described in } \\
\text { Chapter } 4 \text { (see Table 4.3) }\end{array}$} \\
\hline & STS & $2.6 \mathrm{cP}$ & W147, S151 & \\
\hline \multirow{2}{*}{$\mathrm{G},<32 \mu \mathrm{m}, 200 \mathrm{psi}, 1 \times 10-\mathrm{mm}$ slot } & Water & $1.0 \mathrm{cP}$ & W90 & \\
\hline & STS & $2.6 \mathrm{cP}$ & W147, S151 & \\
\hline \multirow{2}{*}{$\mathrm{G},<102 \mu \mathrm{m}, 200 \mathrm{psi}, 1 \times 10-\mathrm{mm}$ slot } & Water & $1.0 \mathrm{cP}$ & W90 & \\
\hline & STS & $2.6 \mathrm{cP}$ & W147, S151 & \\
\hline \multirow{2}{*}{$\mathrm{G},<10 \mu \mathrm{m}, 380 \mathrm{psi}, 1 \times 10-\mathrm{mm}$ slot } & Water & $1.0 \mathrm{cP}$ & W91, W96, W161 & \\
\hline & STS & $2.6 \mathrm{cP}$ & S148, S149, S152 & \\
\hline \multirow{2}{*}{$\mathrm{G},<32 \mu \mathrm{m}, 380 \mathrm{psi}, 1 \times 10-\mathrm{mm}$ slot } & Water & $1.0 \mathrm{cP}$ & W91, W96, W161 & \\
\hline & STS & $2.6 \mathrm{cP}$ & S148, S149, S152 & \\
\hline \multirow{2}{*}{$\mathrm{G},<102 \mu \mathrm{m}, 380 \mathrm{psi}, 1 \times 10$-mm slot } & Water & $1.0 \mathrm{cP}$ & W91, W96, W161 & \\
\hline & STS & $2.6 \mathrm{cP}$ & S148, S149, S152 & \\
\hline
\end{tabular}


Table B.13. Figure 8.23 Data Sets

\begin{tabular}{|c|c|c|c|c|}
\hline Data Set & Fluid & $\begin{array}{c}\text { Pressure / } \\
\text { Orifice }\end{array}$ & Test in Data Set & Notes \\
\hline Water & Water & \multirow{4}{*}{$\begin{array}{c}380 \mathrm{psi}, \\
\text { S7A (1× } \\
10-\mathrm{mm} \text { slot })\end{array}$} & W91 & \multirow{4}{*}{$\begin{array}{l}\text { "STR" is an abbreviation } \\
\text { for the boehmite/water } \\
\text { slurry described in } \\
\text { Chapter } 4 \text { (see Table 4.4) }\end{array}$} \\
\hline $8 \mathrm{wt} \%$ STR simulant & STR 8 & & S197 & \\
\hline $20 \mathrm{wt} \%$ STR simulant & STR 20 & & S191 & \\
\hline WTP models & Each fluid & & $\begin{array}{l}\text { Prediction based on tests } \\
\text { listed above }\end{array}$ & \\
\hline
\end{tabular}

Table B.14. Figure 8.24 Data Sets

\begin{tabular}{|c|c|c|c|c|}
\hline Data Set & Fluid & $\begin{array}{c}\text { Pressure / } \\
\text { Orifice }\end{array}$ & Test in Data Set & Notes \\
\hline 227.25 in. from splash wall & \multirow{4}{*}{ Water } & \multirow{4}{*}{$\begin{array}{l}380 \mathrm{psi}, \\
\text { S1A (1-mm } \\
\text { hole) }\end{array}$} & W74 & \multirow{4}{*}{$\begin{array}{l}\text { The WTP model does no } \\
\text { account for spray } \\
\text { distance; thus, all test } \\
\text { conditions have the same } \\
\text { model prediction }\end{array}$} \\
\hline 120.25 in. from splash wall & & & W138 & \\
\hline 43.25 in. from splash wall & & & W128 & \\
\hline WTP model & & & Prediction based on W74 & \\
\hline
\end{tabular}

Table B.15. Figure 8.28 Data Sets

\begin{tabular}{|c|c|c|c|c|}
\hline Data at Value & Fluid & $\begin{array}{c}\text { Pressure / } \\
\text { Orifice }\end{array}$ & Test in Data Set & Notes \\
\hline 43.25 in. & \multirow{3}{*}{ Water } & \multirow{3}{*}{$\begin{array}{l}\text { 380, S1D (2-mm } \\
\text { hole) }\end{array}$} & W131, W135 & \multirow{3}{*}{$\begin{array}{l}\text { GRs for }<10 \mu \mathrm{m},<32 \mu \mathrm{m},<102 \mu \mathrm{m} \text { were } \\
\text { averaged if more than one test is included in } \\
\text { the data set; error bars generated according to } \\
\text { description in Section 8.1.6 }\end{array}$} \\
\hline 120.25 in. & & & W141, W143 & \\
\hline 227.25 in. & & & W81, W88 & \\
\hline
\end{tabular}

Table B.16. Figure 9.9 and 9.10 Data Sets

\begin{tabular}{|c|c|c|c|c|}
\hline $\begin{array}{l}\text { Composite } \\
\text { Data Set }\end{array}$ & $\begin{array}{l}\text { Orifice / Target } \\
\text { Dimensions }\end{array}$ & $\begin{array}{c}\text { Fluid / } \\
\text { Pressure }\end{array}$ & $\begin{array}{l}\text { Tests Included in } \\
\text { Composite Data Set }\end{array}$ & Notes \\
\hline \multicolumn{5}{|c|}{ Small-scale data (red) } \\
\hline $0.07 \mathrm{~mm}^{2}$ & $0.3-\mathrm{mm}$ hole & \multirow{5}{*}{$\begin{array}{l}\text { Water, } \\
380 \text { psi }\end{array}$} & OS9 & \multirow{5}{*}{$\begin{array}{l}\text { Small-scale data is shown as individual } \\
\text { tests. The error bars are estimated as } \\
\text { described in Section } 6.3 .1 \text { of Mahoney } \\
\text { et al. ( } 2012 \mathrm{~b})\end{array}$} \\
\hline $0.221 \mathrm{~mm}^{2}$ & 0.5-mm hole & & SO1 & \\
\hline $0.224 \mathrm{~mm}^{2}$ & $\begin{array}{c}\text { 0.5-mm hole [note: } \\
\text { different orifice than } \\
\text { above] }\end{array}$ & & OS8-R1 & \\
\hline $0.75 \mathrm{~mm}^{2}$ & 1-mm hole & & OS7, RT18, RT19 & \\
\hline $3.19 \mathrm{~mm}^{2}$ & 2-mm hole & & OS6 & \\
\hline \multicolumn{5}{|c|}{ Large-scale data (black) } \\
\hline $0.78 \mathrm{~mm}^{2}$ & S1A (1-mm hole) & \multirow{5}{*}{$\begin{array}{l}\text { Water, } \\
380 \text { psi }\end{array}$} & W74, W83 & \multirow{5}{*}{$\begin{array}{l}\text { RFs }(9.9) \text { and GRs }(9.10) \text { for }<10 \mu \mathrm{m}, \\
<32 \mu \mathrm{m},<102 \mu \mathrm{m} \text { were averaged if } \\
\text { more than one test is included in the } \\
\text { data set; error bars generated according } \\
\text { to description in Section 8.1.6 } \\
\\
\text { WTP model values are based on } \\
\text { averages if there is more than one data } \\
\text { set }\end{array}$} \\
\hline $3.50 \mathrm{~mm}^{2}$ & S1D (2-mm hole) & & $\begin{array}{l}\text { W81, W88, W207, } \\
\text { W208, W238 }\end{array}$ & \\
\hline $3.69 \mathrm{~mm}^{2}$ & $\begin{array}{l}\text { S5B (array of five } \\
\text { 1-mm holes) }\end{array}$ & & W104, W105, W112 & \\
\hline $5.89 \mathrm{~mm}^{2}$ & S1C (2.74-mm hole) & & W78 & \\
\hline $17.13 \mathrm{~mm}^{2}$ & S1B (4.46-mm hole) & & W76, W85 & \\
\hline
\end{tabular}


Table B.17. Figure 9.11 and 9.12 Data Sets

\begin{tabular}{|c|c|c|c|c|}
\hline $\begin{array}{c}\text { Composite } \\
\text { Data Set }\end{array}$ & $\begin{array}{c}\text { Orifice / Target } \\
\text { Dimensions }\end{array}$ & $\begin{array}{c}\text { Fluid / } \\
\text { Pressure }\end{array}$ & $\begin{array}{l}\text { Tests Included in } \\
\text { Composite Data Set }\end{array}$ & Notes \\
\hline \multicolumn{5}{|c|}{ Small-scale data (red) } \\
\hline $1.77 \mathrm{~mm}^{2}$ & $0.3 \times 5-\mathrm{mm}$ slot & \multirow{7}{*}{$\begin{array}{l}\text { Water, } \\
380 \text { psi }\end{array}$} & OS13 & \multirow{7}{*}{$\begin{array}{l}\text { Small-scale data is shown as } \\
\text { individual tests. The error bars } \\
\text { are estimated as described in } \\
\text { Section } 6.3 .1 \text { of Mahoney et al. } \\
\text { (2012b) }\end{array}$} \\
\hline $2.61 \mathrm{~mm}^{2}$ & $0.5 \times 5-\mathrm{mm} \mathrm{slot}$ & & OS12-R1-SG, SO2-R1 & \\
\hline $3.52 \mathrm{~mm}^{2}$ & $0.7 \times 5-\mathrm{mm}$ slot & & OS11 & \\
\hline $4.94 \mathrm{~mm}^{2}$ & $0.5 \times 10-\mathrm{mm}$ slot & & SL46B & \\
\hline $5.01 \mathrm{~mm}^{2}$ & $1 \times 5-\mathrm{mm}$ slot & & OS10-R1-SG & \\
\hline $7.92 \mathrm{~mm}^{2}$ & $0.5 \times 15-\mathrm{mm}$ slot & & SL47B & \\
\hline $10.82 \mathrm{~mm}^{2}$ & $0.5 \times 20$-mm slot & & SL48-R1A, SL48-R1B & \\
\hline \multicolumn{5}{|c|}{ Large-scale data (black) } \\
\hline $2.14 \mathrm{~mm}^{2}$ & $\begin{array}{c}\text { S2A }(0.5 \times 5-m m \\
\text { slot })\end{array}$ & \multirow{8}{*}{$\begin{array}{l}\text { Water, } \\
380 \text { psi }\end{array}$} & W121, W125 & \multirow{8}{*}{$\begin{array}{l}\text { RFs }(9.11) \text { and GRs }(9.12) \text { for } \\
<10 \mu \mathrm{m},<32 \mu \mathrm{m},<102 \mu \mathrm{m} \text { were } \\
\text { averaged if more than one test is } \\
\text { included in the data set; error } \\
\text { bars generated according to } \\
\text { description in Section } 8.1 .6\end{array}$} \\
\hline $8.72 \mathrm{~mm}^{2}$ & $\begin{array}{l}\text { S7A }(1 \times 10-\mathrm{mm} \\
\text { slot })\end{array}$ & & W91, W96, W161 & \\
\hline $9.77 \mathrm{~mm}^{2}$ & $\begin{array}{l}\text { S5A }(10 \times 1-\mathrm{mm} \\
\text { slot })\end{array}$ & & W101, W110 & \\
\hline $19.00 \mathrm{~mm}^{2}$ & S7B $(2 \times 10-\mathrm{mm}$ slot $)$ & & W164 & \\
\hline $19.35 \mathrm{~mm}^{2}$ & S5C $(1 \times 20$-mm slot $)$ & & $\begin{array}{l}\text { W108, W114, W170, } \\
\text { W172 }\end{array}$ & \\
\hline $30.83 \mathrm{~mm}^{2}$ & S7C $(3 \times 10-m m$ slot $)$ & & W94, W98, W166, W168 & \\
\hline $73.14 \mathrm{~mm}^{2}$ & $\begin{array}{c}\text { S4A }(1 \times 76.2-\mathrm{mm} \\
\text { slot })\end{array}$ & & W119, W175, W177 & \\
\hline $211.82 \mathrm{~mm}^{2}$ & $\begin{array}{c}\text { S3A }(2.74 \times 76.2-\mathrm{mm} \\
\text { slot })\end{array}$ & & W182 & \\
\hline
\end{tabular}

Table B.18. Figure 9.14 Data Sets

\begin{tabular}{|c|c|c|c|c|}
\hline Data Set & Fluid & $\begin{array}{c}\text { Pressure / } \\
\text { Orifice }\end{array}$ & Test in Data Set & Notes \\
\hline Water & Water & \multirow{4}{*}{$\begin{array}{l}380 \mathrm{psi}, \\
\text { S2A }(0.5 \times \\
5 \text {-mm slot })\end{array}$} & W121 & \multirow{4}{*}{$\begin{array}{l}\text { "STR" is an abbreviation } \\
\text { for the boehmite/water } \\
\text { slurry described in } \\
\text { Chapter } 4 \text { (see Table 4.4) }\end{array}$} \\
\hline $8 \mathrm{wt} \%$ STR simulant & STR 8 & & S203 & \\
\hline $20 \mathrm{wt} \%$ STR simulant & STR 20 & & S188 & \\
\hline WTP models & $\begin{array}{l}\text { Water, STR 8, } \\
\text { STR 20, } \\
\text { respectively }\end{array}$ & & $\begin{array}{l}\text { Prediction based on tests } \\
\text { listed above for respective } \\
\text { fluids }\end{array}$ & \\
\hline
\end{tabular}

Table B.19. Figure 9.16 Data Sets

\begin{tabular}{|c|c|c|c|c|}
\hline Data Set & Fluid & Viscosity & Tests in Data Set & Notes \\
\hline \multirow{2}{*}{$\begin{array}{c}\mathrm{G},<10 \mu \mathrm{m}, 380 \mathrm{psi}, \\
1 \times 10-\mathrm{mm} \text { slot }\end{array}$} & Water & $1.0 \mathrm{cP}$ & W91, W96, W161 & \multirow{4}{*}{$\begin{array}{l}\text { "STS" is an abbreviation for the sodium } \\
\text { thiosulfate simulant, described in Chapter } 4 \\
\text { (see Table 4.3) }\end{array}$} \\
\hline & STS & $2.6 \mathrm{cP}$ & S148, S149, S152 & \\
\hline \multirow{2}{*}{$\begin{array}{c}\mathrm{G},<32 \mu \mathrm{m}, 380 \mathrm{psi}, \\
1 \times 10-\mathrm{mm} \text { slot }\end{array}$} & Water & $1.0 \mathrm{cP}$ & W91, W9 & \\
\hline & STS & $2.6 \mathrm{cP}$ & S148, S & \\
\hline \multirow{2}{*}{$\begin{array}{c}\mathrm{G},<102 \mu \mathrm{m}, 380 \mathrm{psi}, \\
1 \times 10-\mathrm{mm} \text { slot }\end{array}$} & Water & $1.0 \mathrm{cP}$ & W91, W96, W161 & \multirow{2}{*}{$\begin{array}{l}\text { GRs for }<10 \mu \mathrm{m},<32 \mu \mathrm{m},<102 \mu \mathrm{m} \text { were } \\
\text { averaged if more than one test is included in } \\
\text { the data set; error bars generated according to } \\
\text { description in Section 8.1.6 }\end{array}$} \\
\hline & STS & $2.6 \mathrm{cP}$ & S148, S149, S152 & \\
\hline
\end{tabular}


Table B.20. Figure 9.17 Data Sets

\begin{tabular}{|c|c|c|c|c|}
\hline $\begin{array}{l}\text { Composite } \\
\text { Data Set }\end{array}$ & $\begin{array}{c}\text { Orifice / } \\
\text { Target } \\
\text { Dimensions }\end{array}$ & $\begin{array}{c}\text { Fluid / } \\
\text { Pressure }\end{array}$ & $\begin{array}{l}\text { Tests Included in } \\
\text { Composite Data Set }\end{array}$ & Notes \\
\hline \multicolumn{5}{|c|}{ Small-scale data $(\mathrm{red})$} \\
\hline $1 \mathrm{in.}$ & \multirow{5}{*}{ 1-mm hole } & \multirow{5}{*}{$\begin{array}{l}\text { Water, } \\
380 \text { psi }\end{array}$} & $\begin{array}{c}\text { O75-EX1A, O75-EX1B, } \\
\text { O75-EX1C }\end{array}$ & \multirow{2}{*}{$\begin{array}{l}\text { Small-scale data is shown as individual } \\
\text { tests. The error bars are estimated as } \\
\text { described in Section } 6.3 .1 \text { of Mahoney et al. } \\
\text { (2012b) }\end{array}$} \\
\hline 3 in. & & & $\begin{array}{c}\text { O75-EX3A, O75-EX3B, } \\
\text { O75-EX3C }\end{array}$ & \\
\hline 6 in. & & & $\begin{array}{c}\text { O75-EX6A, O75-EX6B, } \\
\text { O75-EX6C }\end{array}$ & \multirow{3}{*}{$\begin{array}{l}\text { WTP model values are calculated for each } \\
\text { test }\end{array}$} \\
\hline $18 \mathrm{in.}$ & & & $\begin{array}{c}\text { O75-EX18A, O75- } \\
\text { EX18B, O75-EX18C }\end{array}$ & \\
\hline 42 in. & & & OS7, RT18, RT19 & \\
\hline \multicolumn{5}{|c|}{ Large-scale data (black) } \\
\hline 43.25 in. & & & W128, W133 & \multirow{3}{*}{$\begin{array}{l}\text { RFs for }<10 \mu \mathrm{m},<32 \mu \mathrm{m},<102 \mu \mathrm{m} \text { were } \\
\text { averaged if more than one test is included in } \\
\text { the data set; error bars generated according } \\
\text { to description in Section 8.1.6 } \\
\text { WTP model values are also based on } \\
\text { averages if there is more than one data set }\end{array}$} \\
\hline 120.25 in. & & & W138, W145 & \\
\hline 227.25 in. & $\begin{array}{l}\text { S1A (1-mm } \\
\text { hole) }\end{array}$ & $\begin{array}{l}\text { Water, } \\
380 \text { psi }\end{array}$ & W74, W83 & \\
\hline
\end{tabular}

Table B.21. Figure 9.18 Data Sets

\begin{tabular}{|c|c|c|c|c|c|}
\hline $\begin{array}{c}\text { Data for } \\
\text { Orifice } \\
\text { Size }\end{array}$ & $\begin{array}{l}\text { Spray } \\
\text { Length }\end{array}$ & Fluid & Pressure & $\begin{array}{c}\text { Test in Data } \\
\text { Set }\end{array}$ & Notes \\
\hline \multirow[t]{3}{*}{ 1-mm hole } & 43.25 in. & \multirow{3}{*}{ Water } & \multirow{3}{*}{200 psi } & W127 & \multirow{24}{*}{$\begin{array}{l}\text { RFs for }<10 \mu \mathrm{m},<32 \mu \mathrm{m},<102 \mu \mathrm{m} \\
\text { were all taken from the first test } \\
\text { performed at each condition }- \text { all of } \\
\text { the tests were repeated at least once } \\
\text { (refer to Appendix A) }\end{array}$} \\
\hline & 120.25 in. & & & W137 & \\
\hline & 227.25 in. & & & W73 & \\
\hline \multirow[t]{3}{*}{ 2-mm hole } & 43.25 in. & \multirow{3}{*}{ Water } & \multirow{3}{*}{200 psi } & W130 & \\
\hline & 120.25 in. & & & W140 & \\
\hline & 227.25 in. & & & W80 & \\
\hline \multirow{2}{*}{$\begin{array}{c}1 \times 10-\mathrm{mm} \\
\text { slot }\end{array}$} & 43.25 in. & \multirow{2}{*}{ Water } & \multirow{2}{*}{200 psi } & W226 & \\
\hline & 227.25 in. & & & W160 & \\
\hline \multirow{2}{*}{$\begin{array}{c}1 \times 20-\mathrm{mm} \\
\text { slot }\end{array}$} & 43.25 in. & \multirow{2}{*}{ Water } & \multirow{2}{*}{200 psi } & W222 & \\
\hline & 227.25 in. & & & W171 & \\
\hline \multirow{2}{*}{$\begin{array}{c}1 \times \\
76.2-\mathrm{mm} \\
\text { slot }\end{array}$} & 43.25 in. & \multirow[b]{2}{*}{ Water } & \multirow[b]{2}{*}{200 psi } & W231 & \\
\hline & 227.25 in. & & & W174 & \\
\hline \multirow[t]{3}{*}{ 1-mm hole } & 43.25 in. & \multirow{3}{*}{ Water } & \multirow{3}{*}{380 psi } & W128 & \\
\hline & 120.25 in. & & & W138 & \\
\hline & 227.25 in. & & & W74 & \\
\hline \multirow[t]{3}{*}{ 2-mm hole } & 43.25 in. & \multirow{3}{*}{ Water } & \multirow{3}{*}{380 psi } & W131 & \\
\hline & 120.25 in. & & & W141 & \\
\hline & 227.25 in. & & & W81 & \\
\hline \multirow{2}{*}{$\begin{array}{c}1 \times 10-\mathrm{mm} \\
\text { slot }\end{array}$} & 43.25 in. & \multirow{2}{*}{ Water } & \multirow{2}{*}{380 psi } & W229 & \\
\hline & 227.25 in. & & & W161 & \\
\hline \multirow{2}{*}{$\begin{array}{c}1 \times 20-\mathrm{mm} \\
\text { slot }\end{array}$} & 43.25 in. & \multirow{2}{*}{ Water } & \multirow{2}{*}{380 psi } & W223 & \\
\hline & 227.25 in. & & & W172 & \\
\hline \multirow{2}{*}{$\begin{array}{c}1 \times \\
76.2-\mathrm{mm} \\
\text { slot }\end{array}$} & $43.25 \mathrm{in}$. & \multirow[b]{2}{*}{ Water } & \multirow[b]{2}{*}{380 psi } & W234 & \\
\hline & $227.25 \mathrm{in}$. & & & W175 & \\
\hline
\end{tabular}


Table B.22. Figure 9.19 Data Sets

\begin{tabular}{|c|c|c|c|c|}
\hline $\begin{array}{l}\text { Composite } \\
\text { Data Set }\end{array}$ & $\begin{array}{l}\text { Orifice / Target } \\
\text { Dimensions }\end{array}$ & $\begin{array}{c}\text { Fluid / } \\
\text { Pressure }\end{array}$ & $\begin{array}{c}\text { Tests Included in Composite } \\
\text { Data Set }\end{array}$ & Notes \\
\hline $0.78 \mathrm{~mm}^{2}$ & S1A (1-mm hole) & \multirow{13}{*}{$\begin{array}{l}\text { Water, } \\
380 \text { psi }\end{array}$} & W74, W83 & \multirow{13}{*}{$\begin{array}{l}\text { RFs for }<10 \mu \mathrm{m},<32 \mu \mathrm{m} \text {, } \\
<102 \mu \mathrm{m} \text { were averaged if } \\
\text { more than one test is } \\
\text { included in the data set; } \\
\text { error bars generated } \\
\text { according to description in } \\
\text { Section 8.1.6 } \\
\text { The extrapolation lines } \\
\text { (dashed lines) were } \\
\text { generated by performing a } \\
\text { best-fit of the data for each } \\
\text { of the three droplet sizes }\end{array}$} \\
\hline $2.14 \mathrm{~mm}^{2}$ & $\begin{array}{l}\text { S2A }(0.5 \times 5-\mathrm{mm} \\
\text { slot })\end{array}$ & & W121, W125 & \\
\hline $3.50 \mathrm{~mm}^{2}$ & S1D (2-mm hole) & & $\begin{array}{l}\text { W81, W88, W207, W208, } \\
\text { W238 }\end{array}$ & \\
\hline $3.69 \mathrm{~mm}^{2}$ & $\begin{array}{l}\text { S5B (array of five } \\
\text { 1-mm holes) }\end{array}$ & & W104, W105, W112 & \\
\hline $5.89 \mathrm{~mm}^{2}$ & S1C (2.74-mm hole) & & W78 & \\
\hline $8.72 \mathrm{~mm}^{2}$ & $\begin{array}{l}\text { S7A }(1 \times 10-\mathrm{mm} \\
\text { slot })\end{array}$ & & W91, W96, W161 & \\
\hline $9.77 \mathrm{~mm}^{2}$ & $\begin{array}{l}\text { S5A }(10 \times 1-\mathrm{mm} \\
\text { slot })\end{array}$ & & W101, W110 & \\
\hline $17.13 \mathrm{~mm}^{2}$ & S1B (4.46-mm hole) & & W76, W85 & \\
\hline $19.00 \mathrm{~mm}^{2}$ & $\begin{array}{c}\text { S7B }(2 \times 10-\mathrm{mm} \\
\text { slot })\end{array}$ & & W164 & \\
\hline $19.35 \mathrm{~mm}^{2}$ & $\begin{array}{l}\text { S5C }(1 \times 20-\mathrm{mm} \\
\text { slot })\end{array}$ & & W108, W114, W170, W172 & \\
\hline $30.83 \mathrm{~mm}^{2}$ & $\begin{array}{c}\text { S7C }(3 \times 10-\mathrm{mm} \\
\text { slot })\end{array}$ & & W94, W98, W166, W168 & \\
\hline $73.14 \mathrm{~mm}^{2}$ & $\begin{array}{l}\text { S4A }(1 \times 76.2-\mathrm{mm} \\
\text { slot })\end{array}$ & & W119, W175, W177 & \\
\hline $211.82 \mathrm{~mm}^{2}$ & $\begin{array}{c}\text { S3A (2.74× } \\
76.2-\mathrm{mm} \text { slot })\end{array}$ & & W182 & \\
\hline
\end{tabular}

Table B.23. Figure 9.20 Data Sets

\begin{tabular}{|c|c|c|c|c|}
\hline Data Set & Fluid & Pressure & Test in Data Set & Notes \\
\hline S7A $(1 \times 10-m m$ slot $)$ & \multirow{5}{*}{ Water } & \multirow{5}{*}{$380 \mathrm{psi}$} & W161 & \multirow{5}{*}{$\begin{array}{l}\text { *The extrapolation was } \\
\text { performed by using the dashed } \\
\text { lines shown in Figure } 9.19 \text { to } \\
\text { adjust the W175 data for the } \\
\text { change in area from } 73.14 \mathrm{~mm}^{2} \\
\text { to } 589 \mathrm{~mm}^{2}\end{array}$} \\
\hline S5C $(1 \times 20-m m$ slot $)$ & & & W172 & \\
\hline S4A $(1 \times 76.2-\mathrm{mm}$ slot $)$ & & & W175 & \\
\hline $\begin{array}{l}\text { Postulated slot of } 589 \mathrm{~mm}^{2} \\
\quad(4.64 \times 127-\mathrm{mm} \text { slot })\end{array}$ & & & $\begin{array}{l}\text { Extrapolation based on } \\
\text { Figure } 9.19 \text { curves* }\end{array}$ & \\
\hline WTP models & & & $\begin{array}{l}\text { Predictions based on } \\
\text { conditions for test } \\
\text { numbers above }\end{array}$ & \\
\hline
\end{tabular}

Table B.24. Figure 9.22 Data Sets

\begin{tabular}{|c|c|c|c|c|}
\hline Data Set & Fluid & Pressure/Orifice & Test in Data Set & Notes \\
\hline 227.25 in. from splash wall & \multirow{4}{*}{ Water } & \multirow{4}{*}{$\begin{array}{l}380 \mathrm{psi}, \mathrm{S} 1 \mathrm{D} \\
\text { (2-mm hole) }\end{array}$} & W81 & \multirow{4}{*}{$\begin{array}{l}\text { The in-spray data is the } \\
\text { same as is shown in } \\
\text { Figure } 8.32\end{array}$} \\
\hline $\begin{array}{l}\text { In-spray, } 23.5 \text { in. from } \\
\text { orifice }\end{array}$ & & & W219, M1854 & \\
\hline $\begin{array}{l}\text { In-spray, } 59 \text { in. from } \\
\text { orifice }\end{array}$ & & & W219, M1852 & \\
\hline WTP model & & & Prediction based on W81 & \\
\hline
\end{tabular}


Table B.25. Figure 9.23 Data Sets

\begin{tabular}{|c|c|c|c|c|}
\hline Data Set & Fluid & Pressure/Orifice & Test in Data Set & Notes \\
\hline 227.25 in. from splash wall & \multirow{4}{*}{ Water } & \multirow{4}{*}{$\begin{array}{l}380 \mathrm{psi}, \mathrm{S} 1 \mathrm{~A} \\
\text { (1-mm hole) }\end{array}$} & W74 & \multirow{4}{*}{$\begin{array}{l}\text { The in-spray data is the } \\
\text { same as is shown in } \\
\text { Figure } 8.31\end{array}$} \\
\hline $\begin{array}{l}\text { In-spray, } 23.5 \text { in. from } \\
\text { orifice }\end{array}$ & & & $\begin{array}{l}\text { W215, measured by } \\
\text { M1854 }\end{array}$ & \\
\hline $\begin{array}{l}\text { In-spray, } 59 \text { in. from } \\
\text { orifice }\end{array}$ & & & $\begin{array}{c}\text { W215, measured by } \\
\text { M1852 }\end{array}$ & \\
\hline WTP model & & & Prediction based on W74 & \\
\hline
\end{tabular}



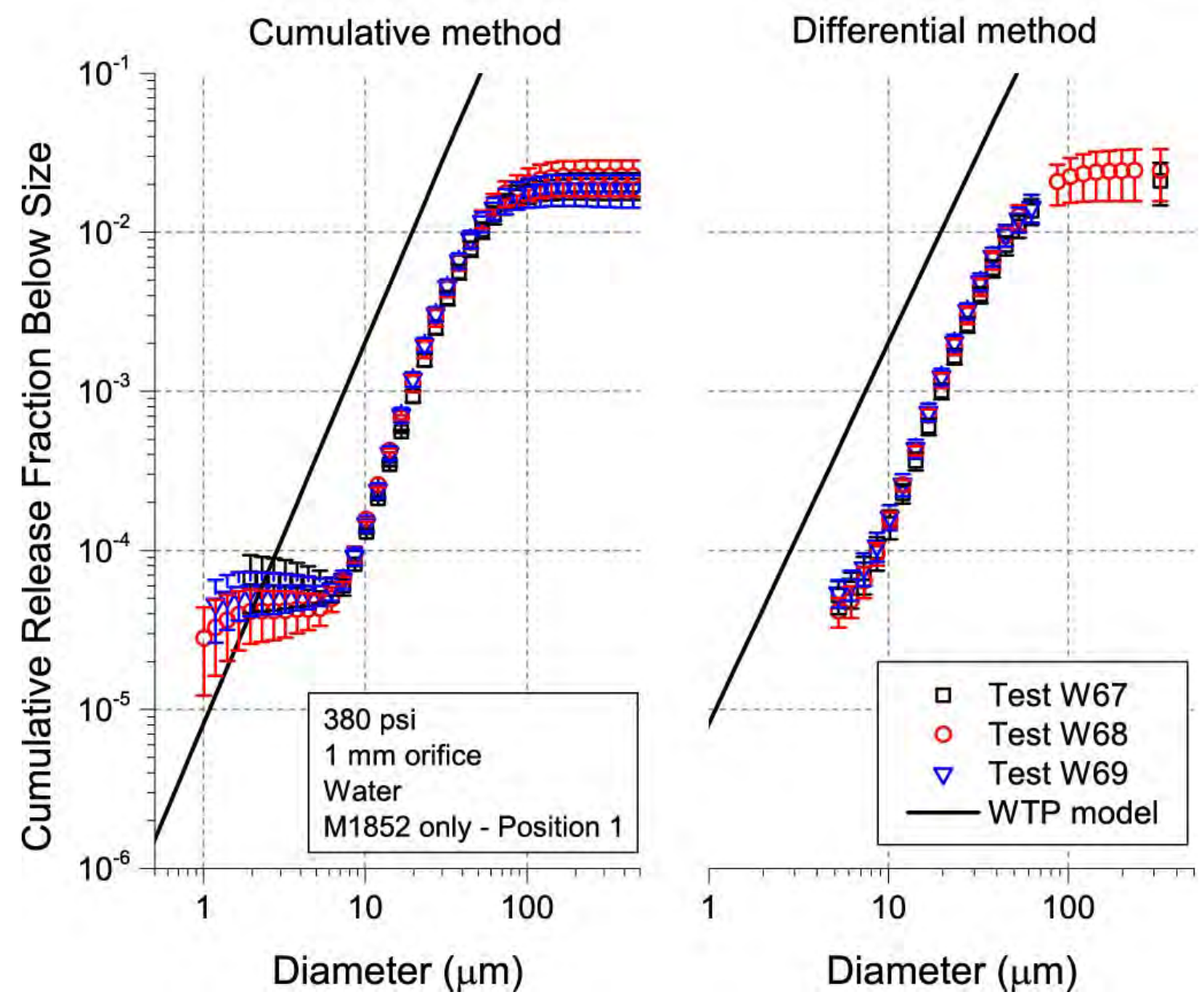

Figure B.1. Release Fractions Determined From Triplicate Experiments Using a Single Malvern Instrument (M1852) in Position 1. The tests were conducted consecutively with water at 380 psig using a 1-mm orifice. 


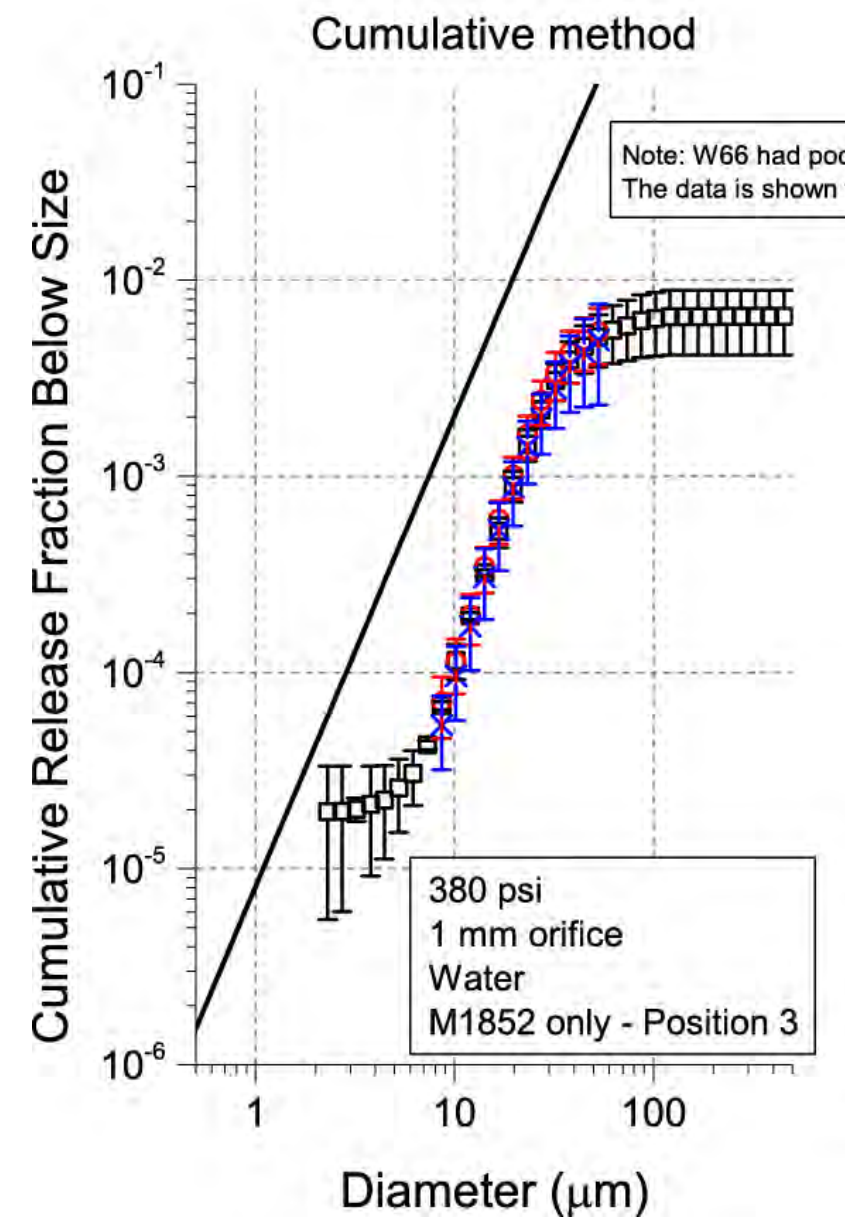

\section{Differential method}

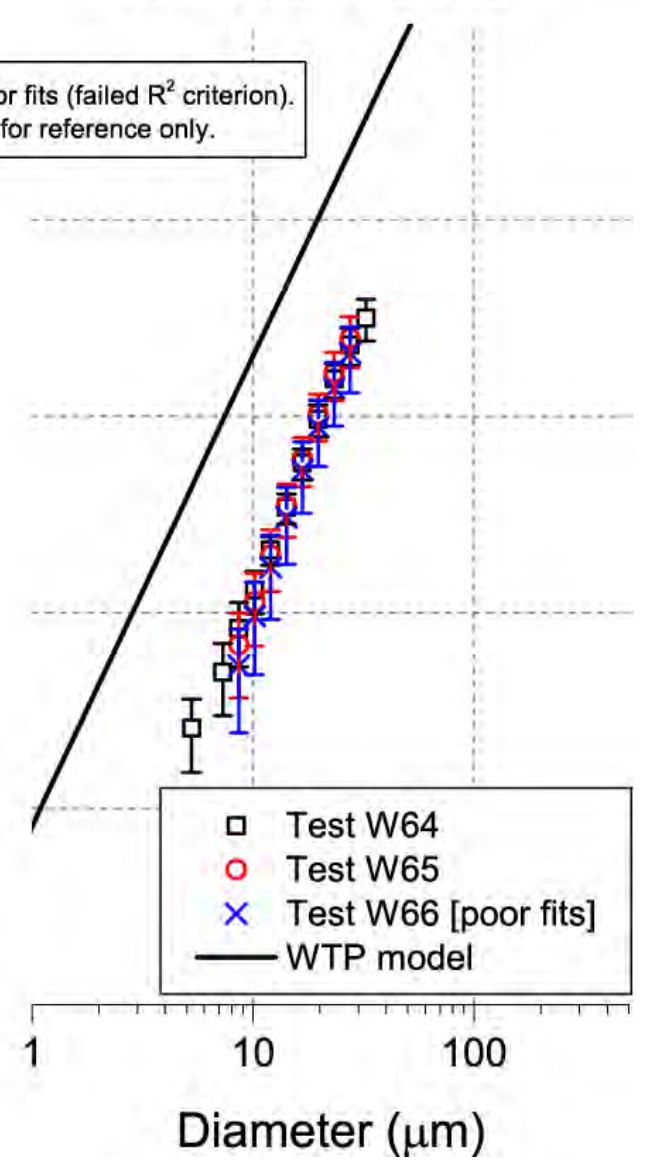

Figure B.2. Release Fractions Determined From Triplicate Experiments Using a Single Malvern Instrument (M1852) in Position 3. The tests were conducted consecutively with water at 380 psig using a 1-mm orifice. 

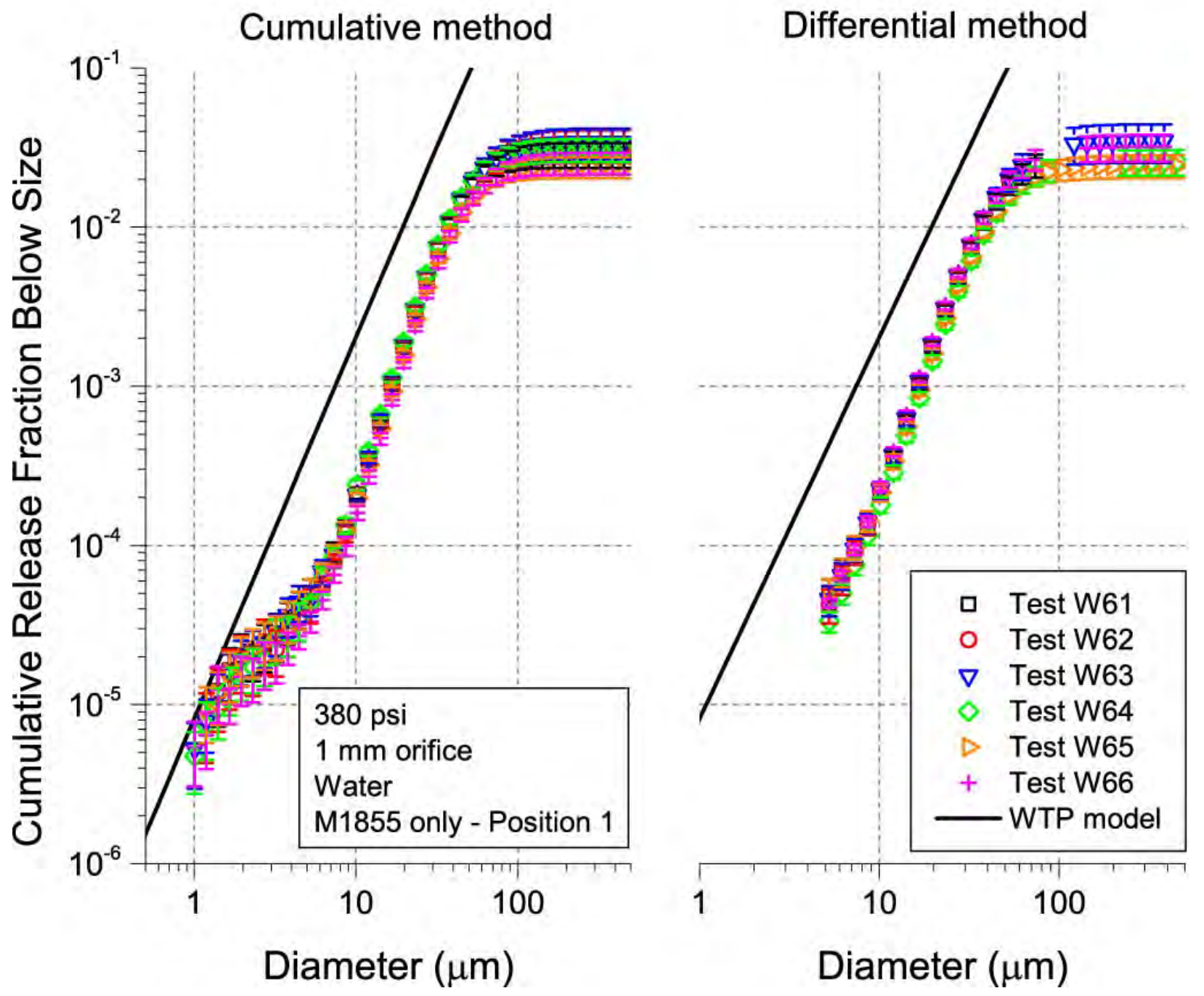

Figure B.3. Release Fractions Determined from Six Experiments Using a Single Malvern Instrument (M1855) in Position 1. The tests were conducted consecutively with water at $380 \mathrm{psig}$ using a 1-mm orifice. 

Test W61 [Malvern 1852]: 380 psi, Orifice S1A - round, 1 mm

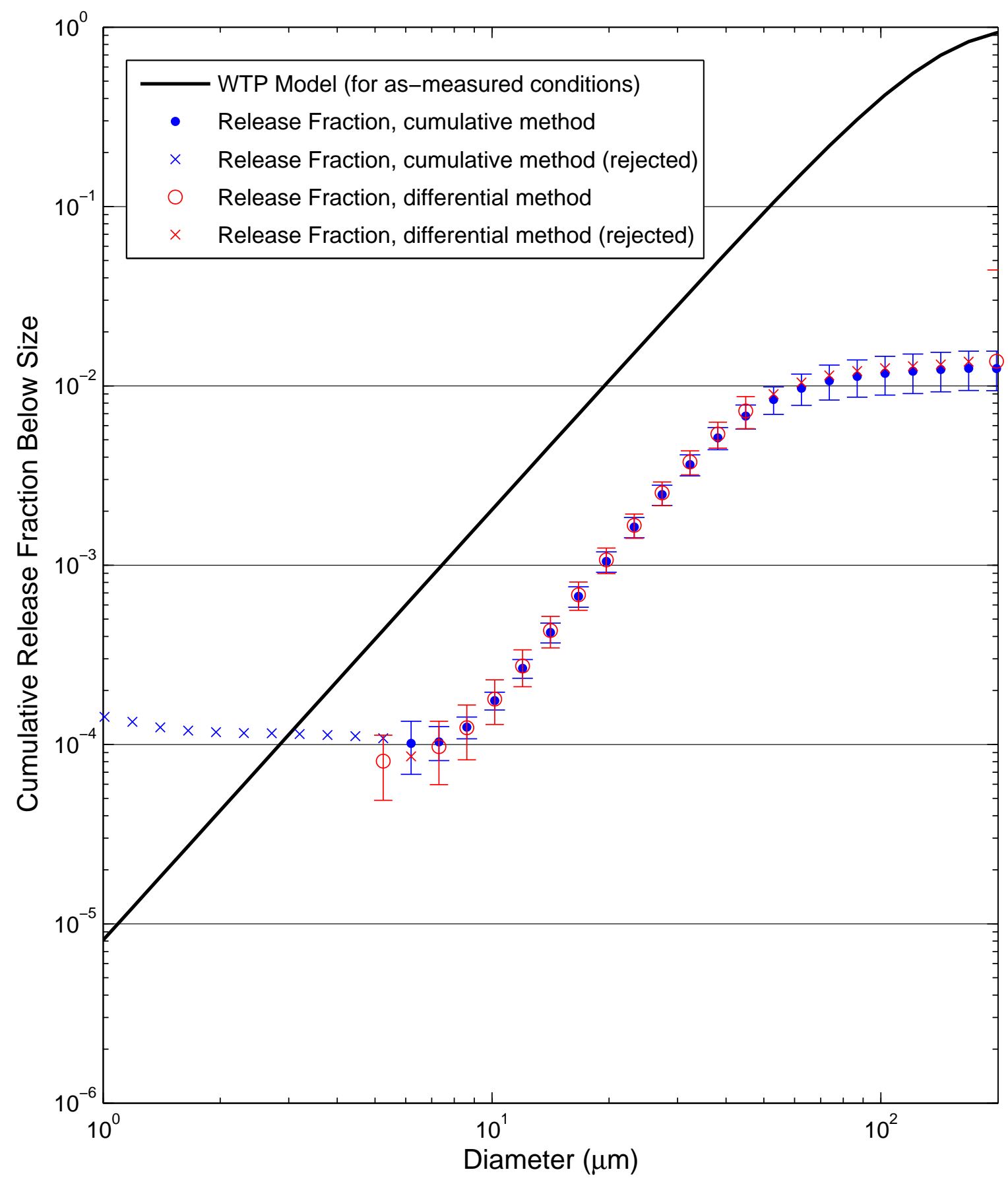


Test W61 [Malvern 1855]: 380 psi, Orifice S1A - round, 1 mm

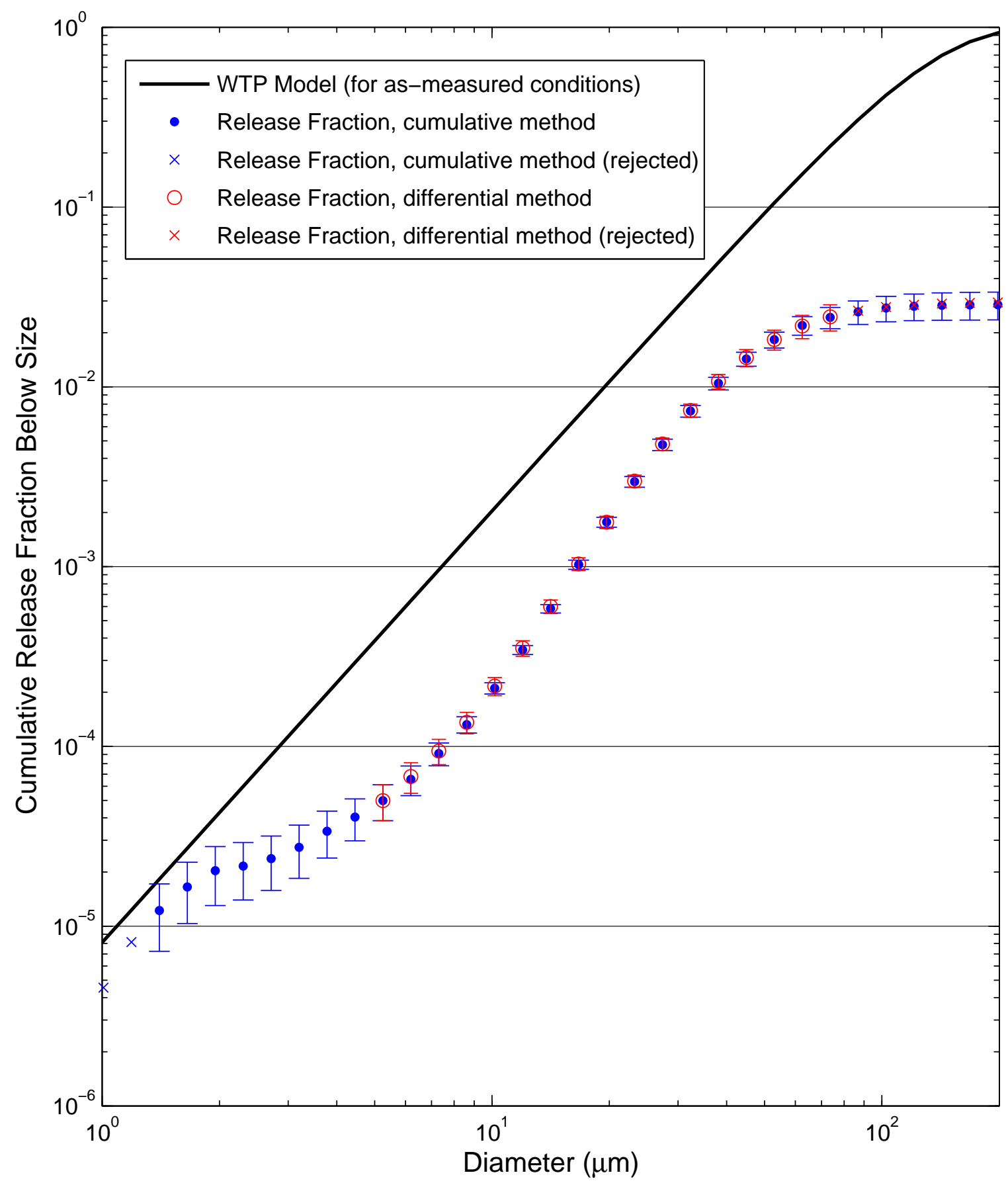


Test W62 [Malvern 1852]: 380 psi, Orifice S1A - round, 1 mm

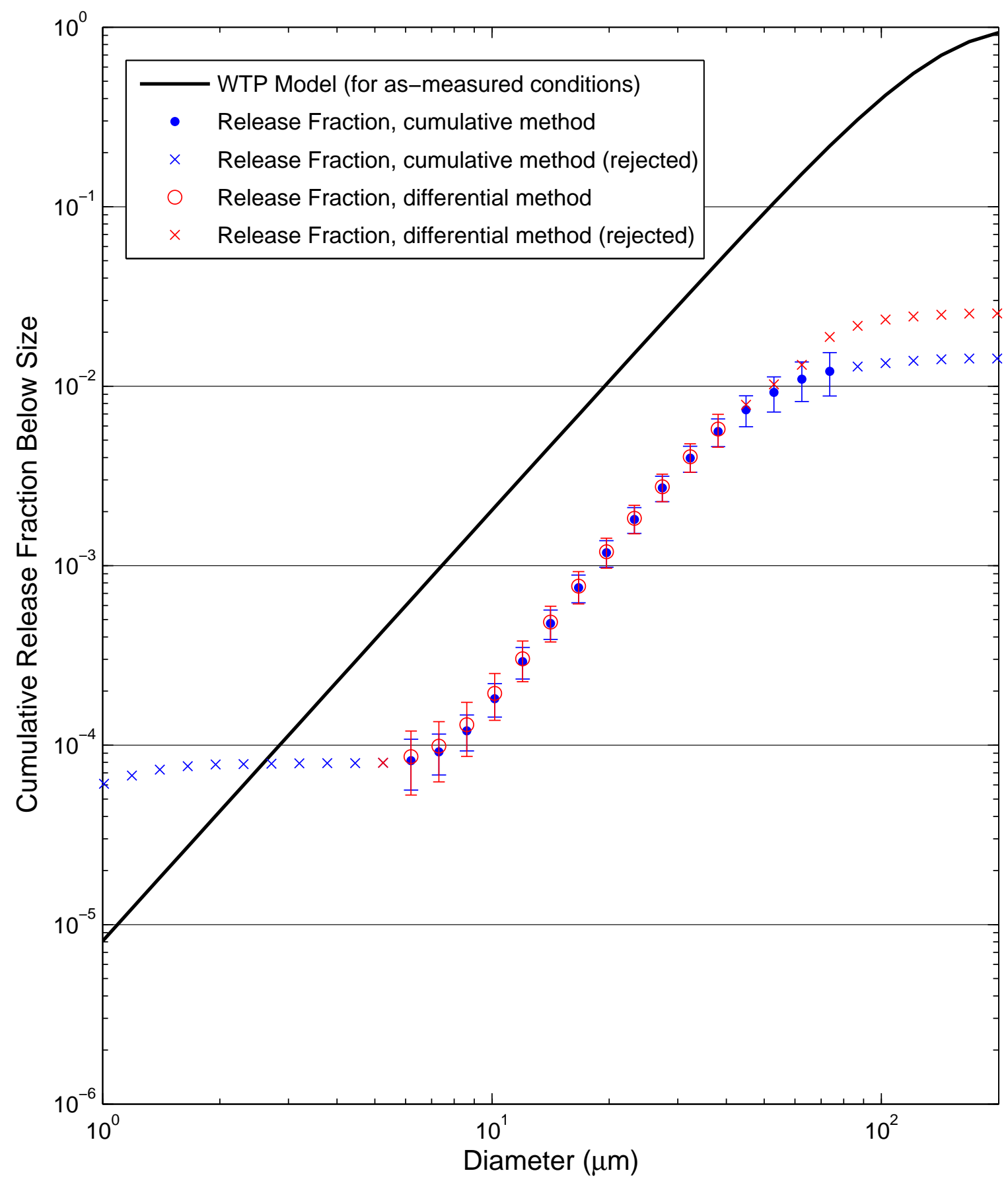


Test W62 [Malvern 1855]: 380 psi, Orifice S1A - round, 1 mm

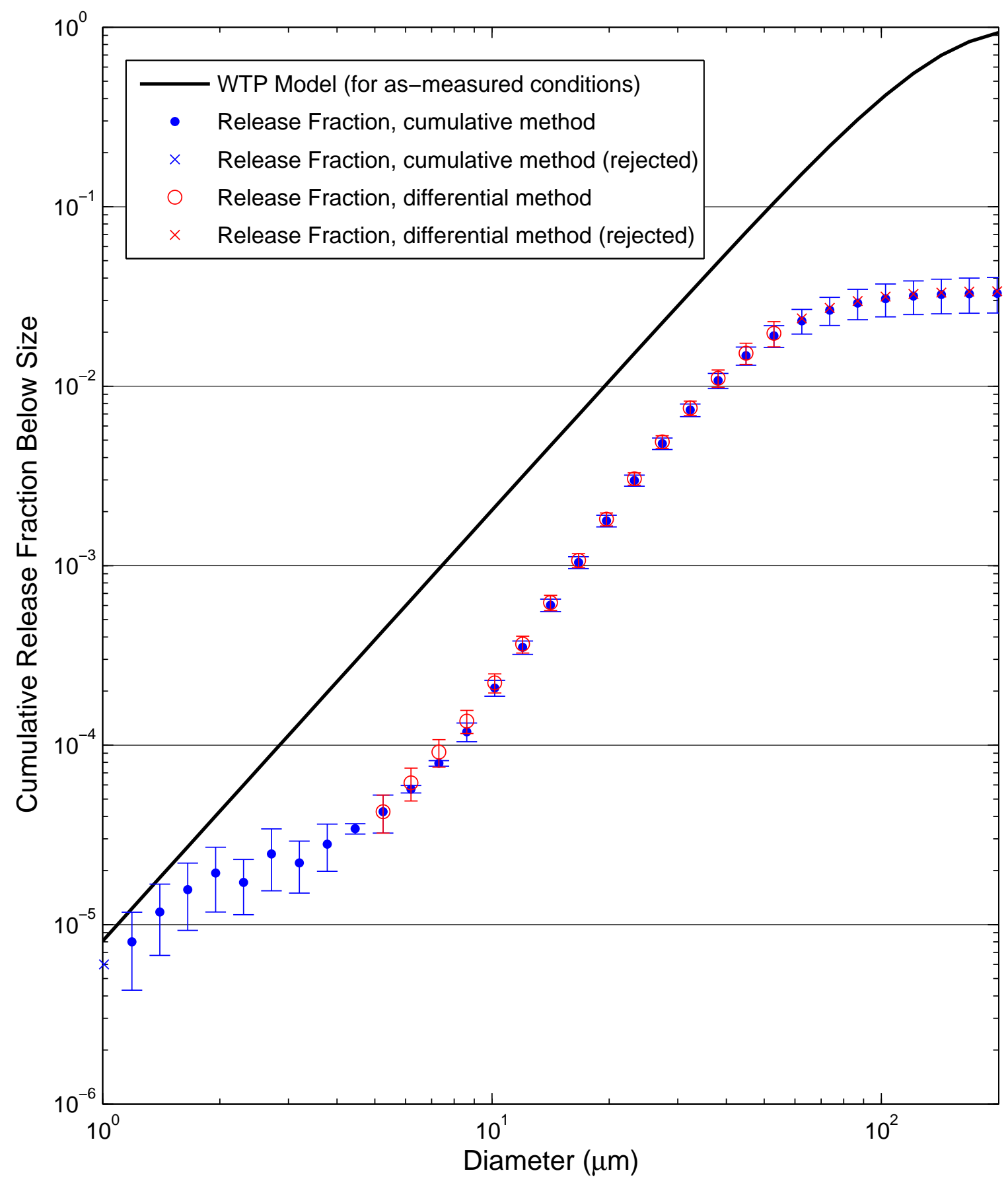


Test W63 [Malvern 1852]: 380 psi, Orifice S1A - round, 1 mm

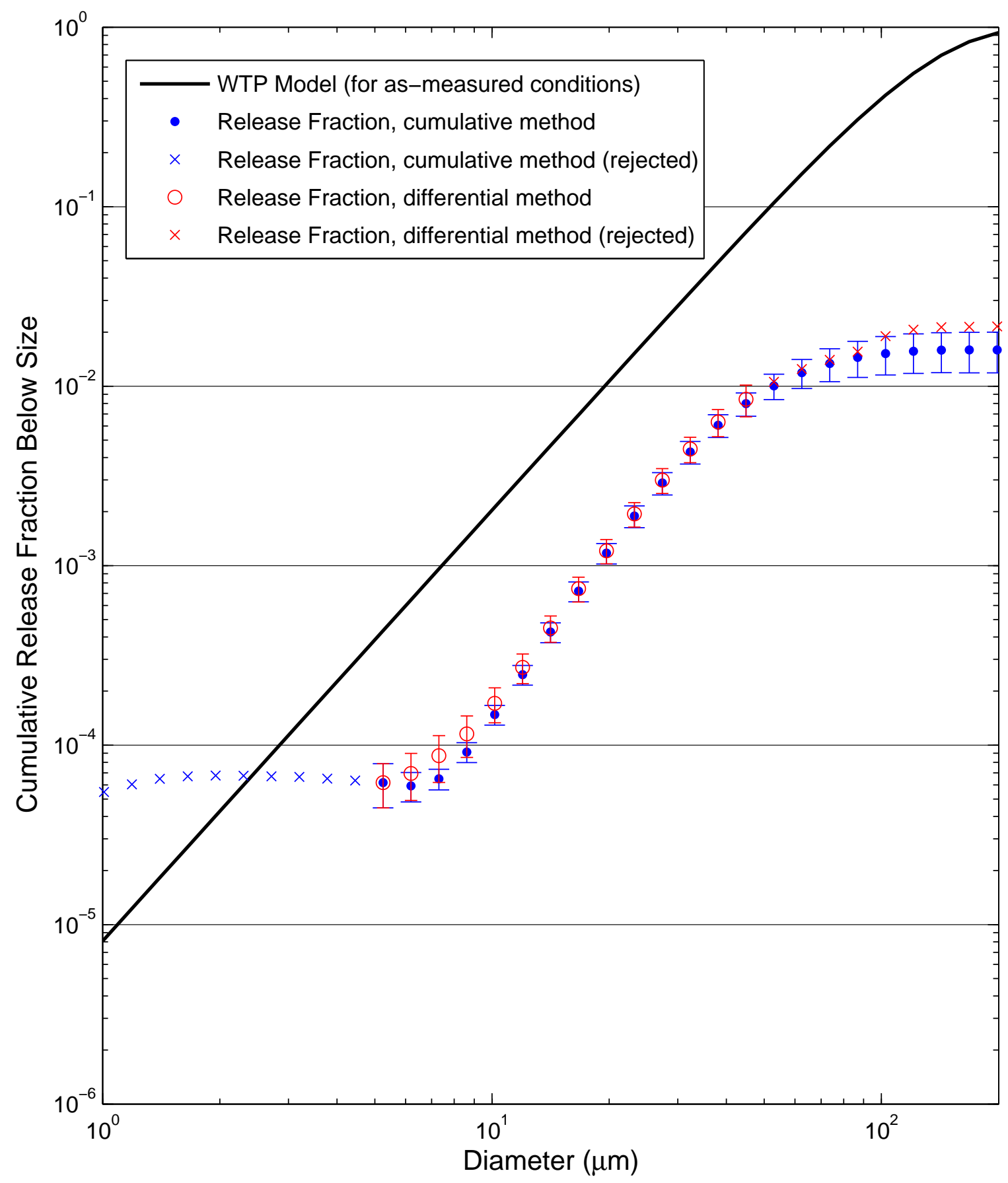


Test W63 [Malvern 1855]: 380 psi, Orifice S1A - round, 1 mm

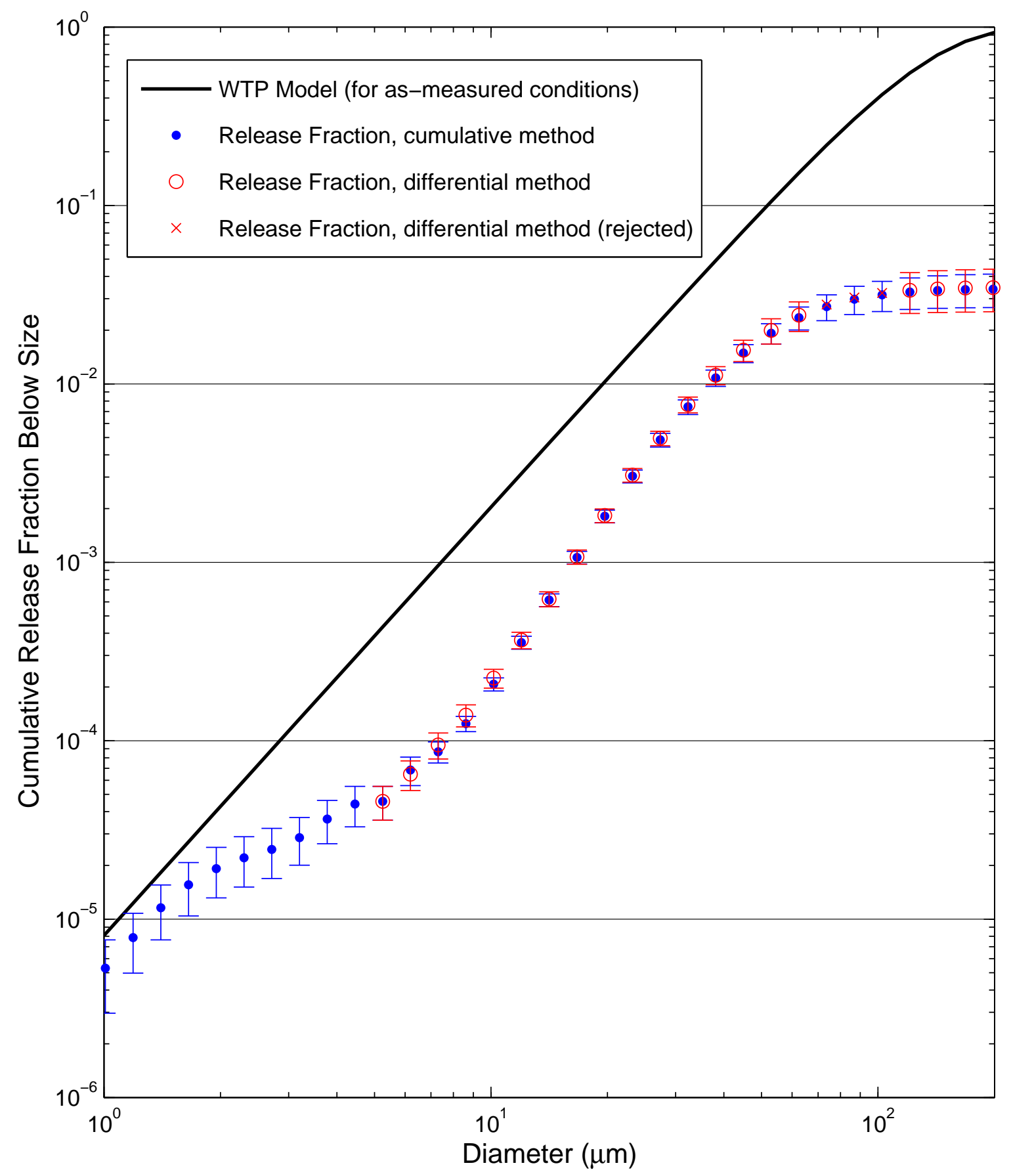


Test W64 [Malvern 1852]: 380 psi, Orifice S1A - round, 1 mm

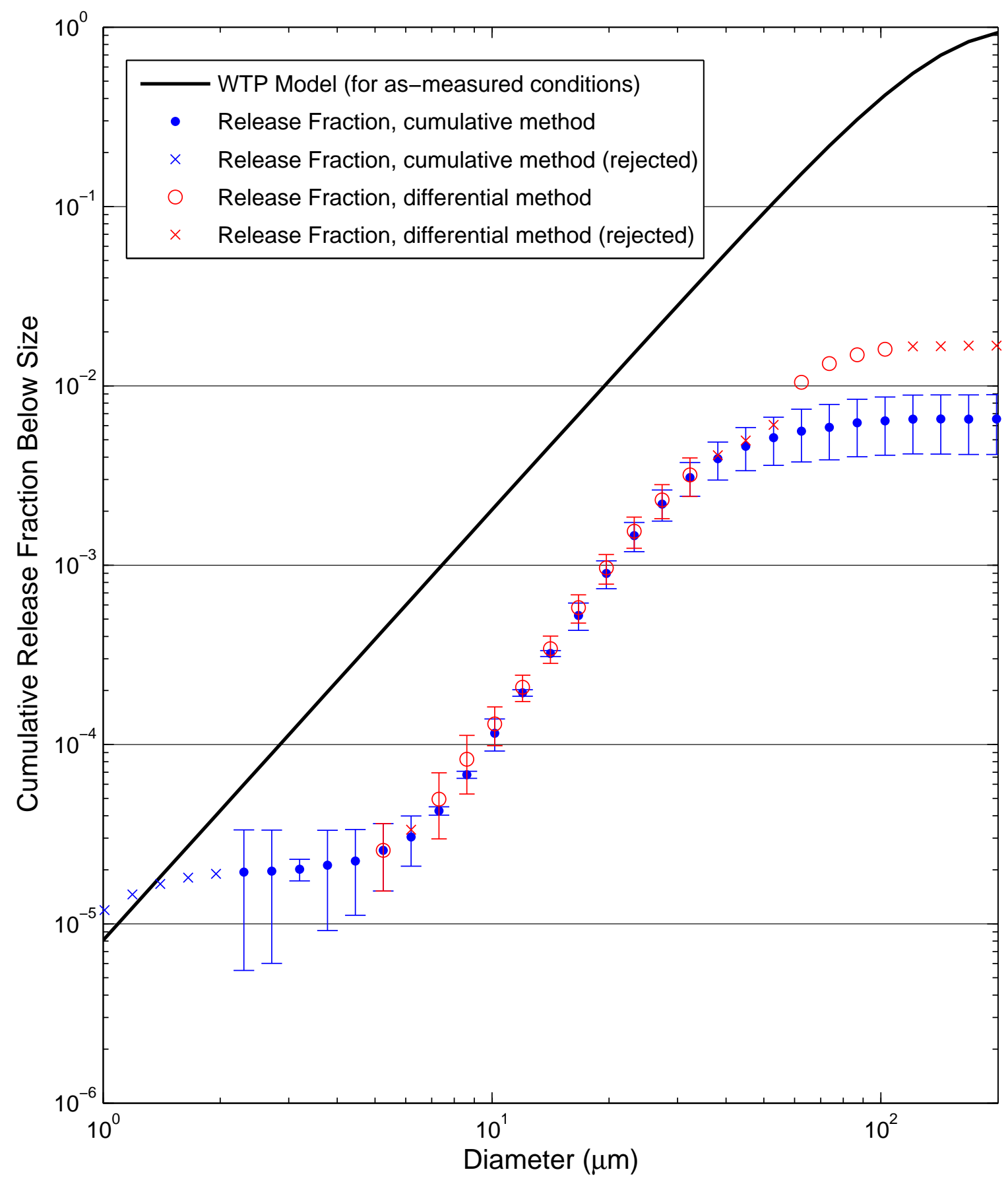


Test W64 [Malvern 1855]: 380 psi, Orifice S1A - round, 1 mm

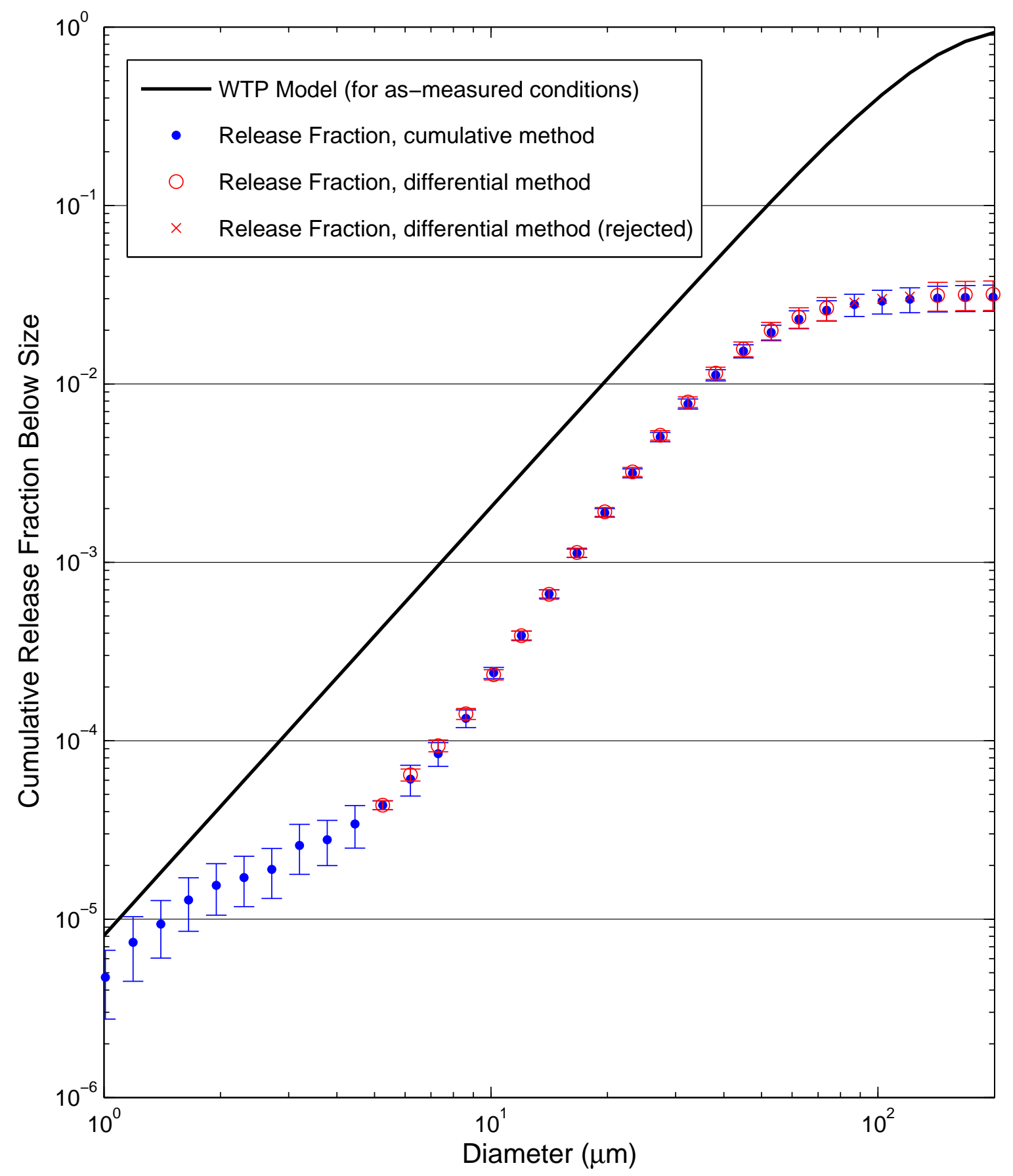


Test W65 [Malvern 1852]: 380 psi, Orifice S1A - round, 1 mm

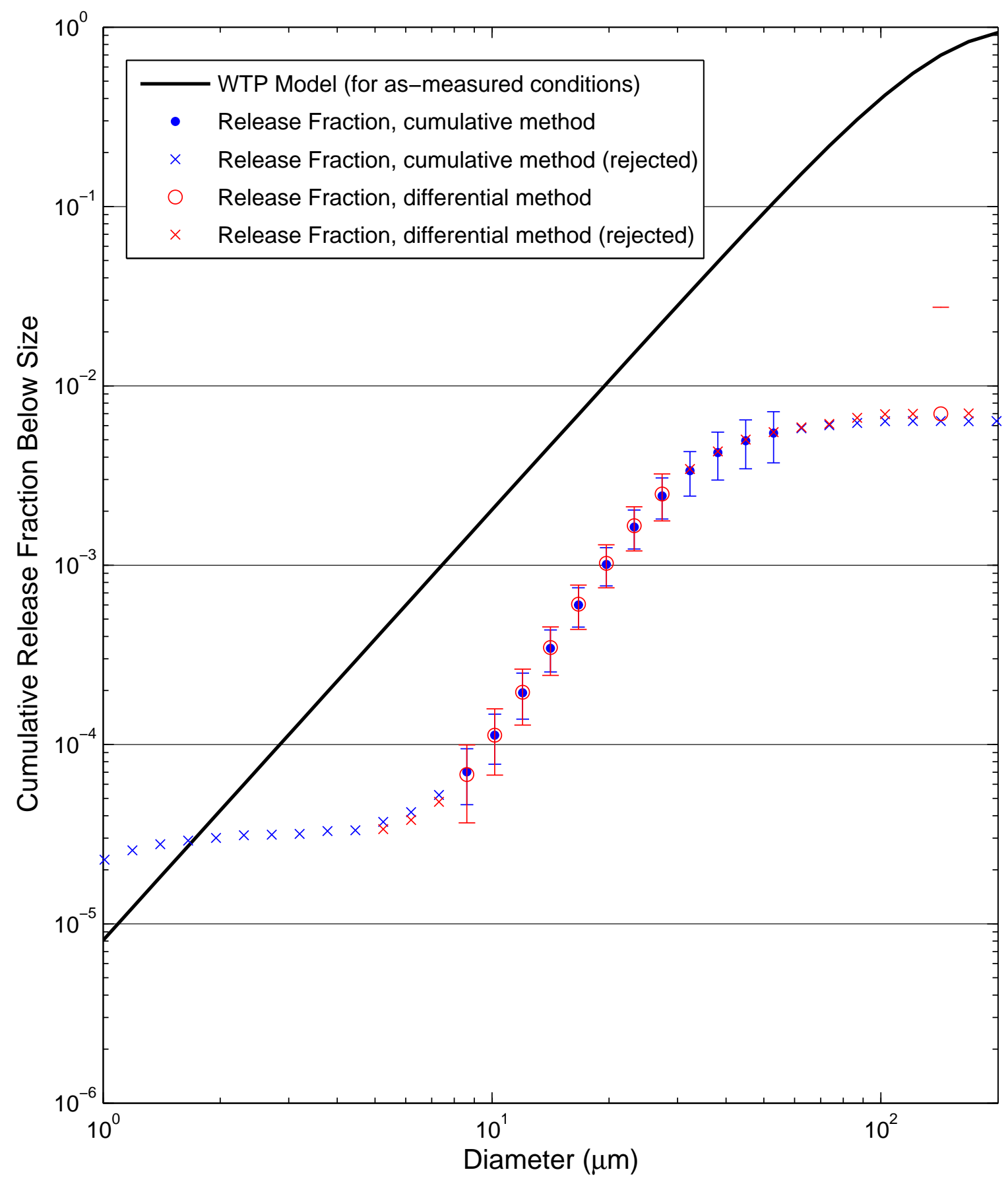


Test W65 [Malvern 1855]: 380 psi, Orifice S1A - round, 1 mm

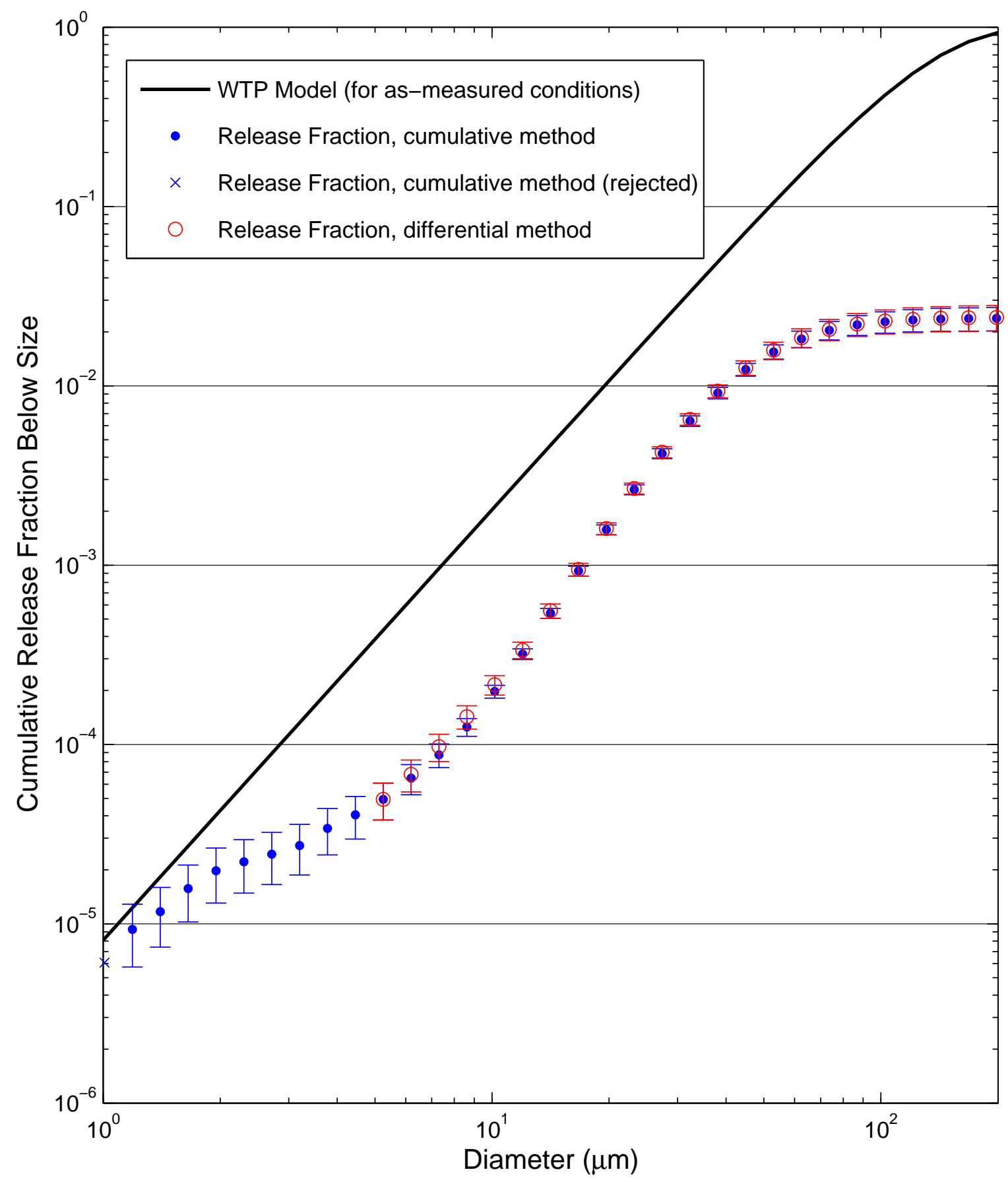


Test W66 [Malvern 1852]: 380 psi, Orifice S1A - round, 1 mm

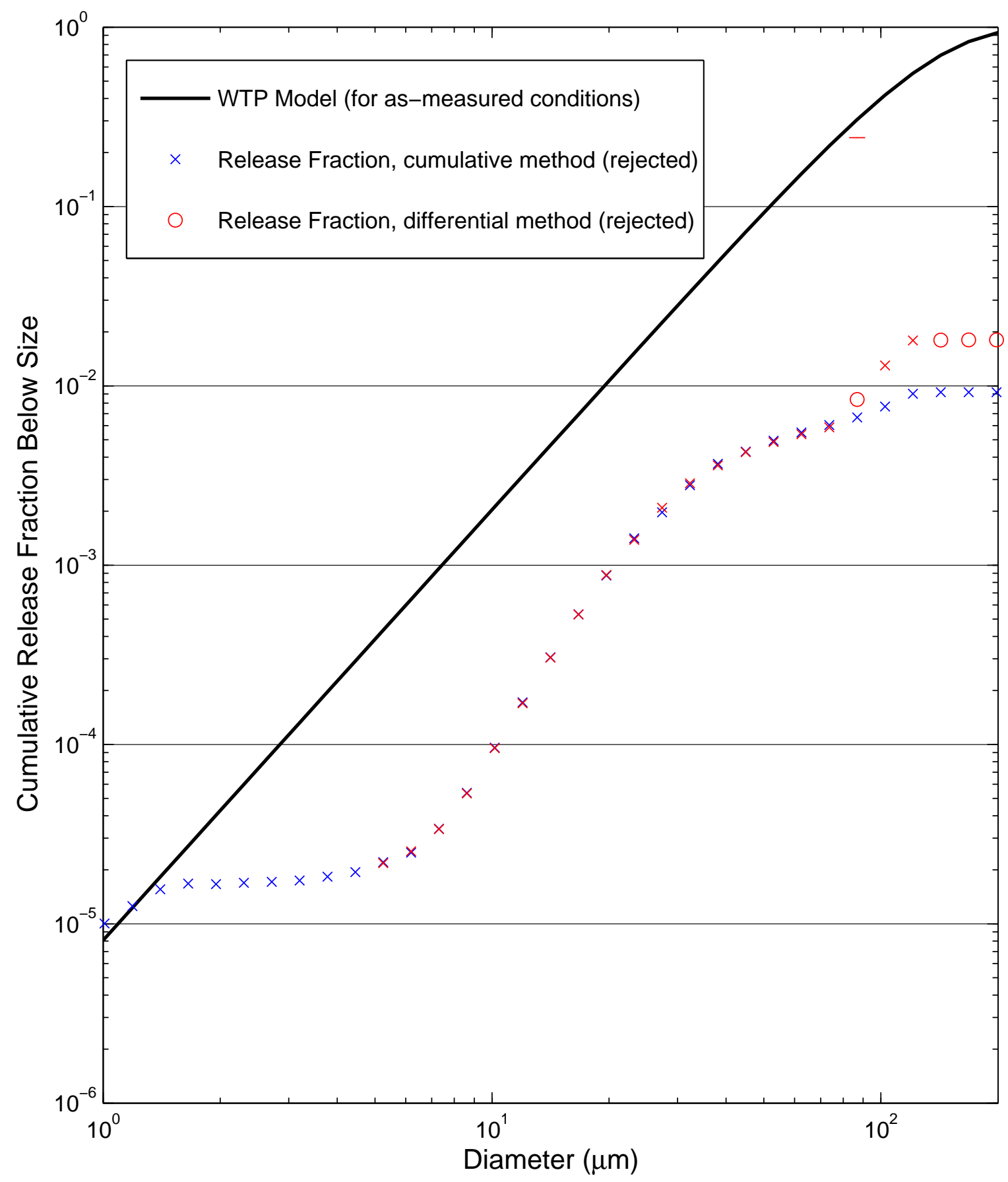


Test W66 [Malvern 1855]: 380 psi, Orifice S1A - round, 1 mm

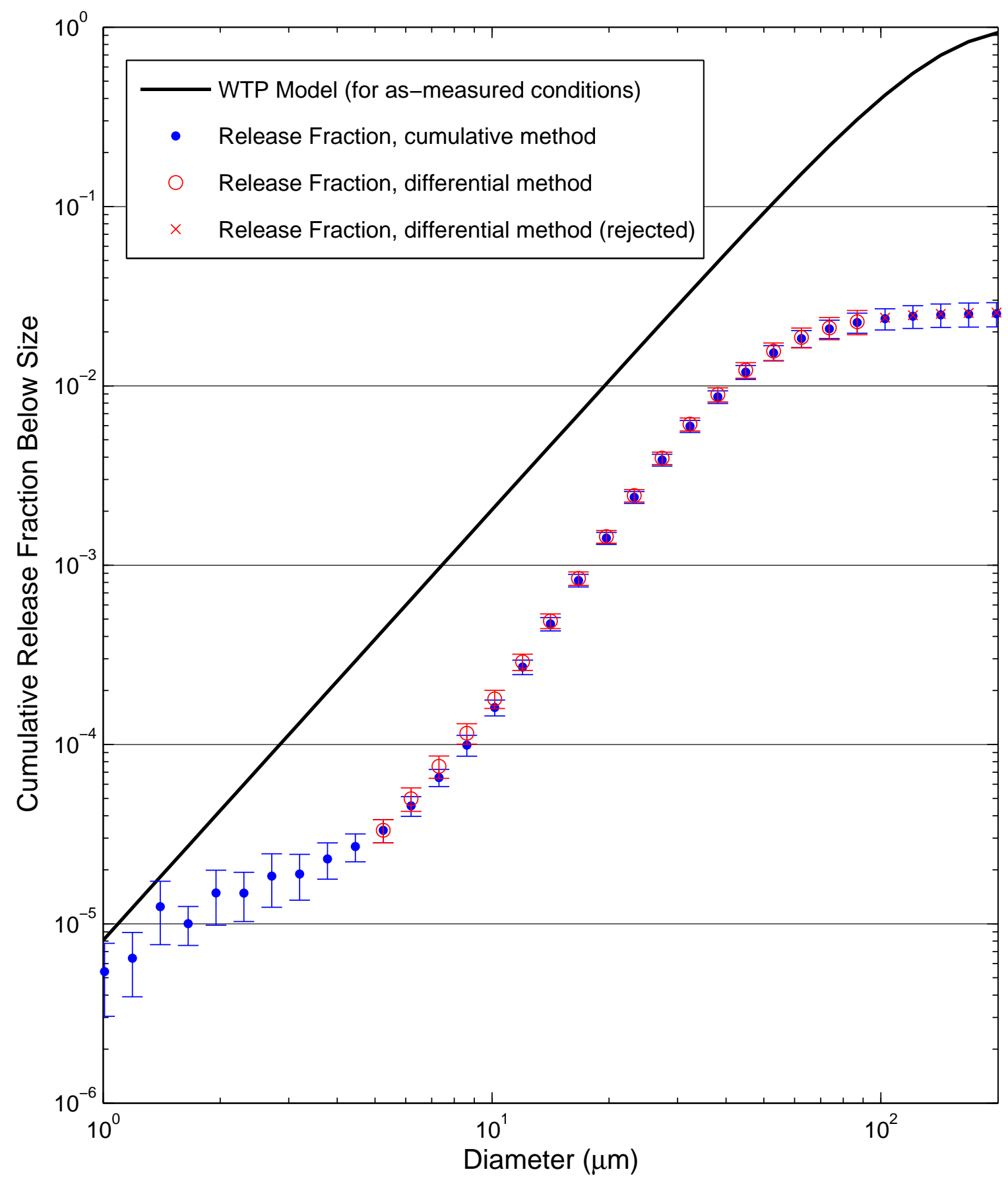


Test W67 [Malvern 1852]: 380 psi, Orifice S1A - round, 1 mm

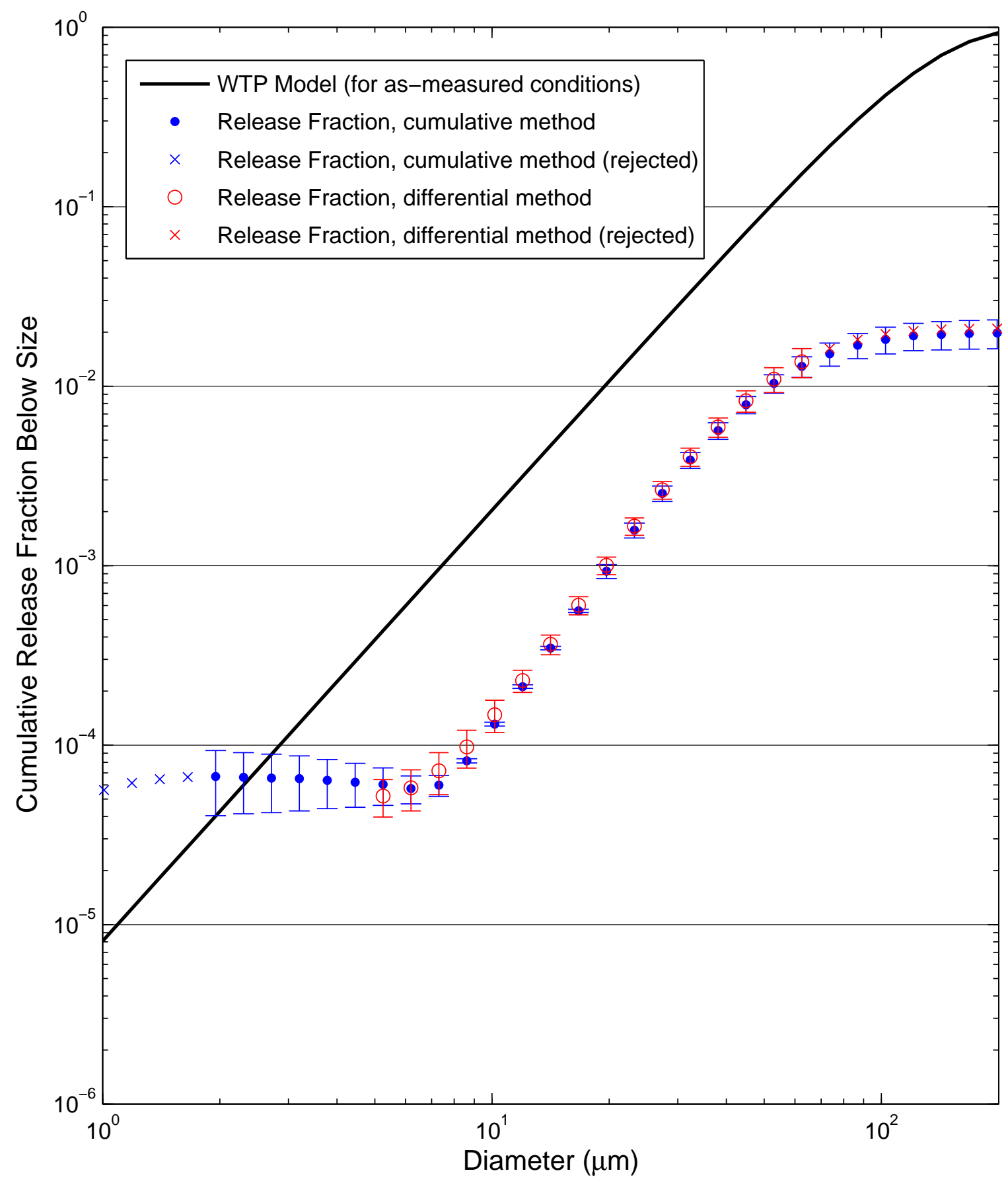


Test W68 [Malvern 1852]: 380 psi, Orifice S1A - round, 1 mm

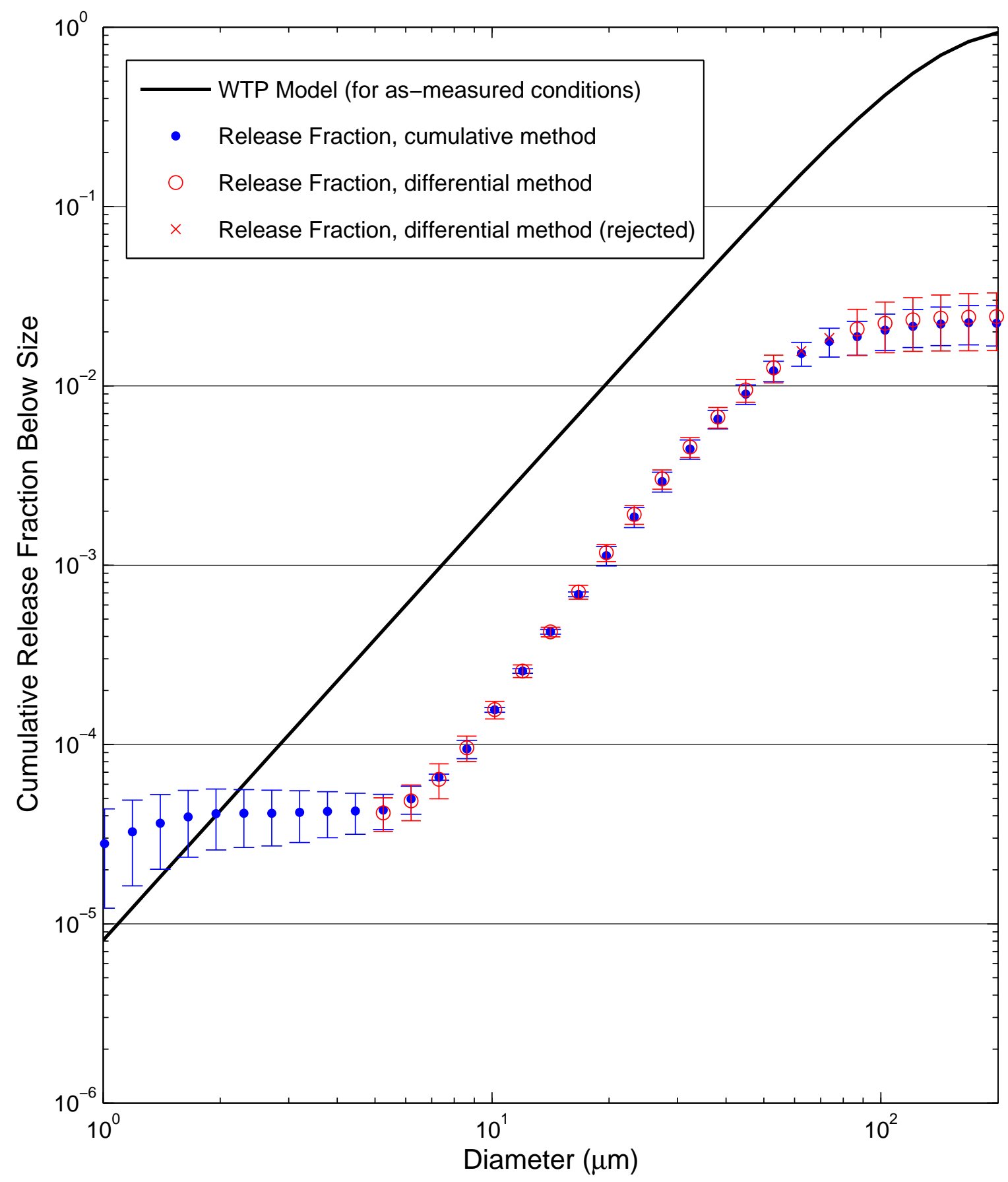


Test W69 [Malvern 1852]: 380 psi, Orifice S1A - round, 1 mm

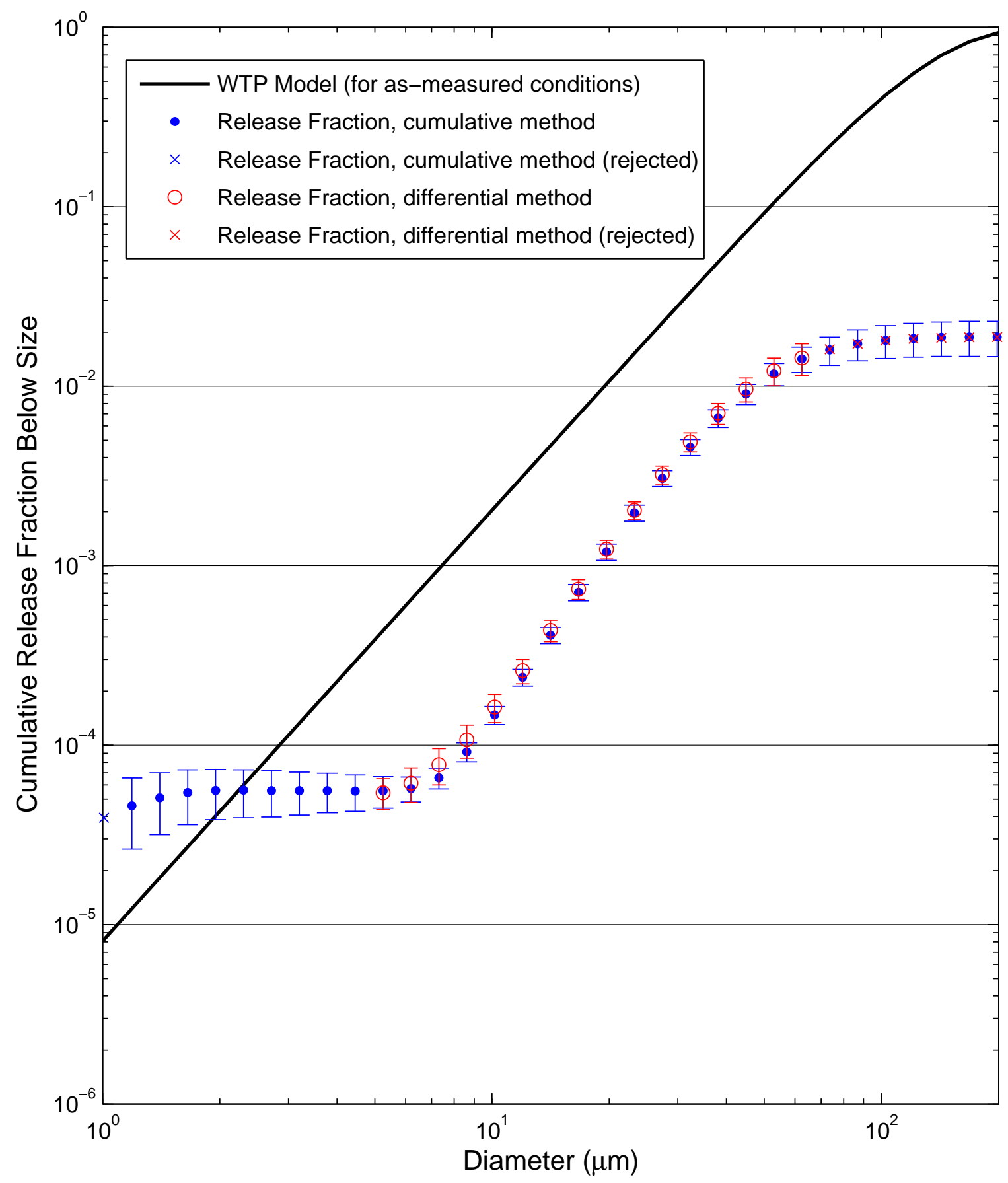


Test W70 [Malvern 1852]: 380 psi, Orifice S1D - round, 2 mm

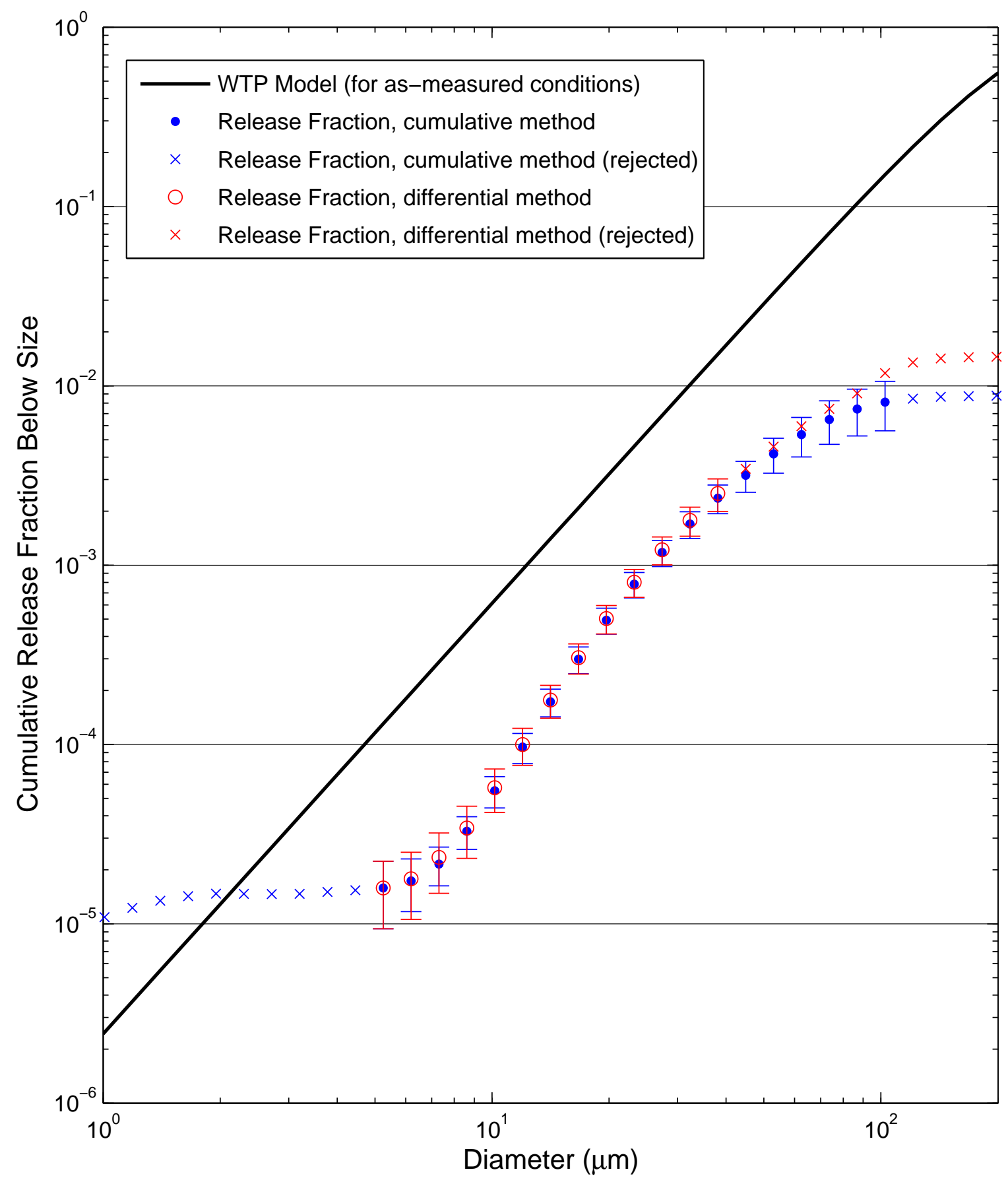


Test W72 [Malvern 1852]: 380 psi, Orifice S1B - round, $4.46 \mathrm{~mm}$

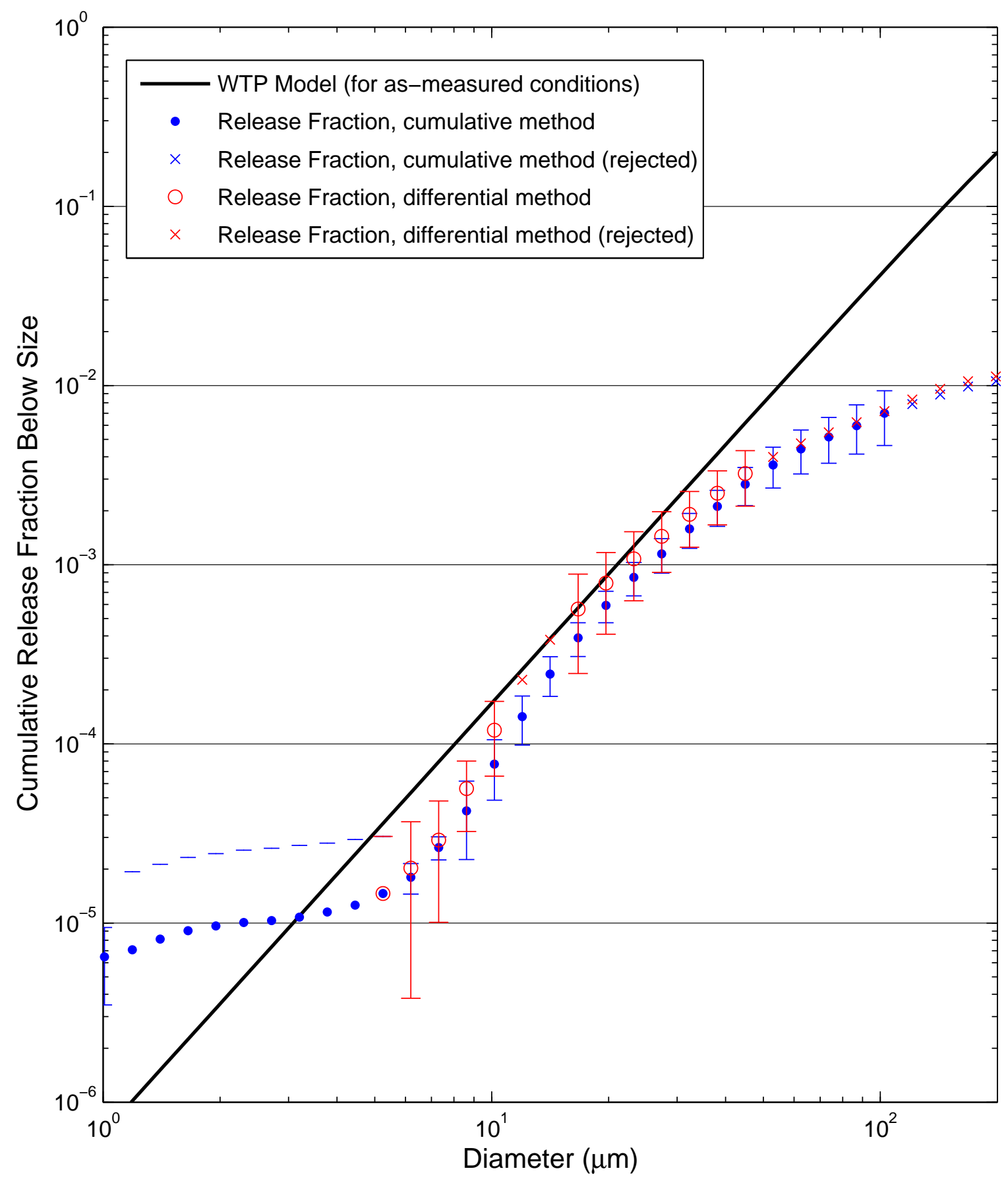


Test W73 [3 Malvern Average]: 200 psi, Orifice S1A - round, $1 \mathrm{~mm}$

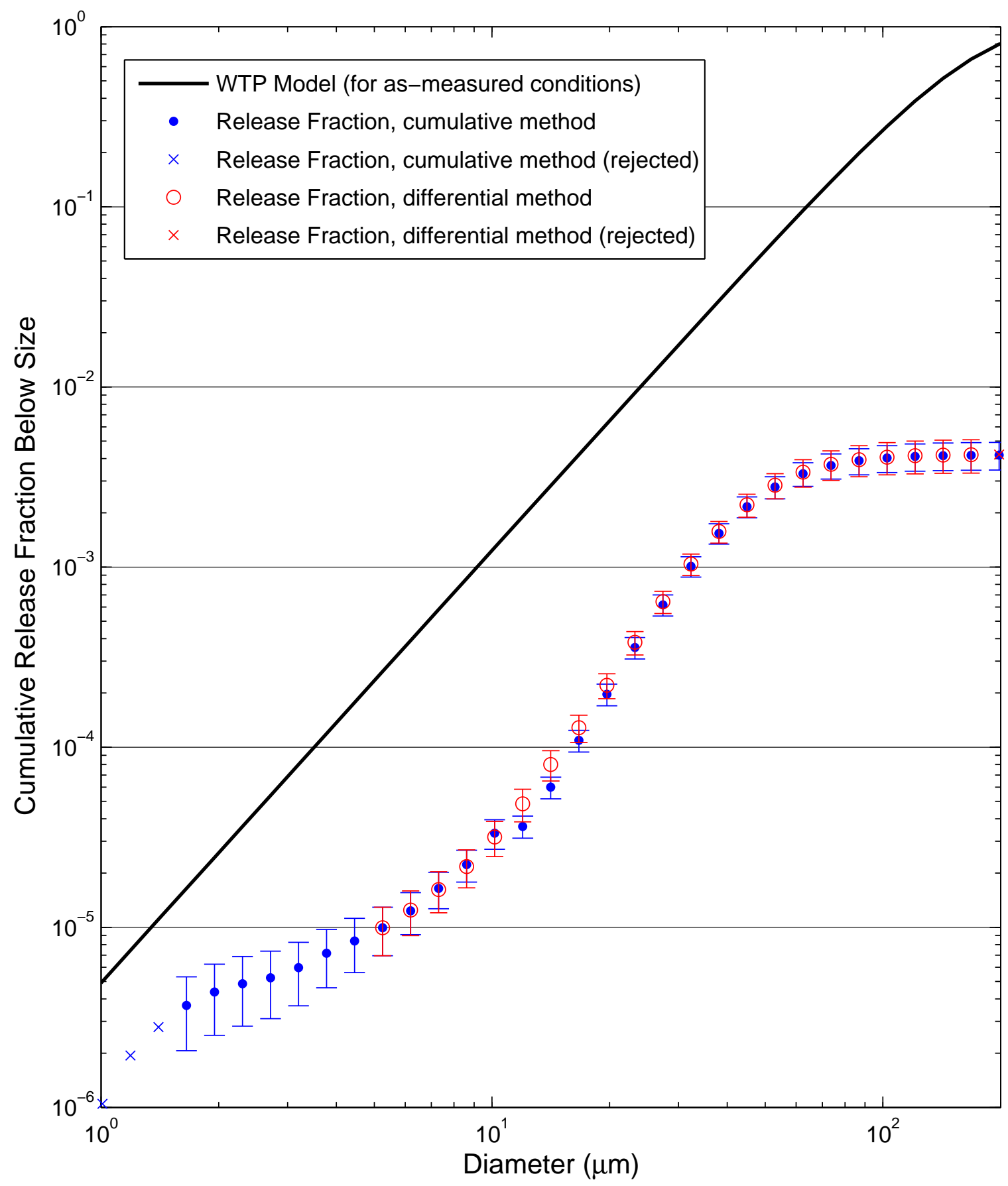


Test W74 [3 Malvern Average]: 380 psi, Orifice S1A - round, $1 \mathrm{~mm}$

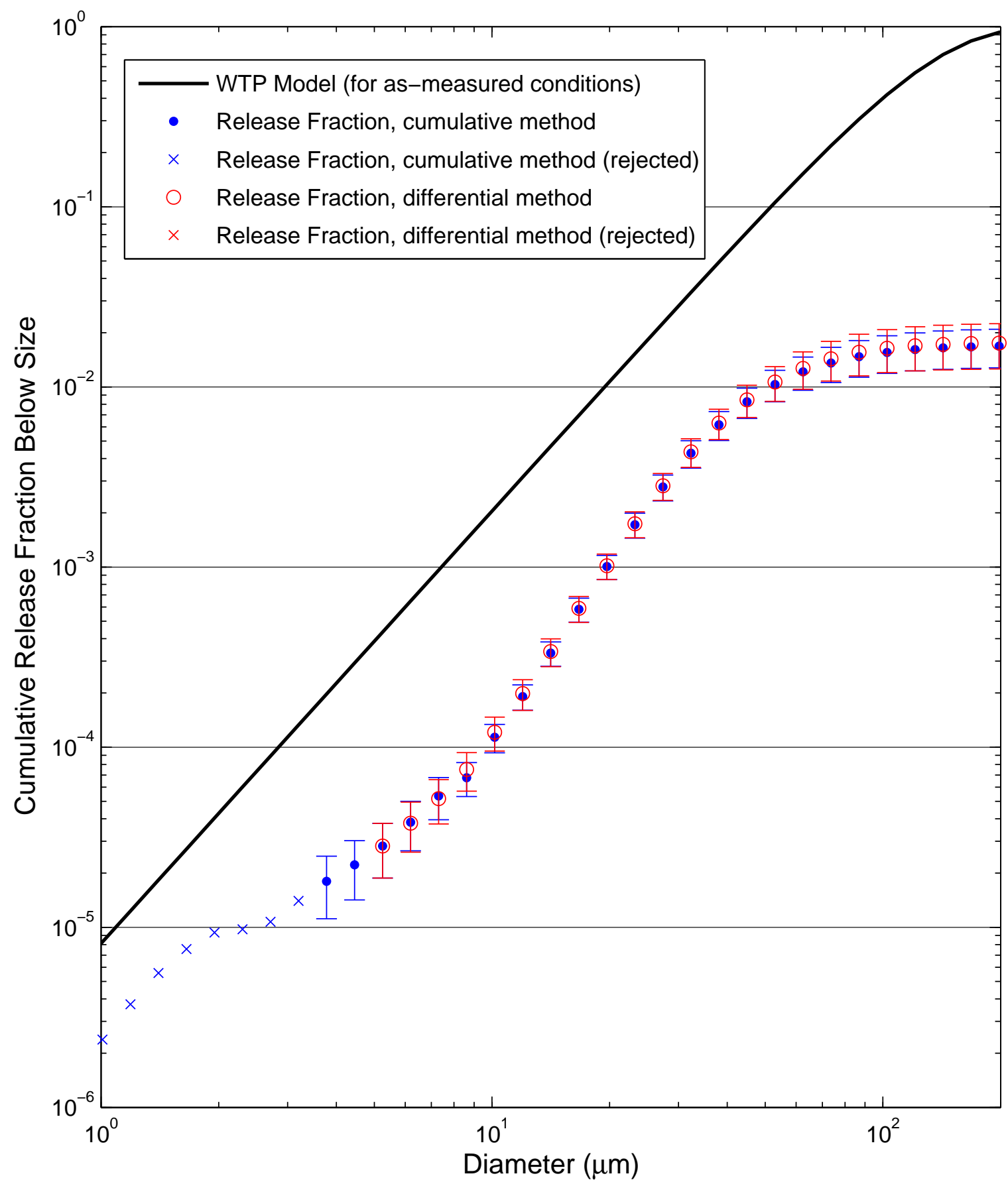


Test W75 [3 Malvern Average]: 200 psi, Orifice S1B - round, $4.46 \mathrm{~mm}$

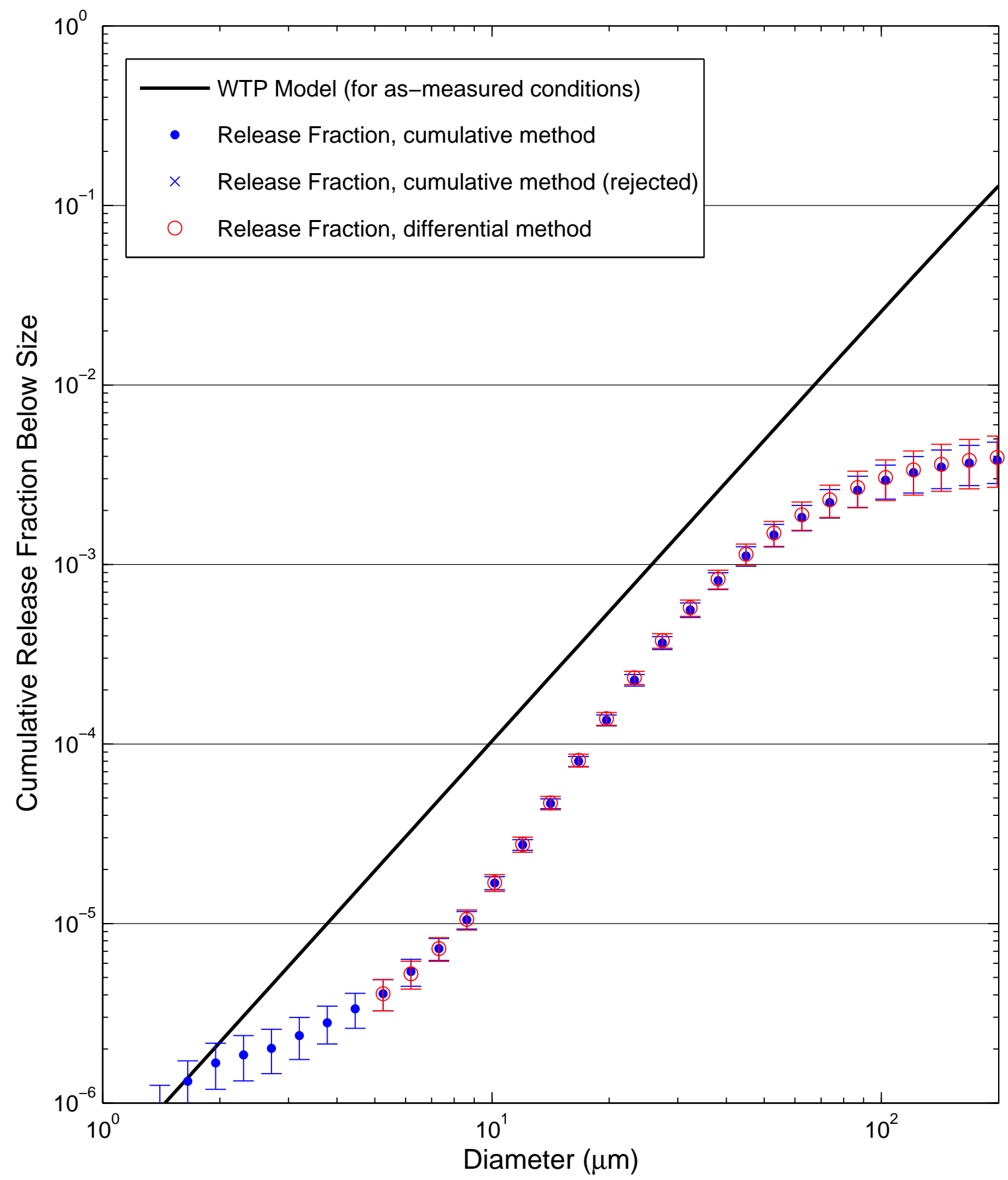


Test W76 [3 Malvern Average]: 380 psi, Orifice S1B - round, $4.46 \mathrm{~mm}$

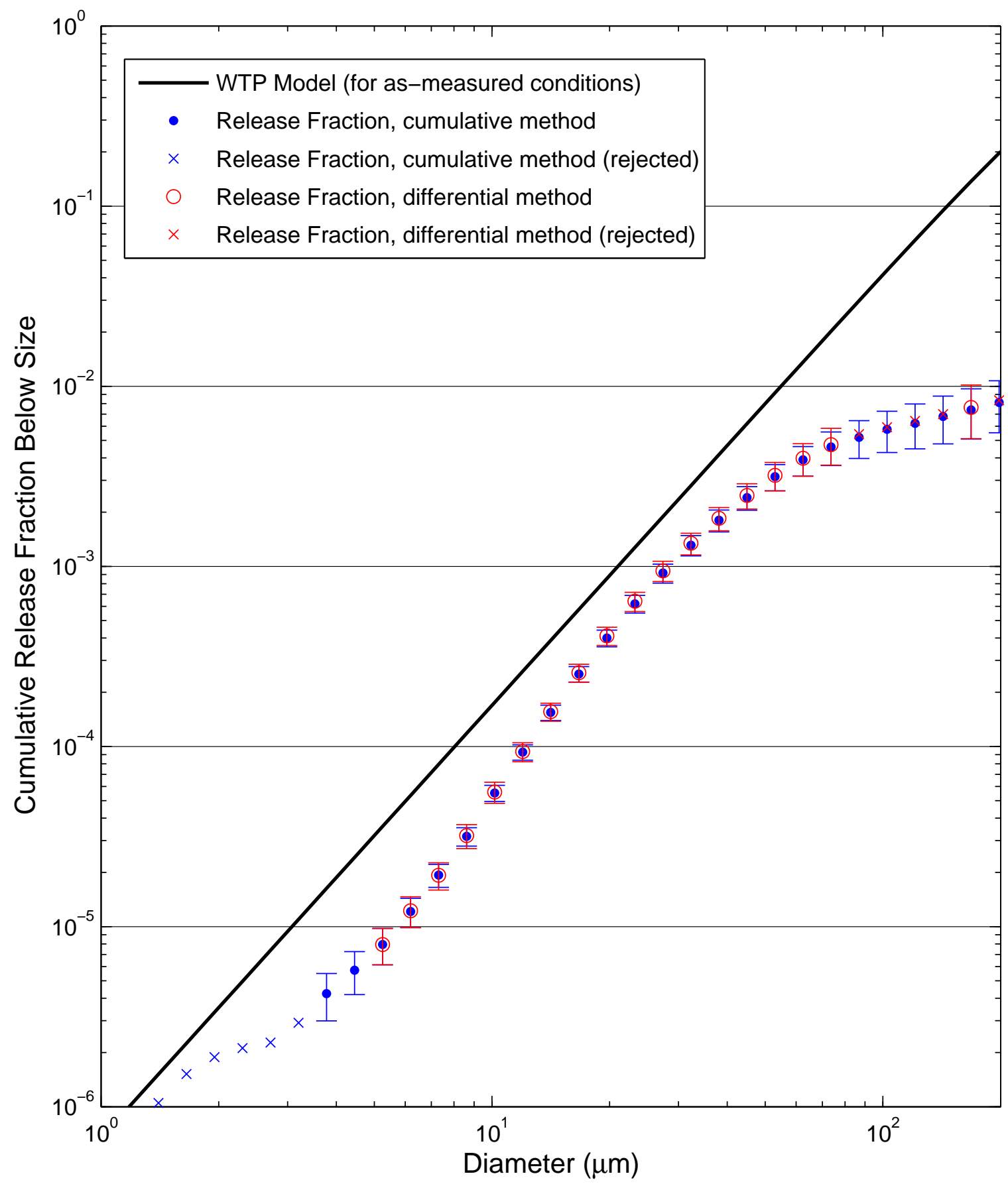


Test W77 [3 Malvern Average]: 200 psi, Orifice S1C - round, 2.74 mm

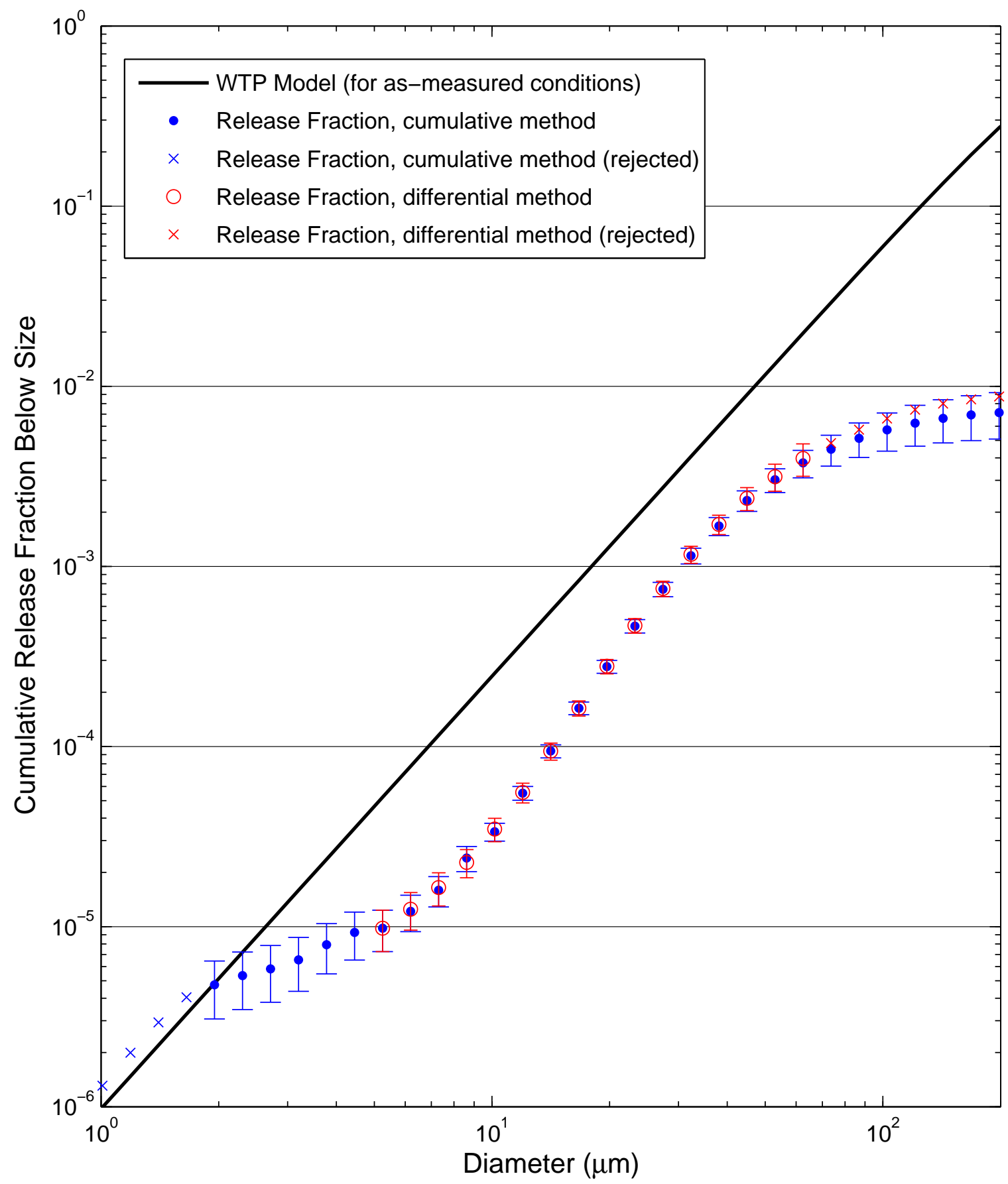


Test W78 [3 Malvern Average]: 380 psi, Orifice S1C - round, $2.74 \mathrm{~mm}$

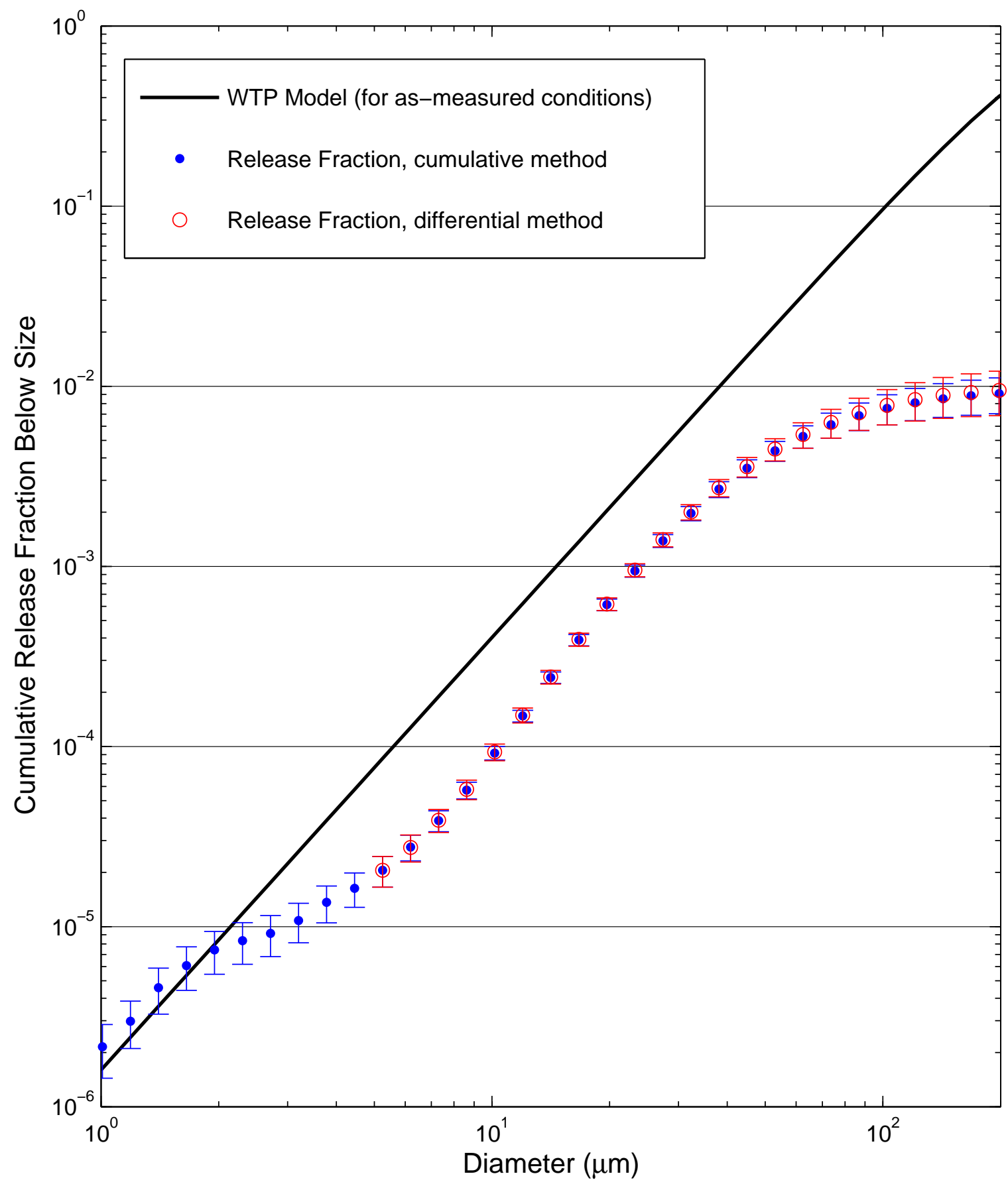


Test W79 [3 Malvern Average]: 100 psi, Orifice S1D - round, 2 mm

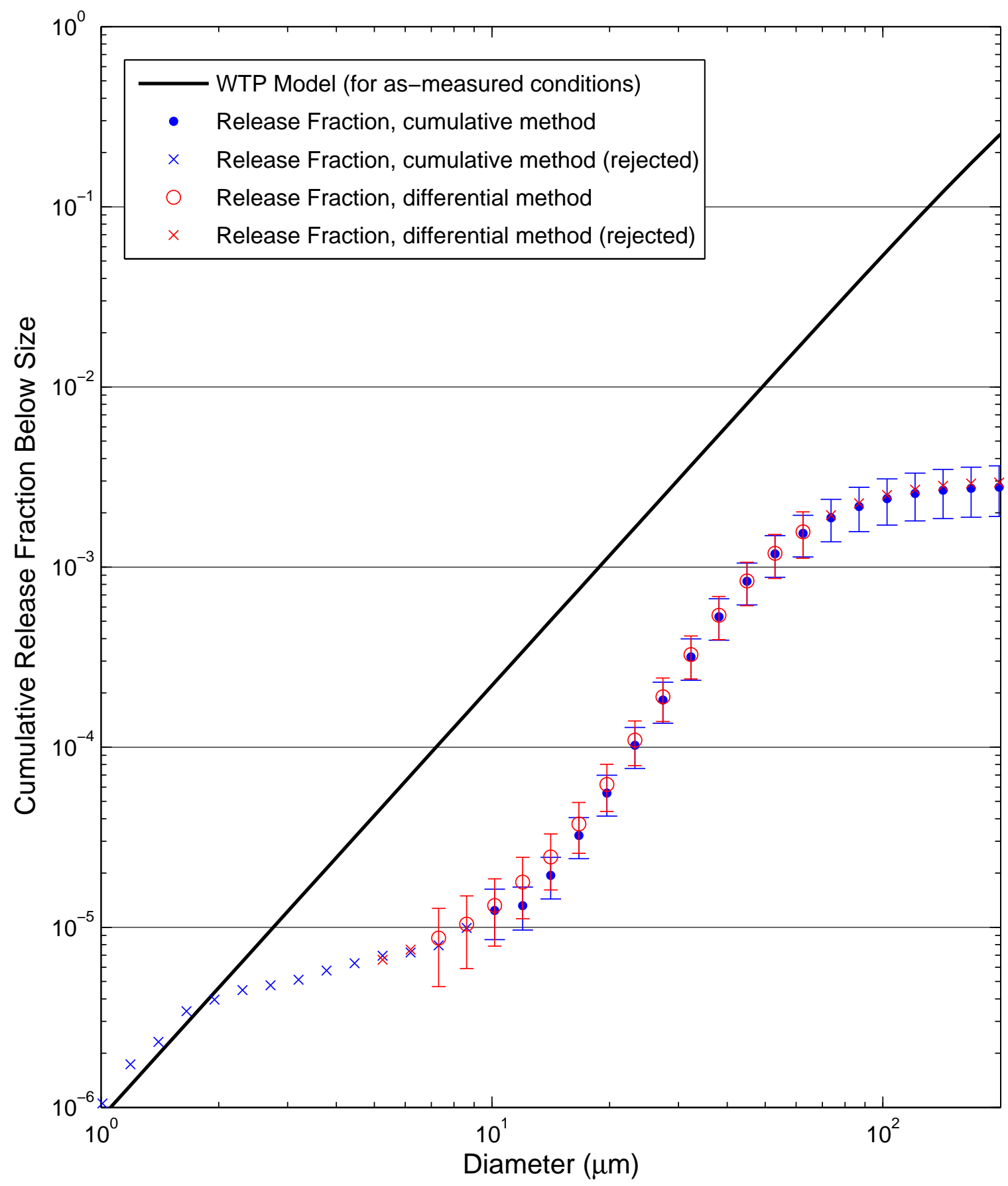


Test W80 [3 Malvern Average]: 200 psi, Orifice S1D - round, 2 mm

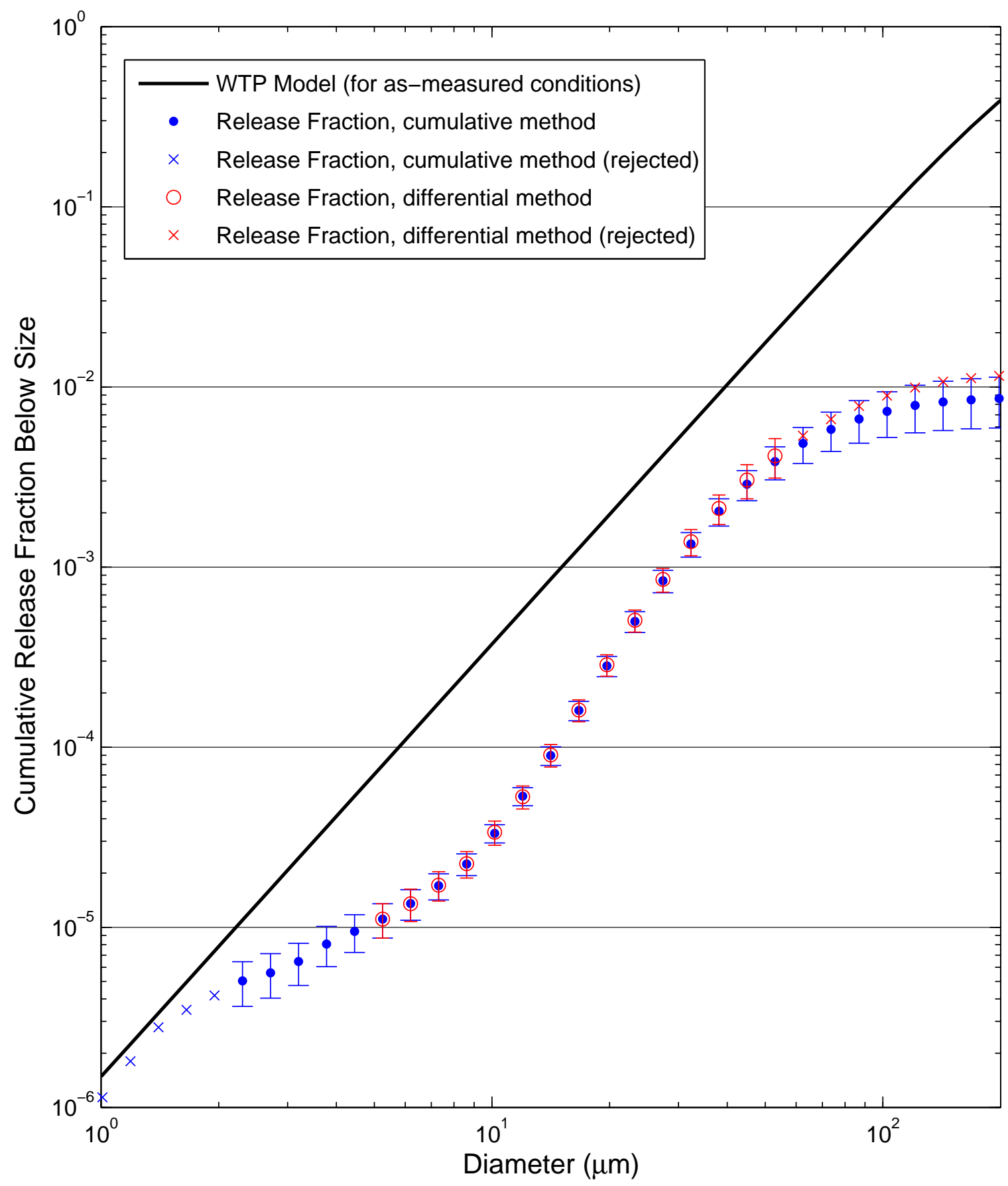


Test W81 [3 Malvern Average]: 380 psi, Orifice S1D - round, 2 mm

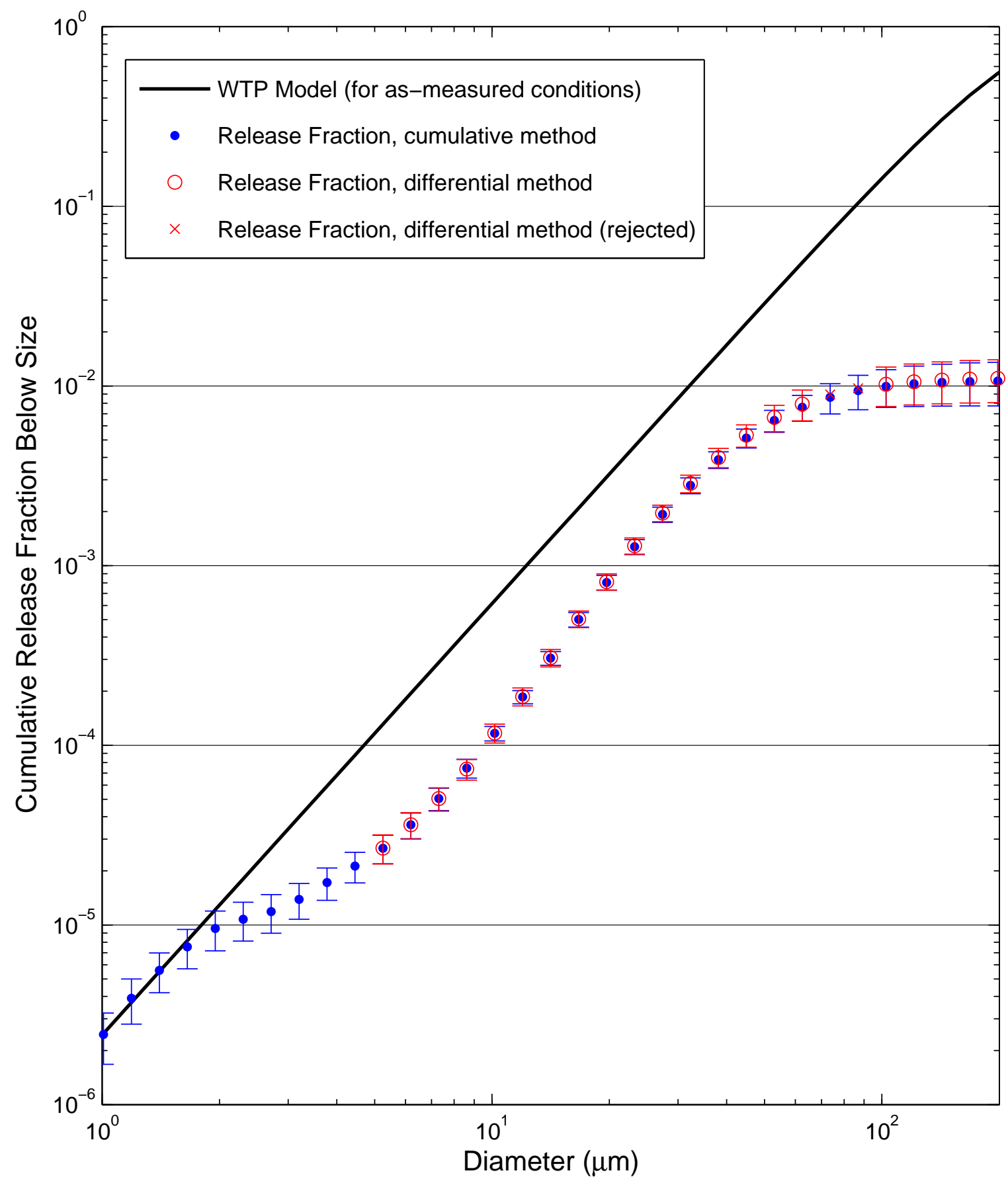


Test W82 [3 Malvern Average]: 200 psi, Orifice S1A - round, $1 \mathrm{~mm}$

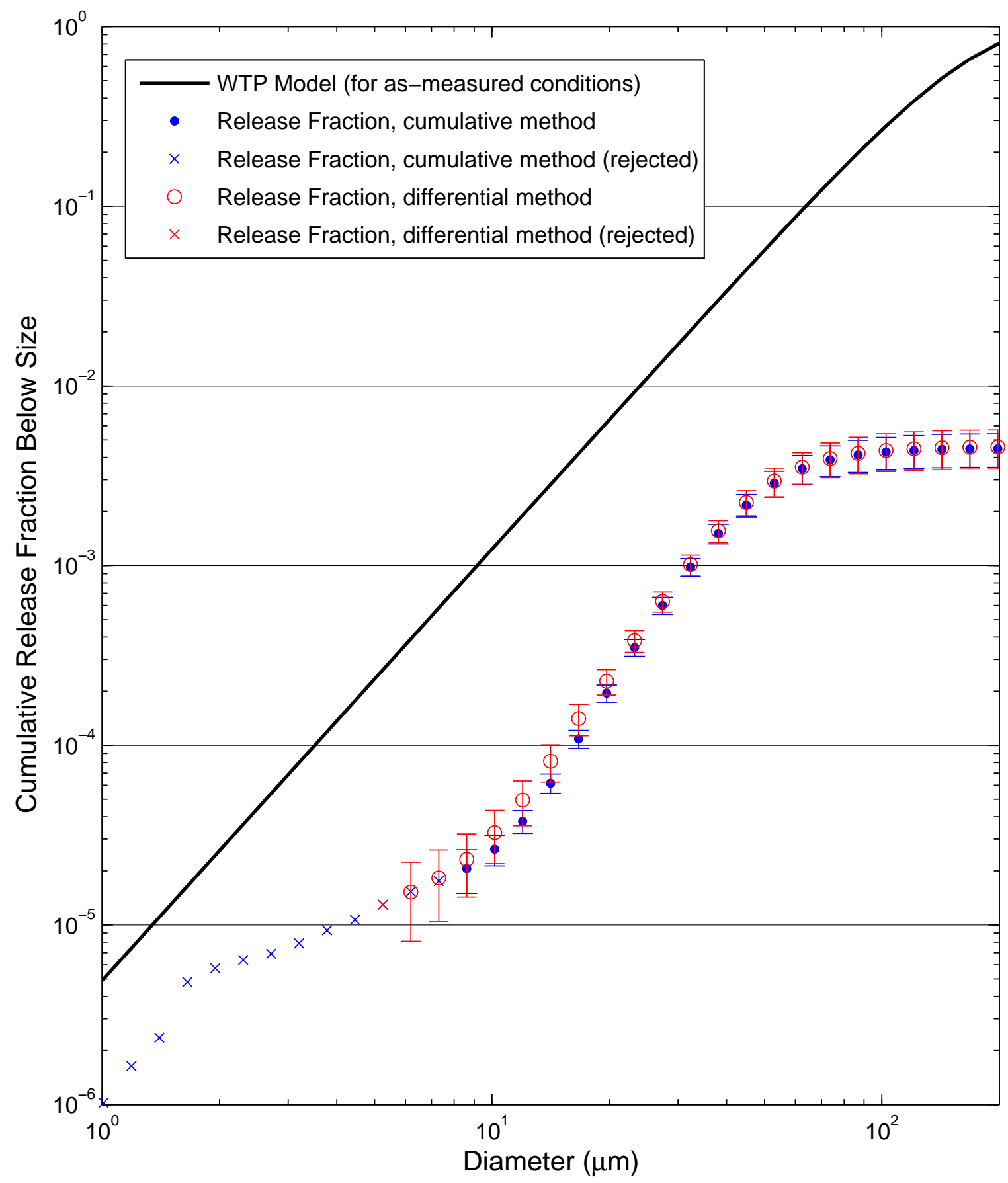


Test W83 [3 Malvern Average]: 380 psi, Orifice S1A - round, $1 \mathrm{~mm}$

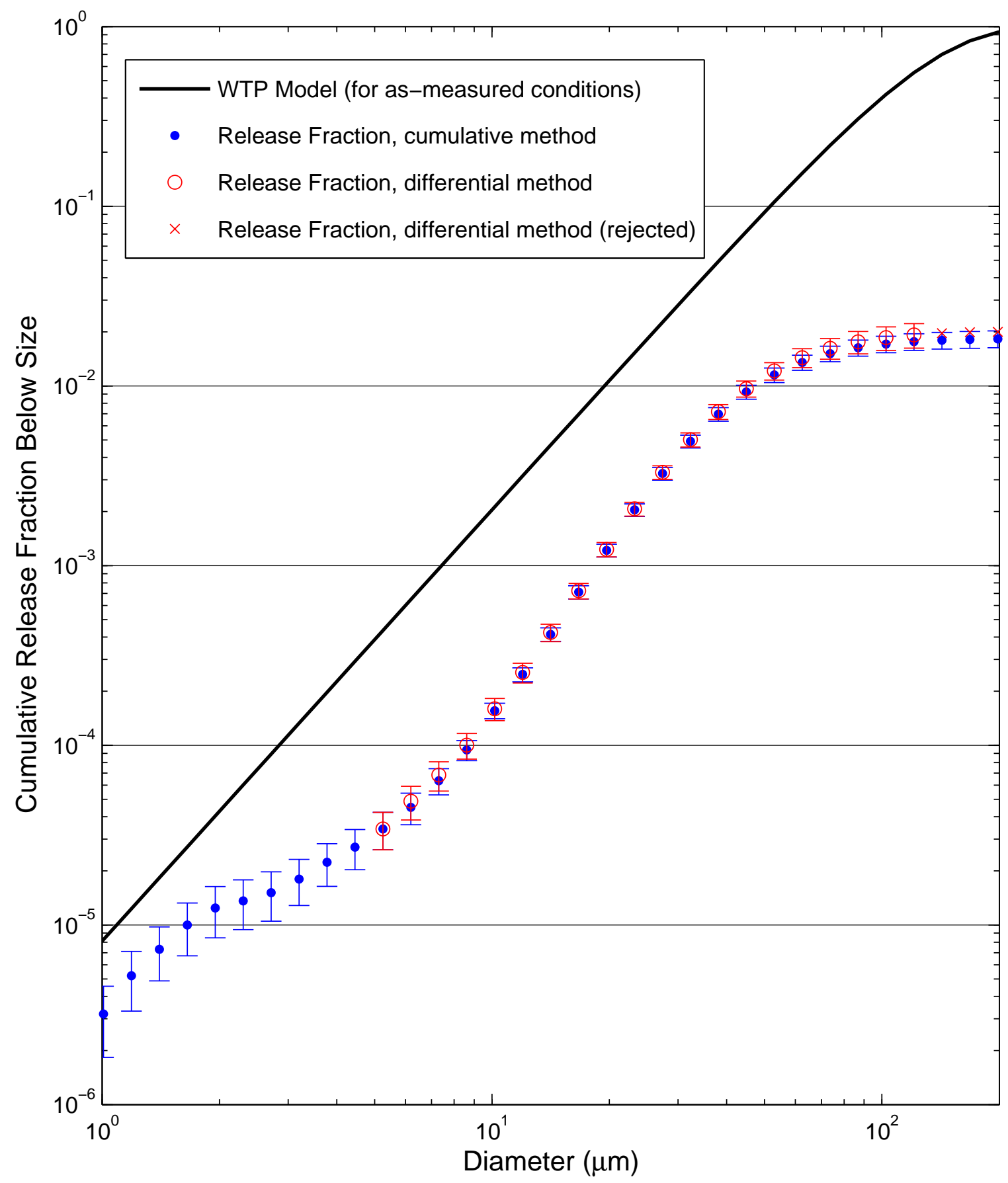


Test W84 [3 Malvern Average]: 200 psi, Orifice S1B - round, $4.46 \mathrm{~mm}$

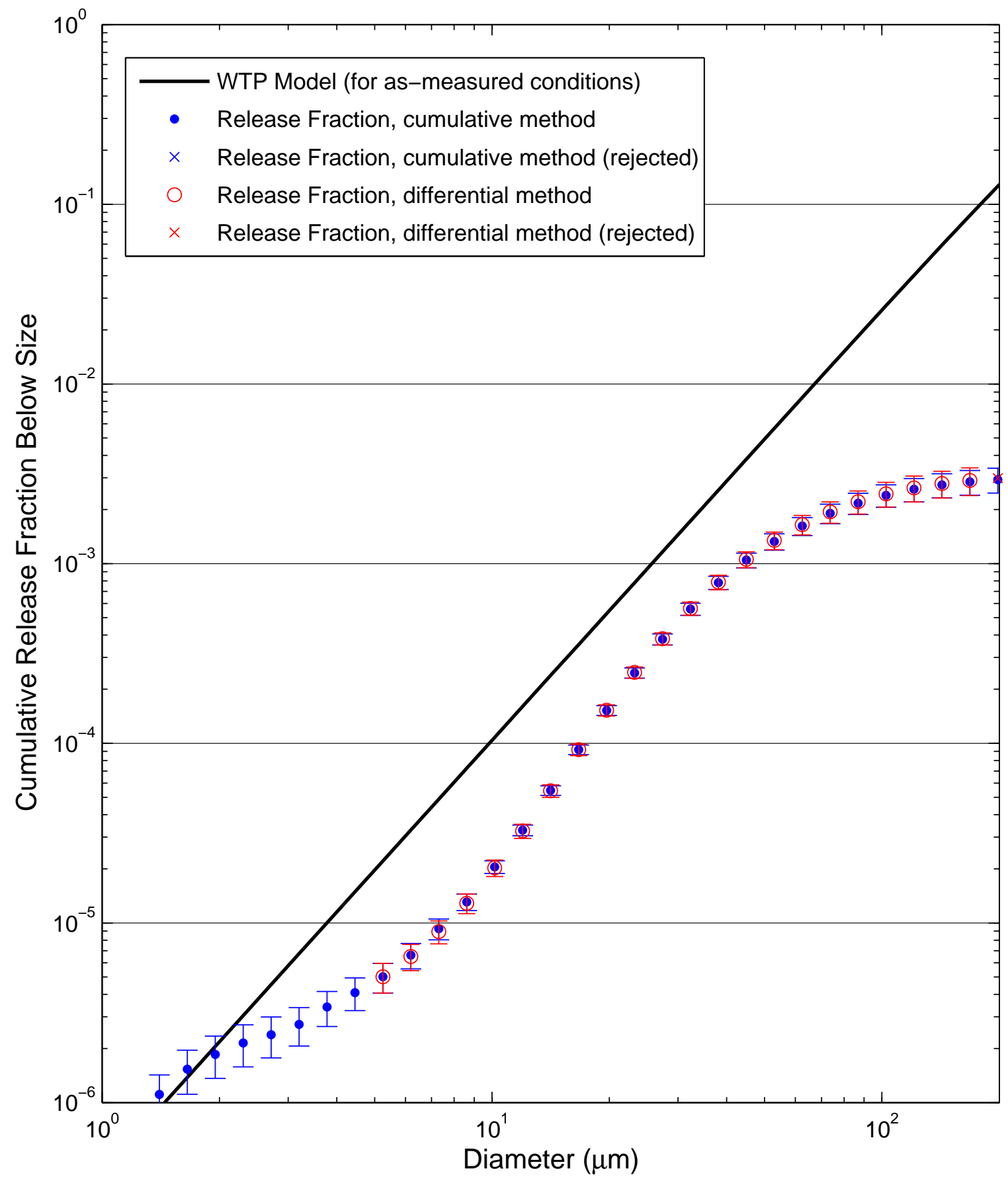


Test W85 [3 Malvern Average]: 380 psi, Orifice S1B - round, $4.46 \mathrm{~mm}$

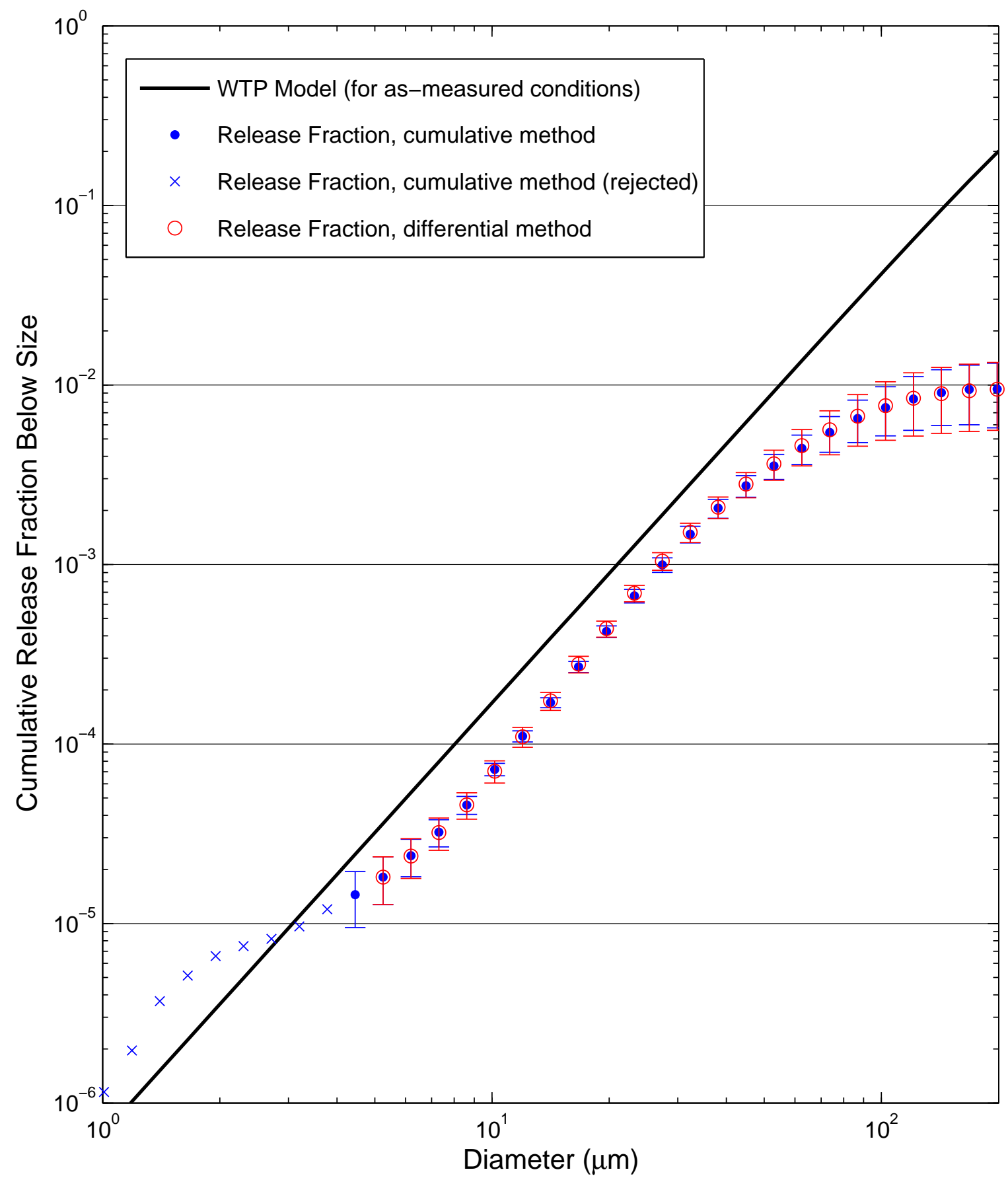


Test W86 [3 Malvern Average]: 100 psi, Orifice S1D - round, 2 mm

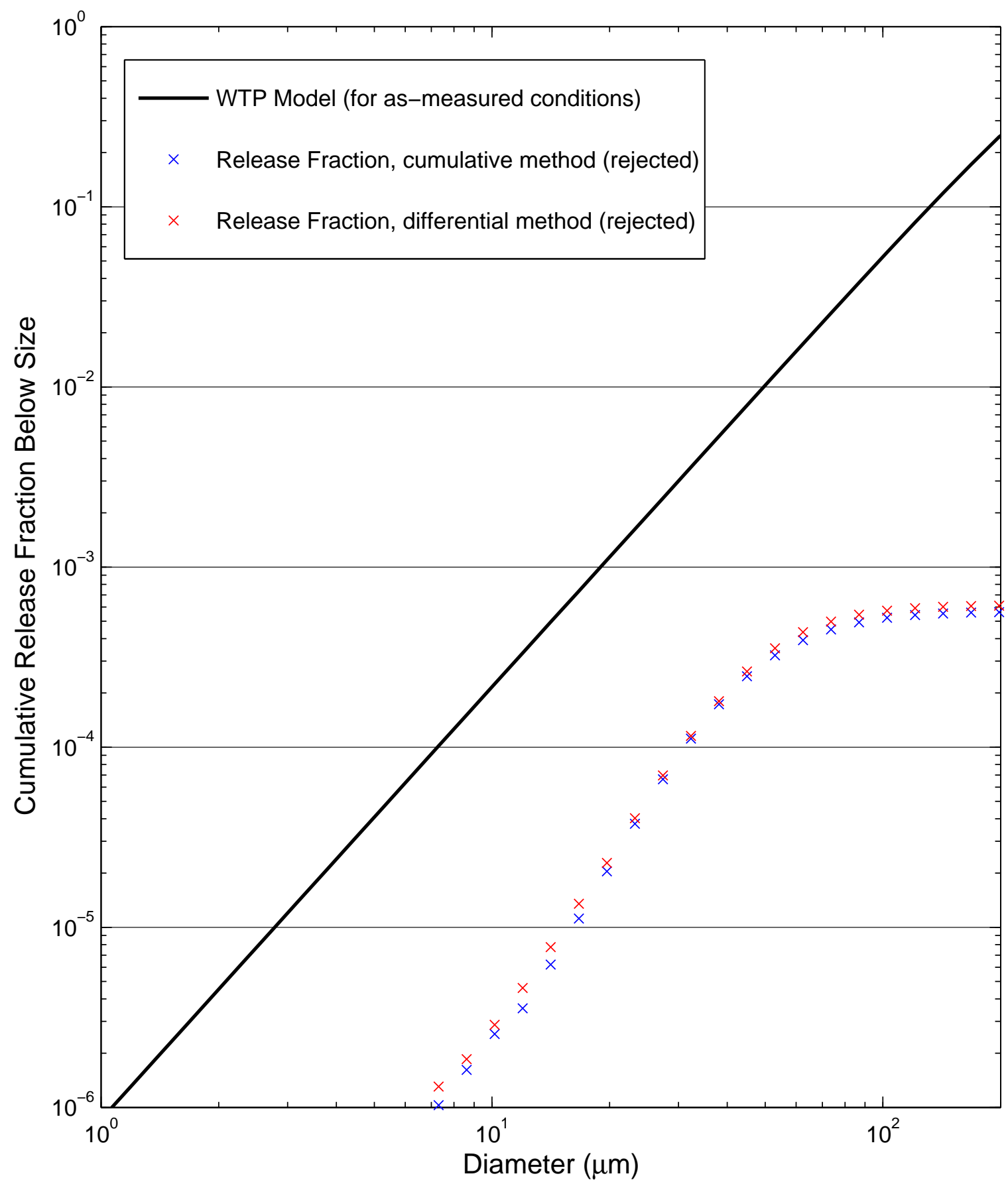


Test W87 [3 Malvern Average]: 200 psi, Orifice S1D - round, 2 mm

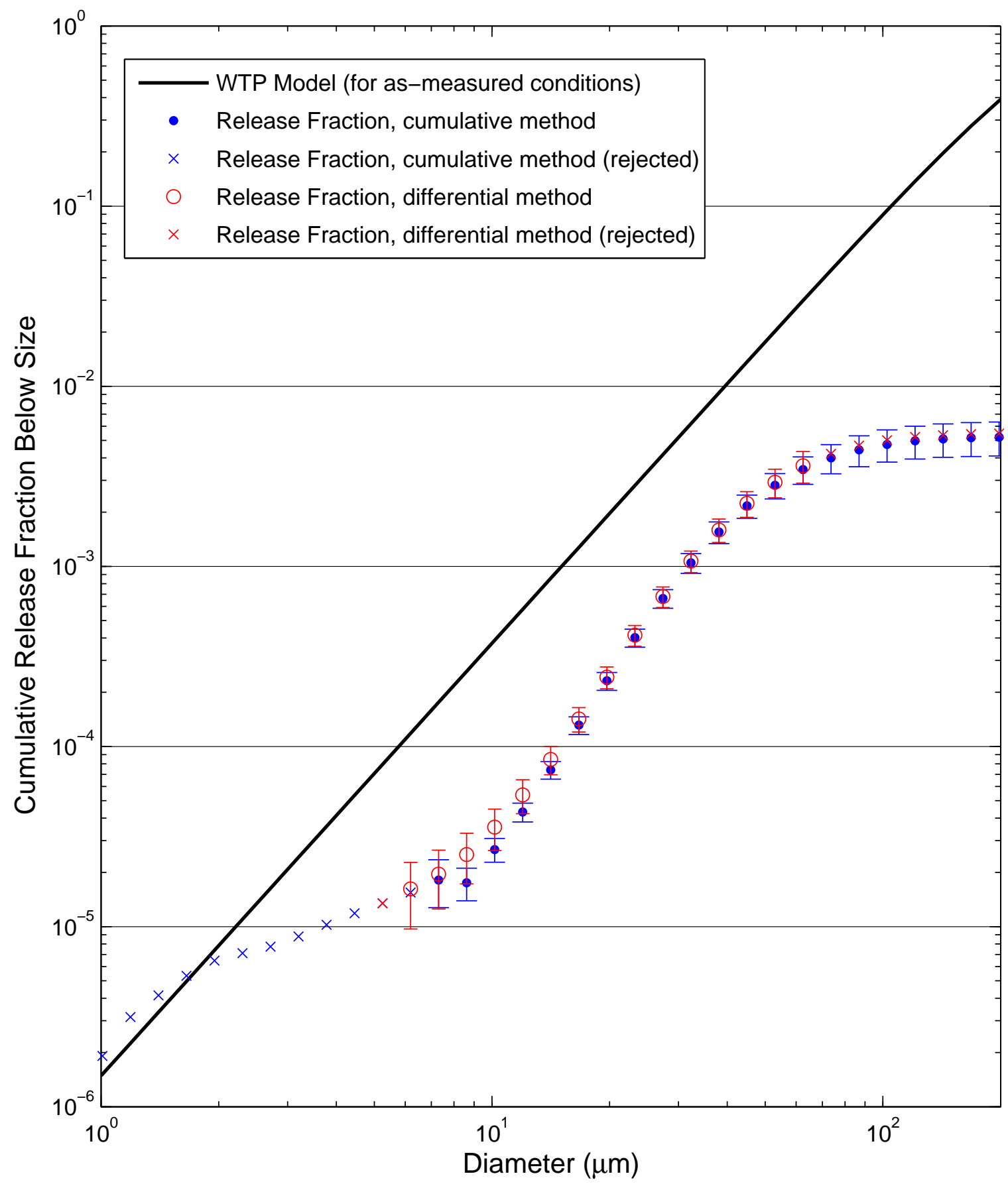


Test W88 [3 Malvern Average]: 380 psi, Orifice S1D - round, 2 mm

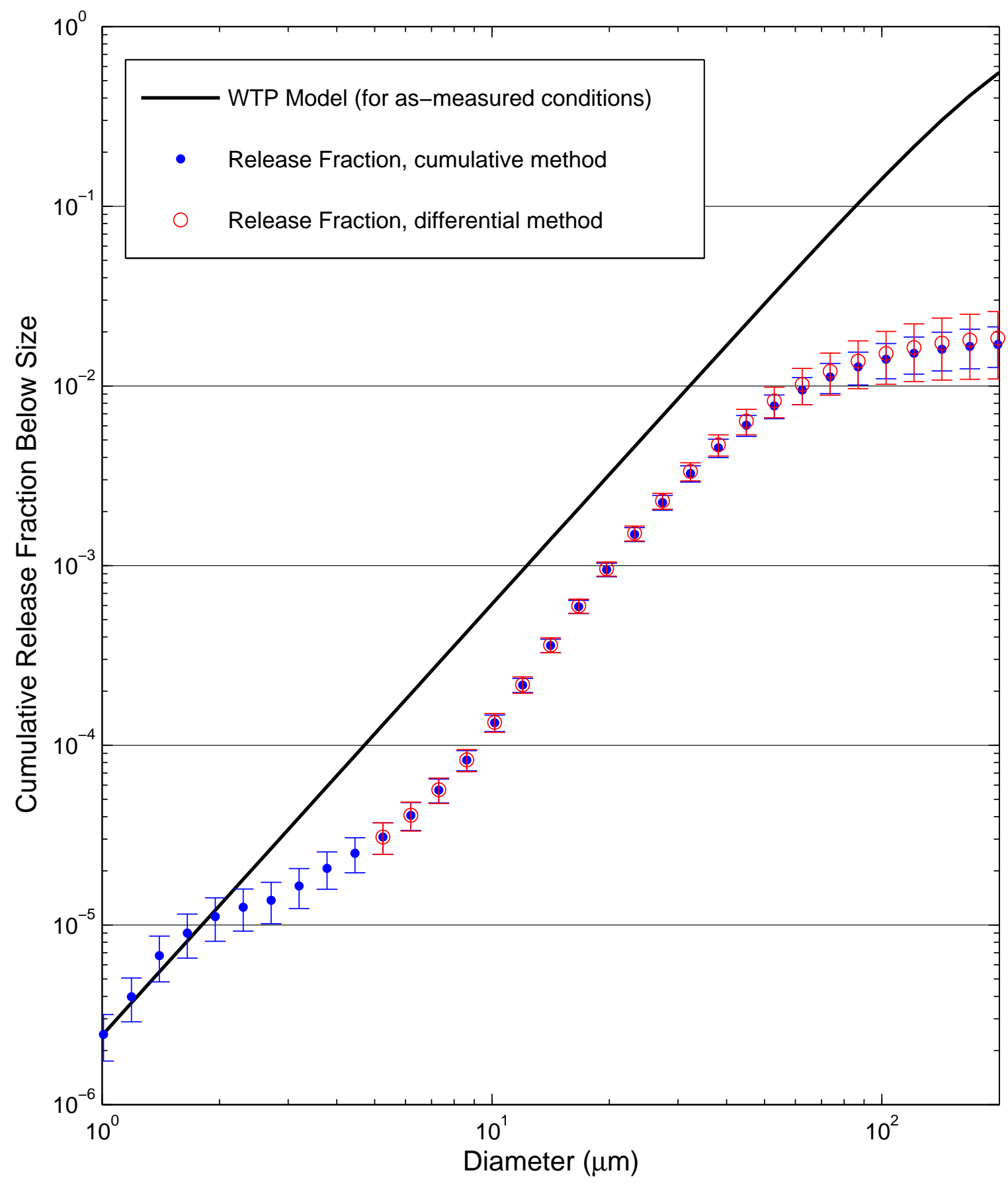


Test W89 [3 Malvern Average]: 100 psi, Orifice S7A - rectangular, $1 \times 10 \mathrm{~mm}$

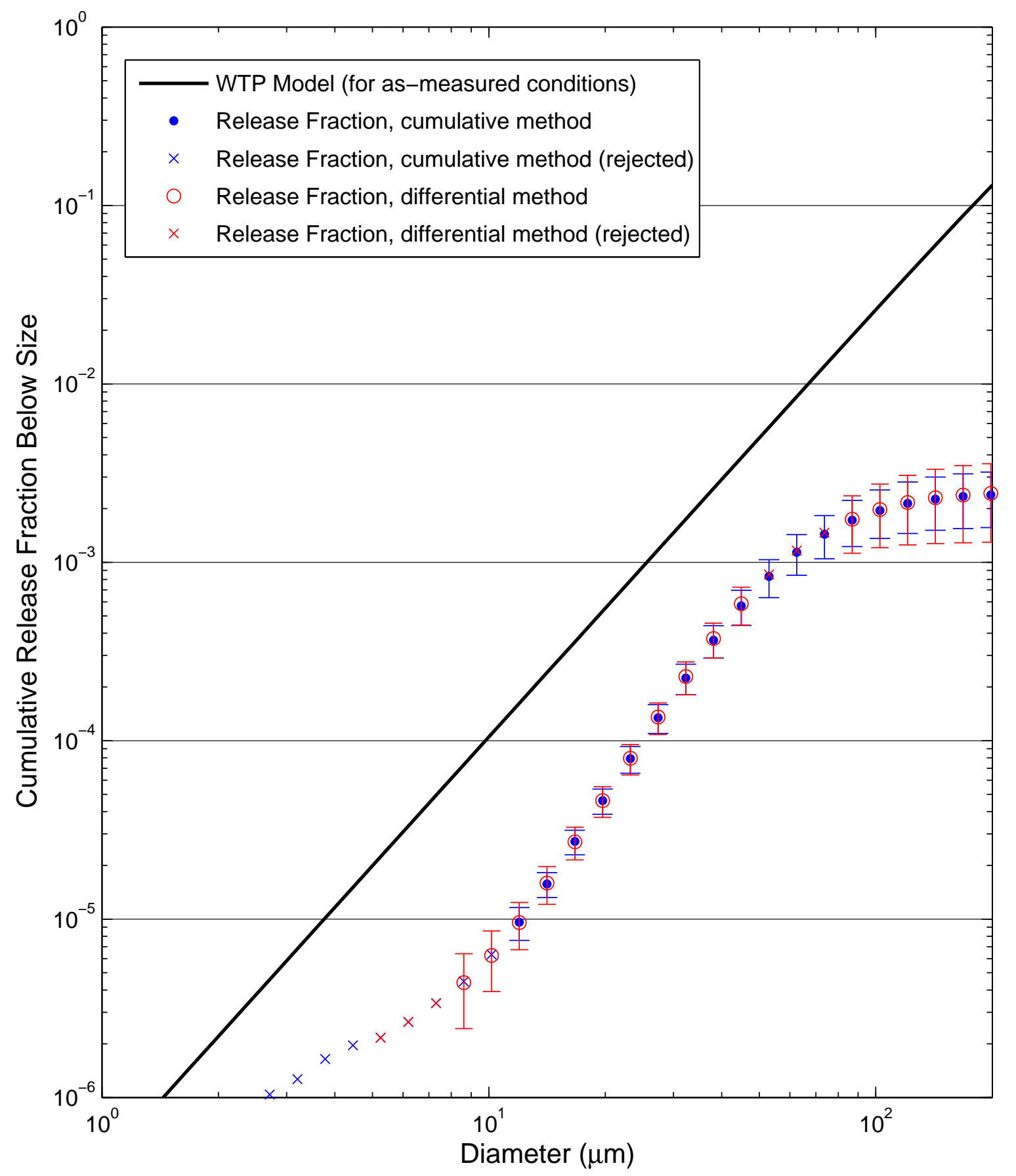


Test W90 [3 Malvern Average]: 200 psi, Orifice S7A - rectangular, $1 \times 10 \mathrm{~mm}$

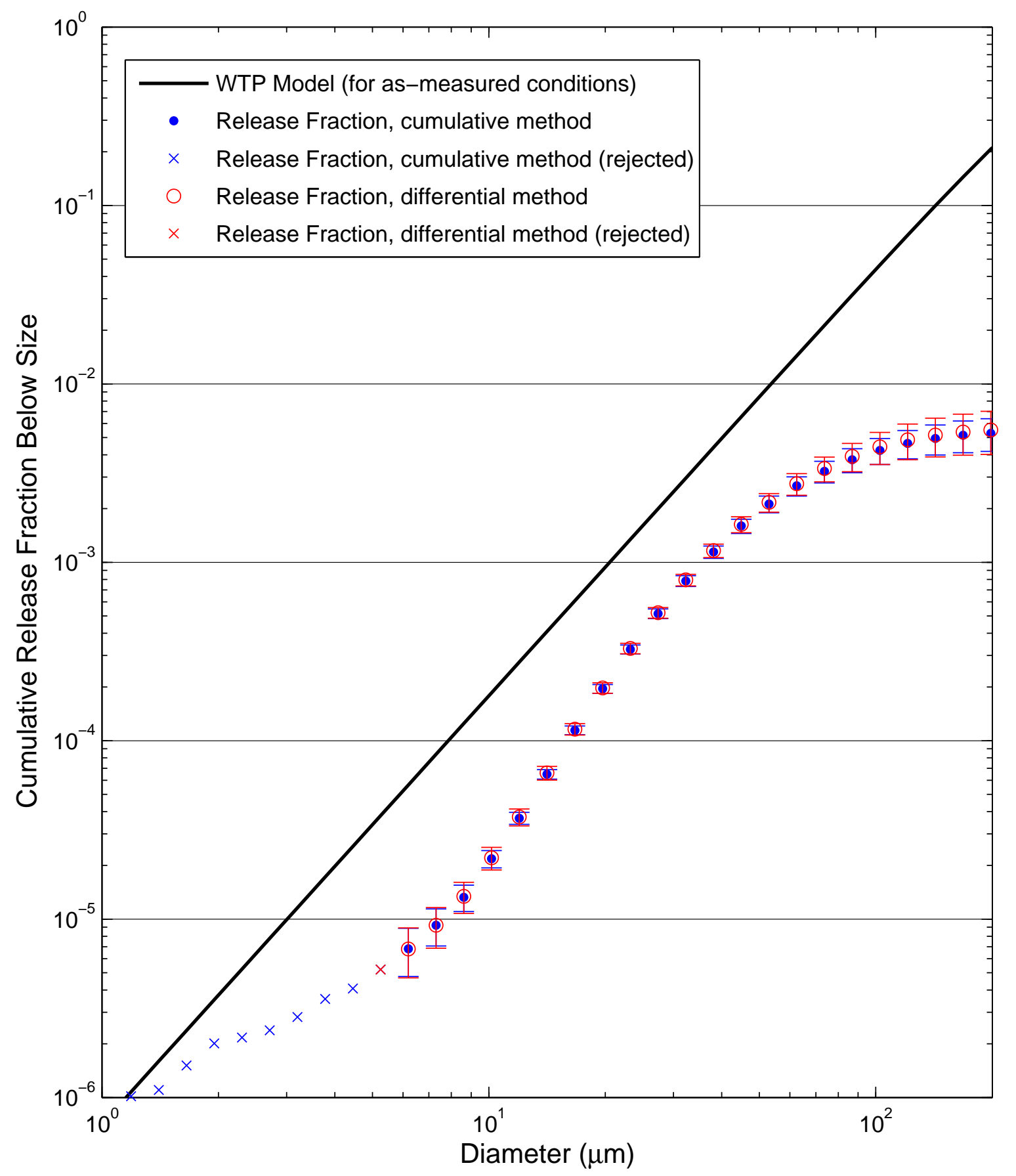


Test W91 [3 Malvern Average]: 380 psi, Orifice S7A - rectangular, $1 \times 10 \mathrm{~mm}$

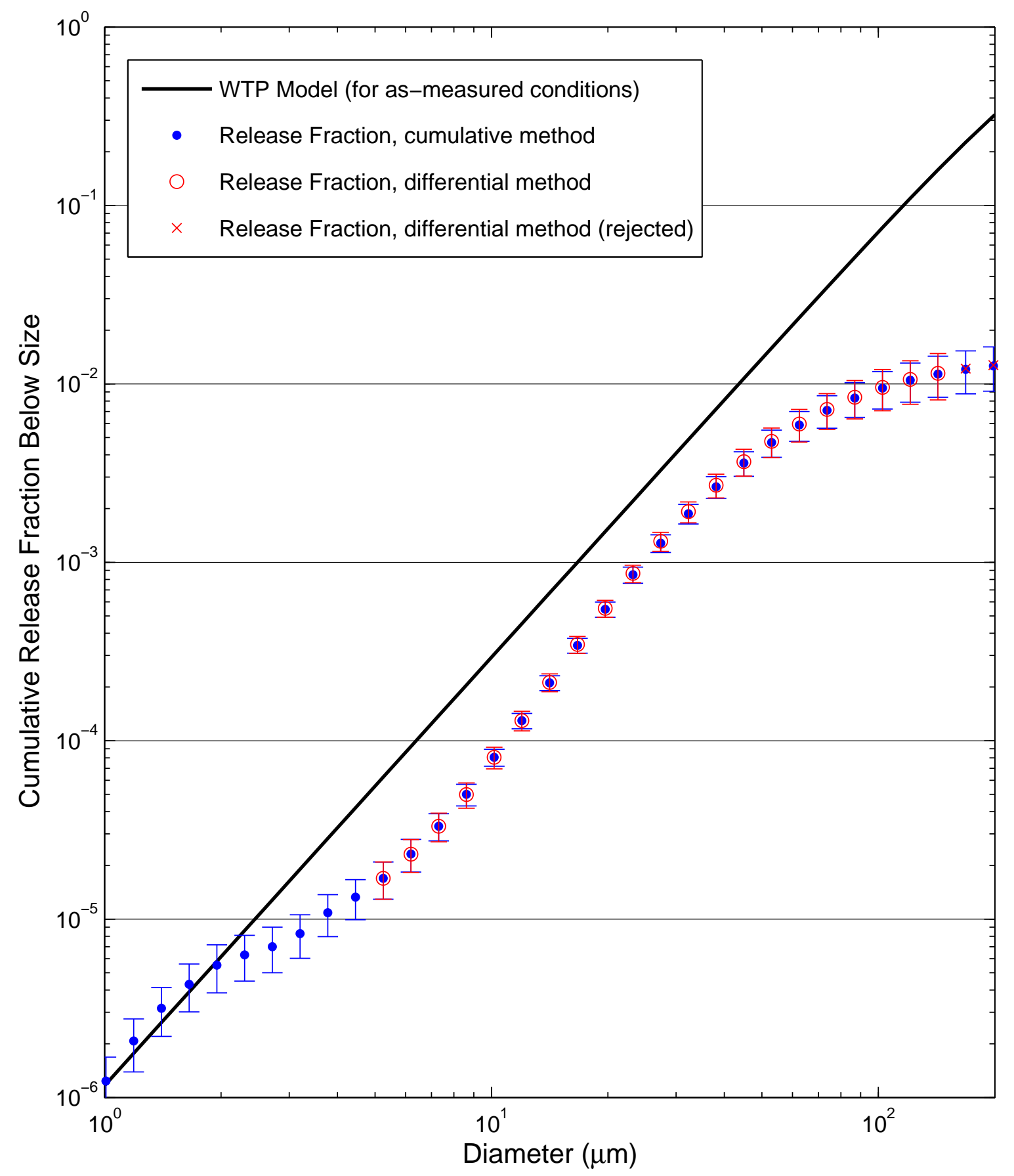


Test W92 [3 Malvern Average]: 100 psi, Orifice S7C - rectangular, 3× $10 \mathrm{~mm}$

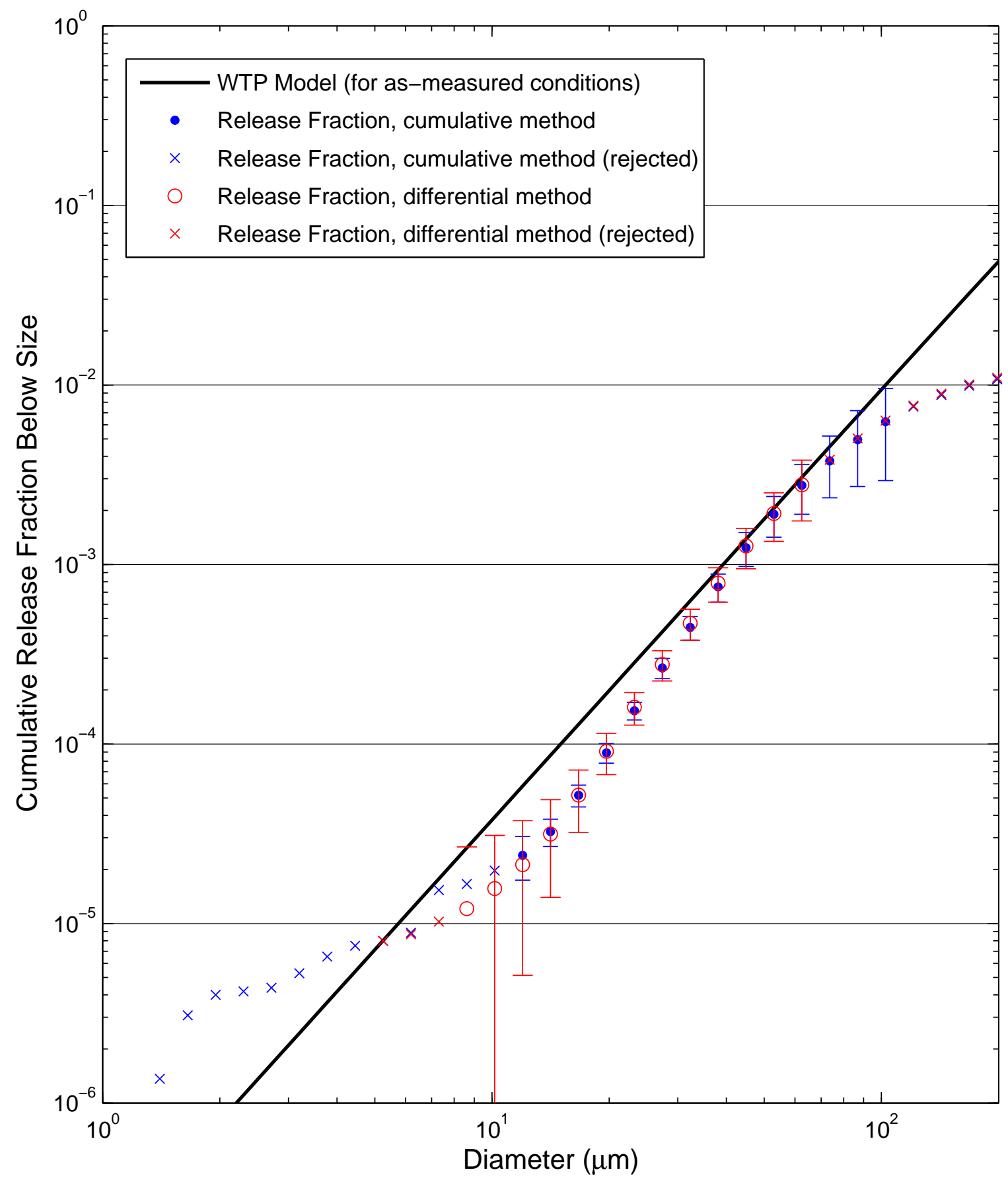


Test W93 [3 Malvern Average]: 200 psi, Orifice S7C - rectangular, $3 \times 10 \mathrm{~mm}$

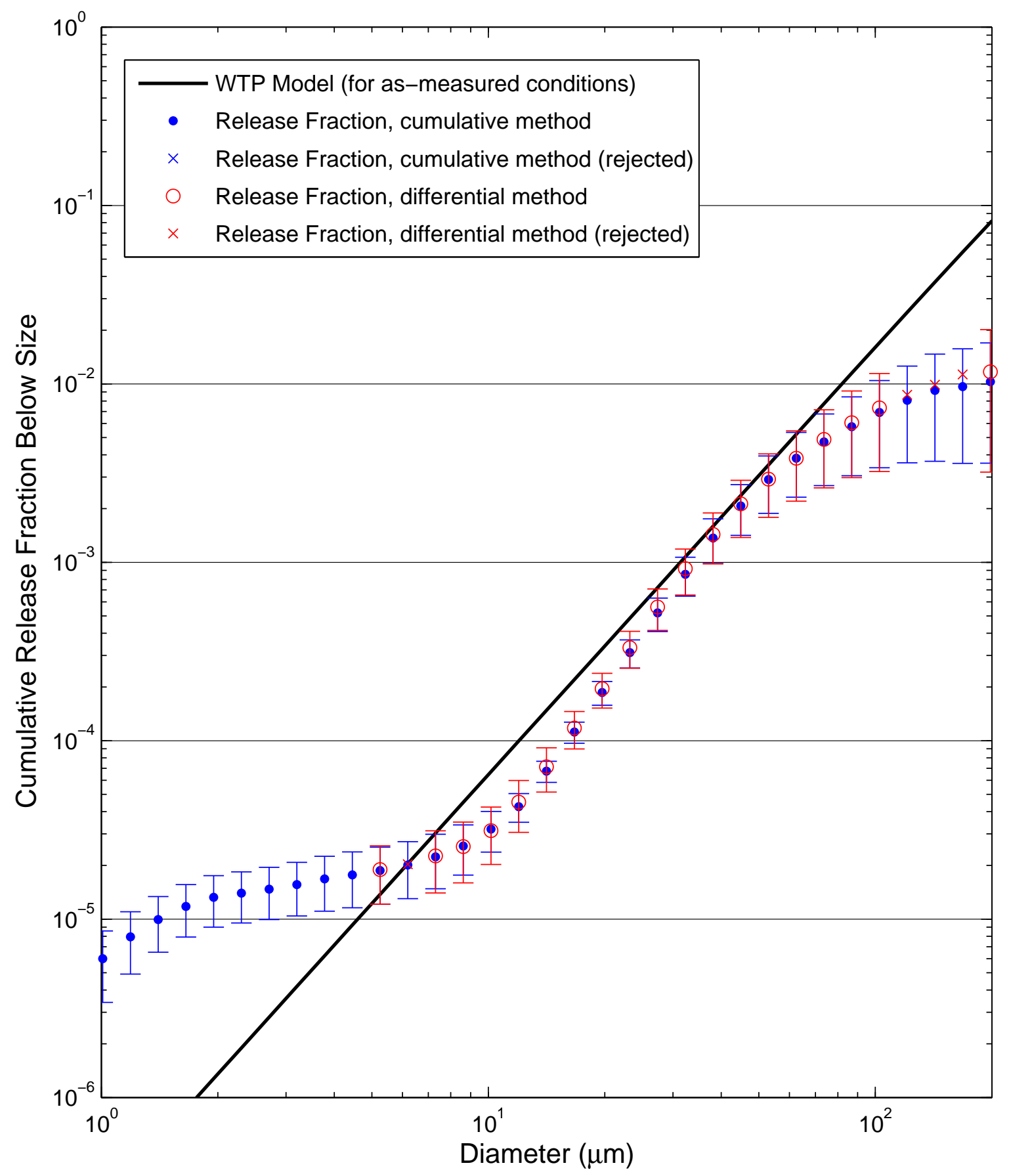


Test W94 [3 Malvern Average]: 380 psi, Orifice S7C - rectangular, 3× $10 \mathrm{~mm}$

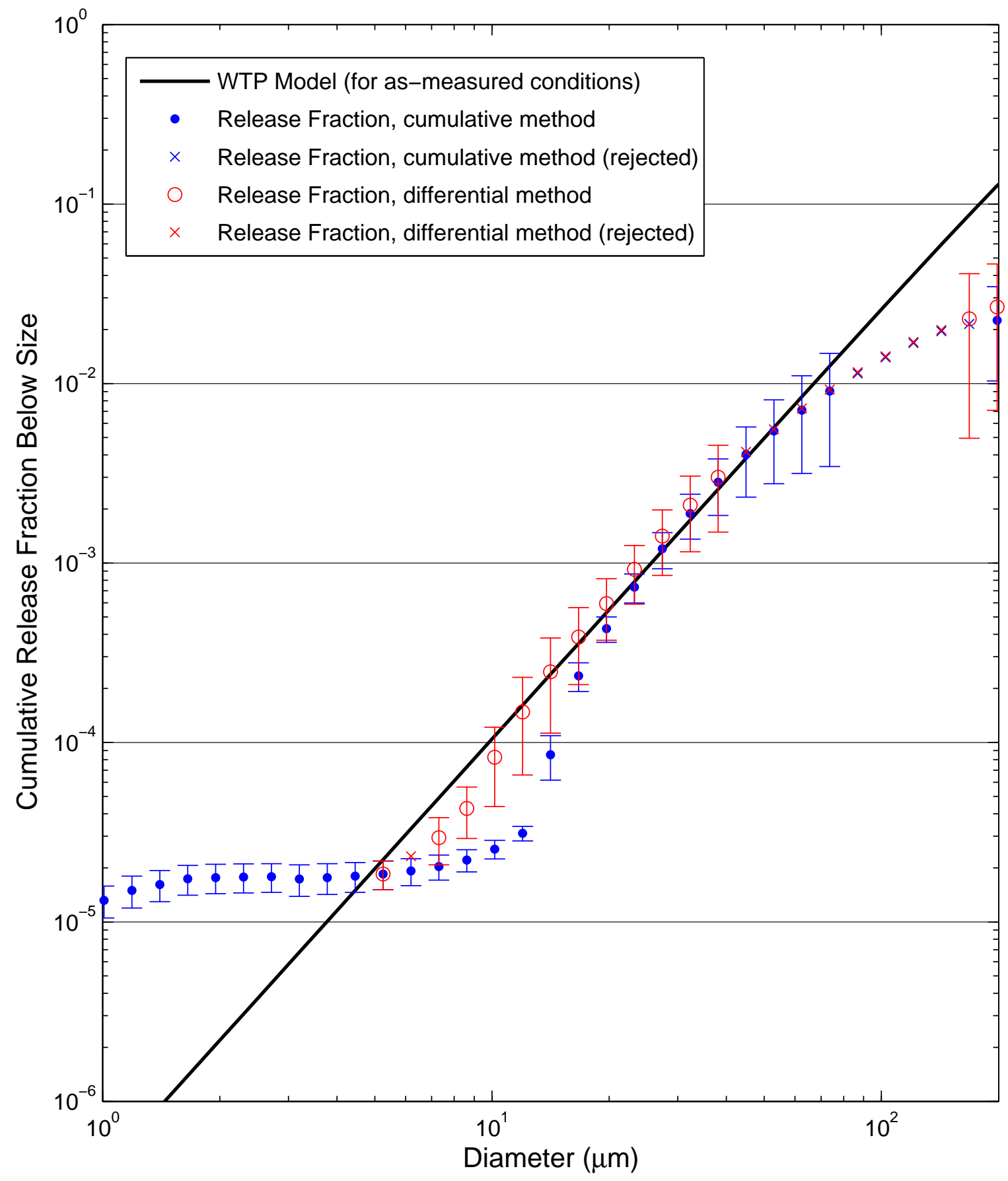


Test W95 [3 Malvern Average]: 100 psi, Orifice S7A - rectangular, $1 \times 10 \mathrm{~mm}$

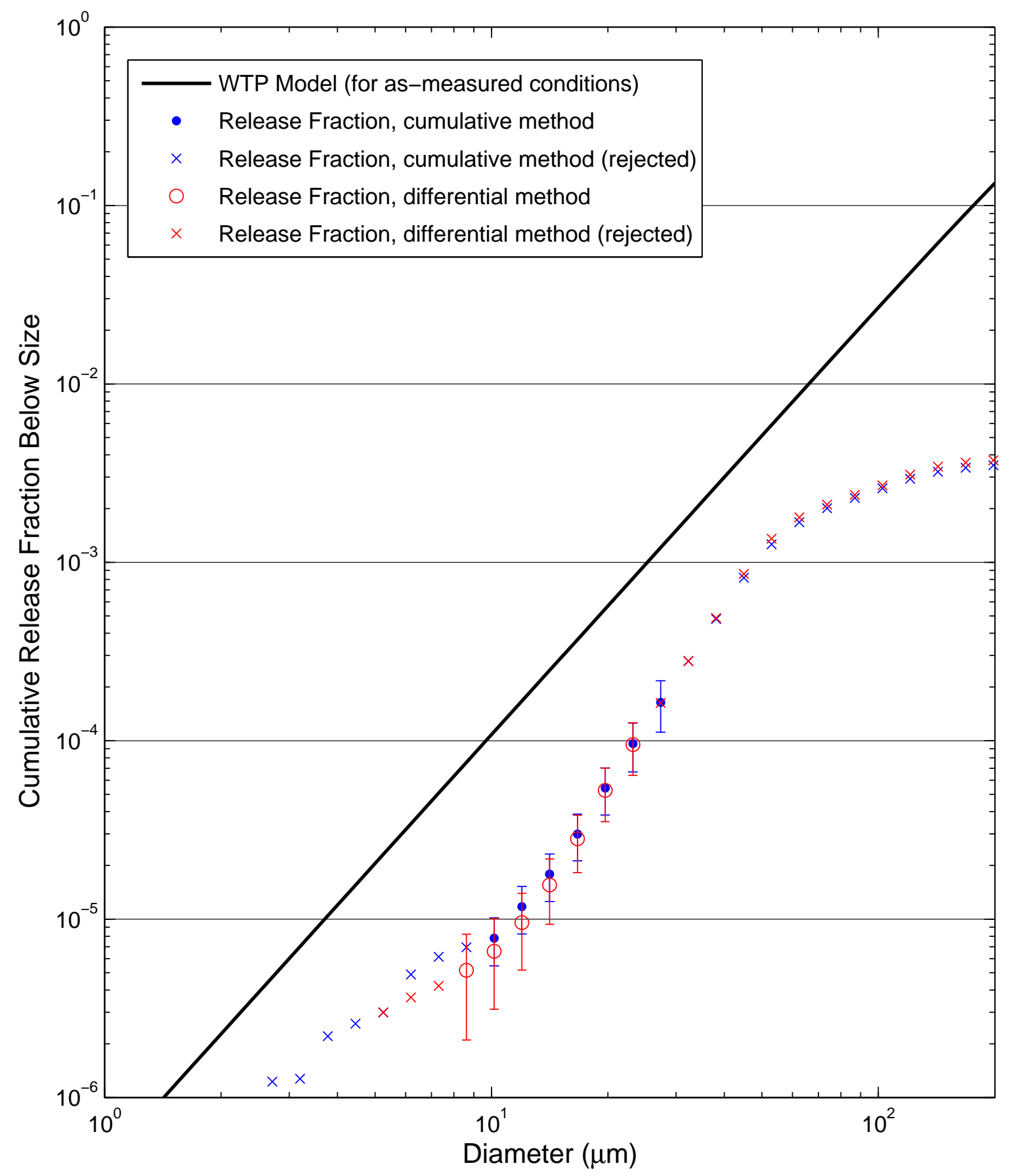


Test W96 [3 Malvern Average]: 380 psi, Orifice S7A - rectangular, $1 \times 10 \mathrm{~mm}$

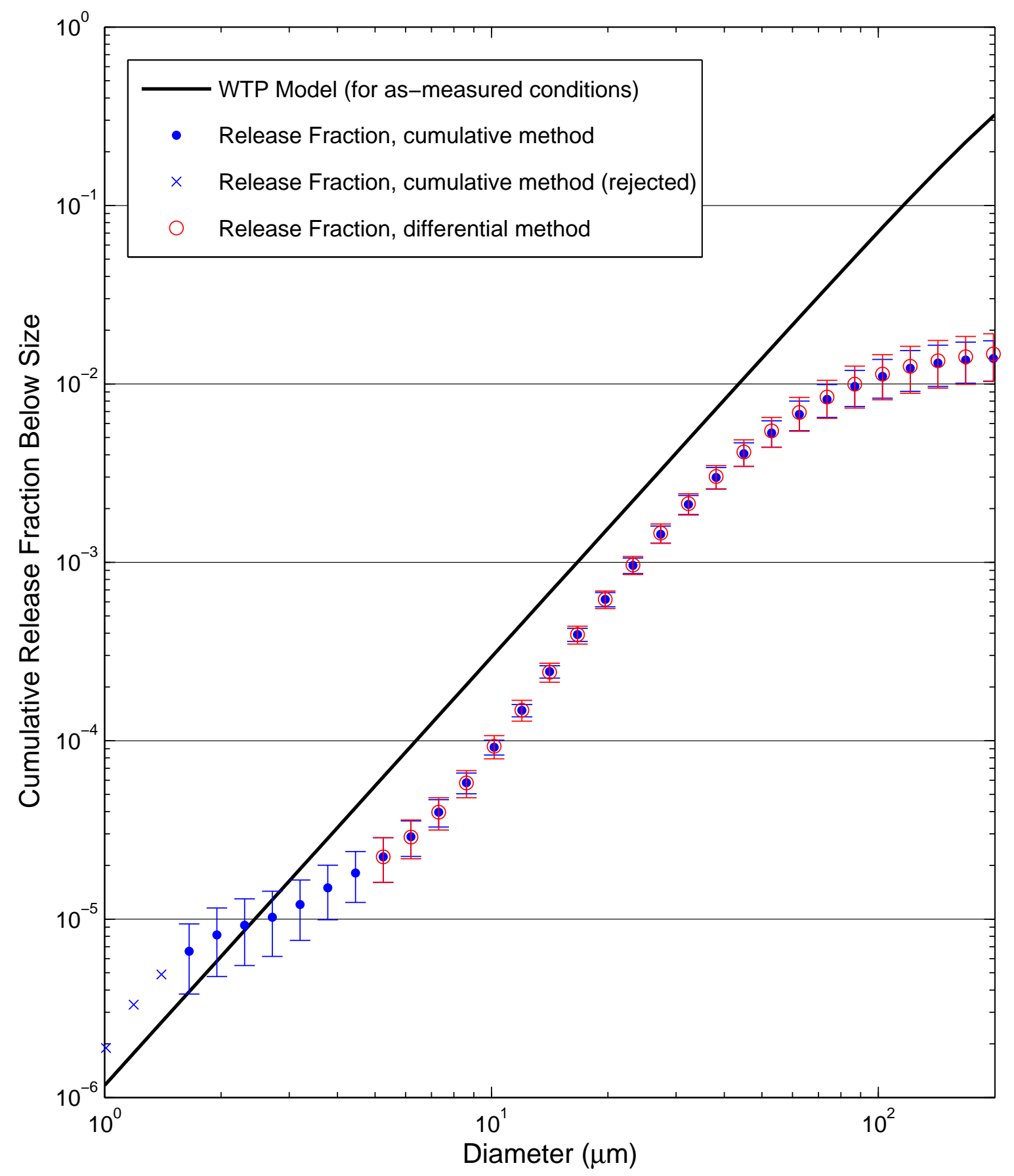


Test W97 [3 Malvern Average]: 100 psi, Orifice S7C - rectangular, $3 \times 10 \mathrm{~mm}$

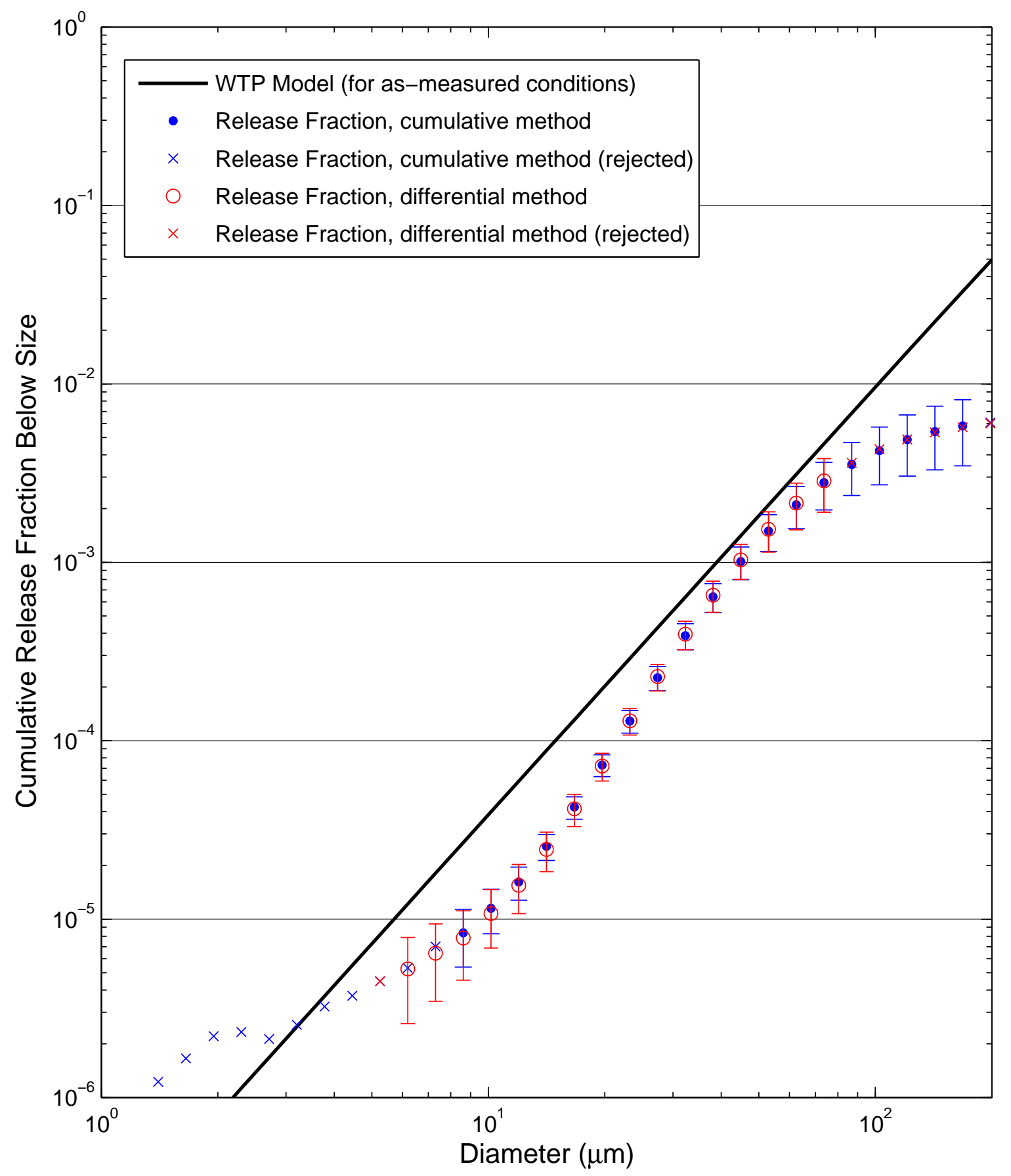


Test W98 [3 Malvern Average]: 380 psi, Orifice S7C - rectangular, $3 \times 10 \mathrm{~mm}$

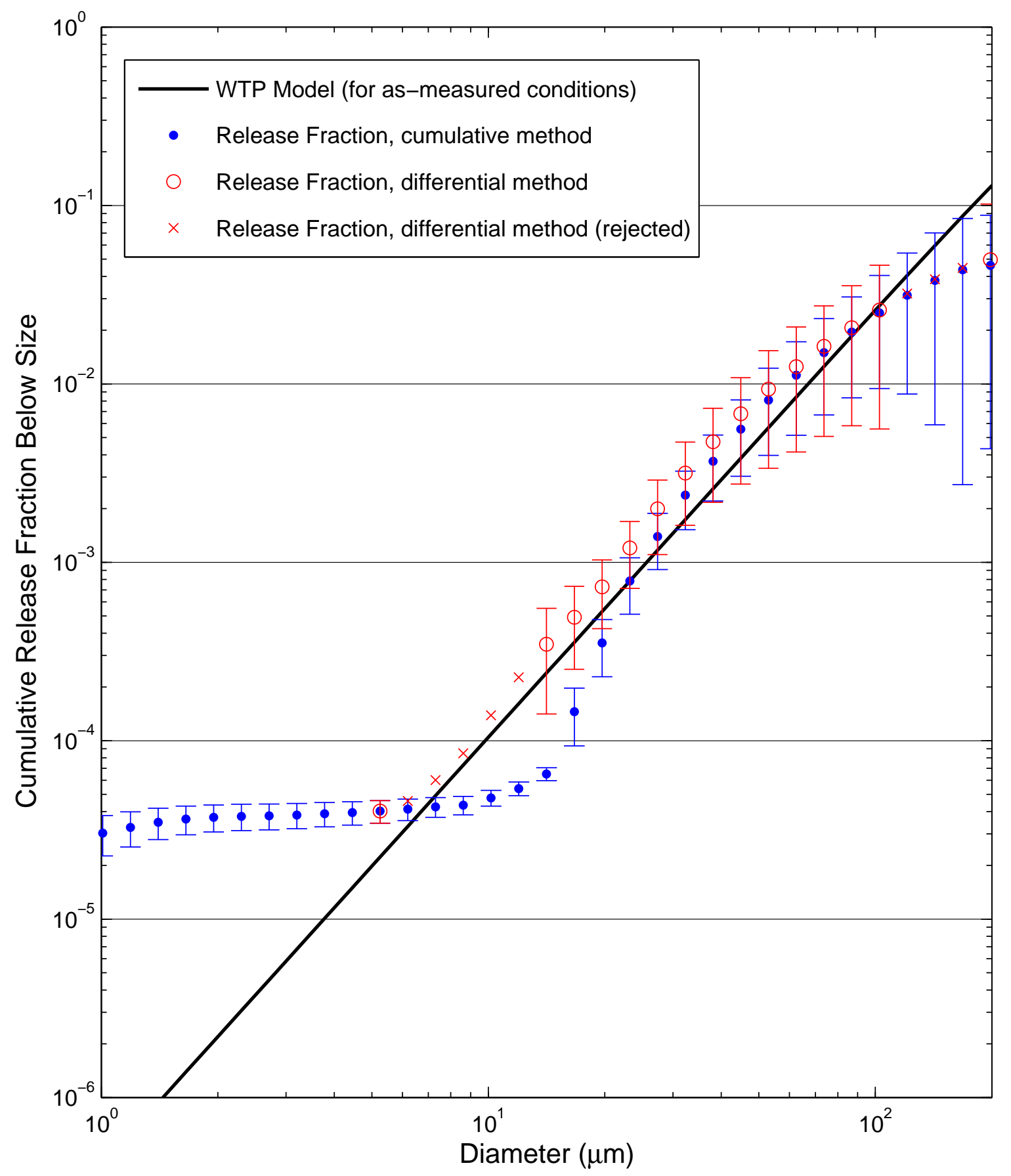


Test W99 [3 Malvern Average]: 100 psi, Orifice S5A - rectangular, 10× $1 \mathrm{~mm}$

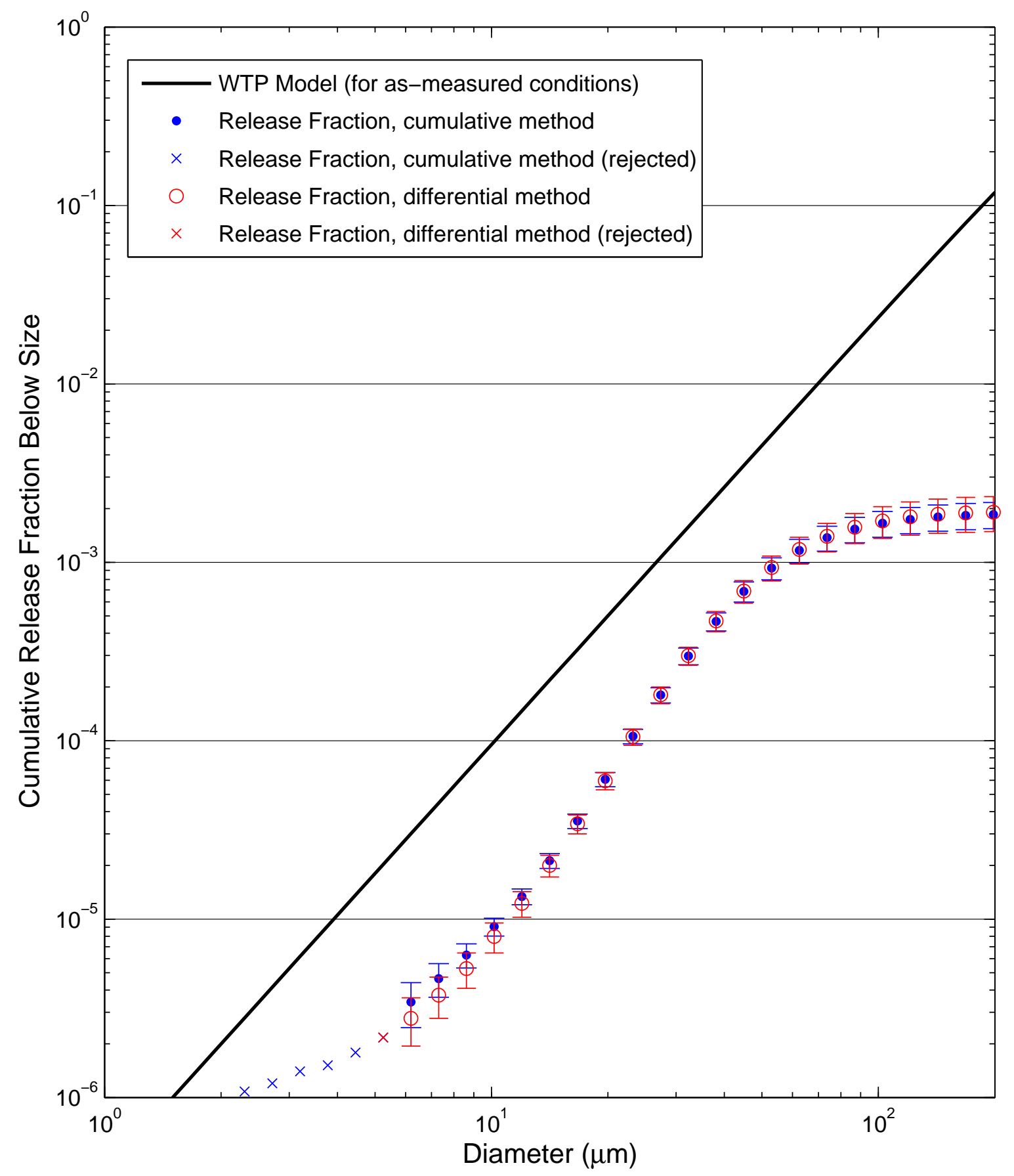


Test W100 [3 Malvern Average]: 200 psi, Orifice S5A - rectangular, 10× $1 \mathrm{~mm}$

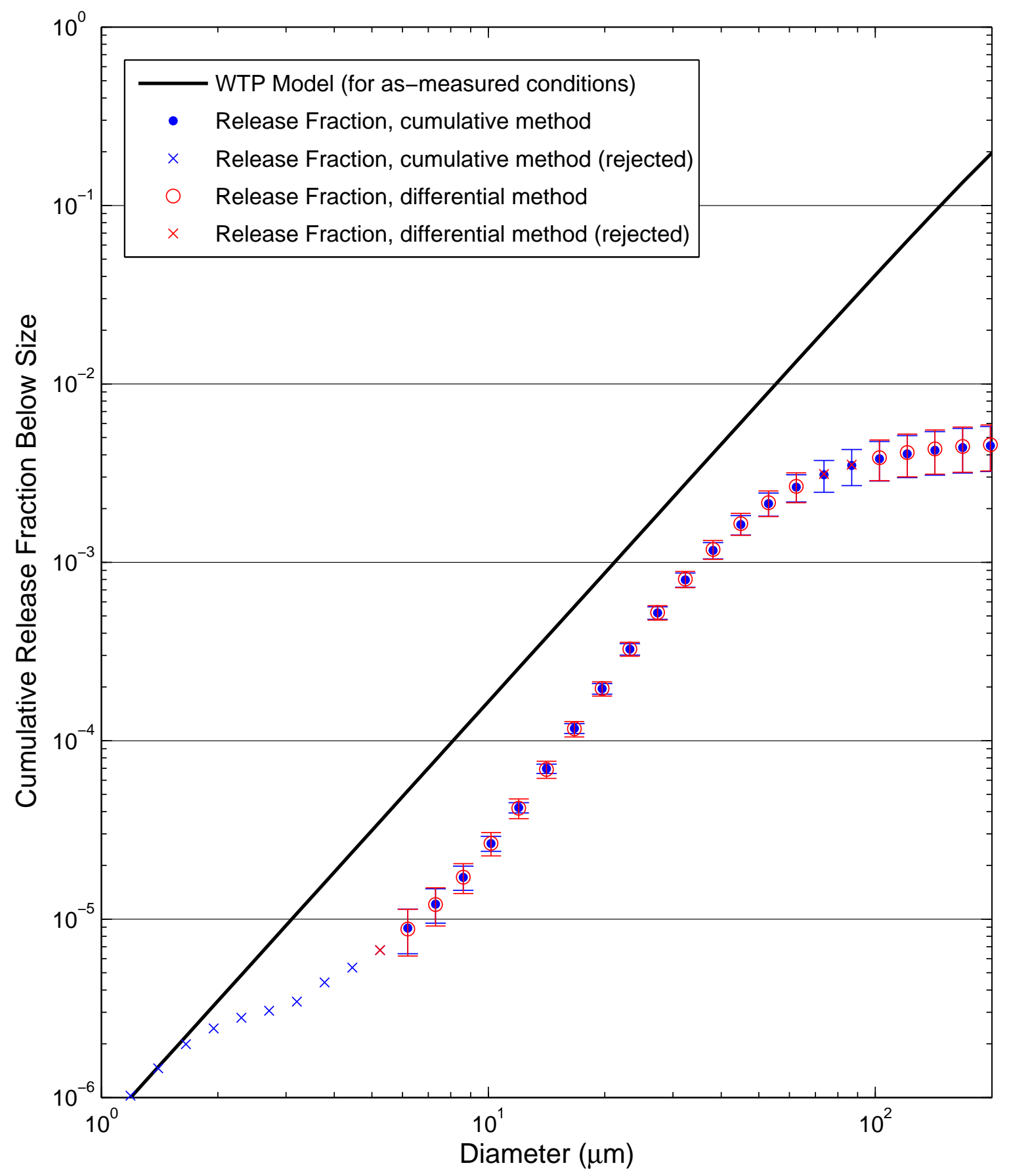


Test W101 [3 Malvern Average]: 380 psi, Orifice S5A - rectangular, 10× $1 \mathrm{~mm}$

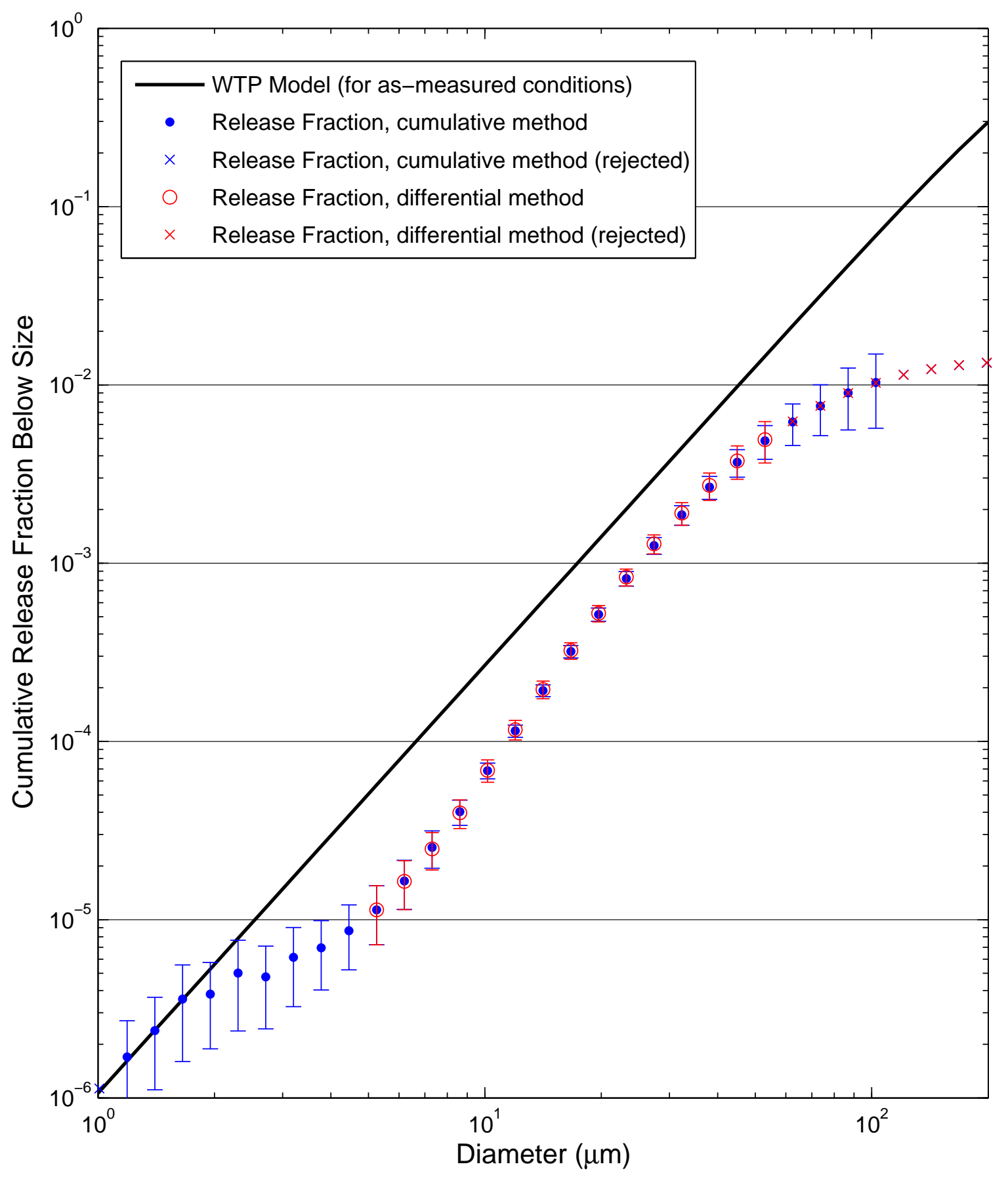


Test W102 [3 Malvern Average]: 100 psi, Orifice S5B - round, $1.982 \mathrm{~mm}$

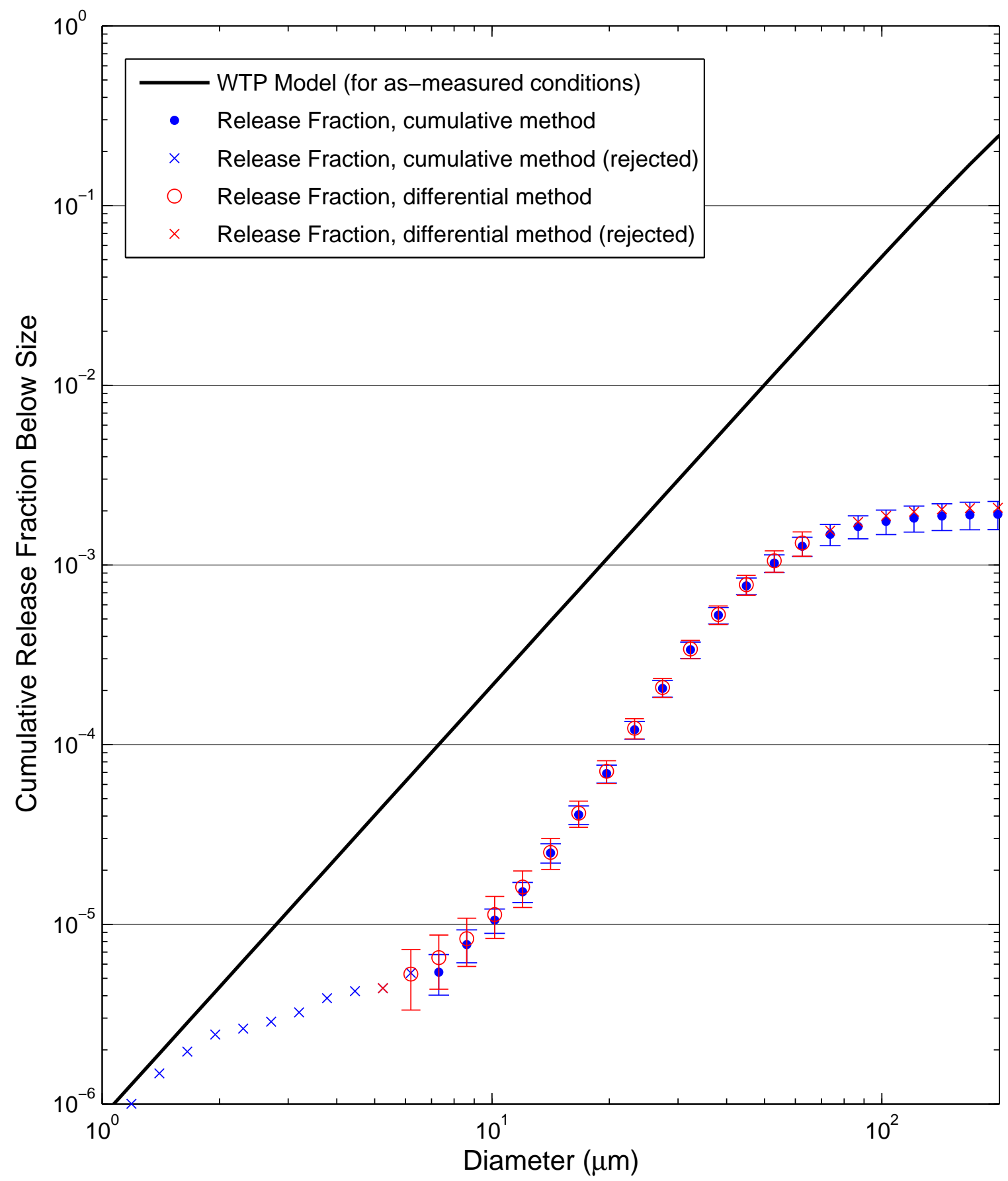


Test W103 [3 Malvern Average]: 200 psi, Orifice S5B - round, $1.982 \mathrm{~mm}$

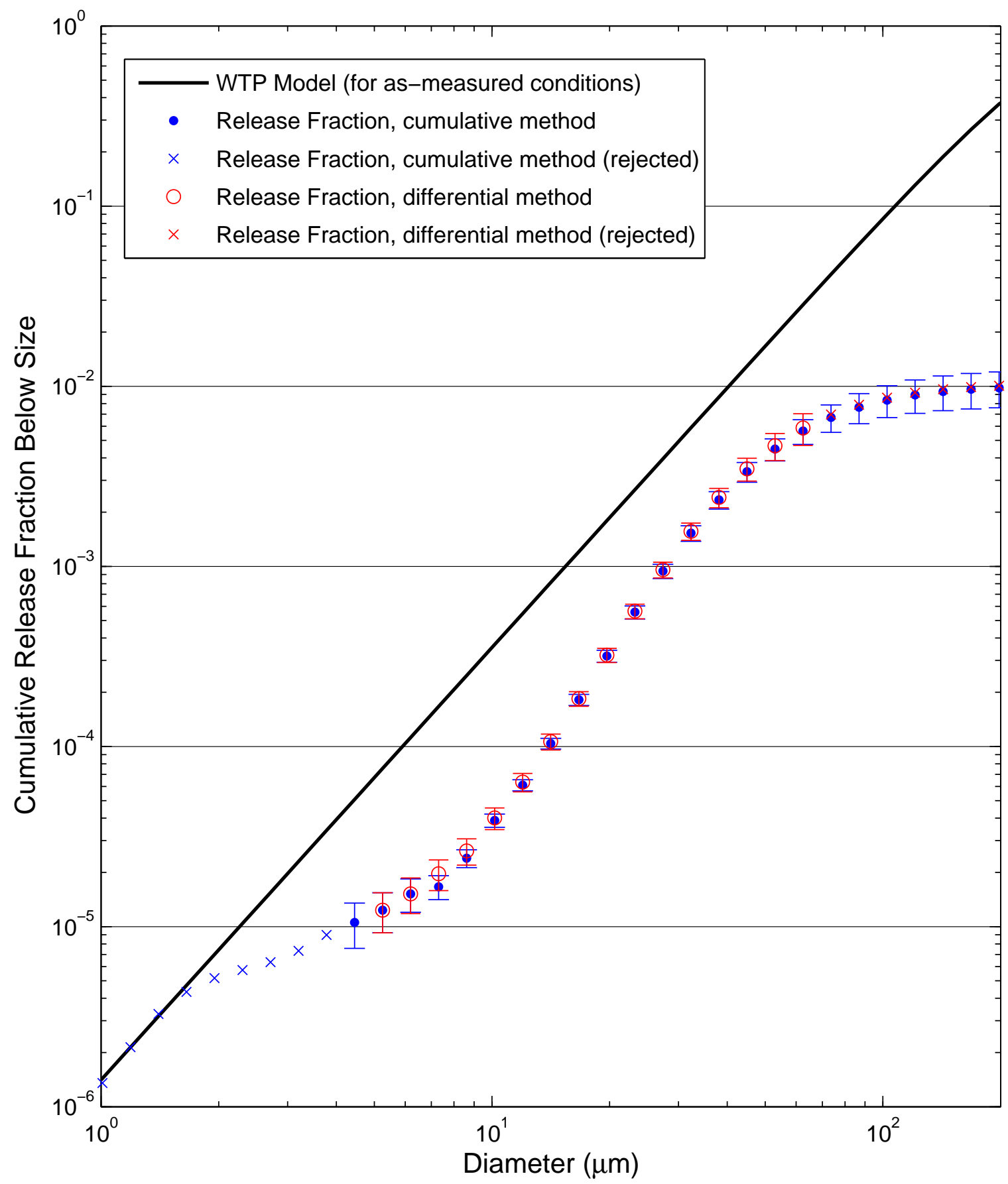


Test W104 [3 Malvern Average]: 380 psi, Orifice S5B - round, $1.982 \mathrm{~mm}$

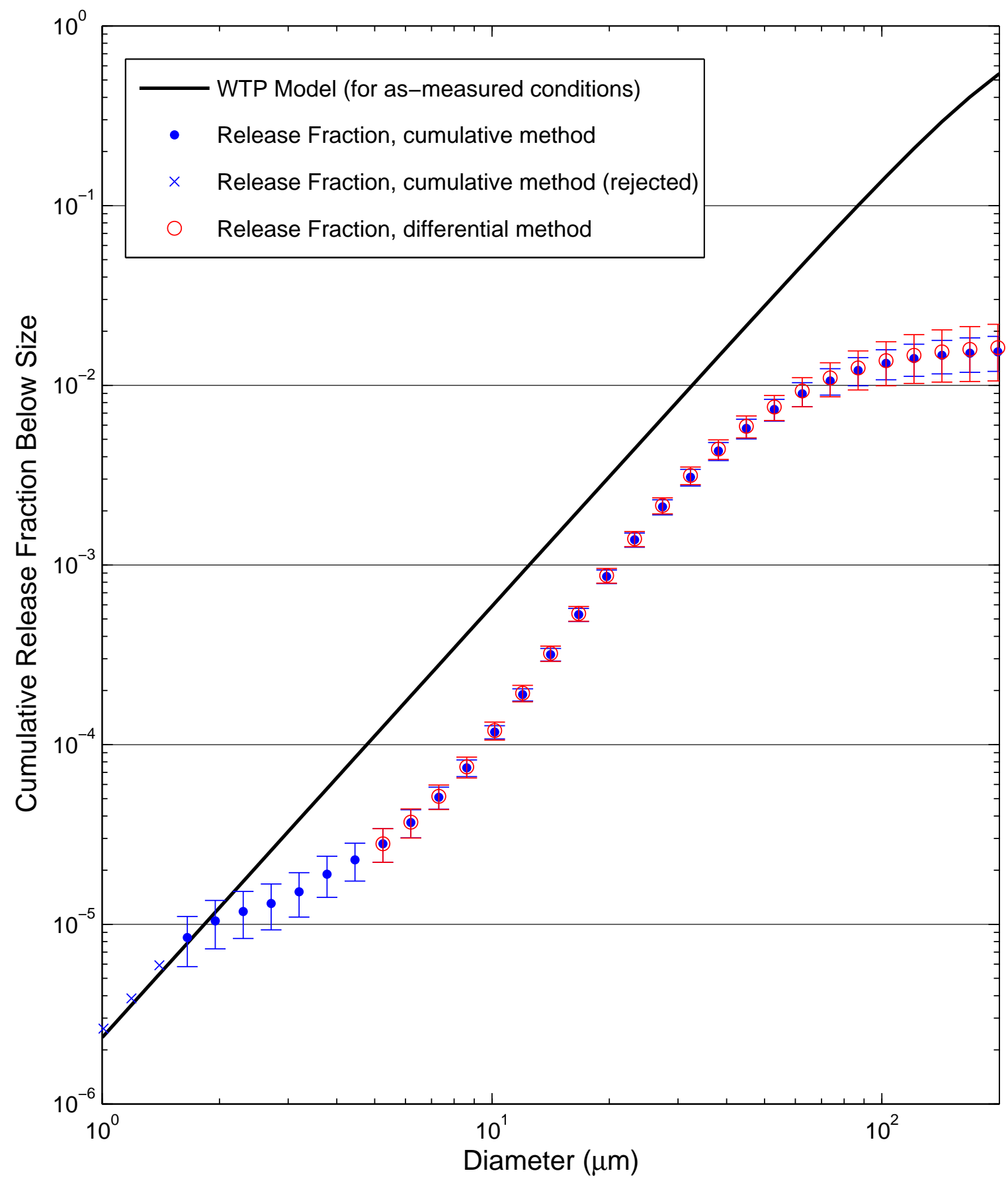


Test W105 [3 Malvern Average]: 380 psi, Orifice S5B - round, $1.982 \mathrm{~mm}$

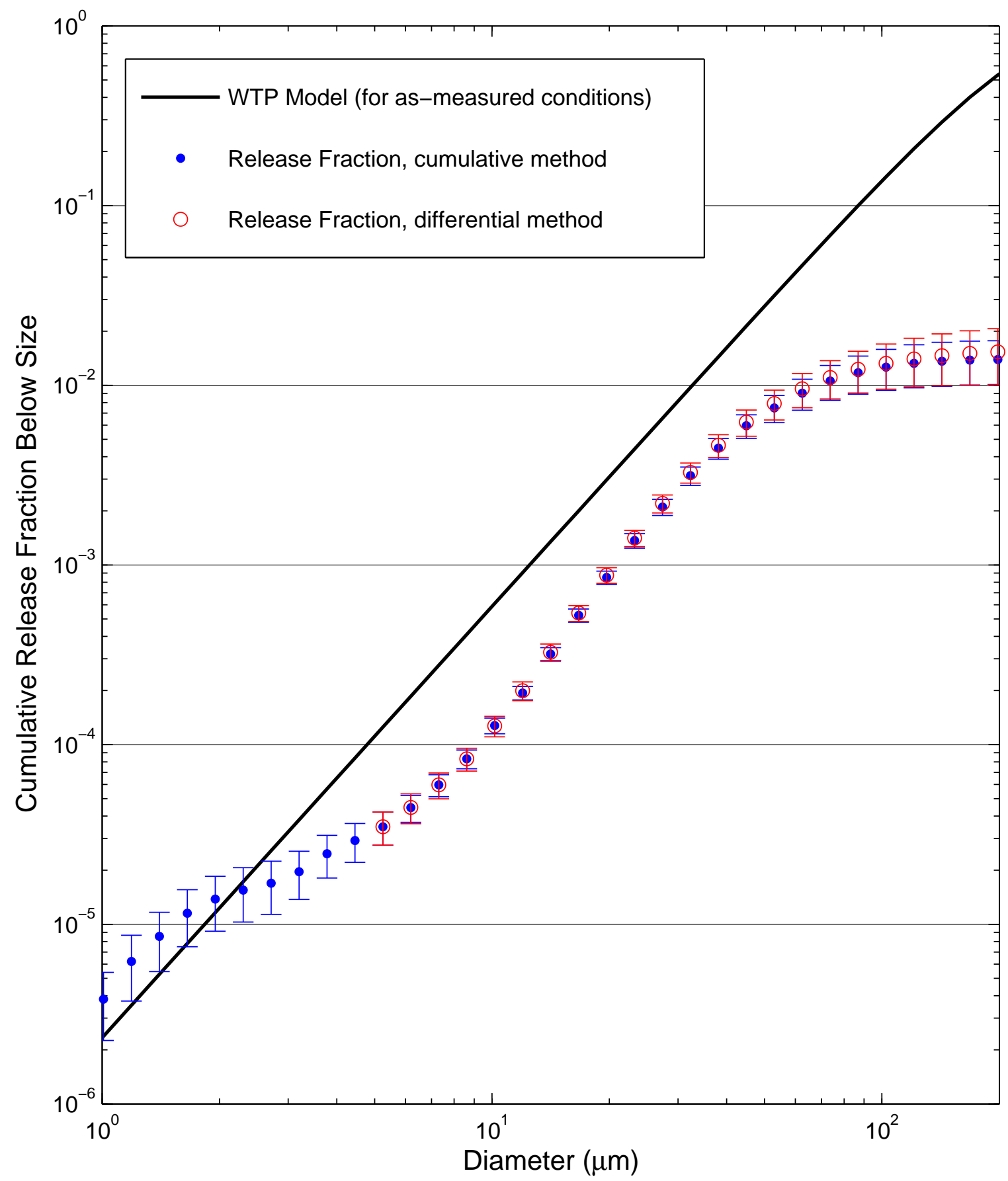


Test W106 [3 Malvern Average]: 100 psi, Orifice S5C - rectangular, $1 \times 20 \mathrm{~mm}$

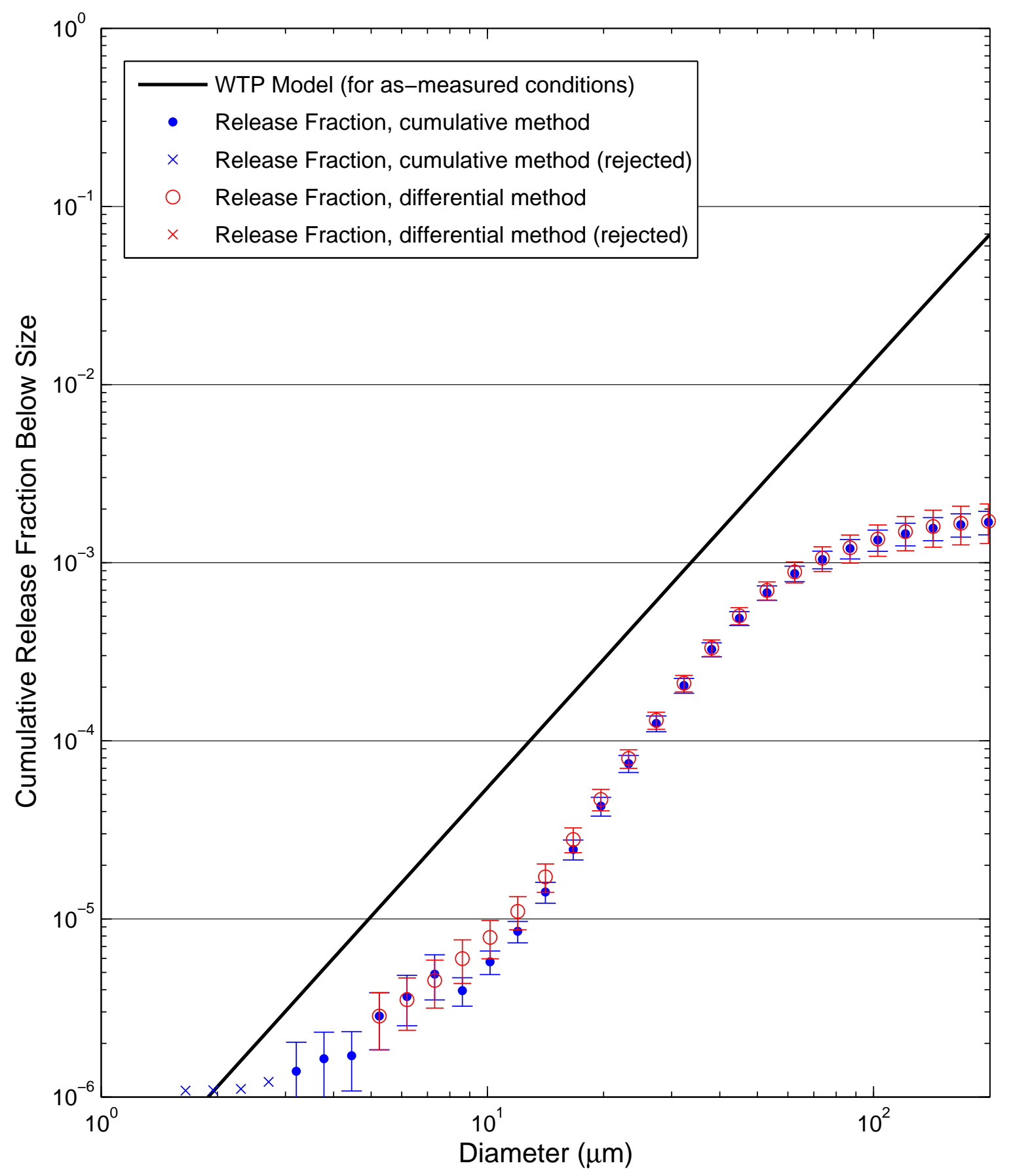


Test W107 [3 Malvern Average]: 200 psi, Orifice S5C - rectangular, $1 \times 20 \mathrm{~mm}$

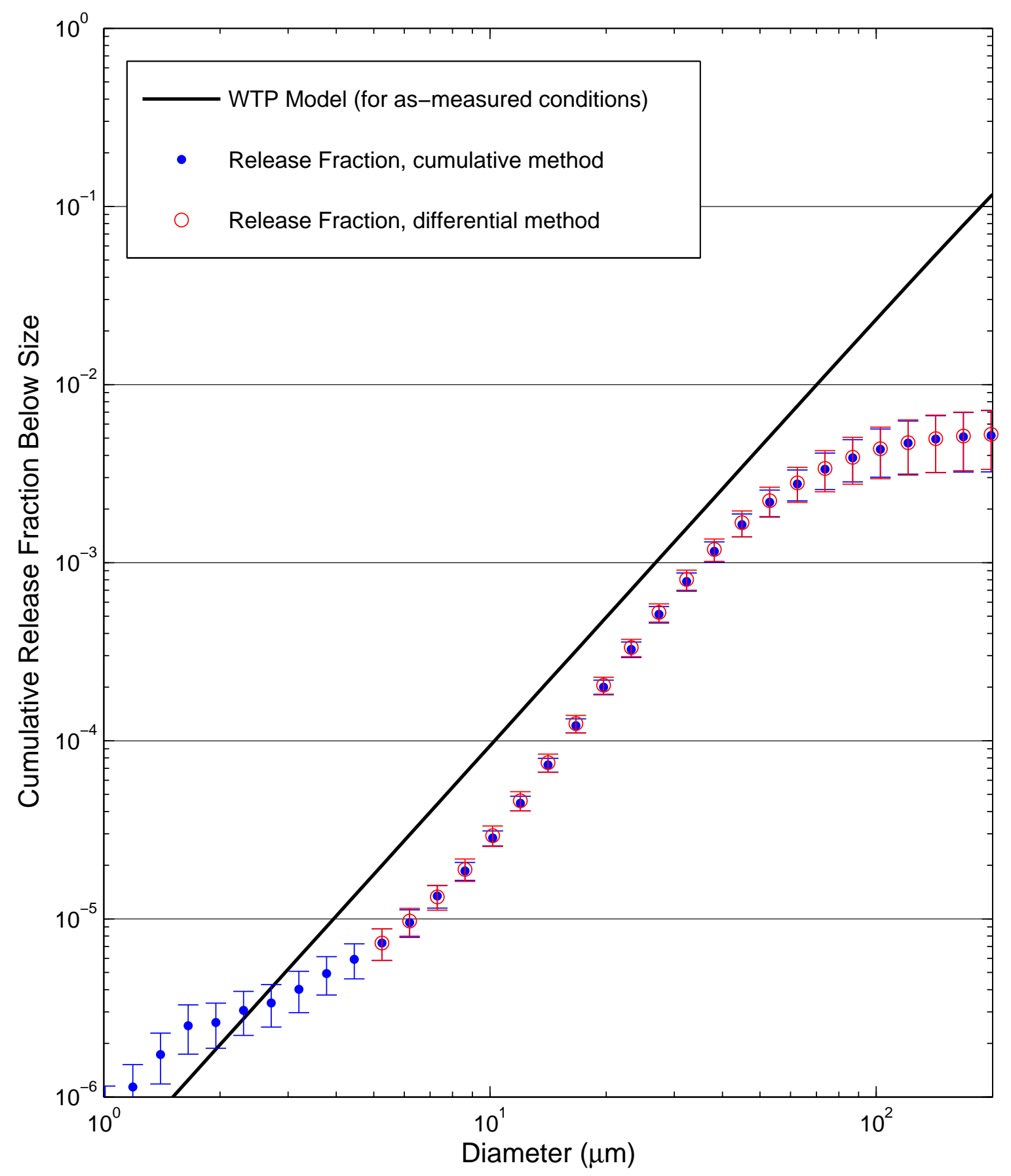


Test W108 [3 Malvern Average]: 380 psi, Orifice S5C - rectangular, $1 \times 20 \mathrm{~mm}$

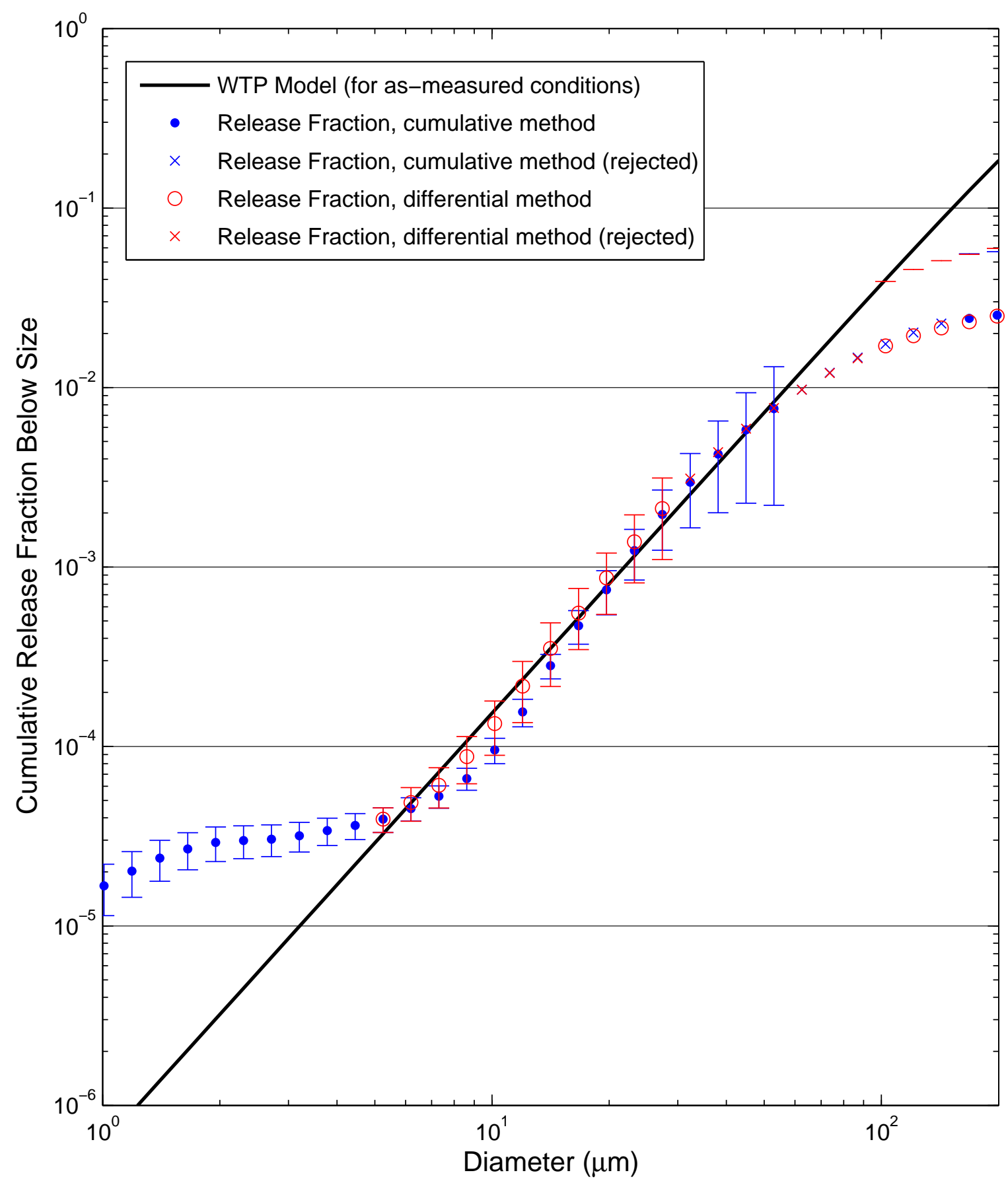


Test W109 [3 Malvern Average]: 100 psi, Orifice S5A - rectangular, 10× $1 \mathrm{~mm}$

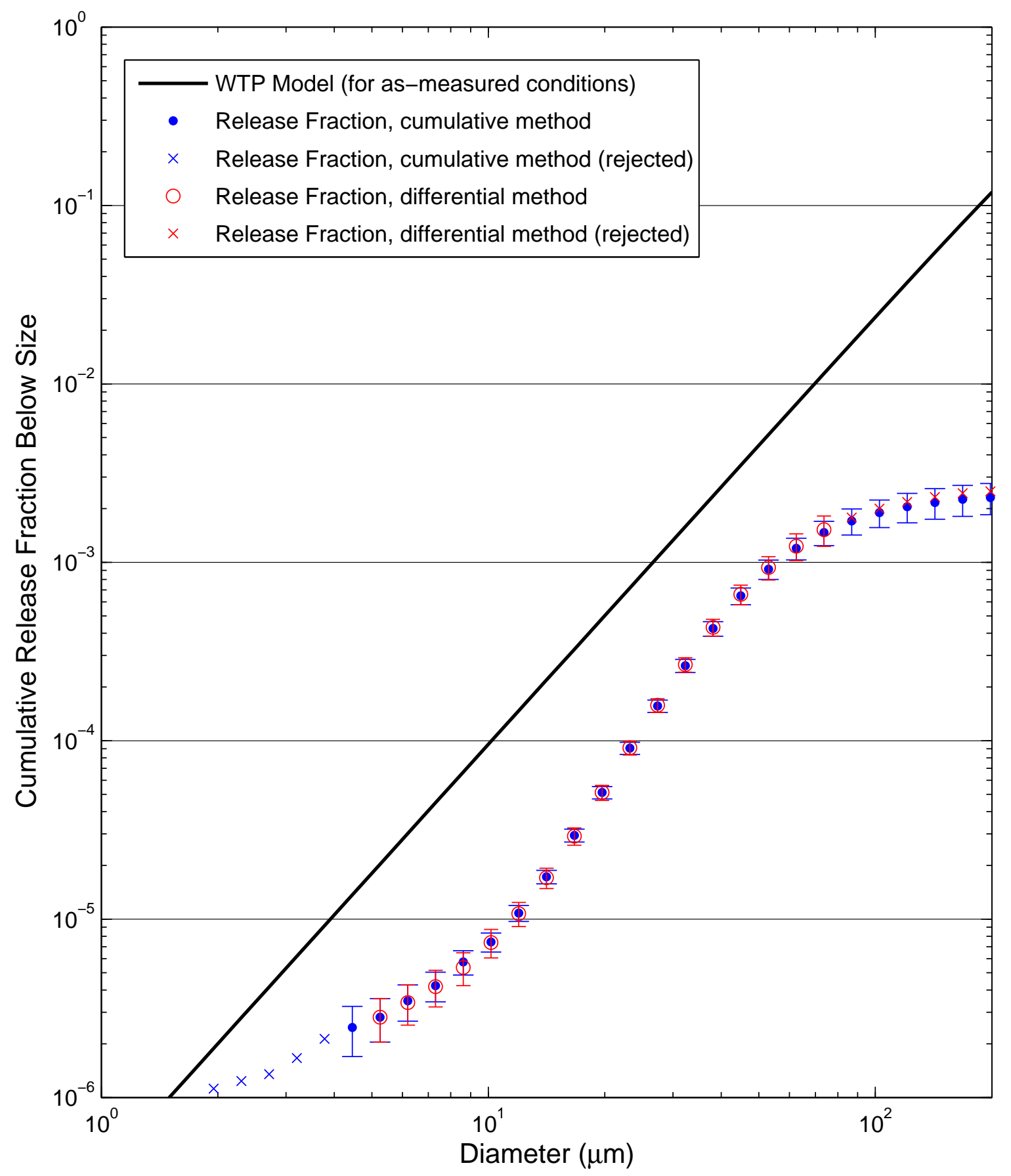


Test W110 [3 Malvern Average]: 380 psi, Orifice S5A - rectangular, 10× $1 \mathrm{~mm}$

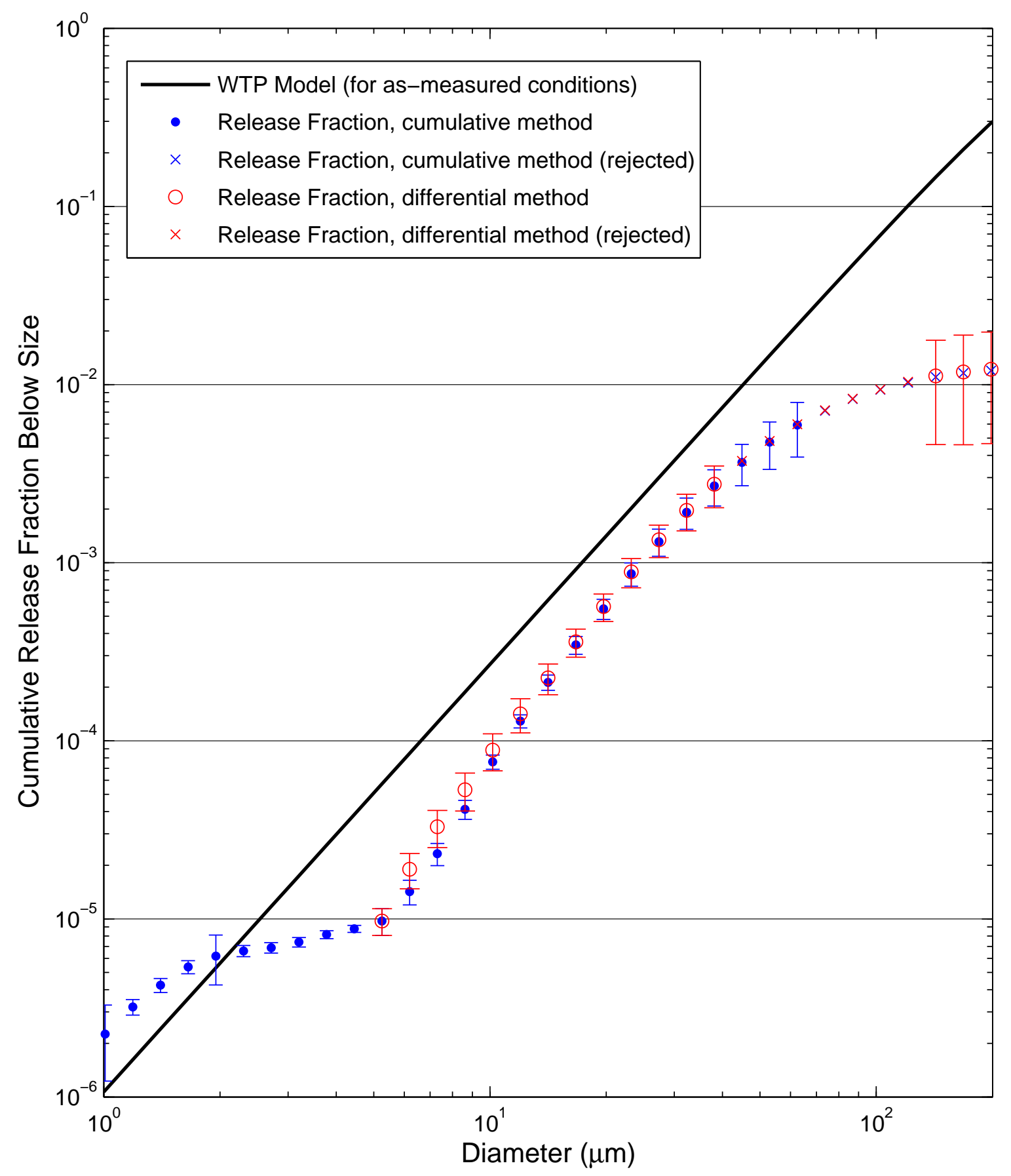


Test W111 [3 Malvern Average]: 100 psi, Orifice S5B - round, $1.982 \mathrm{~mm}$

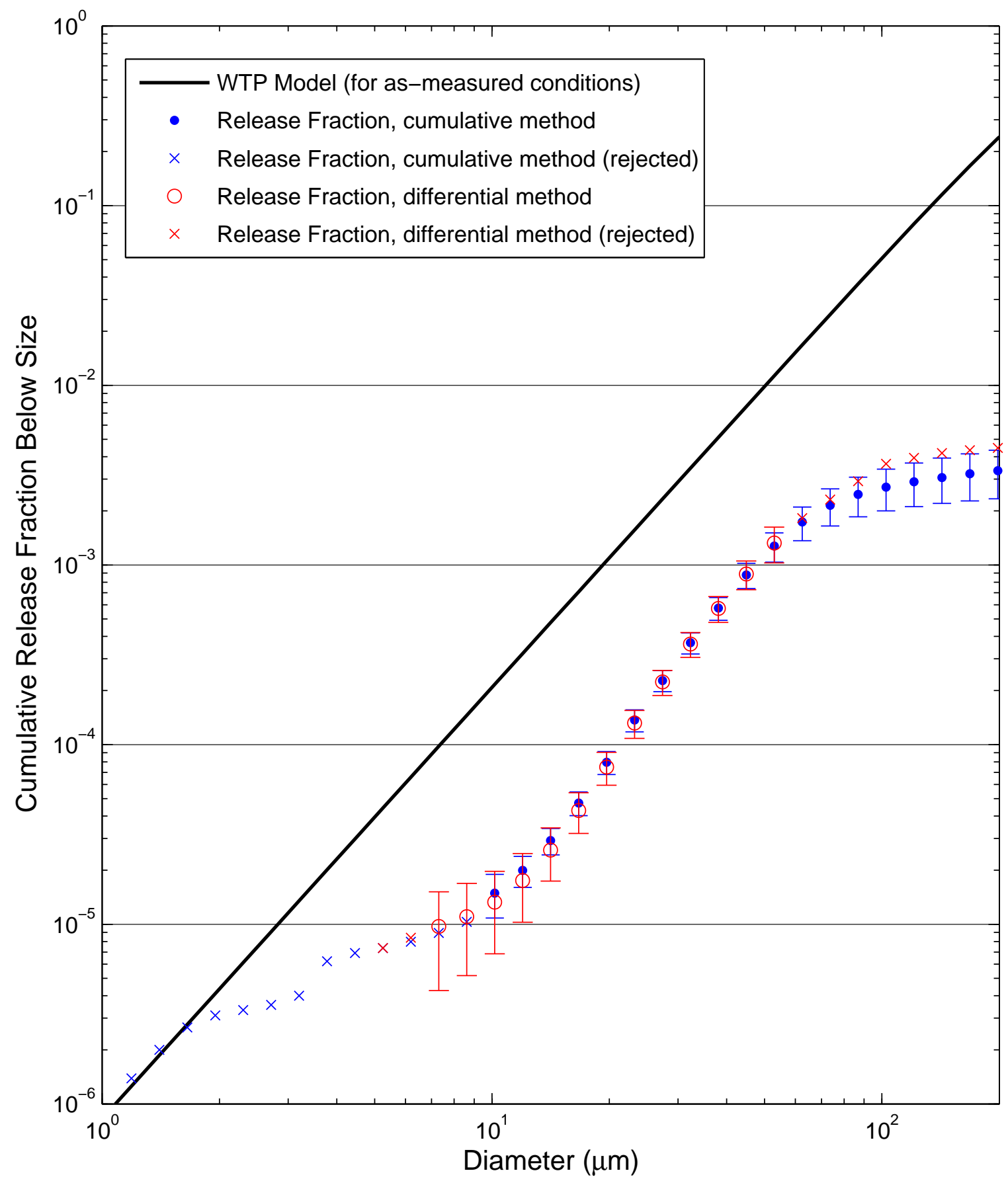


Test W112 [3 Malvern Average]: 380 psi, Orifice S5B - round, $1.982 \mathrm{~mm}$

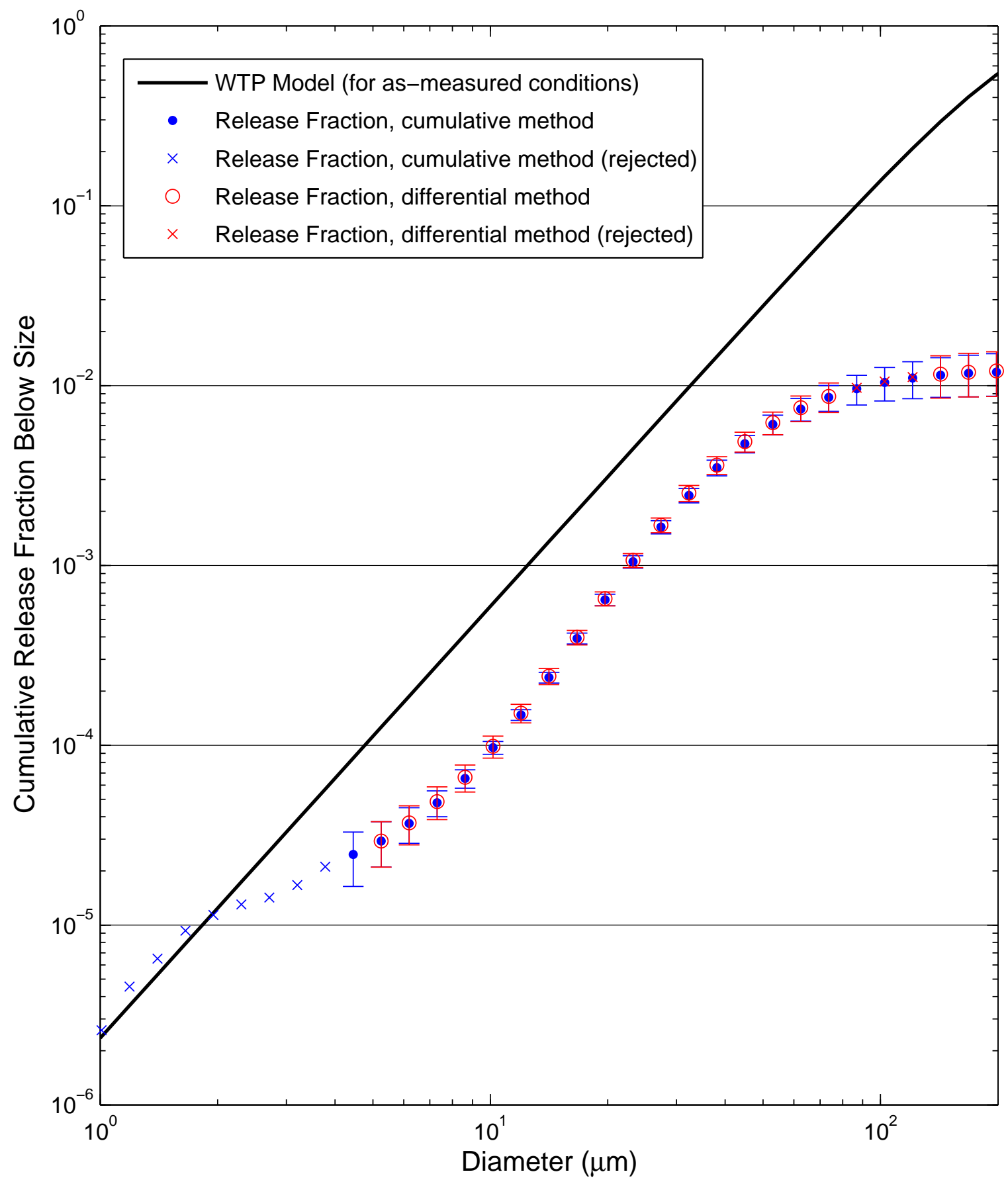


Test W113 [3 Malvern Average]: 100 psi, Orifice S5C - rectangular, $1 \times 20 \mathrm{~mm}$

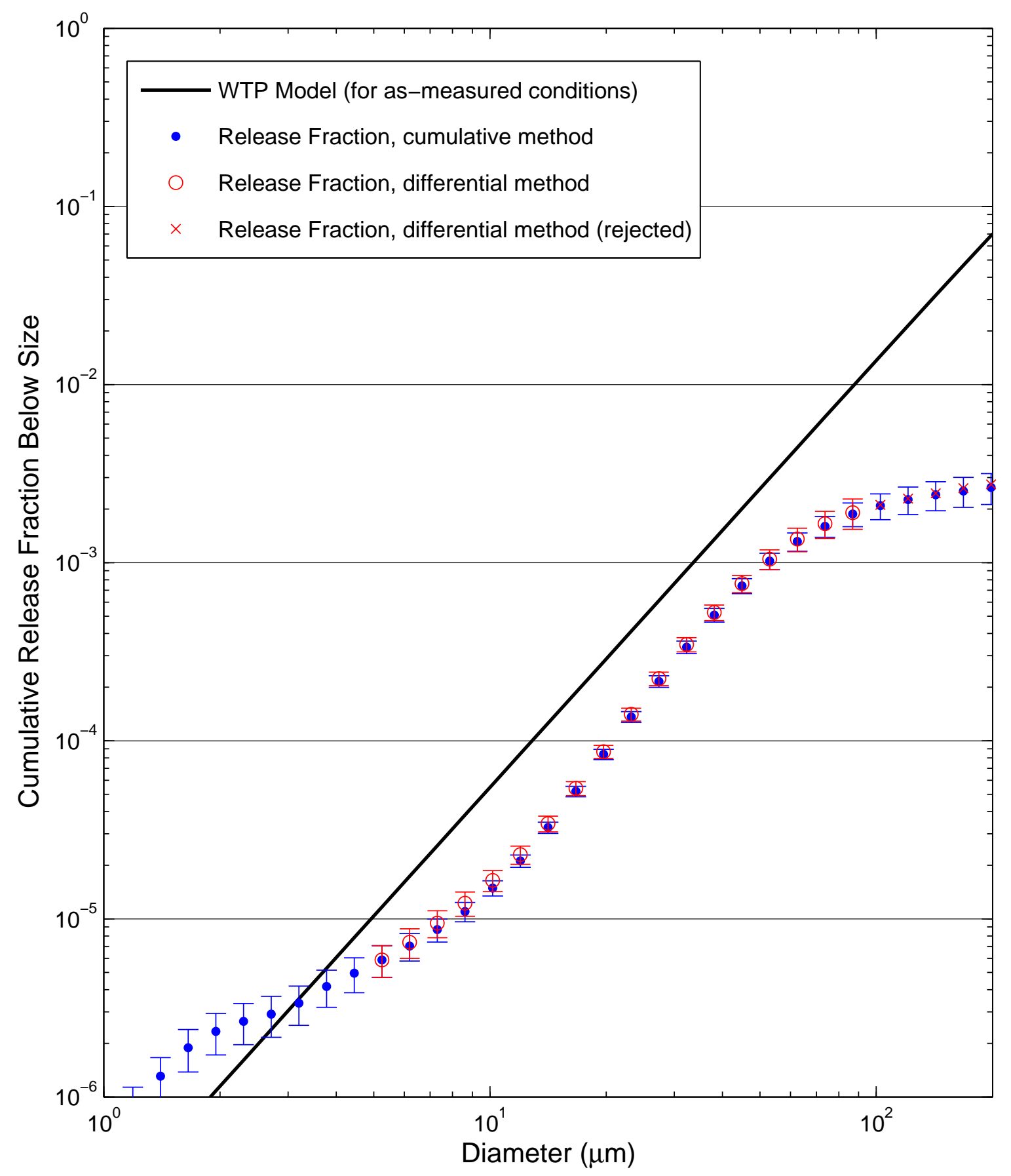

B.72 
Test W114 [3 Malvern Average]: 380 psi, Orifice S5C - rectangular, $1 \times 20 \mathrm{~mm}$

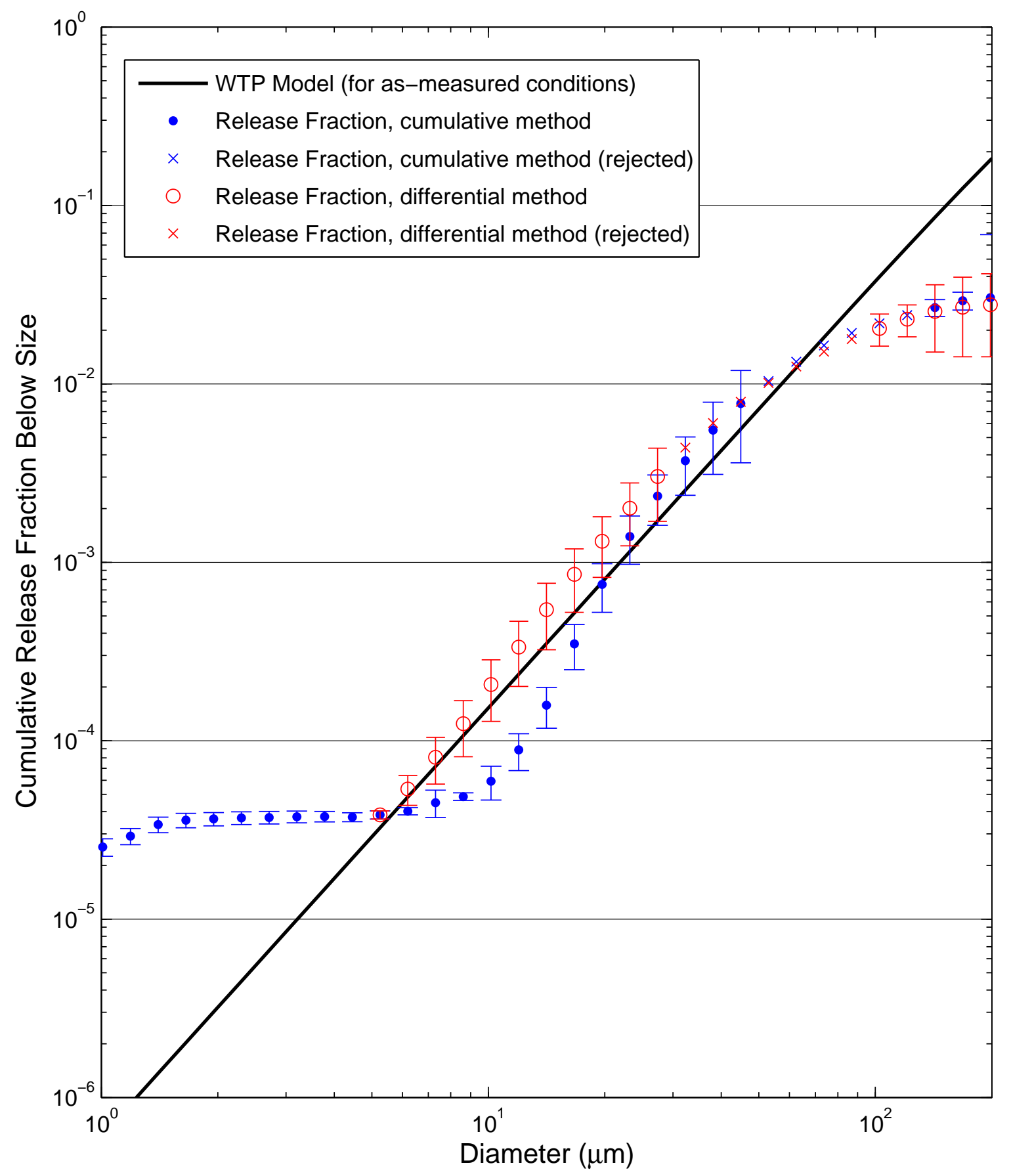


Test W115 [3 Malvern Average]: 100 psi, Orifice S4A - rectangular, 1× $76.2 \mathrm{~mm}$

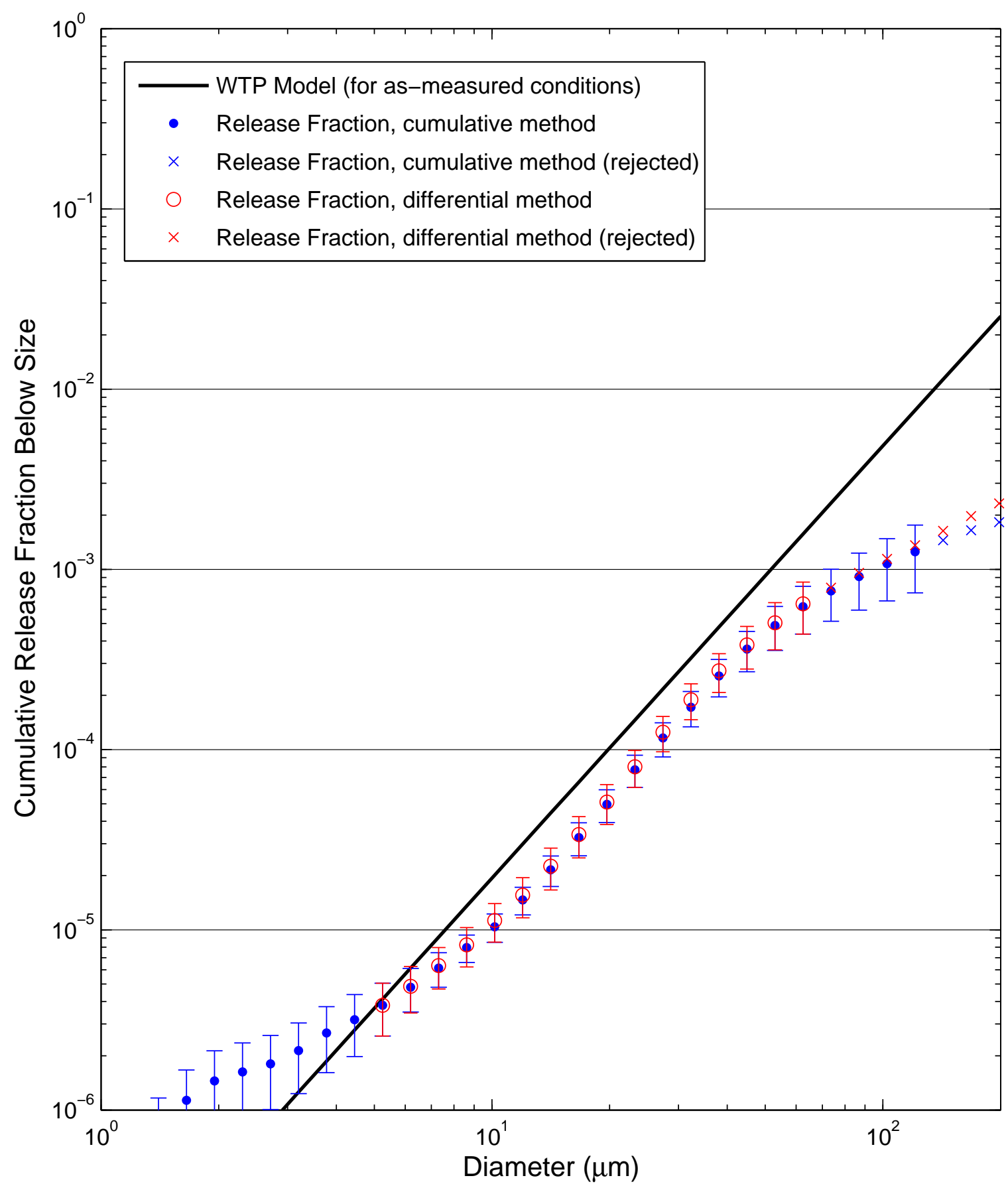


Test W116 [3 Malvern Average]: 200 psi, Orifice S4A - rectangular, $1 \times 76.2$ mm

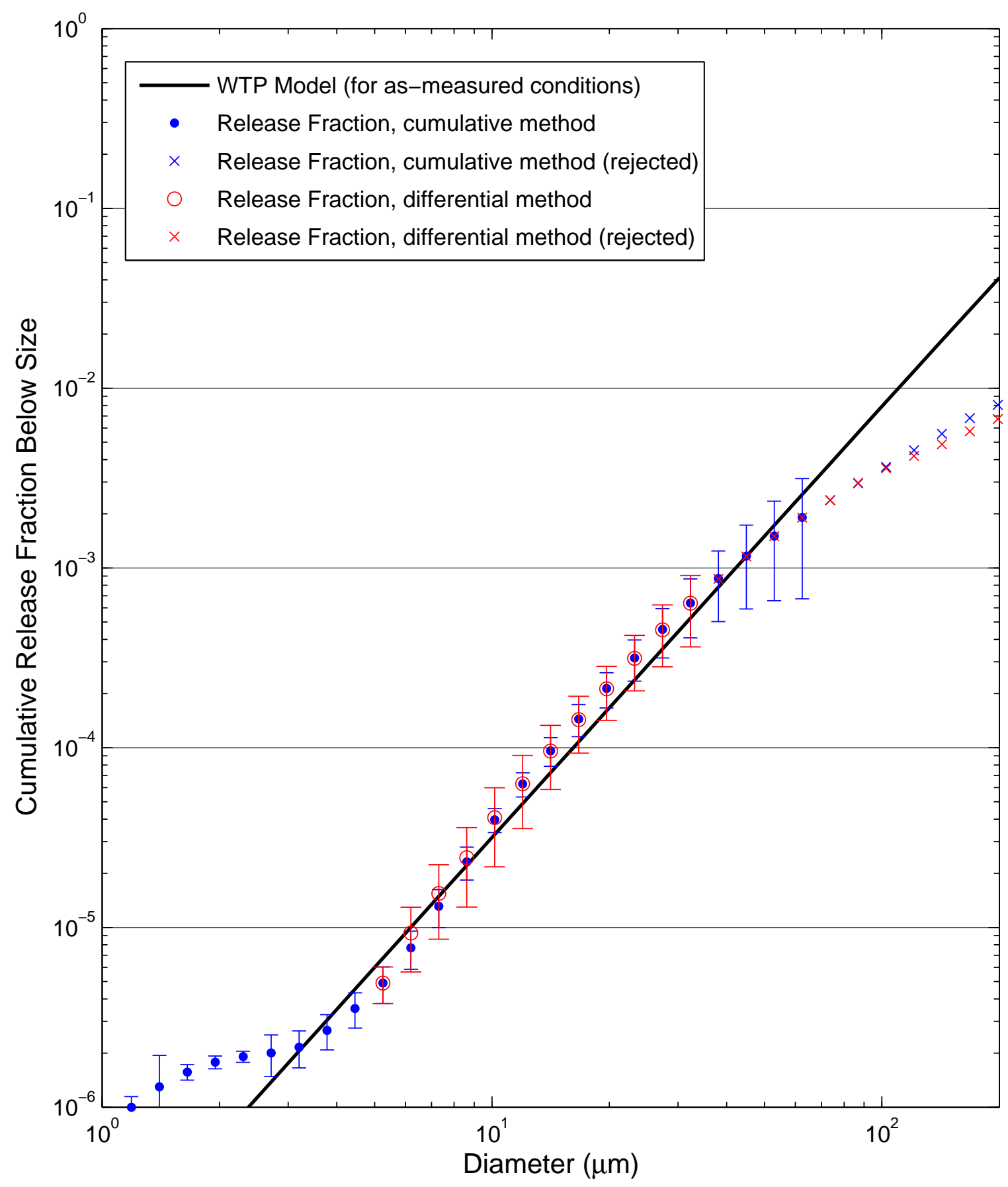


Test W117 [3 Malvern Average]: 100 psi, Orifice S4A - rectangular, 1× $76.2 \mathrm{~mm}$

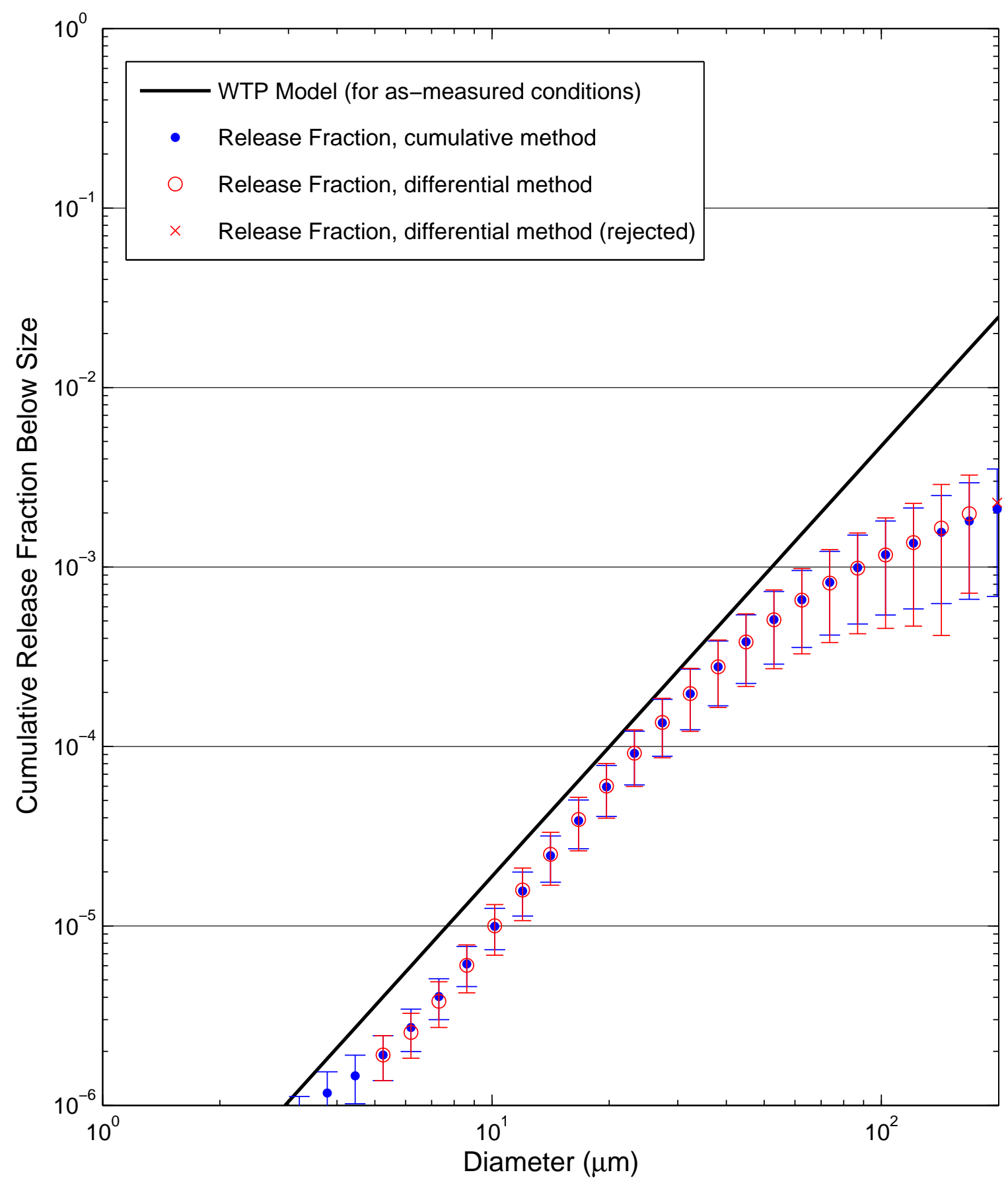


Test W118 [3 Malvern Average]: 200 psi, Orifice S4A - rectangular, $1 \times 76.2$ mm

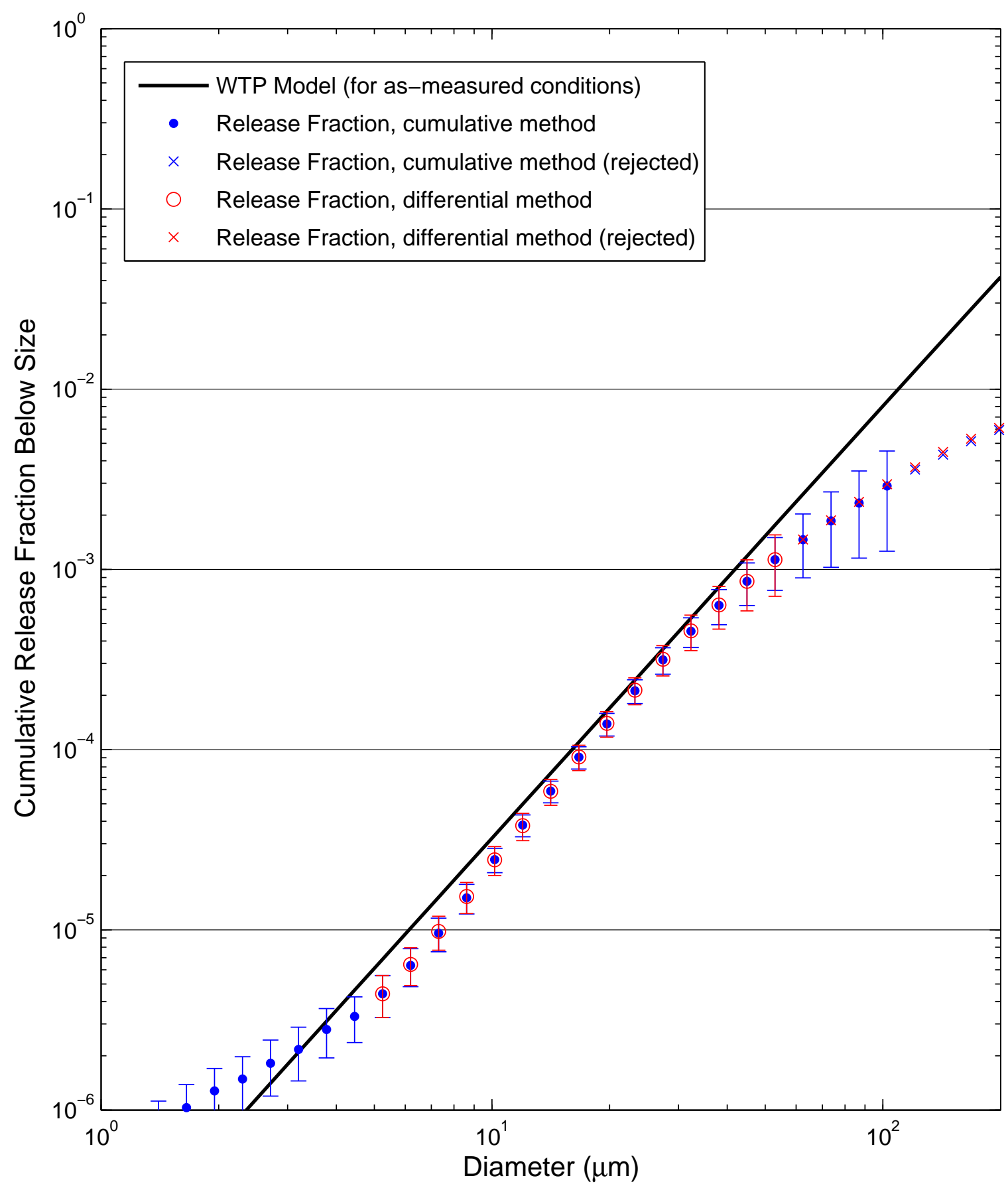


Test W119 [3 Malvern Average]: 380 psi, Orifice S4A - rectangular, 1× $76.2 \mathrm{~mm}$

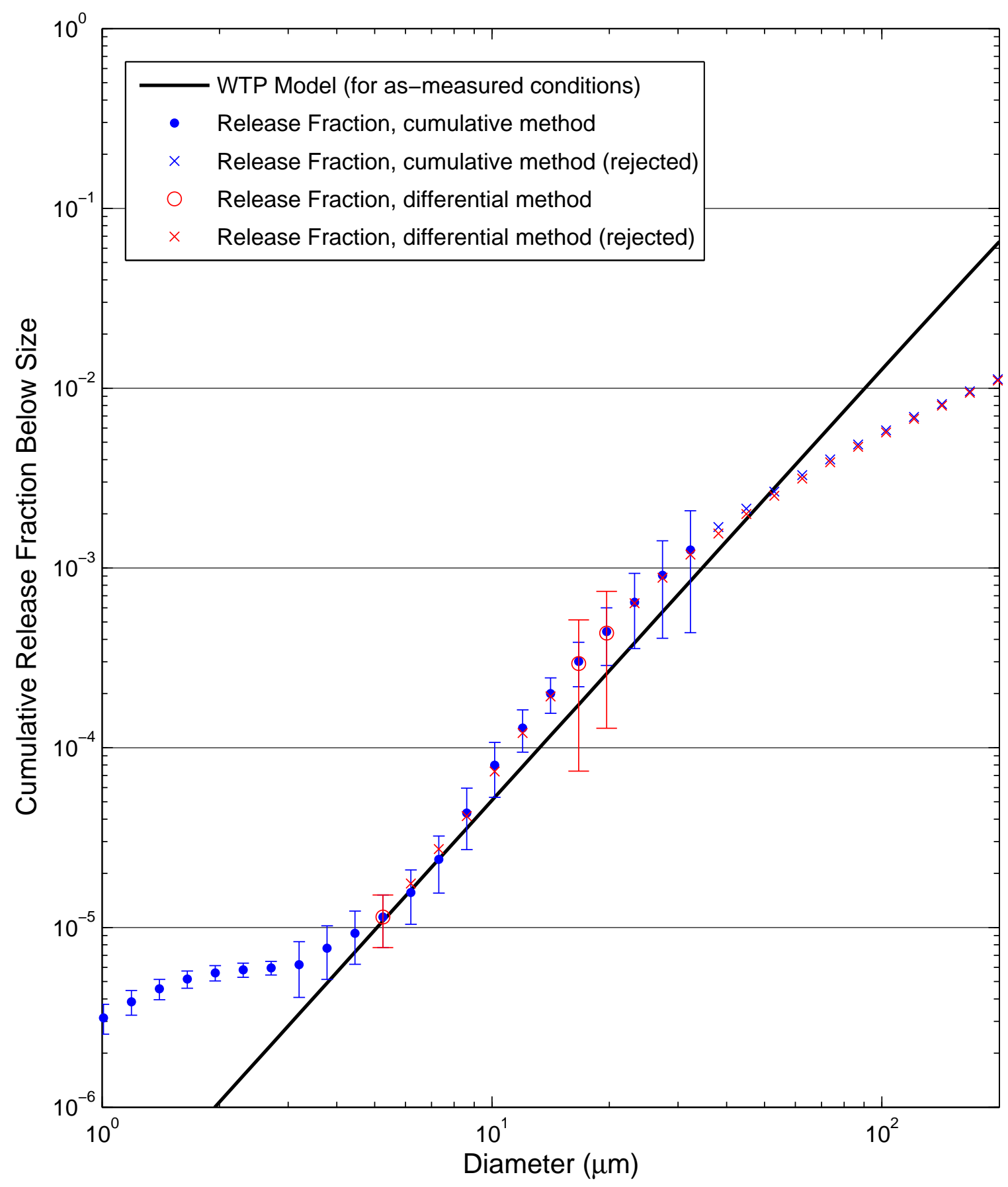


Test W120 [3 Malvern Average]: 100 psi, Orifice S2A - rectangular, $0.5 \times 5 \mathrm{~mm}$

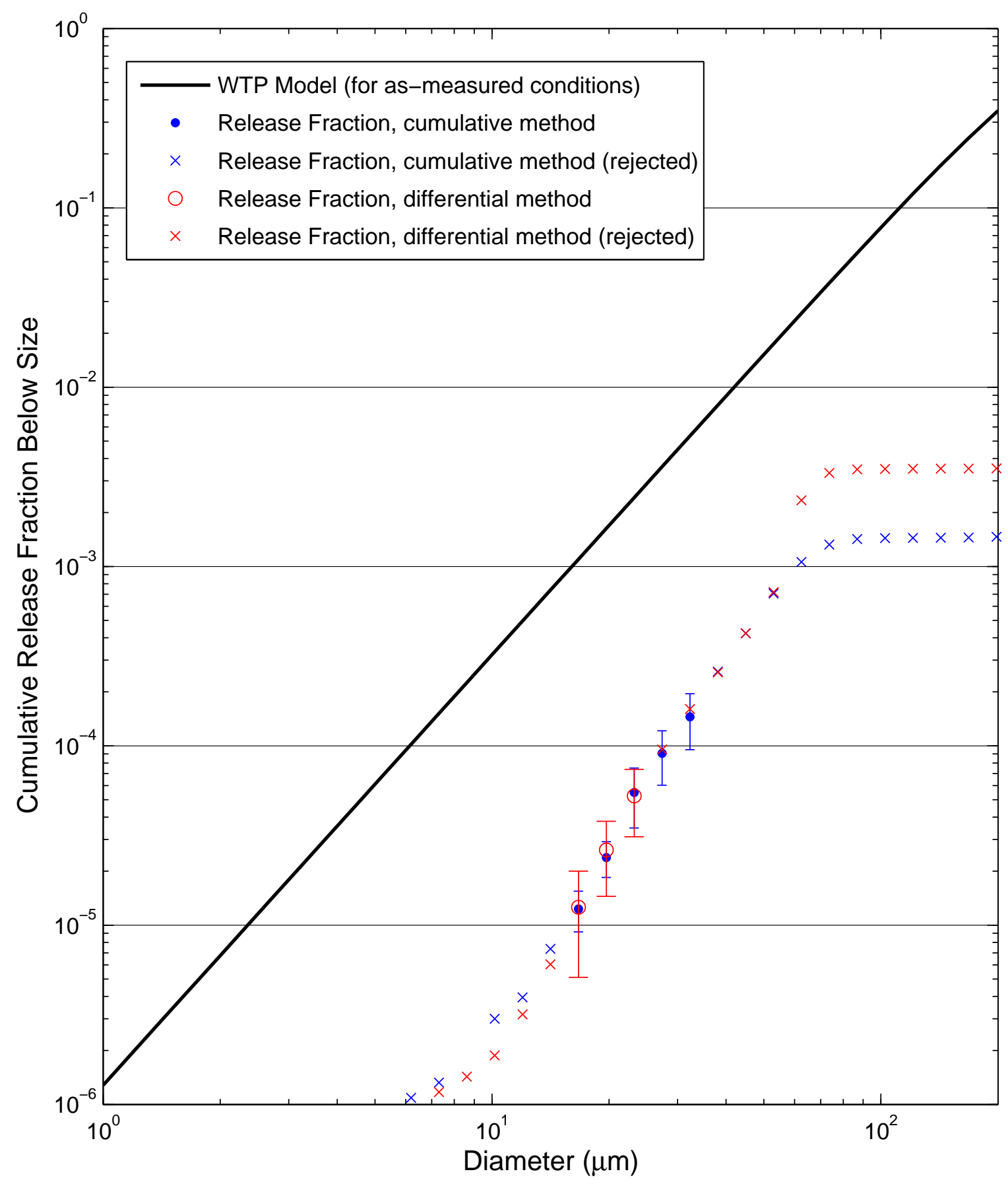


Test W121 [3 Malvern Average]: 380 psi, Orifice S2A - rectangular, $0.5 \times 5 \mathrm{~mm}$

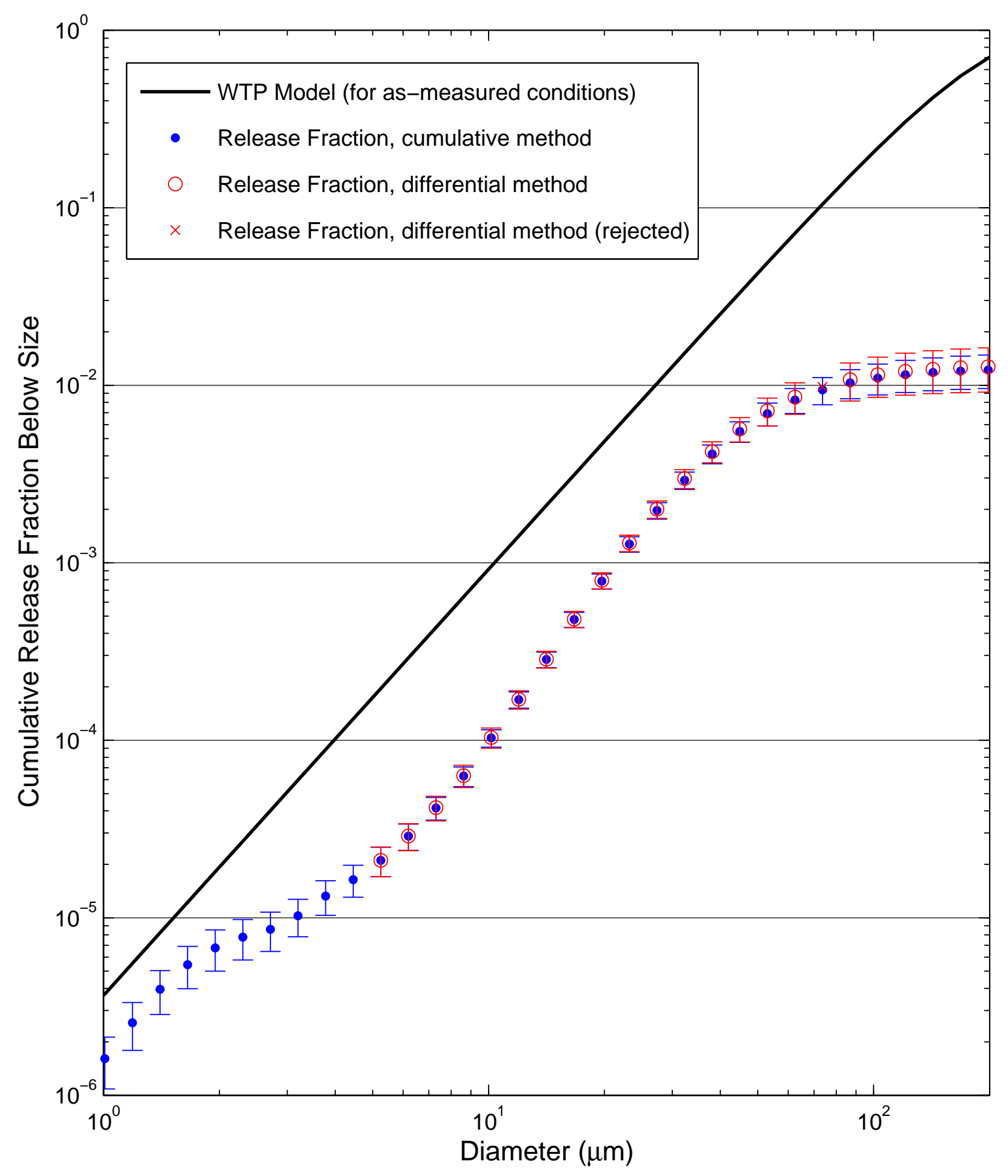


Test W122 [3 Malvern Average]: 200 psi, Orifice S2A - rectangular, $0.5 \times 5 \mathrm{~mm}$

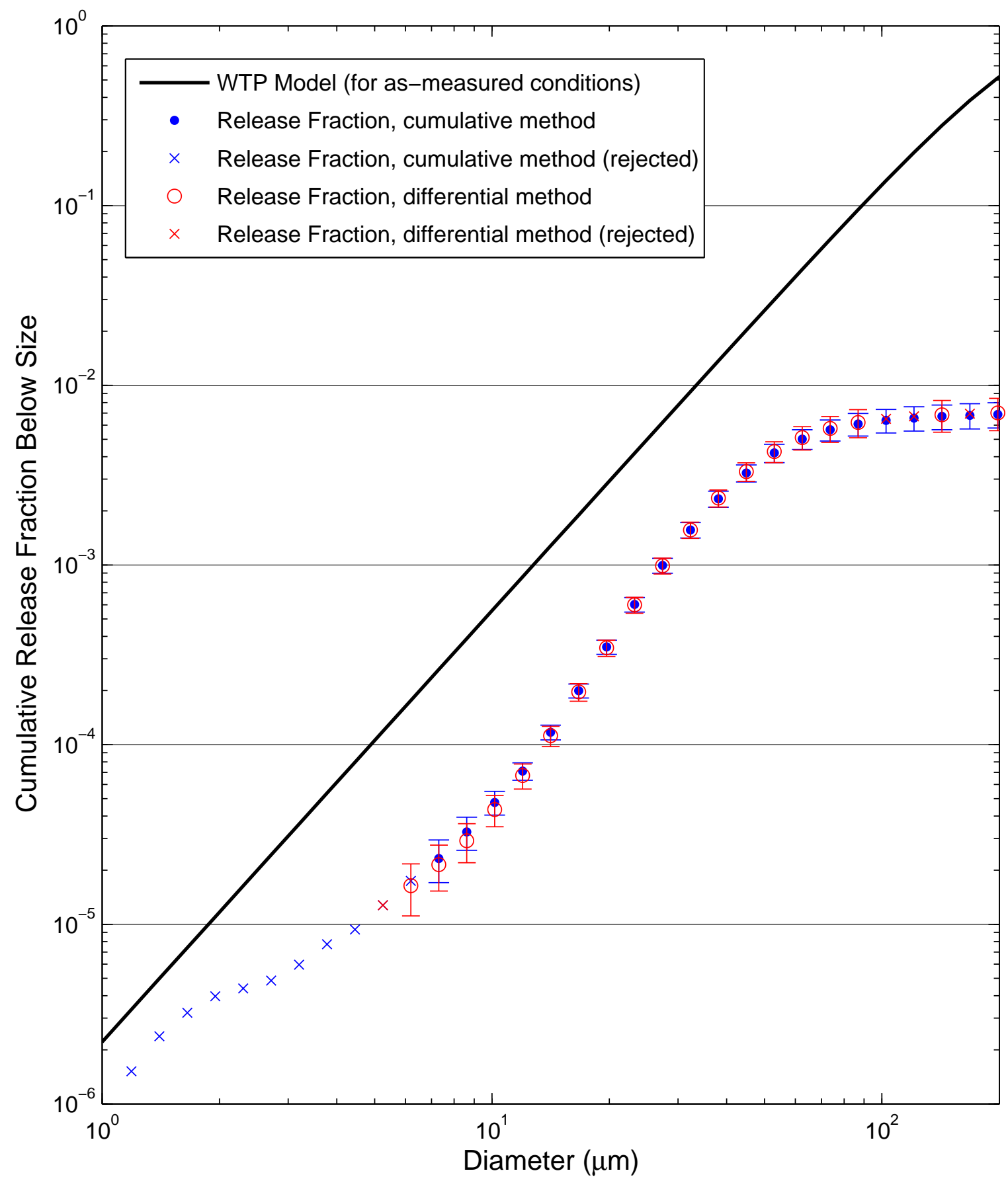


Test W123 [3 Malvern Average]: 200 psi, Orifice S2A - rectangular, $0.5 \times 5 \mathrm{~mm}$

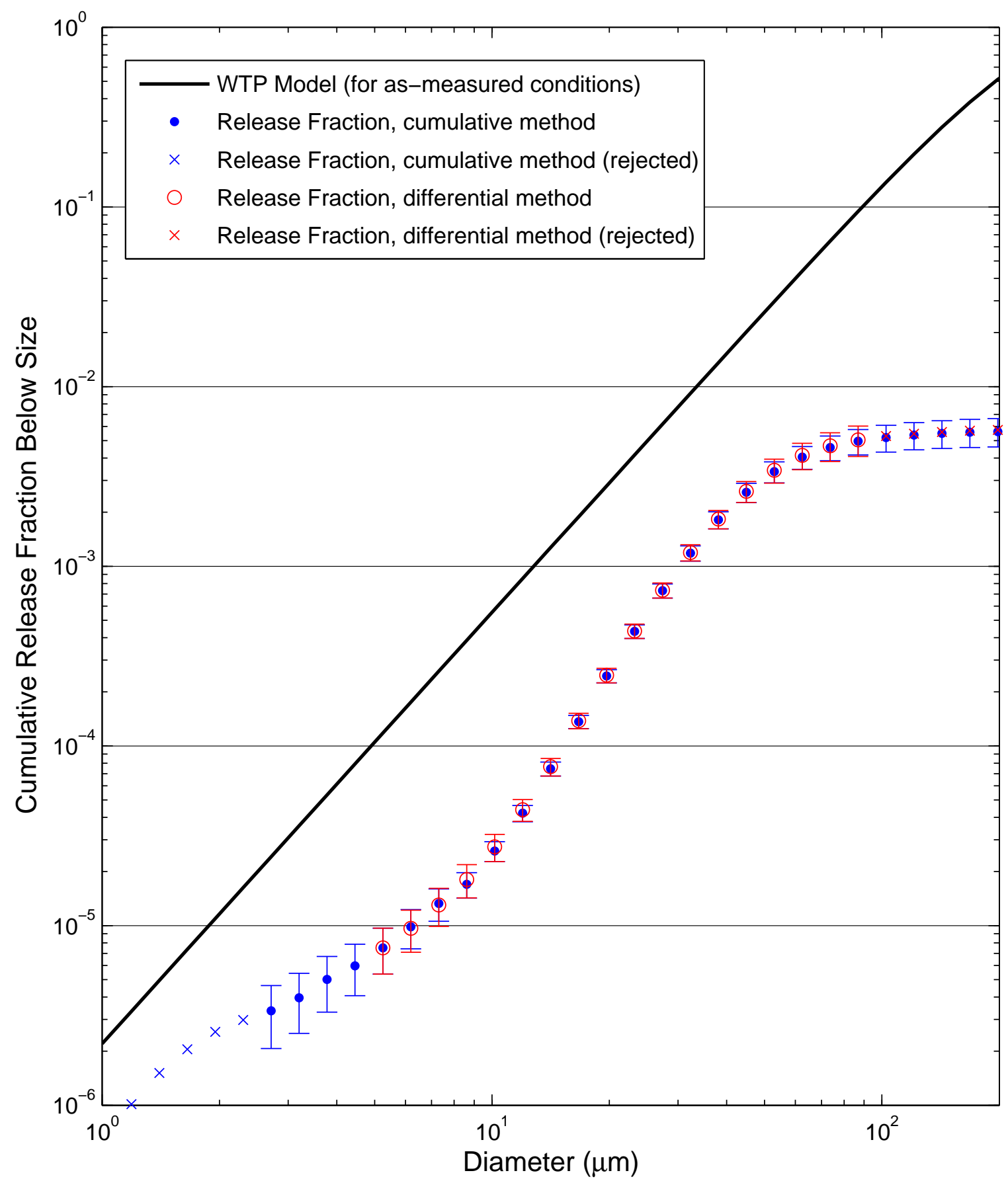


Test W124 [3 Malvern Average]: 100 psi, Orifice S2A - rectangular, $0.5 \times 5 \mathrm{~mm}$

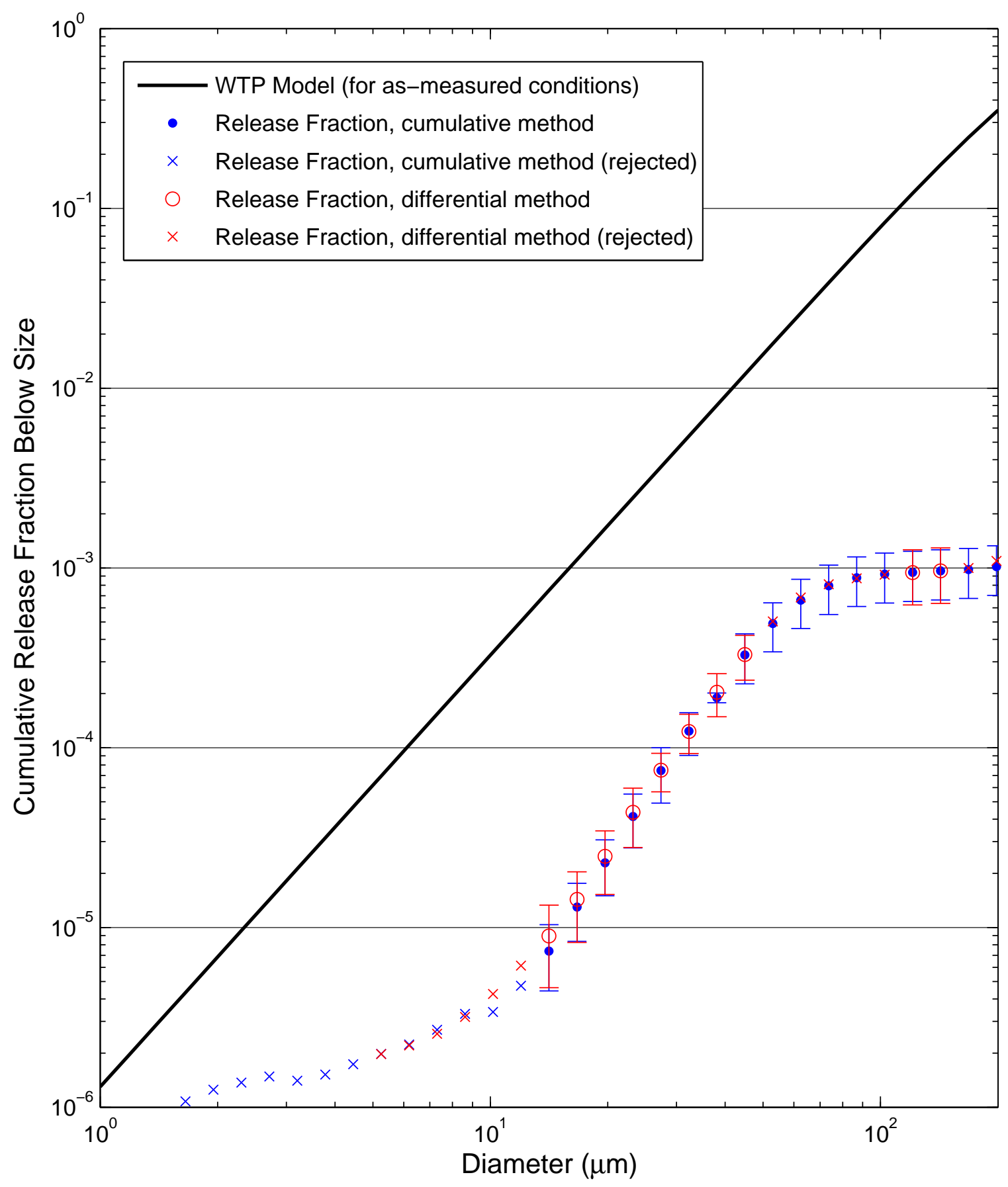


Test W125 [3 Malvern Average]: 380 psi, Orifice S2A - rectangular, $0.5 \times 5 \mathrm{~mm}$

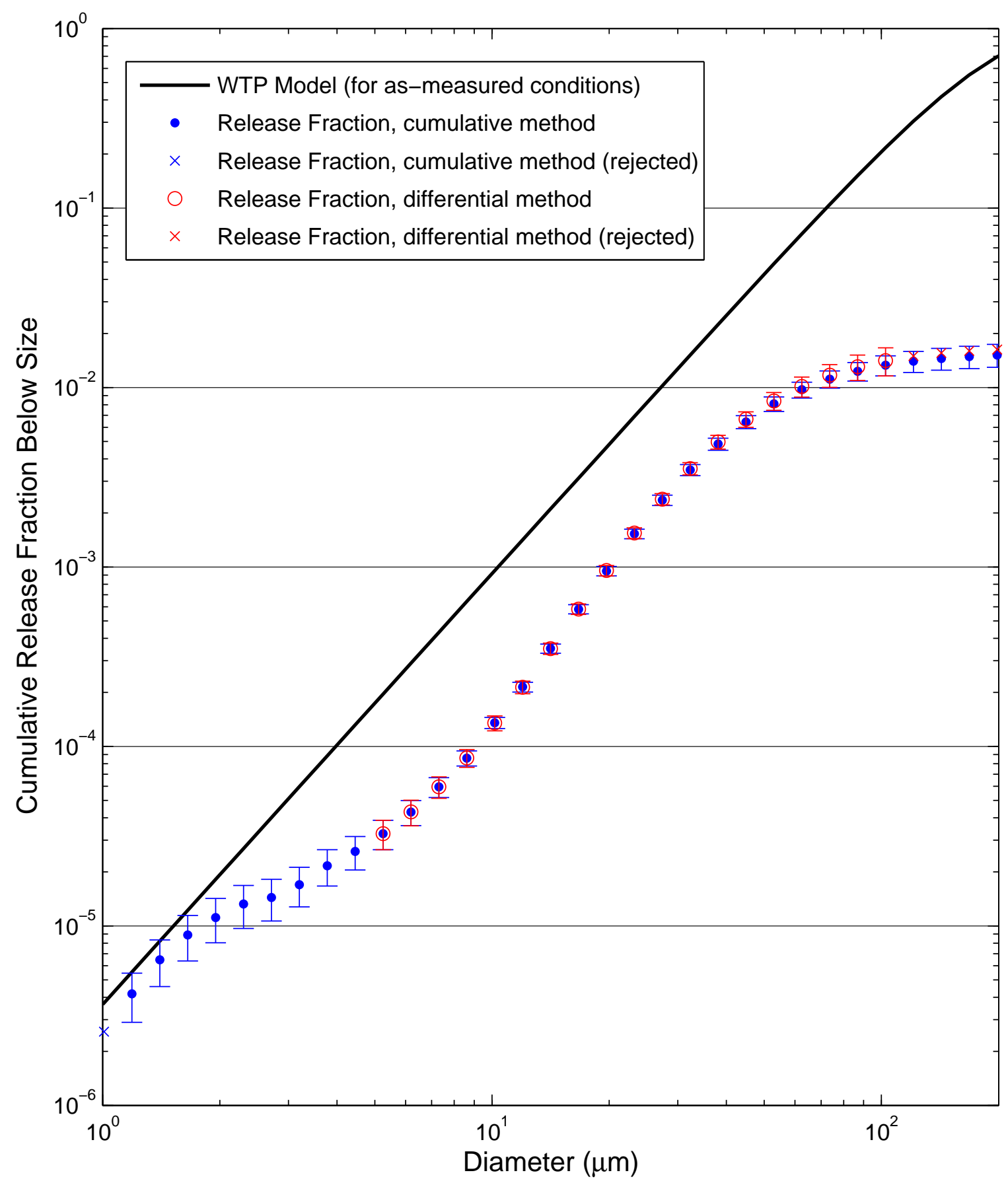


Test W126 [3 Malvern Average]: 100 psi, Orifice S1A - round, $1 \mathrm{~mm}$

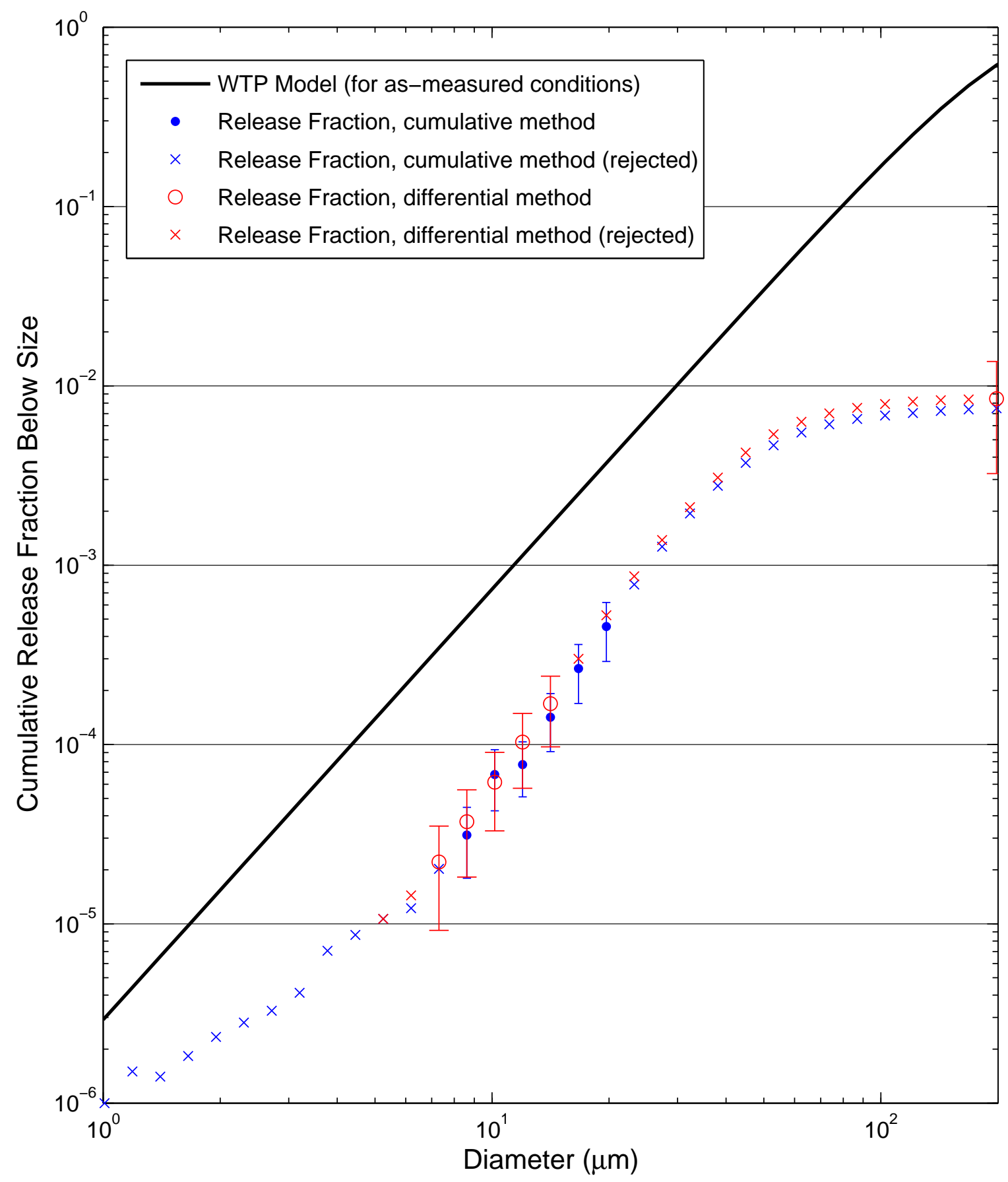


Test W127 [3 Malvern Average]: 200 psi, Orifice S1A - round, $1 \mathrm{~mm}$

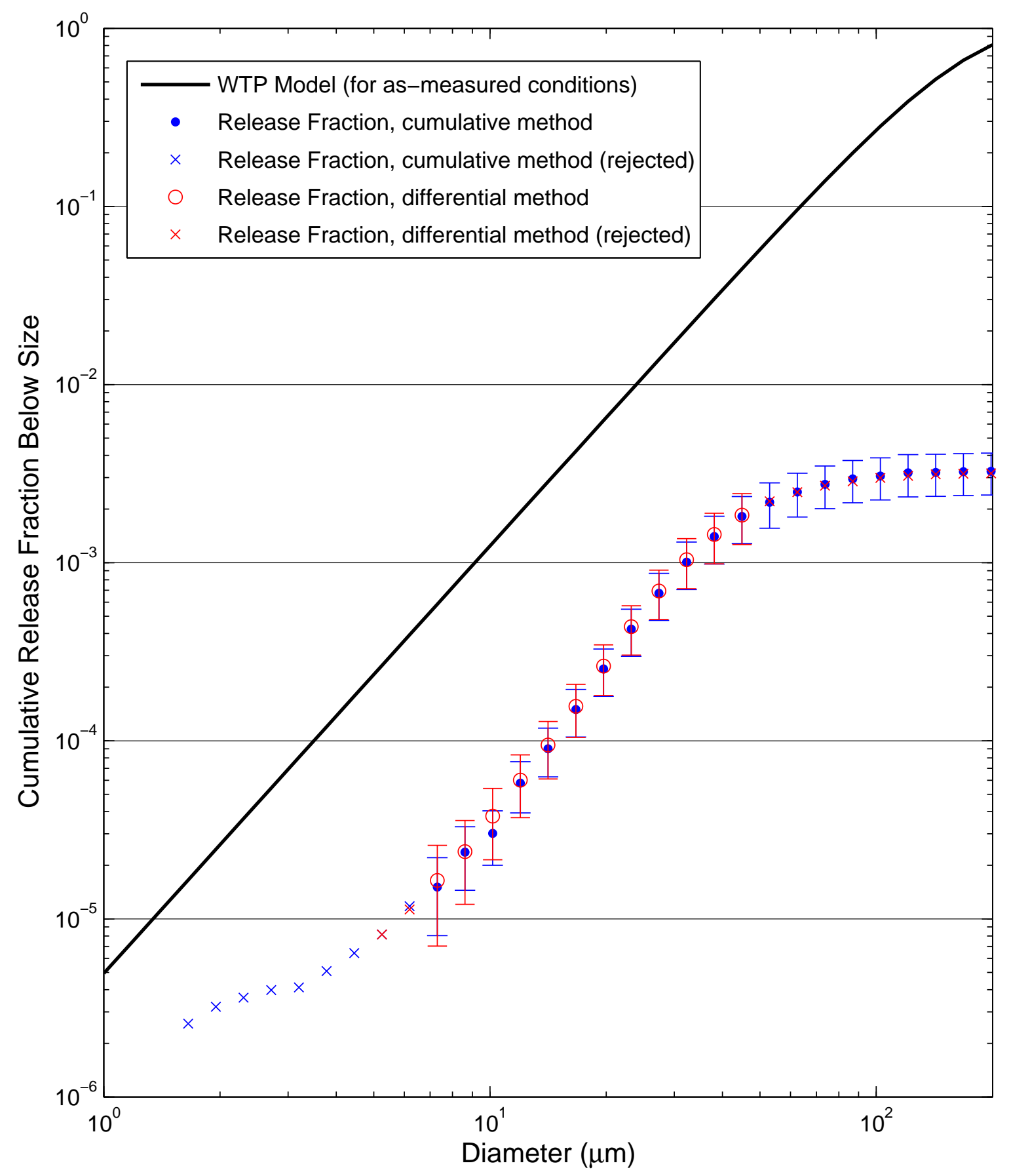


Test W128 [3 Malvern Average]: 380 psi, Orifice S1A - round, $1 \mathrm{~mm}$

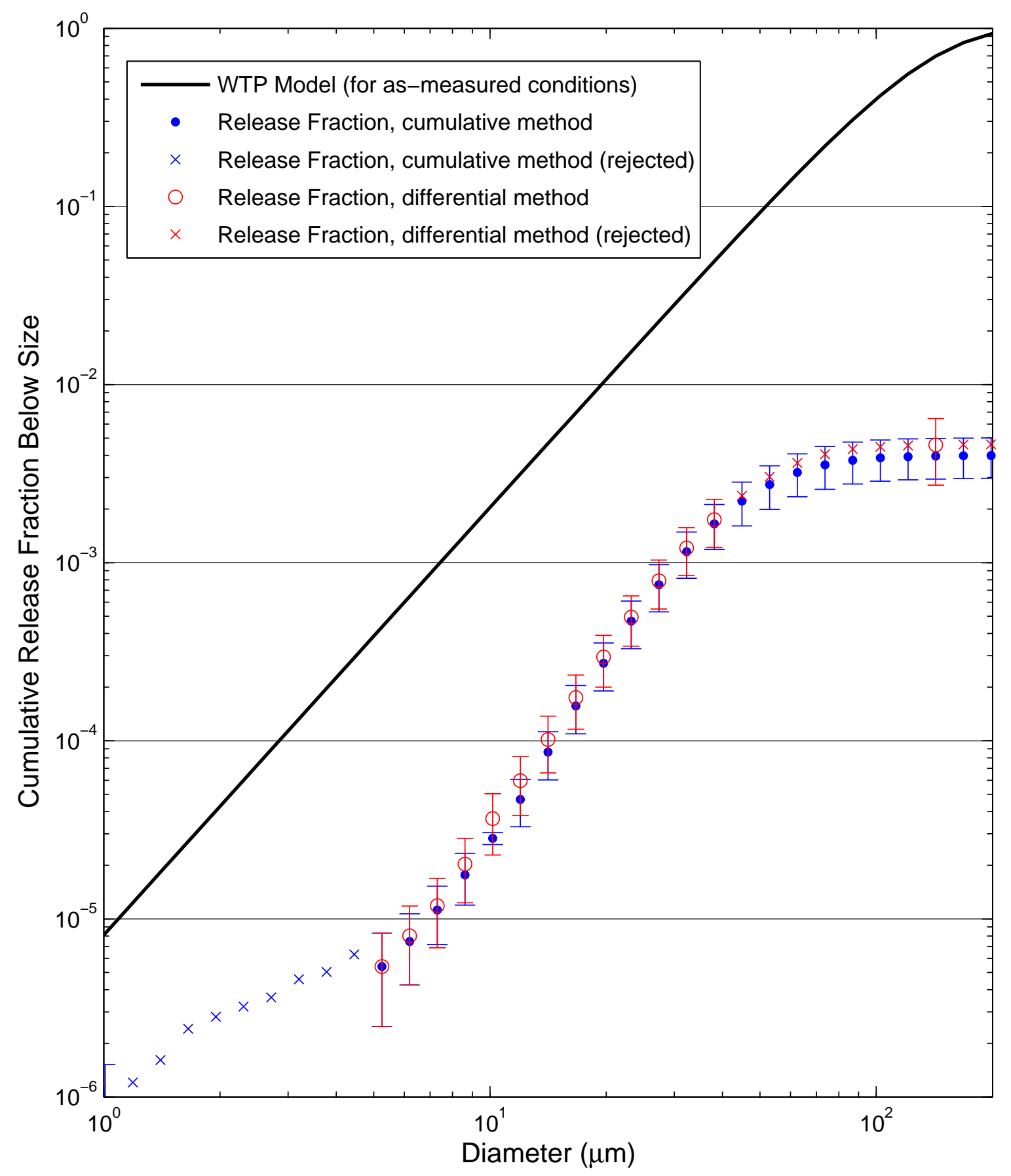


Test W129 [3 Malvern Average]: 100 psi, Orifice S1D - round, 2 mm

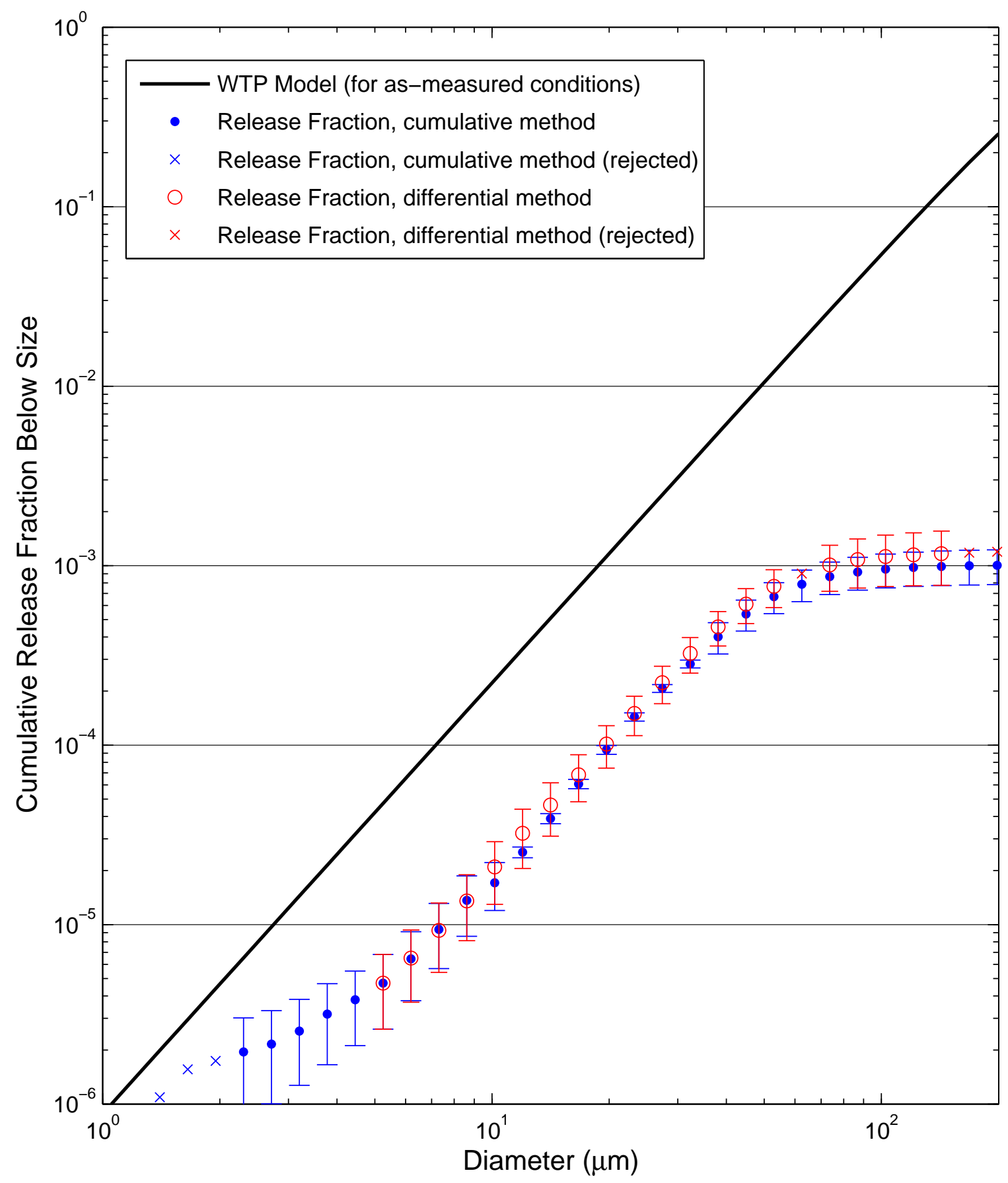


Test W130 [3 Malvern Average]: 200 psi, Orifice S1D - round, 2 mm

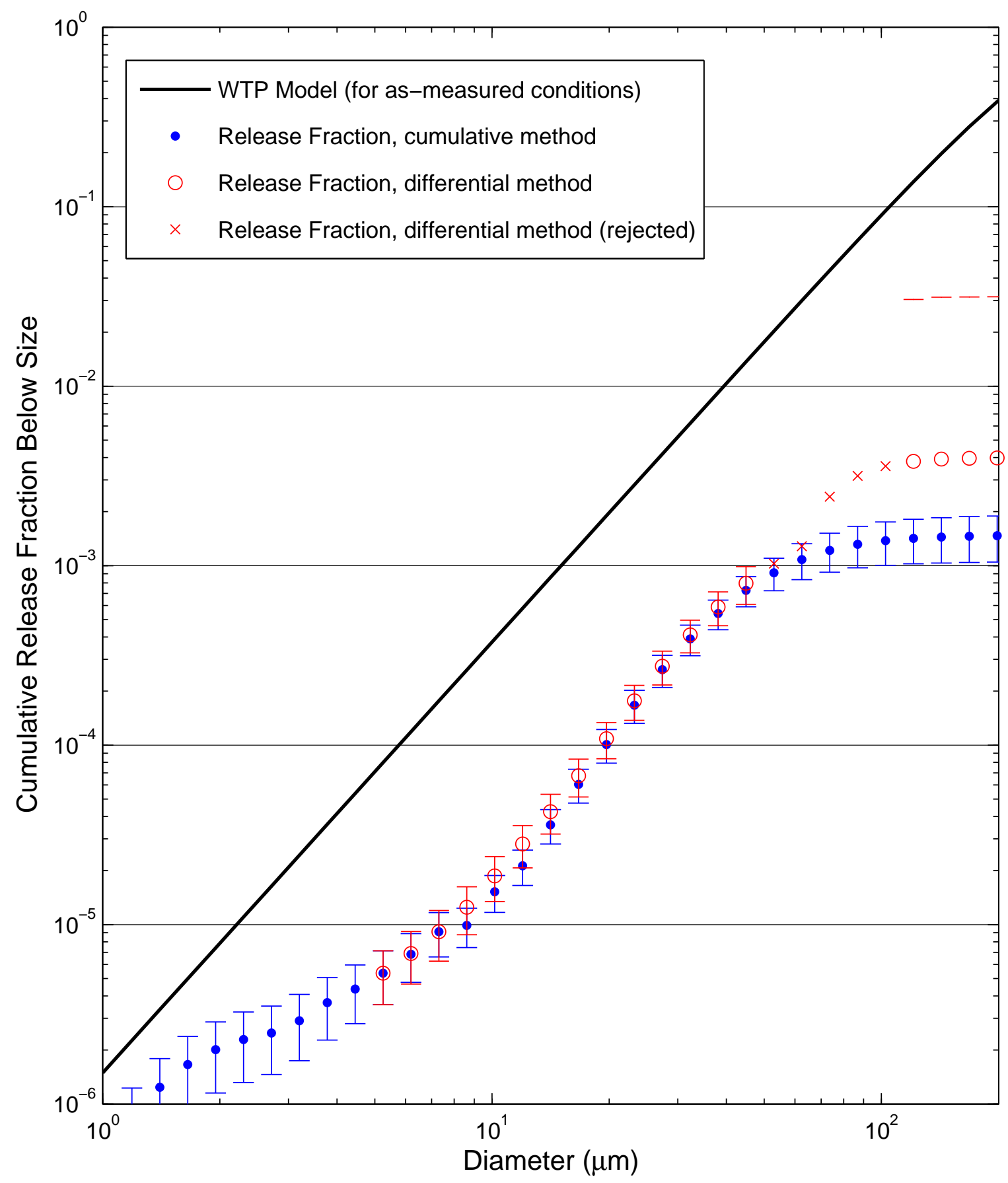


Test W131 [3 Malvern Average]: 380 psi, Orifice S1D - round, 2 mm

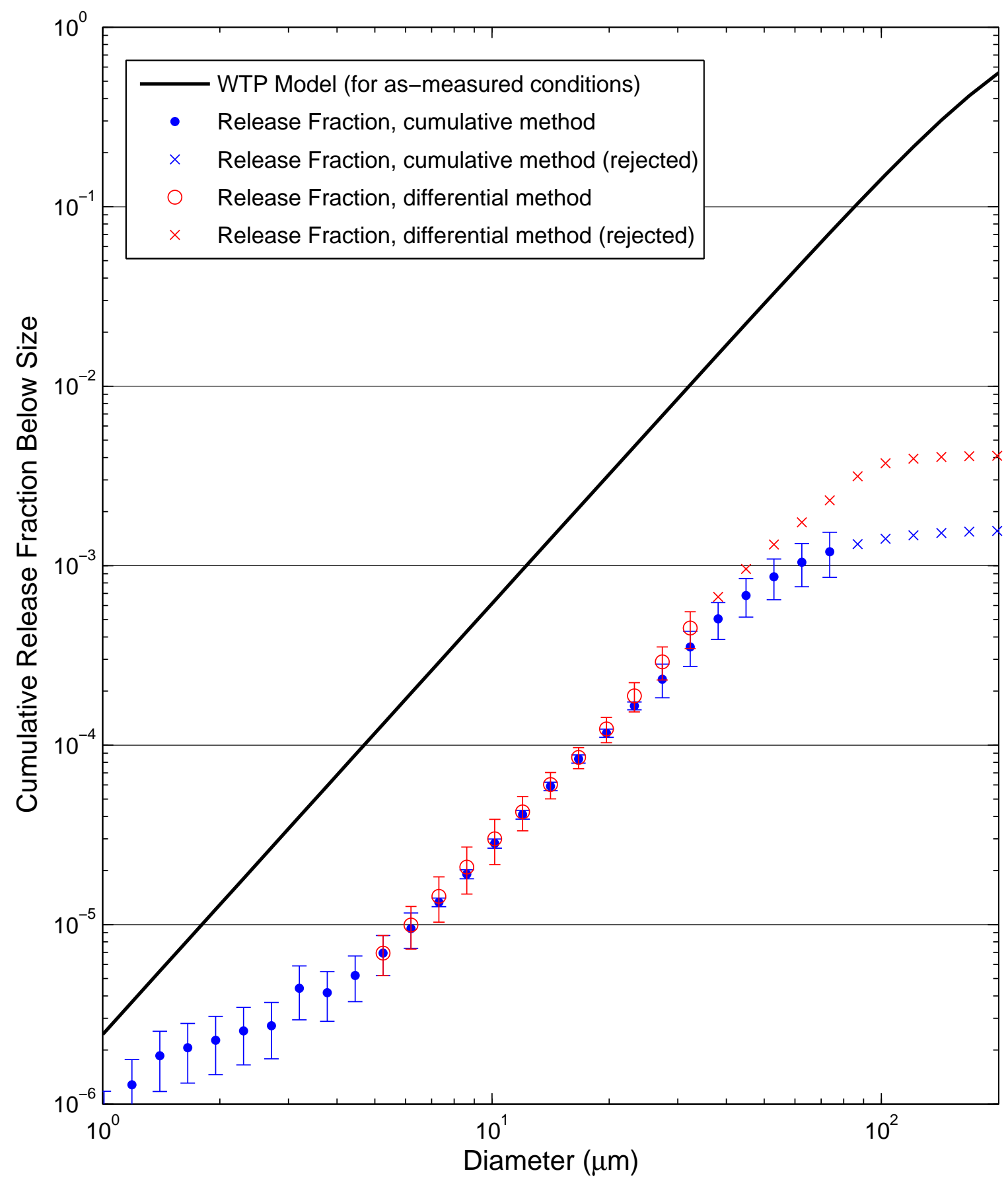


Test W132 [3 Malvern Average]: $100 \mathrm{psi}$, Orifice S1A - round, $1 \mathrm{~mm}$

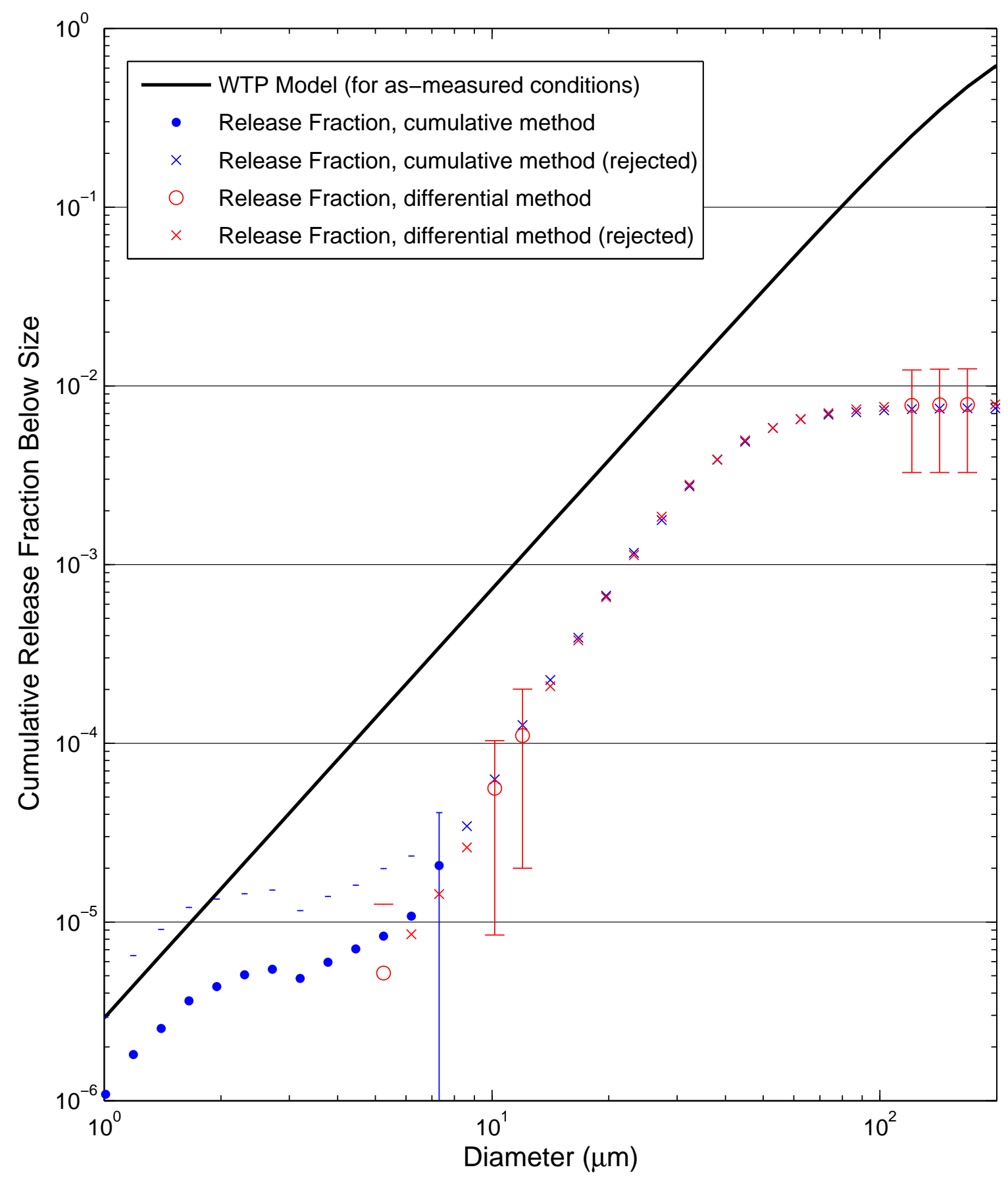


Test W133 [3 Malvern Average]: 380 psi, Orifice S1A - round, $1 \mathrm{~mm}$

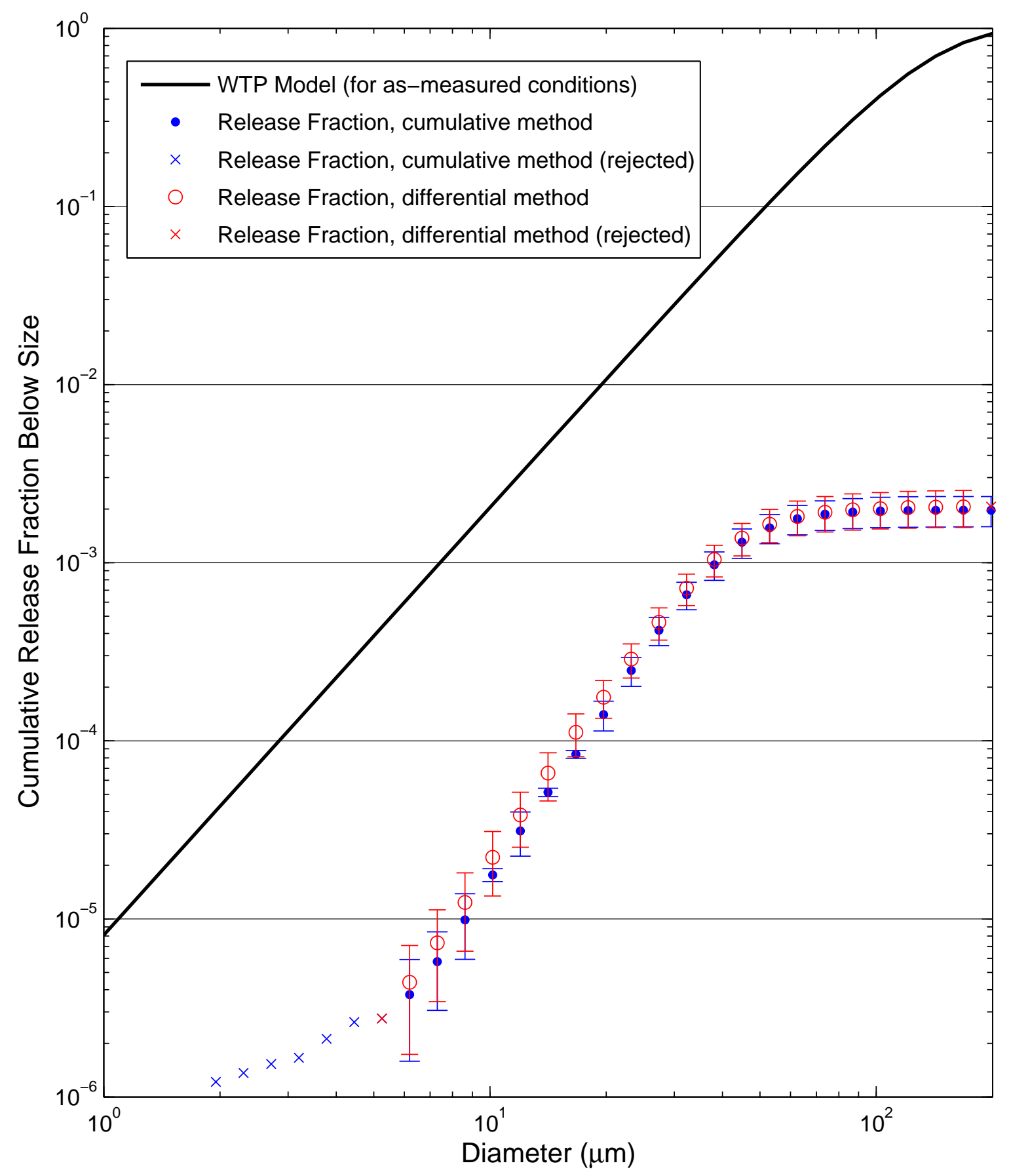


Test W134 [3 Malvern Average]: 100 psi, Orifice S1D - round, 2 mm

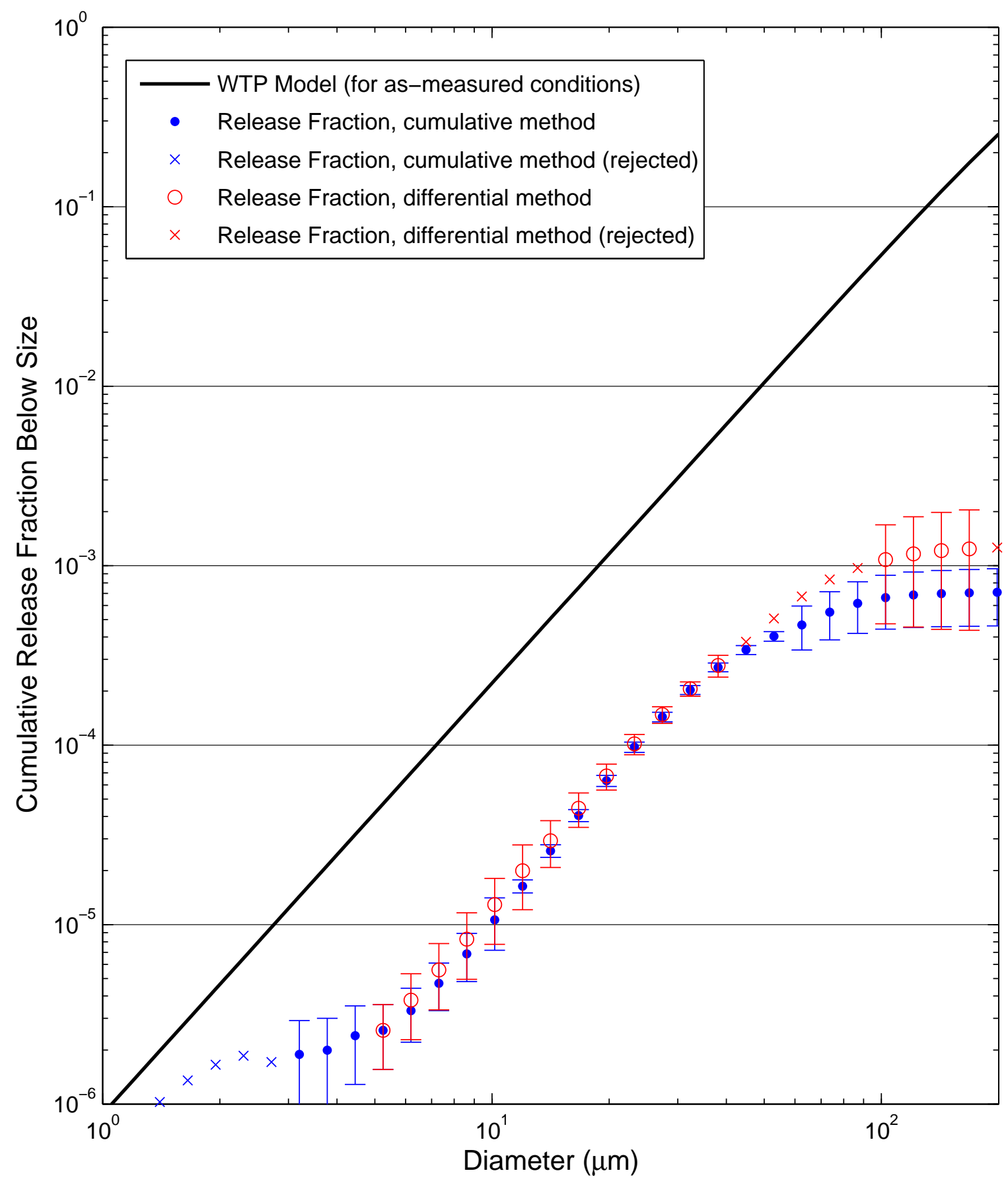


Test W135 [3 Malvern Average]: 380 psi, Orifice S1D - round, 2 mm

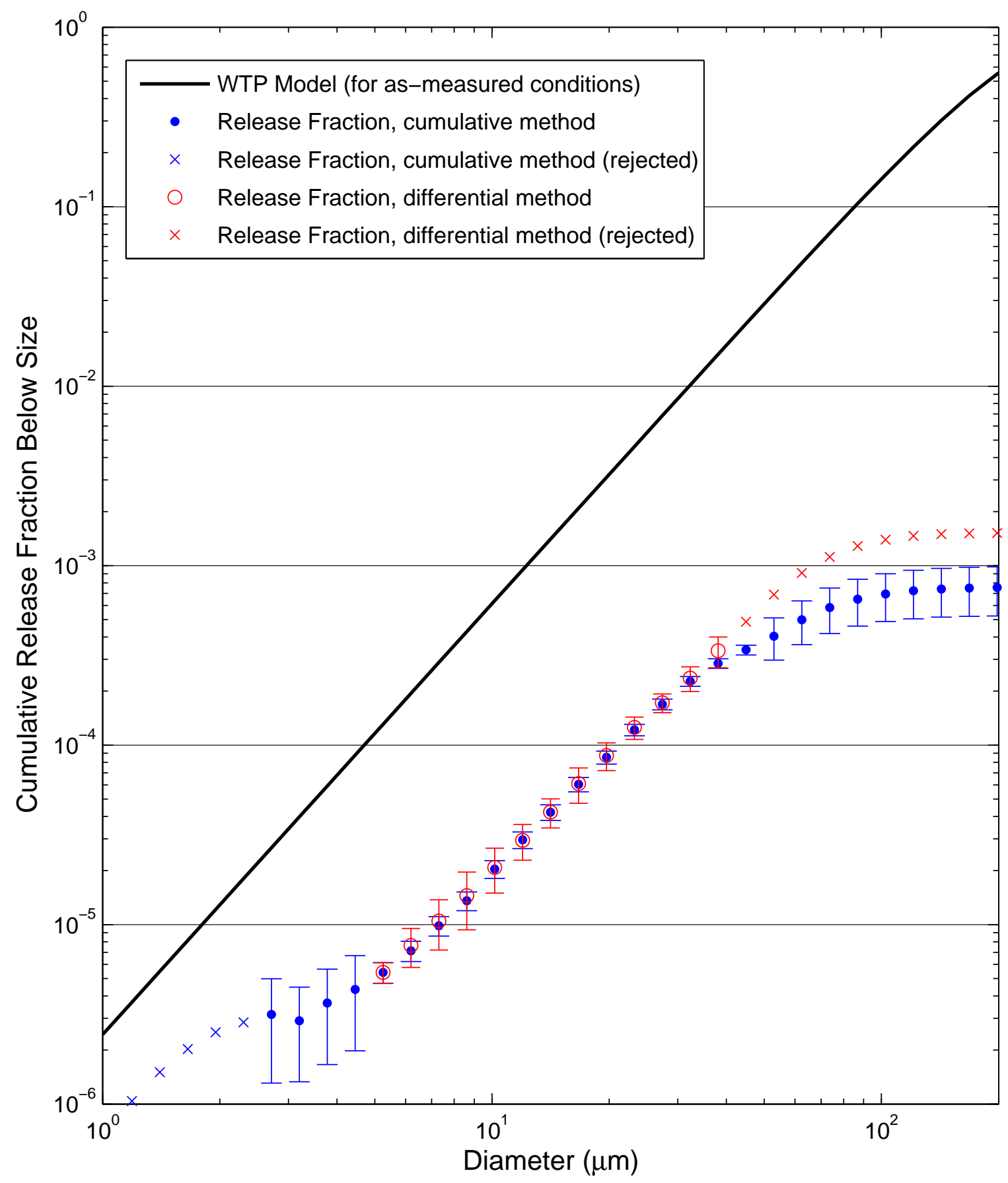


Test W136 [3 Malvern Average]: $100 \mathrm{psi}$, Orifice S1A - round, $1 \mathrm{~mm}$

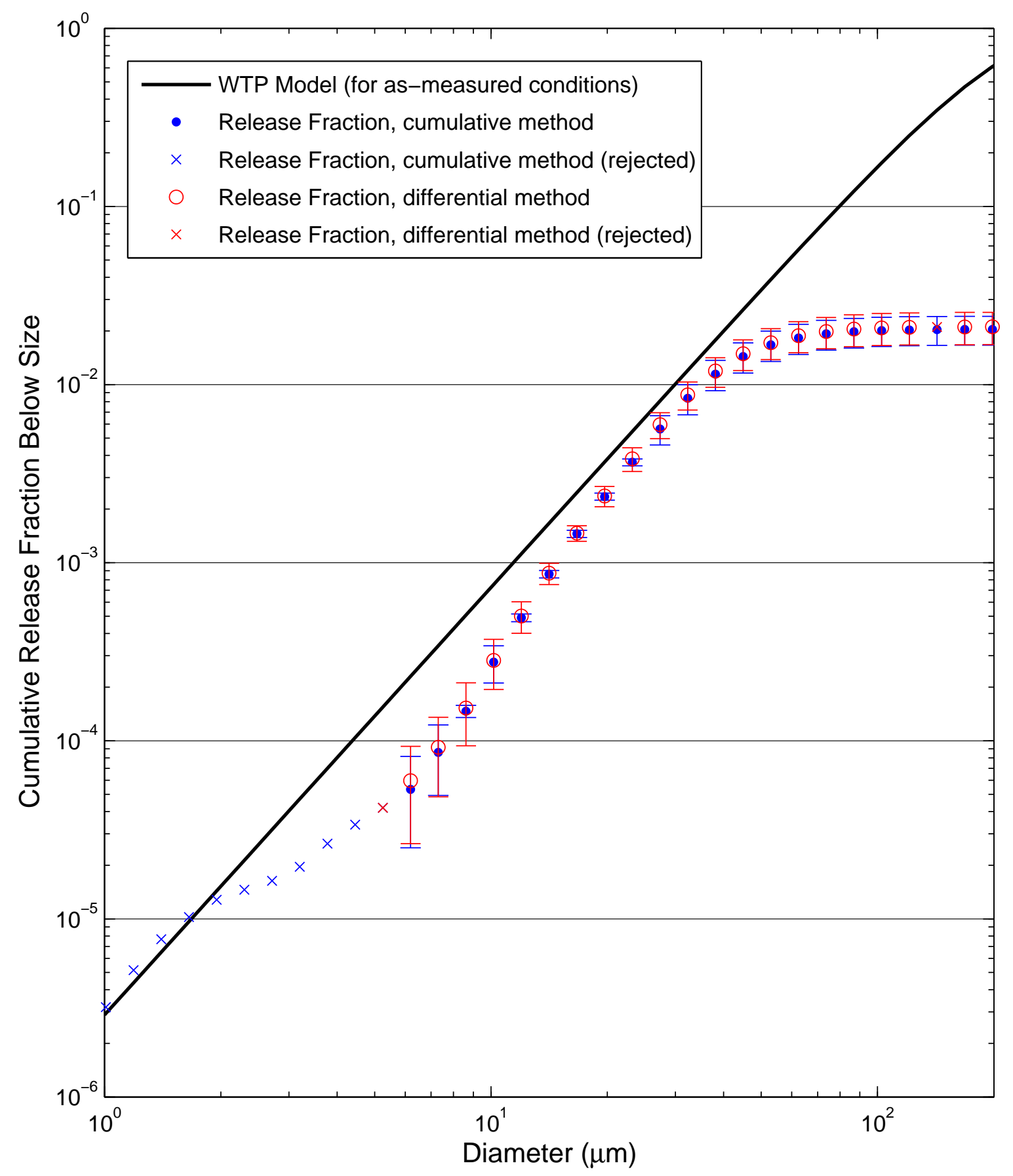


Test W137 [3 Malvern Average]: 200 psi, Orifice S1A - round, $1 \mathrm{~mm}$

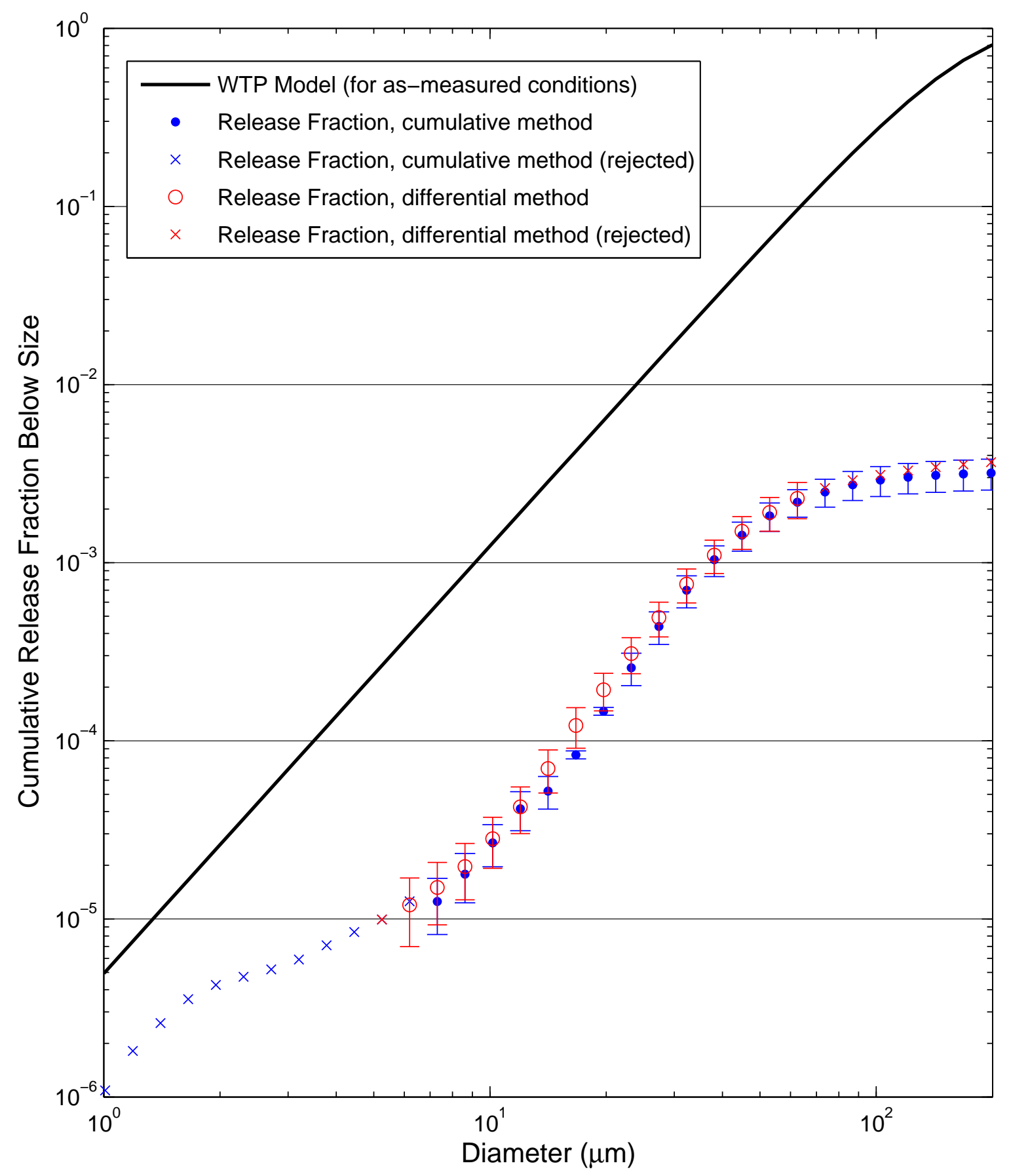


Test W138 [3 Malvern Average]: 380 psi, Orifice S1A - round, $1 \mathrm{~mm}$

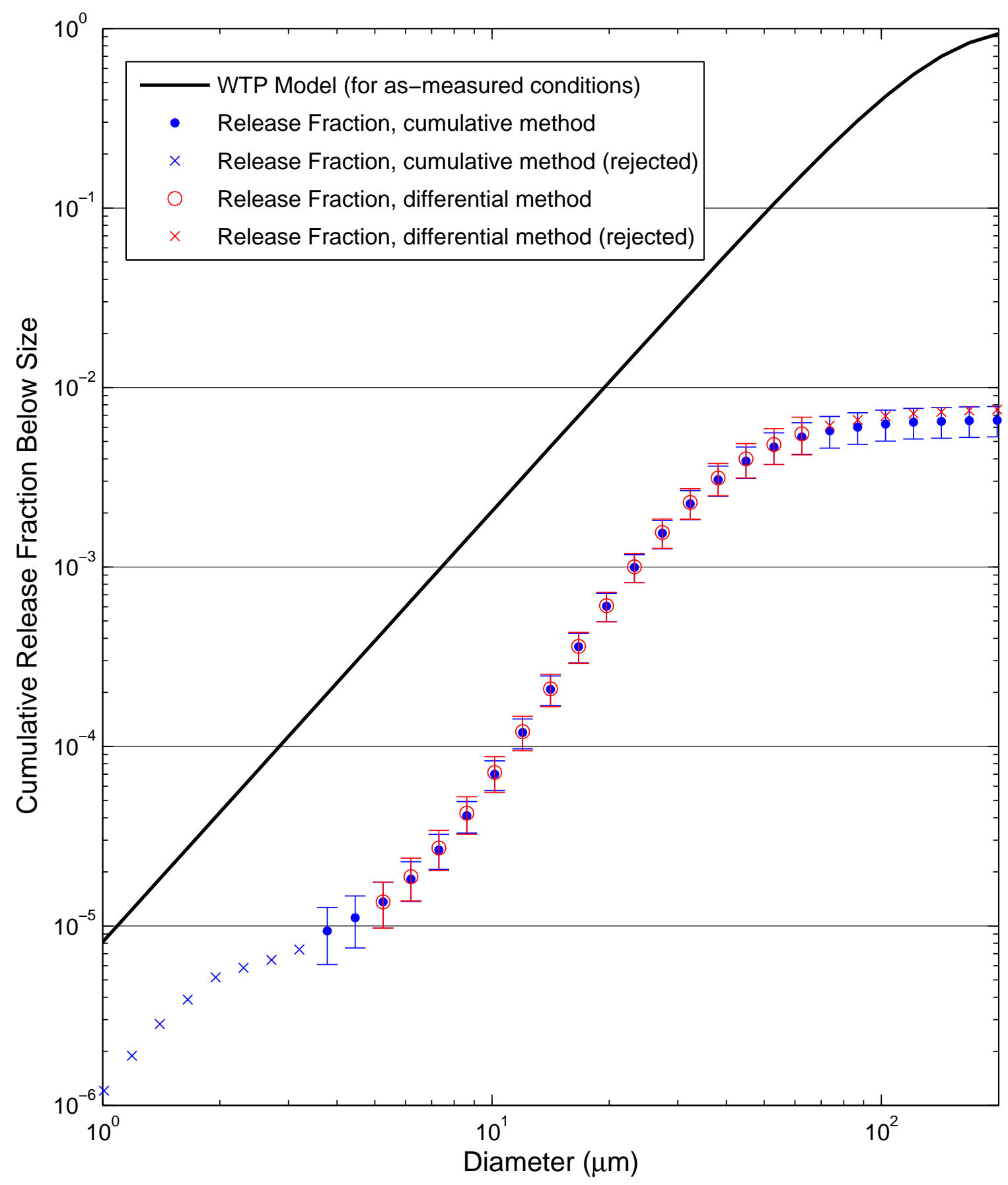


Test W139 [3 Malvern Average]: 100 psi, Orifice S1D - round, 2 mm

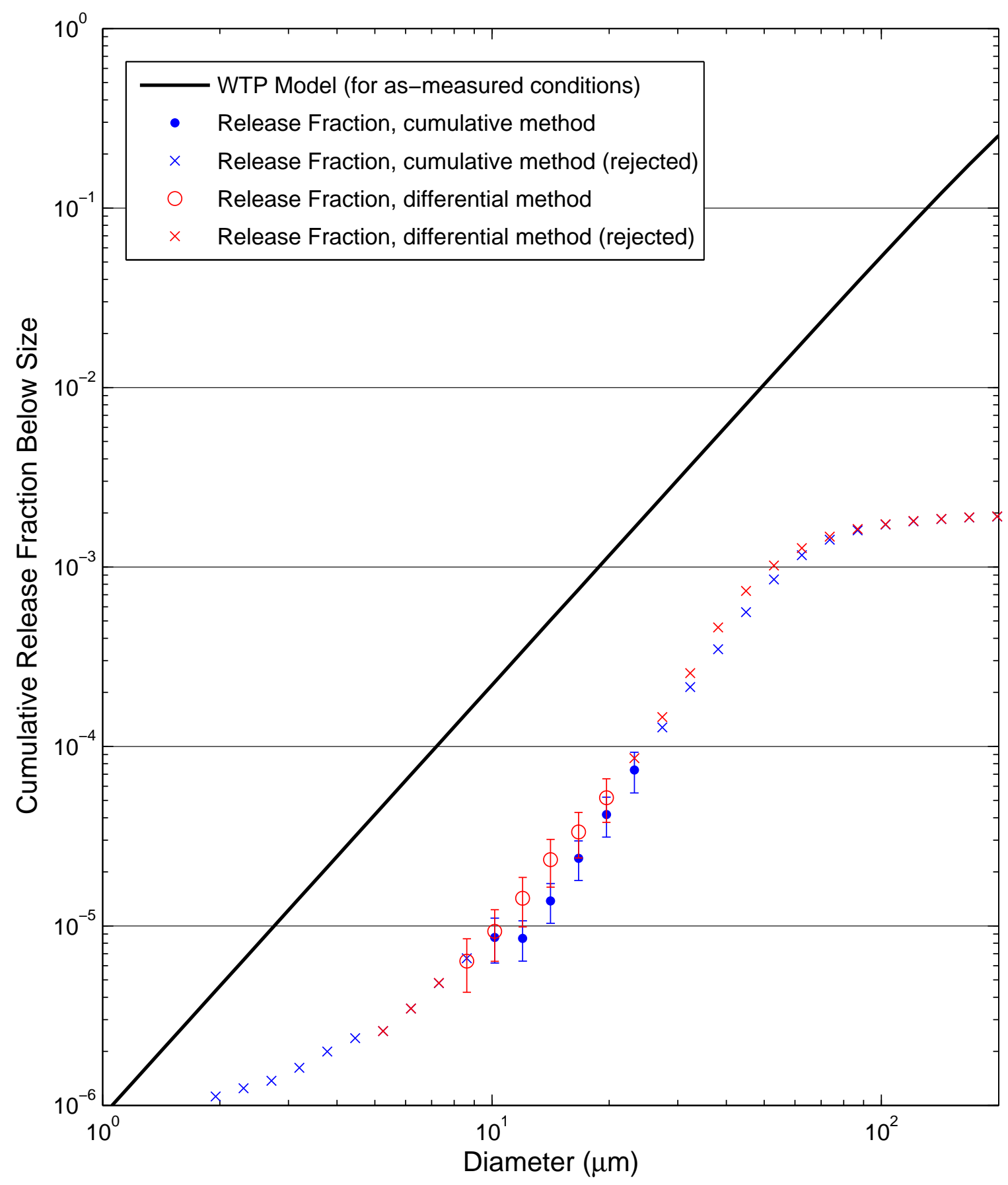


Test W140 [3 Malvern Average]: 200 psi, Orifice S1D - round, 2 mm

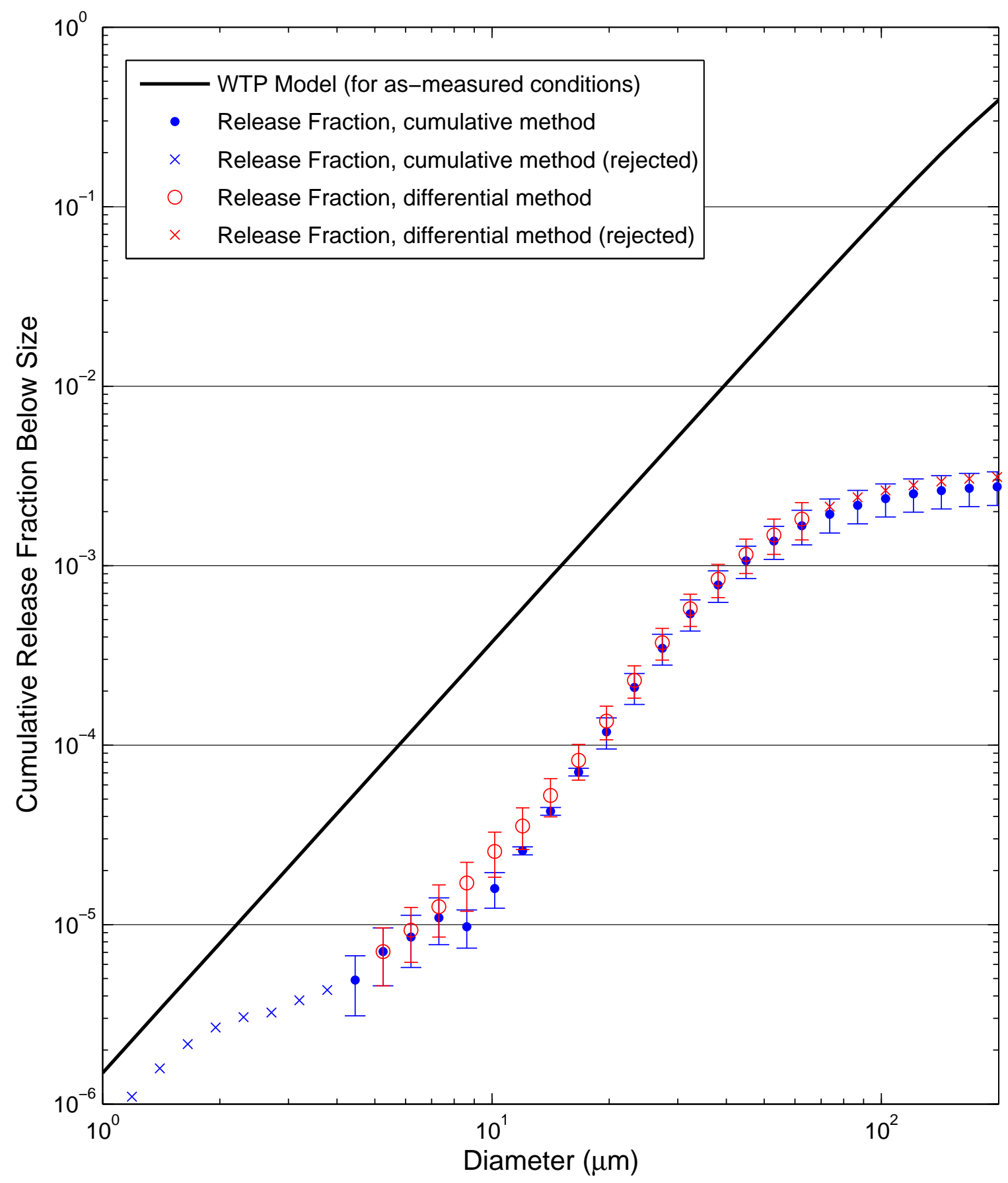


Test W141 [3 Malvern Average]: 380 psi, Orifice S1D - round, 2 mm

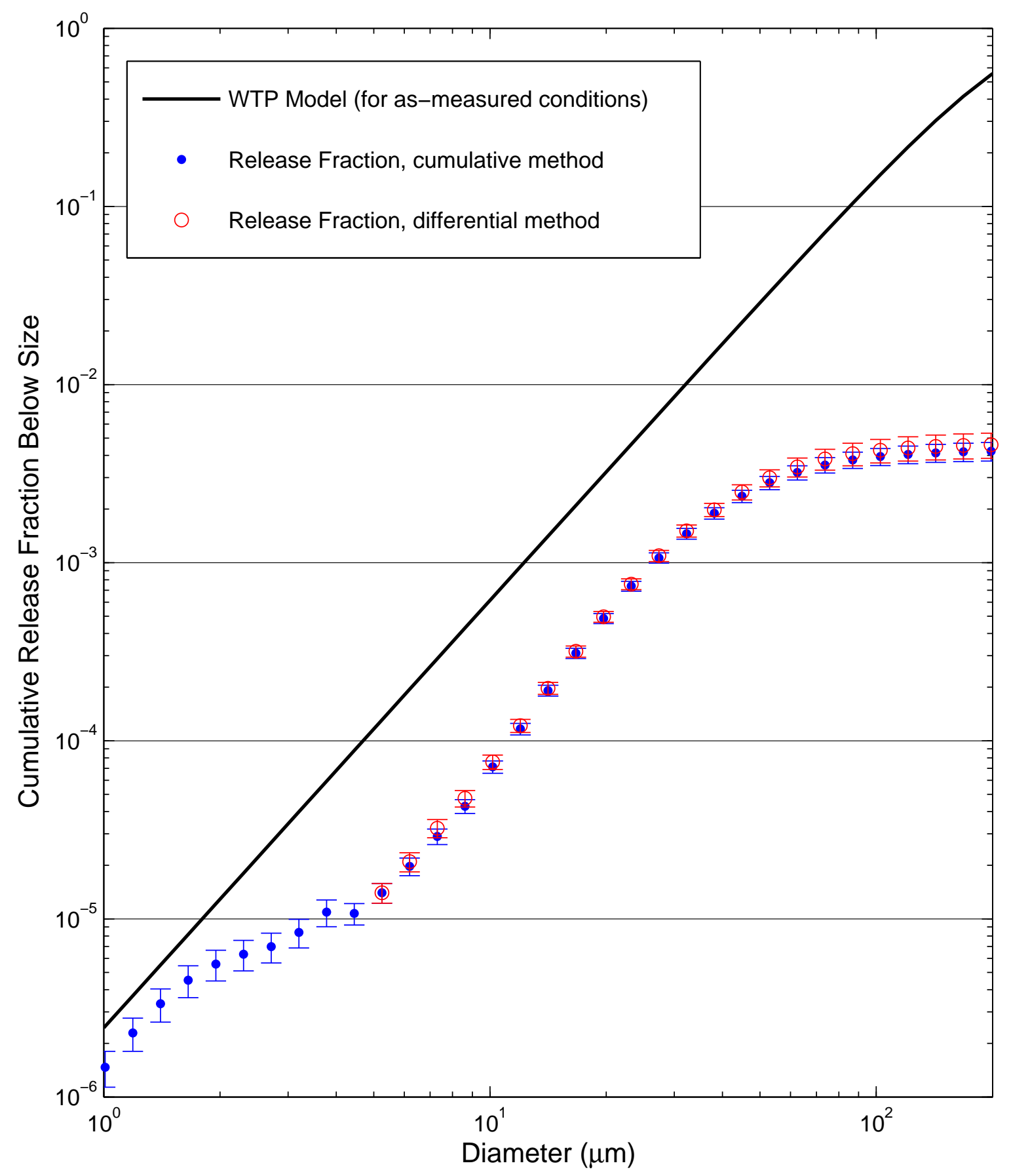

B. 100 
Test W142 [3 Malvern Average]: 100 psi, Orifice S1D - round, 2 mm

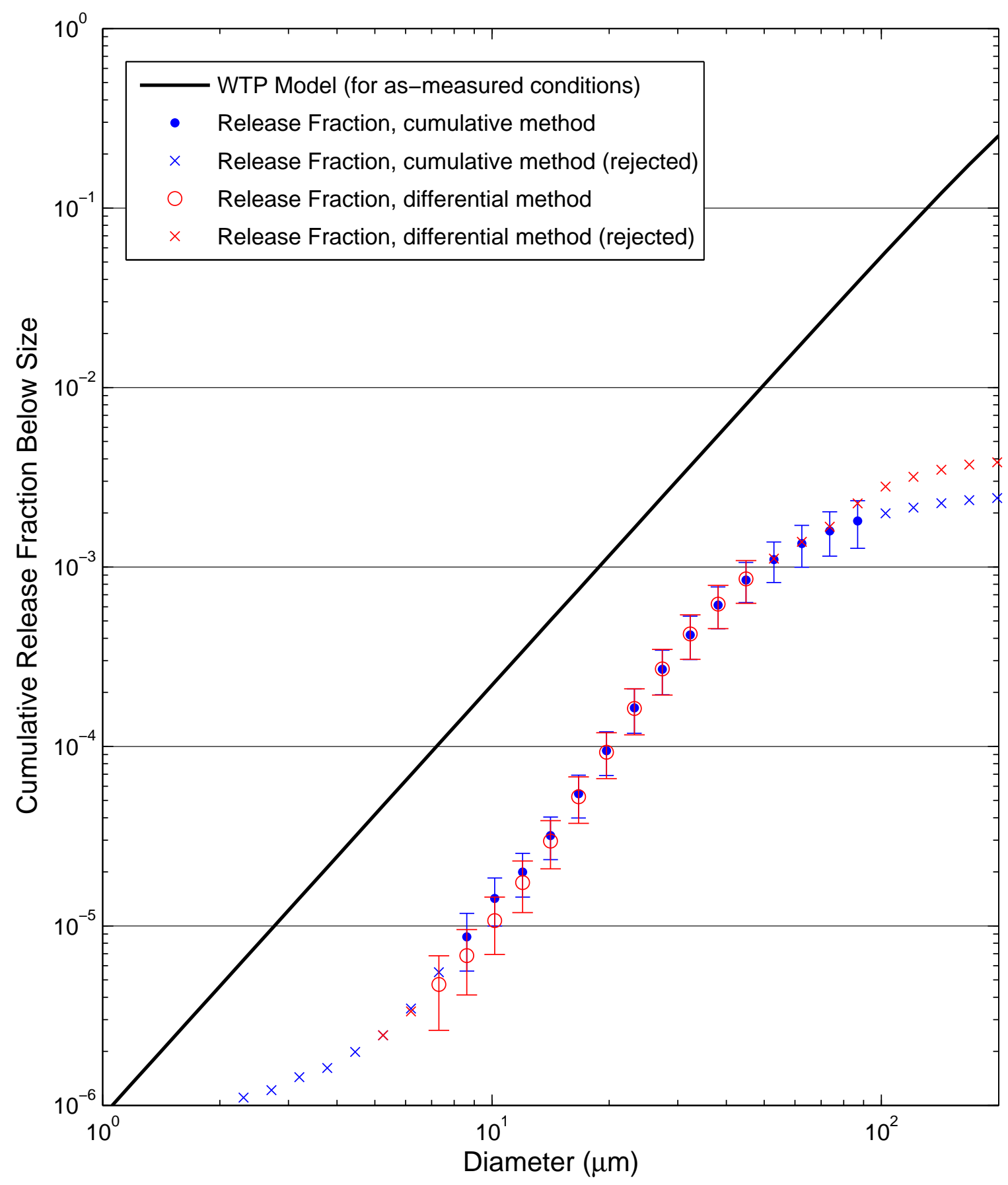


Test W143 [3 Malvern Average]: 380 psi, Orifice S1D - round, 2 mm

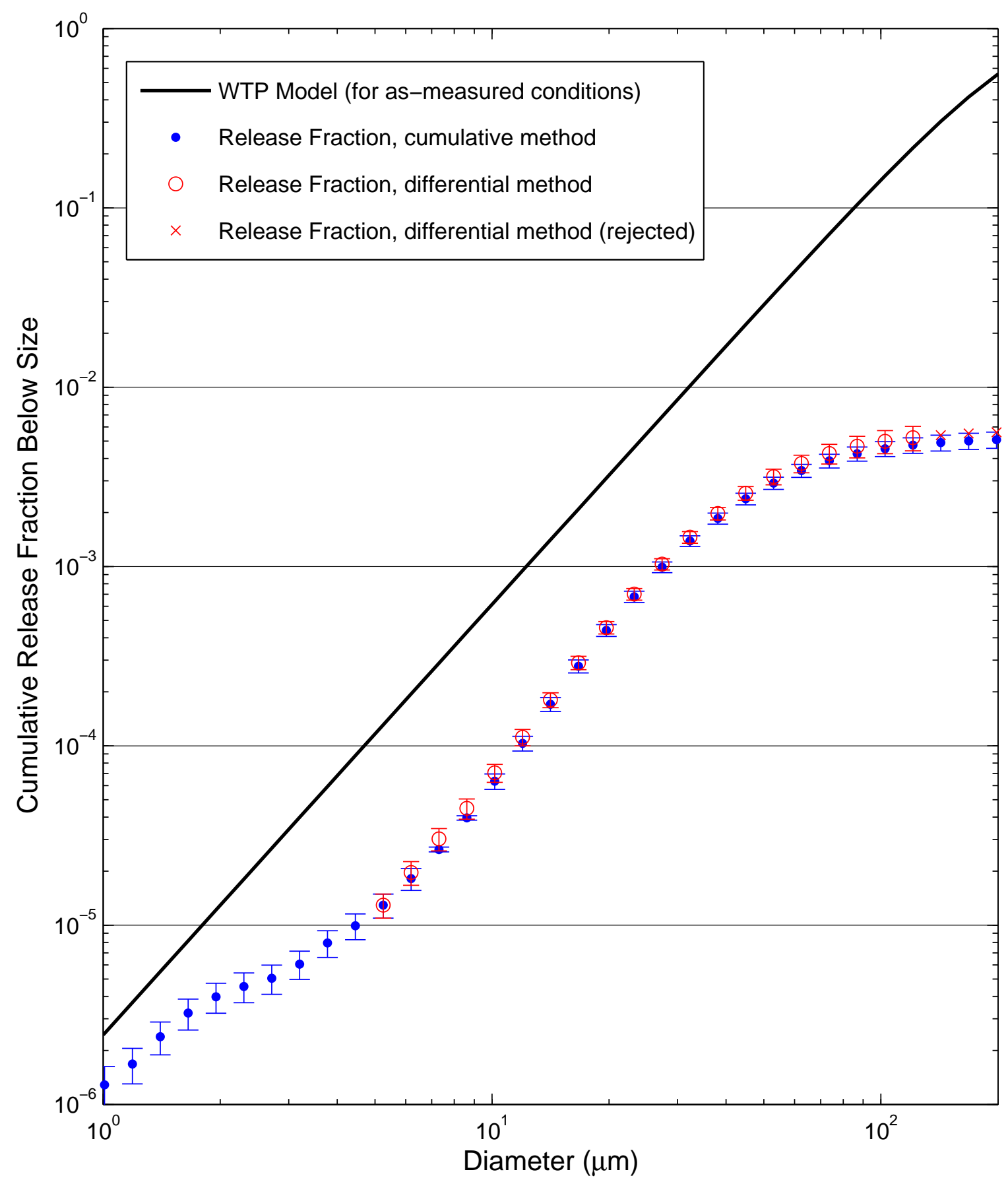

B. 102 
Test W144 [3 Malvern Average]: 100 psi, Orifice S1A - round, $1 \mathrm{~mm}$

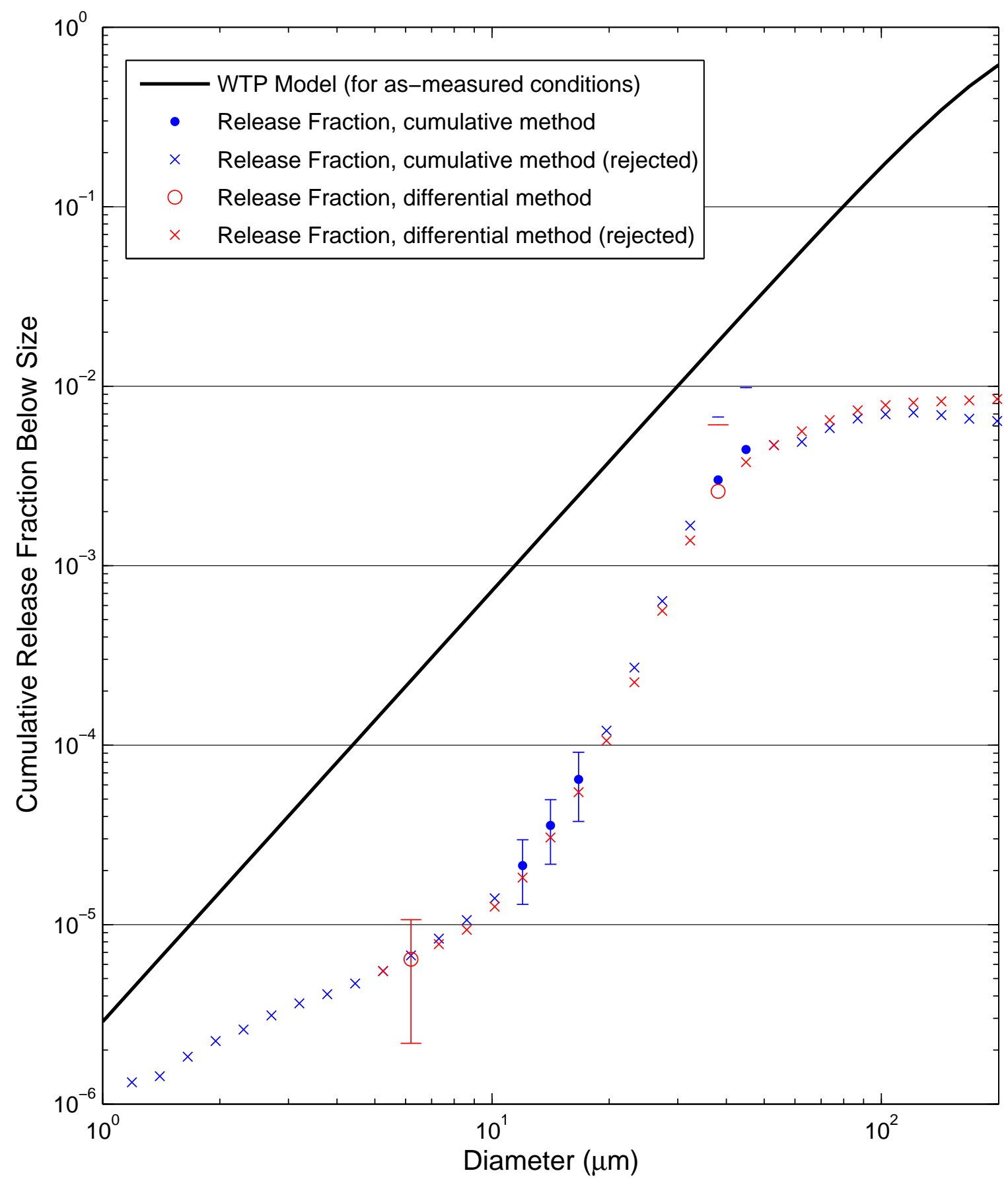


Test W145 [3 Malvern Average]: 380 psi, Orifice S1A - round, $1 \mathrm{~mm}$

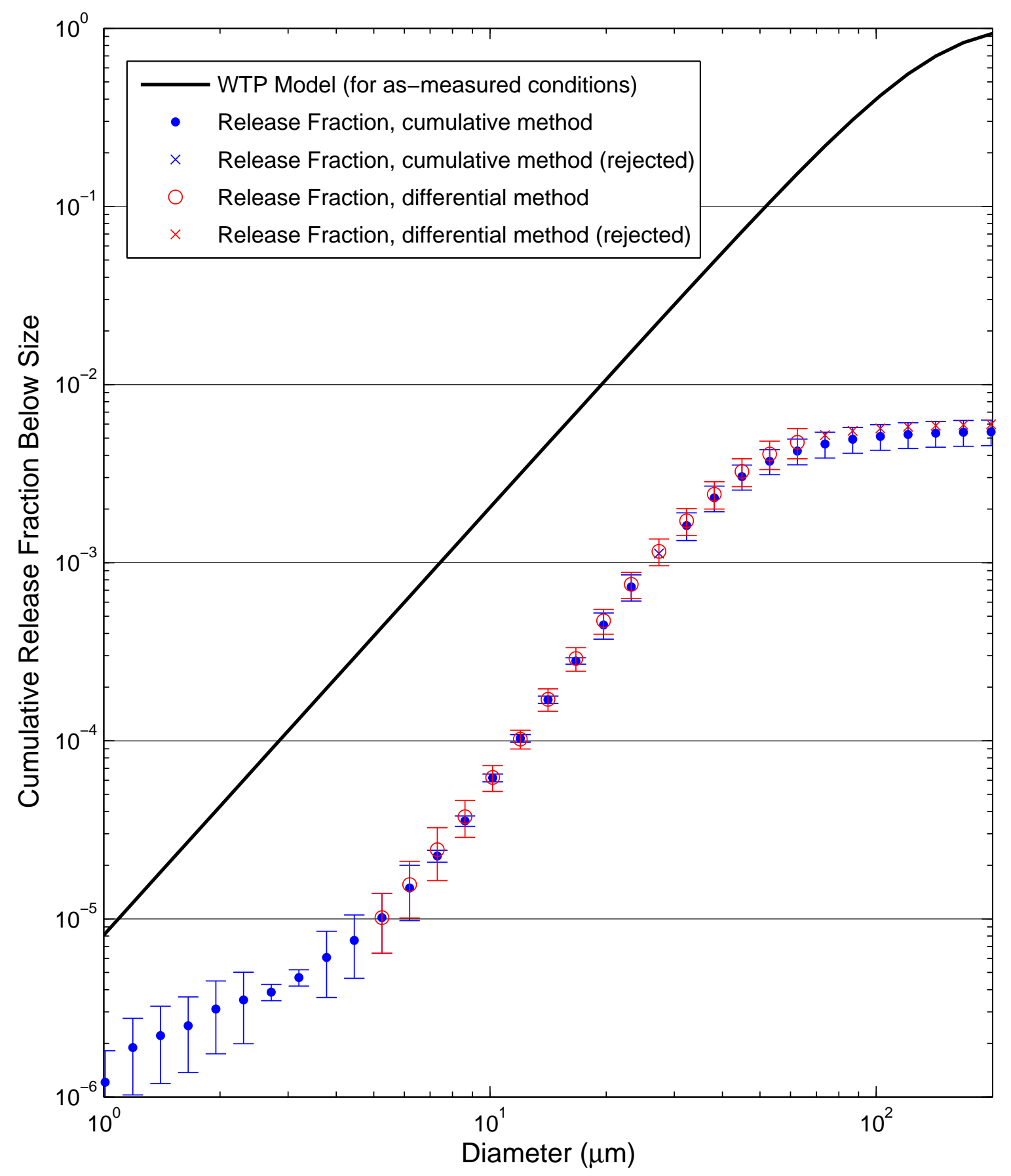


Test S146 [3 Malvern Average]: 100 psi, Orifice S7A - rectangular, 1× $10 \mathrm{~mm}$

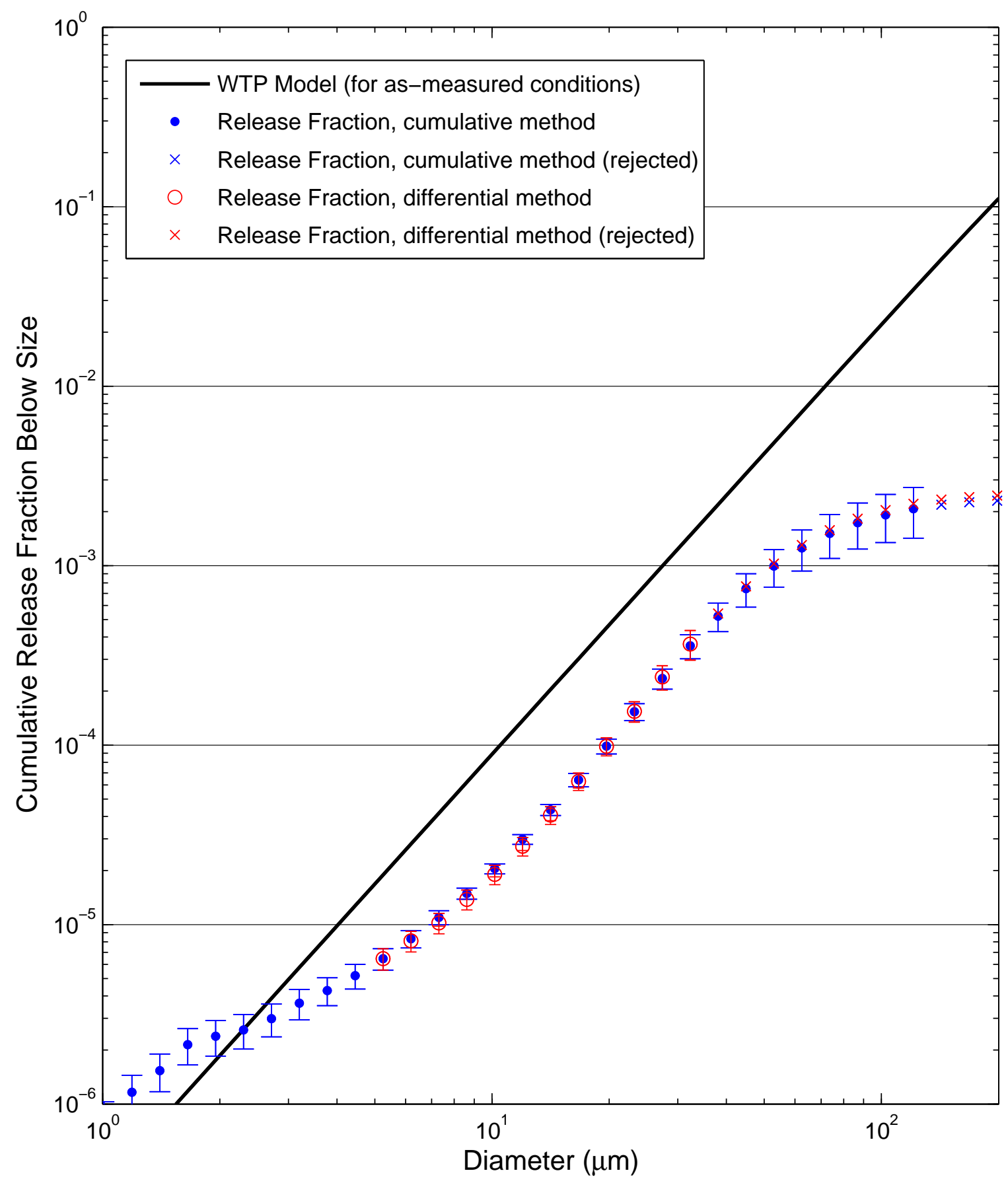


Test S147 [3 Malvern Average]: 200 psi, Orifice S7A - rectangular, $1 \times 10 \mathrm{~mm}$

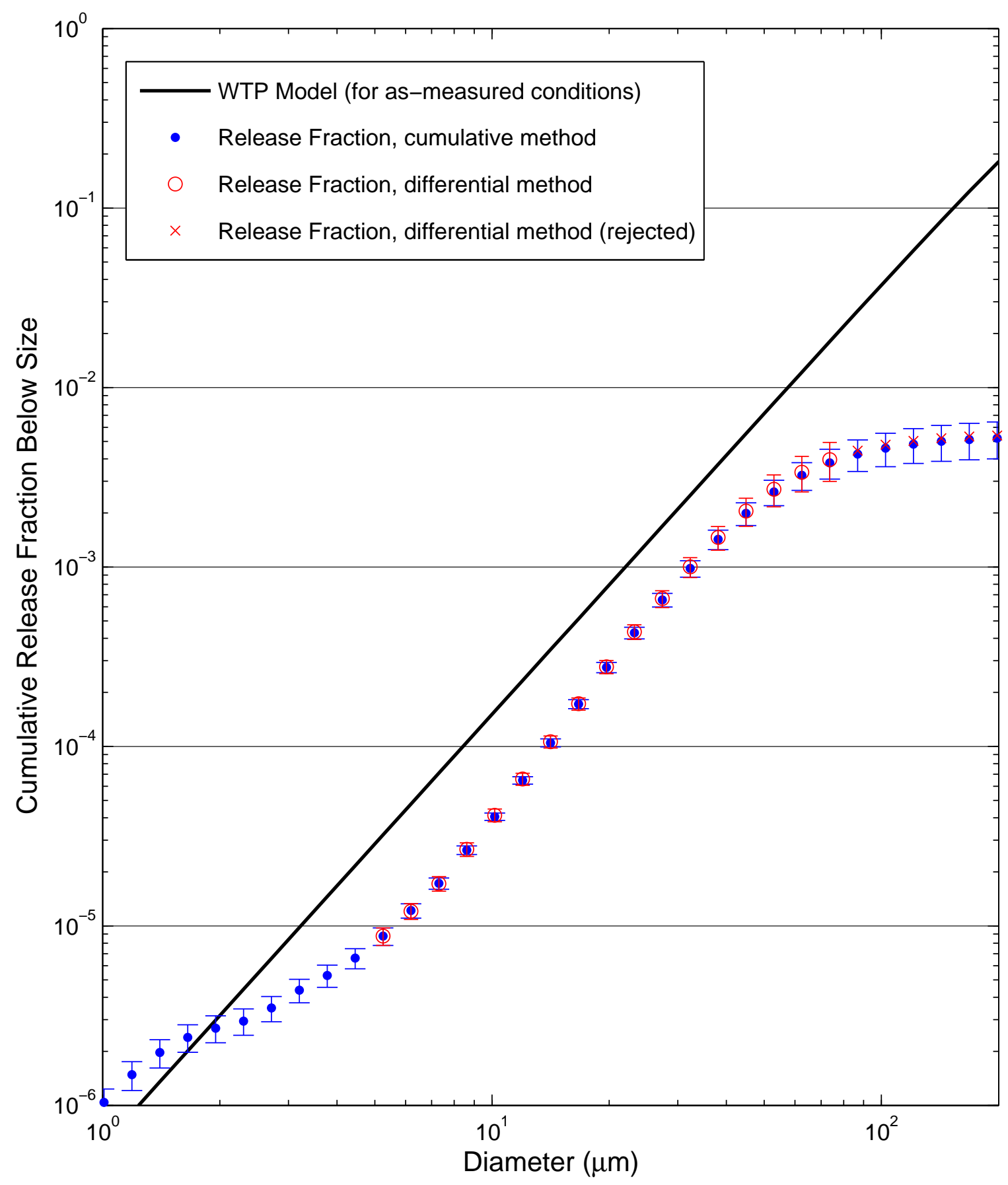


Test S148 [3 Malvern Average]: 380 psi, Orifice S7A - rectangular, $1 \times 10 \mathrm{~mm}$

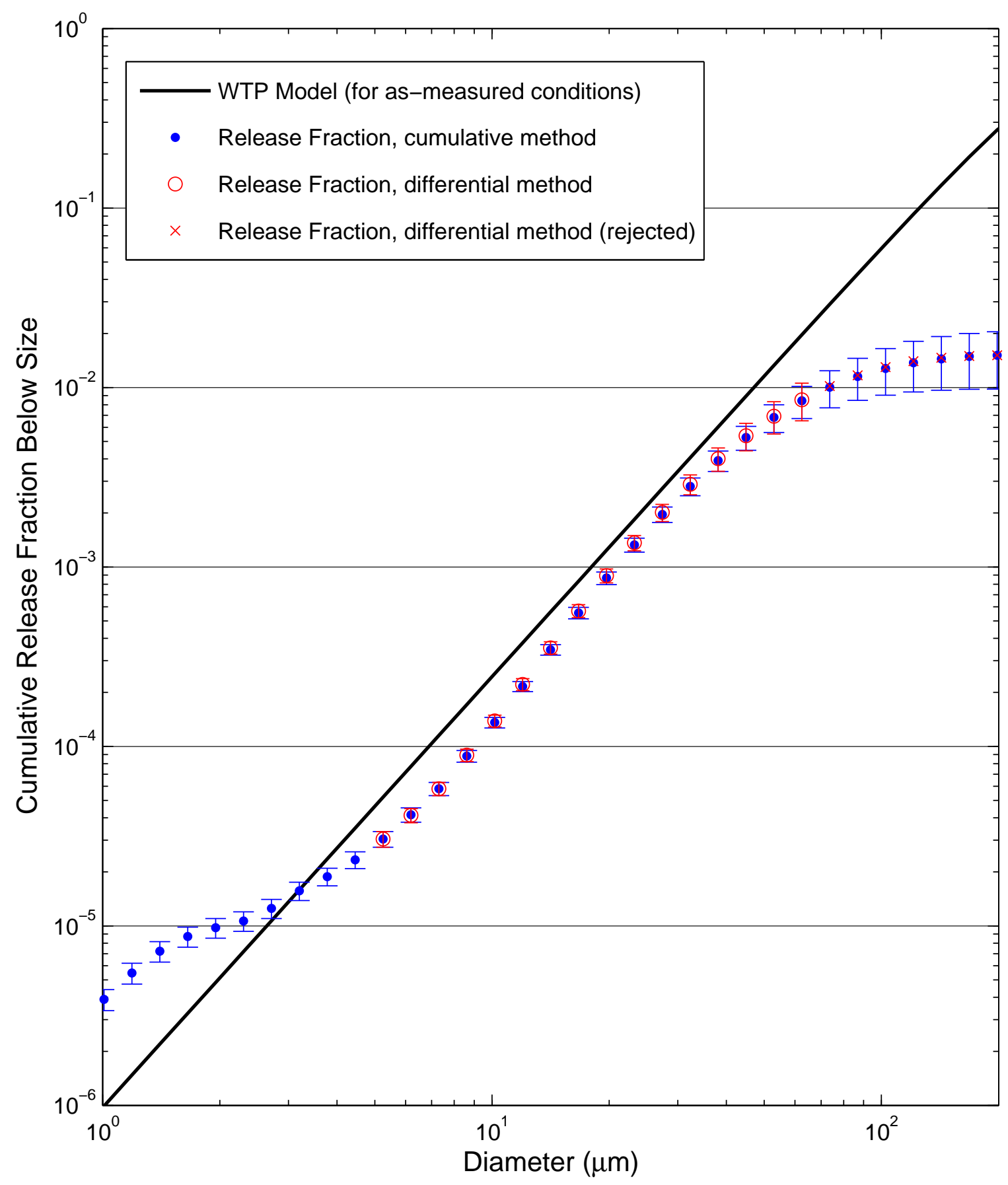


Test S149 [3 Malvern Average]: 380 psi, Orifice S7A - rectangular, $1 \times 10 \mathrm{~mm}$

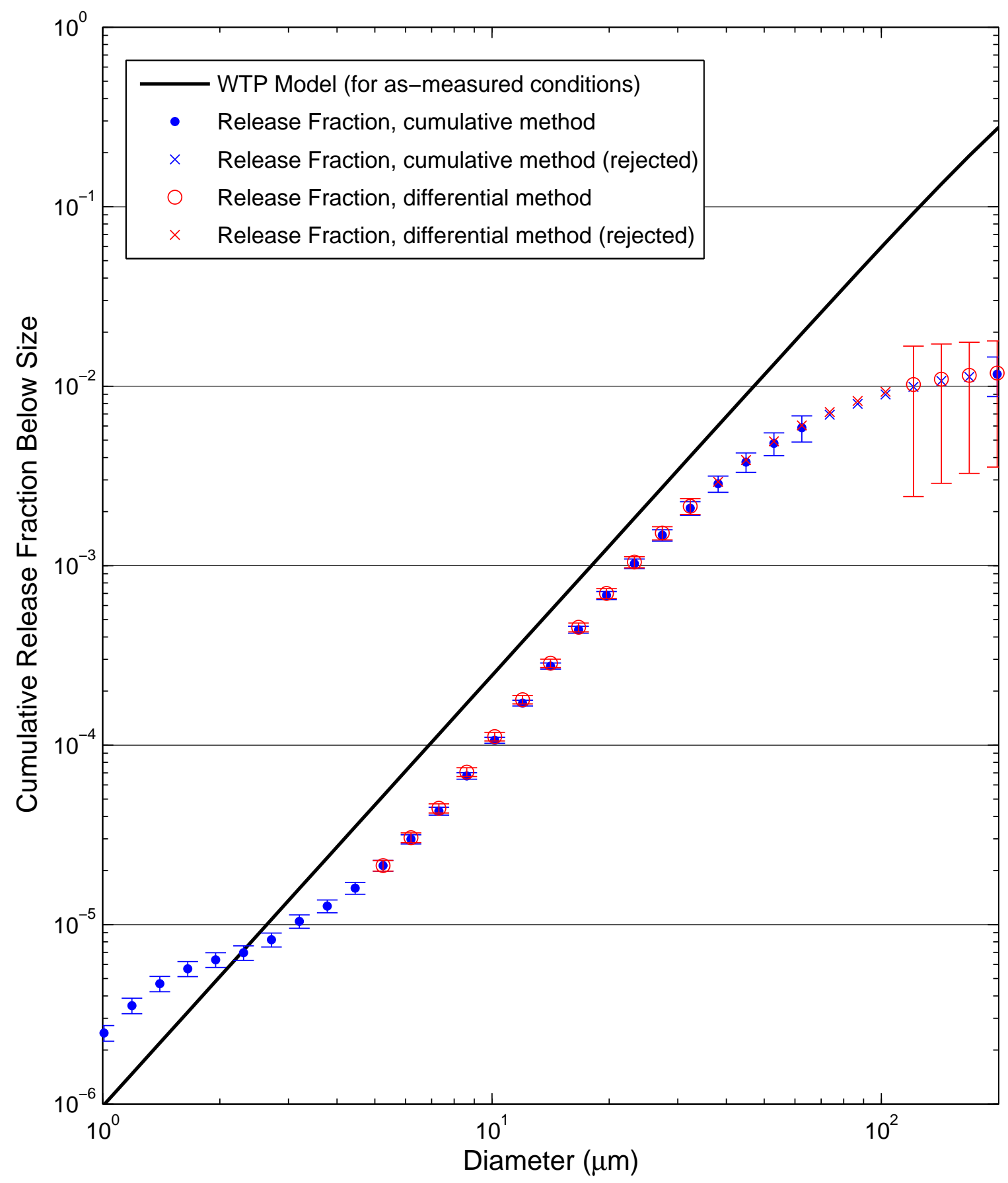


Test S150 [3 Malvern Average]: 100 psi, Orifice S7A - rectangular, $1 \times 10 \mathrm{~mm}$

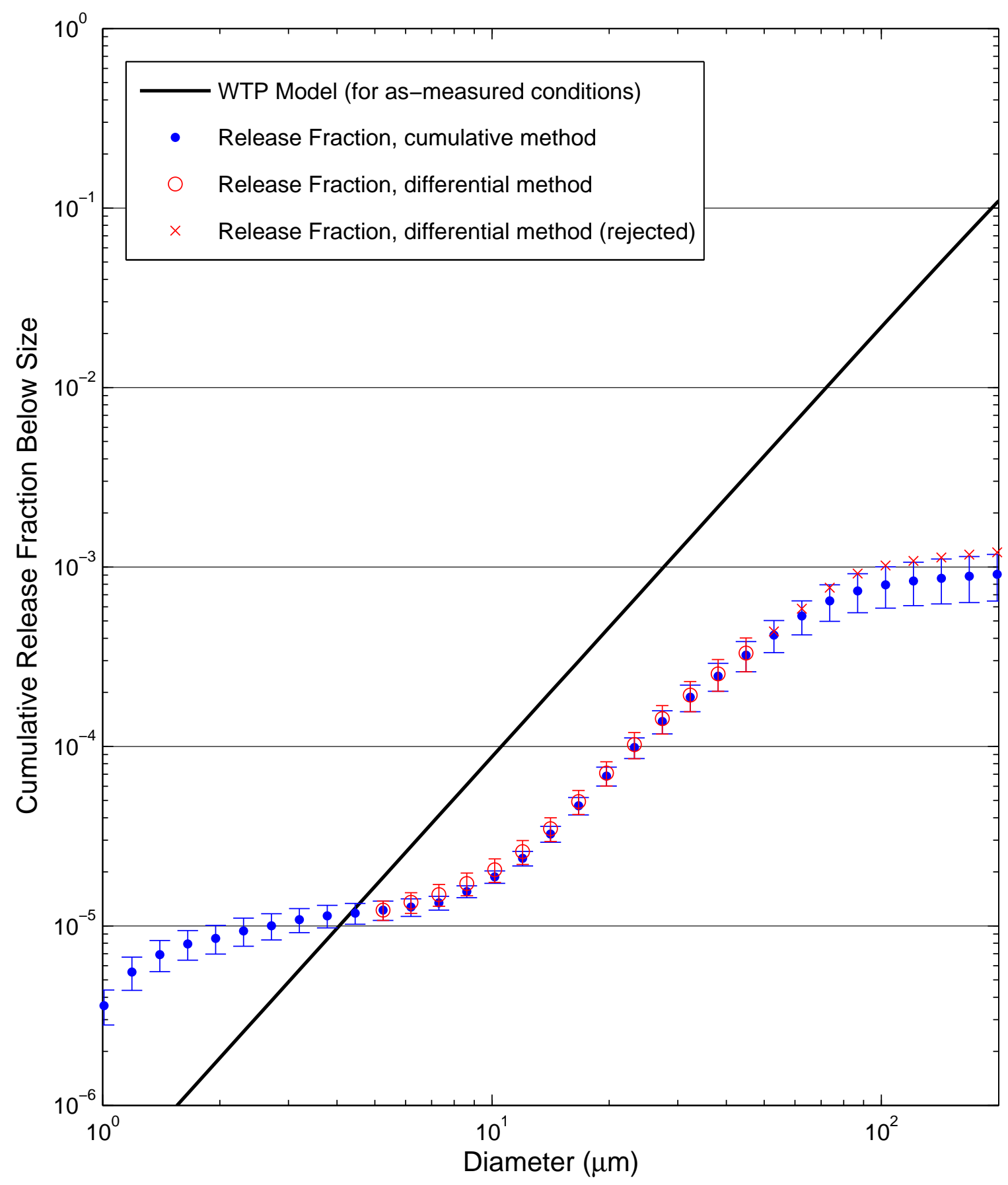

B.109 
Test S151 [3 Malvern Average]: 200 psi, Orifice S7A - rectangular, $1 \times 10 \mathrm{~mm}$

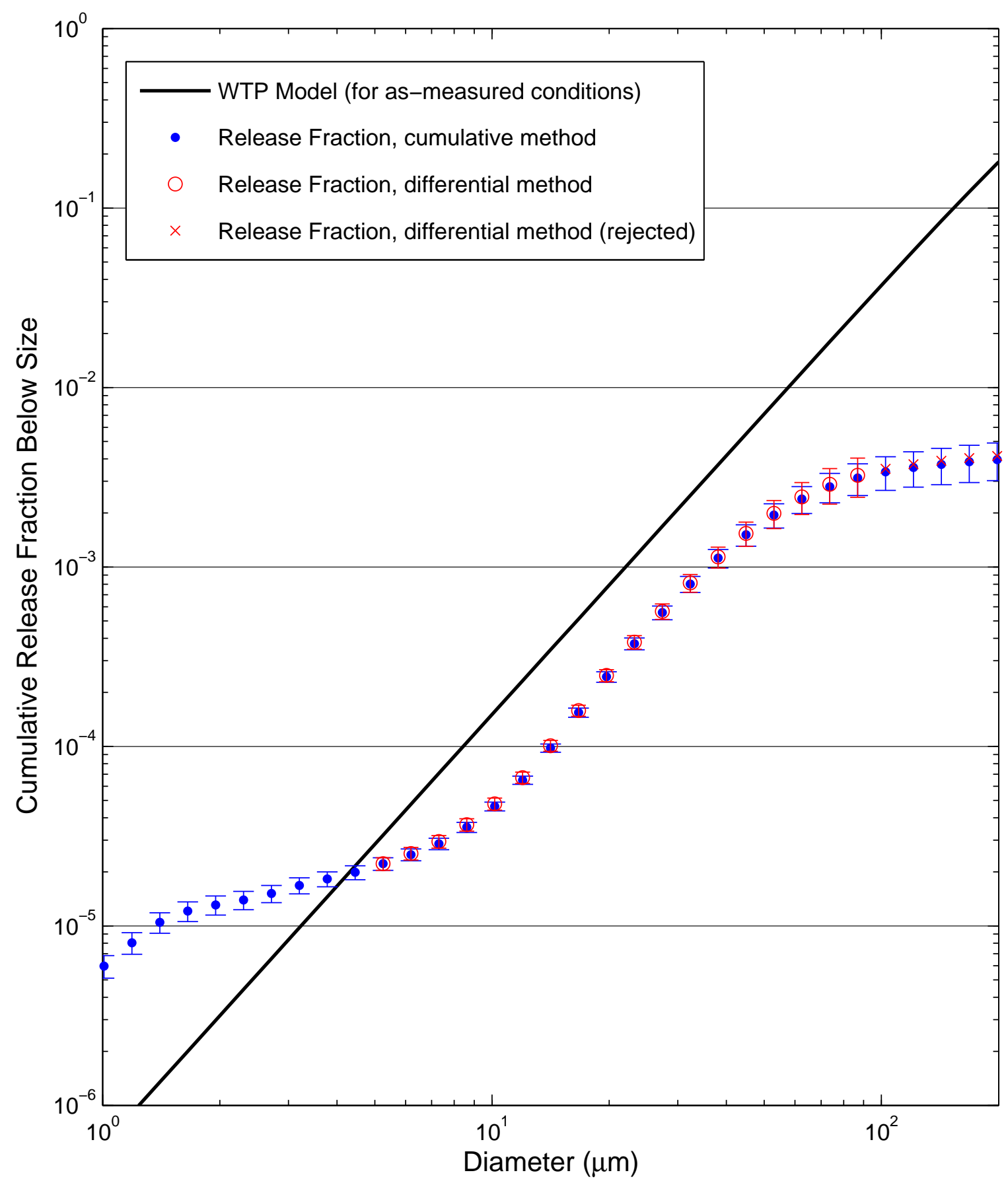

B. 110 
Test S152 [3 Malvern Average]: 380 psi, Orifice S7A - rectangular, $1 \times 10 \mathrm{~mm}$

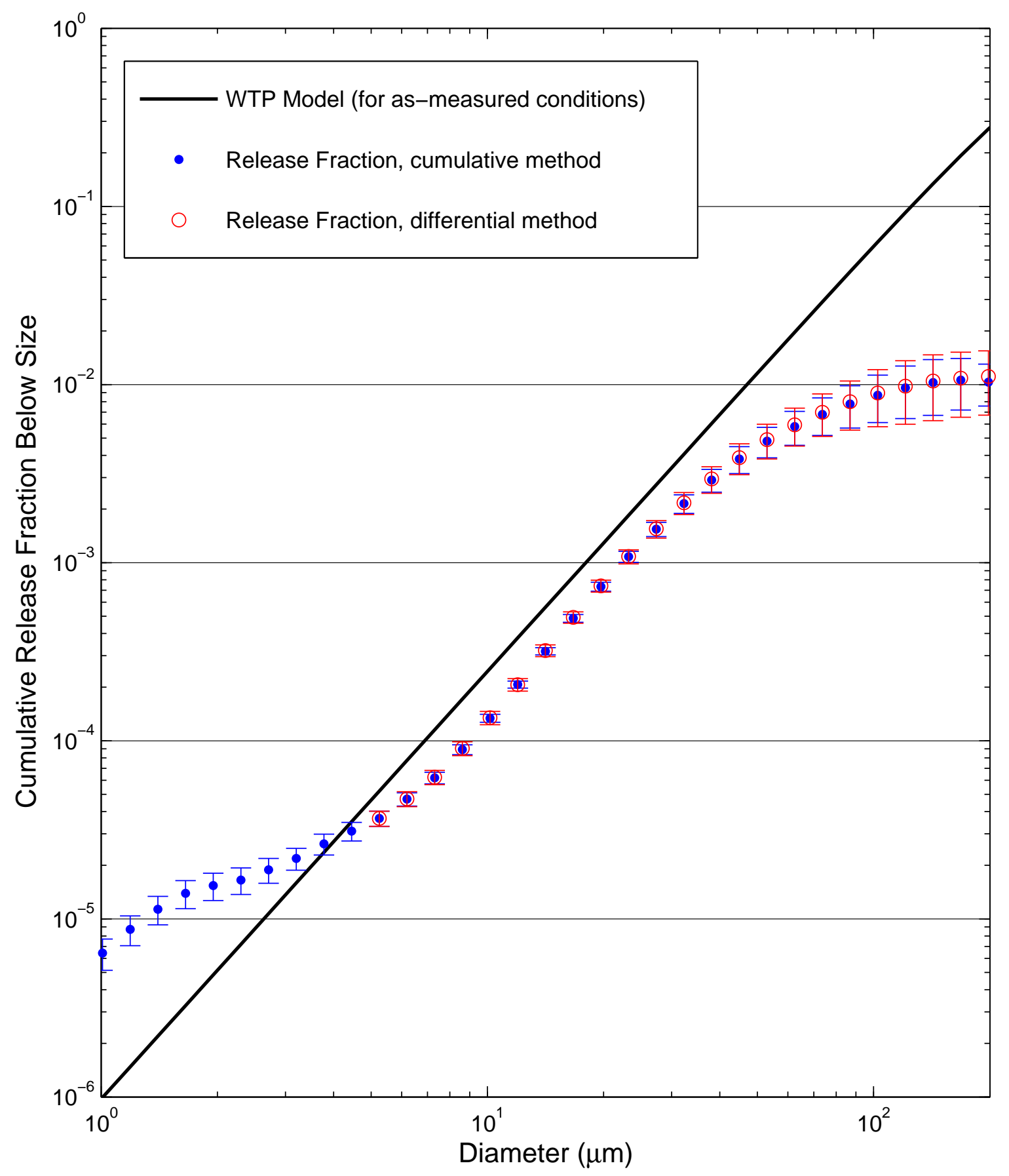


Test S153 [3 Malvern Average]: 100 psi, Orifice S2A - rectangular, $0.5 \times 5 \mathrm{~mm}$

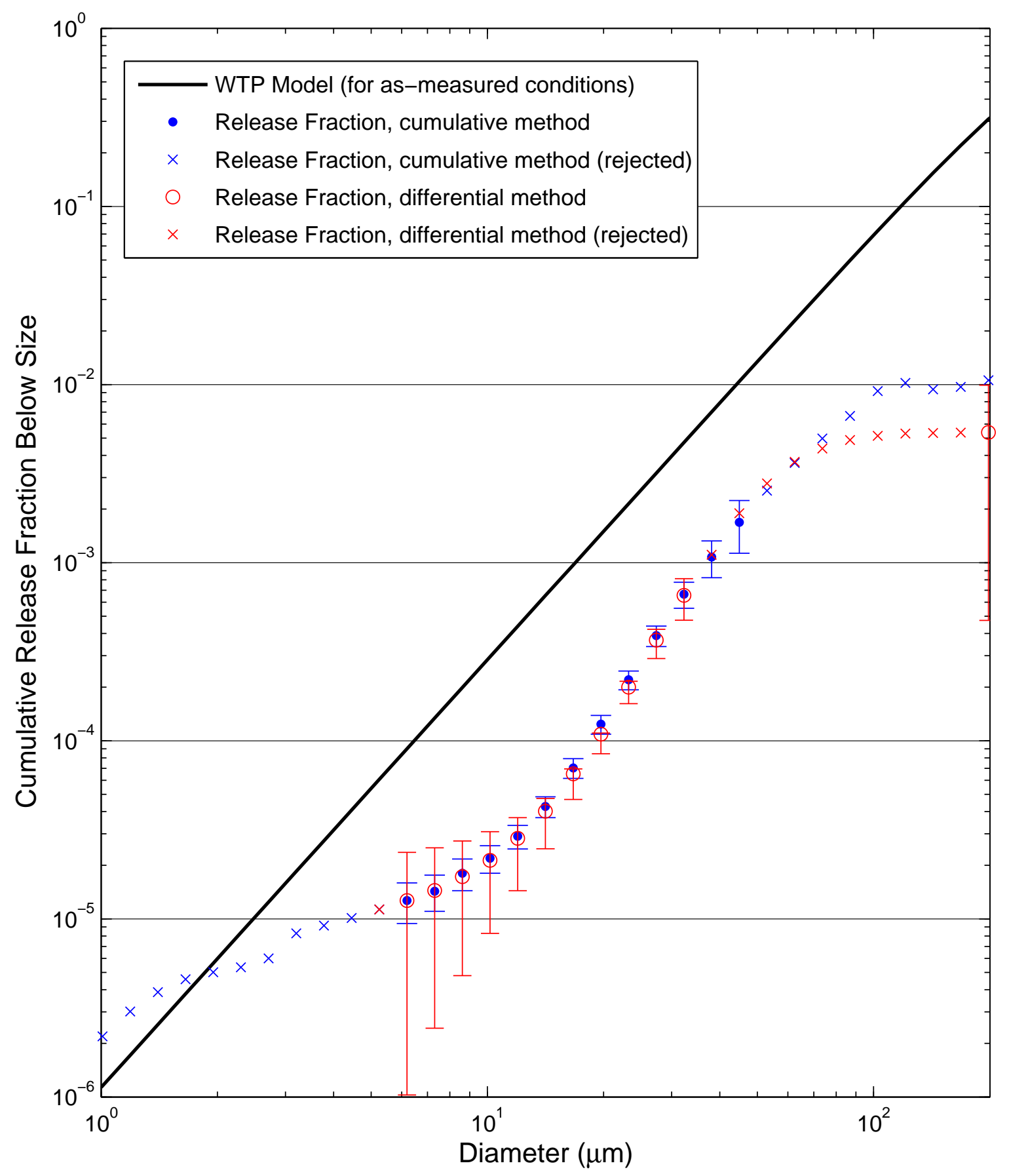

B. 112 
Test S154 [3 Malvern Average]: 200 psi, Orifice S2A - rectangular, $0.5 \times 5 \mathrm{~mm}$

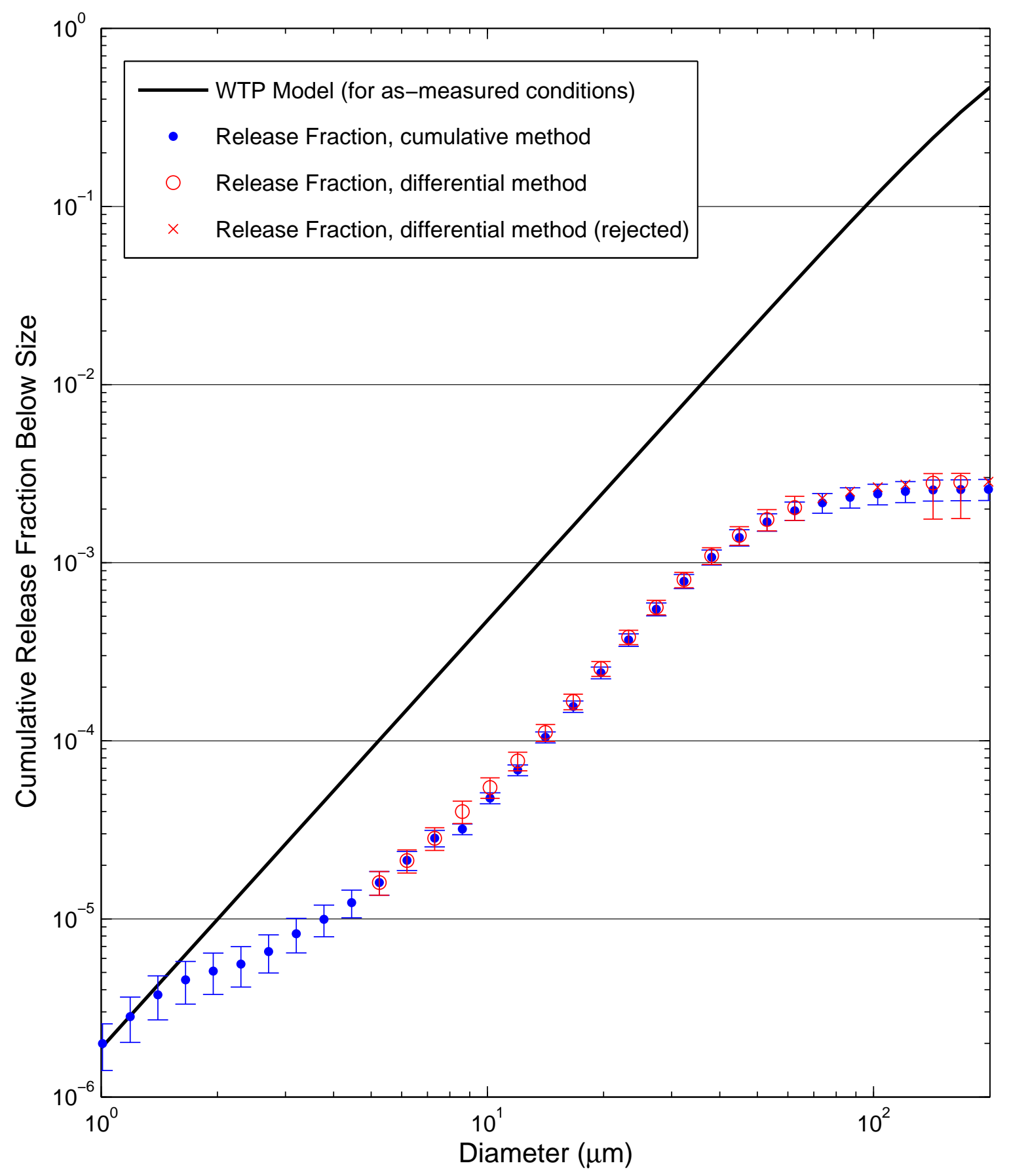


Test S155 [3 Malvern Average]: 380 psi, Orifice S2A - rectangular, $0.5 \times 5 \mathrm{~mm}$

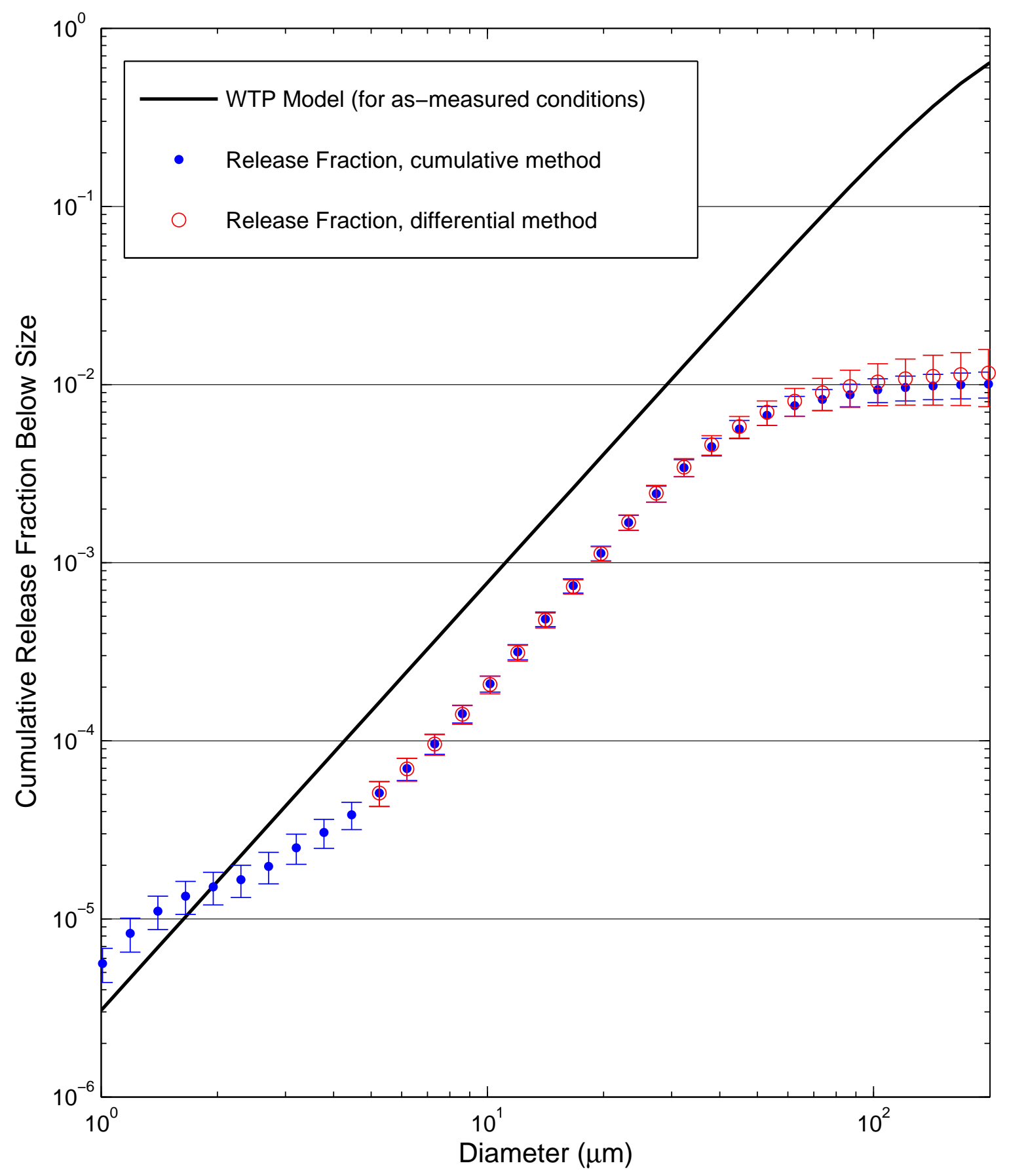


Test S156 [3 Malvern Average]: 100 psi, Orifice S2A - rectangular, 0.5× $5 \mathrm{~mm}$

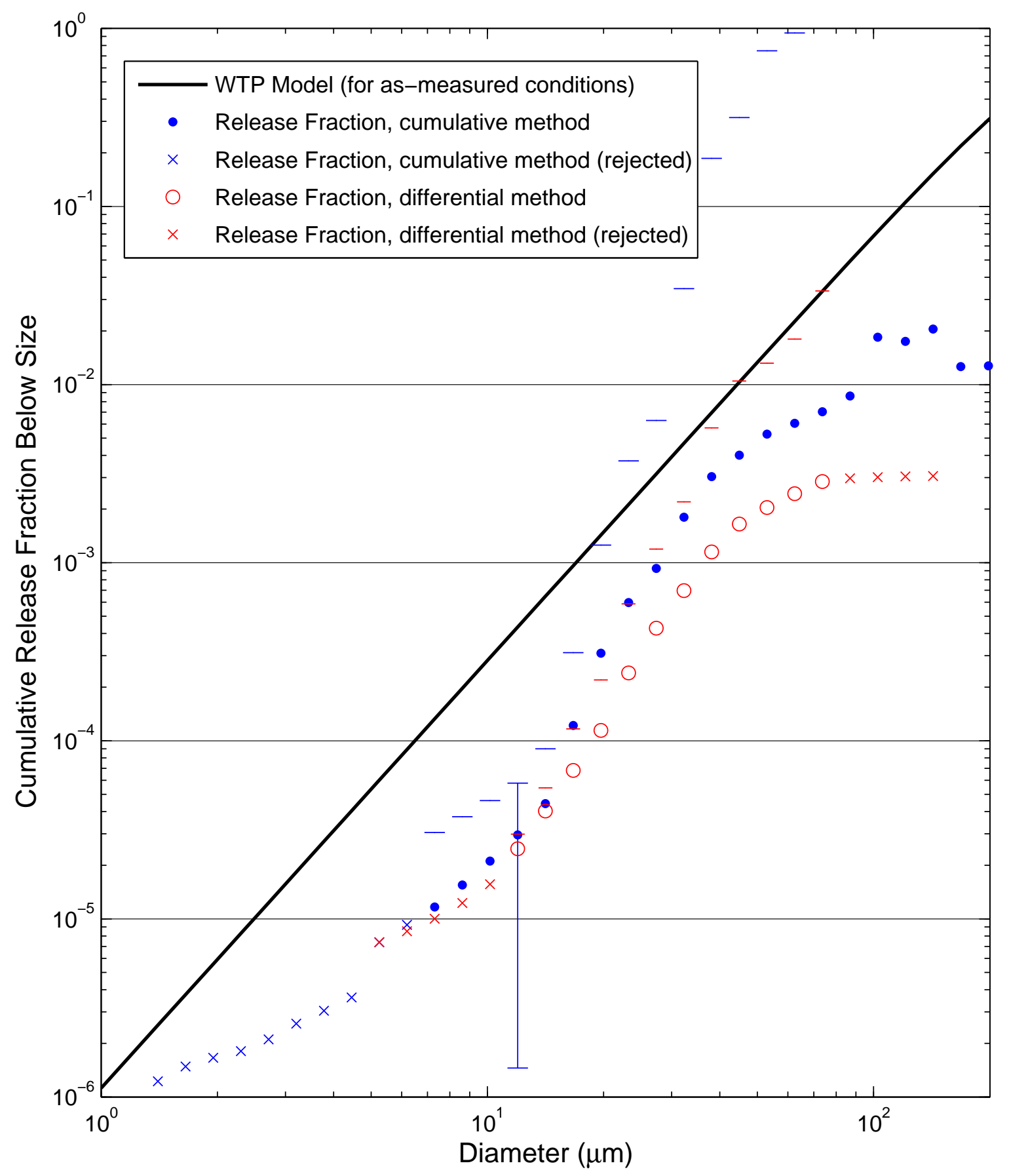


Test S157 [3 Malvern Average]: 200 psi, Orifice S2A - rectangular, $0.5 \times 5 \mathrm{~mm}$

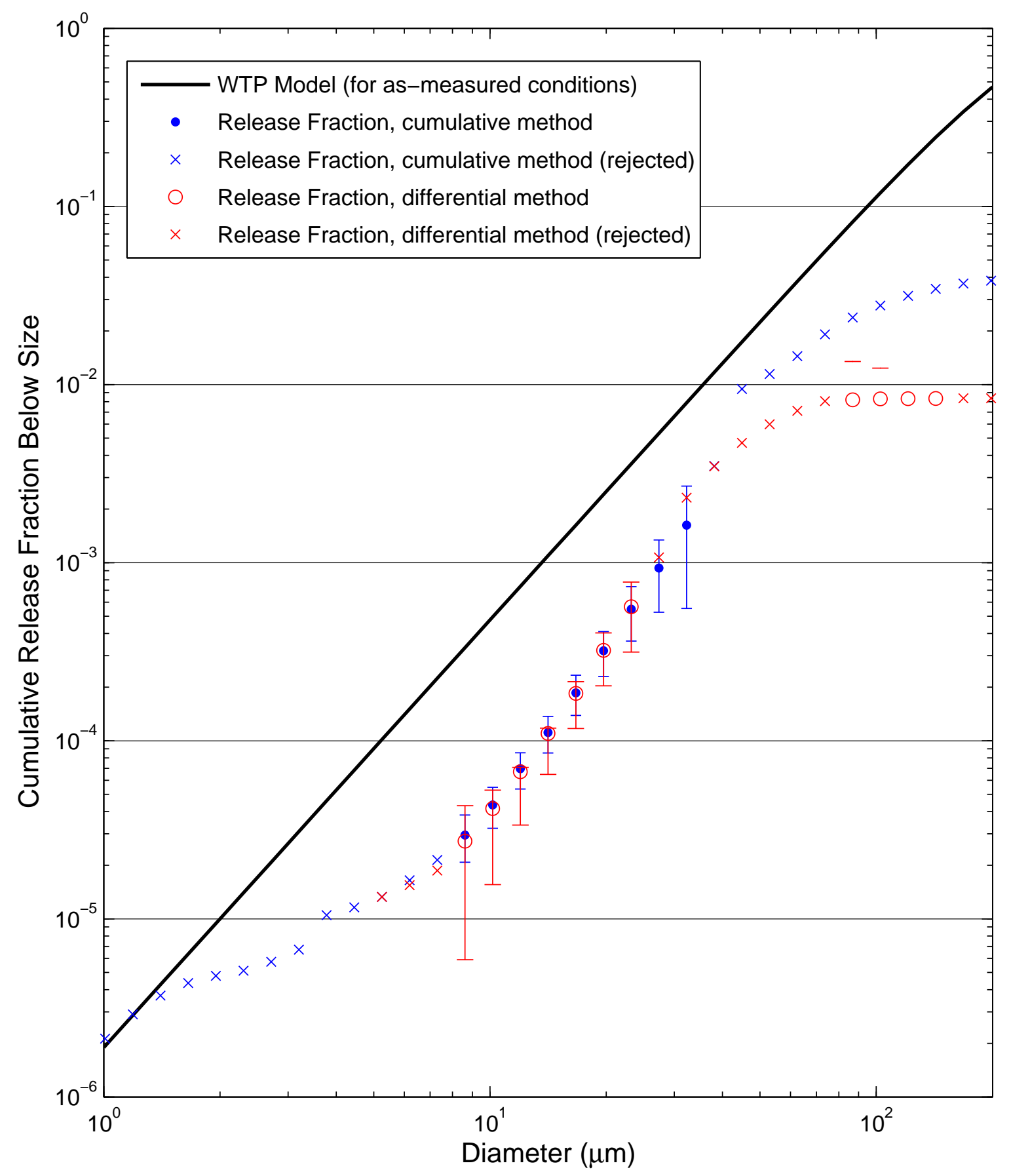


Test S158 [3 Malvern Average]: 380 psi, Orifice S2A - rectangular, $0.5 \times 5 \mathrm{~mm}$

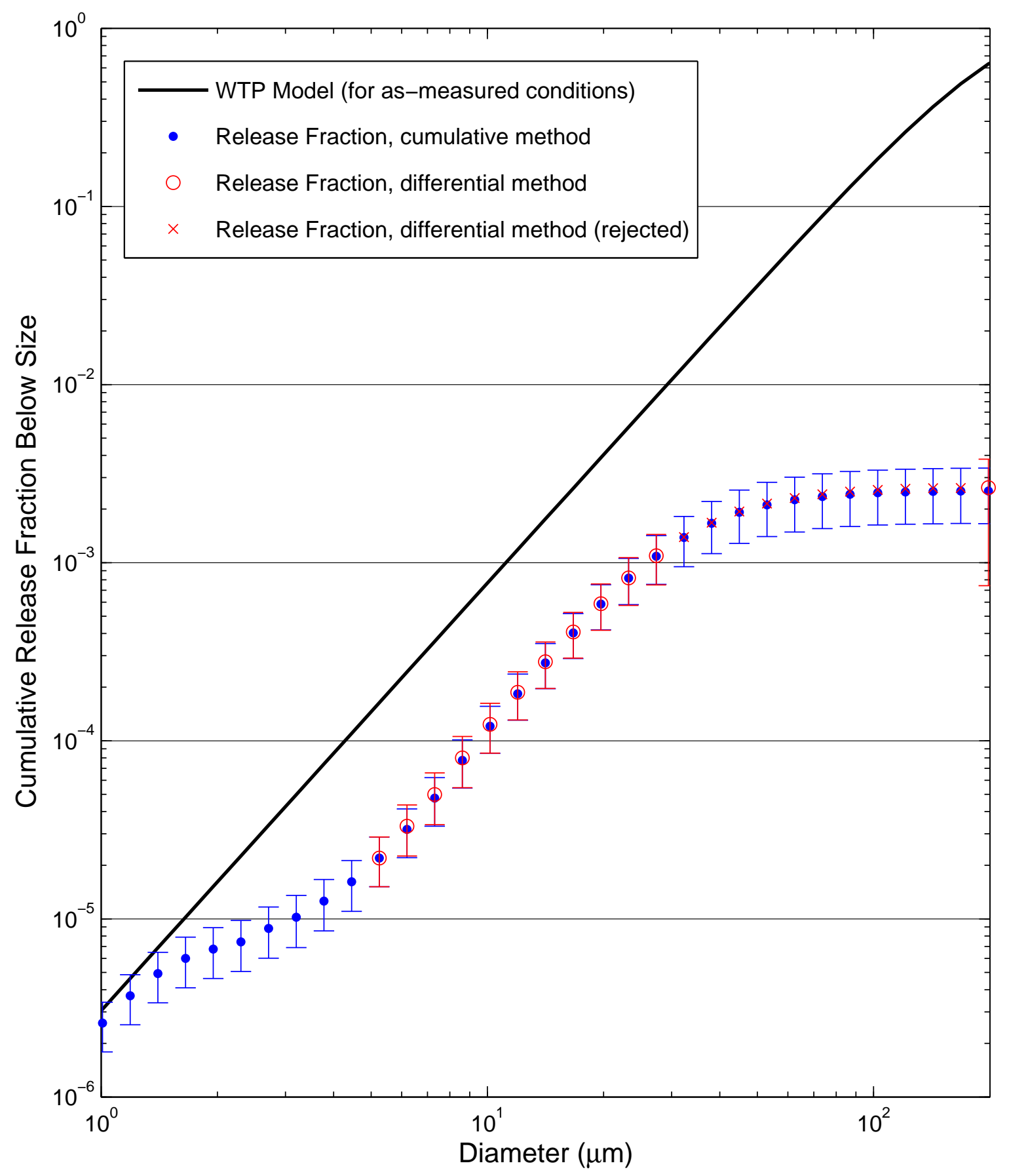


Test W159 [3 Malvern Average]: 100 psi, Orifice S7A - rectangular, $1 \times 10 \mathrm{~mm}$

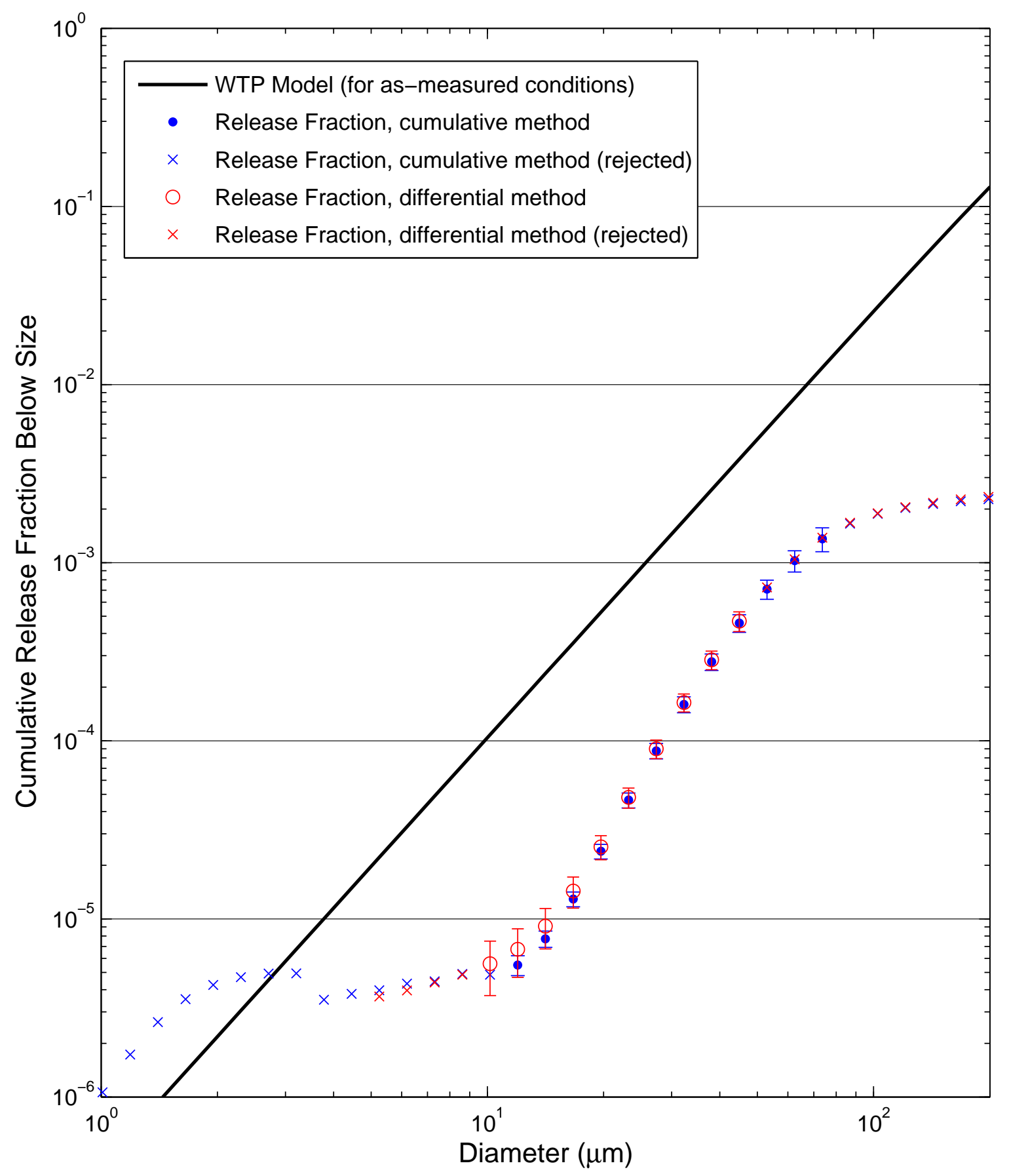


Test W160 [3 Malvern Average]: 200 psi, Orifice S7A - rectangular, $1 \times 10 \mathrm{~mm}$

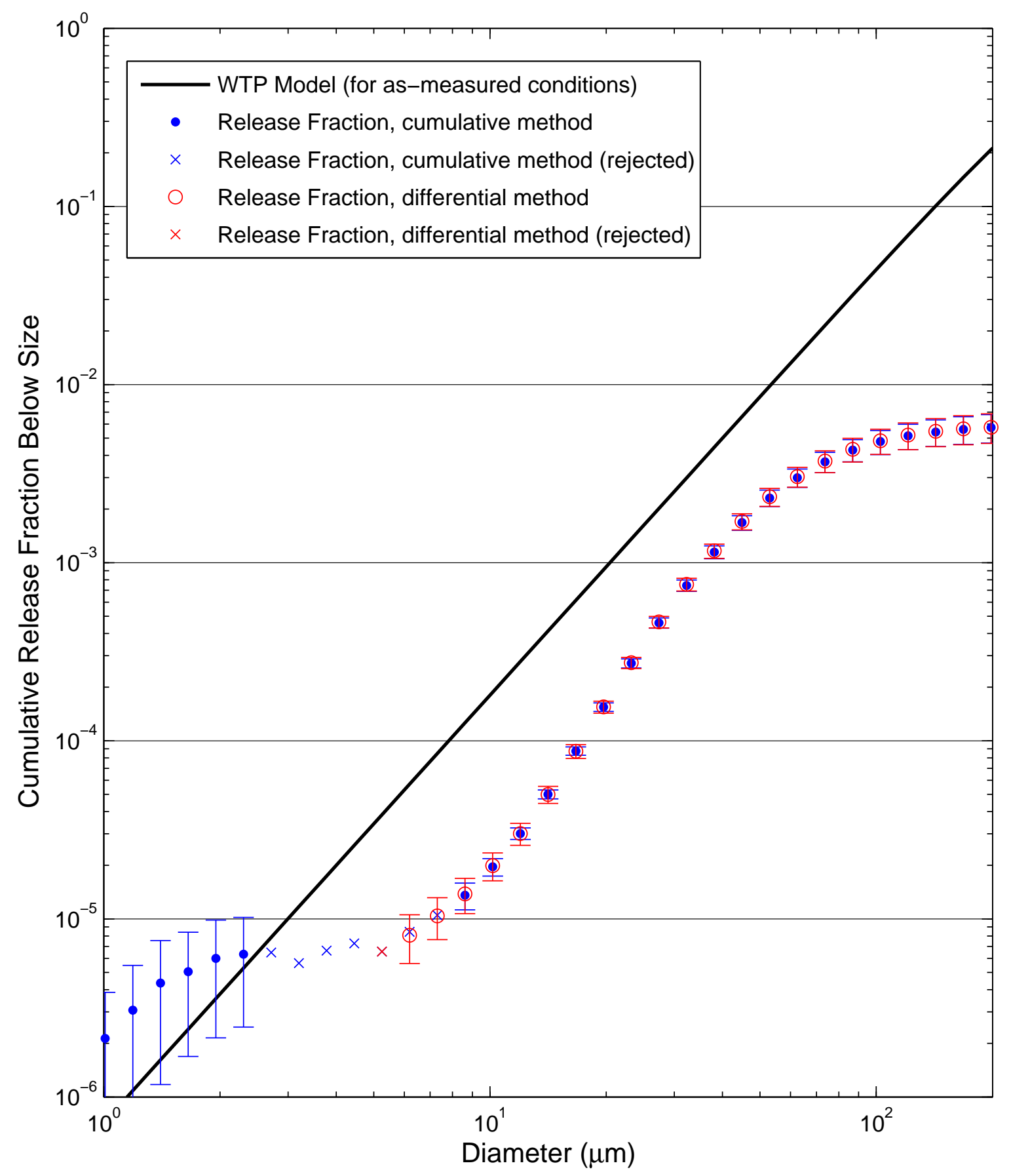


Test W161 [3 Malvern Average]: 380 psi, Orifice S7A - rectangular, $1 \times 10 \mathrm{~mm}$

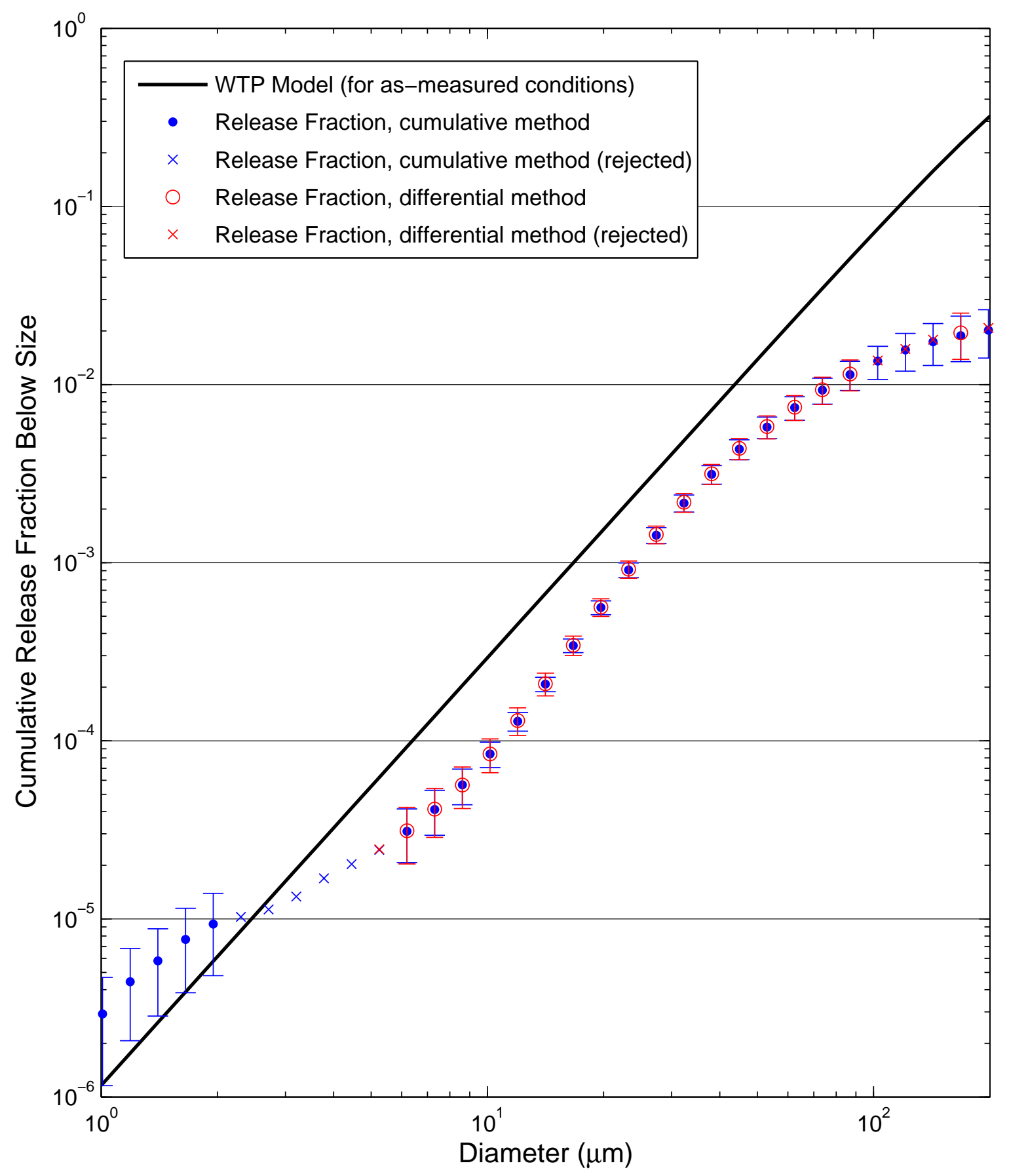

B. 120 
Test W162 [3 Malvern Average]: 100 psi, Orifice S7B - rectangular, $2 \times 10 \mathrm{~mm}$

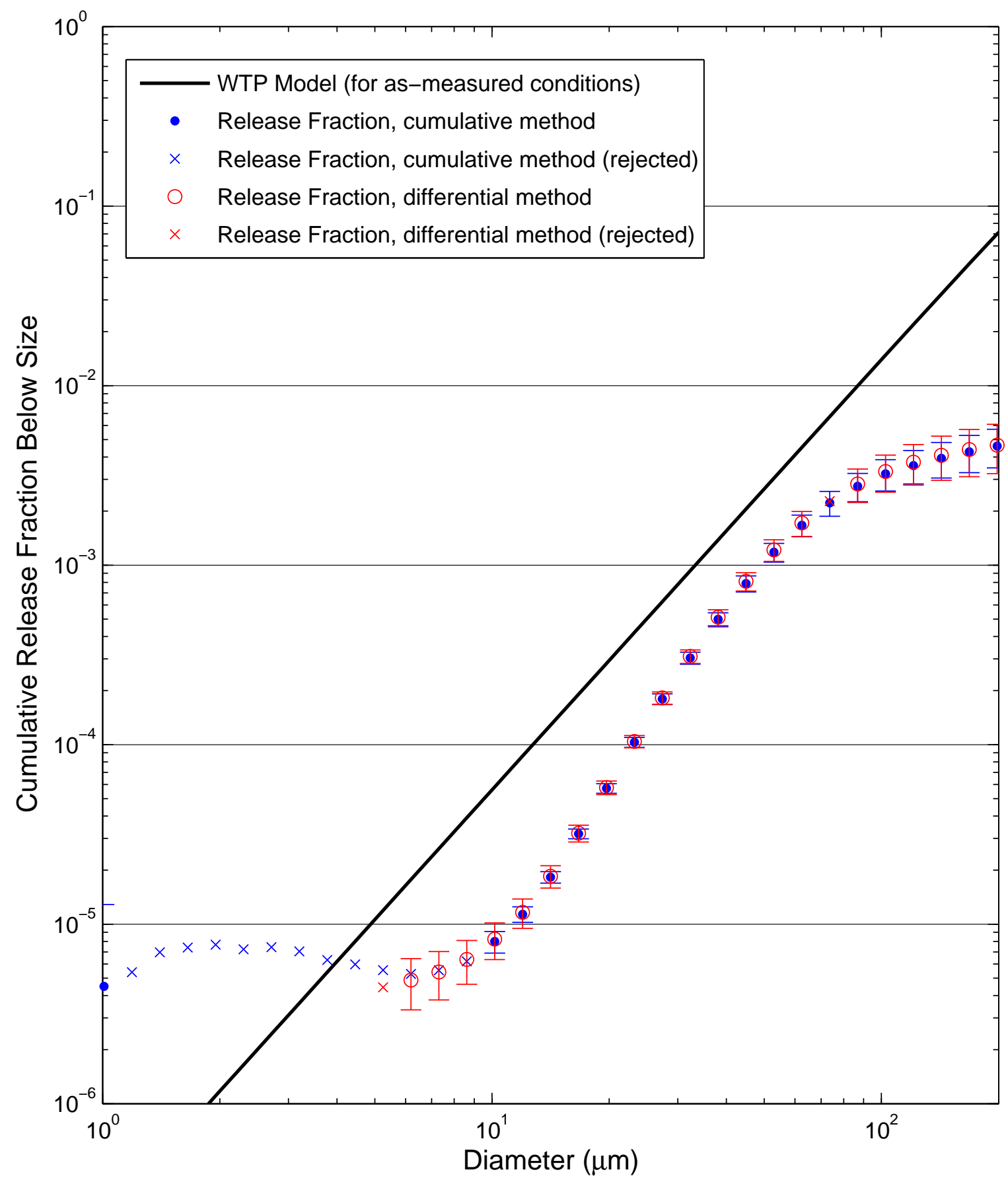

B. 121 
Test W163 [3 Malvern Average]: 200 psi, Orifice S7B - rectangular, $2 \times 10 \mathrm{~mm}$

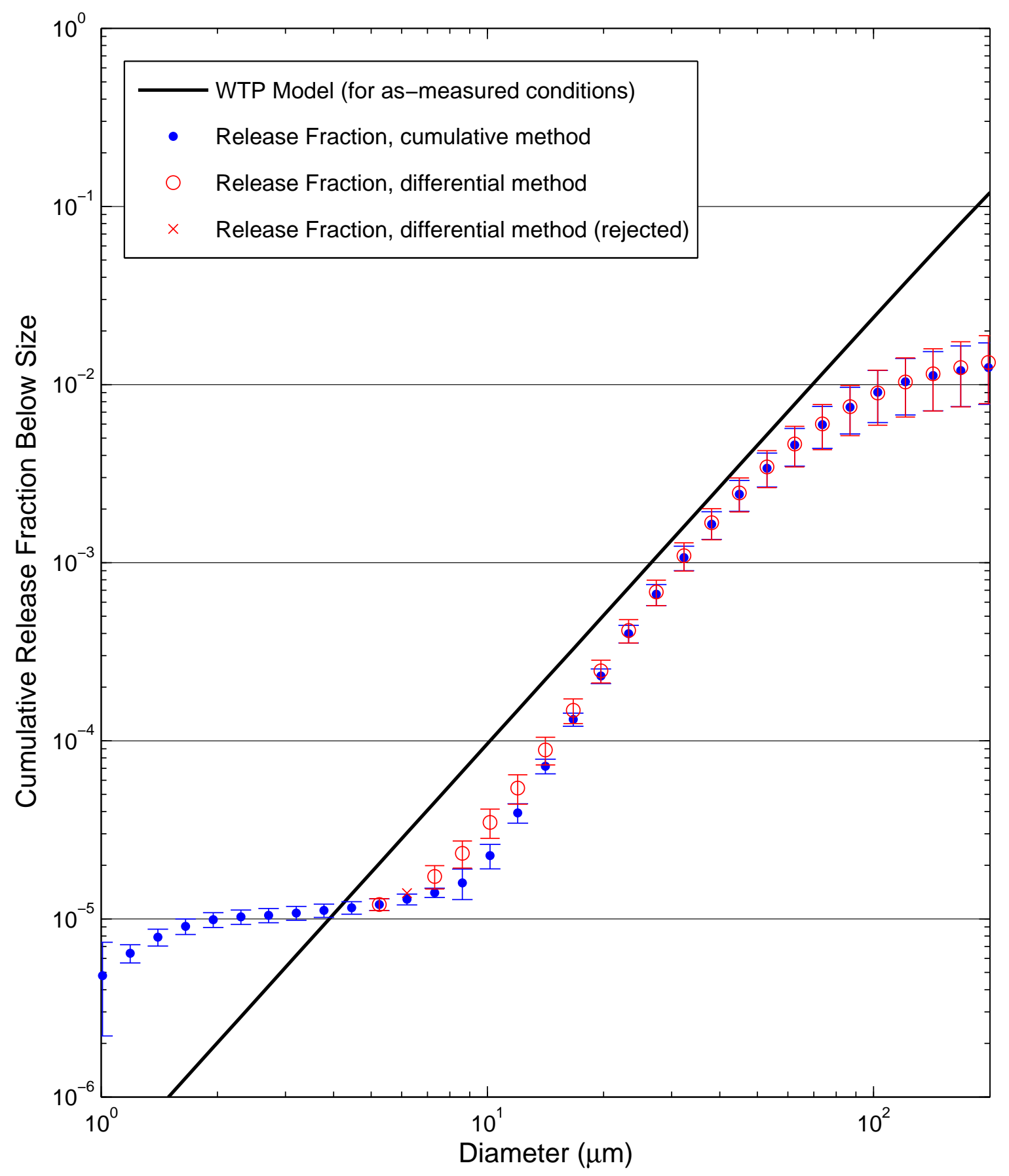

B. 122 
Test W164 [3 Malvern Average]: 380 psi, Orifice S7B - rectangular, $2 \times 10 \mathrm{~mm}$

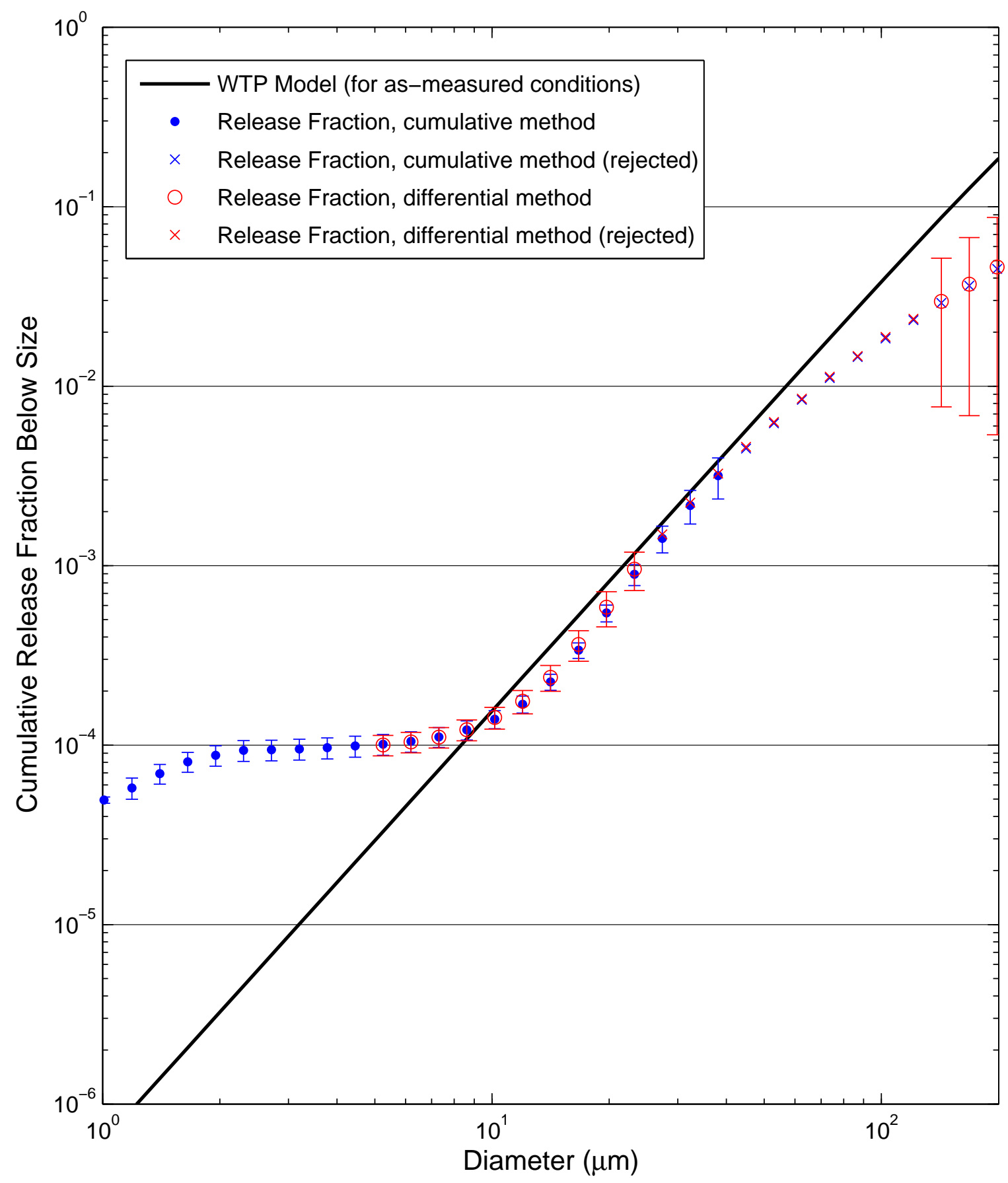


Test W165 [3 Malvern Average]: 200 psi, Orifice S7C - rectangular, 3× $10 \mathrm{~mm}$

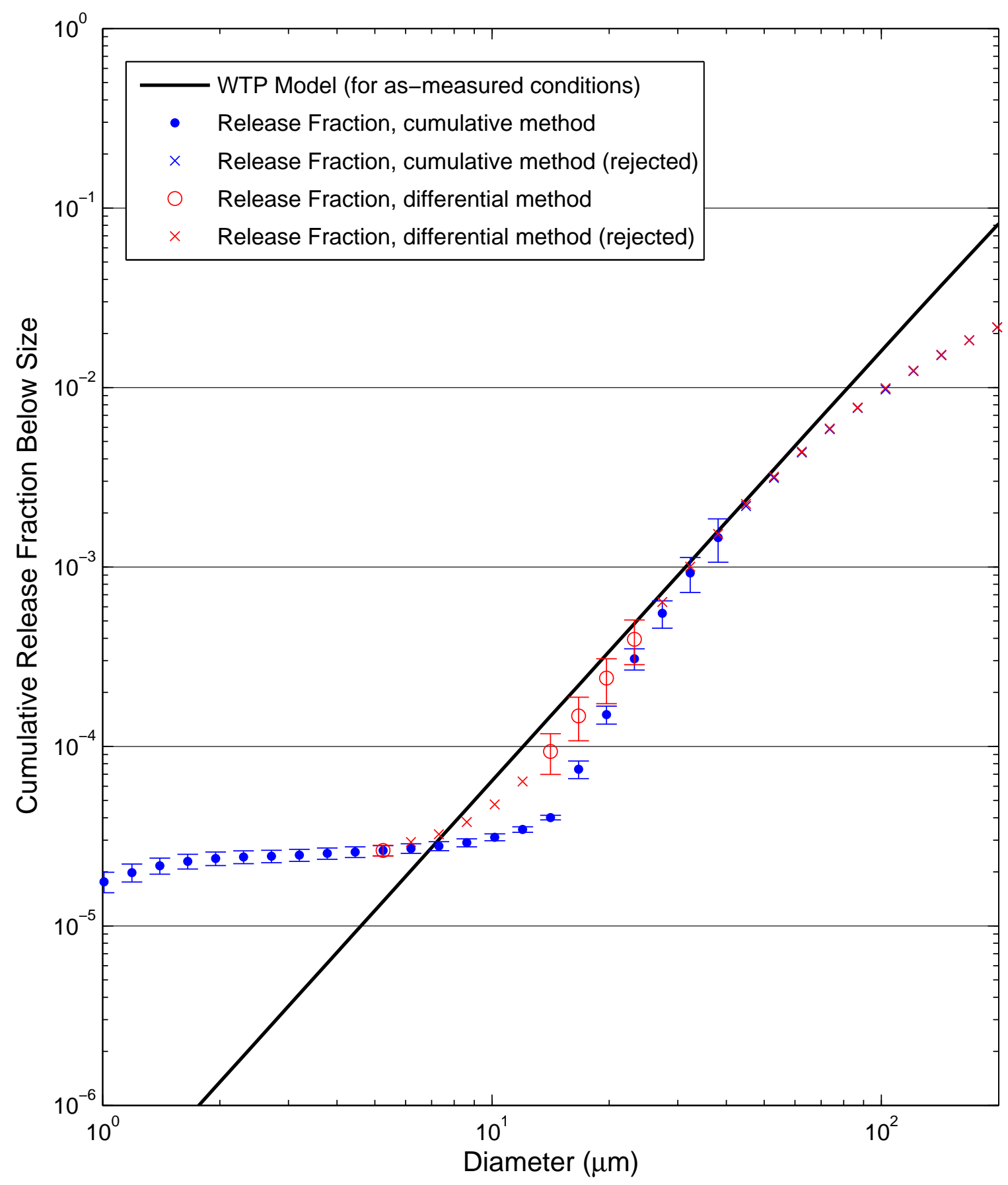


Test W166 [3 Malvern Average]: 380 psi, Orifice S7C - rectangular, $3 \times 10 \mathrm{~mm}$

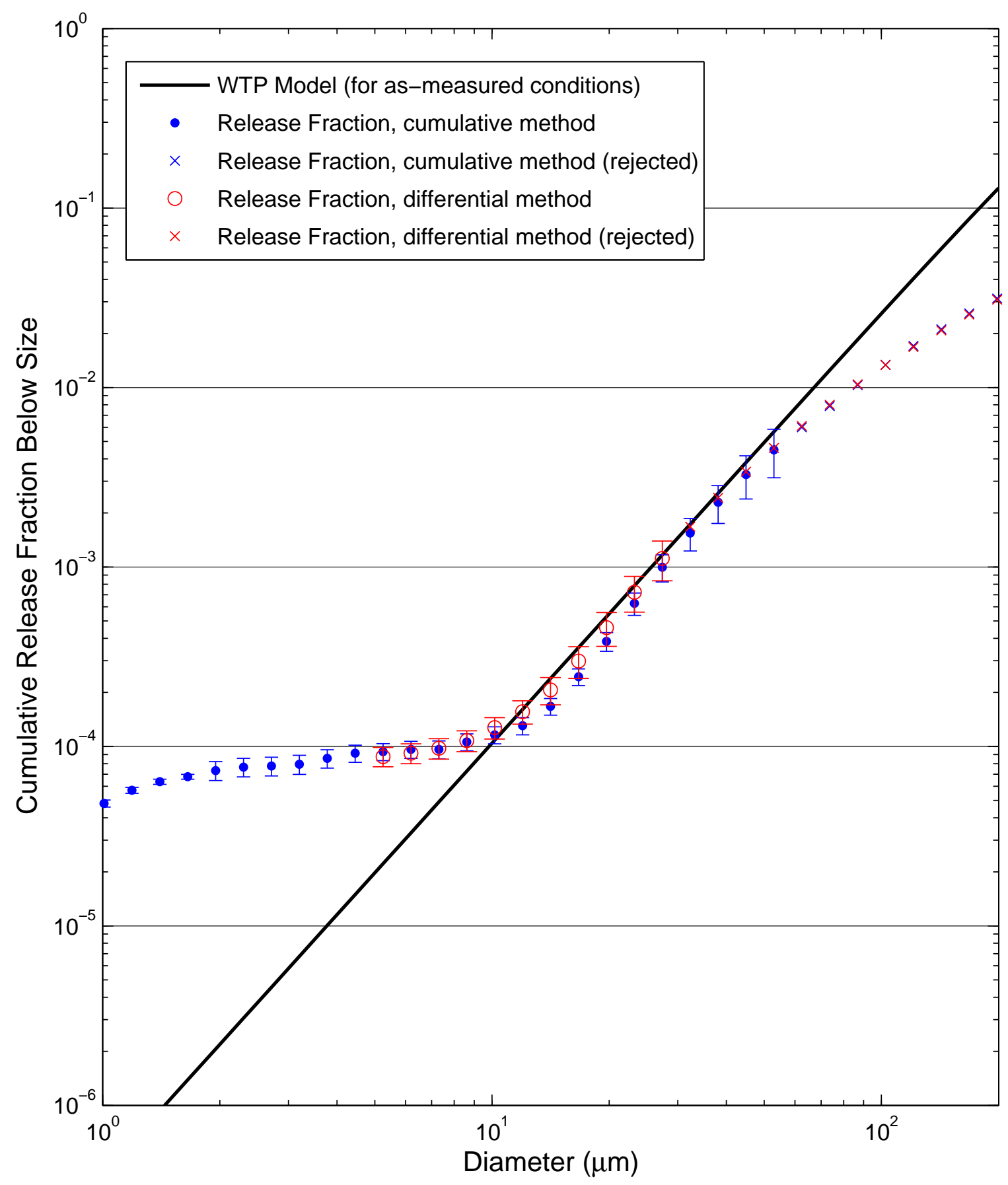

B. 125 
Test W167 [3 Malvern Average]: 200 psi, Orifice S7C - rectangular, 3× $10 \mathrm{~mm}$

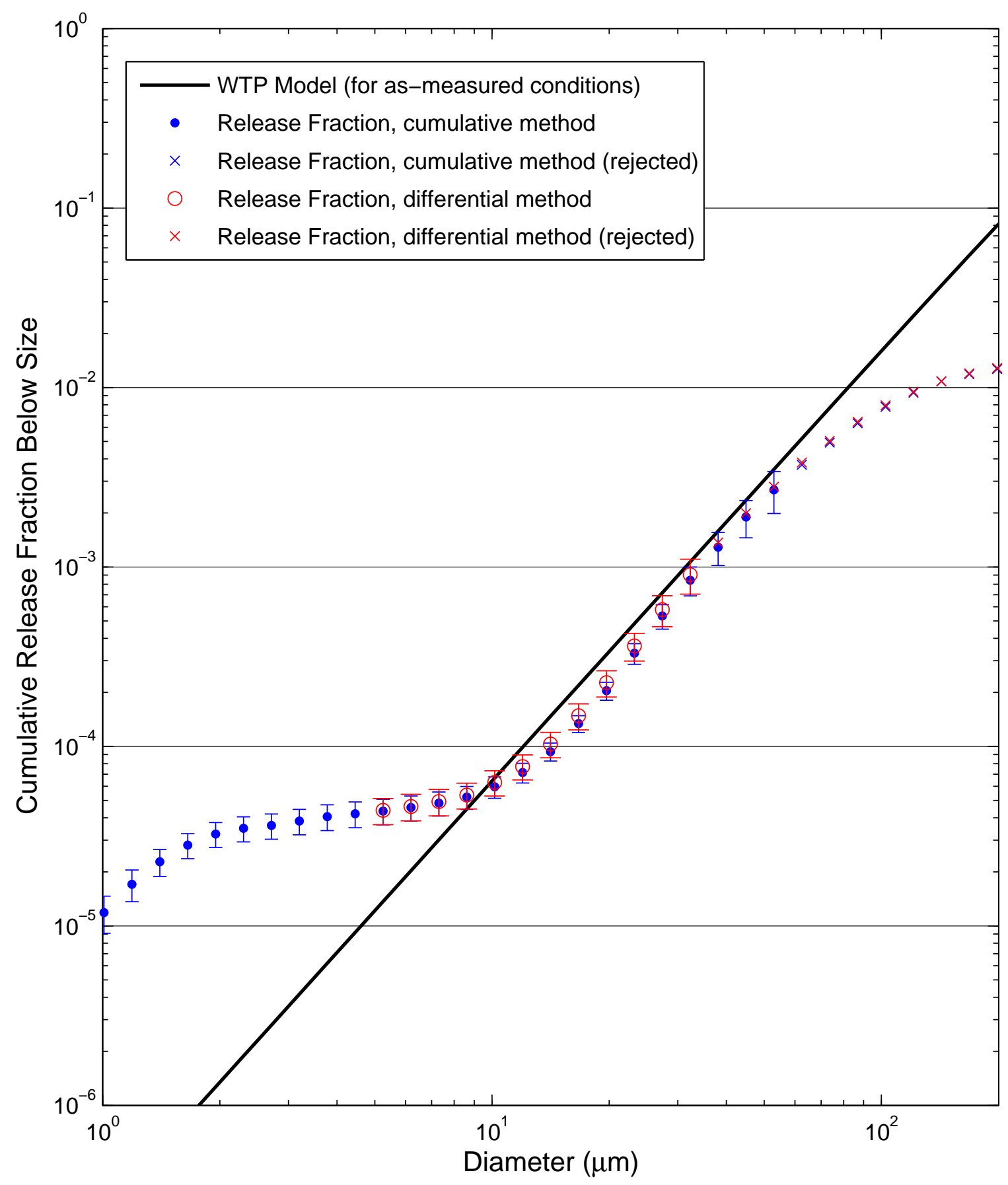

B. 126 
Test W168 [3 Malvern Average]: 380 psi, Orifice S7C - rectangular, $3 \times 10 \mathrm{~mm}$

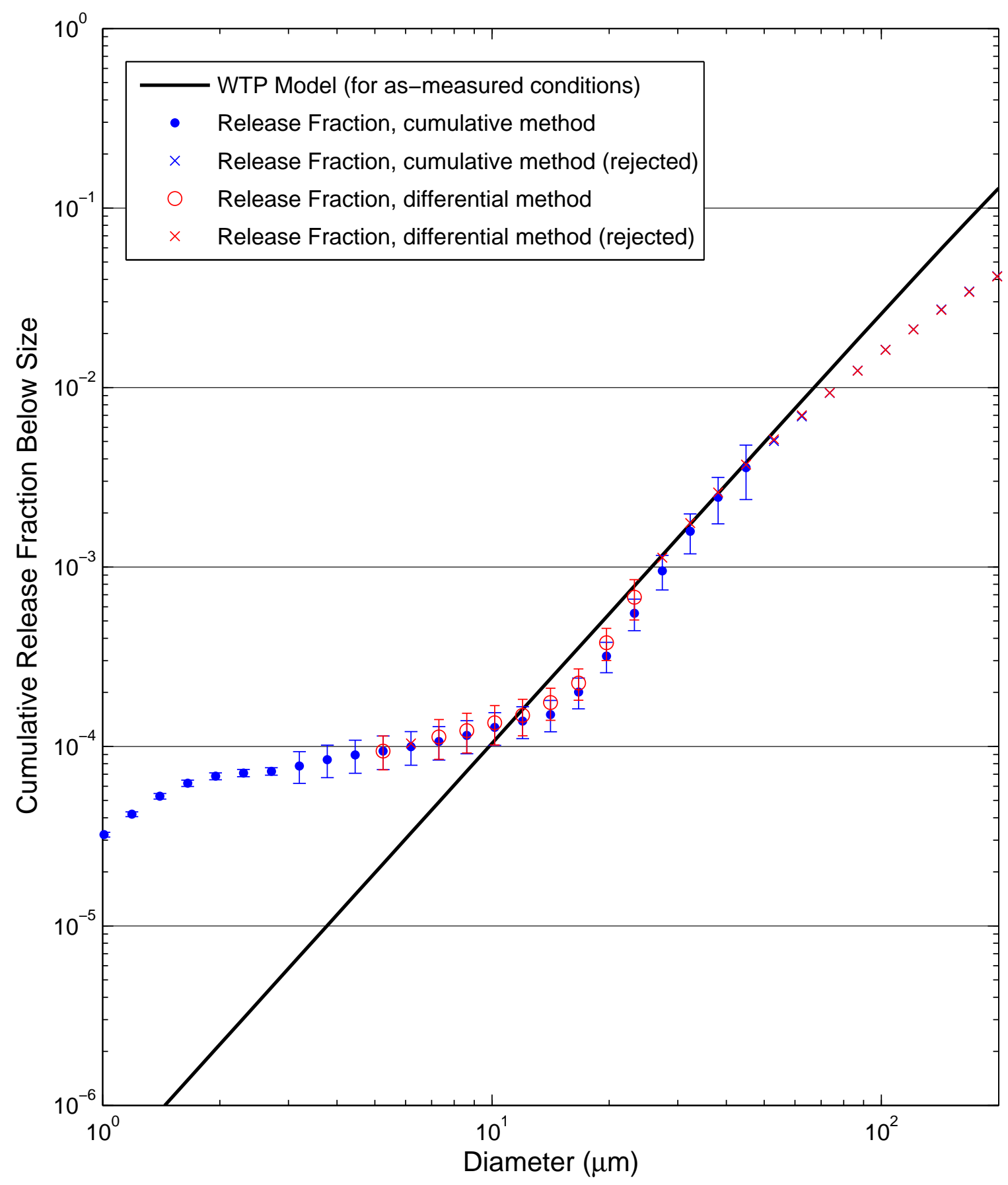

B. 127 
Test W169 [3 Malvern Average]: 200 psi, Orifice S5C - rectangular, $1 \times 20 \mathrm{~mm}$

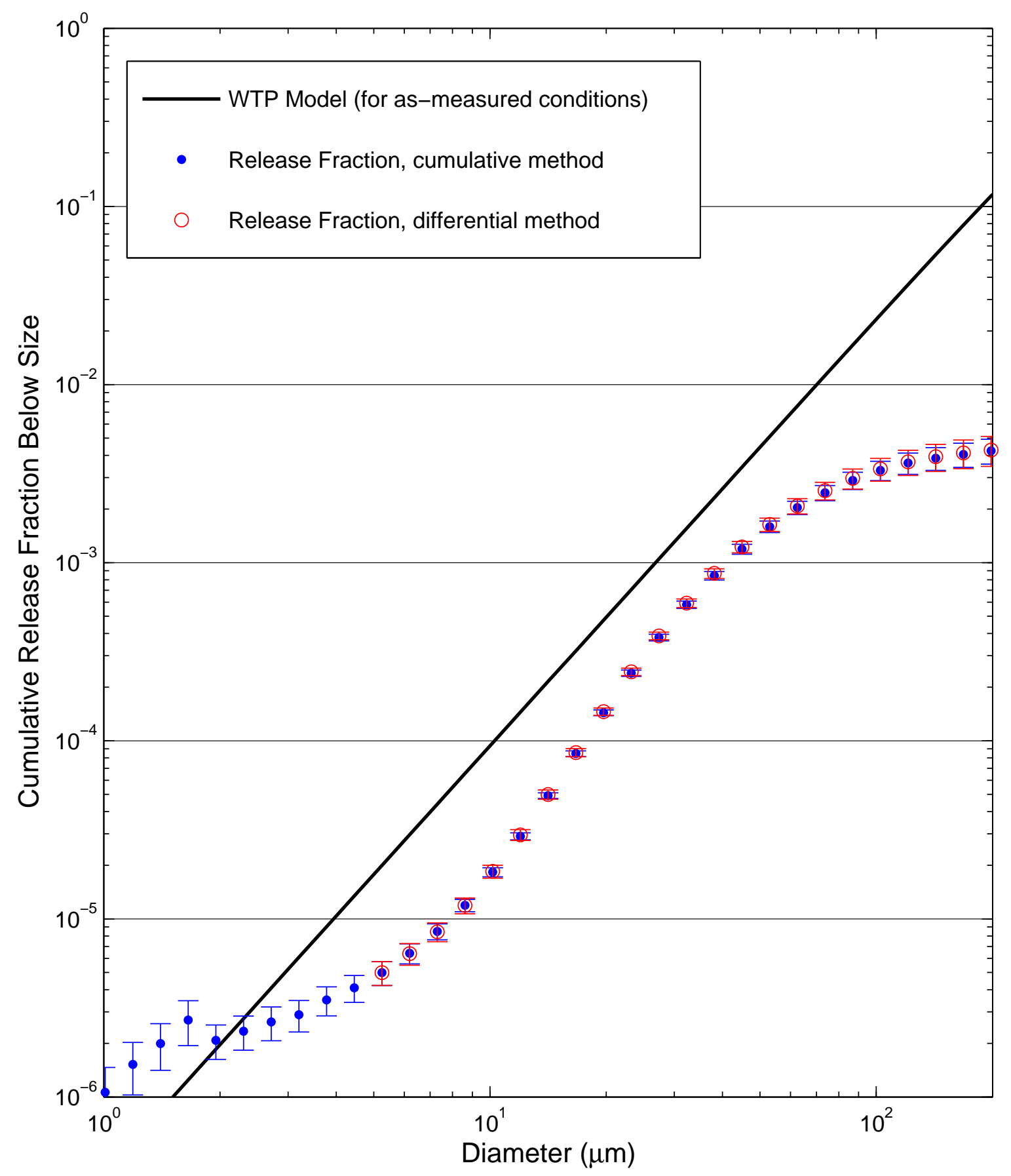

B. 128 
Test W170 [3 Malvern Average]: 380 psi, Orifice S5C - rectangular, $1 \times 20 \mathrm{~mm}$

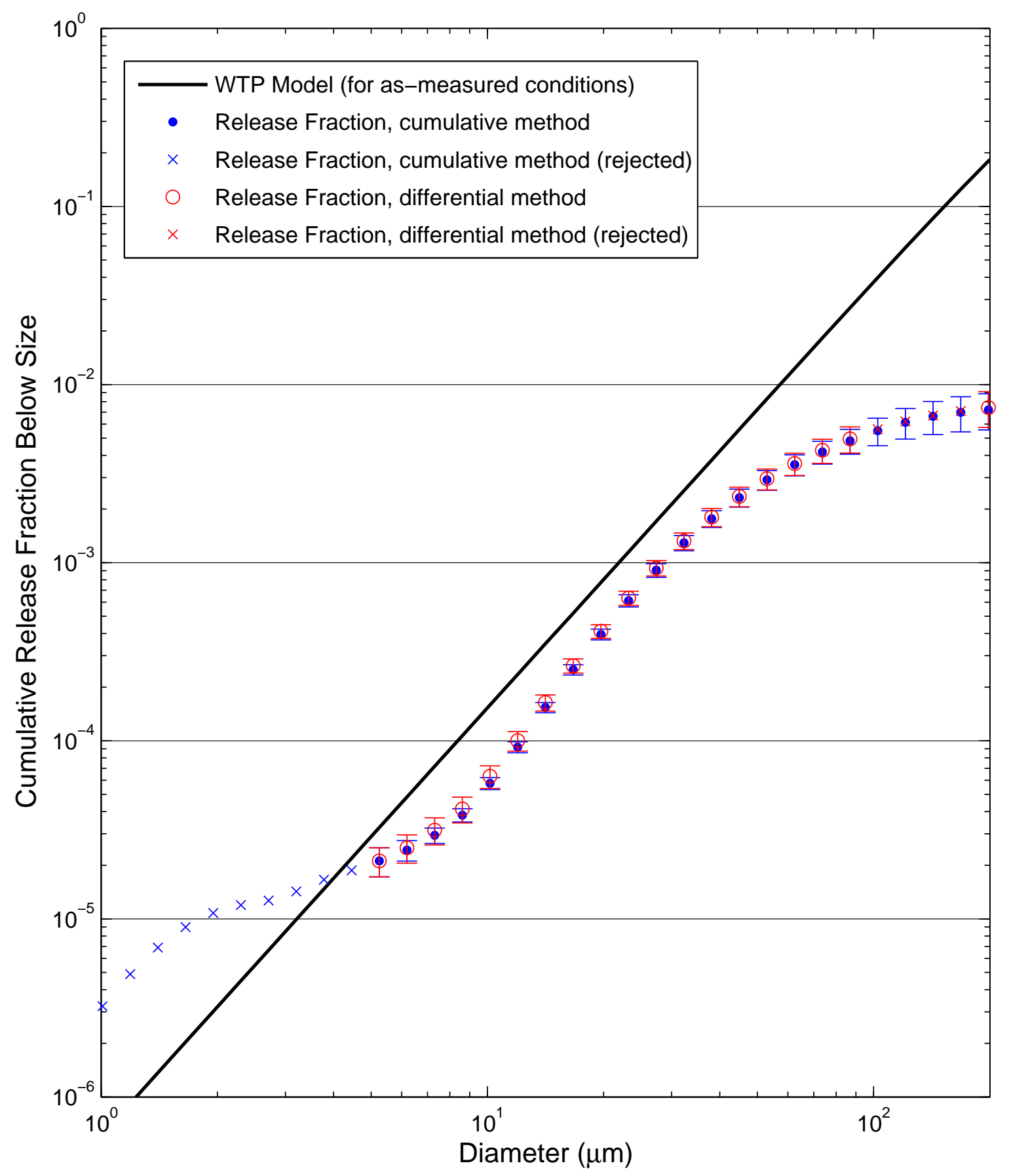


Test W171 [3 Malvern Average]: 200 psi, Orifice S5C - rectangular, $1 \times 20 \mathrm{~mm}$

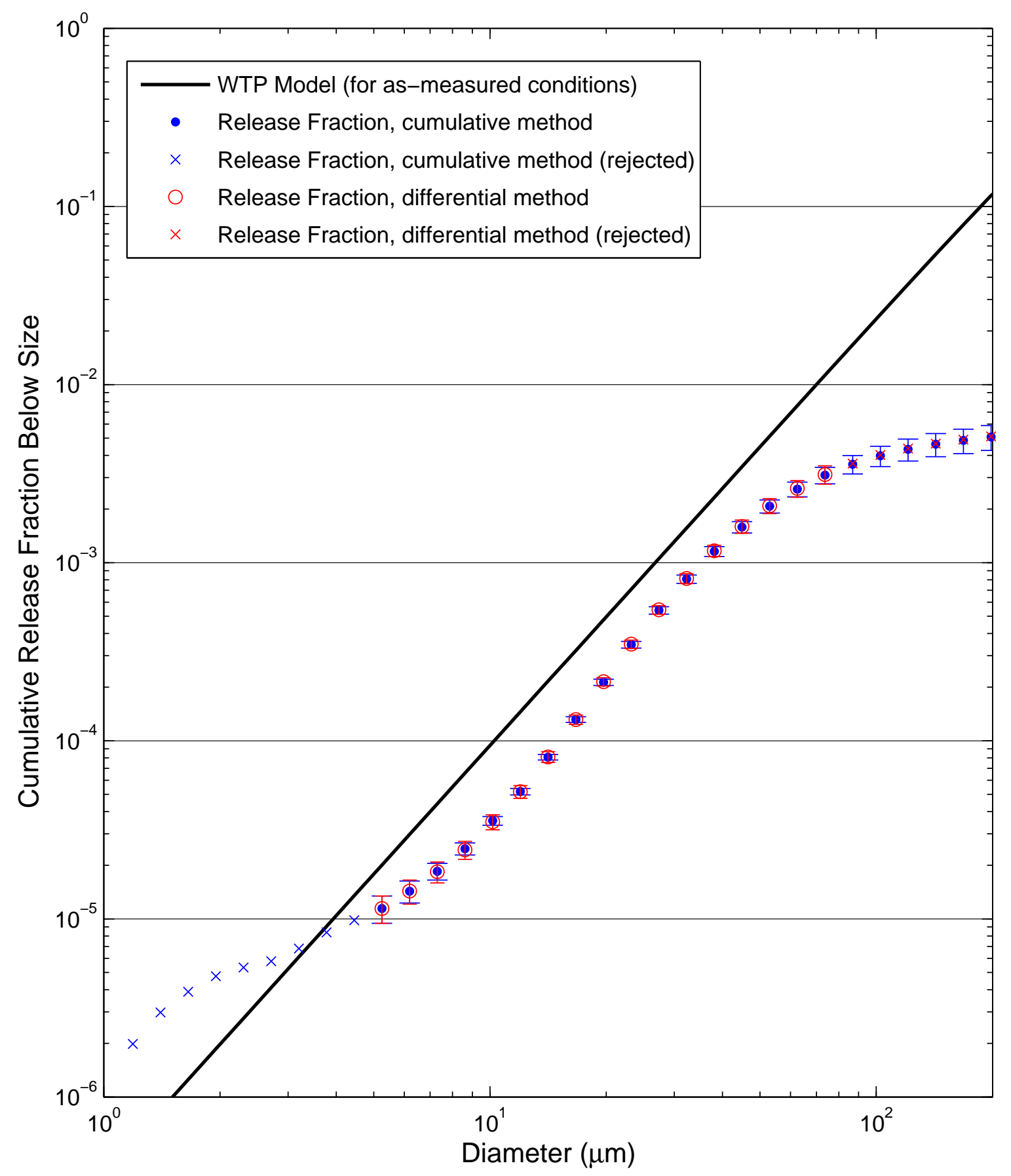


Test W172 [3 Malvern Average]: 380 psi, Orifice S5C - rectangular, $1 \times 20 \mathrm{~mm}$

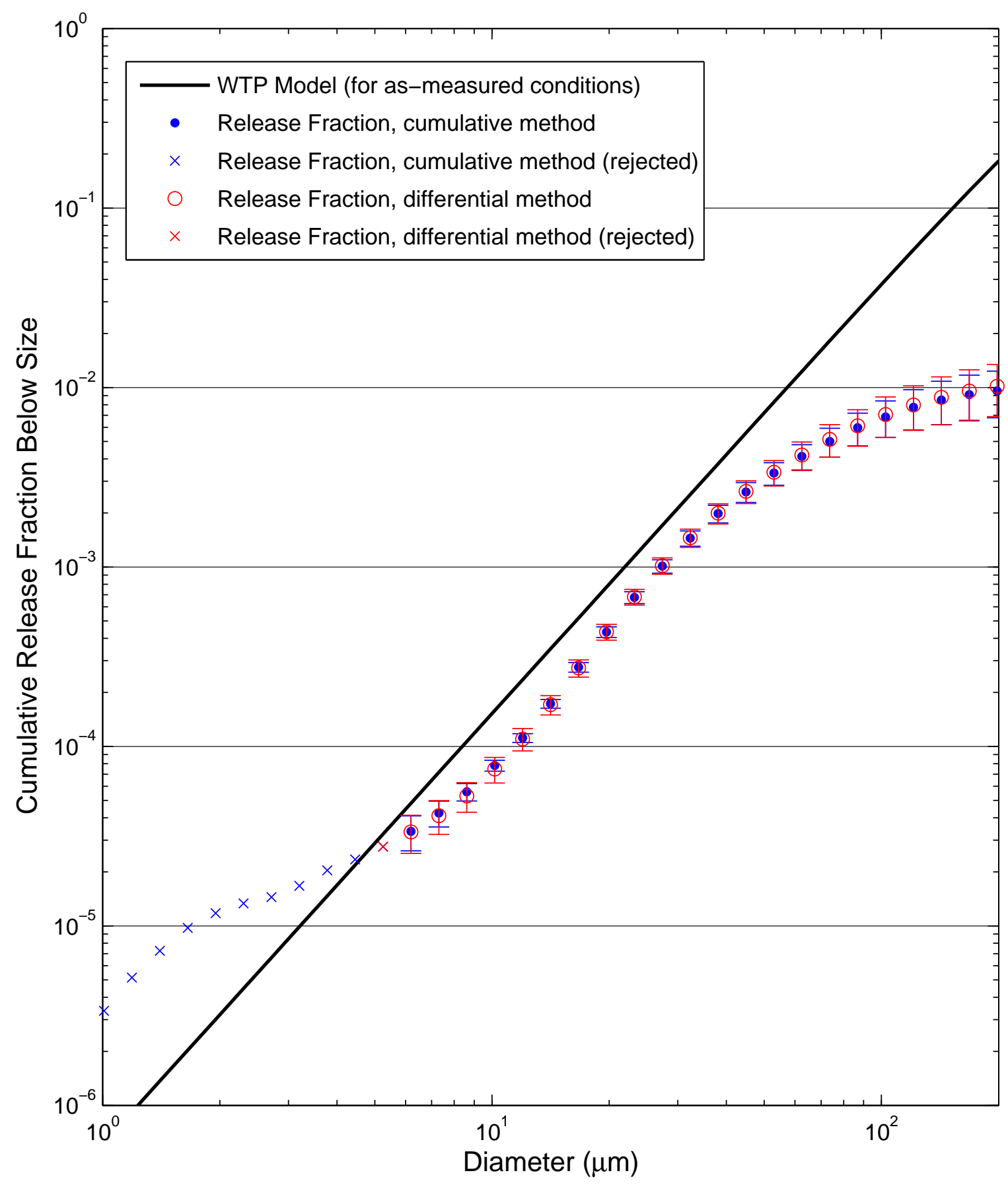


Test W173 [3 Malvern Average]: 100 psi, Orifice S4A - rectangular, 1× $76.2 \mathrm{~mm}$

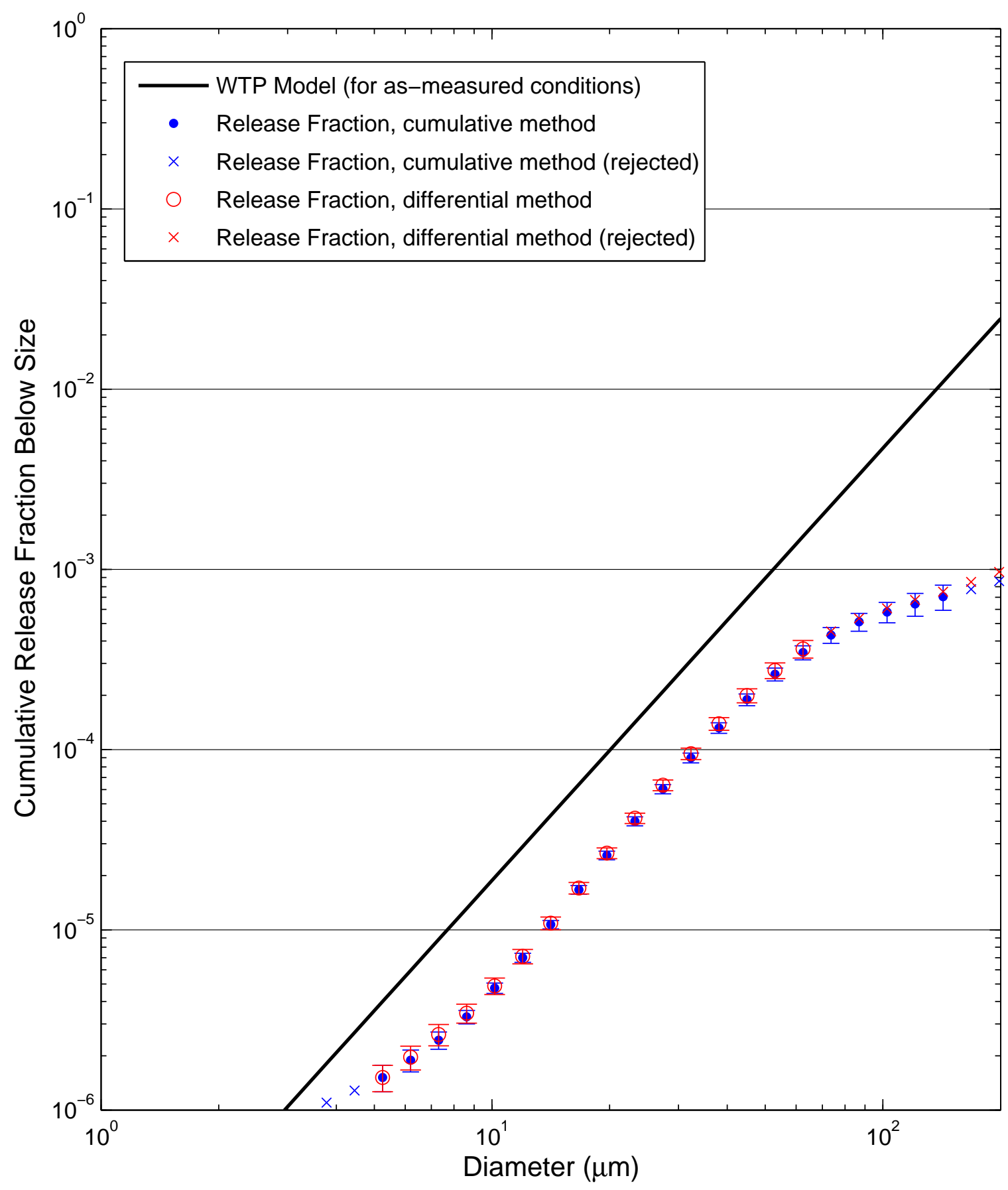


Test W174 [3 Malvern Average]: 200 psi, Orifice S4A - rectangular, 1× $76.2 \mathrm{~mm}$

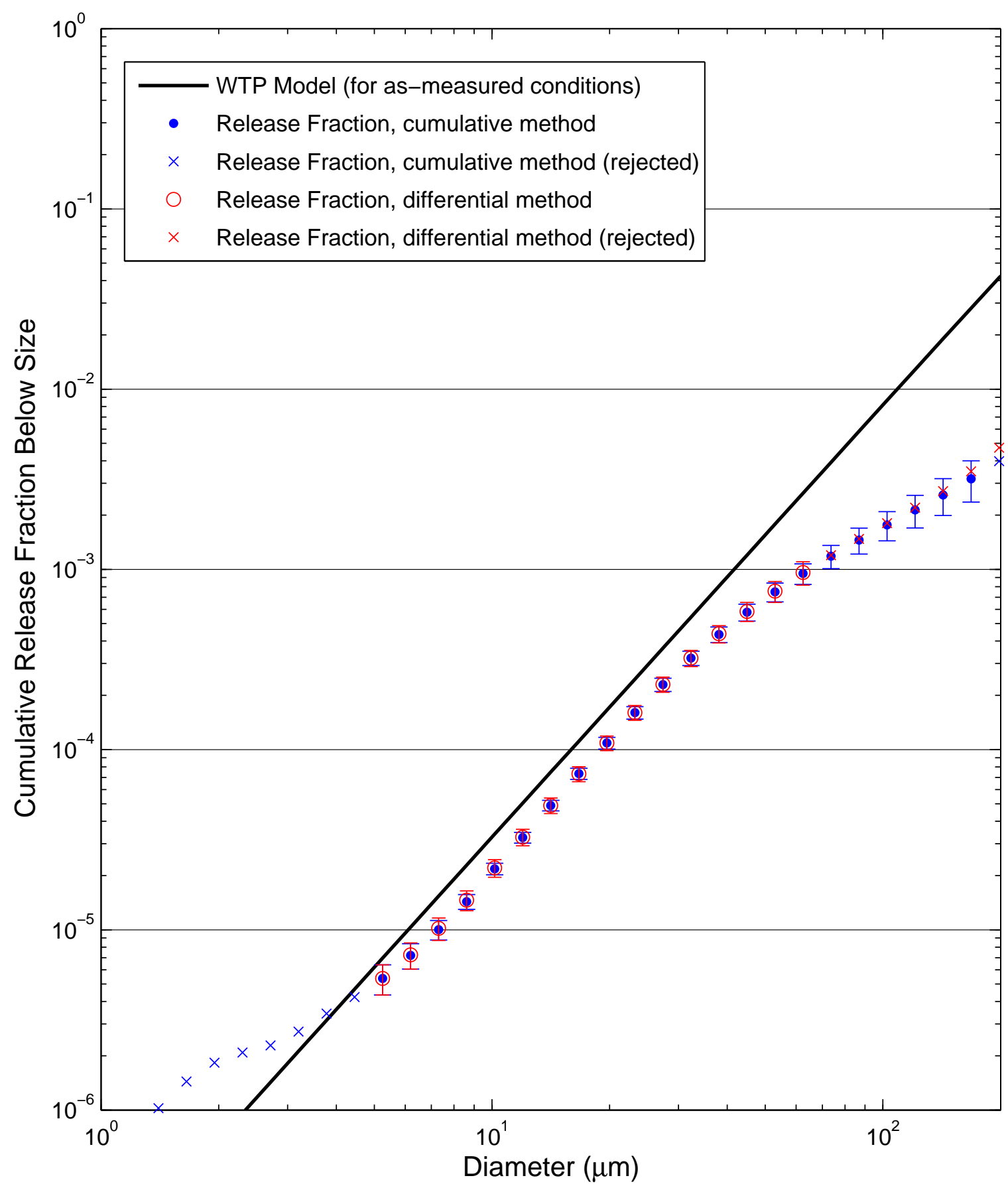


Test W175 [3 Malvern Average]: 380 psi, Orifice S4A - rectangular, $1 \times 76.2$ mm

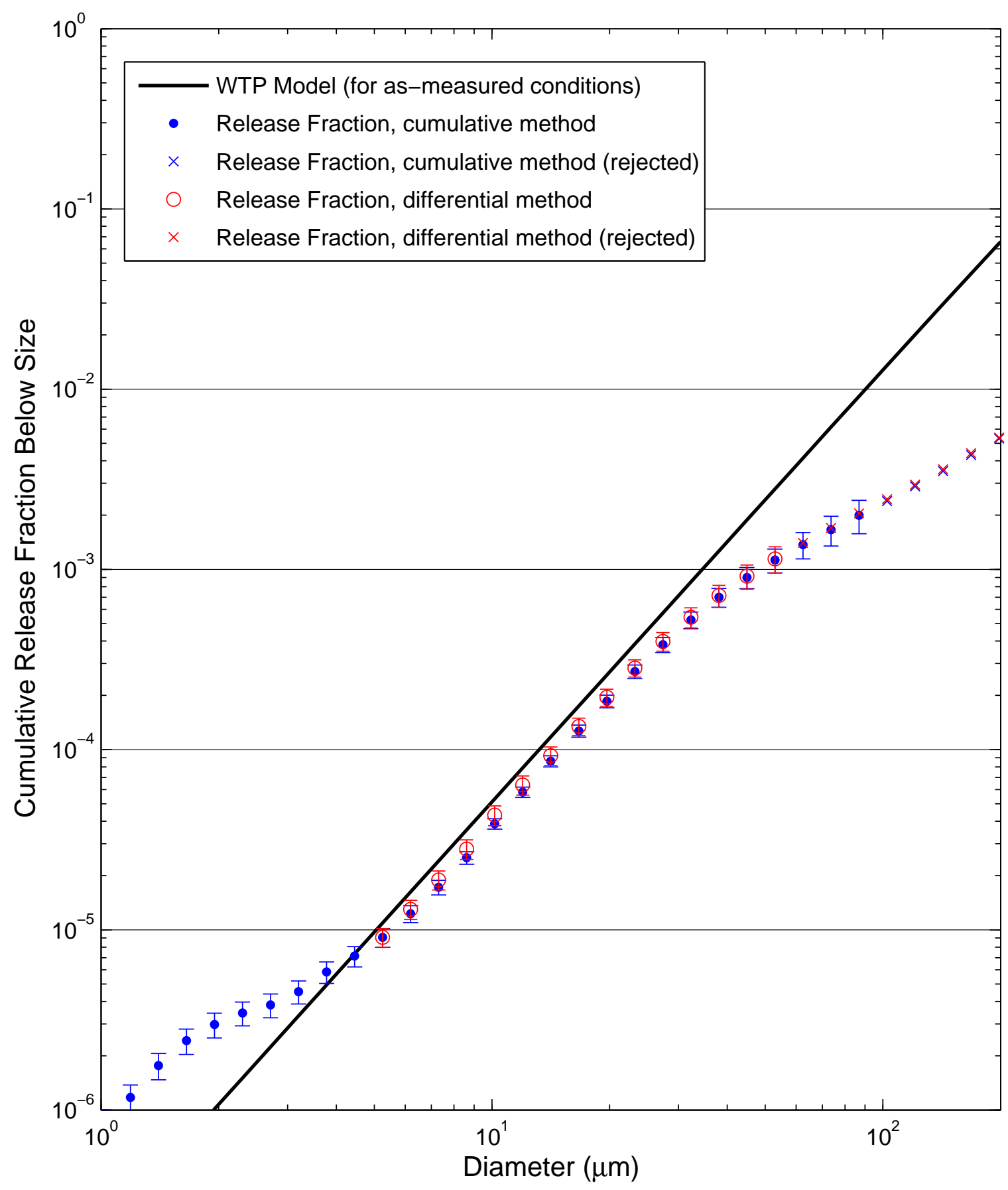


Test W176 [3 Malvern Average]: 200 psi, Orifice S4A - rectangular, $1 \times 76.2$ mm

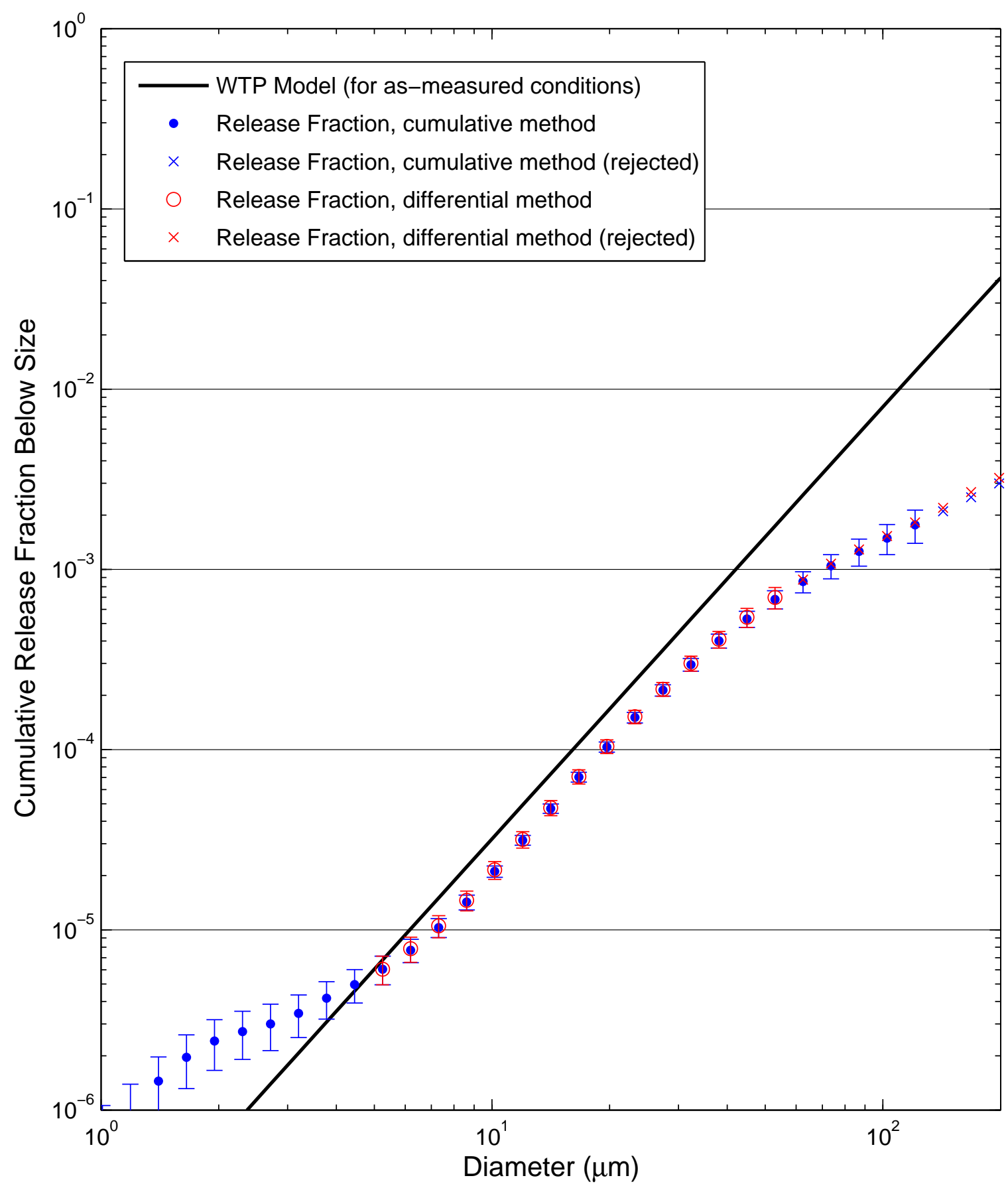


Test W177 [3 Malvern Average]: 380 psi, Orifice S4A - rectangular, 1× $76.2 \mathrm{~mm}$

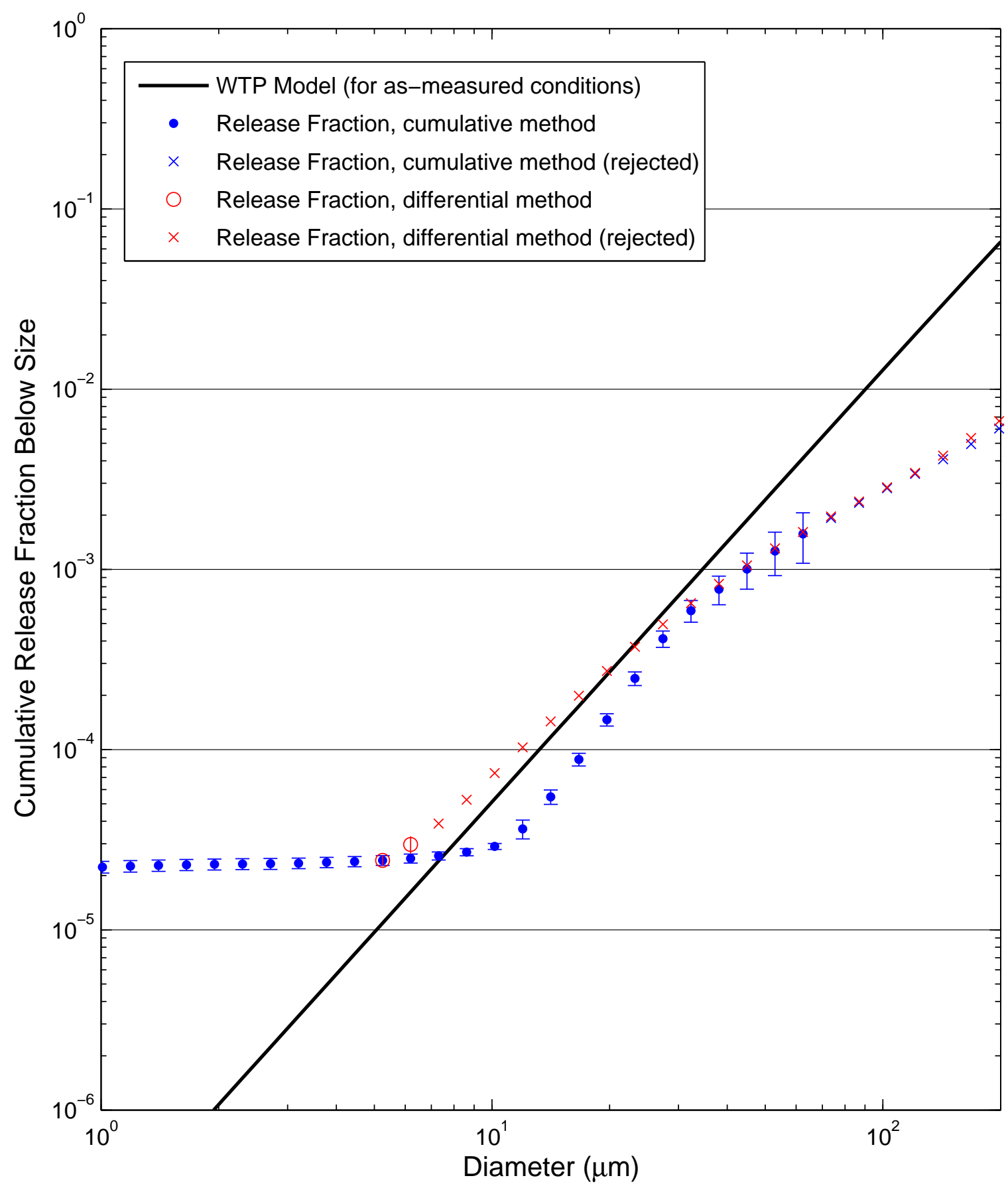


Test W178 [3 Malvern Average]: 100 psi, Orifice S3A - rectangular, $2.74 \times 76.2 \mathrm{~mm}$

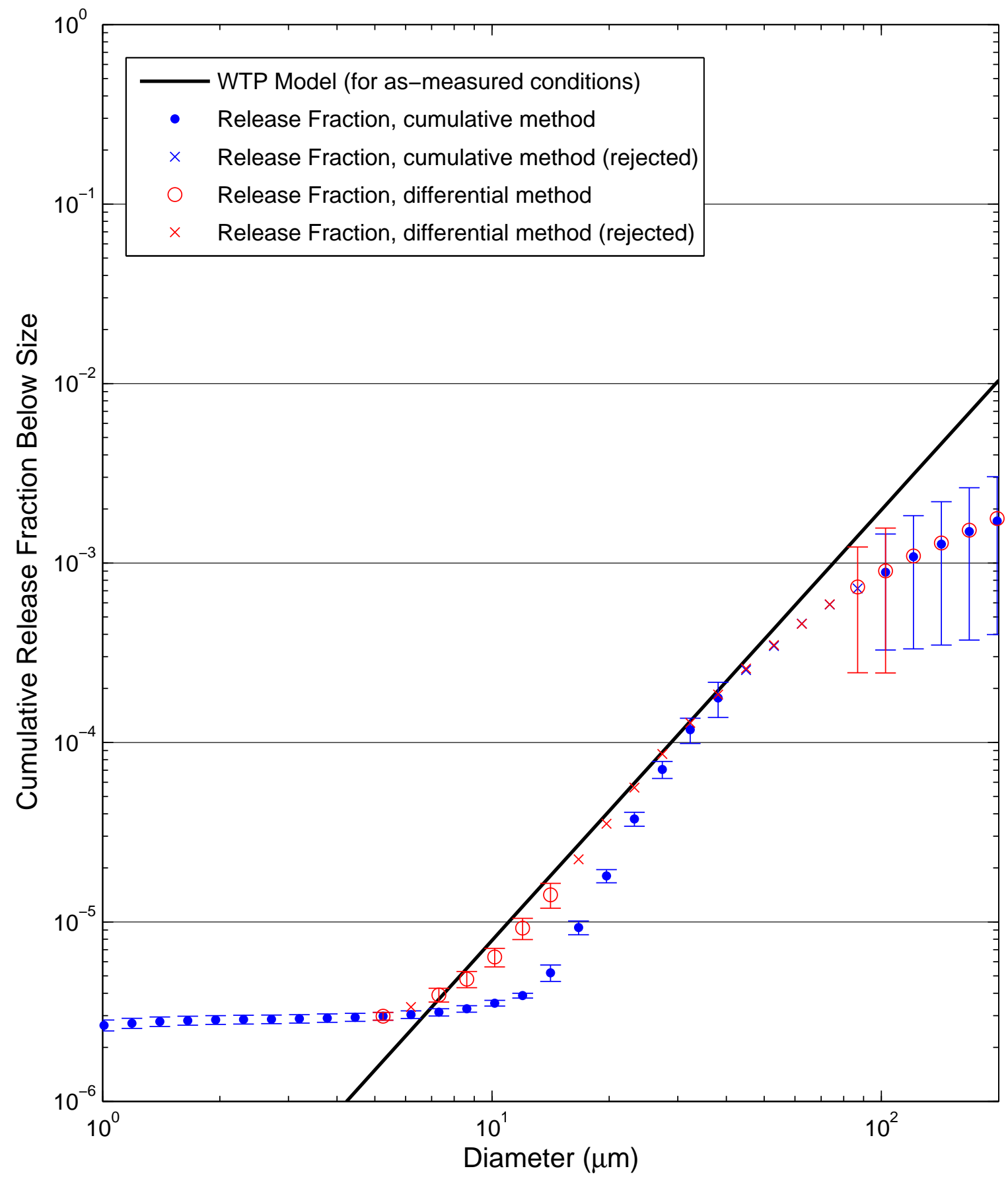


Test W179 [3 Malvern Average]: 100 psi, Orifice S3A - rectangular, 2.74× $76.2 \mathrm{~mm}$

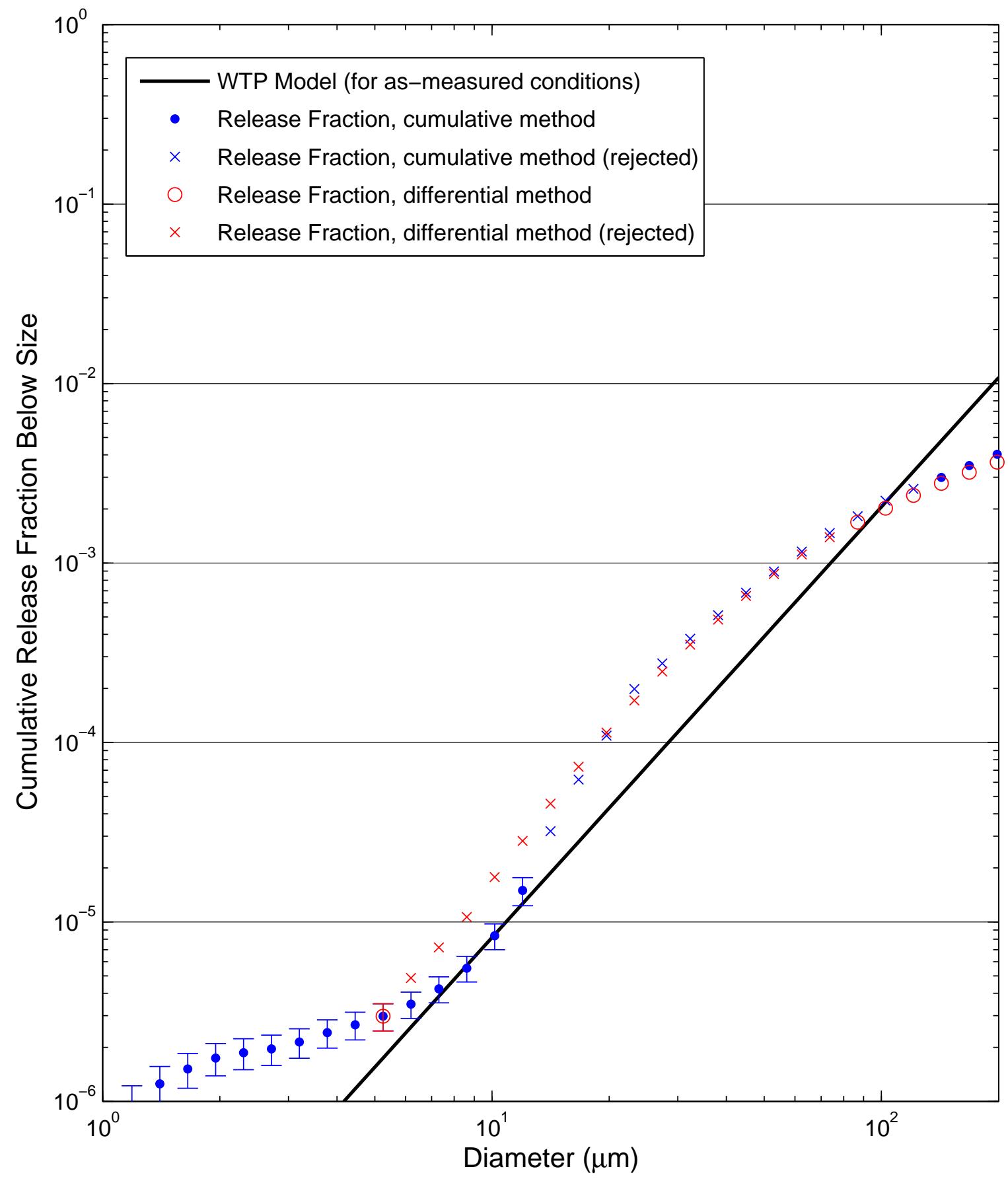


Test W180 [3 Malvern Average]: 200 psi, Orifice S3A - rectangular, $2.74 \times 76.2 \mathrm{~mm}$

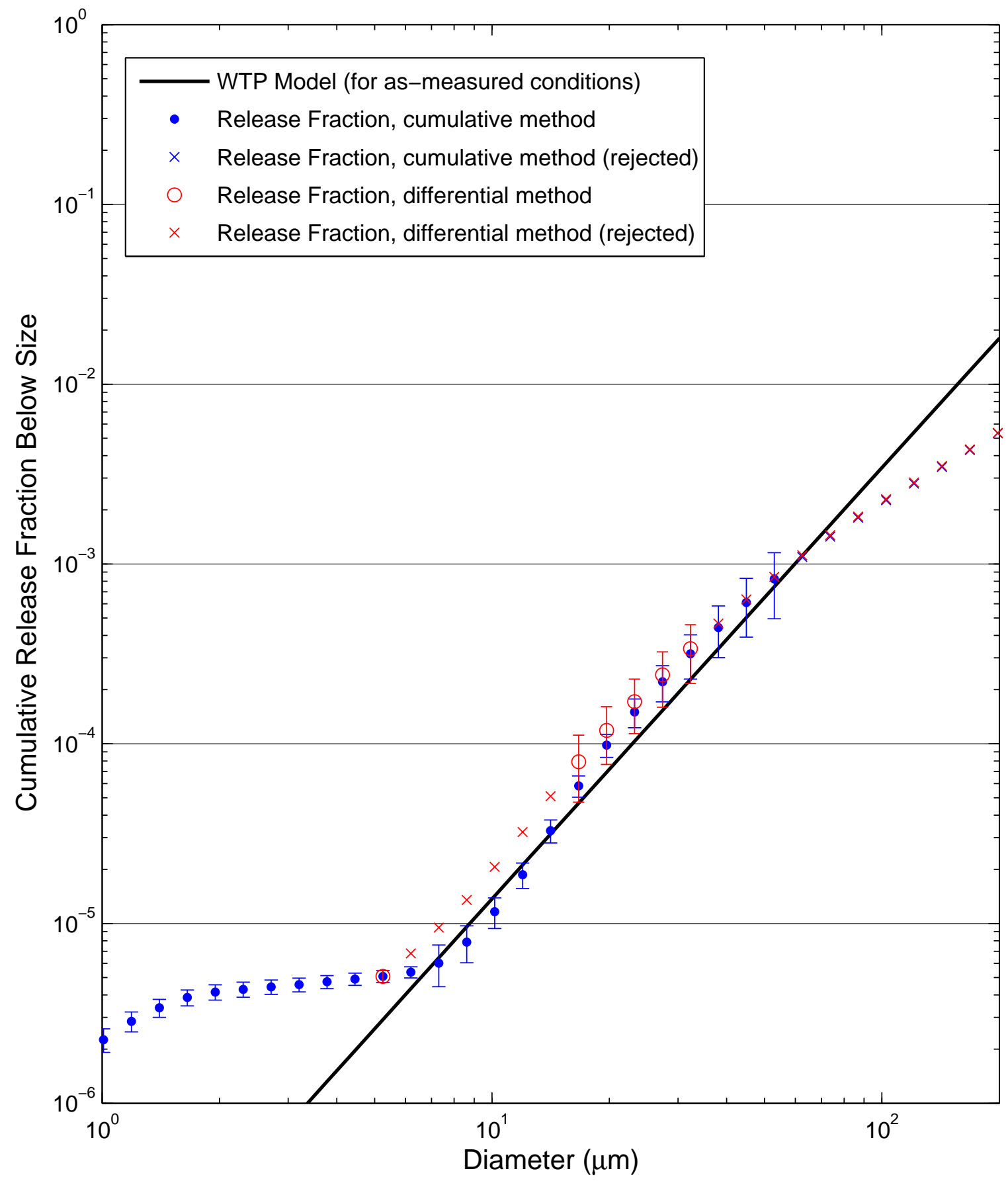


Test W181 [3 Malvern Average]: 200 psi, Orifice S3A - rectangular, 2.74× $76.2 \mathrm{~mm}$

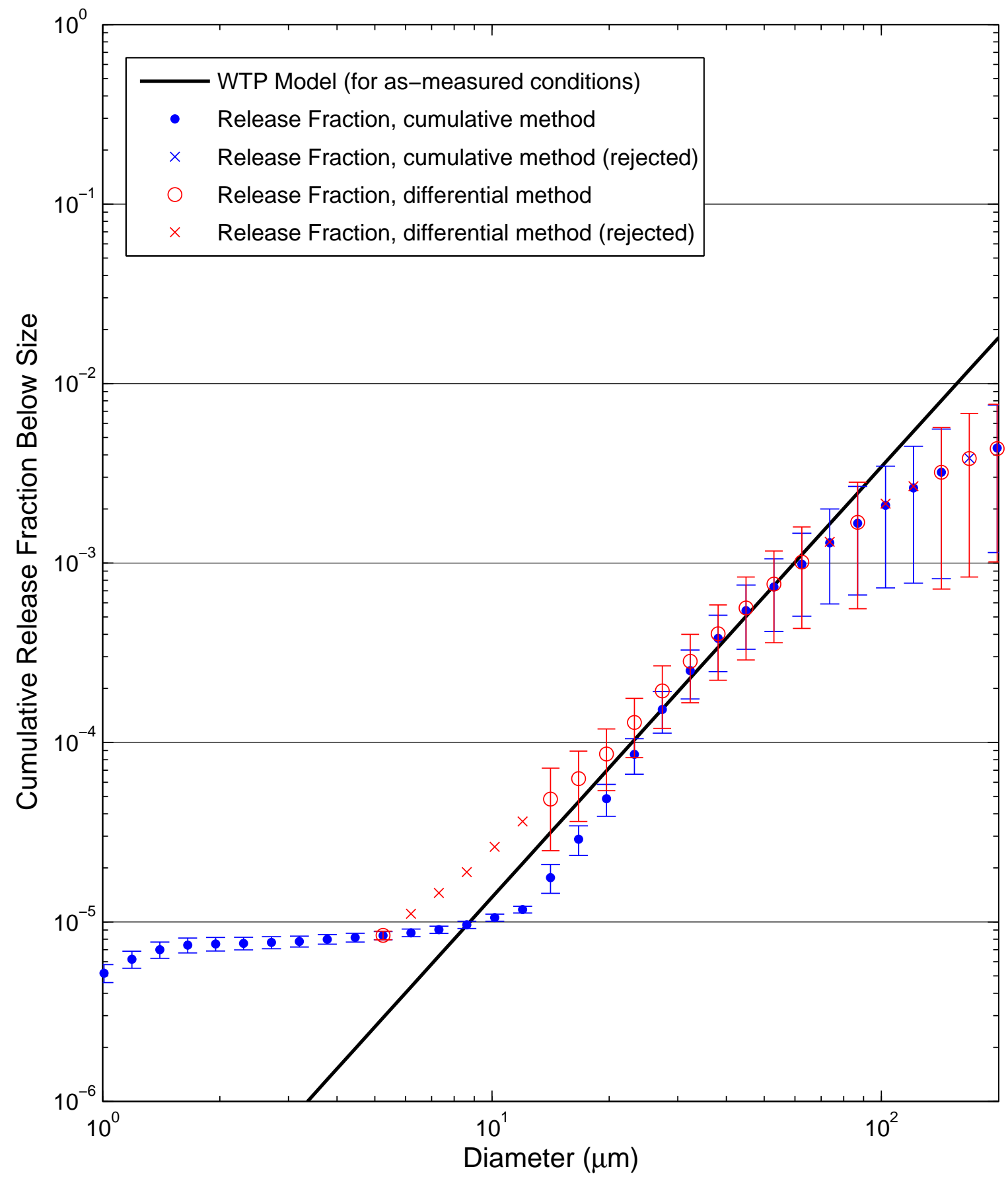


Test W182 [3 Malvern Average]: 380 psi, Orifice S3A - rectangular, $2.74 \times 76.2 \mathrm{~mm}$

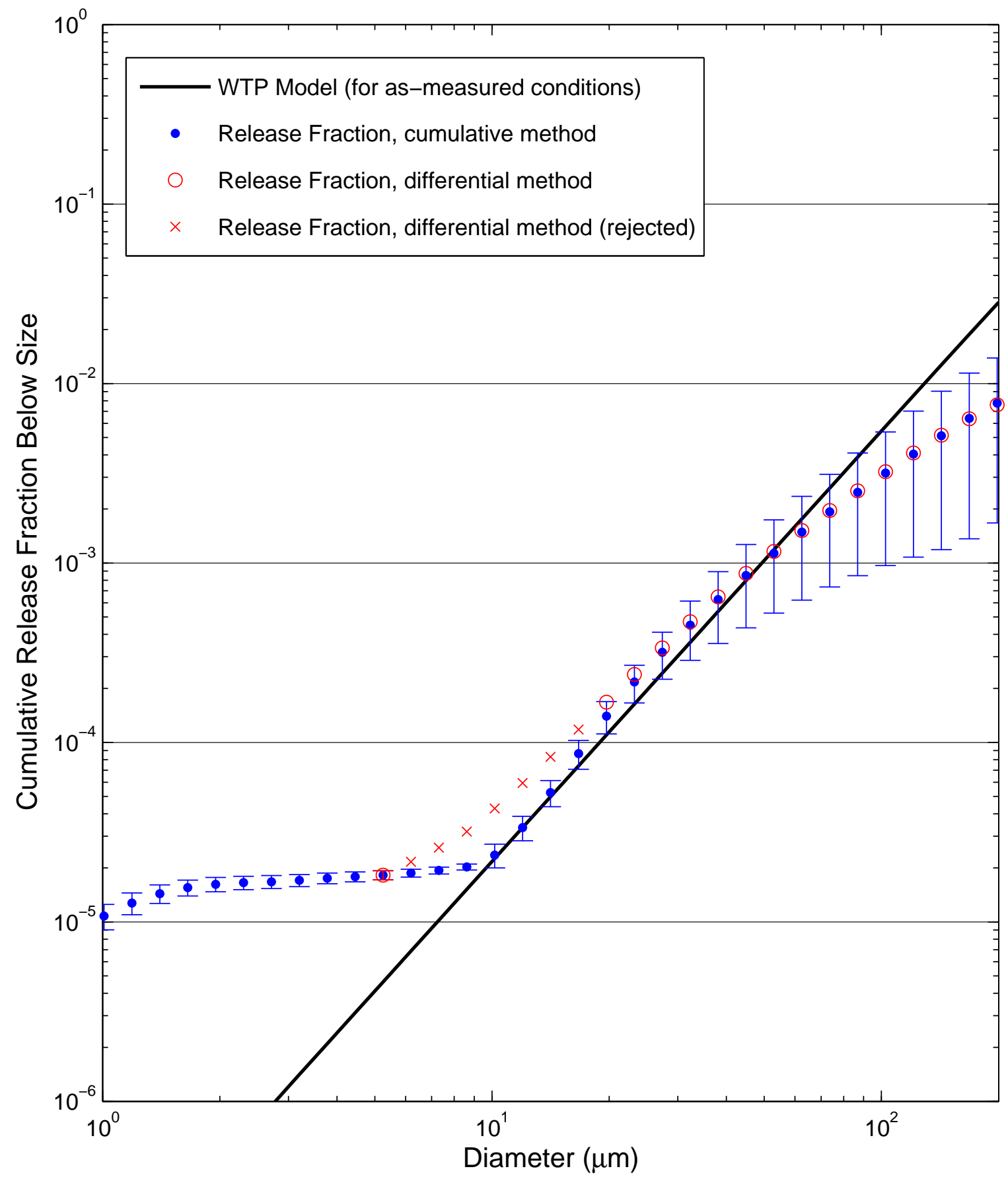


Test S183R [3 Malvern Average]: $100 \mathrm{psi}$, Orifice S2A - rectangular, $0.5 \times 5 \mathrm{~mm}$

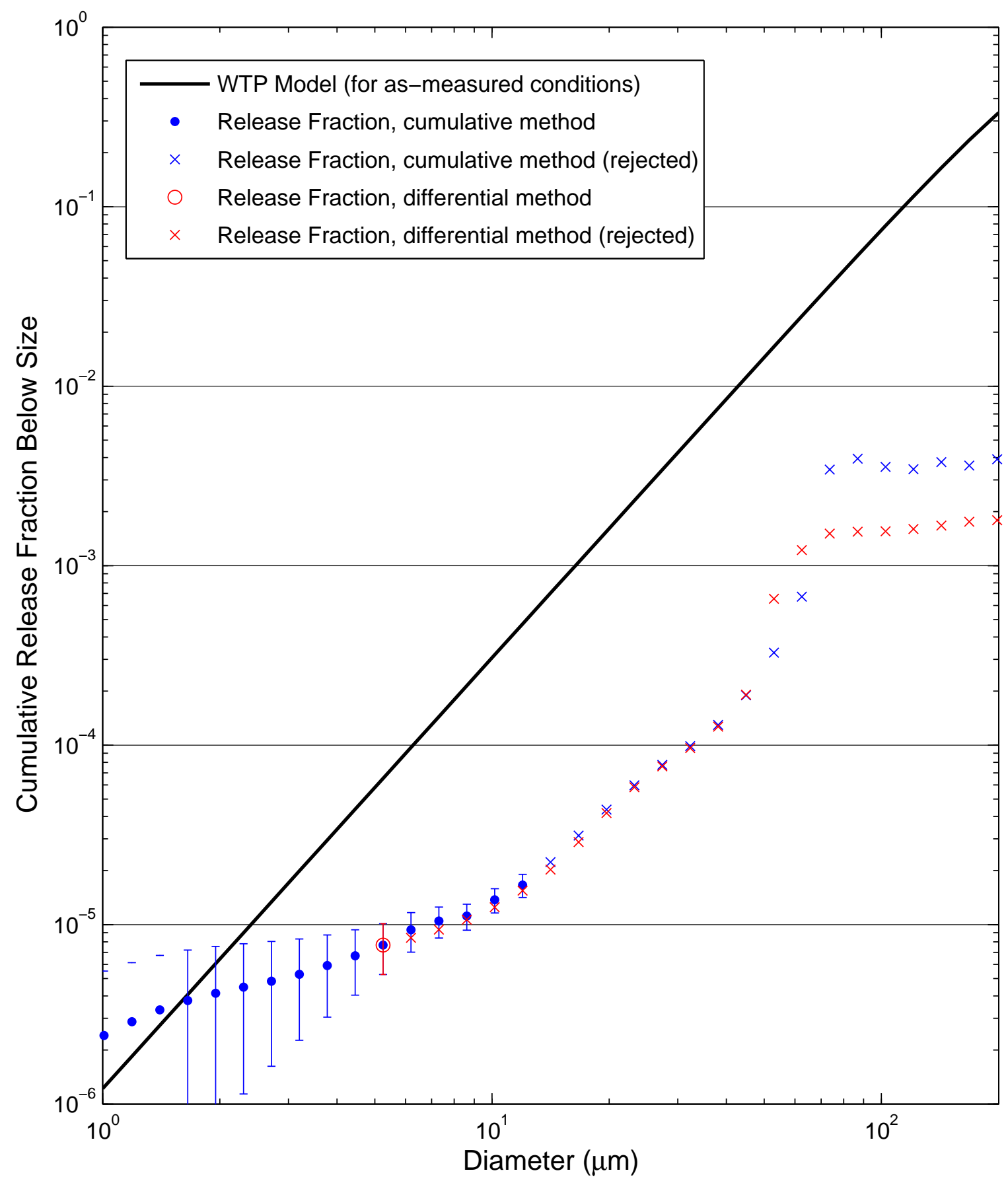


Test S184 [3 Malvern Average]: 200 psi, Orifice S2A - rectangular, $0.5 \times 5 \mathrm{~mm}$

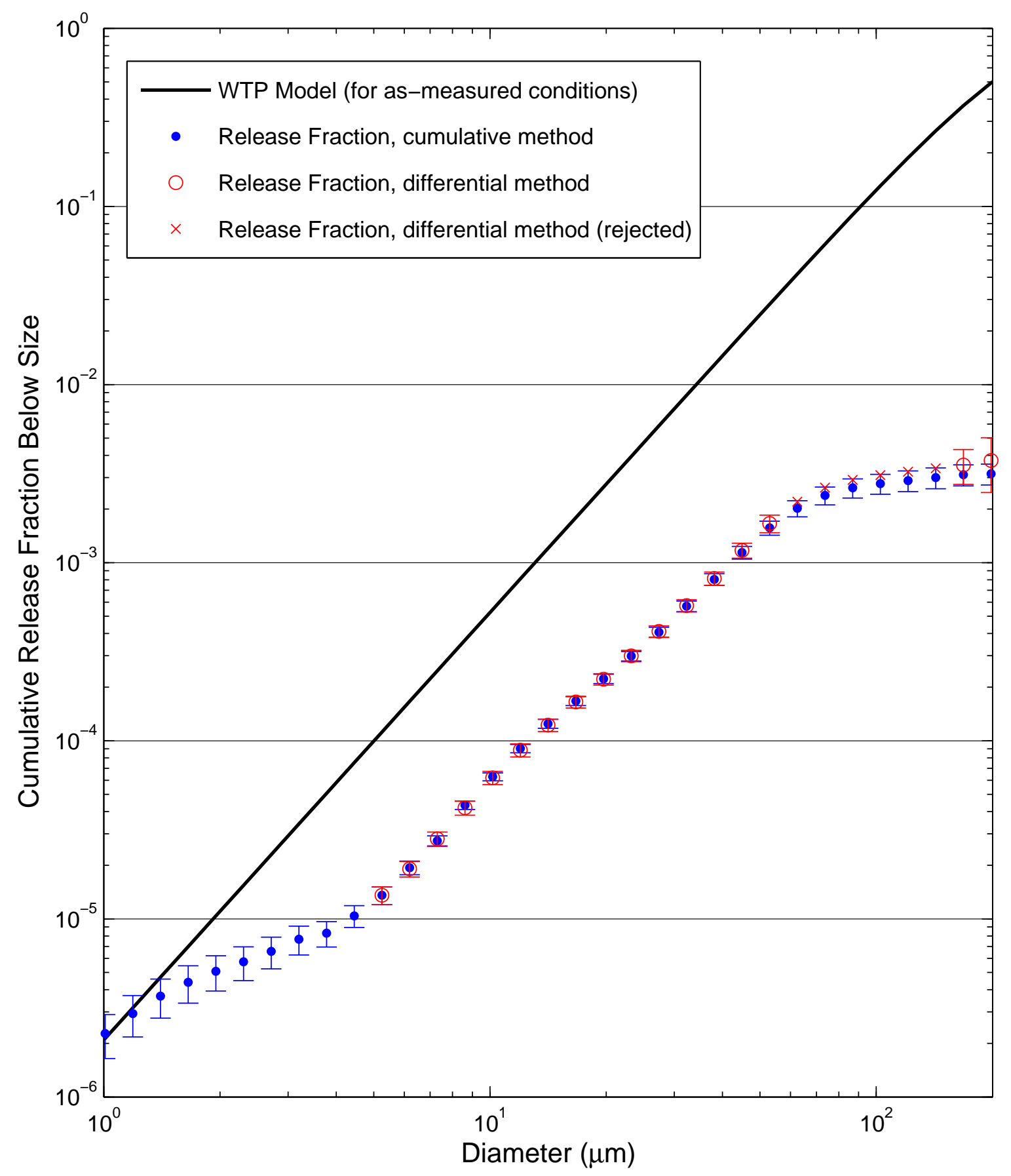


Test S185 [3 Malvern Average]: 380 psi, Orifice S2A - rectangular, $0.5 \times 5 \mathrm{~mm}$

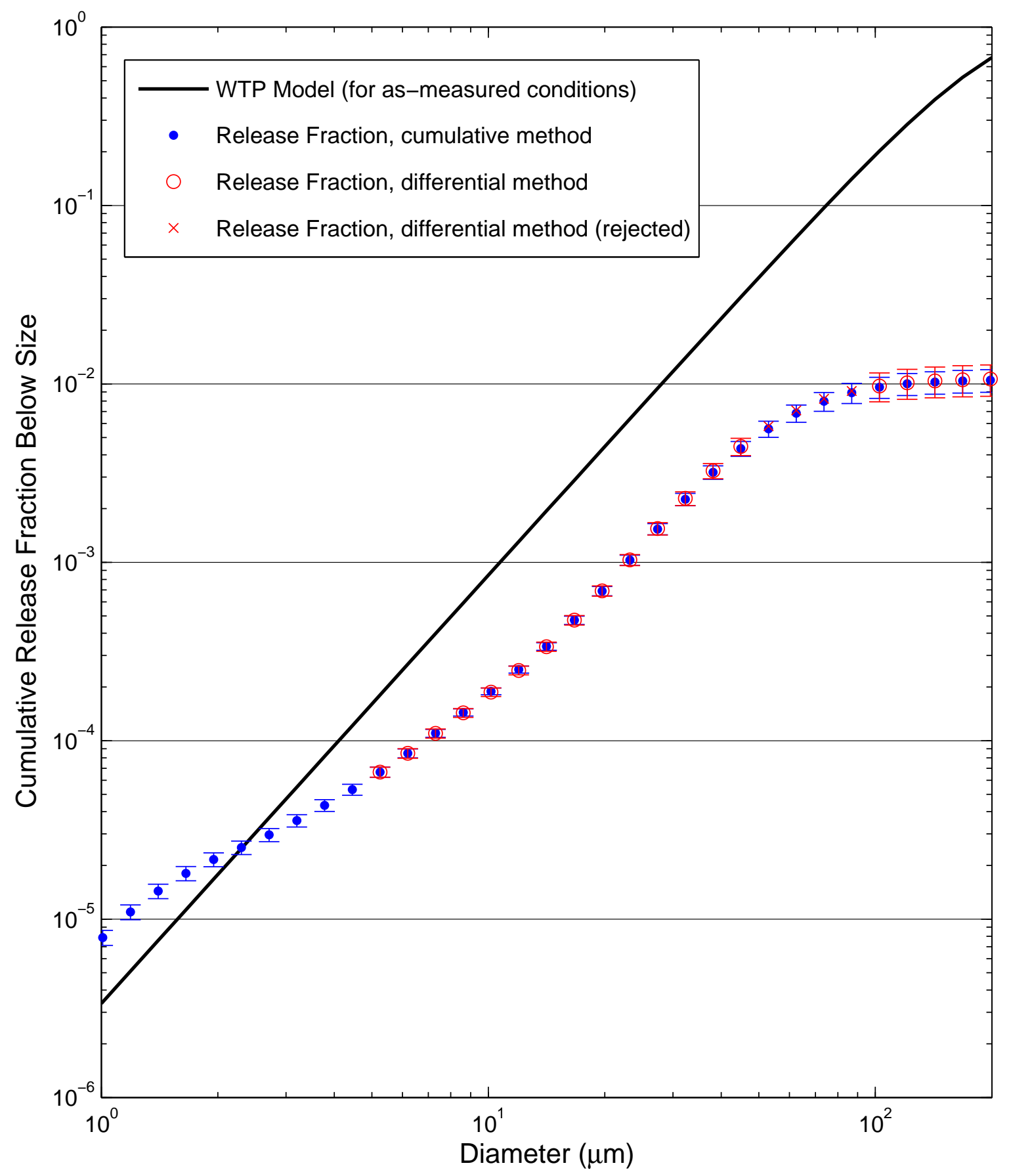


Test S186 [3 Malvern Average]: 100 psi, Orifice S2A - rectangular, 0.5× $5 \mathrm{~mm}$

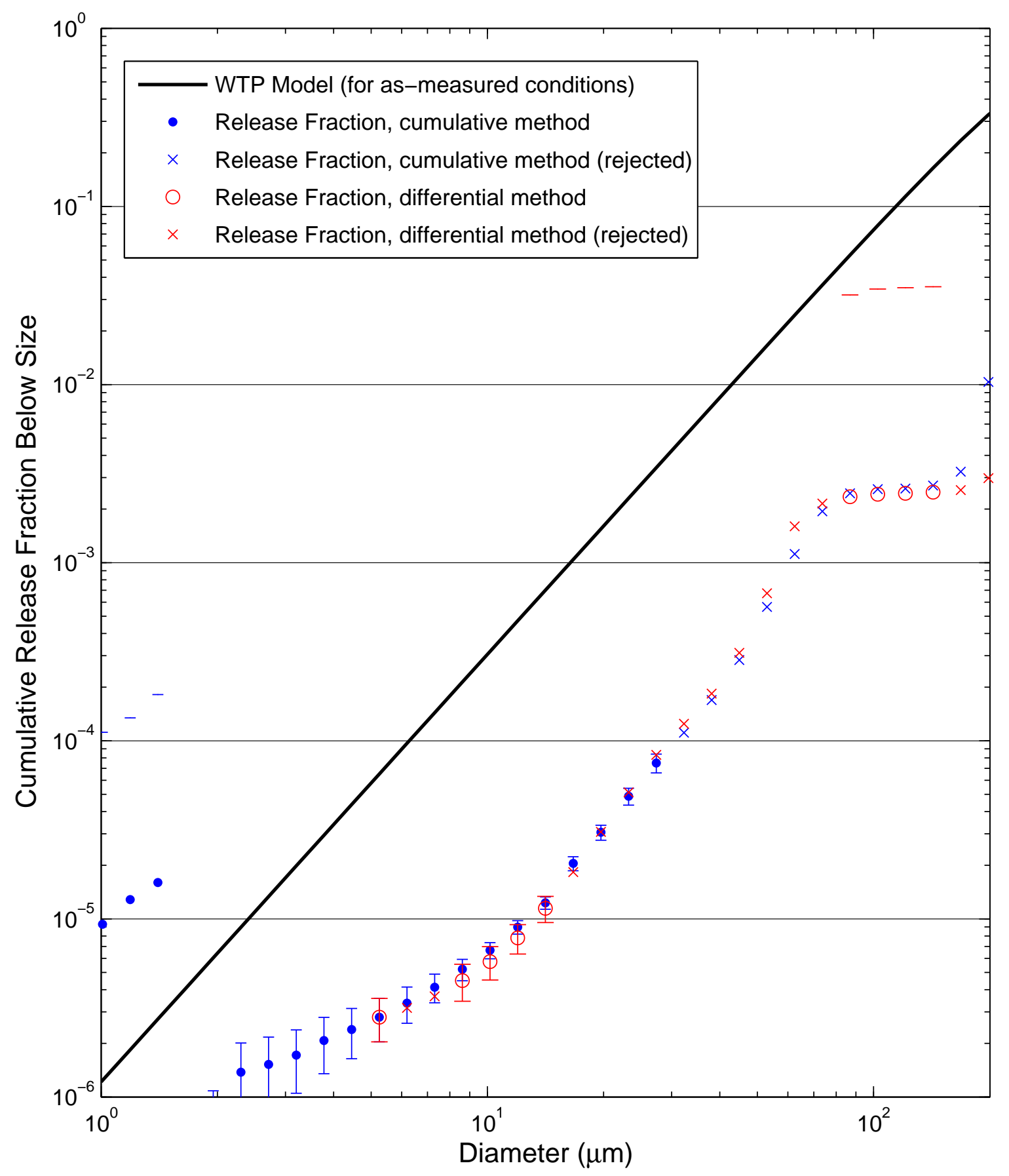


Test S187 [3 Malvern Average]: 200 psi, Orifice S2A - rectangular, $0.5 \times 5 \mathrm{~mm}$

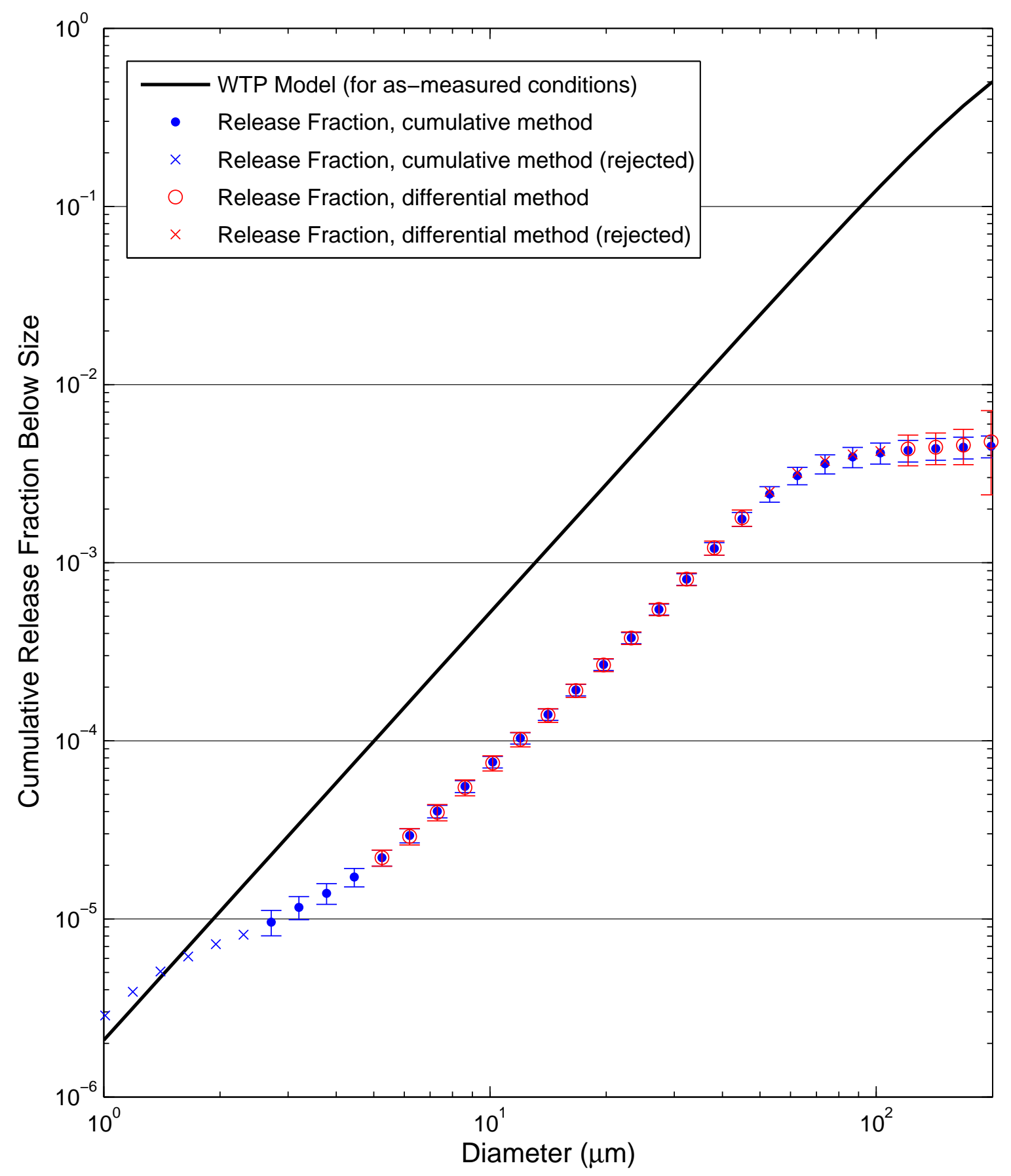


Test S188 [3 Malvern Average]: 380 psi, Orifice S2A - rectangular, $0.5 \times 5 \mathrm{~mm}$

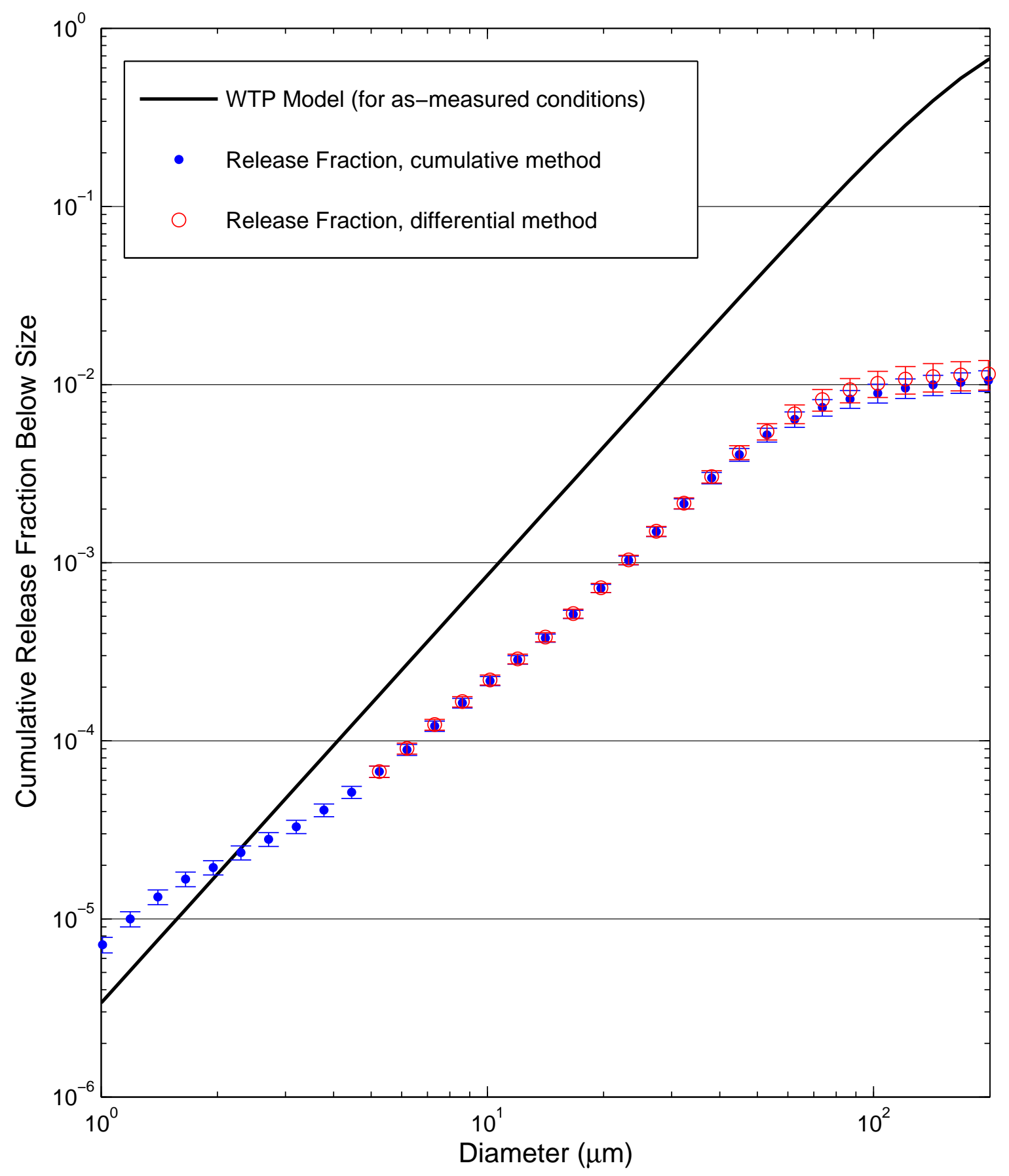


Test S189 [3 Malvern Average]: 100 psi, Orifice S7A - rectangular, $1 \times 10 \mathrm{~mm}$

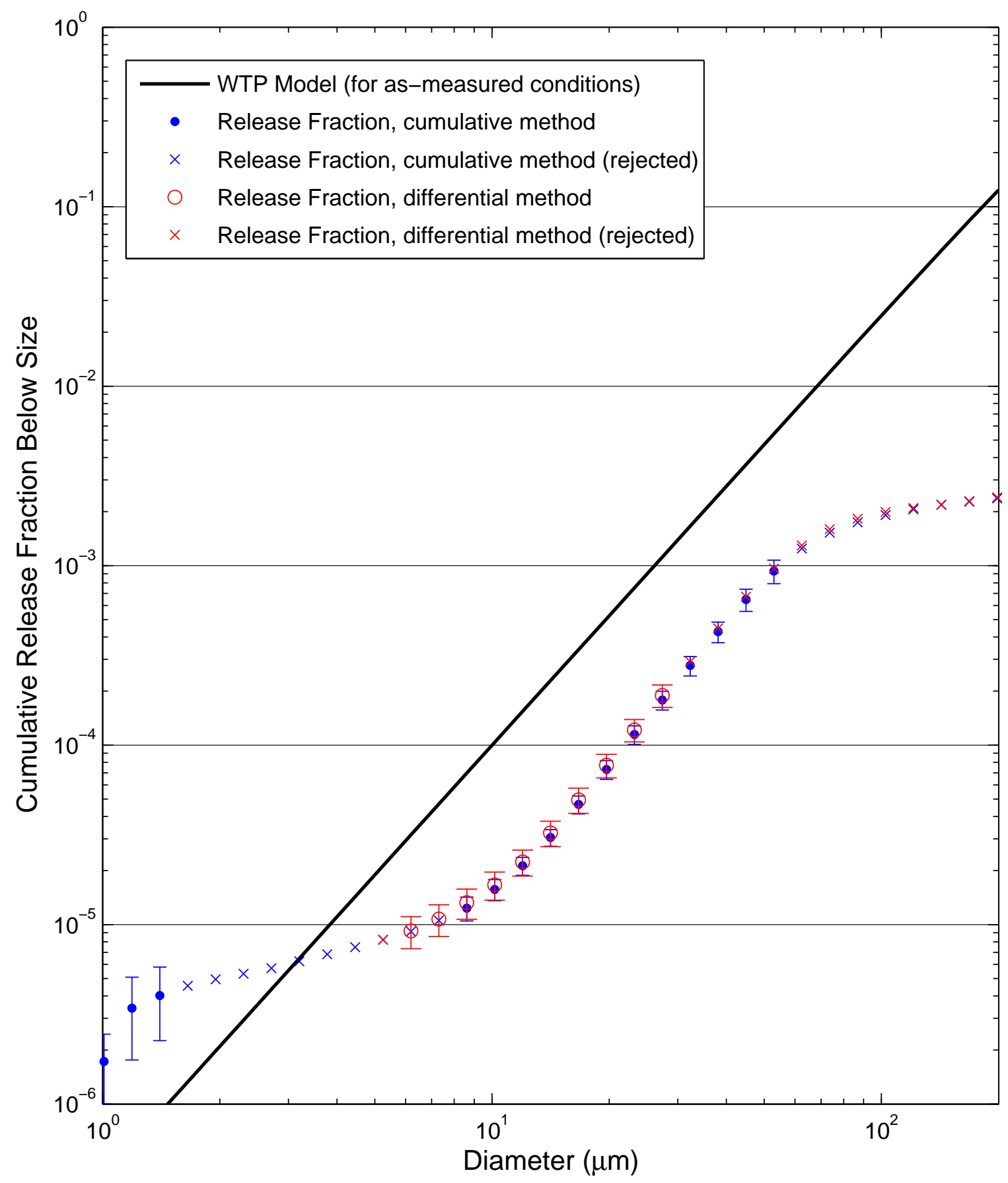


Test S190 [3 Malvern Average]: 200 psi, Orifice S7A - rectangular, $1 \times 10 \mathrm{~mm}$

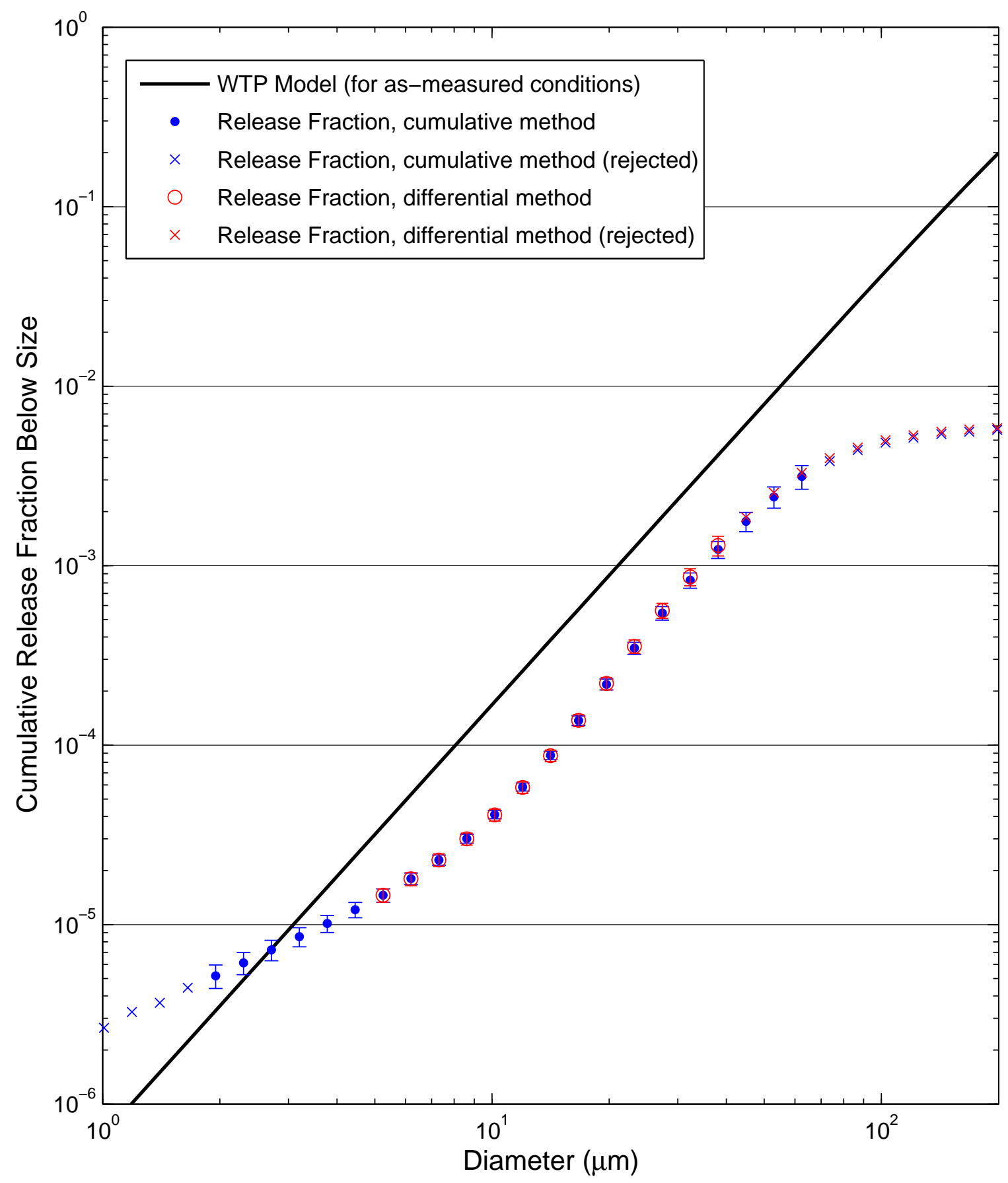


Test S190 [3 Malvern Average]: 200 psi, Orifice S7A - rectangular, $1 \times 10 \mathrm{~mm}$

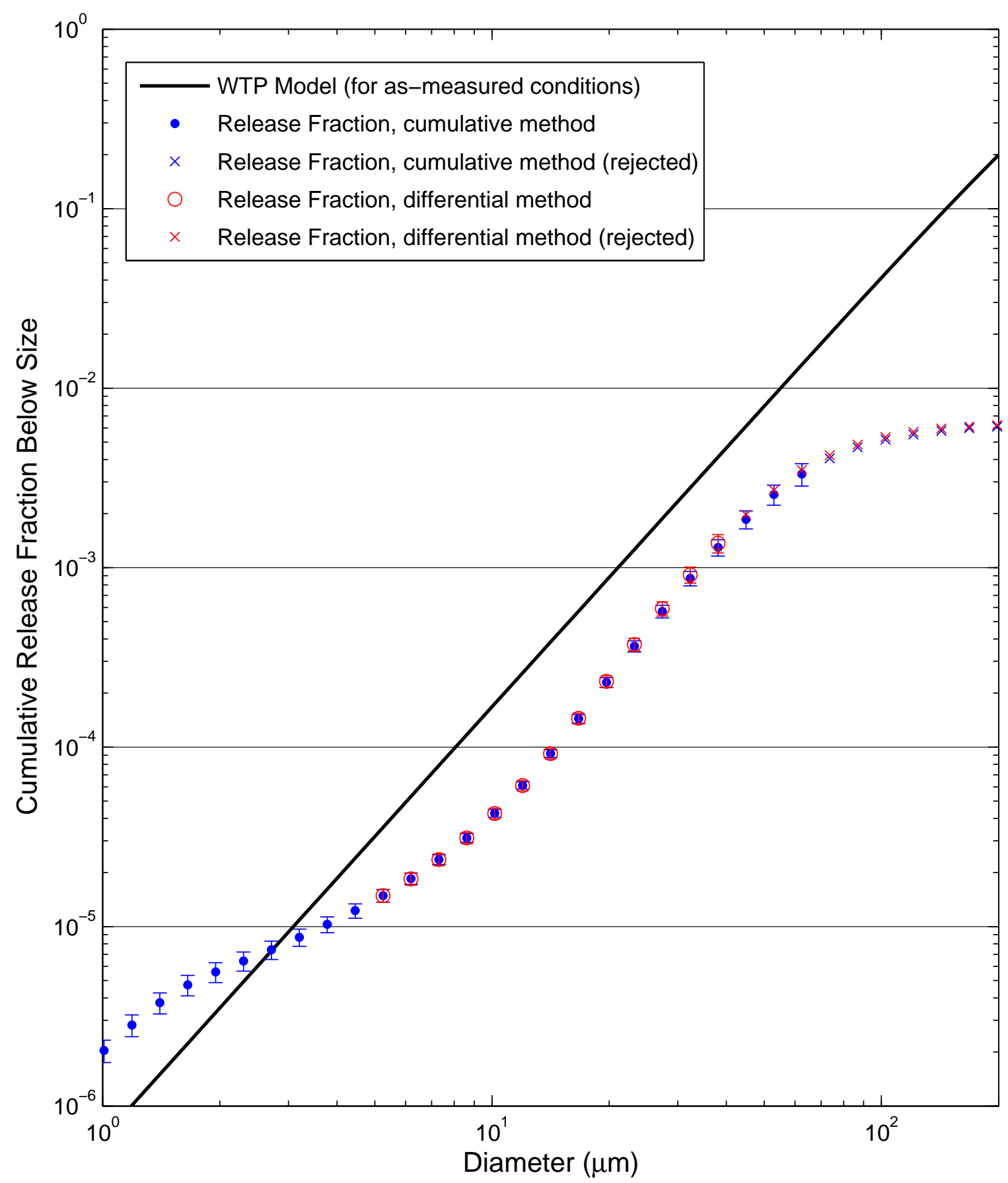

B. 150 
Test S190 [3 Malvern Average]: 200 psi, Orifice S7A - rectangular, $1 \times 10 \mathrm{~mm}$

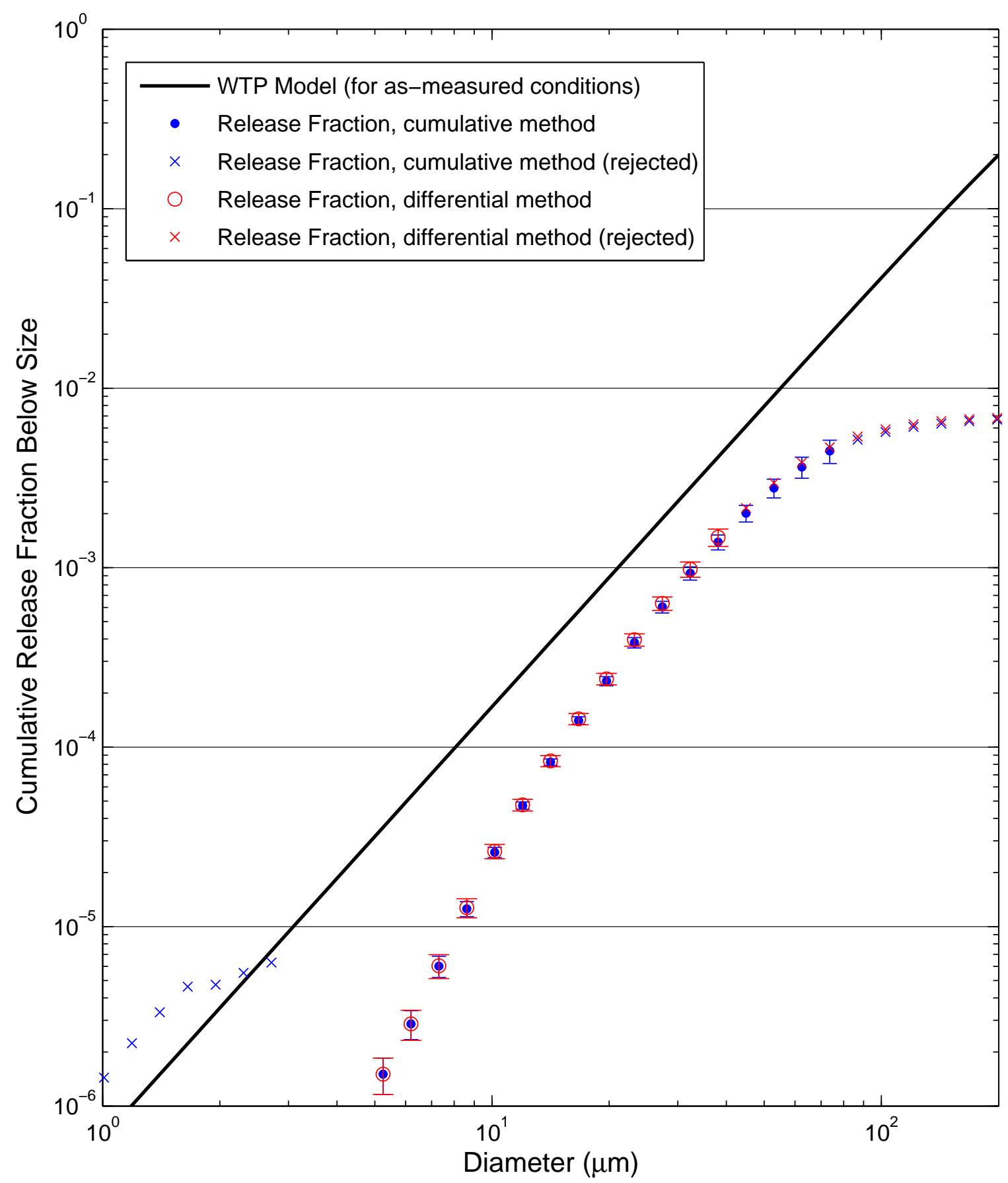


Test S191 [3 Malvern Average]: 380 psi, Orifice S7A - rectangular, $1 \times 10 \mathrm{~mm}$

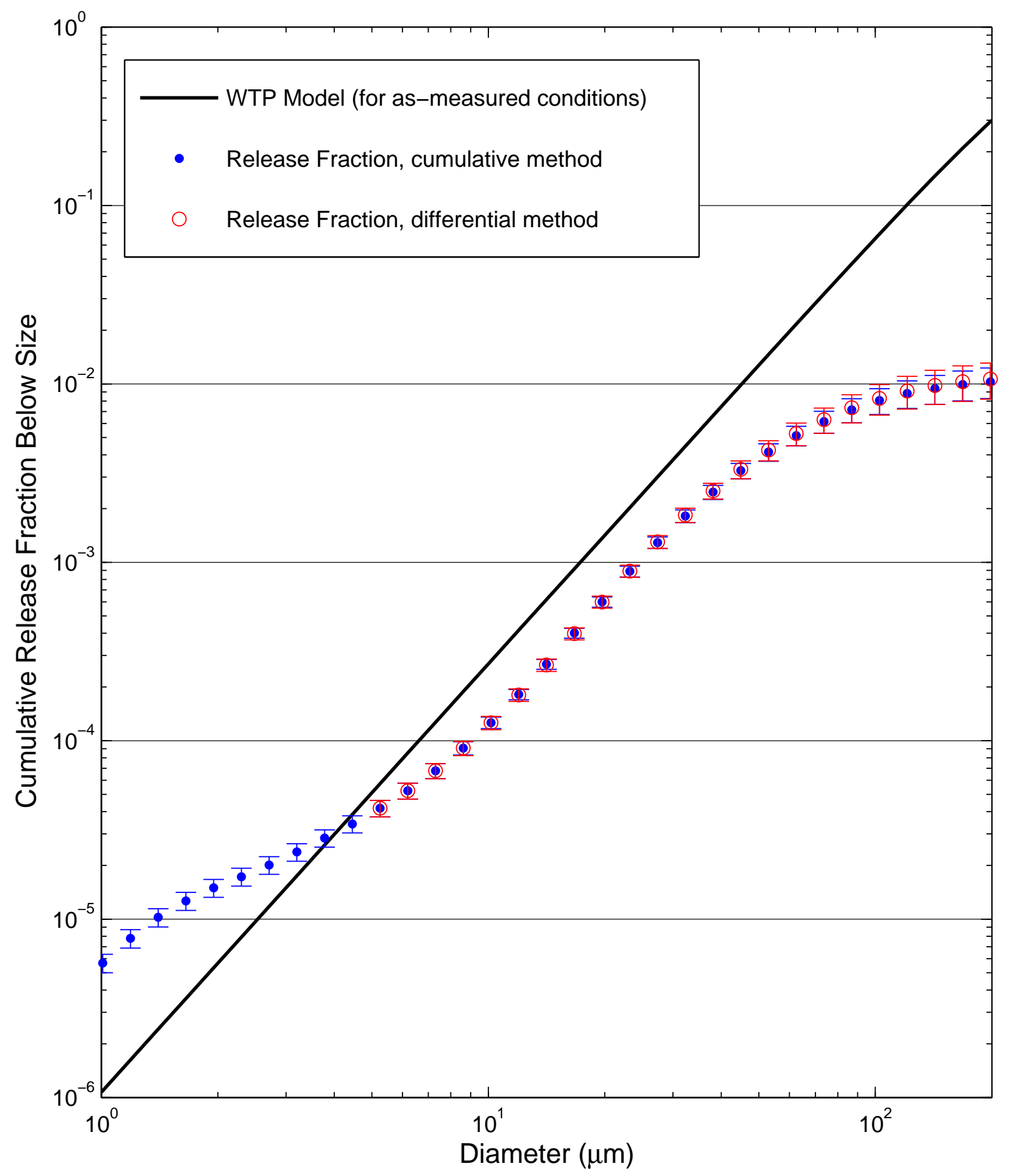


Test S192 [3 Malvern Average]: 100 psi, Orifice S7A - rectangular, $1 \times 10 \mathrm{~mm}$

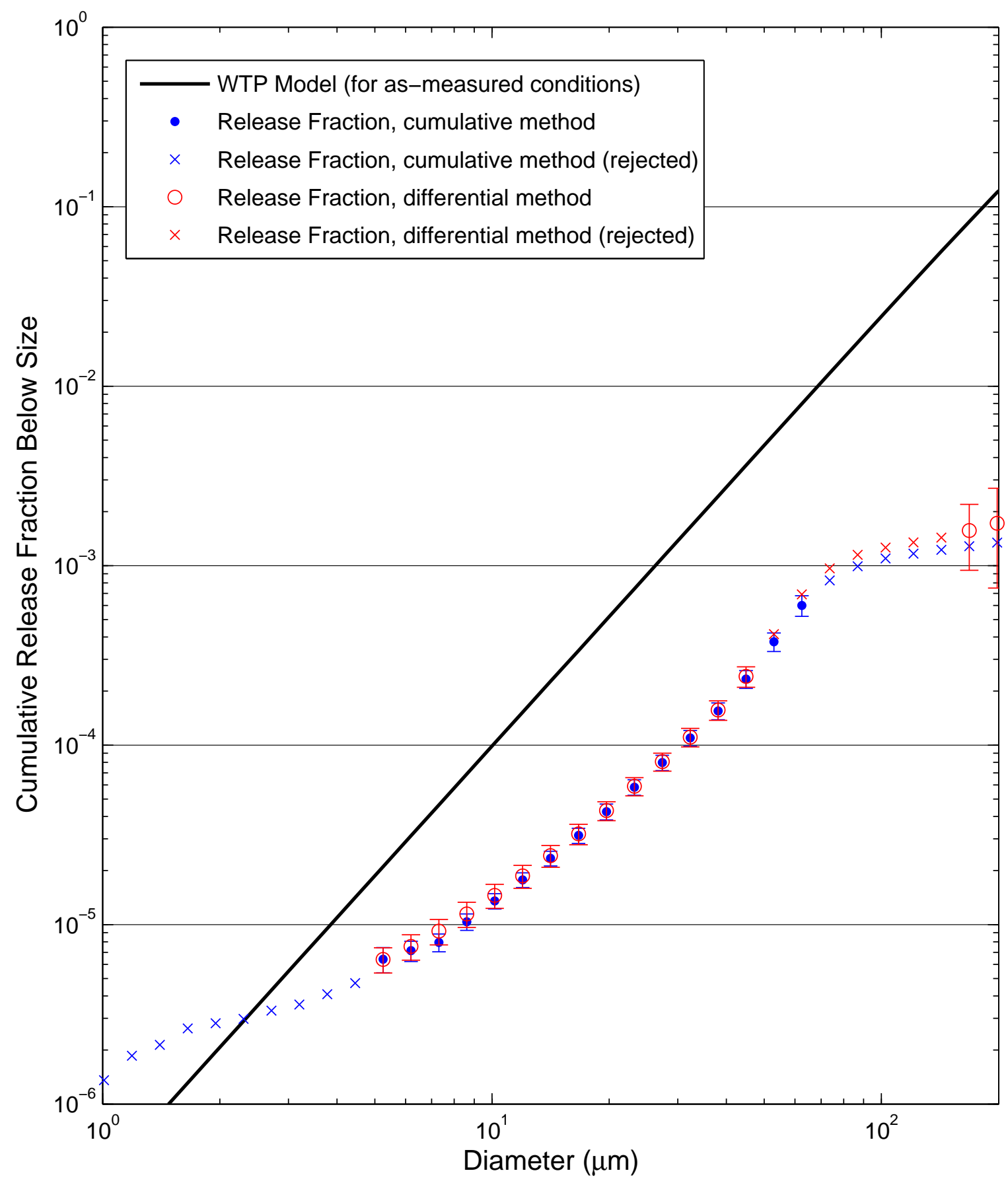


Test S193 [3 Malvern Average]: 200 psi, Orifice S7A - rectangular, $1 \times 10 \mathrm{~mm}$

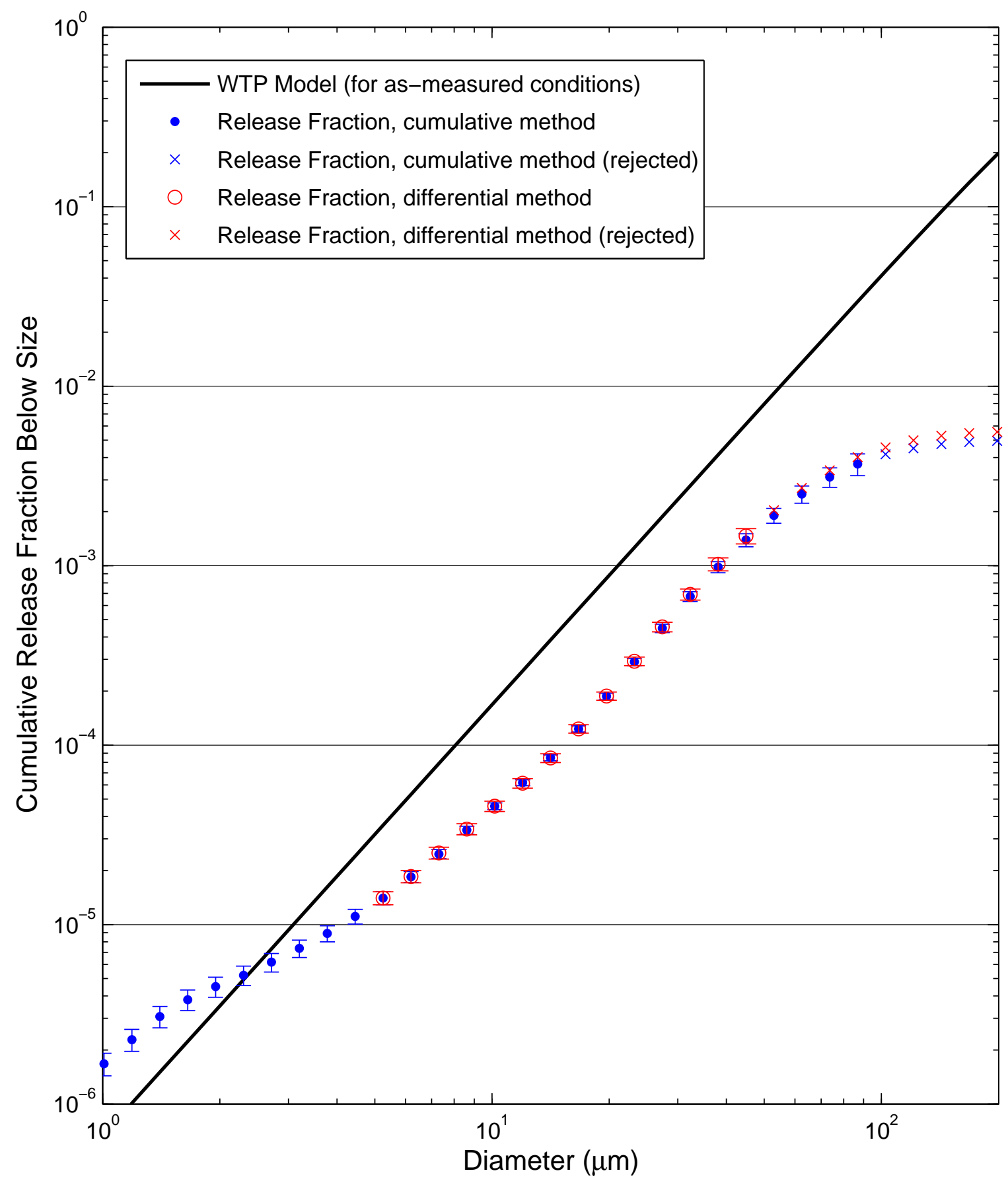


Test S194 [3 Malvern Average]: 380 psi, Orifice S7A - rectangular, $1 \times 10 \mathrm{~mm}$

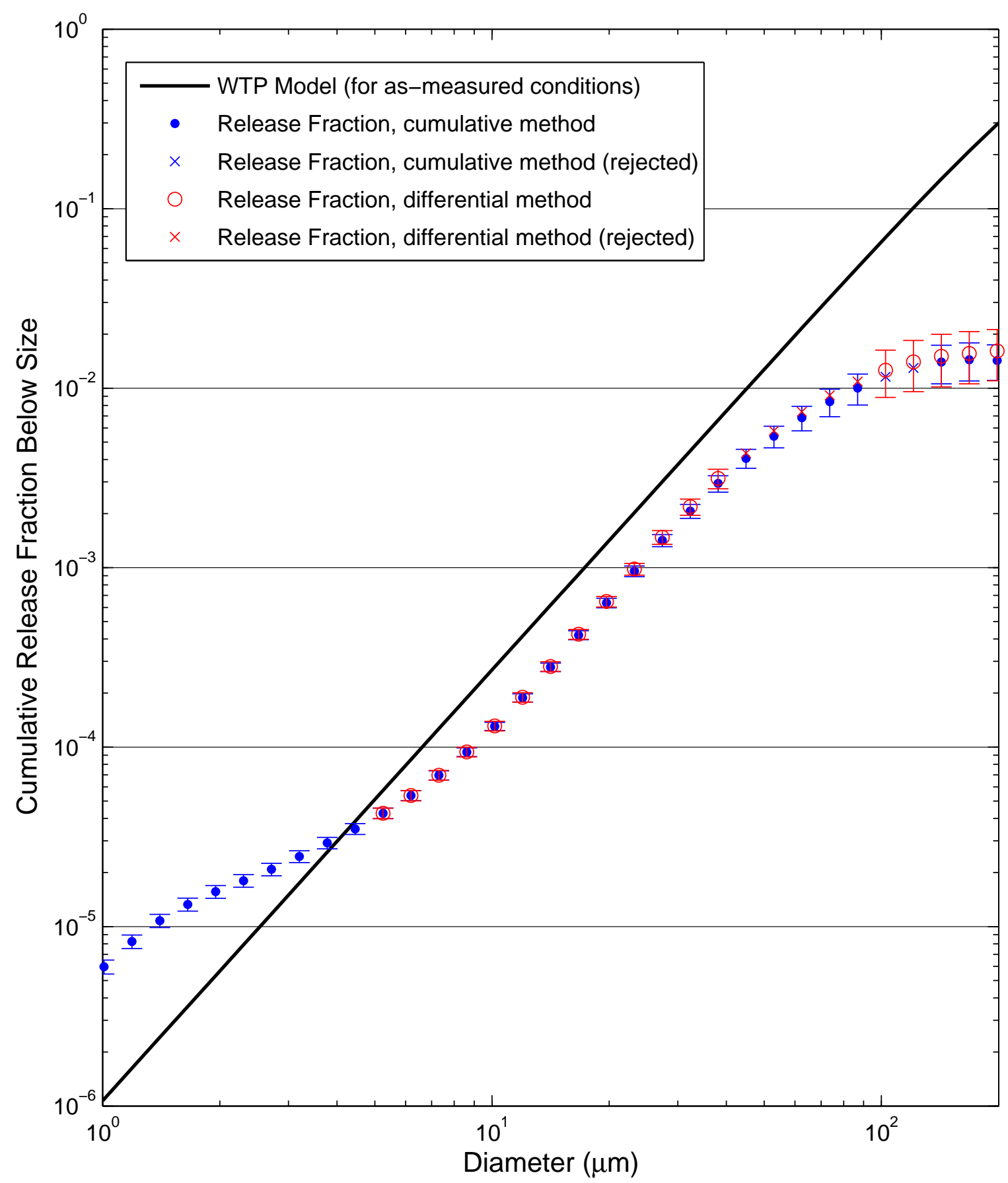


Test S195 [3 Malvern Average]: 100 psi, Orifice S7A - rectangular, $1 \times 10 \mathrm{~mm}$

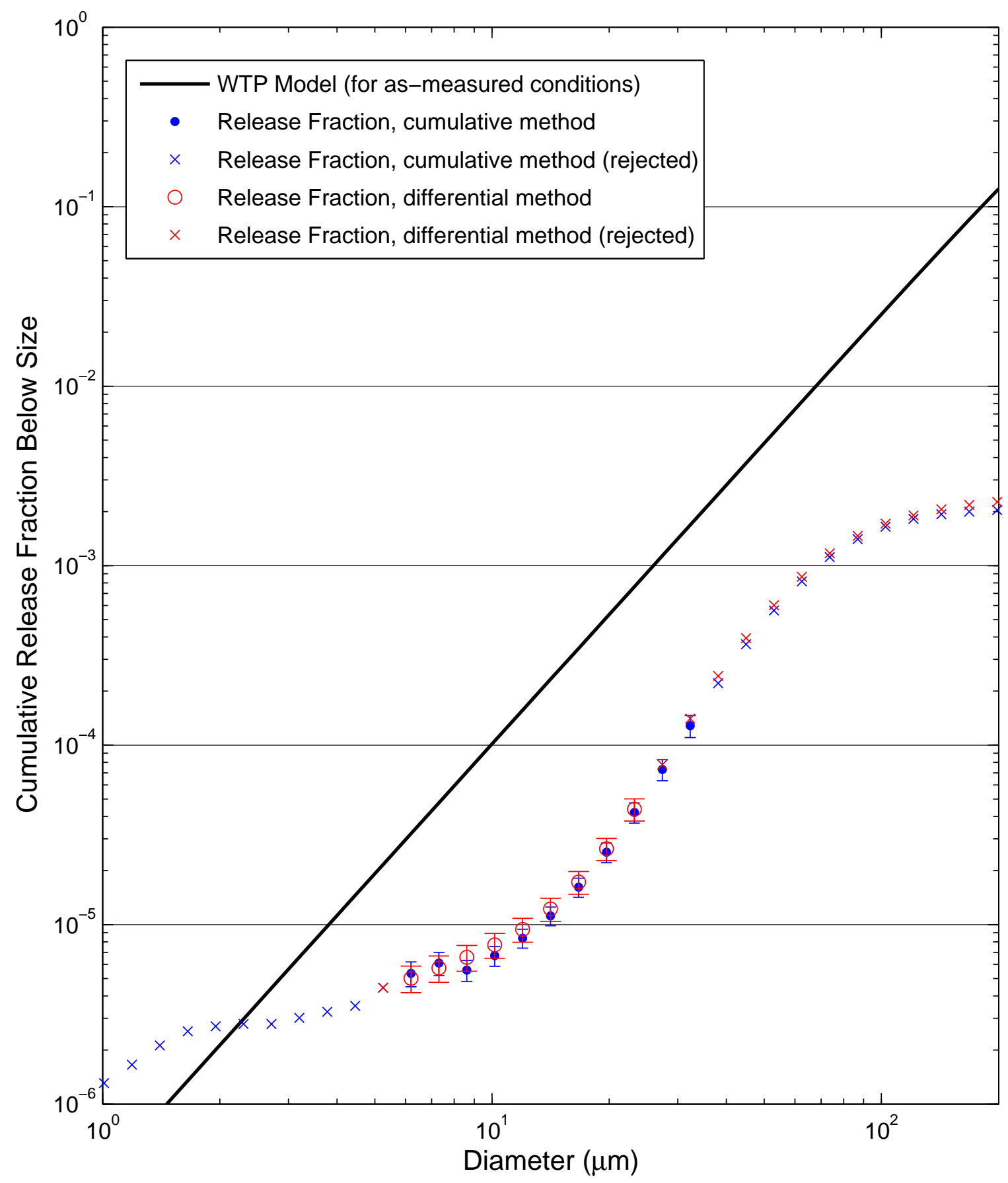


Test S196 [3 Malvern Average]: 200 psi, Orifice S7A - rectangular, $1 \times 10 \mathrm{~mm}$

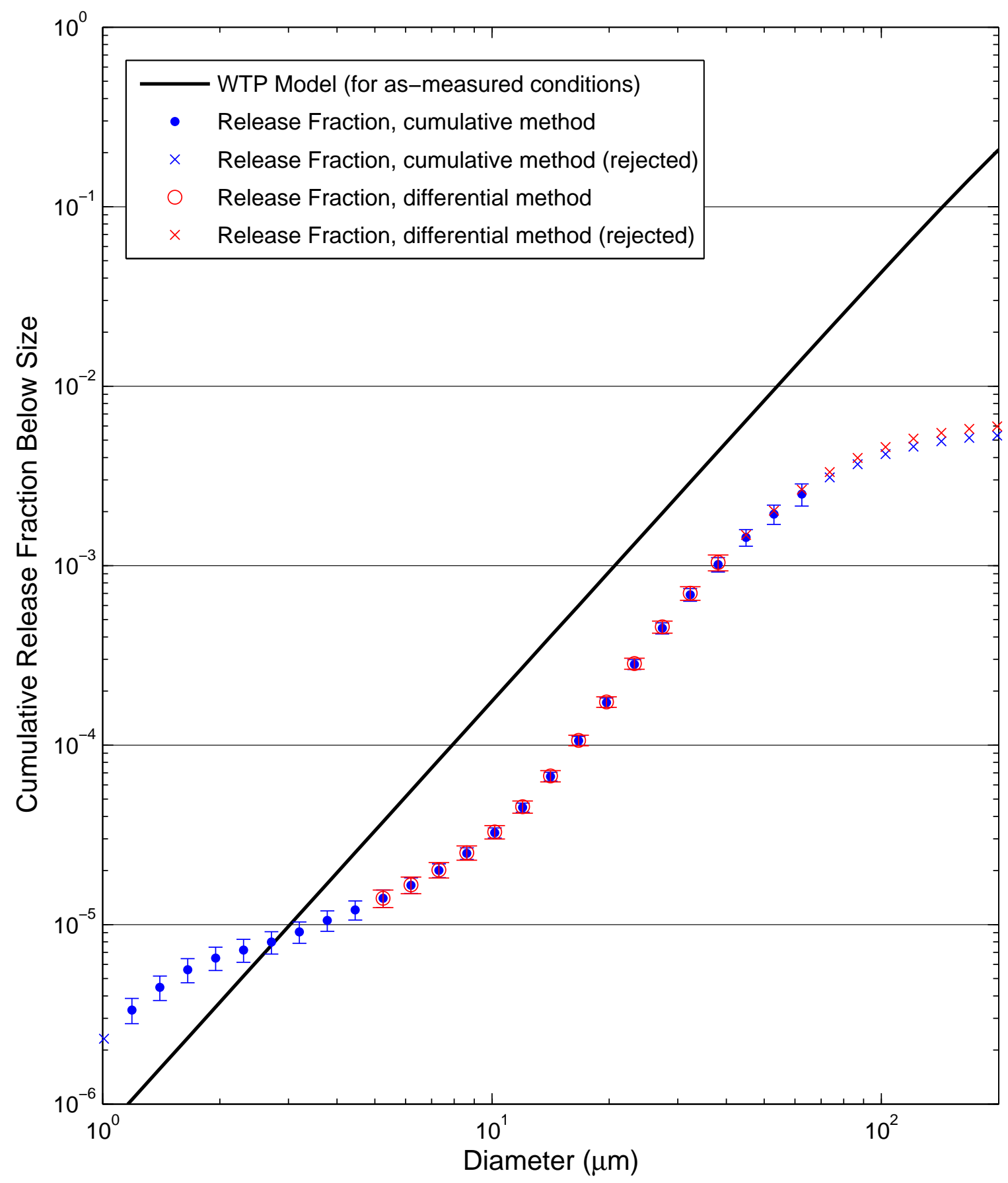


Test S197 [3 Malvern Average]: 380 psi, Orifice S7A - rectangular, $1 \times 10 \mathrm{~mm}$

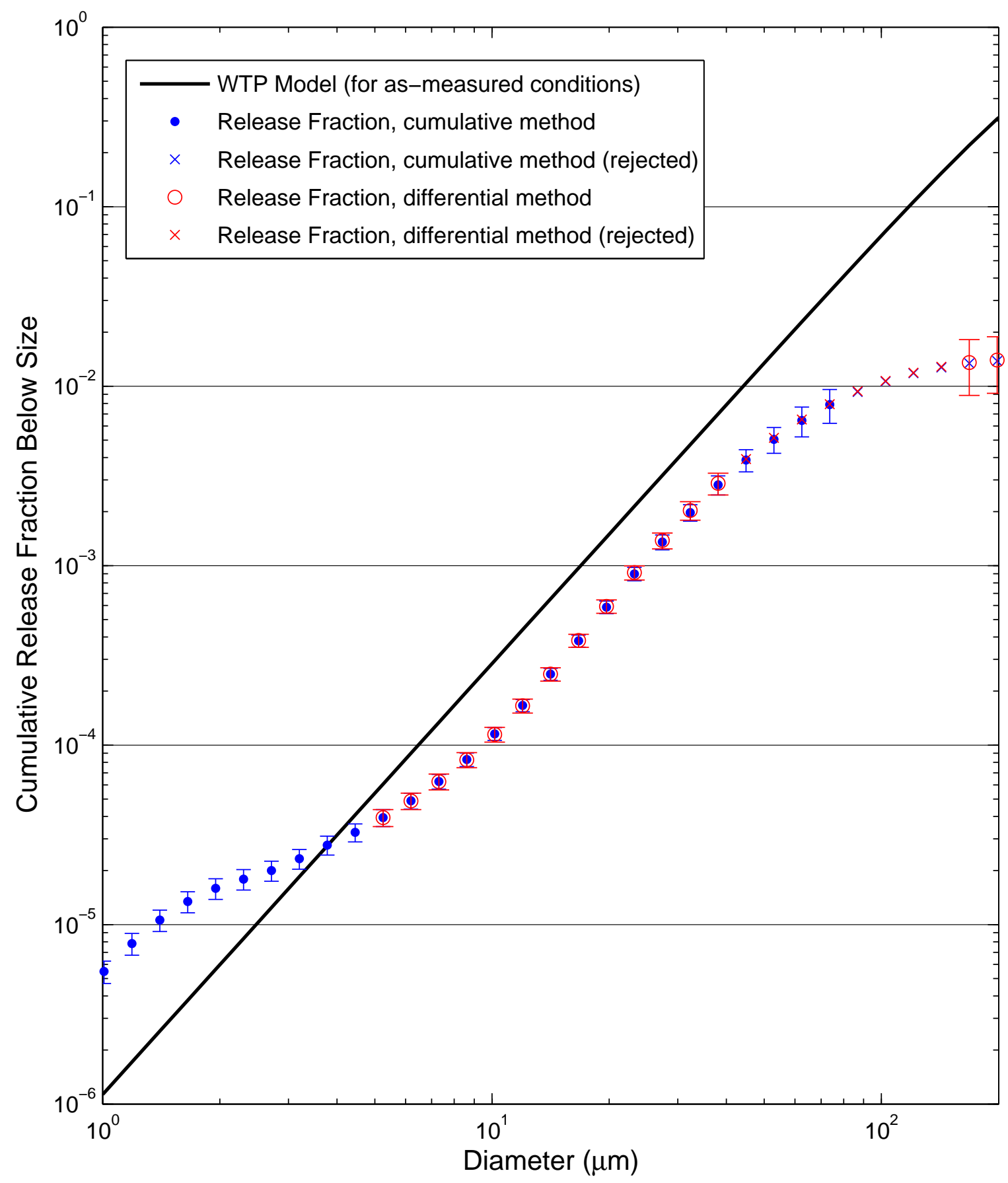


Test S198 [3 Malvern Average]: 100 psi, Orifice S7A - rectangular, $1 \times 10 \mathrm{~mm}$

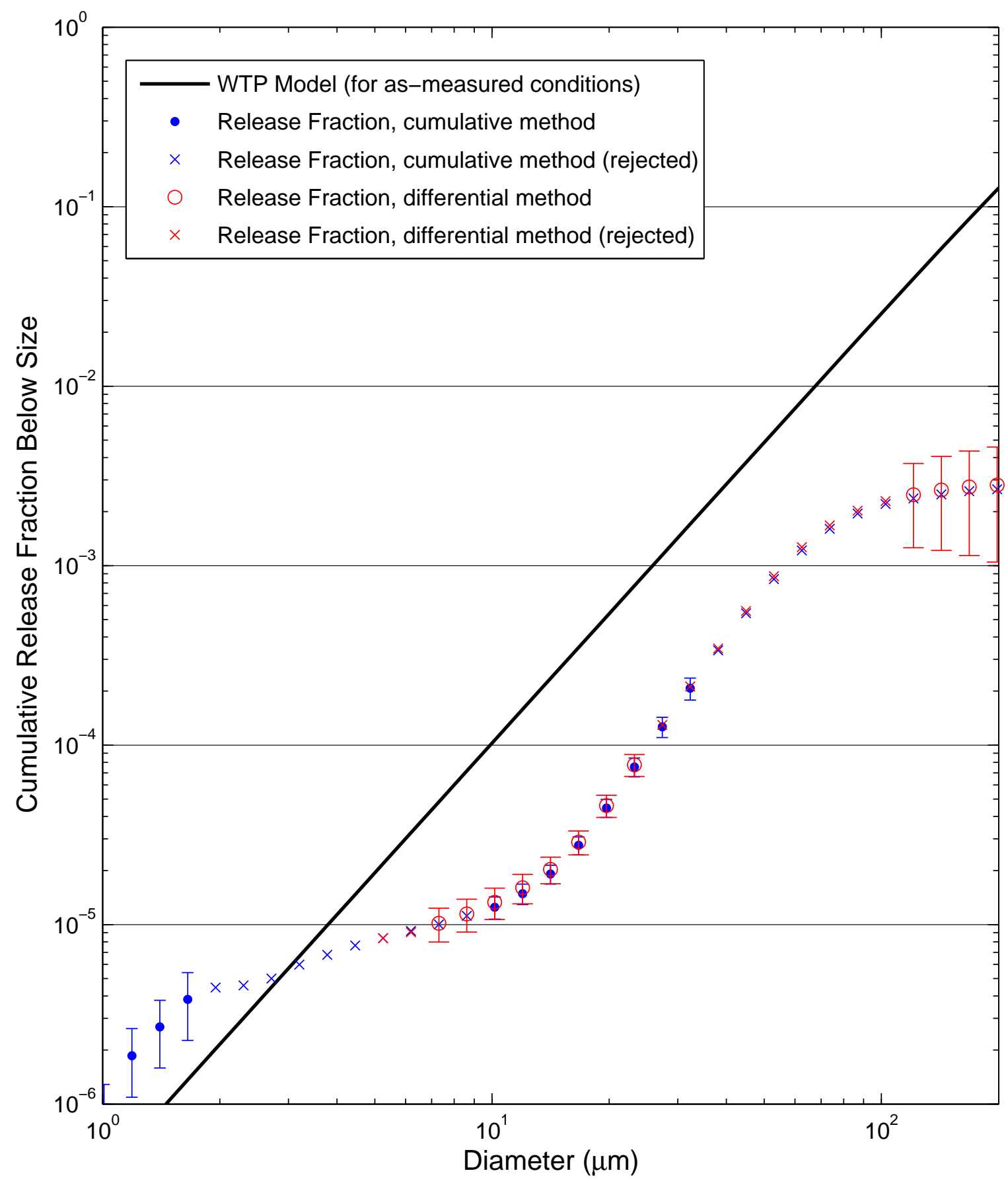


Test S199 [3 Malvern Average]: 200 psi, Orifice S7A - rectangular, $1 \times 10 \mathrm{~mm}$

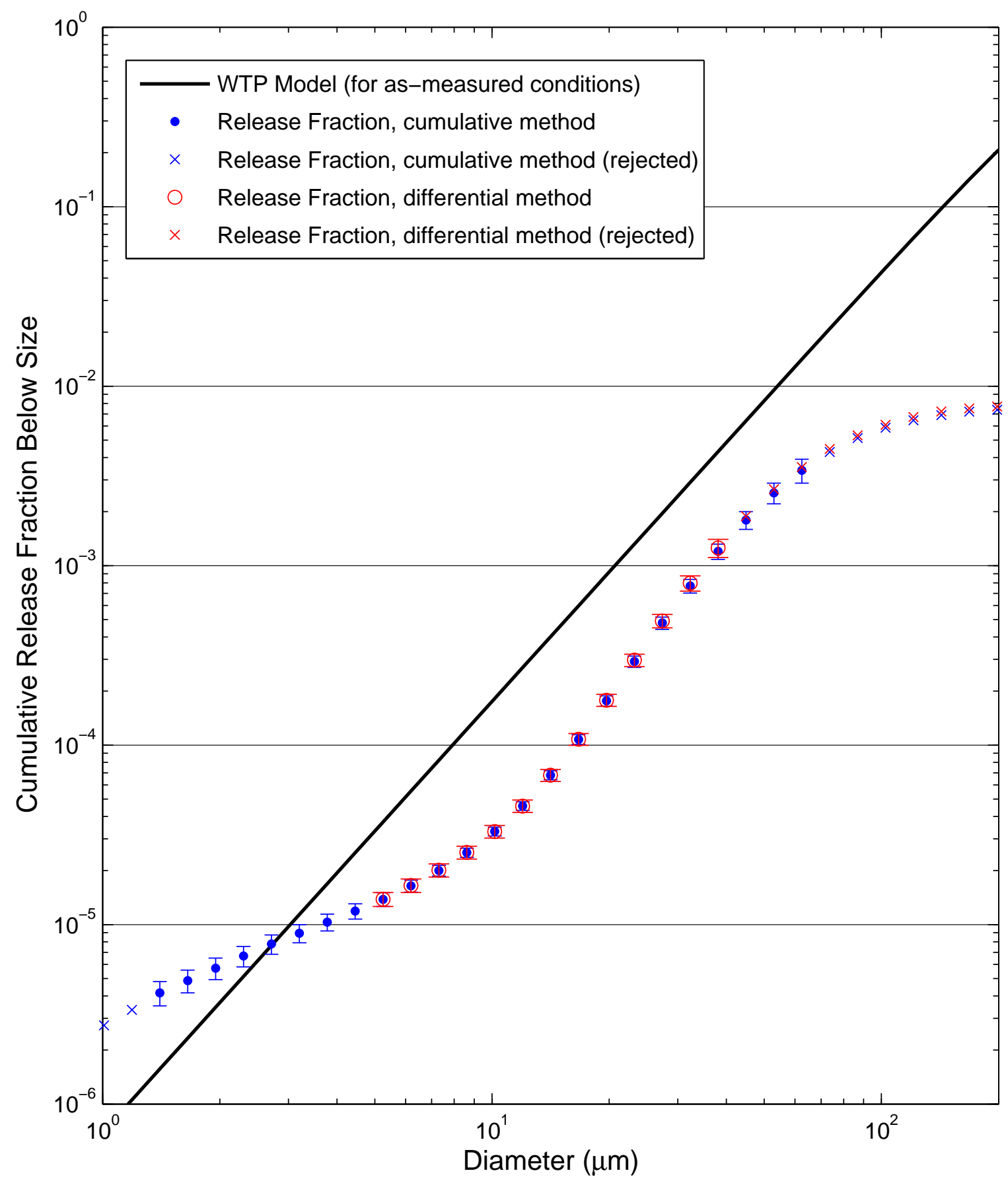


Test S199 [3 Malvern Average]: 200 psi, Orifice S7A - rectangular, $1 \times 10 \mathrm{~mm}$

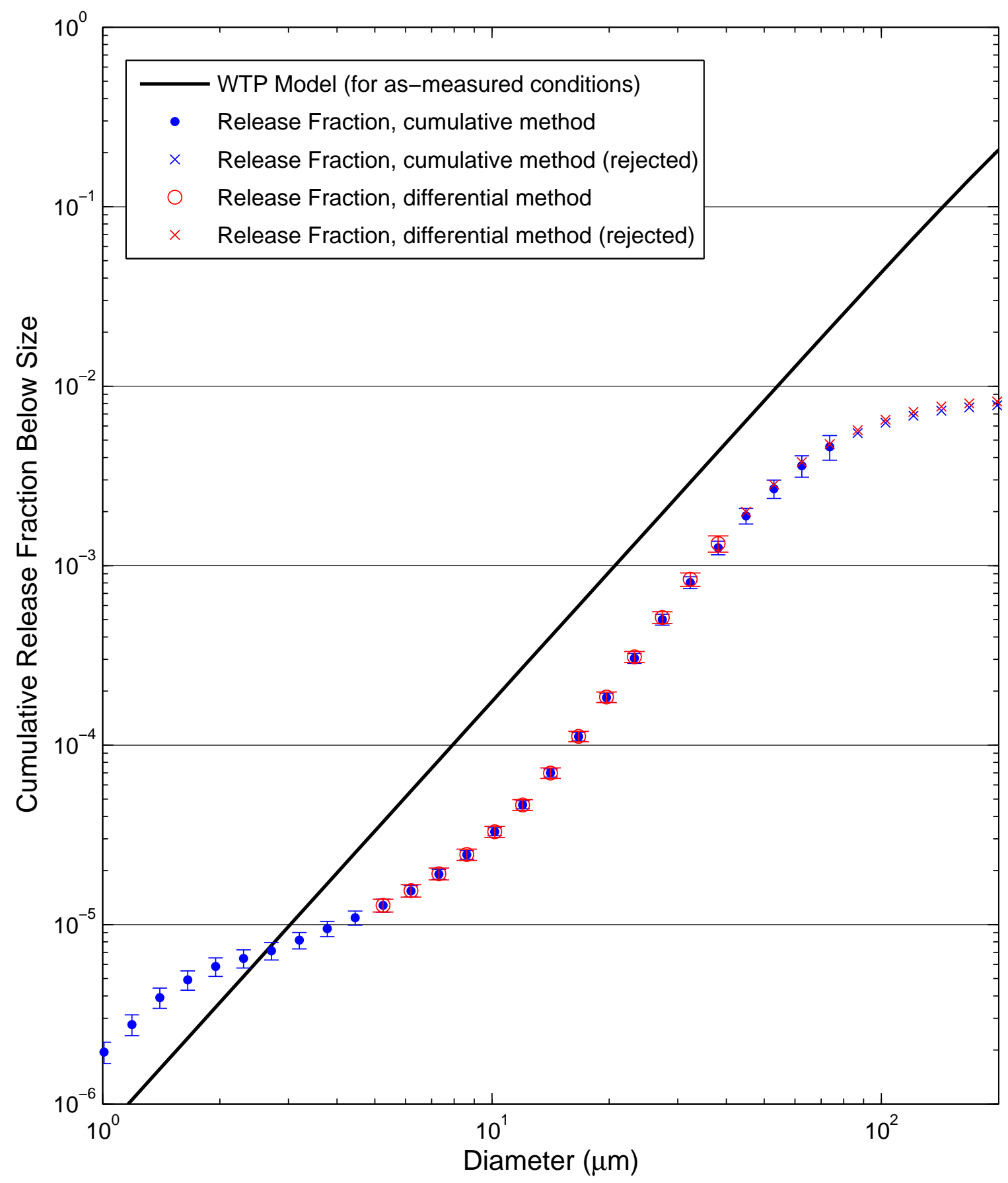


Test S199 [3 Malvern Average]: 200 psi, Orifice S7A - rectangular, $1 \times 10 \mathrm{~mm}$

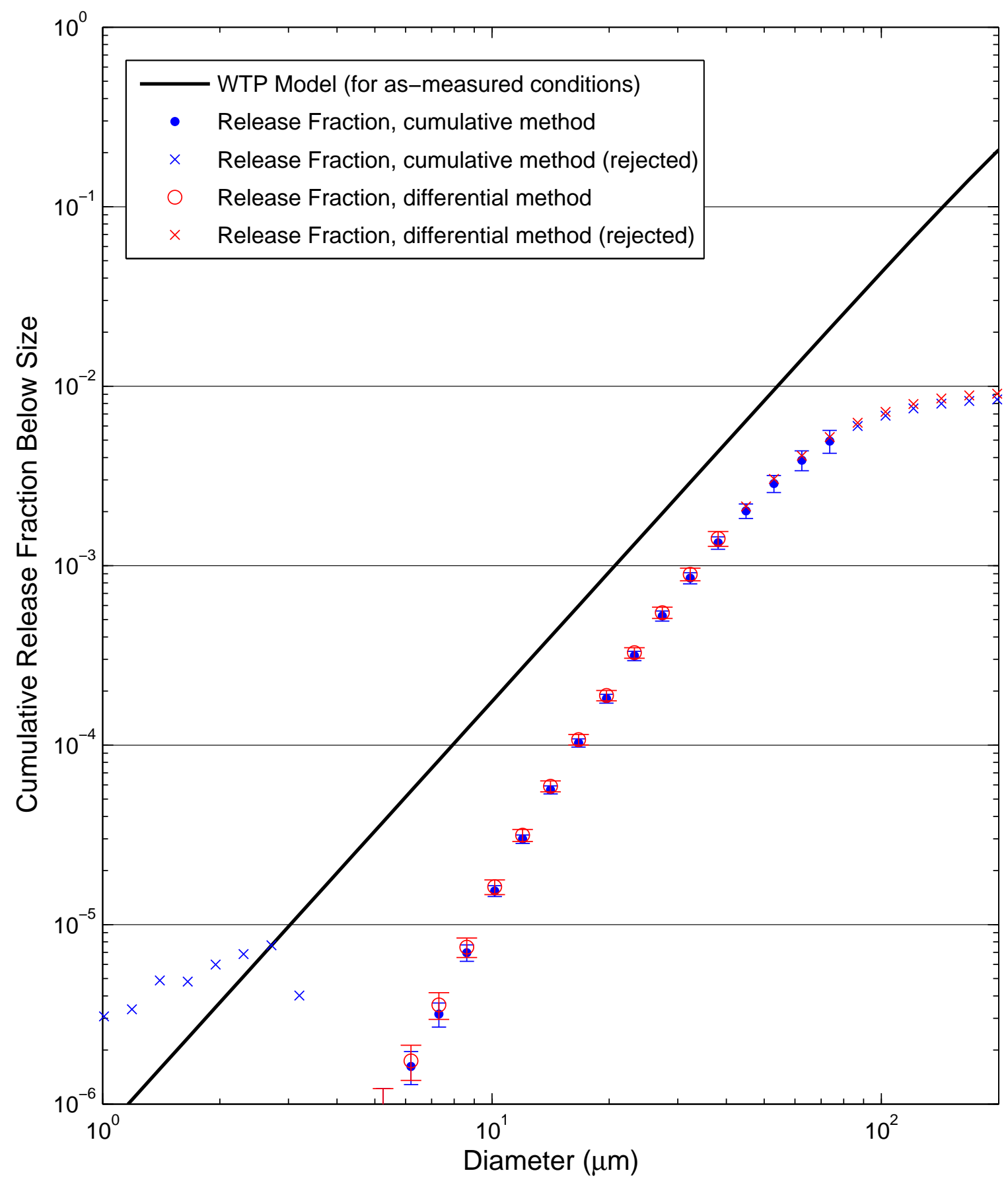

B. 162 
Test S200 [3 Malvern Average]: 380 psi, Orifice S7A - rectangular, $1 \times 10 \mathrm{~mm}$

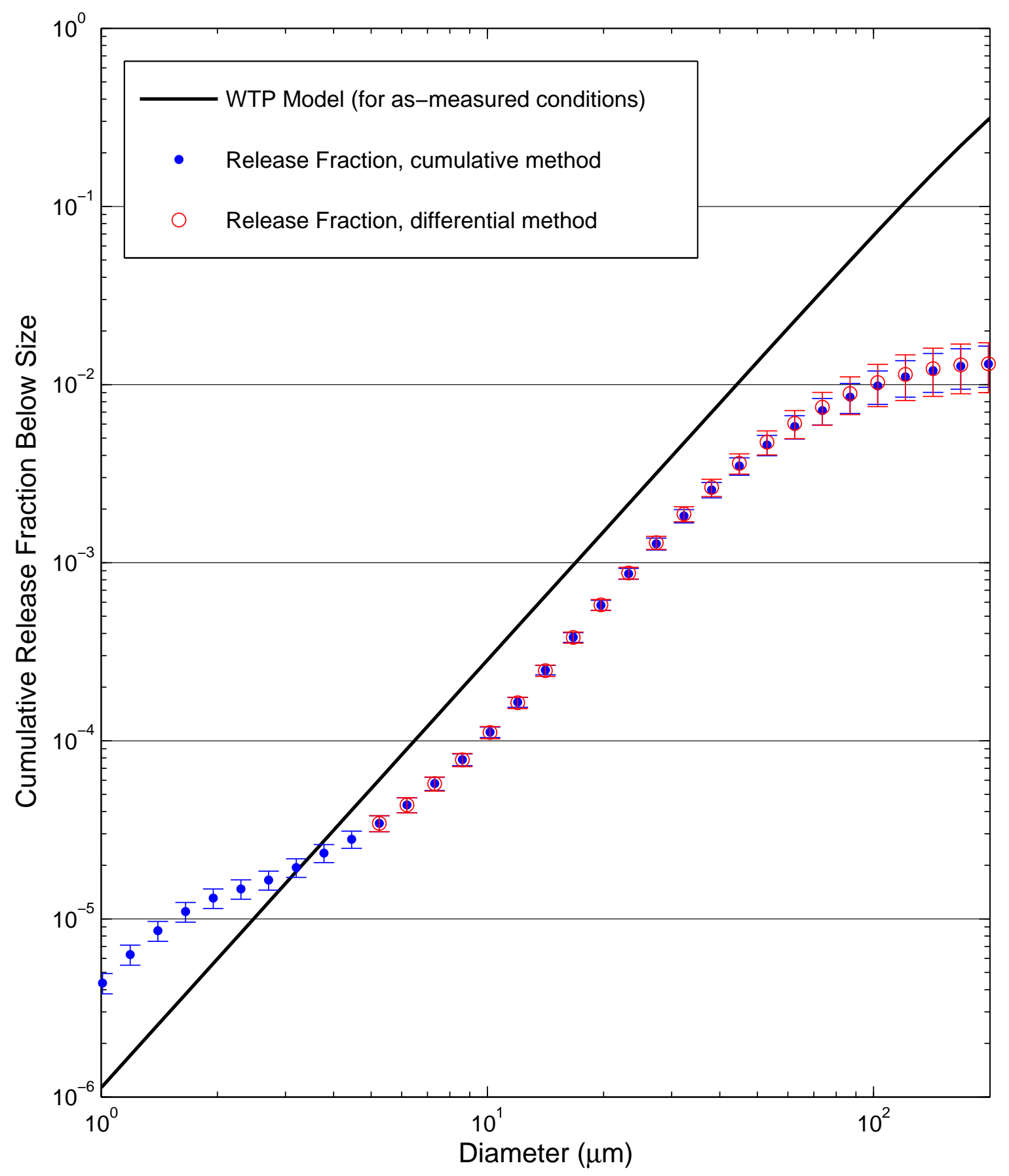


Test S201 [3 Malvern Average]: 100 psi, Orifice S2A - rectangular, $0.5 \times 5 \mathrm{~mm}$

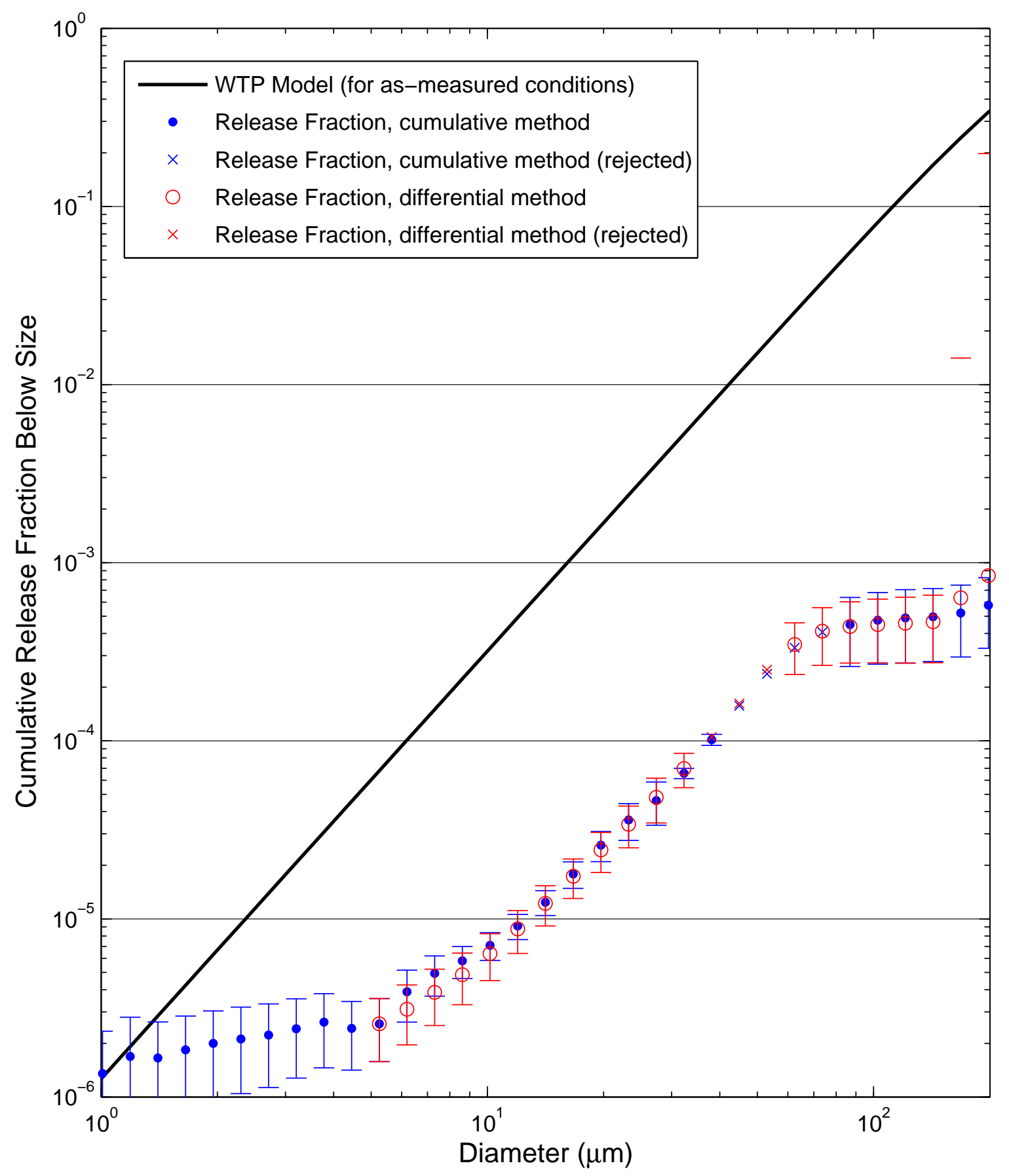


Test S202 [3 Malvern Average]: 200 psi, Orifice S2A - rectangular, $0.5 \times 5 \mathrm{~mm}$

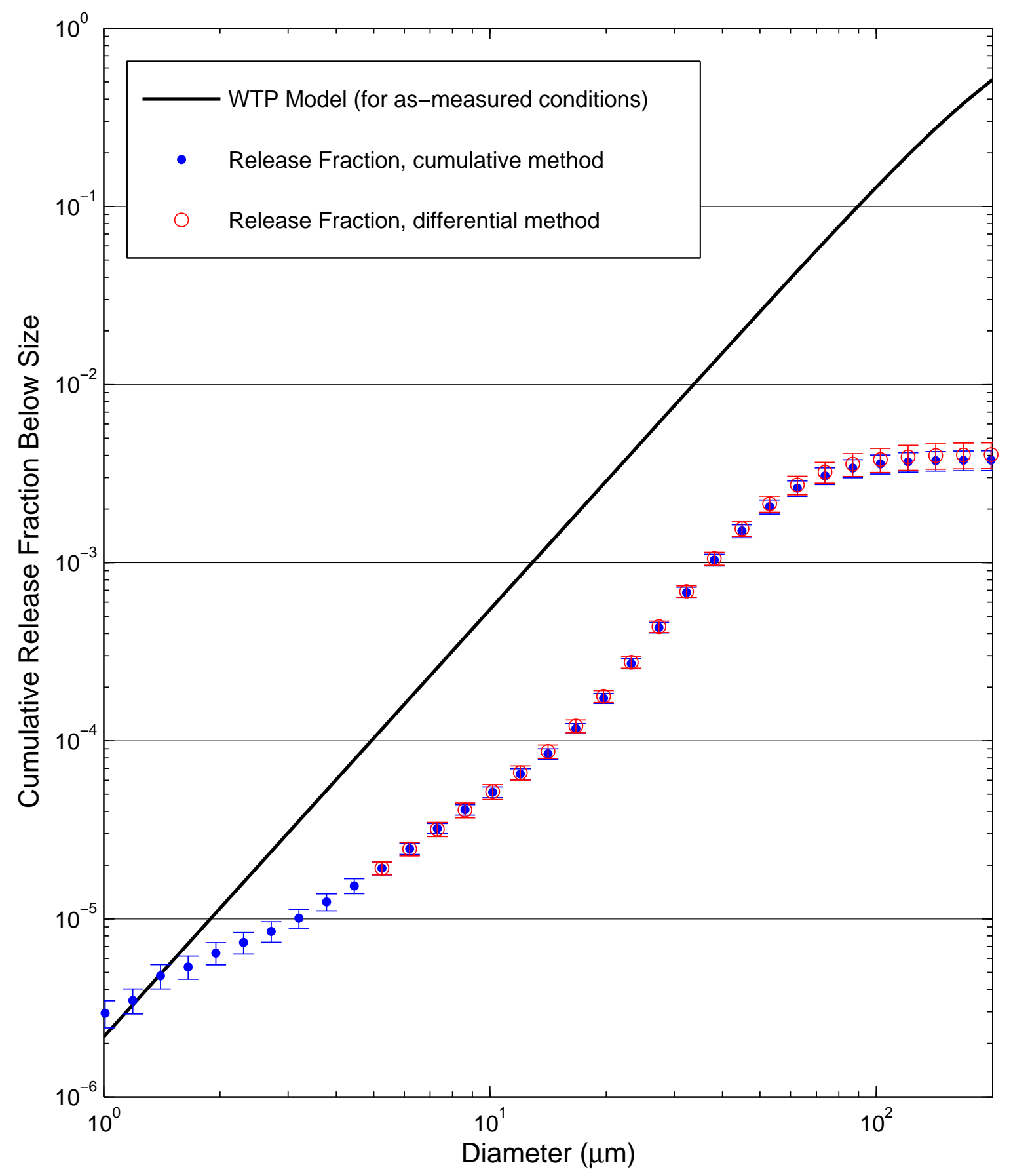


Test S203 [3 Malvern Average]: 380 psi, Orifice S2A - rectangular, $0.5 \times 5 \mathrm{~mm}$

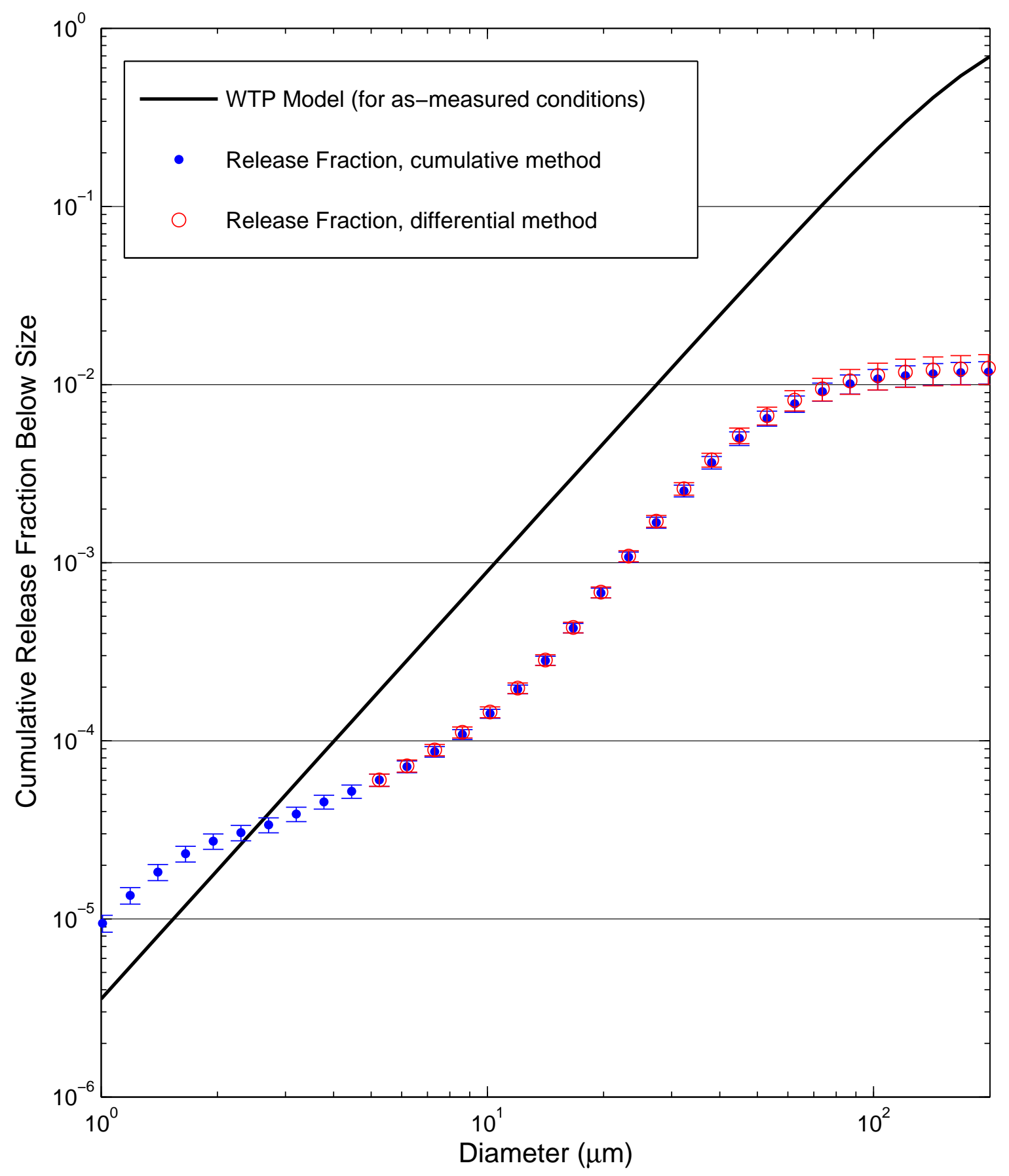


Test S204 [3 Malvern Average]: 100 psi, Orifice S2A - rectangular, $0.5 \times 5 \mathrm{~mm}$

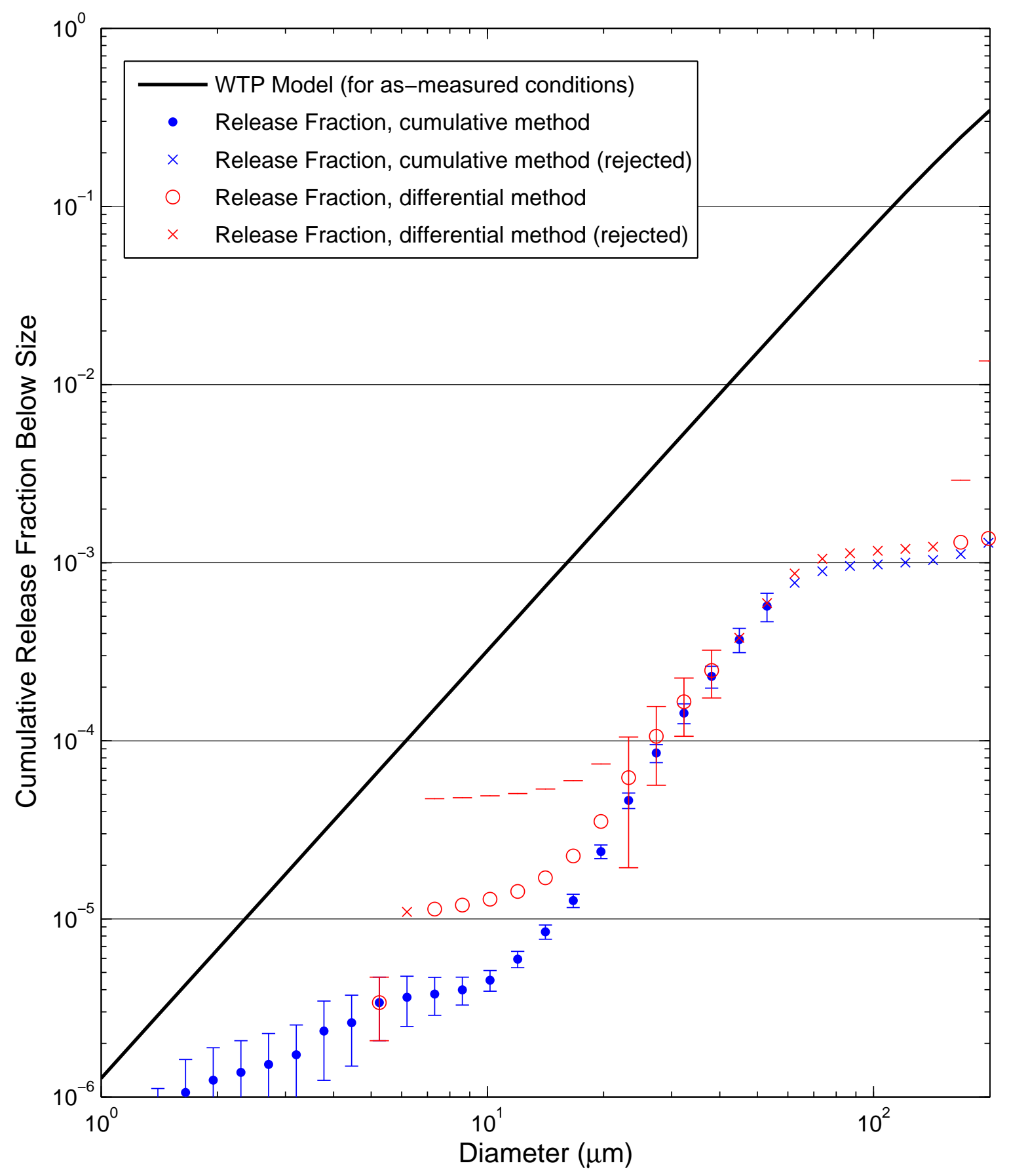


Test S205 [3 Malvern Average]: 200 psi, Orifice S2A - rectangular, $0.5 \times 5 \mathrm{~mm}$

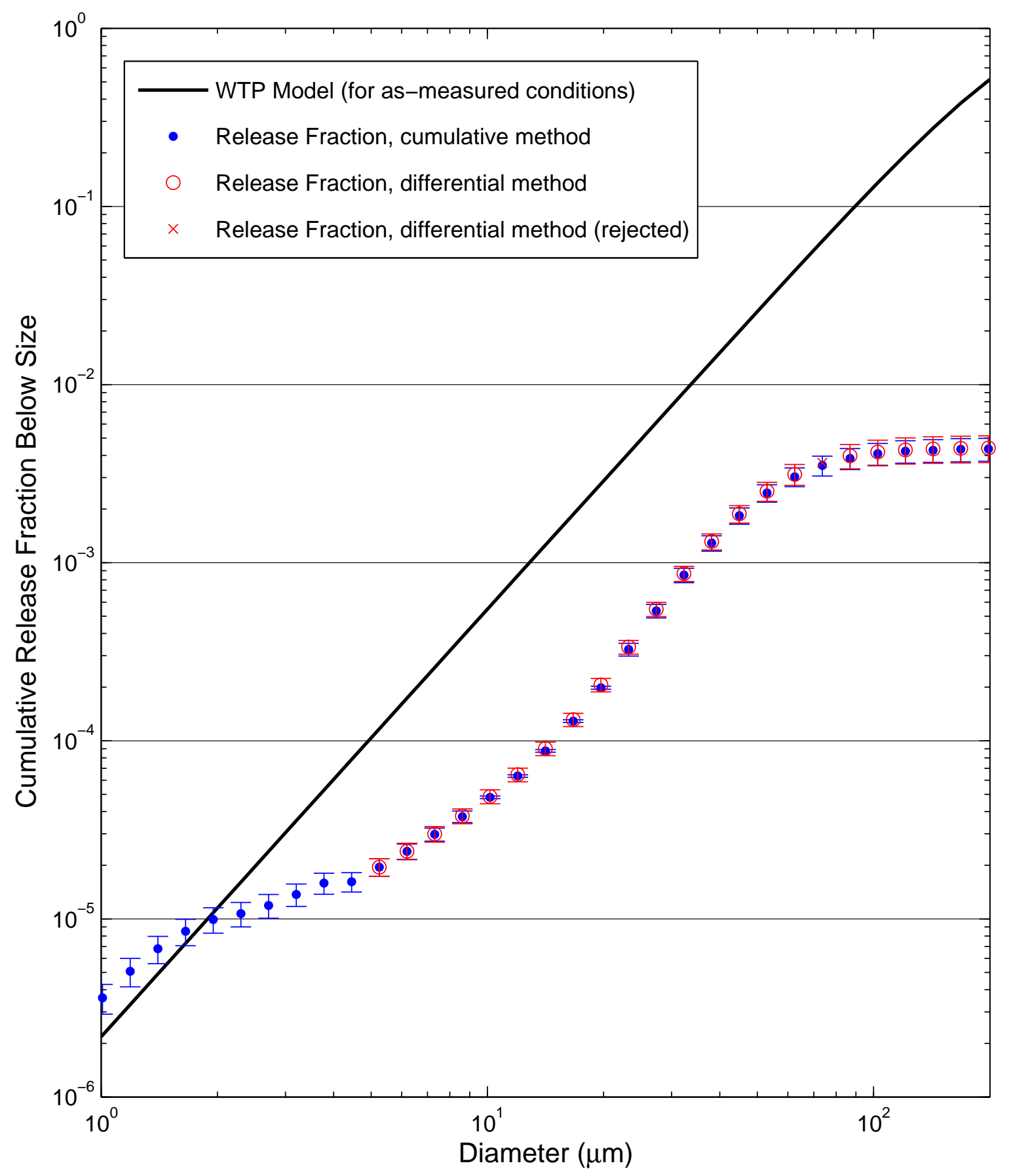


Test S206 [3 Malvern Average]: 380 psi, Orifice S2A - rectangular, $0.5 \times 5 \mathrm{~mm}$

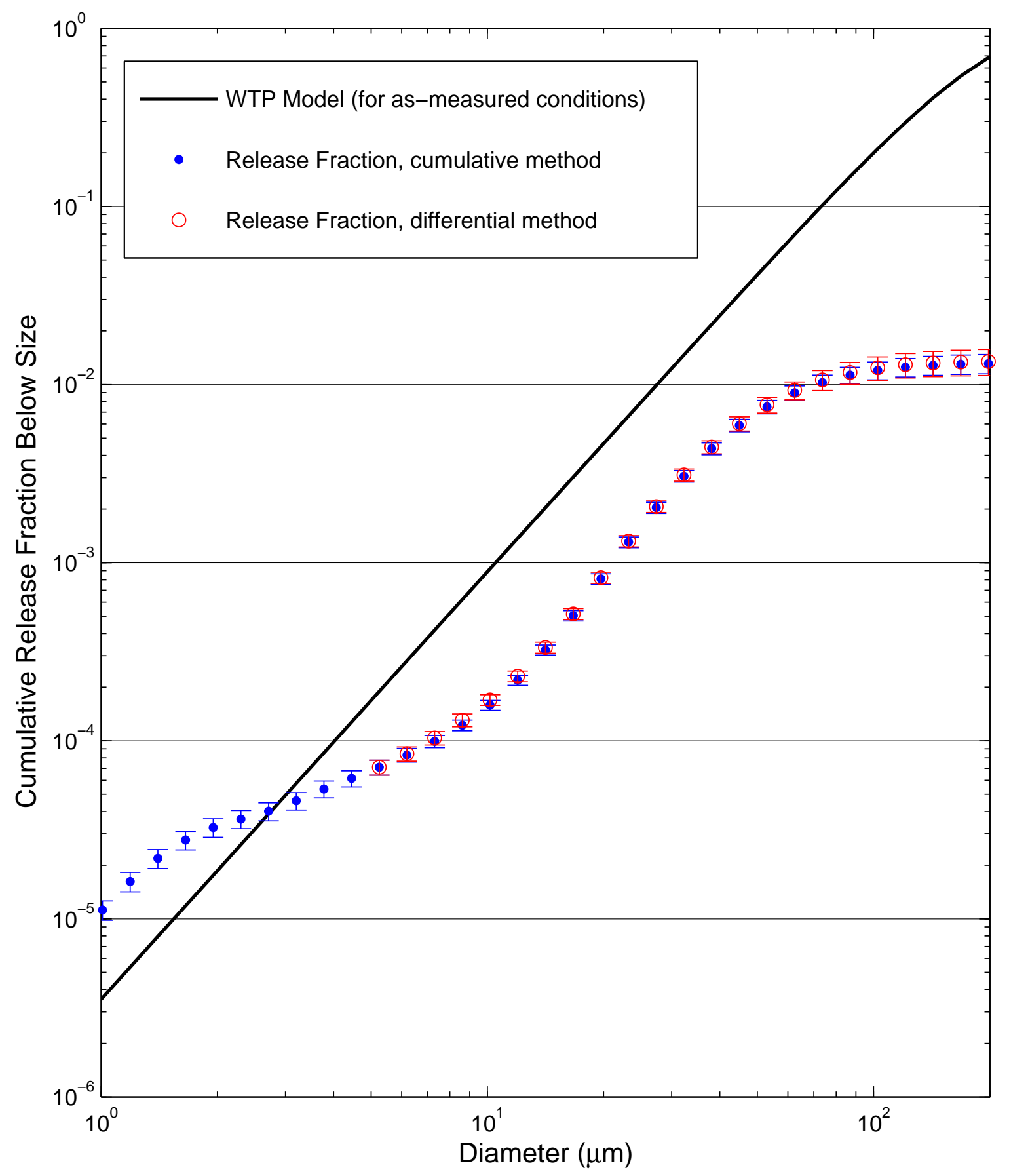


Test W207 [3 Malvern Average]: 380 psi, Orifice S1D - round, 2 mm

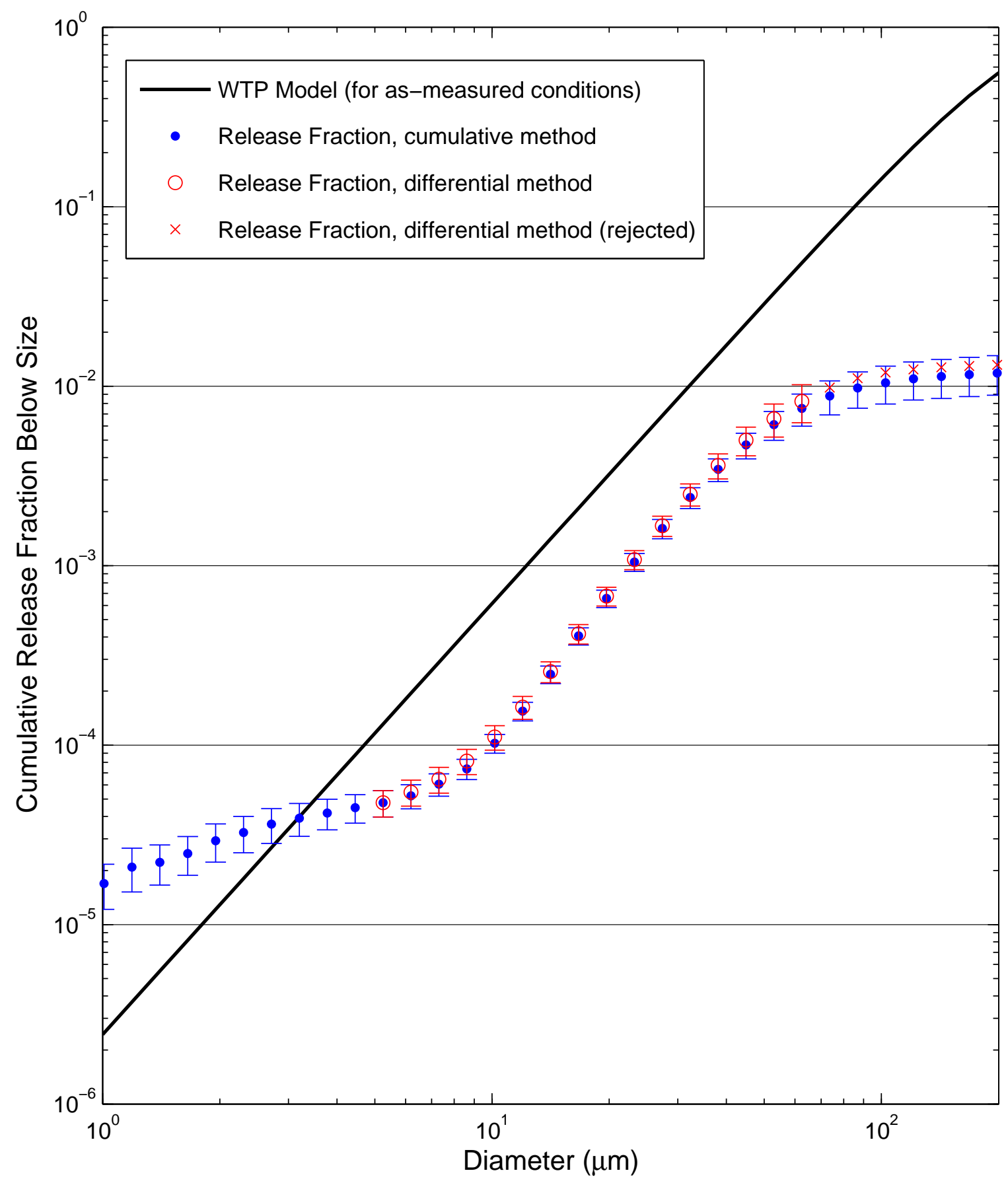

B. 170 
Test W208 [3 Malvern Average]: 380 psi, Orifice S1D - round, 2 mm

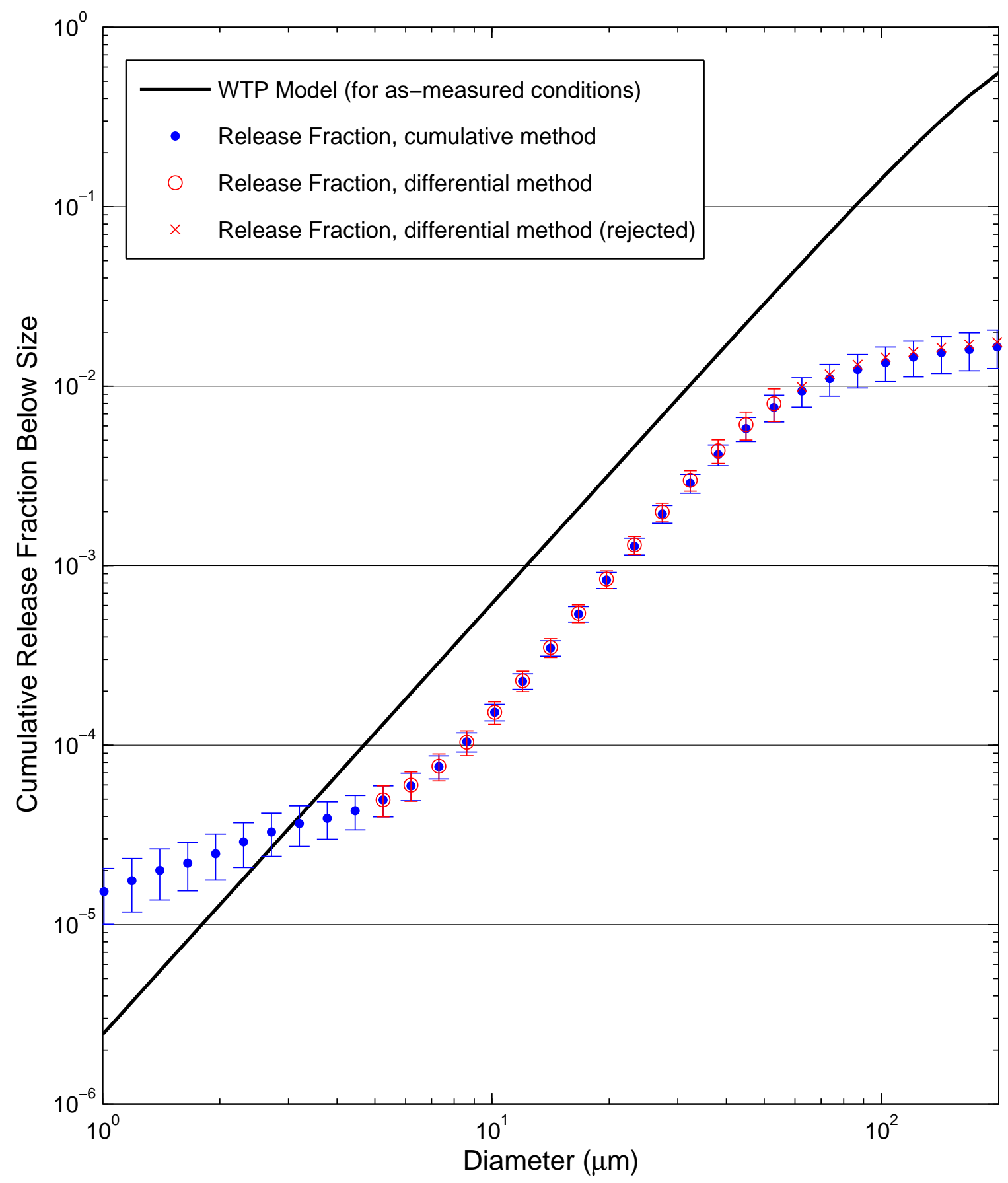


Test W209 [3 Malvern Average]: 380 psi, Orifice S1D - round, 2 mm

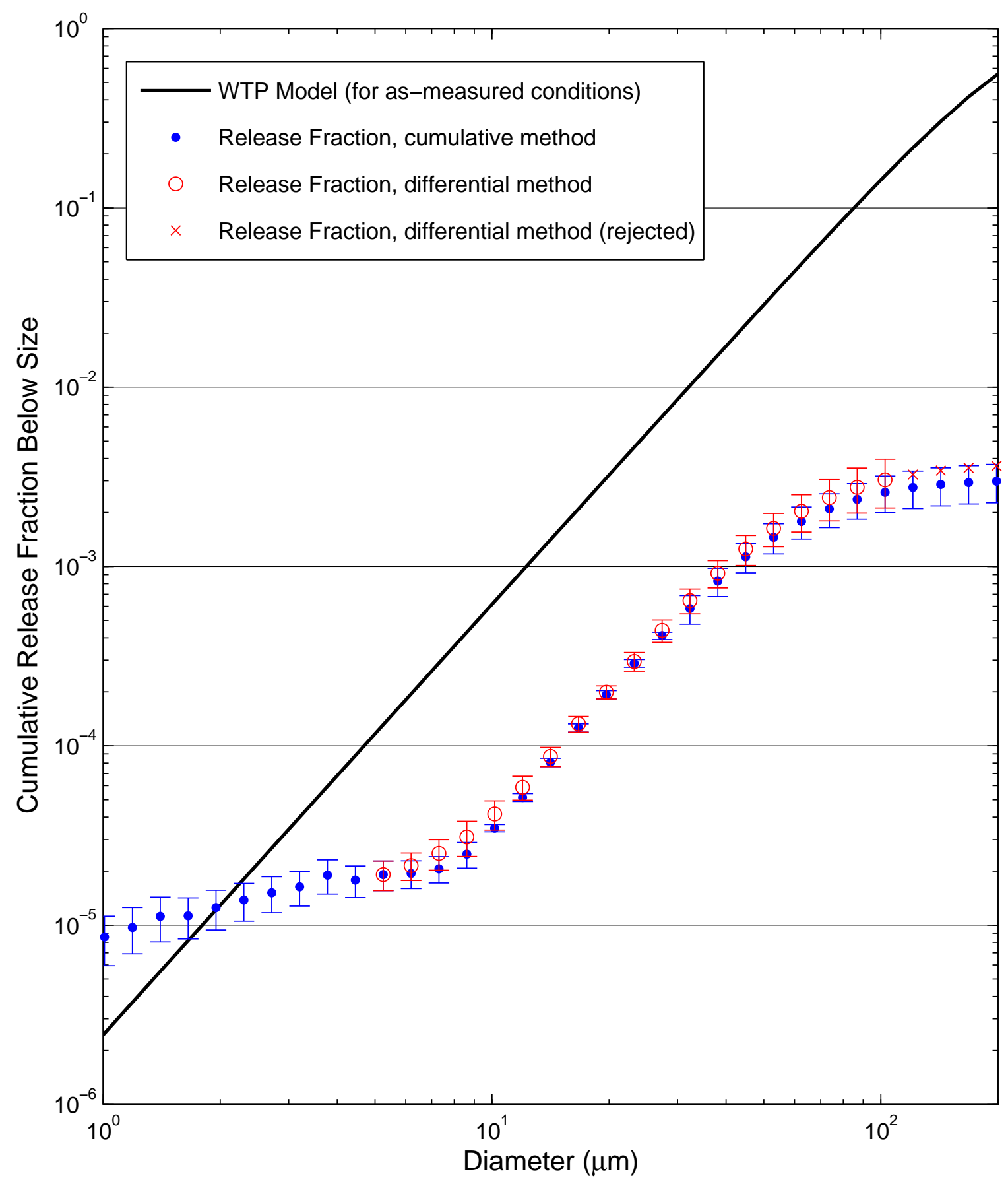

B. 172 
Test W210 [3 Malvern Average]: 380 psi, Orifice S1D - round, 2 mm

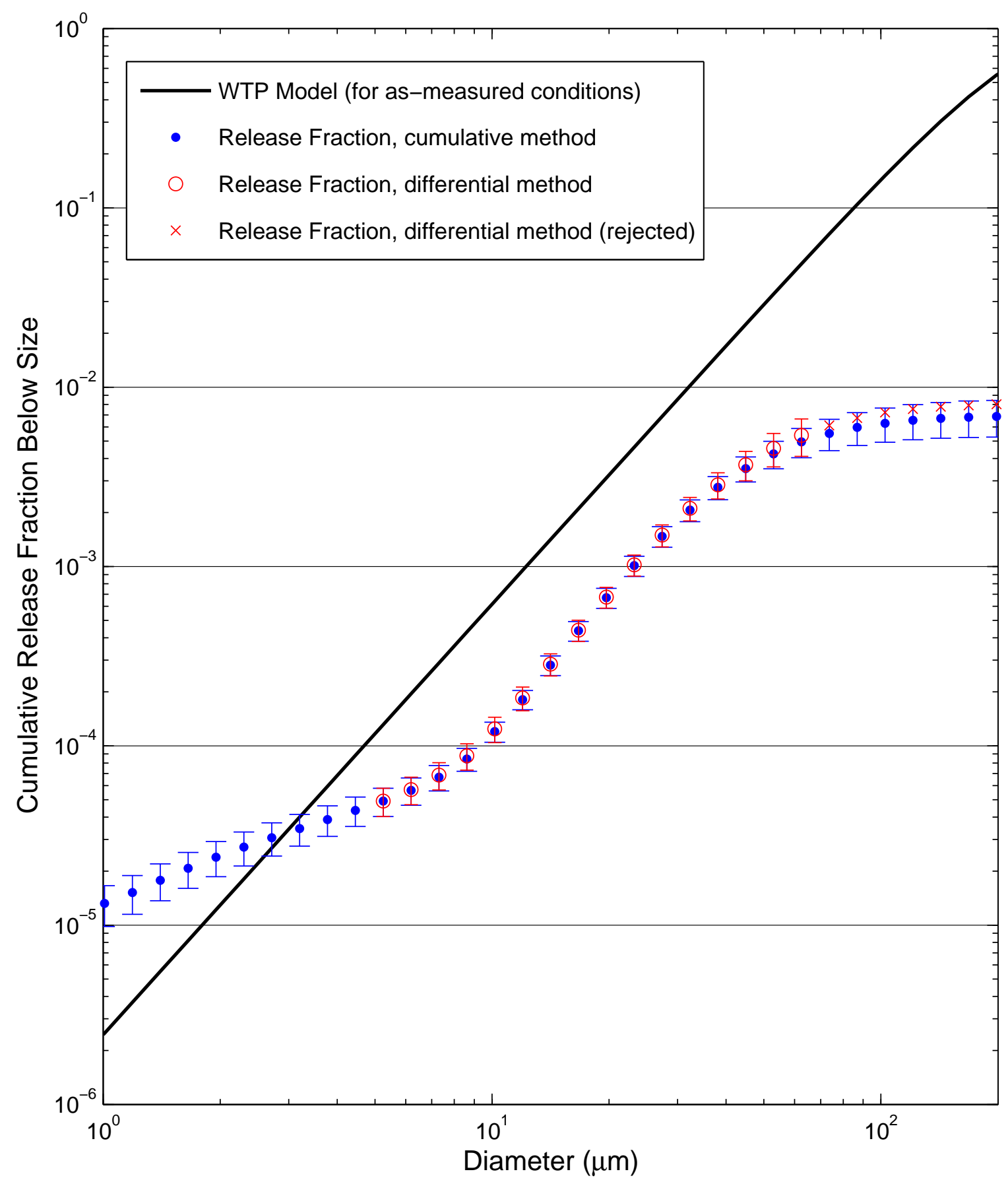


Test W211 [3 Malvern Average]: 380 psi, Orifice S1D - round, 2 mm

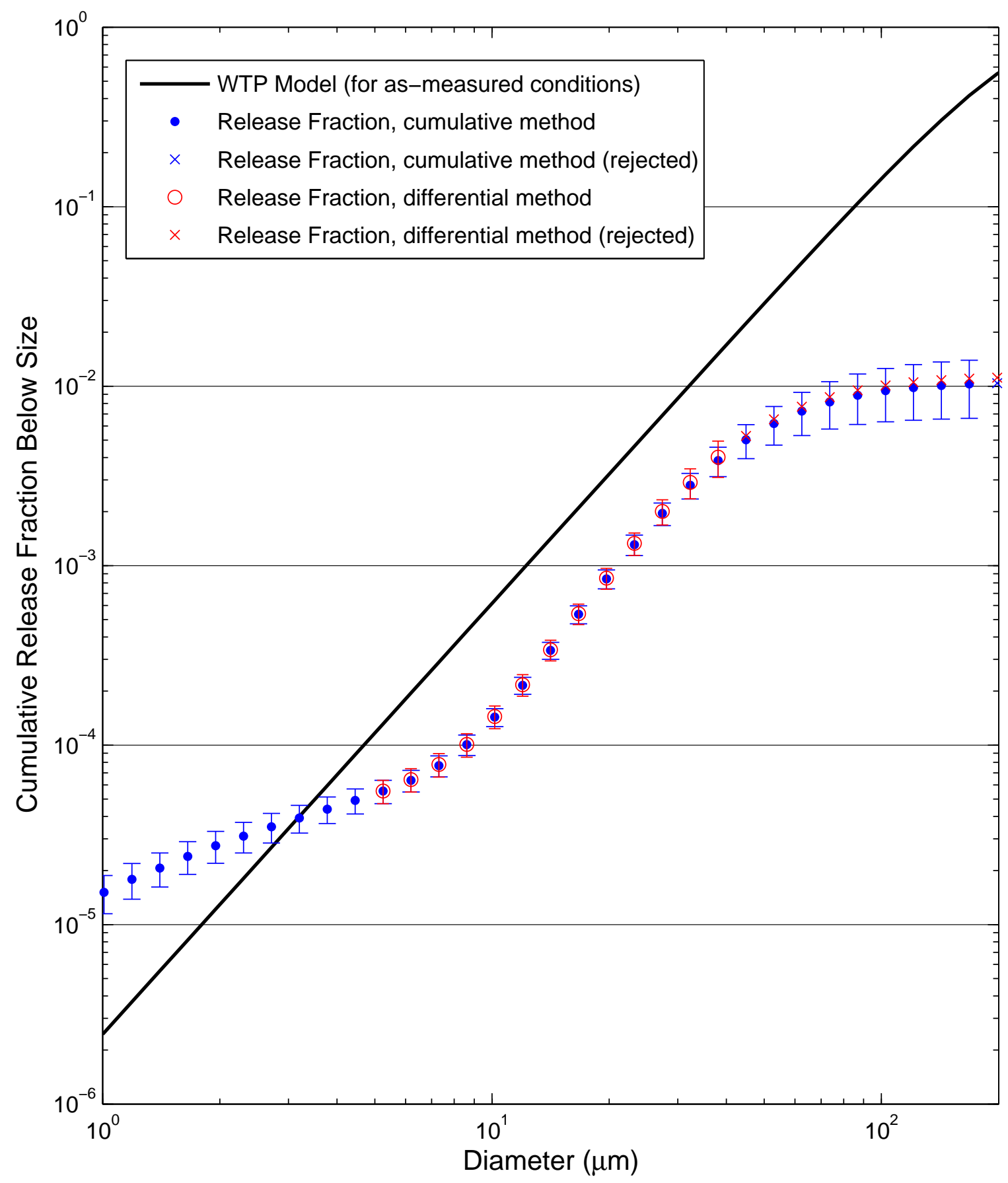


Test W222 [3 Malvern Average]: 200 psi, Orifice S5C - rectangular, $1 \times 20 \mathrm{~mm}$

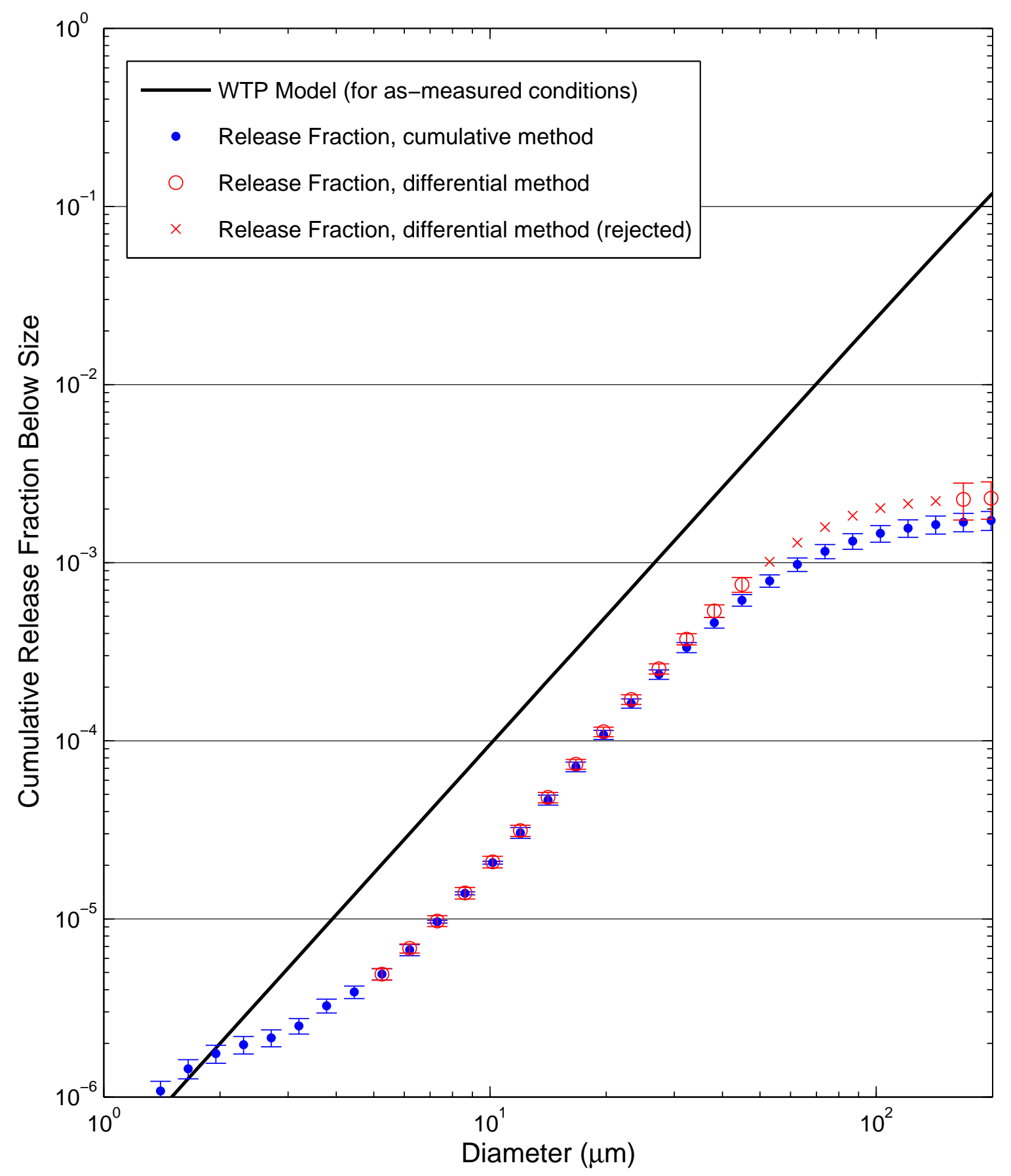


Test W223 [3 Malvern Average]: 380 psi, Orifice S5C - rectangular, $1 \times 20 \mathrm{~mm}$

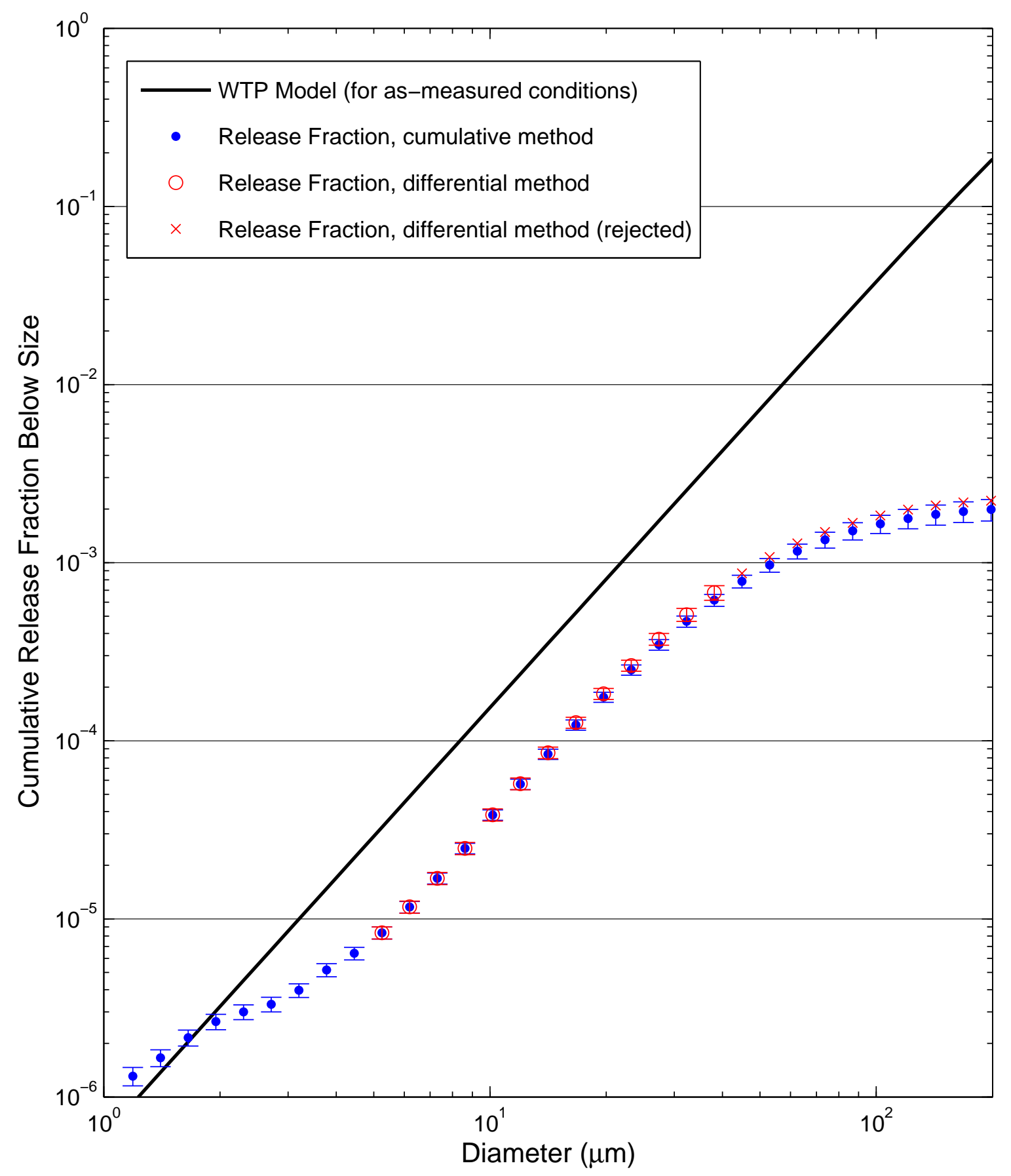


Test W224 [3 Malvern Average]: 200 psi, Orifice S5C - rectangular, $1 \times 20 \mathrm{~mm}$

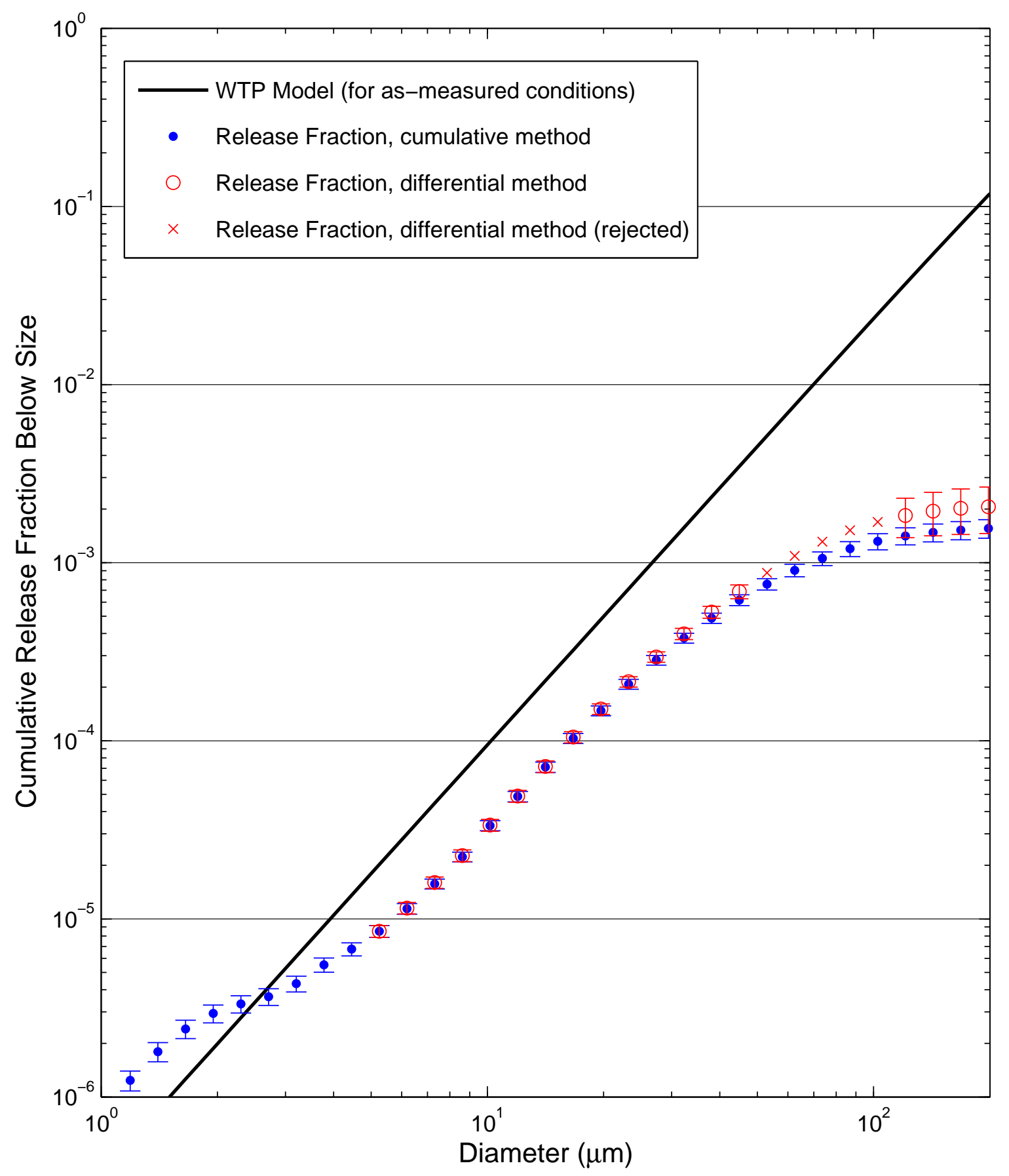


Test W225 [3 Malvern Average]: 380 psi, Orifice S5C - rectangular, $1 \times 20 \mathrm{~mm}$

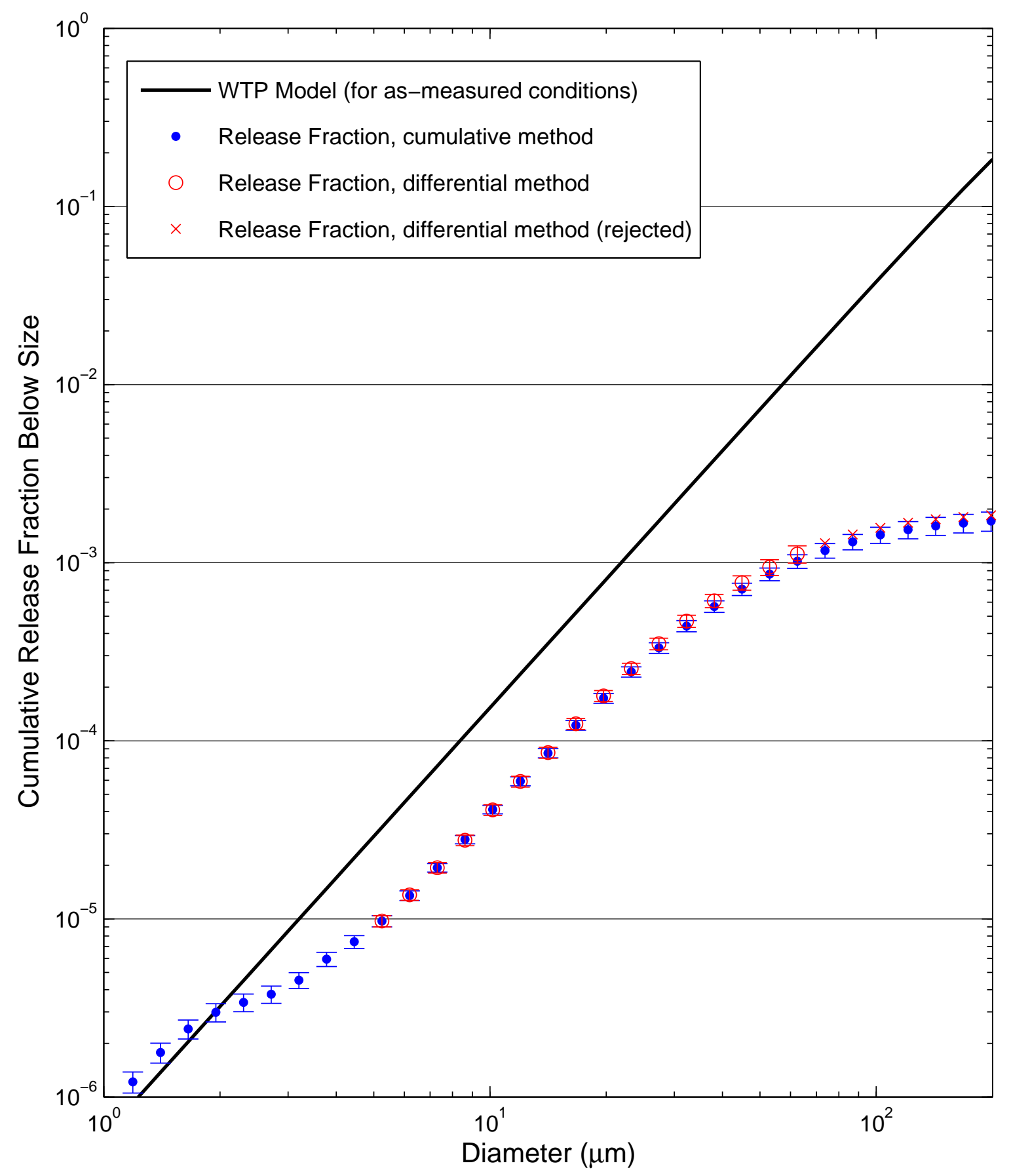


Test W226 [2 Malvern Average]: 200 psi, Orifice S7A - rectangular, $1 \times 10 \mathrm{~mm}$

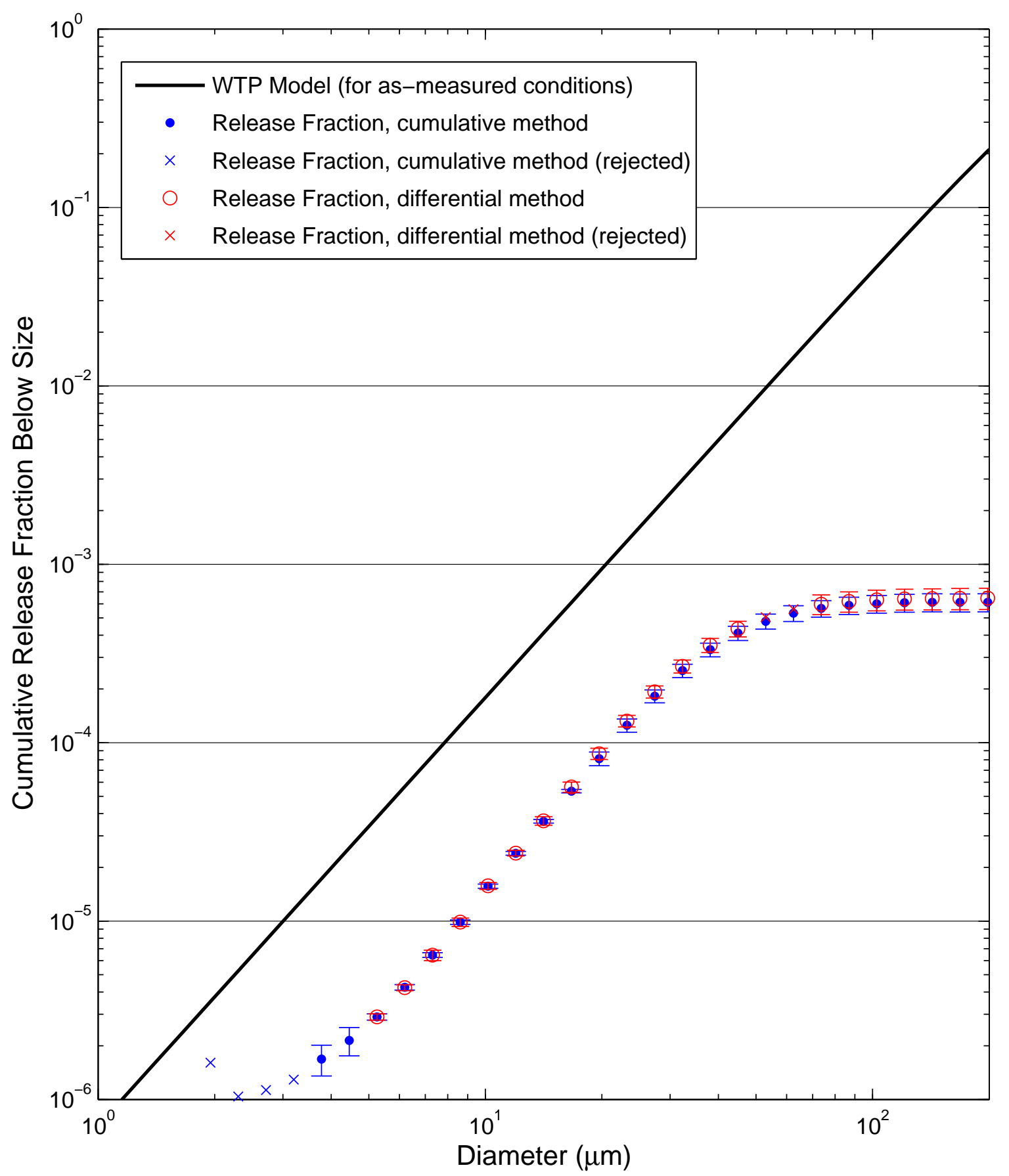


Test W227 [3 Malvern Average]: 380 psi, Orifice S7A - rectangular, $1 \times 10 \mathrm{~mm}$

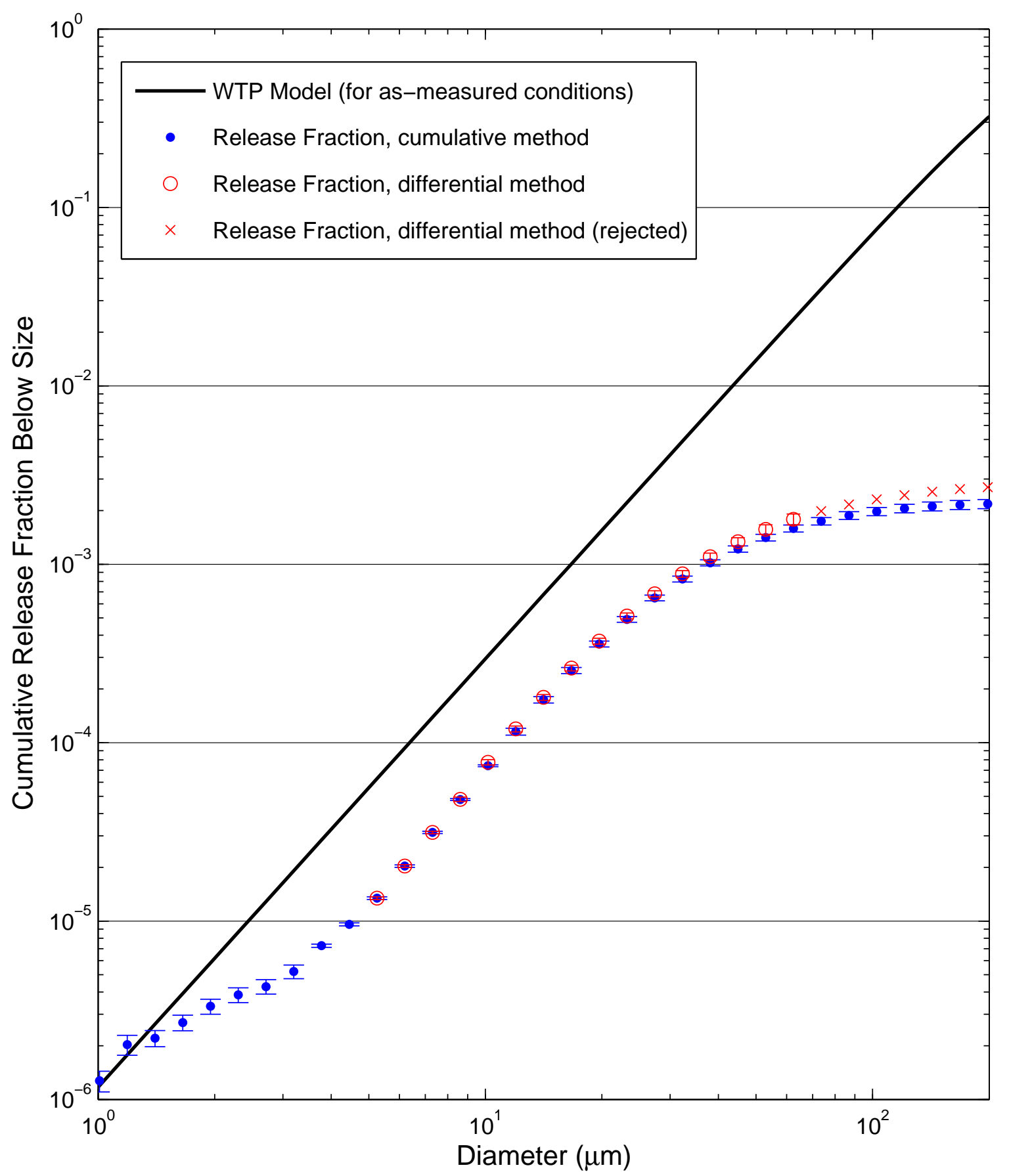


Test W228 [3 Malvern Average]: 200 psi, Orifice S7A - rectangular, $1 \times 10 \mathrm{~mm}$

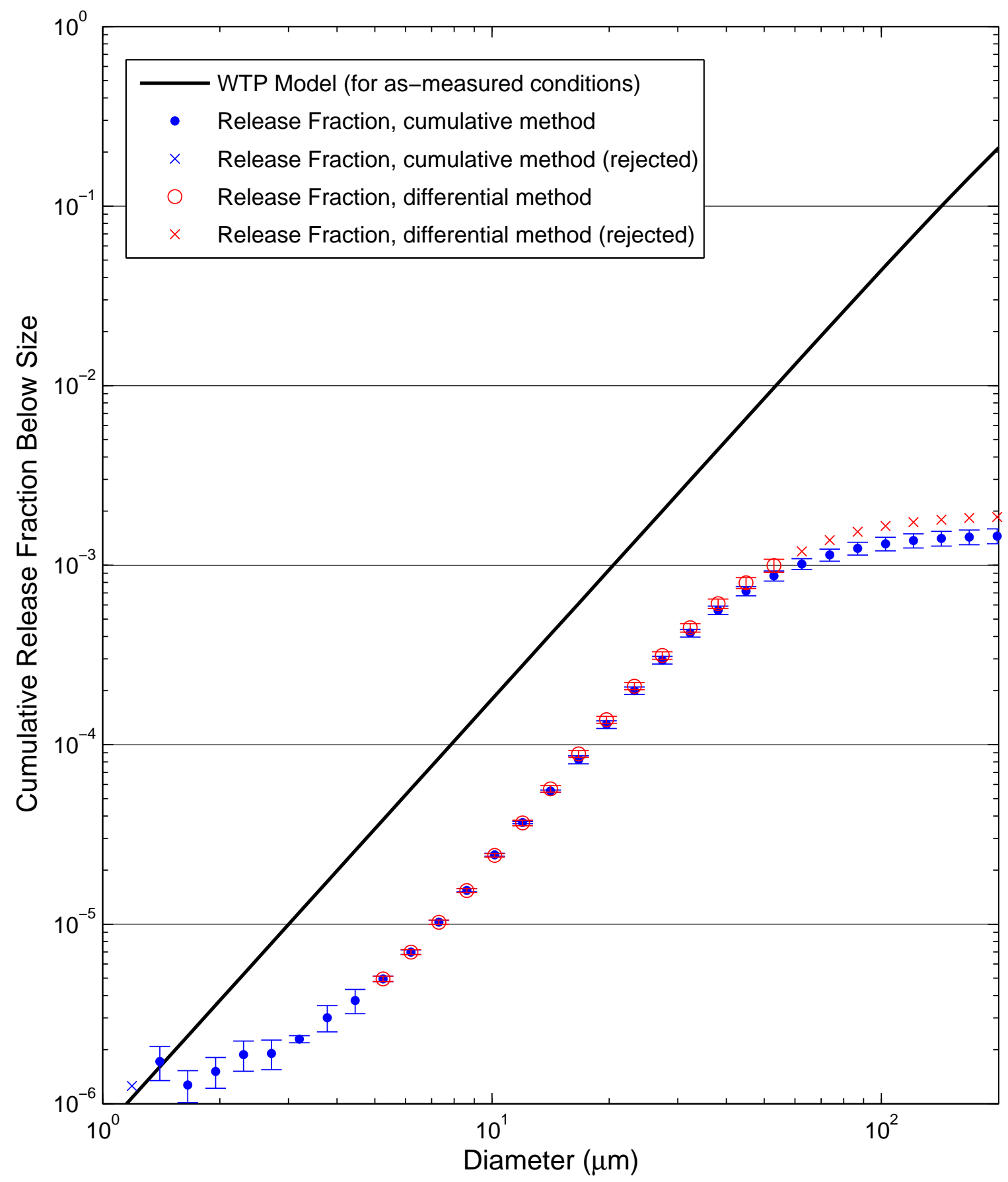


Test W229 [3 Malvern Average]: 380 psi, Orifice S7A - rectangular, $1 \times 10 \mathrm{~mm}$

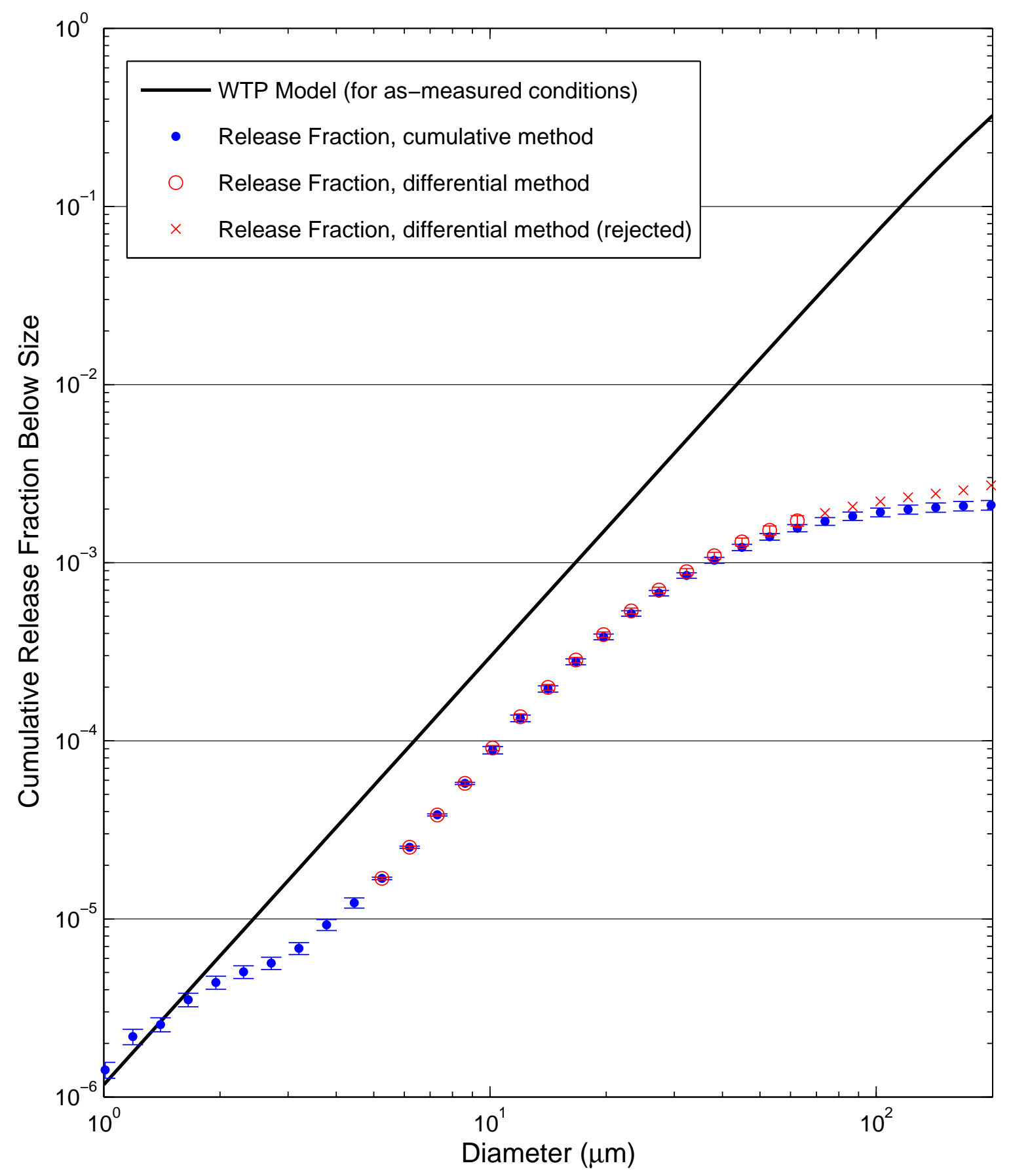

B. 182 
Test W230 [3 Malvern Average]: 200 psi, Orifice S7A - rectangular, $1 \times 10 \mathrm{~mm}$

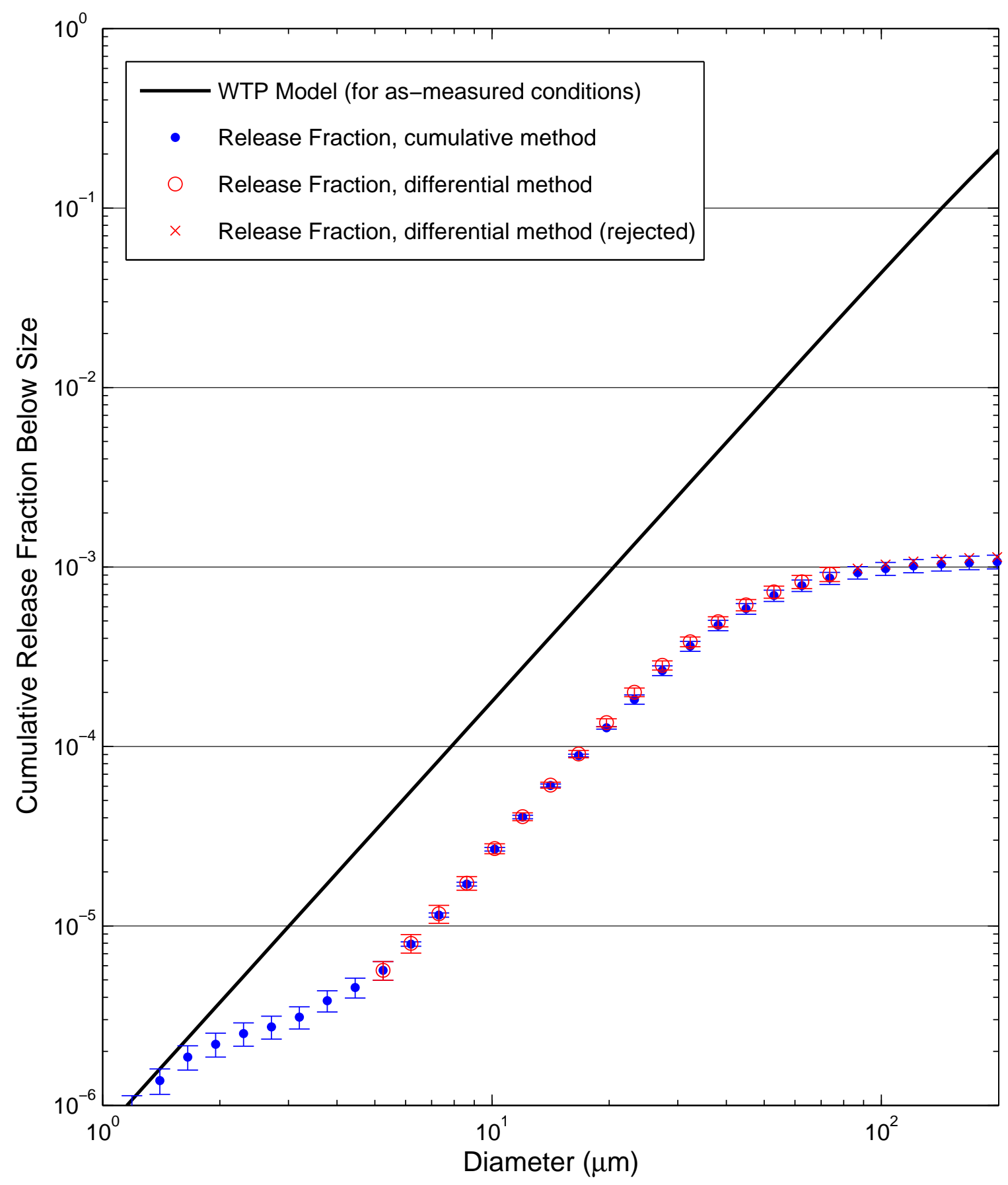


Test W231 [3 Malvern Average]: 200 psi, Orifice S4A - rectangular, $1 \times 76.2 \mathrm{~mm}$

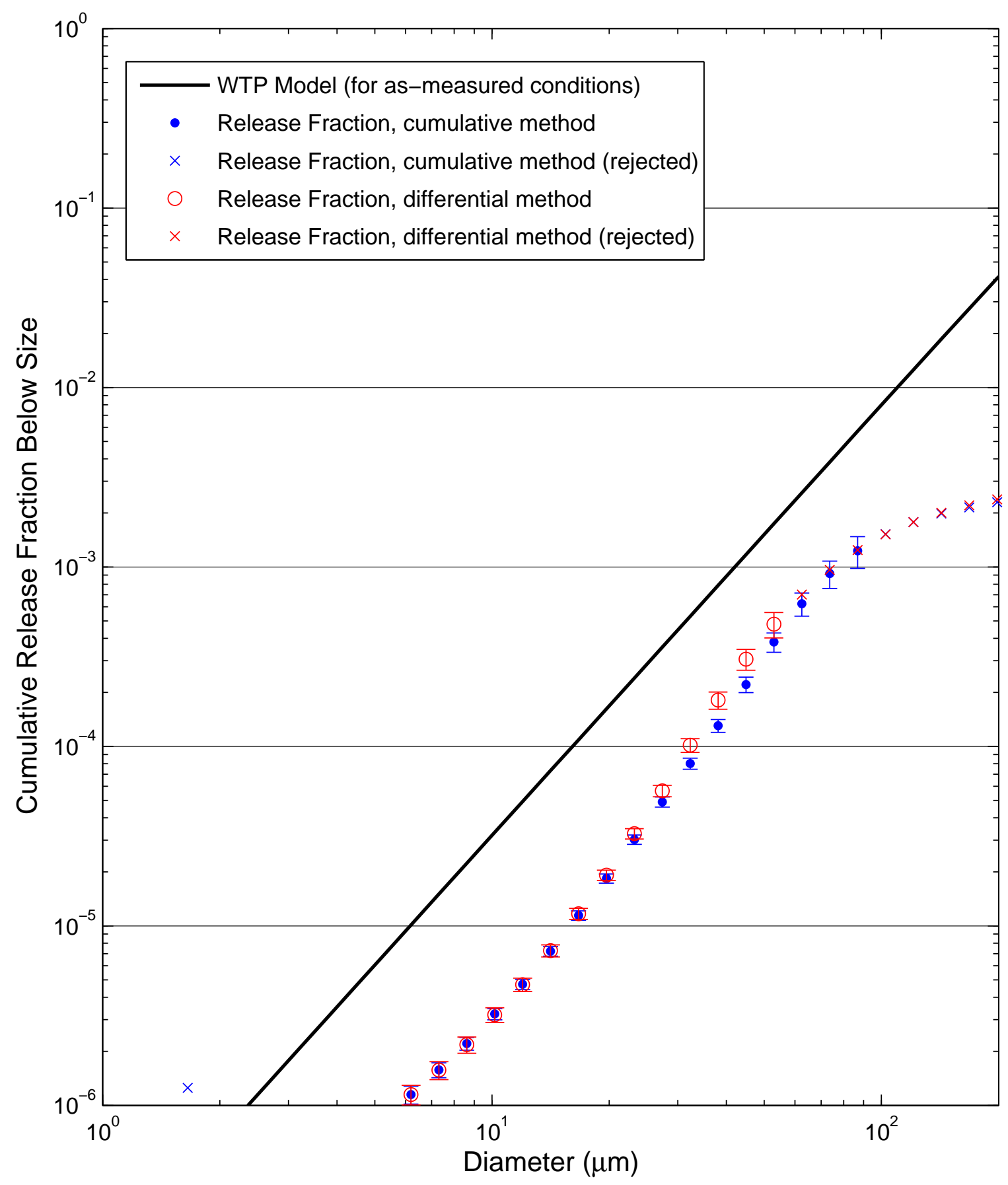


Test W232 [3 Malvern Average]: 380 psi, Orifice S4A - rectangular, 1× $76.2 \mathrm{~mm}$

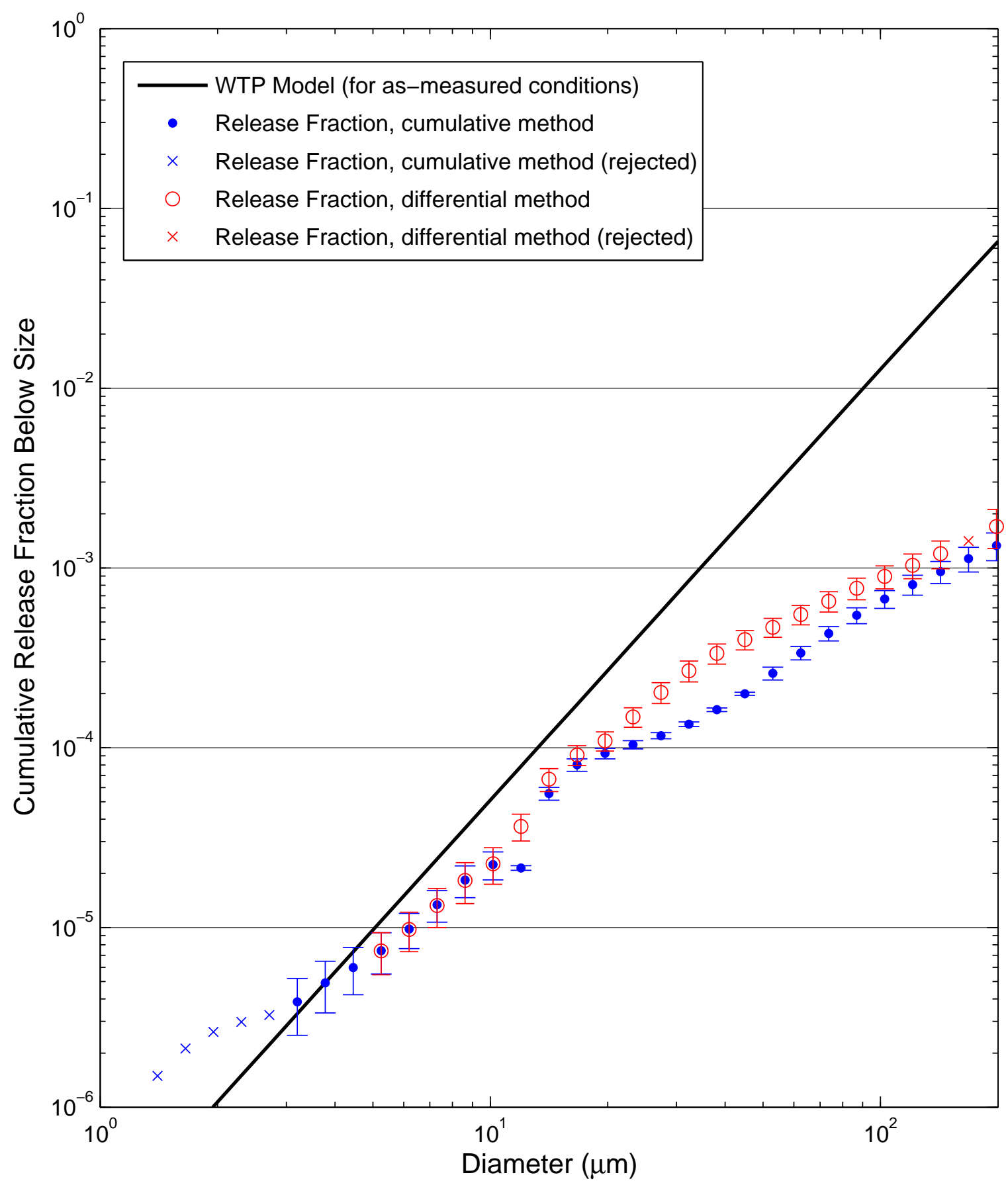


Test W233 [3 Malvern Average]: 200 psi, Orifice S4A - rectangular, 1× $76.2 \mathrm{~mm}$

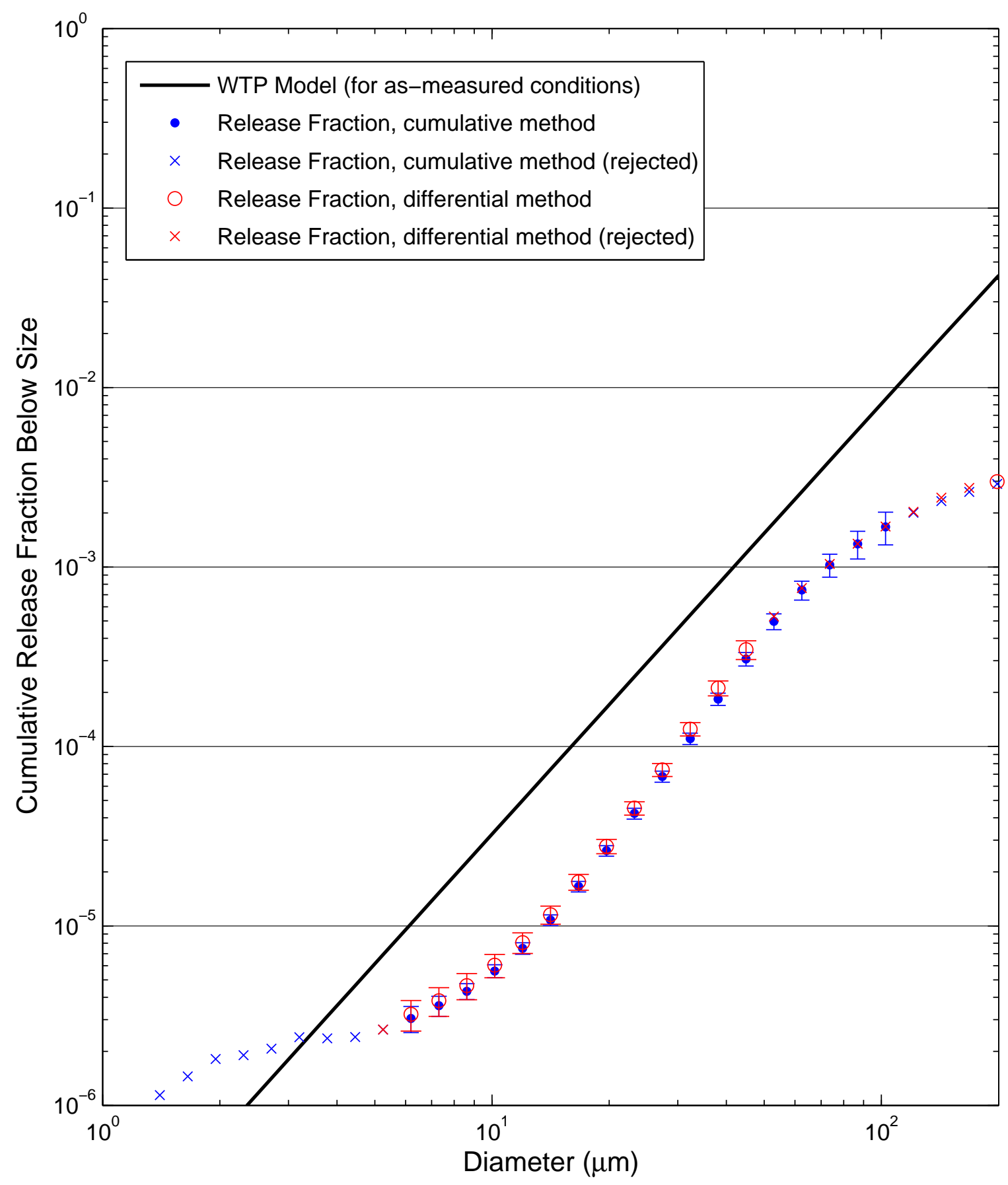


Test W234 [3 Malvern Average]: 380 psi, Orifice S4A - rectangular, 1× $76.2 \mathrm{~mm}$

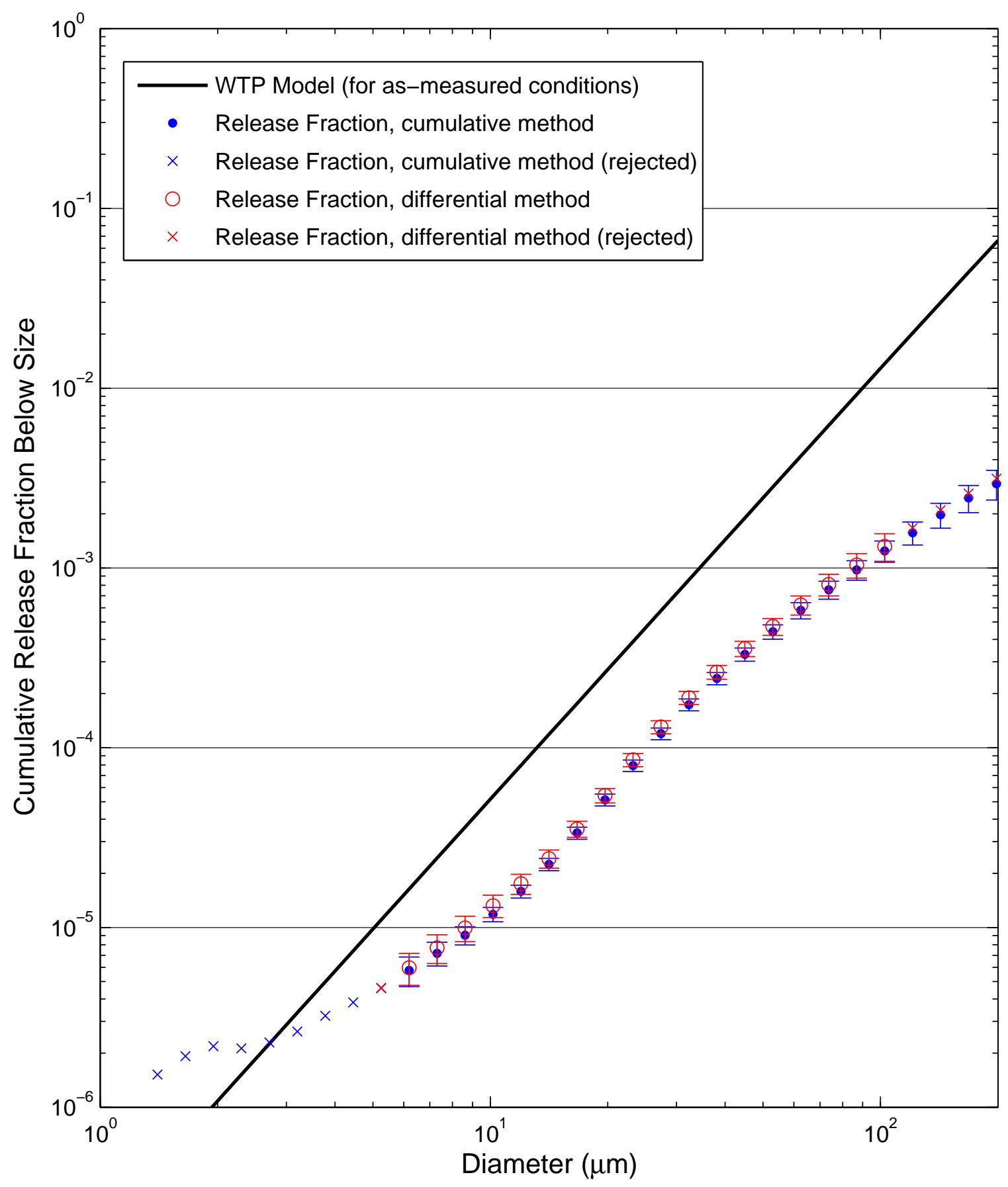


Test W235 [3 Malvern Average]: 380 psi, Orifice S1D - round, 2 mm

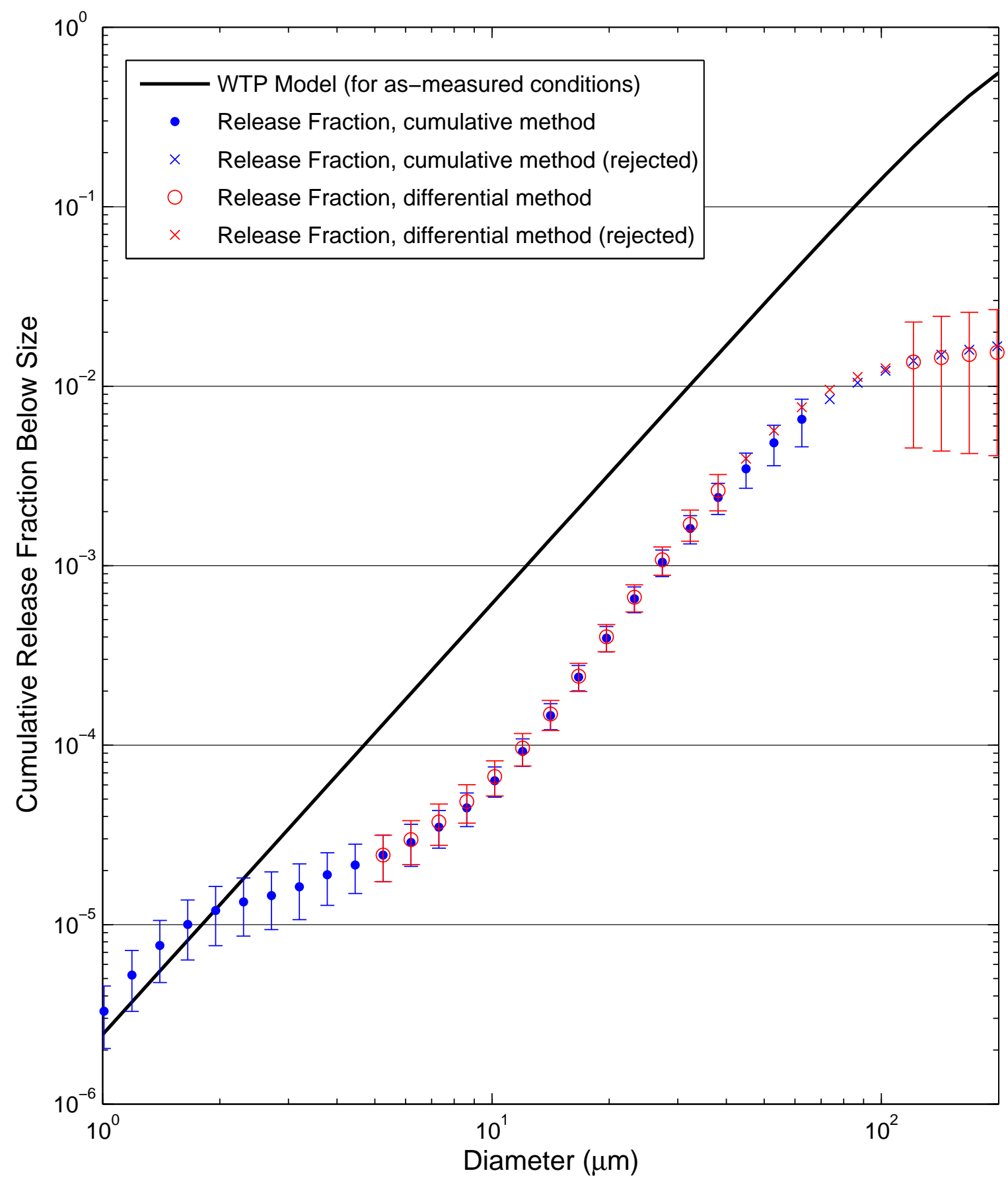


Test W236 [3 Malvern Average]: 380 psi, Orifice S1D - round, 2 mm

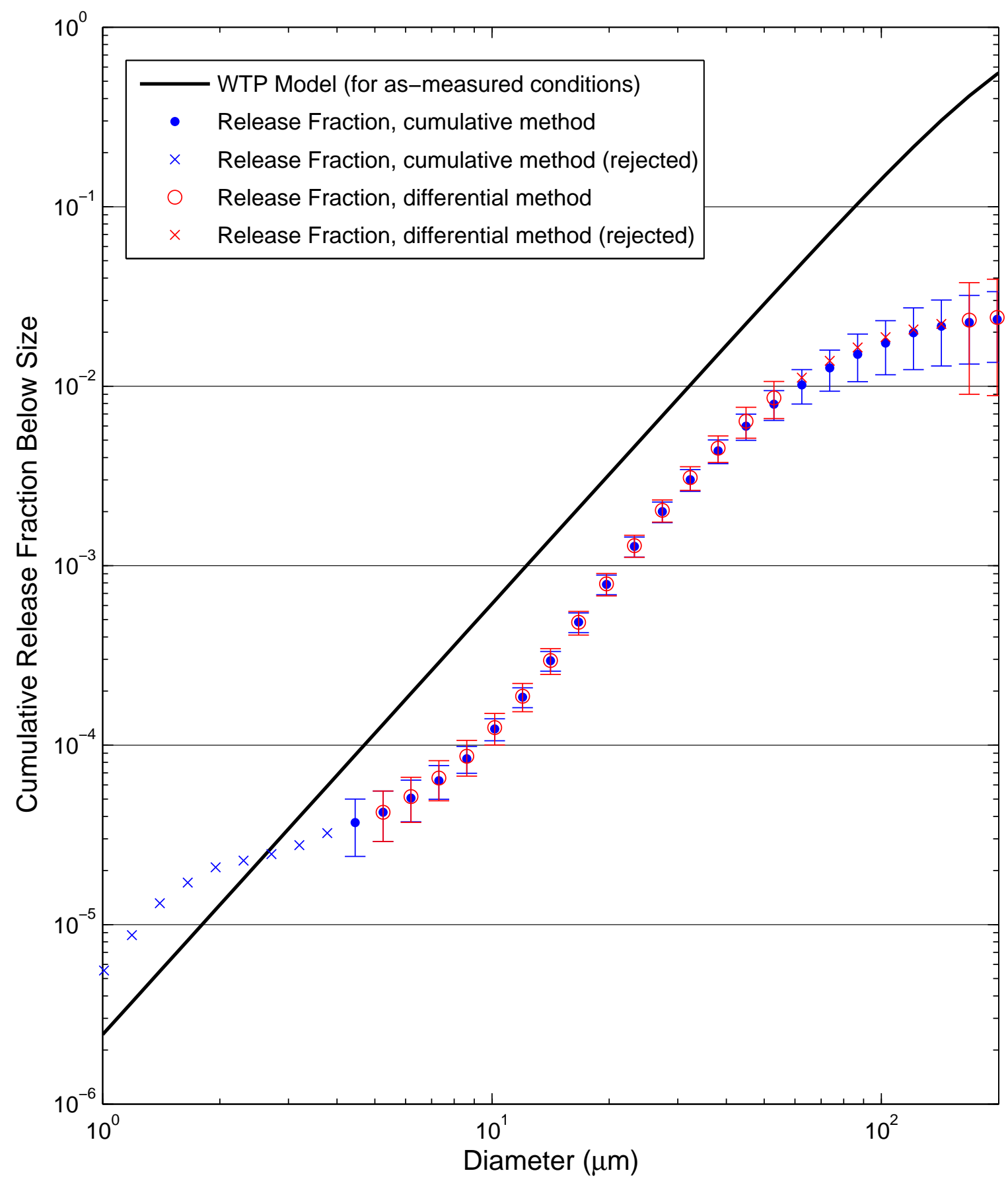


Test W237 [3 Malvern Average]: 380 psi, Orifice S1D - round, 2 mm

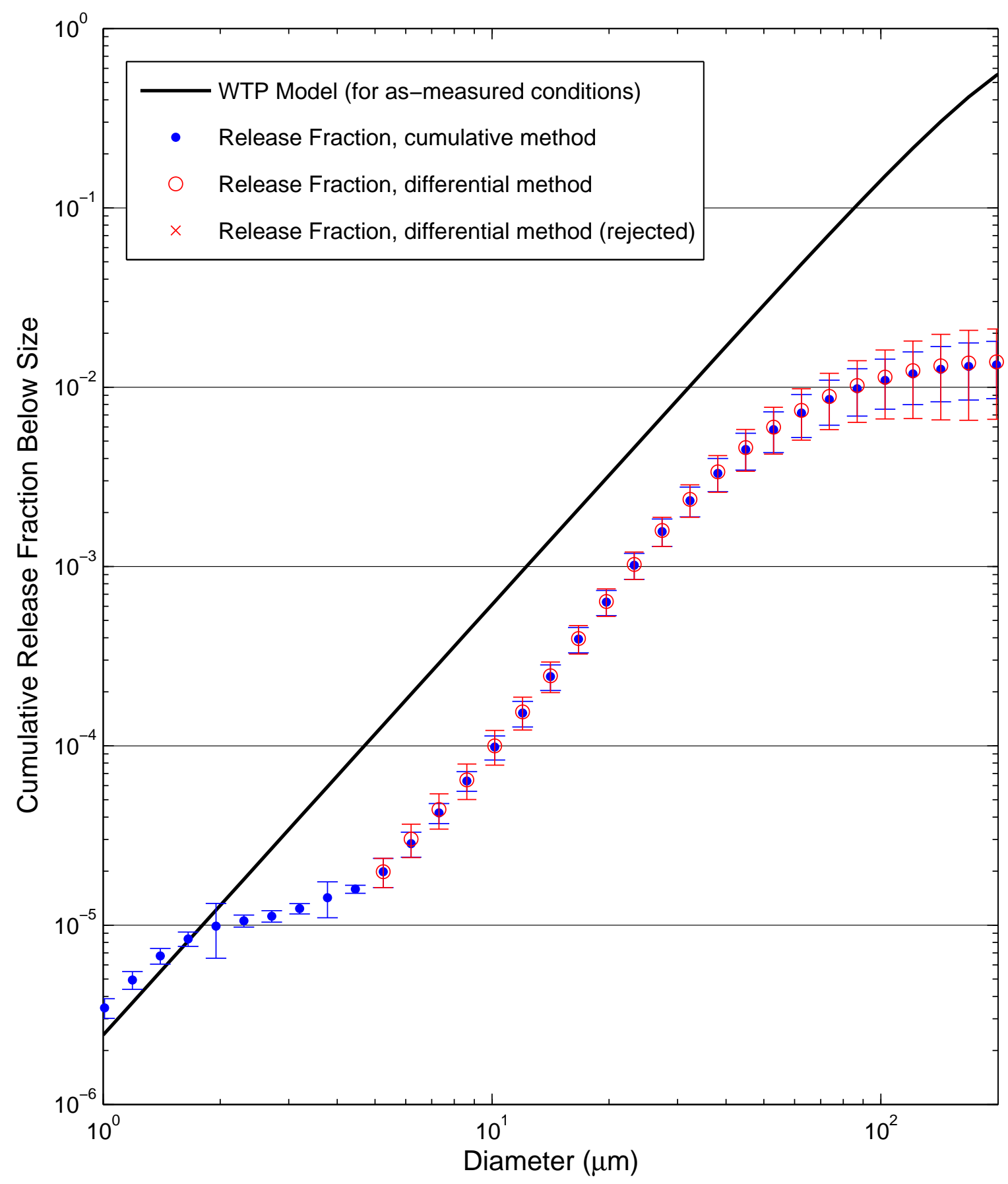


Test W238 [3 Malvern Average]: 380 psi, Orifice S1D - round, 2 mm

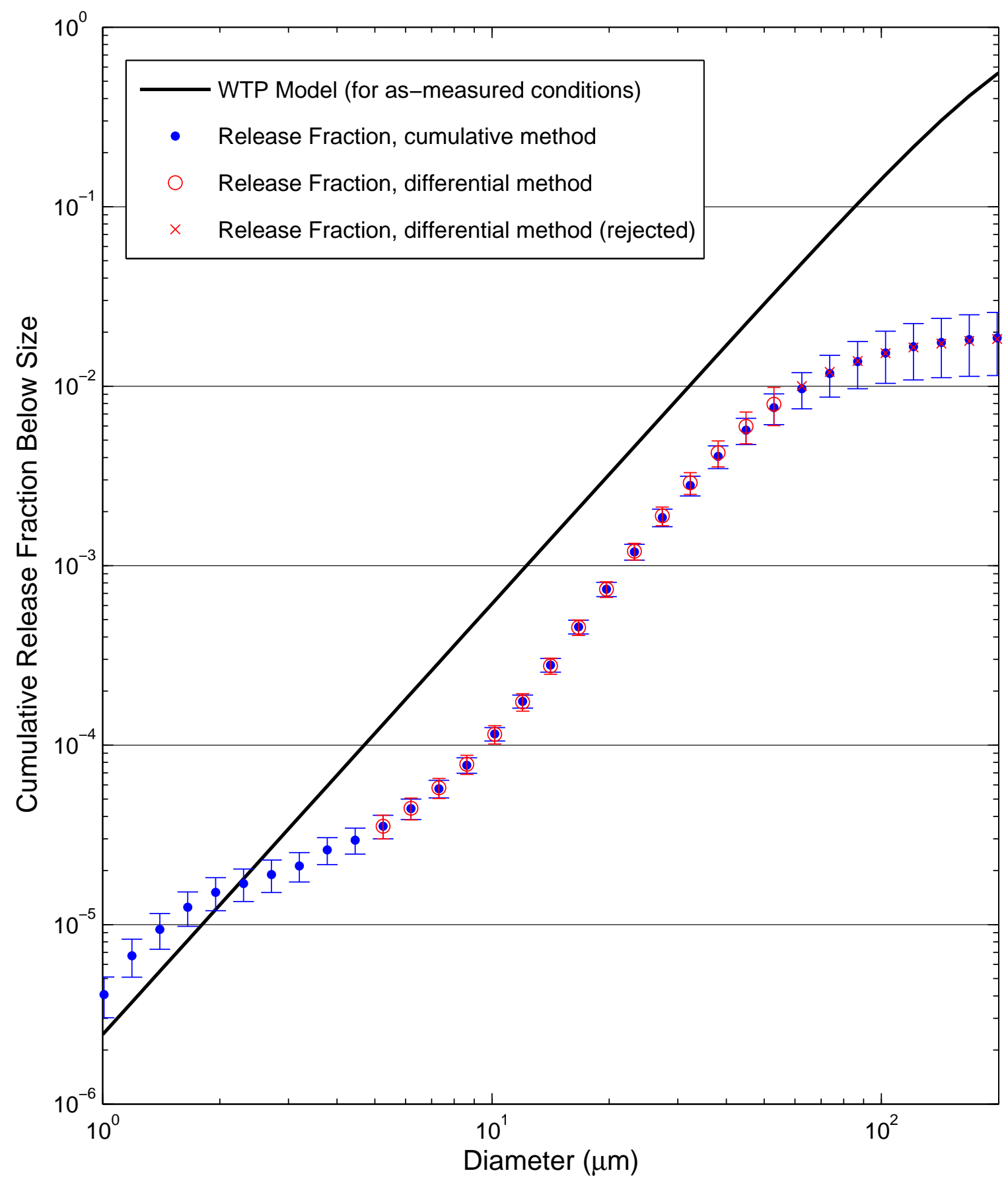


Test W239 [3 Malvern Average]: 380 psi, Orifice S1D - round, 2 mm

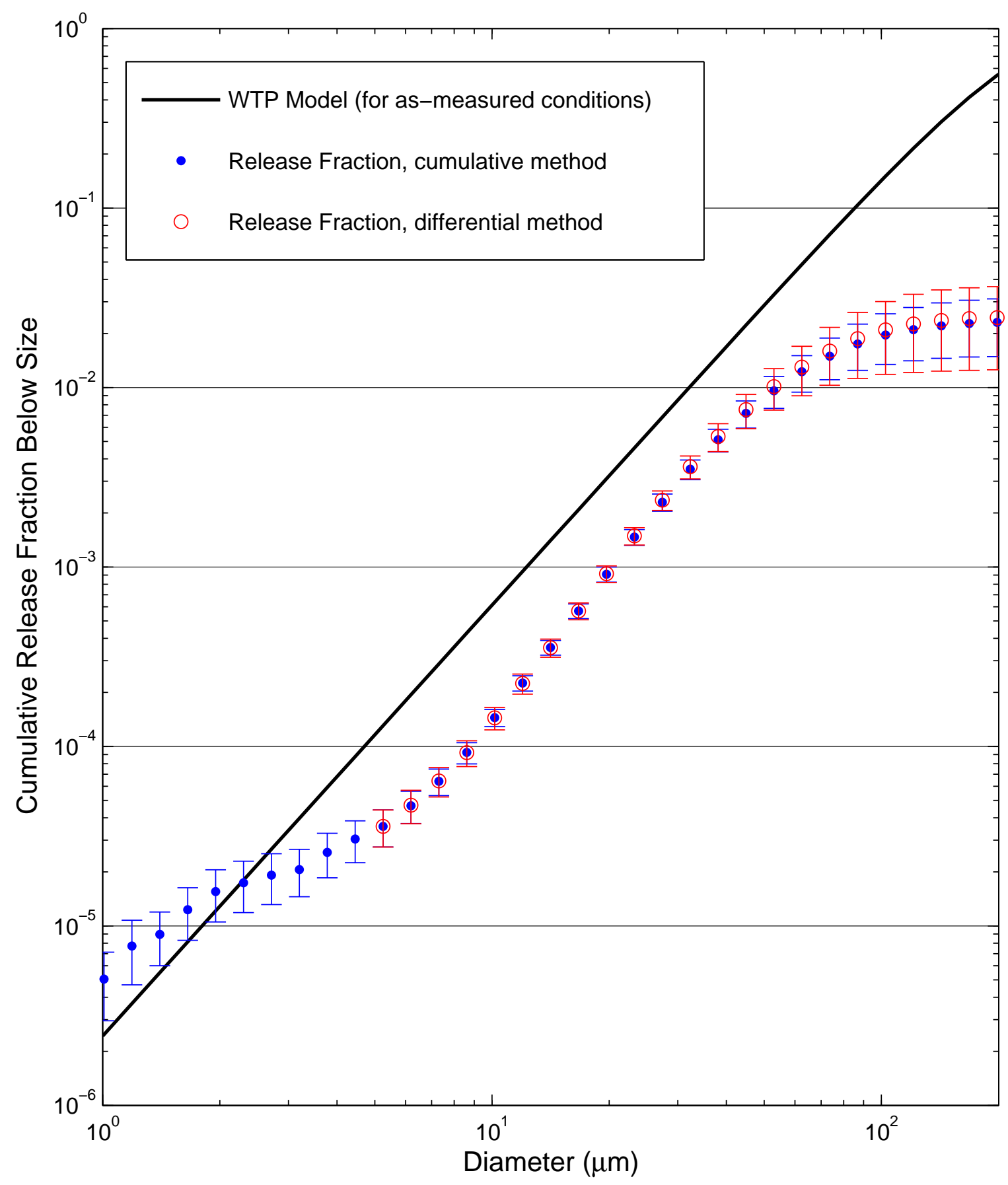


Test W240 [3 Malvern Average]: 380 psi, Orifice S1D - round, 2 mm

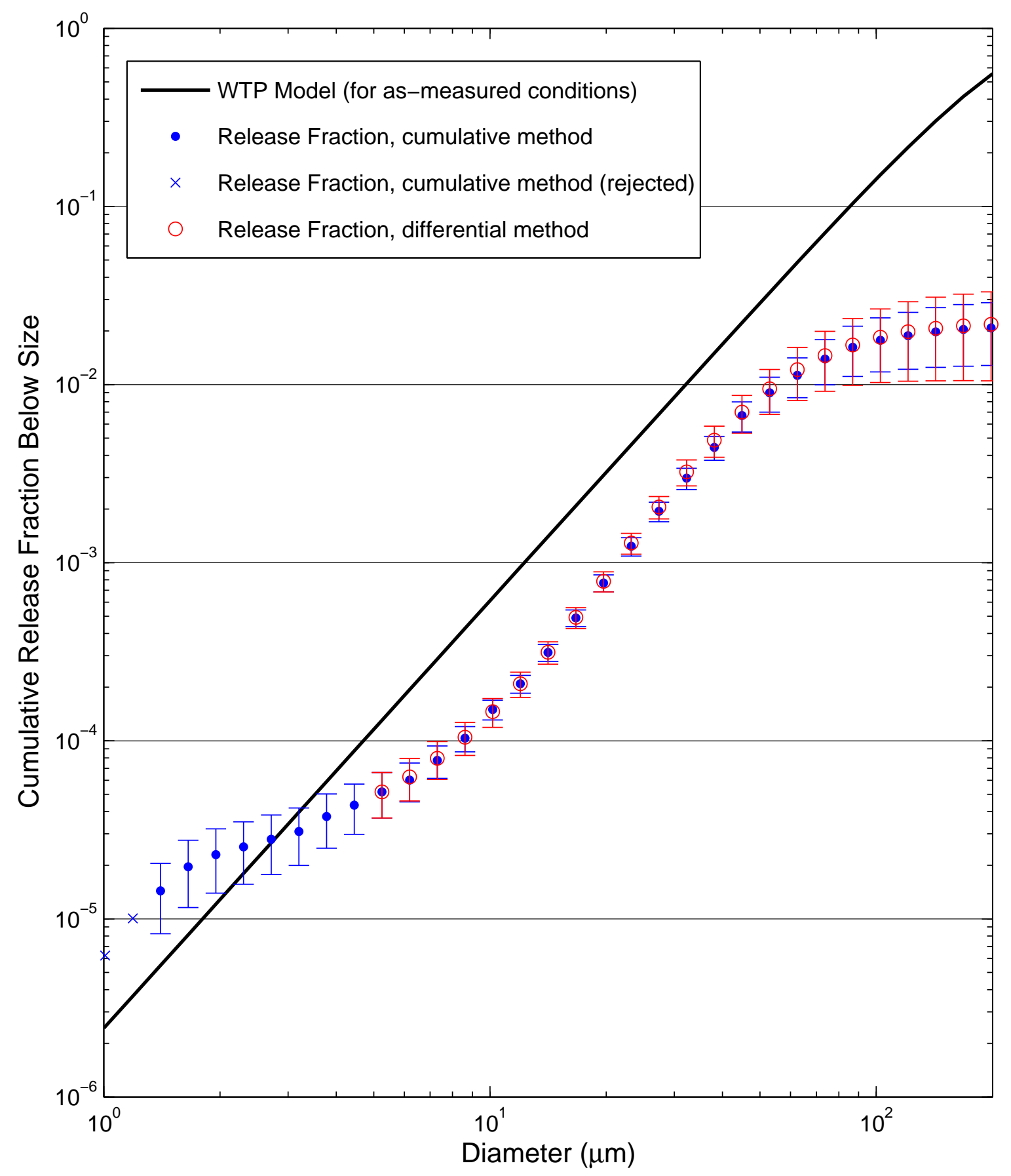


Test W242 [3 Malvern Average]: 380 psi, Orifice S1D - round, 2 mm

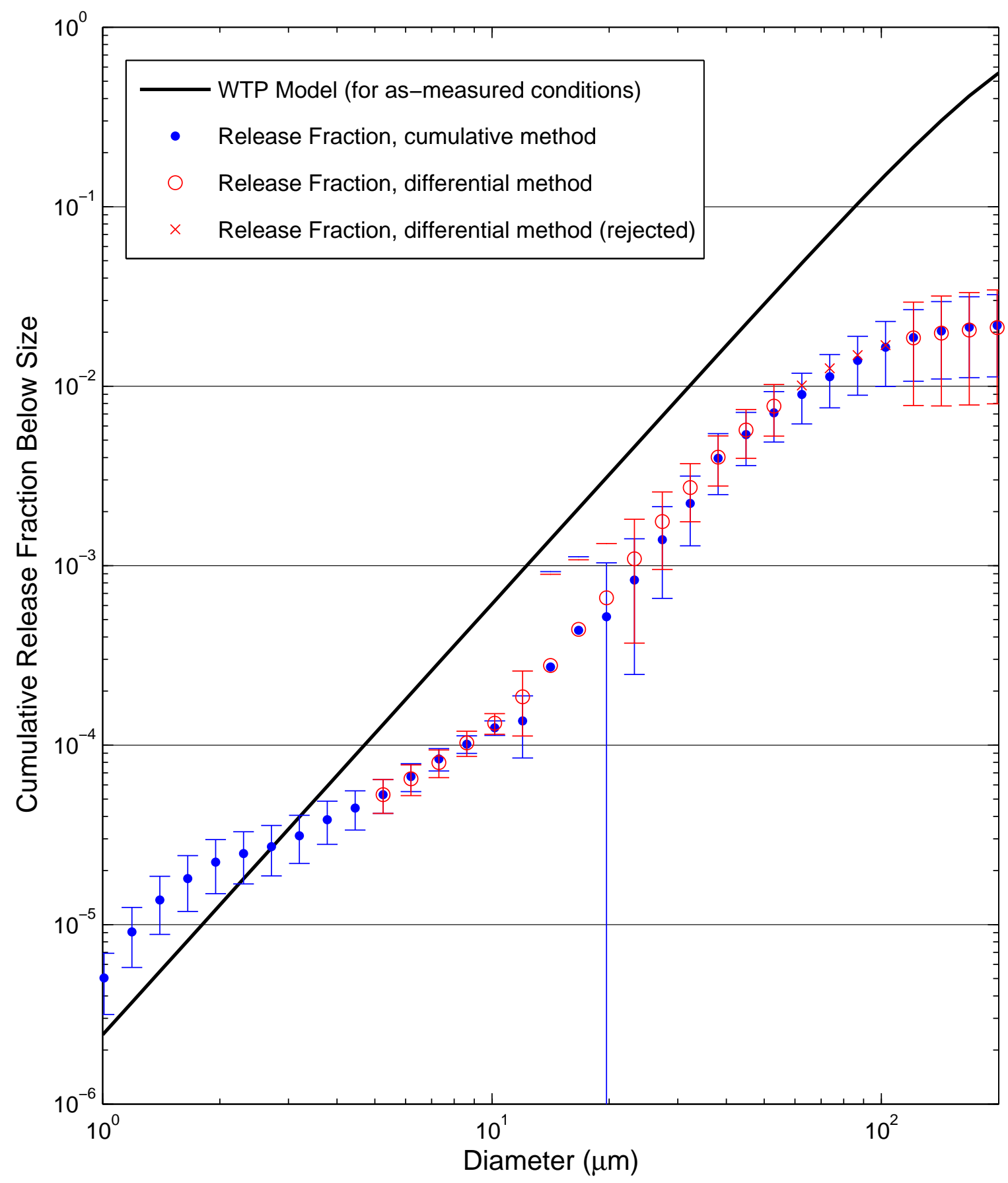


Test W243 [3 Malvern Average]: 380 psi, Orifice S1D - round, 2 mm

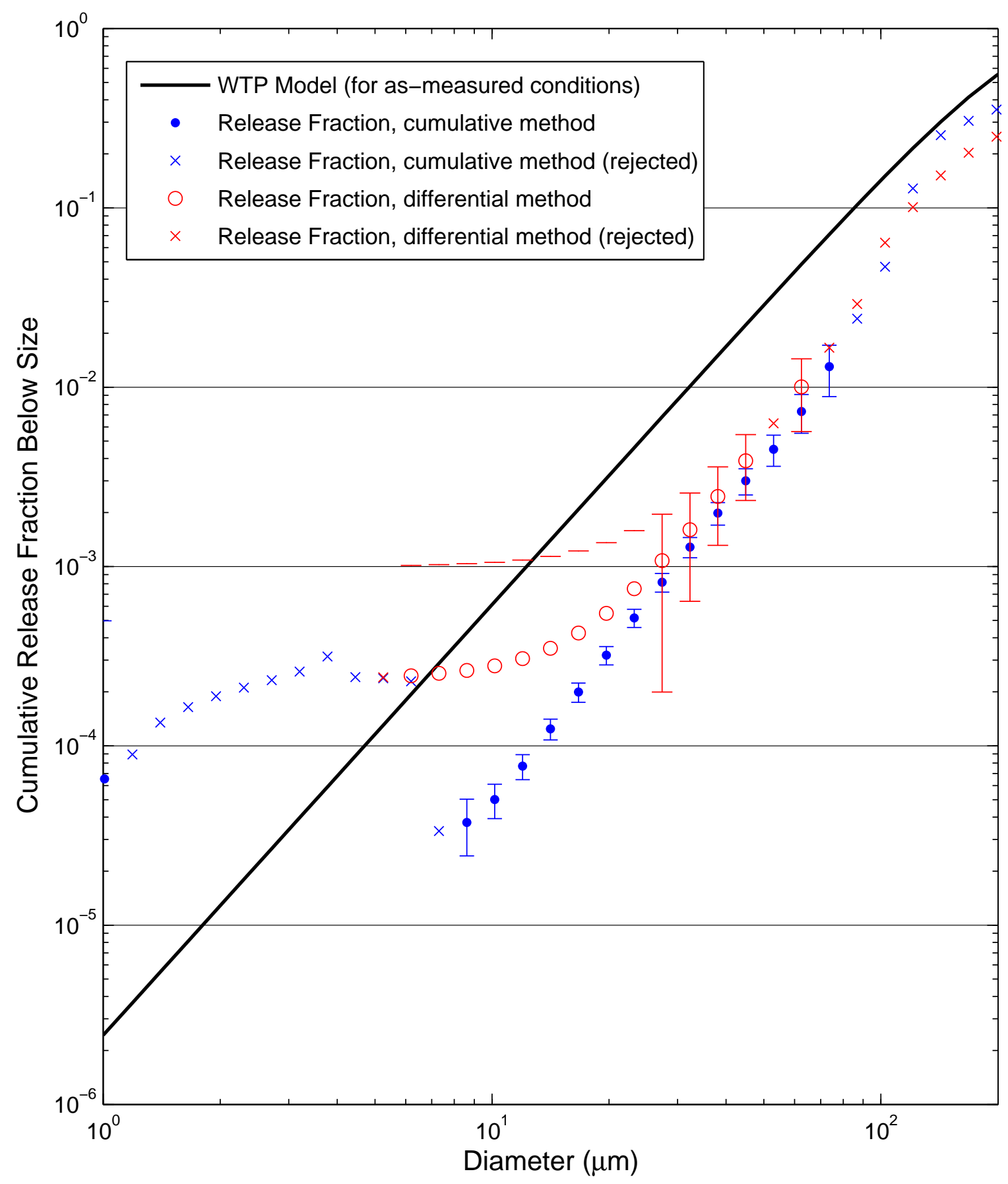


Test W244 [3 Malvern Average]: 380 psi, Orifice S1D - round, 2 mm

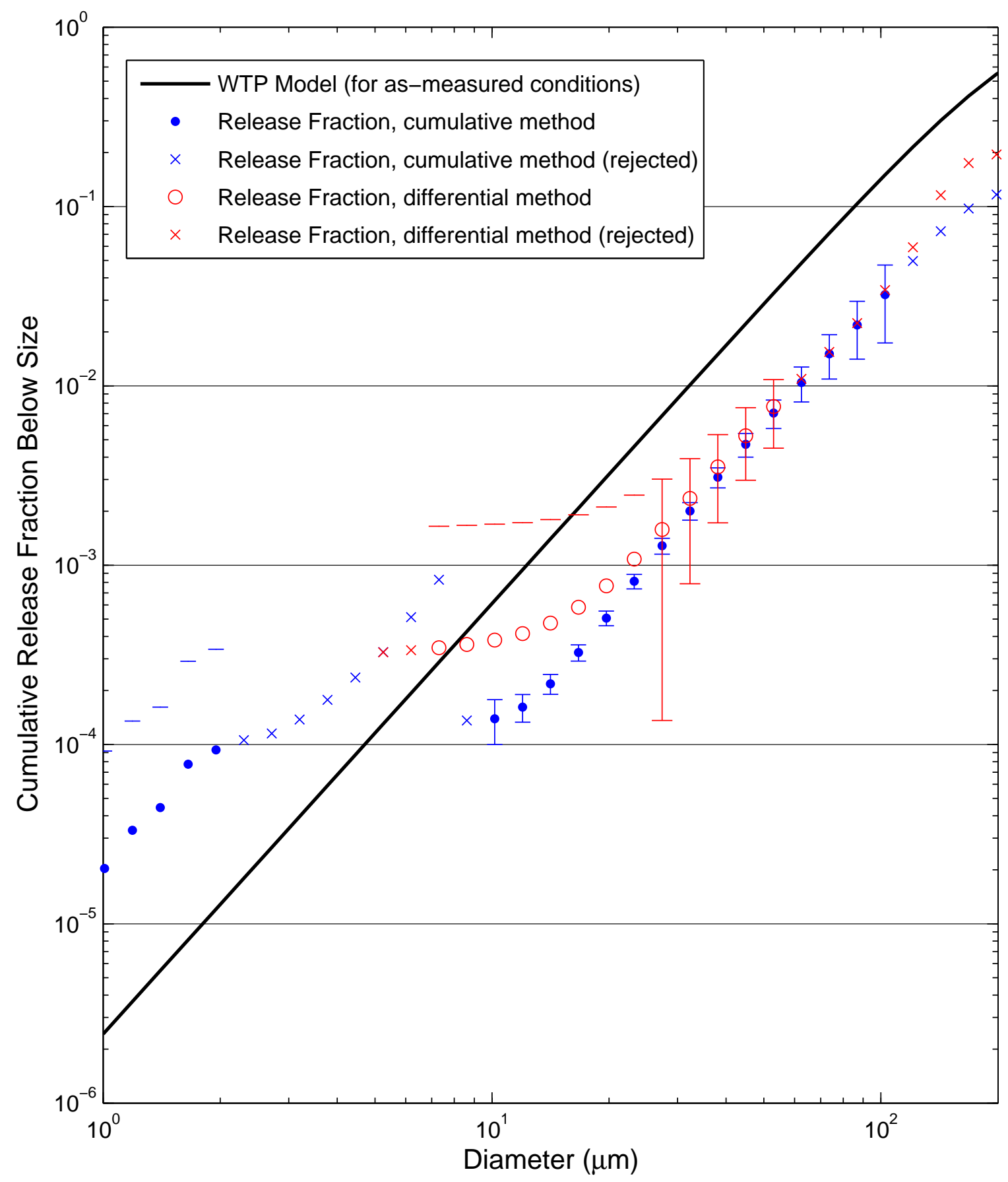


Test W245 [3 Malvern Average]: 380 psi, Orifice S1D - round, 2 mm

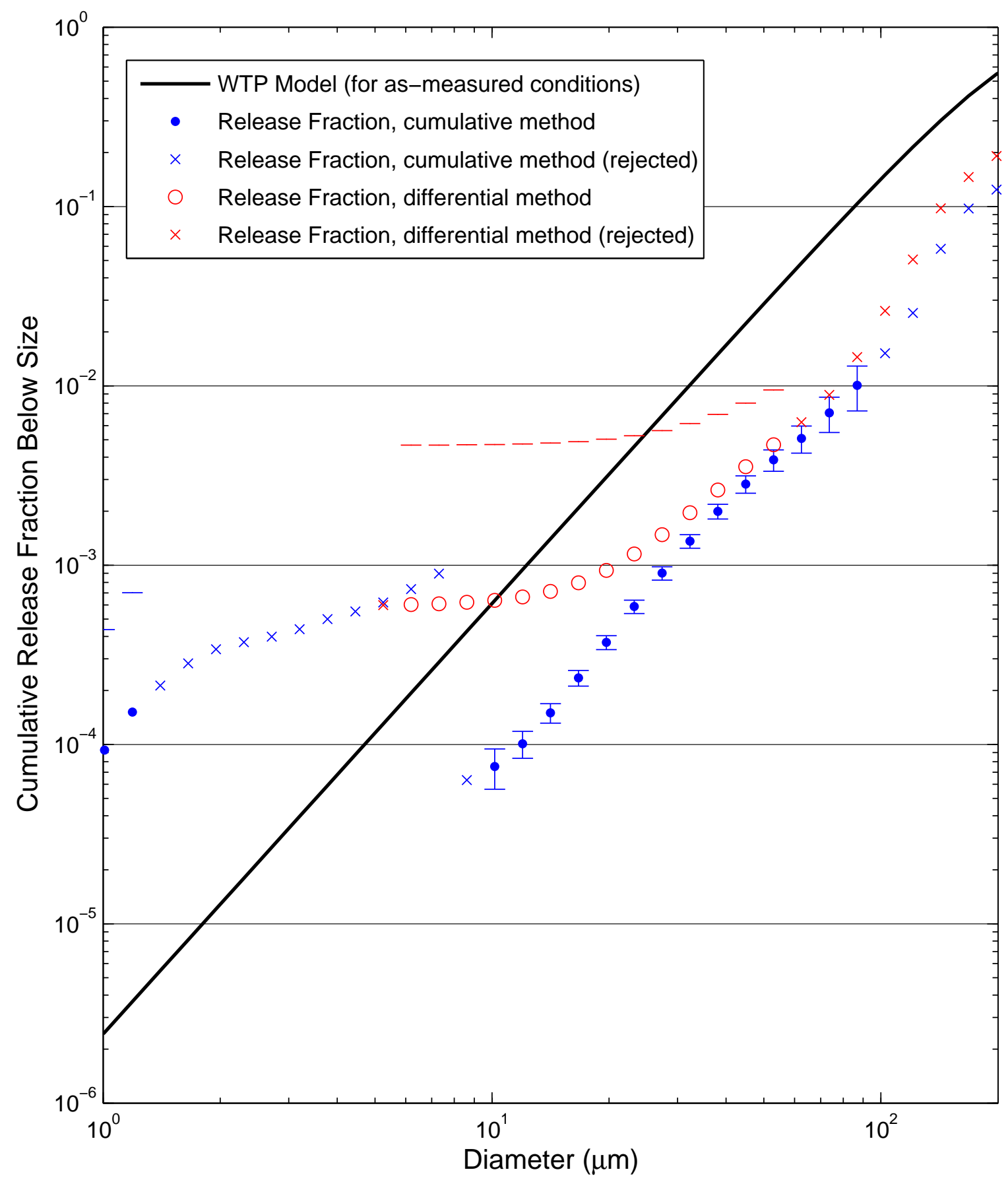


Appendix C

\section{Basis for Concentration Averaging}





\section{Appendix C}

\section{Basis for Concentration Averaging}

In this Appendix, a detailed derivation of the model used to evaluate release fraction for sprays where loss of aerosol cannot be neglected is presented. For the purposes of this derivation, the notation used may differ from that provided in the main body of this report. The overall goal of this section is to develop a more complete model (relative to that presented in the main body of the report) by which to evaluate the impact of concentration averaging. The need for averaging derives from the fact that in large-scale, the aerosol concentration is not uniform along the length of the test chamber. It is believed that this non-uniformity results from imperfect mixing and/or mass transport from one end of the chamber to the other. Mass transport and mixing are assumed to result from convective air currents created by momentum transfer from the spray. In an attempt to account for imperfect mixing or mass transport, the large-scale chamber is considered using a multi-zone model, where each zone can transfer aerosol to the adjacent zone.

Consider a three zone model for aerosol generation in the large-scale test chamber (see Figure C.1). Each zone represents a volume of perfect, instantaneous mixing. Any aerosol generated therein is immediately and fully mixed (relative to generation time scales) by turbulence in the volume of the zone, $V_{k}$, where $k$ is the zone number $(k)$. Aerosol is transported from zone to zone by convective transport. Aerosol is removed from the system by loss to the walls and settling. Loss attributable to these mechanisms take the forms derived in Chapter 3 of the report and are generally first order.

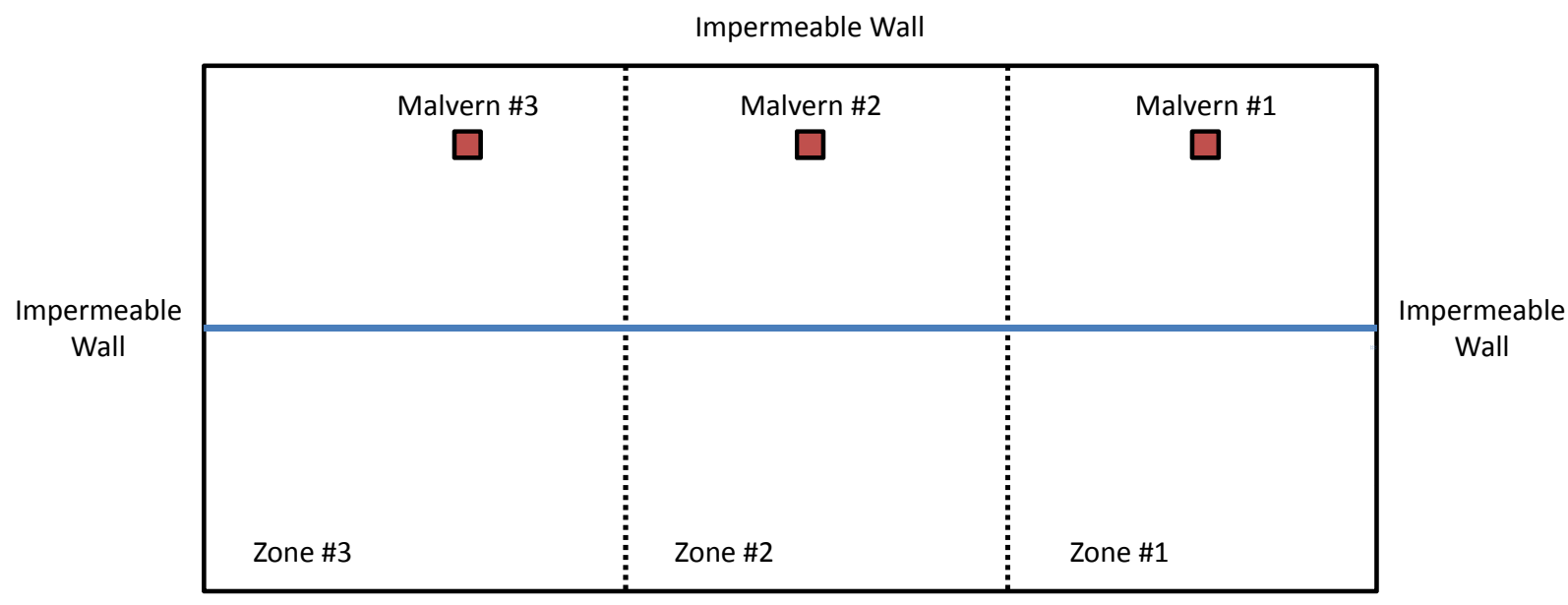

Impermeable Wall

Figure C.1. Four Zone Model of the Large-Scale Test Chamber Showing Three Malvern Insitec-S Measuring Positions

Using the far-field approximation for aerosolization derived in Chapter 3, the aerosol mass balance for a three zone system will take the form: 


$$
V_{k} \frac{d x_{k}}{d t}=r_{k} \pm \sum_{j} q_{k j} x_{j}-\lambda_{k} x_{k}
$$

Here, $x_{k}$ is the concentration in zone $k, q_{k j}$ is the loss term from convective transport into zone $k$ from zone $j(+)$ and out of zone $k$ to zone $j(-), \lambda_{k}$ is the loss coefficient encompassing all forms of aerosol loss in zone $k$ (e.g., deposition and settling), and $r_{k}$ represents in the zone aerosol generation.

To model the large-scale system using the three zone approximation, spray breakup is assumed to yield apparent generation in all four zones. As shown in Figure C.1, the spray travels to and impinges on an impermeable "splash" wall. Momentum transfer from the spray to the surrounding air creates a convective back-transport that moves aerosol toward zone \#3. Aerosol concentration and size analyzers are located in zone \#1, \#2, and \#3 and measure the perfectly mixed concentration in each zone. Under these assumptions, the mass balance becomes:

$$
\begin{gathered}
V_{1} \frac{d x_{1}}{d t}=r_{1}-\lambda_{1} x_{1}-q_{12} x_{1} \\
V_{2} \frac{d x_{2}}{d t}=r_{2}-\lambda_{2} x_{2}+q_{12} x_{1}-q_{23} x_{2} \\
V_{3} \frac{d x_{3}}{d t}=r_{3}-\lambda_{3} x_{3}+q_{23} x_{2}
\end{gathered}
$$

The goal of aerosol analysis is to determine the total generation rate of aerosol in the chamber. Based on the model framework developed above, the total generation rate of aerosol in the three zone model is:

$$
r=r_{1}+r_{2}+r_{3}
$$

The approach used in large-scale analysis is to average aerosol concentration measured at three locations in the large-scale chamber. If the aerosol concentration in each zone is averaged volumetrically, then,

$$
V\langle x\rangle=V_{1} x_{1}+V_{2} x_{2}+V_{3} x_{3}
$$

where $\langle x\rangle$ is the average volumetric concentration of aerosol and $V$ is the total chamber volume defined by:

$$
V=V_{1}+V_{2}+V_{3}
$$

Combining Equations C.2 through C.7 yields:

$$
V \frac{d\langle x\rangle}{d t}=r-\left(\lambda_{1} x_{1}+\lambda_{2} x_{2}+\lambda_{3} x_{3}\right)
$$

Note that in obtaining Equation C.8, the convective transport terms $\left(\mathrm{q}_{12}\right.$ and $\left.\mathrm{q}_{23}\right)$ can be time-dependent and the time-dependence does not need to be known because these terms cancel when Equations C.2 through C.4 are combined. Direct solution of Equation C.8 is not possible; however, the resulting curve will be the sum of exponential rise curve of differing time scales. If all time scales can be accessed experimentally, then initial slope analysis of the average aerosol concentration curve, $\langle x\rangle(t)$, should yield:

$$
\lim _{t \rightarrow \infty} \frac{d\langle x\rangle}{d t}=\frac{r}{V}
$$


From which it is clear that the total generation rate of aerosol is given by:

$$
r=V\left(\lim _{t \rightarrow \infty} \frac{d\langle x\rangle}{d t}\right)
$$

It should be noted that the issue of multiple growth time scales affecting the average is eliminated if the loss coefficients are assumed to be a fixed quantity per unit volume of chamber, such that,

$$
\lambda_{k}=\Lambda V_{k}
$$

Then,

$$
V \frac{d\langle x\rangle}{d t}=r-V \Lambda\langle x\rangle
$$

which can be integrated to:

$$
\langle x\rangle=\frac{r}{\Lambda V}[1-\exp (-\Lambda t)]
$$

Equation C.13 is independent of any individual zone concentration $\left(x_{k}\right)$ and of any of the convective transport terms. This means that the simple exponential model holds for the average aerosol concentration even when the convective terms show complex functionality and are not constant (such as aerosol concentration or time-dependent convection). Under the limiting assumptions used to reach this result, Equation C.13 provides a basis for averaging chamber aerosol concentrations and fitting the result to an exponential model. 

PNNL-21333

WTP-RPT-217 Rev. 0

\section{Distribution*}

No. of

$\underline{\text { Copies }}$

ONSITE

3 DOE Office of River Protection

JS Fox

CC Harrington

DL Noyes

7 Bechtel National Inc.

A Hassan

HR Hazen

J Mauss

S Omberg-Carro

RJ Van Vleet

JL Weamer

WTP PETD Docs
No. of

Copies
H6-60

H4-02

15 Pacific Northwest National Laboratory

H6-60

H6-60

H4-02

H4-02

H4-02

H4-02

H4-02

H4-02

HE Adkins, Jr
JM Billing
JR Bontha
GN Brown
CA Burns
RC Daniel
CW Enderlin
PA Gauglitz
JJ Jenks
ML Kimura
DE Kurath
PP Schonewill
DN Tran
Information Release (pdf)
Project File

K7-15

P7-25

P7-25

P7-25

P7-25

P7-22

K7-15

K7-15

K7-15

K6-28

K3-52

P7-25

K6-24

P8-55

K3-52

*All distribution will be made electronically

Dist. 1 




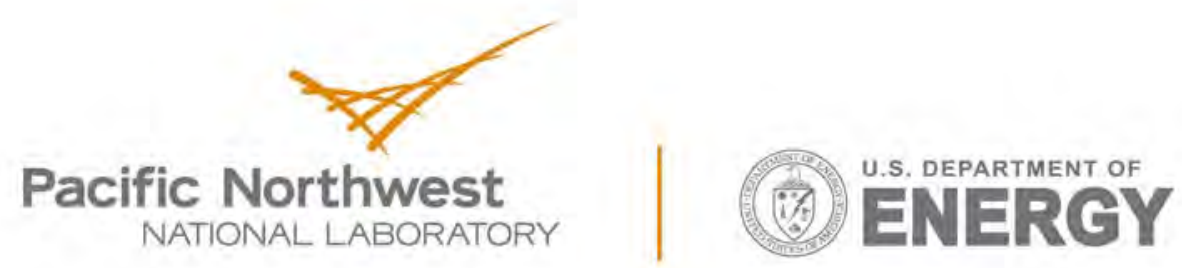

Proudly Operated by Battelle Since 1965

902 Battelle Boulevard

P.O. Box 999

Richland, WA 99352

1-888-375-PNNL (7665)

www.pnnl.gov 